LBNL-42856

\title{
Study of Pair and Many-Body Interactions in Rare-Gas Halide Atom Clusters Using Negative Ion Zero Electron Kinetic Energy (ZEKE) and Threshold Photodetachment Spectroscopy
}

\author{
Ivan Yourshaw \\ Ph.D. Thesis \\ Department of Chemistry \\ University of California, Berkeley \\ and \\ Chemical Sciences Division \\ Ernest Orlando Lawrence Berkeley National Laboratory \\ University of California \\ Berkeley, CA 94720
}

July 1998

This work was supported by the Director, Office of Science, Office of Basic Energy Sciences, Chemical Sciences Division, of the U.S. Department of Energy under Contract No. DE-AC03-76SF00098, and the National Science Foundation. 


\section{DISCLAIMER}

This report was prepared as an account of work sponsored by an agency of the United States Government. Neither the United States Government nor any agency thereof, nor any of their employees, make any warranty, express or implied, or assumes any legal liability or responsibility for the accuracy, completeness, or usefulness of any information, apparatus, product, or process disclosed, or represents that its use would not infringe privately owned rights. Reference herein to any specific commercial product, process, or service by trade name, trademark, manufacturer, or otherwise does not necessarily constitute or imply its endorsement, recommendation, or favoring by the United States Government or any agency thereof. The views and opinions of authors expressed herein do not necessarily state or reflect those of the United States Government or any agency thereof. 


\section{DISCLAIMER}

Portions of this document may be illegible in electronic image products. Images are produced from the best available original document. 
Study of Pair and Many-Body Interactions in Rare-Gas Halide Atom Clusters Using Negative Ion Zero Electron Kinetic Energy (ZEKE)

and Threshold Photodetachment Spectroscopy

by

Ivan Yourshaw

B.S. (University of Iowa) 1991

A dissertation submitted in partial satisfaction of the

requirements for the degree of

Doctor of Philosophy

in

Chemistry

in the

GRADUATE DIVISION

of the

UNIVERSITY OF CALIFORNIA, BERKELEY

Committee in charge:

Professor Daniel M. Neumark

Professor Richard J. Saykally

Professor Arup K. Chakraborty

Fall 1998 


\begin{abstract}
Study of Pair and Many-Body Interactions in Rare-Gas Halide Atom Clusters Using Negative Ion Zero Electron Kinetic Energy (ZEKE) and Threshold

Photodetachment Spectroscopy
\end{abstract}

by

Ivan Yourshaw

Doctor of Philosophy in Chemistry

University of California, Berkeley

Professor Daniel M. Neumark, Chair

The diatomic halogen atom-rare gas diatomic complexes $\mathrm{KrBr}^{-}, \mathrm{XeBr}^{-}$, and $\mathrm{KrCl}^{-}$ are studied in this work by zero electron kinetic energy (ZEKE) spectroscopy in order to characterize the weak intermolecular diatomic potentials of these species. Also, the ZEKE and threshold photodetachment spectra of the polyatomic clusters $\mathrm{Ar}_{n} \mathrm{Br}^{-}(n=2-9)$ and $\mathrm{Ar}_{\mathrm{n}} \mathrm{I}^{\cdot}(\mathrm{n}=2-19)$ are studied to obtain information about the non-additive effects on the interactions among the atoms. This work is part of an ongoing effort to characterize the pair and many-body potentials of the complete series of rare gas halide clusters. In these studies we obtain information about both the anionic and neutral clusters.

In the spectra of the diatomic complex we observe well-resolved vibrational structure which is used in conjunction with scattering results from the literature to 
construct accurate intermolecular potentials. We also obtain accurate electron affinities for these species.

From the spectra of the polyatomic species we obtain accurate electron affinities and electronic state term values. We observe some partially resolved vibrational structure for $\mathrm{Ar}_{n} \mathrm{Br}^{-}(n=2-3)$ and $\mathrm{Ar}_{n} \mathrm{I}^{-}(n=2-3)$. The global minimum energy structures of the clusters are found using a molecular-dynamics based simulated annealing algorithm. The electron affinities calculated using these structures and the pair potentials obtained from previous $\mathrm{ZEKE}$ studies of the $\mathrm{ArBr}^{-}$and $\mathrm{ArI}^{-}$complexes are found to be inconsistent with the experimentally observed electron affinities, indicating the importance of non-additive effects in these clusters. Various non-additive interactions are considered, and the most important are found to be non-additive induction effects and the effect arising from the interaction of the halide charge with the multipole moments due to the distortion of the rare-gas electron clouds. For the neutral clusters $\operatorname{Ar}_{n} \mathrm{Br}(n=$ 2-3) and $A r_{n} I(n=2-7)$ we also observe many-body effects in the electronic structure due to the presence of the open-shell halogen atom. These effects are successfully modeled using a simple degenerate perturbation theory treatment of the open shell-closed shell interaction. 
Dedicated to my parents

Michael Yourshaw

and

Sarah Mulholland 


\section{Table of Contents}

\section{Abstract}

Dedication iii

Acknowledgments ..$x$

\section{Chapter 1. Introduction}

1.1. Intermolecular forces: importance and brief historical review .2

1.2. Early spectroscopic work on clusters .4

1.3. ZEKE studies of simple van der Waals complexes .5

1.3.1. The interaction of an open-shell $\left({ }^{2} \mathrm{P}\right)$ halogen atom with a closed-shell species 5

1.3.2. Review of previous anion $\mathrm{ZEKE}$ studies of halogen atom-closed shell systems .8

1.4. $\mathrm{Ar}_{\mathrm{n}} \mathrm{X}^{-}$and $\mathrm{Ar}_{\mathrm{n}} \mathrm{X}$ clusters: many-body interactions 9

1.5. References 12

Chapter 2. Experimental techniques and apparatus 14

2.1. Anion PES and ZEKE spectroscopy .14

2.1.1. Anion photoelectron spectroscopy 15

2.1.2. Anion ZEKE and threshold photodetachment spectroscopy .16

2.2.3. Wigner's law .19

2.2. The experimental apparatus 22

2.2.1. "Double skimmer" setup for large cluster production .25

2.2.2. Electron deceleration field .29 
Chapter 3. Zero electron kinetic energy spectroscopy (ZEKE)

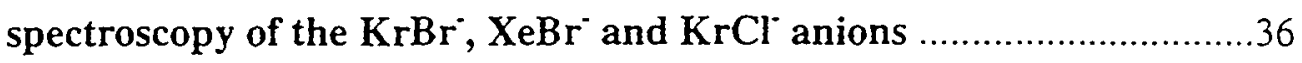

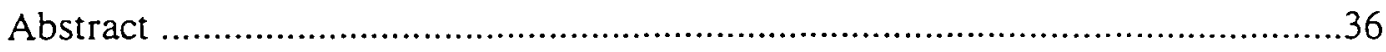

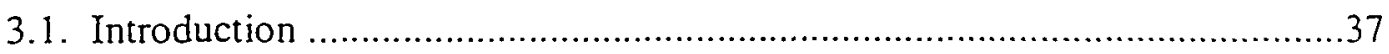

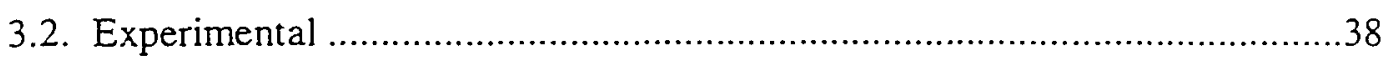

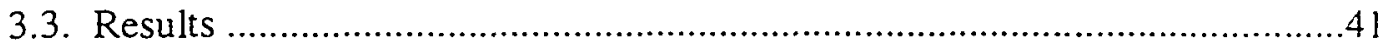

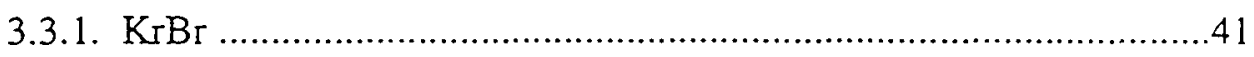

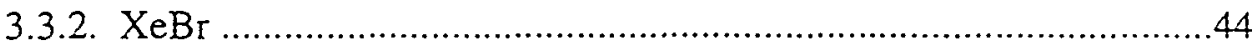

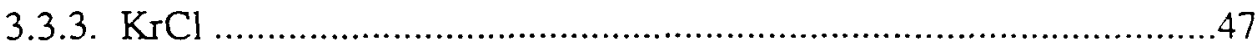

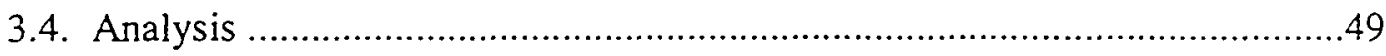

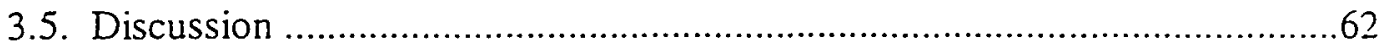

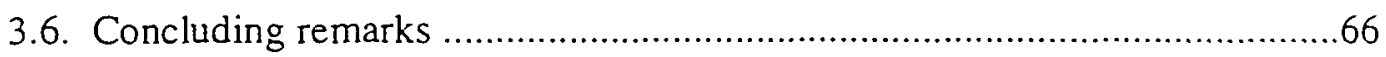

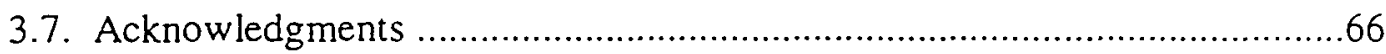

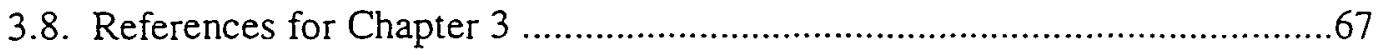

Chapter 4. Observation of many-body effects in the zero electron kinetic energy (ZEKE) and threshold photodetachment

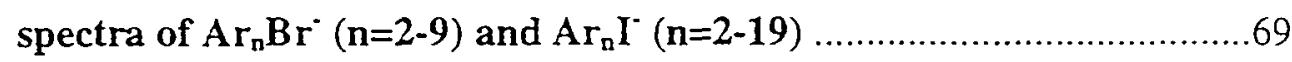

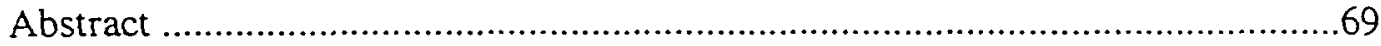

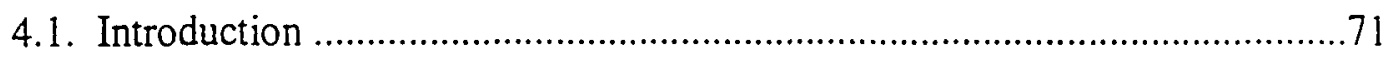

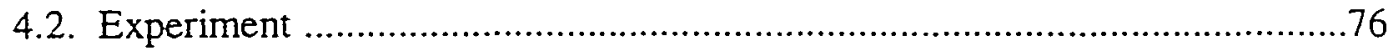




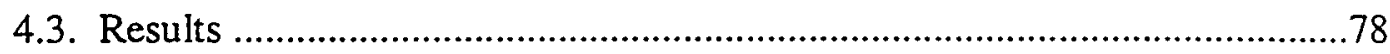

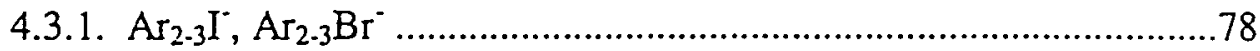

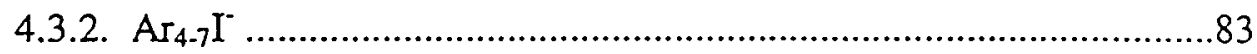

4.3.3. Partially discriminated threshold photodetachment spectra ..........85

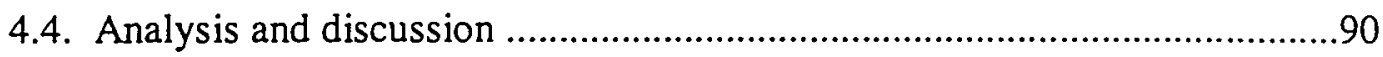

4.4.1. Pair potentials ........................................................................ 92

4.4.2. Simulated annealing method .........................................................96

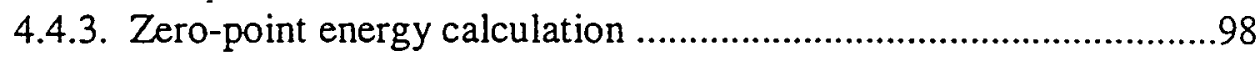

4.4.4. Anion minimum energy geometries .............................................98

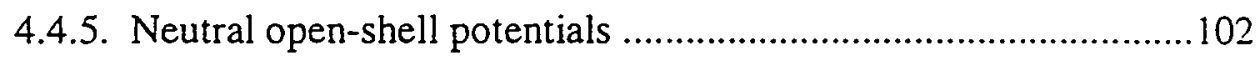

4.4.6. Electron affinities calculated from additive potentials .................109

4.4.7. Many-body interactions ...........................................................113

4.4.7.1. Triple-dipole interaction .............................................114

4.4.7.2. Three-body exchange ………………………….............117

4.4.7.3. Induction non-additivity ...............................................118

4.4.7.4. Exchange and dispersion multipoles .............................122

4.4.8. Electron affinities calculated with many-body potentials .............130

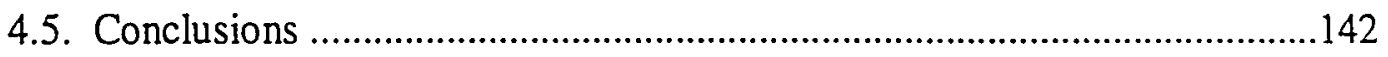

4.6. Acknowledgments .................................................................................. 144

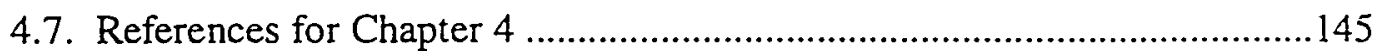




\section{Appendix A. Program for calculating vibrational states and Franck-}

Condon factors for highly anharmonic one-dimensional

potentials based on the eigenfunctions of the Morse

potentials using the DVR method .............................................152

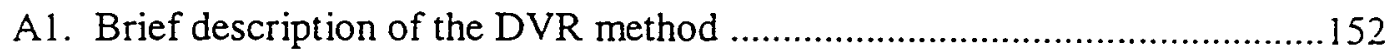

A2. How to implement DVR using Morse basis functions ...............................154

A3. Choosing the parameters for the Morse basis .........................................157

A4. Comparison of Morse and harmonic oscillator basis set

convergence for a highly anharmonic potential ......................................158

A5. Documentation of the Morse DVR program "idvr":

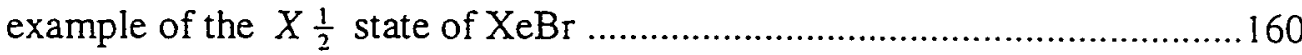

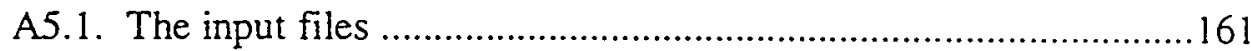

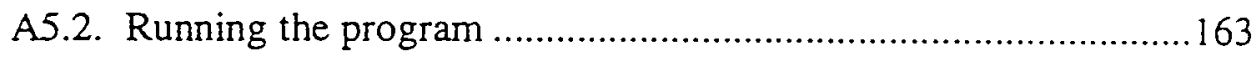

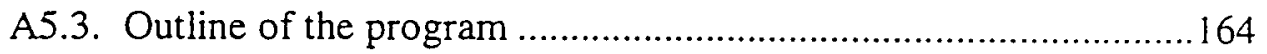

A6. Source code for the Morse DVR program "idvr.f" ..................................166

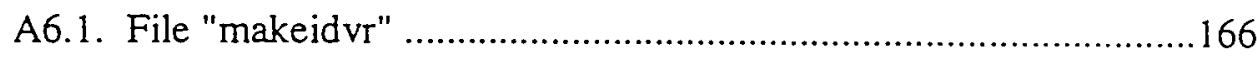

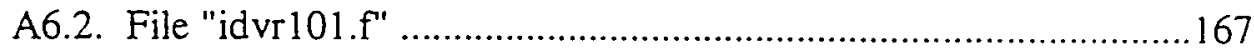

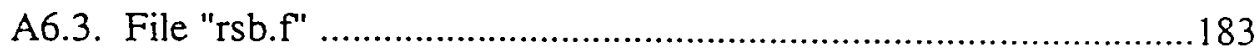

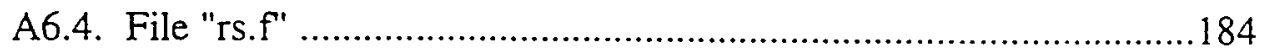

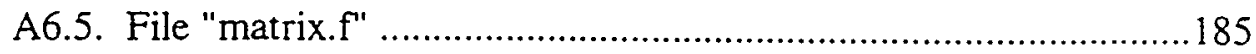

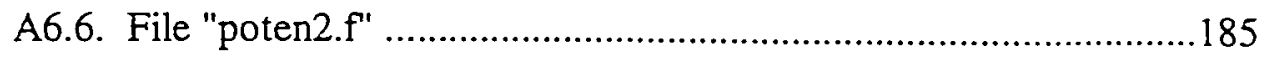

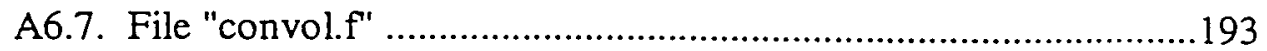

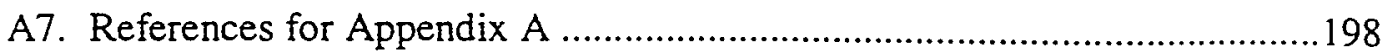




\section{Appendix B. Program for fitting rovibronic transitions}

in $\mathrm{RgX}$ photodetachment

B1. Line strength factors for photodetachment

of Hund's case (c) molecules 199

B2. Documentation of the rotational fitting program "rfit":

example of the $X \frac{1}{2}$ state of $\mathrm{XeBr}$

B2.1. The input files 206

B2.2. Running the program 210

B2.3. Outline of the program 210

B3. Source code for the rotational fitting program "rfit" .212

B4. References for Appendix B

Appendix C. Simulated annealing .230

C1. Introduction .230

C2. Brief survey of methods of finding global minima of clusters 230

C3. Molecular dynamics simulated annealing .235

C3.1. Microcanonical molecular dynamics .236

C3.2. Constant temperature molecular dynamics .238

C3.3. Using the rescaling of velocities for simulated annealing 239

C4. Documentation of the simulated annealing program "amain. $f$ " 239

C4.1. The input file 240

C4.2. The atomic configuration files .250

C4.3. Running the program: example of $\mathrm{Ar}_{6} \mathrm{I}^{\circ}$ 250

C4.3.1. Gradient minimization when near a local minimum .....251 viii 
C4.3.2. Reheating and annealing 252

C4.4. Outline of the program .256

C5. Source code for the simulated annealing program ......................................258

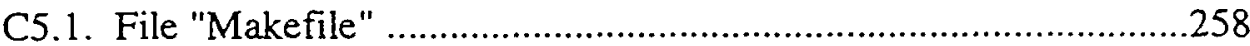

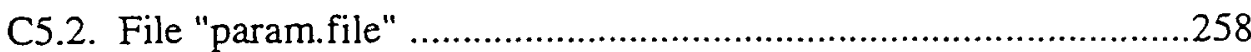

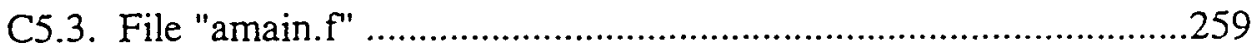

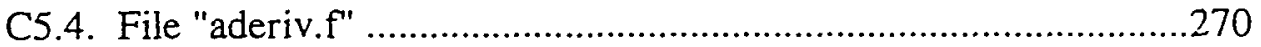

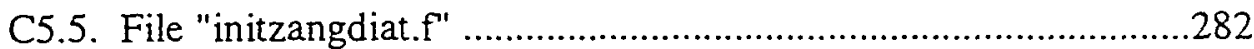

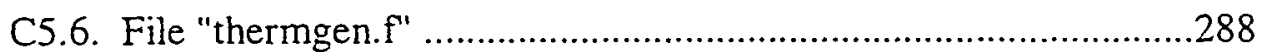

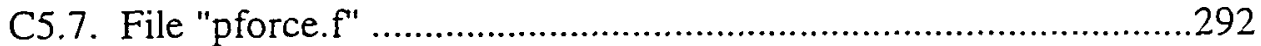

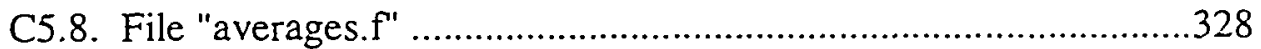

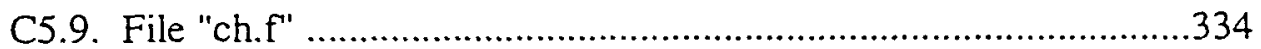

C6. Documentation of the "normal" program

for finding zero point energies ...............................................................335

C6.1. Example: Zero-point energy calculation for $\operatorname{Ar}_{6}{ }^{\top}$.......................335

C6.1.1. The input file ………………………………….............35

C6.1.2. Running the program ...................................................339

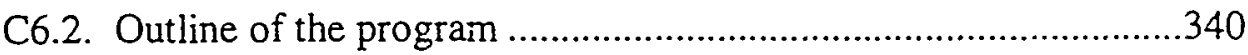

C6.3. Source code for the program "normal" .........................................342

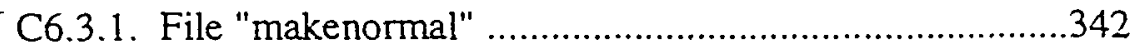

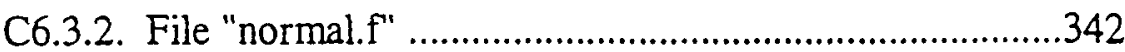

C7. References for Appendix C ..................................................................376 


\section{Acknowledgments}

I would first of all like to thank my advisor Dan Neumark for hiring me, and for being a constant source of creative inspiration. I have learned a lot about science and about life from his mentorship.

The other members of the Neumark group all also contributed to this work in one way or another. Primary thanks go to the past and present students and post-docs who have worked with me in the "second lab," as the ZEKE lab is affectionately known. Yuexing Zhao taught me almost everything I needed to know about running the experiment, and his amiable good nature made him a pleasure to work with. Caroline Arnold and Georg Reiser also provided me with valuable insights into the mysteries and rituals of anion ZEKE spectroscopy. Esther DeBeer contributed greatly to the development of the Raman experiment, not covered in this thesis, and patiently dealt with the trials and tribulations of burnt YAG rods and fused silica windows. Thomas Lenzer "the radioactive post-doc" joined the group recently, and his quick mind and easy-going nature have made him a pleasure to work with and valuable addition to the ZEKE team. Thomas has also patiently and uncritically endured my attempts to practice my very rudimentary German on him. Mike Furlanetto has contributed a great deal to the ZEKE experiment recently, and his organizational skills have been especially appreciated. He and the newest member of the ZEKE team Nick Provinka are currently engaged in an

overhaul and transfiguration of the ZEKE machine into a Raman photoelectron spectrometer, a task which they have undertaken with enthusiasm and skill. I would wish them good luck, but I don't believe luck will be necessary with their talent. 
I would also like to acknowledge the friendship and support of several other group members. Former group members Dave Osborn and Don Arnold deserve special mention because it was through talking with them and Dan that I was first persuaded to join the group, and I continued to look to them for advice and as valuable role models during my stay at Berkeley. Knut Asmis, "the yodeling post-doc" has been of great help in tutoring me in some of the finer points of the German language. Two group members, Travis Taylor and Jeff Greenblatt have been special friends in times of distress. Travis has been inspiration by example in how to stay true to oneself in an often chaotic environment. Jeff has also been a special friend, and I have benefited a lot from our discussions of what it means to be a scientist at the end of the 20th Century. Special thanks also to my office mates Ryan Bise and Hyeon Choi for their companionship during the final phase of my thesis writing, and for not complaining about my insistence that the office door be kept locked.

I also wish to thank my family. My parents Michael Yourshaw and Sarah Mulholland, to whom this thesis is dedicated, instilled in me from an early age a love of learning. For their emotional and financial support throughout my educational career I will always be grateful. Special thanks are also due to my step-father Dan Mulholland for all the email, and the support of my step-mother Kathy Yourshaw.

These acknowledgments would not me complete without thanking the fine professional staff at Alta Bates Herrick Hospital and my fellow patients there, for restoring me to sanity and literally saving my life.

Finally I would like to thank Gilda Mark for her love and unfailing faith in me even at the times when I had lost faith in myself. 


\section{Chapter 1. Introduction}

The spectroscopic study of weakly bound van der Waals clusters has experienced an explosive period of growth in the past two decades, prompted mainly by innovations in laser and molecular beam technology. At the same time, a tremendous amount of complementary theoretical work has been done on these systems. This work is still underway, and it is probably not an understatement to describe the current era as a "golden age" of cluster research. In this introductory chapter, we will summarize the reasons for this outpouring of interest in this field, briefly review some highlights of what has been learned so far, and review the results previous work in the Neumark group in this field. In particular we will focus on recent advances in the understanding of "manybody" interactions, i.e., interactions involving synergistic effects among three or more atoms or molecules.

Here, and in the rest of this work, we will restrict our attention to weakly bound clusters interacting by dispersion or induction forces, and leave aside discussion of covalently bound or "metallic" clusters, a field of study which has also experienced a massive amount of attention during this same time period, but is beyond the scope of the present work.

\subsection{Intermolecular forces: importance and brief historical review}

"Since the end of the nineteenth century a considerable amount of work has been devoted to the exact formulation of the connection between the properties of matter in bulk and intermolecular forces. Such a formulation represents the ultimate aim of the molecular theory of matter since, when a theory of this kind is established, a knowledge of the intermolecular forces is sufficient for 
the evaluation of all the properties of the bulk materials." -G. C. Maitland, M. Rigby. E. B. Smith. and W. A. Wakeham in Intermolecular Forces, p. 3. ${ }^{1}$

Interest in the weak interactions of atoms and molecules actually preceded these recent spectroscopic and theoretical advances by many years. The importance of the concept of intermolecular interactions with both attractive and repulsive components, and their connection with the bulk properties of gases was first made clear by van der Waals in 1873 with his formulation of the van der Waals equation of state. (See Ref.1, p. 2.) Subsequently, the term "van der Waals forces" was adopted as a blanket phrase for all of these weak interactions. An understanding of van der Waals forces is necessary to understand many bulk properties of matter, such of heats of sublimation and evaporation, ${ }^{2}$ and solvation. The existence of the inductive dipole-induced dipole and dipole-dipole interactions were first proposed to explain these bulk properties by Debye (1920), and Keesom (1921). However it was not until the work of London in 1930 that dispersion forces were proposed to explain the bulk properties of atoms which do not posses multipole moments, such as rare gas atoms. For reviews of this early work, see the articles by Margenau ${ }^{3}$ and London. ${ }^{4}$ For a very basic and readable introduction to van der Waals forces, see Chapter 13 of Kauzmann ${ }^{2}$ as well as Chapter 1 of Maitland et. $a l .1$

With the advent of the full development of quantum mechanics, the conceptual framework to understand van der Waals forces was established. However, because of the practical intractability of exact quantum mechanical solution of even the simplest problem involving van der Waals interactions, it was necessary to take a more empirical approach to the problem. And thus was born what Maitland et al. refer to as the 
"Lennard-Jones era" in the study of intermolecular forces. (See Chapter 9 of Ref. 1.) Under this paradigm, the Born-Oppenheimer approximation was assumed to separate electronic from nuclear motions and simple analytic forms for the internuclear potential-such as the familiar Lennard-Jones 12-6 potential--were used, with the Coulomb interactions of the electrons implicitly included in this potential function. Also, pairwise additivity of the potentials was usually assumed. The methodology under this paradigm was then to attempt to reconcile these model potentials with the experimentally observed properties of the bulk substances. For a comprehensive treatment of these early efforts to understand the relation between intermolecular forces and bulk properties see Hirschfelder, et. al., Molecular Theory of Gases and Liquids. ${ }^{5}$

During this time some theoretical work was done to advance knowledge about the non-pairwise interactions among atoms and molecules which are essential to understand if one is to bridge the gap between molecular and bulk properties. In 1943 Axilrod and Teller ${ }^{6}$ proposed a three-body dispersion force, which was long accepted, with little direct experimental confirmation, as the primary many-body effect in bulk substances. In the early 1960 S Jansen $^{7}$ proposed a model of many-body interactions based on exchange effects, which seemed to account for discrepancies between pairwise additive potential predictions and experimental measurements of the binding energies of alkali-halide crystals. For a review and critique of this early work on many-body forces see Meath and Aziz. ${ }^{8}$ For a recent, comprehensive review of experimental and theoretical research in this field see Elrod and Saykally. ${ }^{9}$ 


\subsection{Early spectroscopic work on clusters}

It became apparent that in order to gain a truly firm understanding of van der Waals interactions one must begin by studying the potentials of small clusters, beginning with diatomic complexes, in order to avoid the confounding factors involved with extrapolating from the properties of bulk materials to fundamental intermolecular forces. Such work began in earnest in the late sixties and early seventies. The earliest work in this field did not involve the use of molecular beams to produce clusters, but relied on spectroscopic probes of the high vibrational levels of molecules such as $\mathrm{Mg}_{2}$ to determine the (Rydberg-Klein-Rees) RKR potential at long range in order to characterize the van der Waals interactions, ${ }^{10}$ and vacuum ultraviolet absorption spectroscopy of dilute rare gases with very long absorption path lengths, such as the work of Tanaka et al. on the rare-gas dimers such as $\mathrm{Ar}_{2}{ }^{11}$ Klemperer and co-workers pioneered the use of supersonic expansions to form molecular beams of clusters in conjunctions with electric resonance spectroscopy, to study van der Waals systems with strong dipole moments, such as Ar-HCl. ${ }^{12}$ For a review of this early spectroscopic work see Chapter 7 of Maitland et al. ${ }^{1}$

The field of cluster research began its period of exponential growth in the late seventies with the landmark LIF studies by Levy and co-workers on the $I_{2}-R g(R g=$ rare gas) complexes. ${ }^{13}$ Since then the field of cluster research has expanded to such an extent that it is not possible to begin a summary here. For a comprehensive treatment of recent cluster research we refer the reader to the review by Castleman and Bowen. ${ }^{14}$ 


\subsection{ZEKE studies of simple van der Waals complexes}

The focus of the present work in on anion ZEKE and threshold photodetachment spectroscopy of halide and halogen atoms with rare gas atoms. This work is of fundamental importance because we are able to study a very simple example of the interaction of an open-shell species with a rare gas atom. We also gain important information about the anionic interaction. In this section we review the basic nature of the interaction of an open-shell $\left({ }^{2} \mathrm{P}\right)$ halogen atom with a single rare gas atom or closed shell molecule, and then review the results of previous anion ZEKE studies of these systems.

\subsubsection{The interaction of an open-shell $\left({ }^{2} \mathrm{P}\right)$ halogen atom with a closed-shell species.}

In this section we describe the electronic states that arise when an open-shell $\left({ }^{2} \mathrm{P}_{1 / 2,3 / 2}\right)$ halogen atom interacts with a ${ }^{1} \mathrm{~S}$ rare gas atom. First we consider the case when the spin orbit coupling of the halogen is negligible. In this case we have two electronic states, pictured schematically in Figure 1.1. When the p-orbital containing the "hole" is aligned along the internuclear axis, we have a ${ }^{2} \Sigma$ state $(\Lambda=0)$, and when this p-orbital is oriented perpendicular to the internuclear axis we have two ${ }^{2} \Pi$ states $(\Lambda=1)$. 


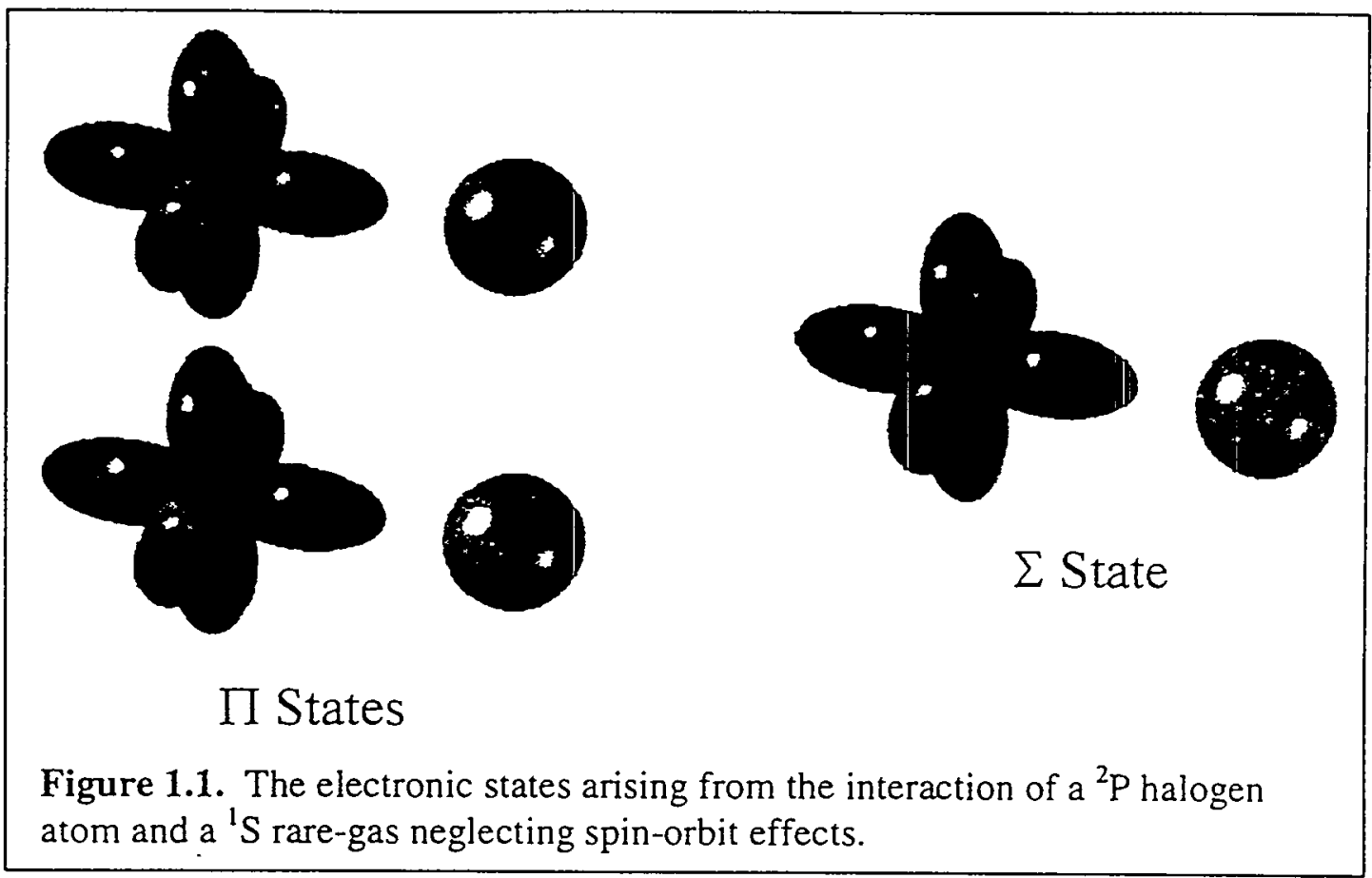

When spin orbit coupling is included and is small relative to the electrostatic interaction Hund's case (a) prevails, and the two states are split into the ${ }^{2} \Pi_{1 / 2}$ and ${ }^{2} \Pi 3 n$ states, which in the case of ${ }^{2} s^{5} p$ atoms like the halogens would lie higher in energy than the ground ${ }^{2} \Sigma_{1 / 2}$ state. This situation is shown on the left-hand side of Figure 1.2 on the following page. As the spin-orbit interaction increases--or as the internuclear nuclear increases so that the electrostatic interaction is relatively smaller--the ${ }^{2} \Pi{ }_{3 / 2}$ and ${ }^{2} \Sigma_{1 / 2}$ states mix, and the complex is then described by Hund's coupling case (c) as $\Lambda$ ceases to be a good quantum number. This is shown on the right-hand side of Figure 1.2. The ${ }^{2} \Pi_{1 / 2}$ and ${ }^{2} \Sigma_{1 / 2}$ states have mixed to form the lower $X \frac{1}{2}\left(j=\frac{3}{2}, \Omega=\frac{1}{2}\right)$ state and the upper $I I \frac{1}{2}\left(j=\frac{1}{2}, \Omega=\frac{1}{2}\right)$ state, separated by an amount similar to the atomic spin orbit splitting. The ${ }^{2} \Pi_{3 / 2}$ state remains as a pure $\Pi$ state, called the $I \frac{1}{2}\left(j=\frac{3}{2}, \Omega=\frac{3}{2}\right)$ state in case (c) 
notation, higher in energy than the $X \frac{1}{2}$ state by an amount related to the electrostatic interaction between the atoms.

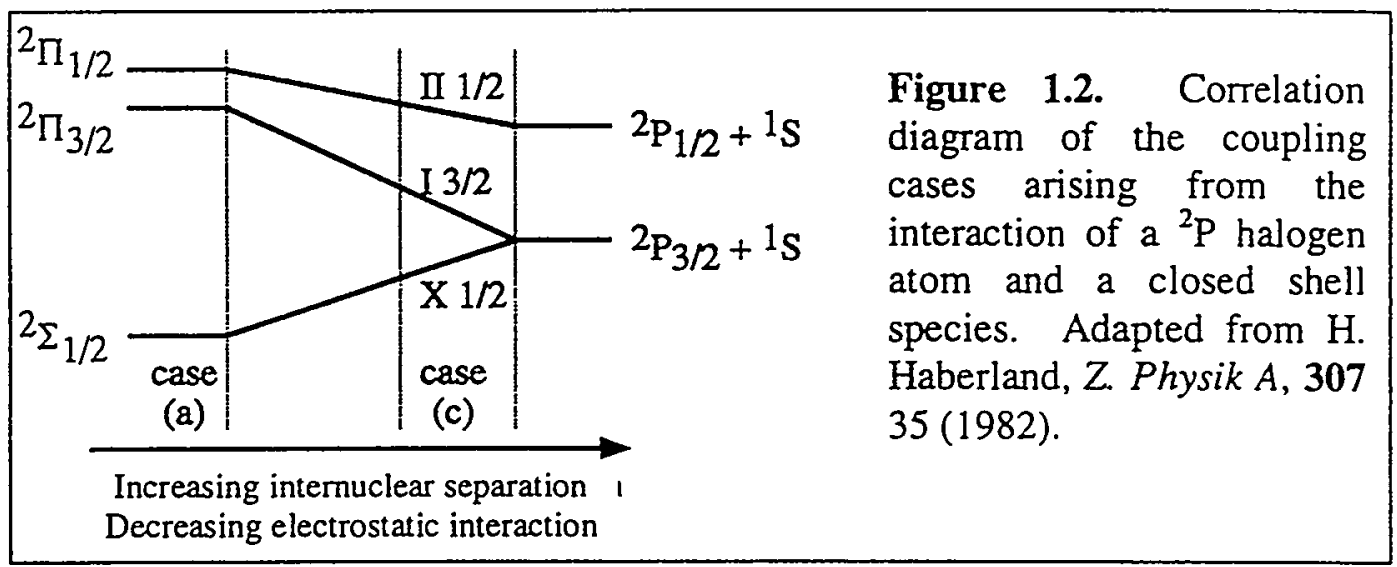

The Hund's case (c) limit applies to the well and long-range region of most of the raregas halogens. The amount of $\Sigma-\Pi$ mixing in the three electronic states is shown in Figure 1.3 as a function of internuclear separation.

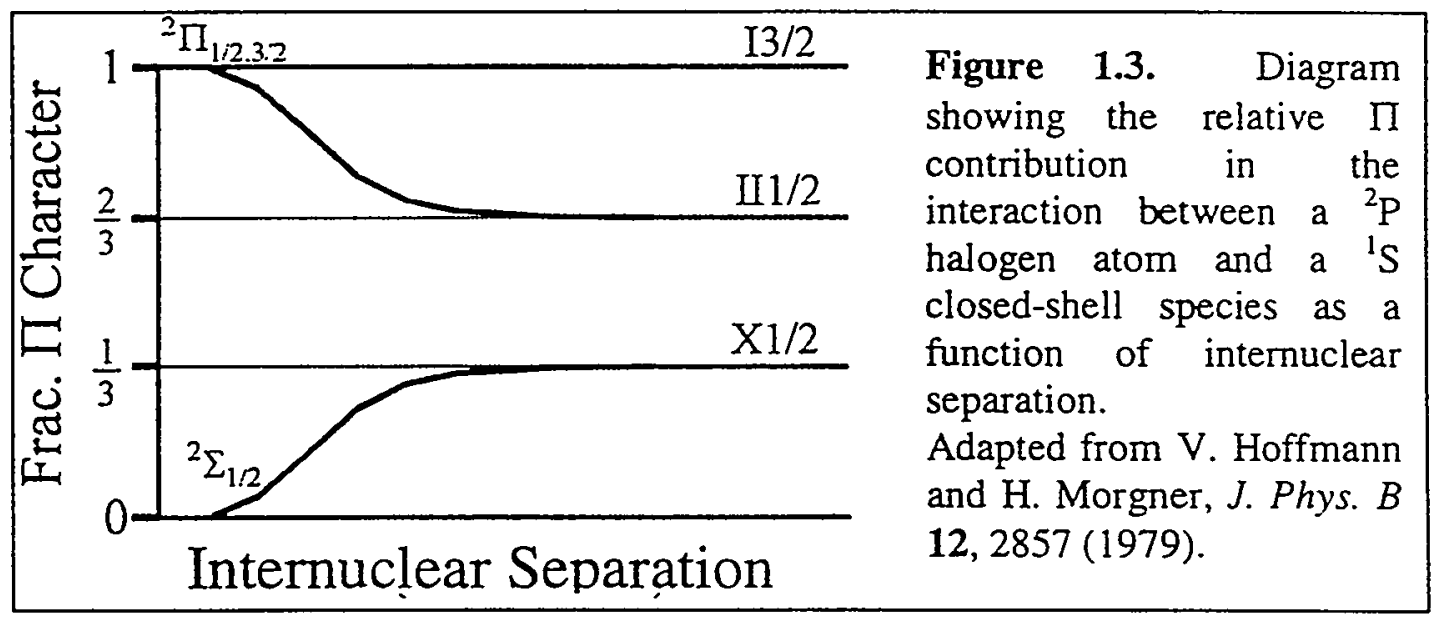

Again the situation on the right-hand side of this figure [case (c)] is the most pertinent to the complexes studied in this work, where the $X \frac{1}{2}$ state has $\frac{2}{3} \sum$ character and $\frac{1}{3}$ $\Pi$ character, and the $I I \frac{1}{2}$ state has $\frac{1}{3} \sum$ and $\frac{2}{3} \Pi$ character. As already mentioned the $I \frac{3}{2}$ state has pure $\Pi$ character. 
It should also be mentioned here that all three of the neutral electronic states can in principle be observed by ZEKE spectroscopy, since the transitions involve removal of a single electron from a p-orbital of the halide anion. A detailed derivation of the rovibronic transition strengths for the anion $\rightarrow$ neutral transition can be found in Appendix B. For more detailed information about the interaction between open-shell and closed shell atoms see Refs. 15 and 16.

\subsubsection{Review of previous anion ZEKE studies of halogen atom-closed shell systems}

In this section we review previous applications of anion ZEKE spectroscopy to study the complexes of halide/halogen atoms with closed shell species. The first such study was the ZEKE spectrum observed in the Neumark group of the $\mathrm{I}^{-}-\mathrm{CO}_{2}$ complex. ${ }^{17}$ In this work it was found that the complex is "T"-shaped, with the $\mathrm{CO}_{2}$ slightly bent. However, the interaction between the I atom and the $\mathrm{CO}_{2}$ molecule is sufficiently weak (44.5 $\mathrm{meV}$ for the $X \frac{1}{2}$ state) that the interaction resembles a van der Waals interaction much more than a covalent bond (in contrast to $\mathrm{FCO}_{2}$, which is a covalently bound molecule. ${ }^{18}$ ) Low frequency vibrational progressions were observed in the van der Waals stretching mode for each electronic state, and the spectra were fit to model onedimensional anion and neutral potentials. The binding energy of the anionic complex was found to be $212.0 \mathrm{meV}$. Structure was also observed corresponding to the $\mathrm{CO}_{2}$ internal bending vibration. Also in this laboratory, the $\mathrm{I}-\mathrm{CH}_{3} \mathrm{I}$ complex was studied by ZEKE spectroscopy. ${ }^{19}$

Schlag and co-workers have studied the $\mathrm{I}^{-}-\mathrm{H}_{2} \mathrm{O}$ complex by ZEKE spectroscopy. ${ }^{20}$ They observed the three electronic states discussed above, as well as two 
low frequency vibrational progressions. However, due to the uncertainty in the cluster geometry and the possibility of observable rotational structure, they were not able to conclusively assign the vibrational features or fit the spectrum to a model potential.

The work of most direct relevance here was our previous anion ZEKE study of the $\mathrm{ArI}^{\circ}, \mathrm{ArBr}^{\circ}$, and $\mathrm{KrI}^{-}$complexes. ${ }^{21}$ Because of the simple nature of the interaction compared to the studies involving molecular solvents, we were able to observe well resolved vibrational progressions in all three of the electronic states and fit the spectra to model potentials, obtaining accurate vibrational frequencies, well depths and bond lengths for the anion as well as neutral states. The potential parameters for the ArI and $\mathrm{ArBr}$ systems can be found in Chapter 4 (Table 4.3). The work described in Chapter 3 is a continuation of this study.

\section{4. $\operatorname{Ar}_{\mathrm{n}} \mathrm{X}^{\cdot}$ and $A r_{\mathrm{n}} \mathrm{X}$ clusters: many-body interactions}

As mentioned above, the study of polyatomic clusters is ideal to directly observe the many-body effects which are of importance in bulk phenomena, such as solvation in liquids and the binding of crystals. In Chapter 4 we will delve in great depth into the nature of the many-body interactions among a halide neutral or anion and a number of rare gas atoms. In this introductory section, therefore, we will give a brief, more intuitive overview of the nature of the most important of these interactions.

In the case of the neutral $\mathrm{Rg}_{\mathrm{n}} \mathrm{X}$ clusters, the non-additivity of the potentials arises because of the open-shell nature of the halogen atom. Consider again the spinless states shown in Figure 1.1. The axis of symmetry is defined by the two nuclei. Because the electronic structure of the halogen atom is not spherically symmetric, the potential 
functions differ according to the orientation of the singly occupied p-orbital. Now consider the situation when a second rare-gas atom is brought near the $\mathrm{Rg}-\mathrm{X}$ pair. The symmetry of the diatomic complex is broken, and the p-orbital must reorient to accommodate the presence of the second $\mathrm{Rg}$ atom. Considering this, it seems rather unlikely that the potentials would add in a simple pairwise fashion. In this sense, that one must consider the orientation of the orbitals involved, this type of open shell-closed shell interaction may be considered something of an intermediate case between a "pure" van der Waals interaction and a covalent bond. This is only meant to be an intuitive discussion of the topic; for all the "gory details" of how this calculation is accomplished, we refer the reader to Chapter 4 .

The many-body forces at work in $\operatorname{Rg}_{n} \mathrm{X}^{-}$anionic clusters are of fundamental interest as models of the forces involved in solvation of ions. The two most important non-additive interactions in these systems are the induction and "exchange charge" forces.

The induction non-additivity is the dominant many body effect in the $\mathrm{Rg}_{\mathrm{n}} \mathrm{X}$ clusters, and is quite easy to understand. In Figure 1.4 we show the induction interaction between a halide anion and two rare gas atoms. The halide induces dipole moments in both rare gas atoms. If the rare gases are next to each other, as shown, the induced dipoles repel, reducing the net binding energy of the cluster from what it would be without this effect. 


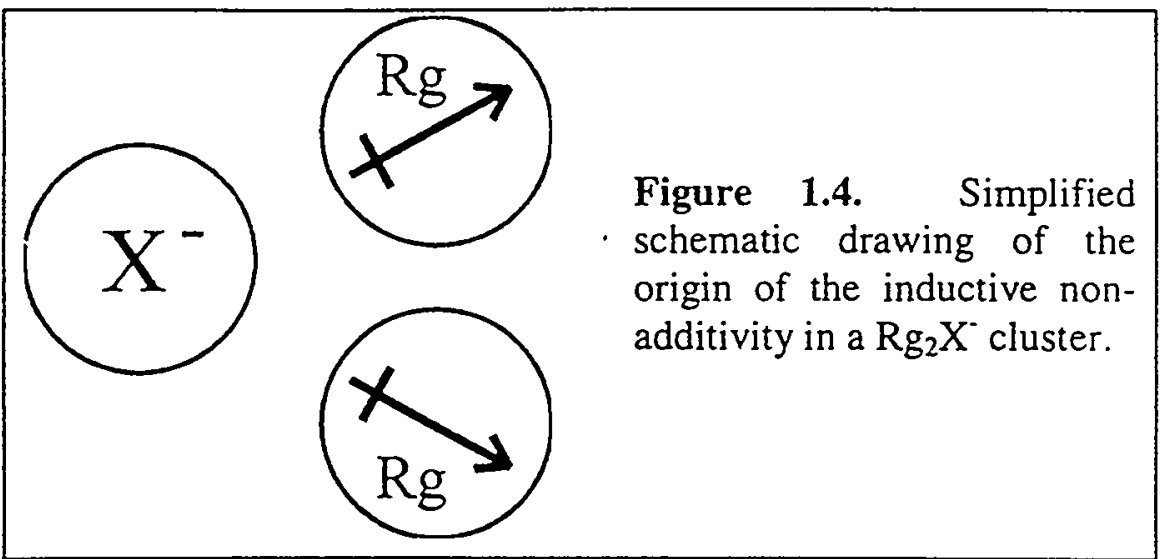

Again, we will wait until Chapter 4 to discuss a more complete model of the induction non-additivity.

The other type of non-additive interaction we have found to be important arises from the distortion of the electron clouds of two $\mathrm{Rg}$ atoms when they are brought close together with a nearby halide anion. This effect is pictured in Figure 1.5. The exchange repulsion between the $\mathrm{Rg}$ electron clouds causes a net positive charge, $\delta^{+}$, to appear at the midpoint along the $\mathrm{Rg}-\mathrm{Rg}$ axis, and partial negative charges, each half the magnitude of $\delta^{+}$, to appear at the $\mathrm{Rg}$ nuclei. Because the positive charge is closer to the halide than the negative charges, the net effect is an attractive force, and an increase in the binding energy over what it would be without this effect.

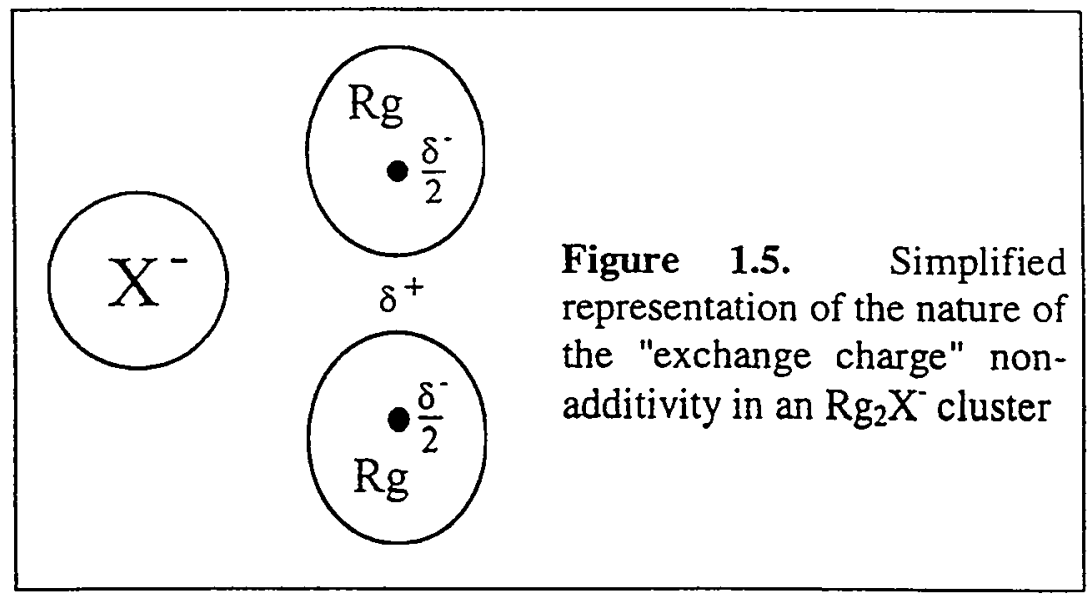


We have found, from the experiments and calculations described in Chapter 4, that this "exchange-charge" effect makes a quite significant contribution to the non-additive energy of the cluster anions, being about half as large as the induction non-additivity with opposite sign.

\subsection{References}

1 G. C. Maitland, M. Rigby, E. B. Smith, and W. A. Wakeham, Intermolecular Forces: Their Origin and Determination (Oxford University Press, Oxford, 1981).

2 W. Kauzmann, Quantum Chemistry: An Introduction (Academic Press Inc., New York, 1957).

3 H. Margenau, Revs. Mod. Pys. 11, 1 (1939).

${ }^{4}$ F. London, Trans. Faraday Soc. 33, 8 (1937).

5 J. O. Hirschfelder, C. F. Curtiss, and R. B. Bird, Molecular Theory of Gases and Liquids (Wiley, New York, 1954).

6 B. M. Axilrod and E. Teller, J. Chem. Phys. 11, 299 (1943).

7 L. Jansen, Adv. Quantum Chem. 2, 119 (1965).

8 W. J. Meath and R. A. Aziz, Mol. Phys. 52, 225 (1984).

${ }^{9}$ M. J. Elrod and R. J. Saykally, Chem. Rev. 94, 1975 (1994).

10 W. C. Stwalley, Chem. Phys. Lett. 7, 600 (1970).

11 Y. Tanaka and K. Yoshino, J. Chem. Phys. 53, 2012 (1970).

12 S. E. Novick, K. C. Janda, S. L. Holmgren, M. Waldman, and W. Klemperer, J. Chem. Phys. 65, 1114 (1976). 
13 M. S. Kim, R. E. Smalley, L. Wharton, and D. H. Levy, J. Chem. Phys. 65, 1216 (1976).

14 A. W. Castleman and K. H. Bowen, J. Phys. Chem. 100, 12911 (1996).

15 V. Aquilanti, G. Liuti, F. Pirani, and F. Vecchiocattivi, J. Chem. Soc. Faraday Trans 2 $85,955(1989)$.

16 V. Aquilanti and G. Grossi, J. Chem. Phys. 73, 1165 (1980).

17 Y. Zhao, C. C. Arnold, and D. M. Neumark, J. Chem. Soc. Faraday Trans. 89, 1449 (1993).

18 D. W. Arnold, S. E. Bradforth, E. H. Kim, and D. M. Neumark, J. Chem. Phys. 102, 3493 (1995).

19 C. C. Arnold, Ph.D. Thesis, University of California, 1994.

20 C. Bassmann, U. Boesl, D. Yang, G. Drechsler, and E. W. Schlag, International Journal of Mass Spectrometry and Ion Processes 159, 153 (1996).

21 Y. Zhao, I. Yourshaw, G. Reiser, C. C. Arnold, and D. M. Neumark, J. Of Chem. Phys. 101, 6538 (1994). 


\section{Chapter 2. Experimental techniques and apparatus}

In this chapter, we describe the experimental techniques used to study $\operatorname{Rg}_{n} X$ clusters, with an emphasis on recent improvements to the experimental apparatus. First we briefly review the basic concepts of anion photoelectron spectroscopy (PES), then describe the closely related techniques of threshold photodetachment and zero electron kinetic energy (ZEKE) spectroscopy used in these studies. Finally we describe in some detail recent improvements to the experimental apparatus which have enabled us to increase production of large van der Waals clusters, as well as improve the signal-tonoise properties of the ZEKE spectrometer.

\subsection{Anion PES and ZEKE spectroscopy}

Two related experimental techniques that have been used to great advantage for the study of van der Waals clusters have been anion PES, and ZEKE spectroscopy. In both of these techniques, one begins by producing an anion and photodetaching the excess electron with a laser pulse. In this way one obtains information about both the anion and neutral species. An advantage of anion studies over other types of spectroscopic probes of clusters, is that because one begins with charged species, one can mass select the species of interest. Another advantage is that because one starts from the anion one can in most cases detach to the ground state of the corresponding neutral, and thus obtain direct spectroscopic information about the ground neutral state. Information about the ground states of neutral species is often difficult to gather from optical spectroscopic studies of neutral clusters, such as laser induced fluorescence (LIF) or resonance-enhanced multi-photon ionization (REMPI), which usually are more 
informative about the excited states than the ground states. Also, from the photoelectron or ZEKE spectrum, one can directly determine the electron affinity $(E A)$ of the neutral species, which provides information about the relative binding energies of the anion and neutral. Finally, both techniques provide valuable information about anionic clusters, which in general are less well studied and understood than neutral systems--for which a more extensive literature exists. (For a recent review of studies of small neutral clusters, see Bačic and Miller. $\left.{ }^{1}\right)$

\subsubsection{Anion photoelectron spectroscopy}

In anion PES, one produces a source of "cold" clusters using a supersonic expansion anion source (described later in more detail), which are then mass-selected and photodetached with a fixed frequency laser. By measuring the kinetic energy of the photoelectrons, one can, knowing the energy of the detachment laser, infer the amount of internal energy remaining in the neutral, and thus interpret the observed spectrum in terms of spectroscopic transitions from the anion to the neutral. The concept of anion PES is shown schematically in Figures 2.1 and 2.2. For an introduction to the literature on anion photoelectron spectroscopy and a detailed description of the anion photoelectron spectrometer used in the Neumark group, see the dissertation of A. Weaver. ${ }^{2}$ In general, the resolution of conventional anion photoelectron spectroscopy is at best about 5-10 $\mathrm{meV}\left(40-80 \mathrm{~cm}^{-1}\right)$. This resolution is sufficient to resolve electronic transitions, and in many cases vibrational transitions of covalently bound molecules, but is insufficient to observe the much finer vibrational structure of weakly bound van der Waals clusters. 

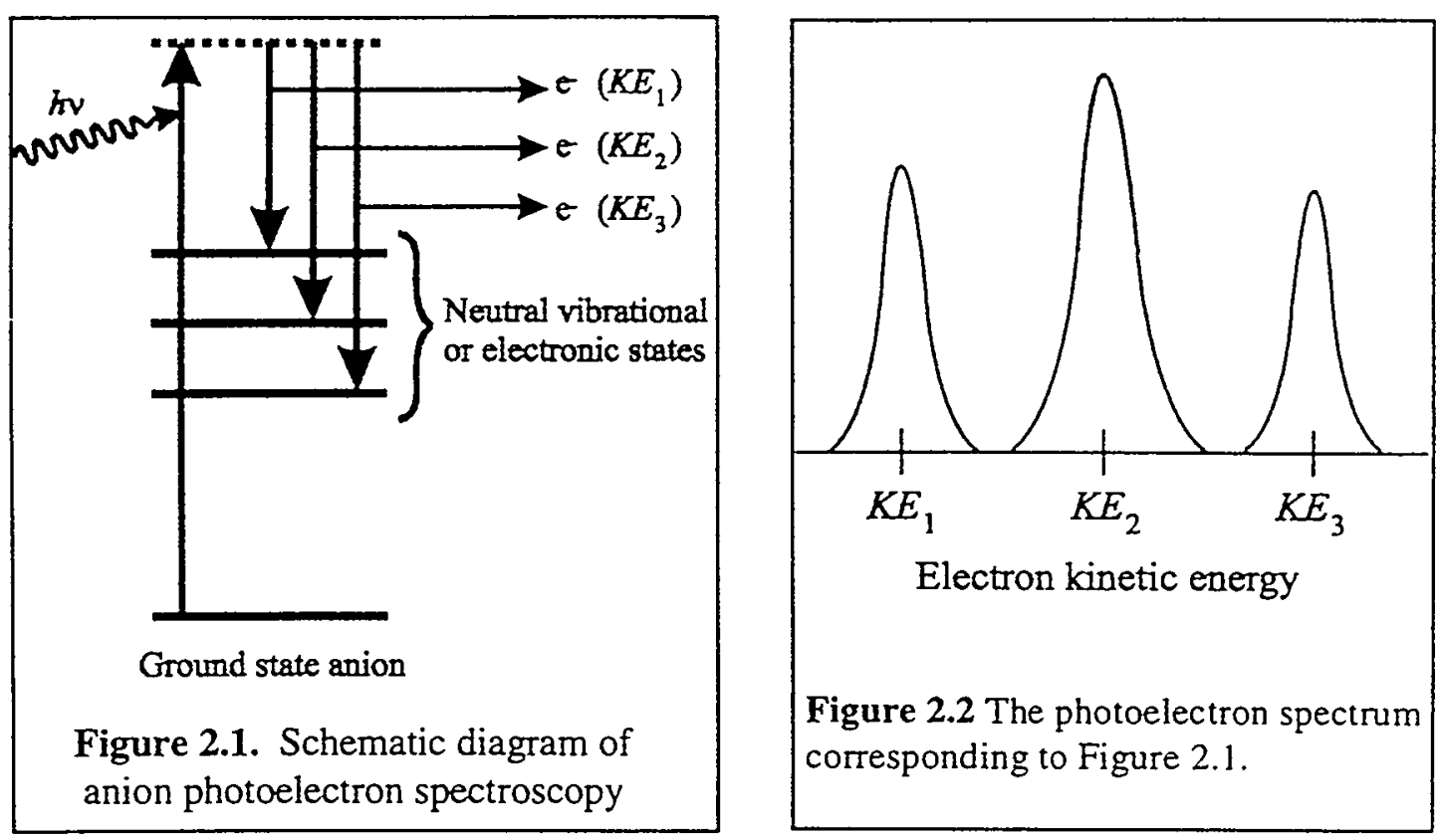

Anion PES has been used in several studies of weakly bound clusters. In the Neumark group, $\mathrm{X}^{-}\left(\mathrm{CO}_{2}\right)_{\mathrm{n}},\left(\mathrm{X}=\mathrm{Cl}, \mathrm{Br}, \mathrm{I}^{3}{ }^{3,},{ }^{4}\right.$, and $\mathrm{I}^{-}\left(\mathrm{N}_{2} \mathrm{O}\right)_{\mathrm{n}}{ }^{4}$ clusters have been studied with this technique. More recently, time-resolved PES has been performed in this group on the $\mathrm{I}_{2}^{-}(\mathrm{Ar})_{\mathrm{n}}^{5}$ and $\mathrm{I}_{2}^{-}\left(\mathrm{CO}_{2}\right)_{\mathrm{n}}{ }^{6}$ clusters. Other groups have used anion PES to study $\mathrm{IXe}_{\mathrm{n}}{ }^{7},\left(\mathrm{CO}_{2}\right)_{\mathrm{n}}^{-8} \mathrm{O}^{-} \mathrm{Ar}_{\mathrm{n}},{ }^{9} \mathrm{X}^{-}\left(\mathrm{H}_{2} \mathrm{O}\right)_{\mathrm{n}}{ }^{10},{ }^{11}, 12$ and $\mathrm{X}^{-}\left(\mathrm{CH}_{3} \mathrm{CN}\right)_{\mathrm{n}}{ }^{13}$. From this PES work, one can determine the EAs of the clusters, and from these make inferences about their binding energies, but cannot study the details of the low-frequency van der Waals vibrational structure.

\subsubsection{Anion ZEKE and threshold photodetachment spectroscopy}

Anion zero electron kinetic energy (ZEKE) spectroscopy, the primary focus of this work, offers significant advantages over conventional PES for the study of clusters. With ZEKE spectroscopy we are able to obtain a resolution of at best $1 \mathrm{~cm}^{-1}$, but more 
typically 2-3 $\mathrm{cm}^{-1}$ (or 0.1-0.4 meV), nearly two orders of magnitude better than conventional PES. This resolution is sufficient to observe the vibrational structure of small van der Waals clusters, as well as to determine very accurate EAs. In addition, regardless of the resolution, one can have more confidence in the accuracy of EAs determined from ZEKE (or threshold photodetachment) spectroscopy because the $E A s$ determined from PES are based on measurement of electron kinetic energies, which are subject to various systematic errors (such as space charge effects), whereas in ZEKE and threshold photodetachment the energy calibration depends only on the calibration of the tunable lasers used in these techniques. The technique is not without its limitations, however, as will be discussed below.

The basic concept of ZEKE spectroscopy was first applied to the photoionization of neutral molecules by Müller-Dethlefs, Schlag and co-workers. ${ }^{14,15}$ Since this time ZEKE (also known and pulsed field ionization, or PFI) spectroscopy of neutrals has been employed by many groups to study a wide variety of systems. ${ }^{16}$ There has also been extensive theoretical work undertaken to understand the processes taking place in neutral ZEKE-PFI experiments (see, for example, Ref. 17)

ZEKE spectroscopy was first applied to the photodetachment of anions by Neumark and co-workers. ${ }^{18}$ In contrast to the rapid growth in the field of neutral ZEKEPFI, subsequent work in the field of anion ZEKE spectroscopy has been confined to a small number of other groups. ${ }^{19-21}$ This is due for the most part to the fundamental difference between ZEKE-PFI of neutrals, in which molecules are excited to long-lived Rydberg states and then ionized by a pulsed electric field, and ZEKE of anions, in which the electron is photodetached from the anion in the first step because anions do not 
posess long-lived Rydberg states. However, despite its difficulty, anion ZEKE spectroscopy has proven to be a unique and invaluable tool for the study of clusters, and we exepect it will continue to be in the future.

In anion $\mathrm{ZEKE}$ spectroscopy, as in anion PES, one begins by creating a massselected packet of anions using pulsed molecular beam techniques, so that one may isolate the species of interest. In ZEKE spectroscopy, one studies the photodetachment process of the anions by scanning a tunable laser over the region of spectroscopic interest, with the apparatus arranged so that only electrons detached with very nearly zero kinetic energy are detected. Electrons ejected with excess kinetic energy are not detected. In this way one obtains a spectrum with peaks corresponding to anion $\rightarrow$ neutral transitions as a function of laser energy. This process is shown in schematic form in Figures 2.3 and 2.4.
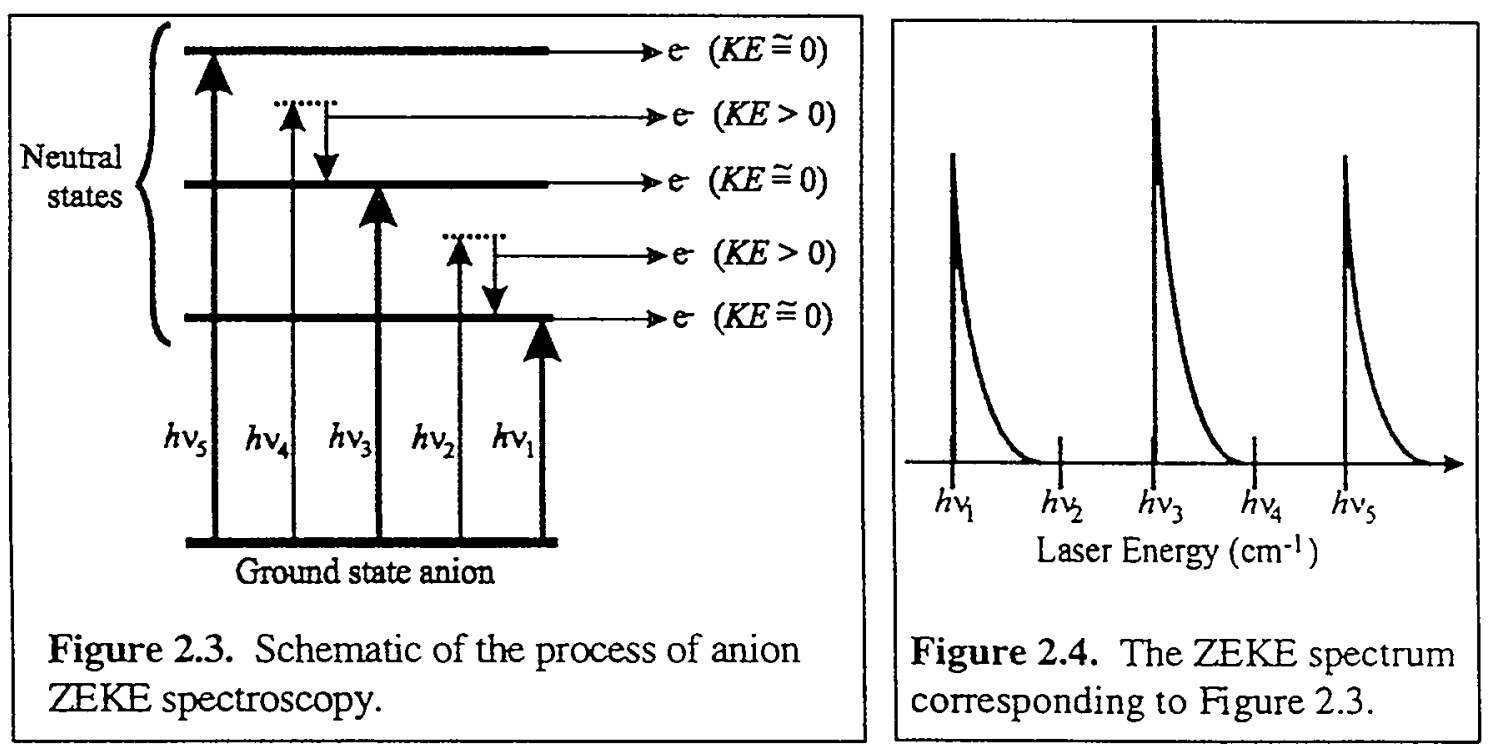

The key to the success of the ZEKE technique is the ability to discriminate against the detection of electrons with excess kinetic energy. This is accomplished through a combination of spatial and temporal filtering of the photoelectrons. These techniques 
will be described in Section 2.2. Here we comment on the asymmetric line shape observed in ZEKE spectra and shown schematically in Figure 2.4. This lineshape appears because the onset of the detachment threshold is much sharper than the discrimination function of the ZEKE spectrometer, so that one tends to see peaks with "tails" at high energy, due to incomplete discrimination against high energy electrons. Refer to Chapter 3 for a discussion of the detailed form of the ZEKE lineshape. In the next section we discuss the nature of the photodetachment cross-sections near threshold and its ramifications for ZEKE spectroscopy, as well as the partially discriminated threshold photodetachment (PDTP) experiments described in Chapter 4.

\subsubsection{Wigner's Law}

The problem of the total photodetachment cross section near threshold was first addressed by Wigner, ${ }^{22}$ who proposed what has become known as "Wigner's Law." If $\ell$ is the orbital angular momentum quantum number of the detached electron, then the photodetachment cross section, $\sigma$, according to Wigner's Law is

$$
\sigma \propto E^{\ell+1 / 2}
$$

where $E$ is the kinetic energy of the detached electron, which is equal to $h v-E_{0}$, where $h v$ is the photon energy and $E_{0}$ is the threshold energy. This relation places restrictions on the systems that may be studied with anion ZEKE spectroscopy. Only those thresholds with sharply rising cross sections can be observed with ZEKE spectroscopy. For the cross section to rise sharply at the threshold, it is necessary to have $\ell=0$. This occurs, for example, when an electron is detached from a p-orbital of an atom. In order for angular momentum to be conserved when the a photon is absorbed, the orbital angular 
momentum of an electron must change according to the selection rule $\Delta \ell= \pm 1$ when it is ejected from an atom. Thus when an electron is photodetached from a p-orbital, it will have $\ell=0$ (s-wave detachment) or $\ell=2$ (p-wave detachment). ZEKE spectroscopy is only sensitive to s-wave electrons.

Wigner's law has been experimentally verified for a number of atoms, 23.27 however the range of validity of the Wigner law has been open to question. A recent study of the total photodetachment cross-section of $\mathrm{Al}^{-}$by Calabrese et. al. ${ }^{28}$ found good agreement with Wigner's law up to $13 \mathrm{meV}\left(105 \mathrm{~cm}^{-1}\right)$ above threshold. Above this, however, these workers found that the experimental cross-section deviates significantly from the prediction of Equation (2.1). They in fact found a leveling off and decrease in the total cross-section, whereas Wigner's law predicts a monotically monotonically increasing cross section. In Figure 2.5 we show the total cross-section of $\mathrm{I}^{-}$measured in this laboratory, and see that it is well fit by the Equation (2.1) up to $150 \mathrm{~cm}^{-1}(19 \mathrm{meV})$ above threshold. 


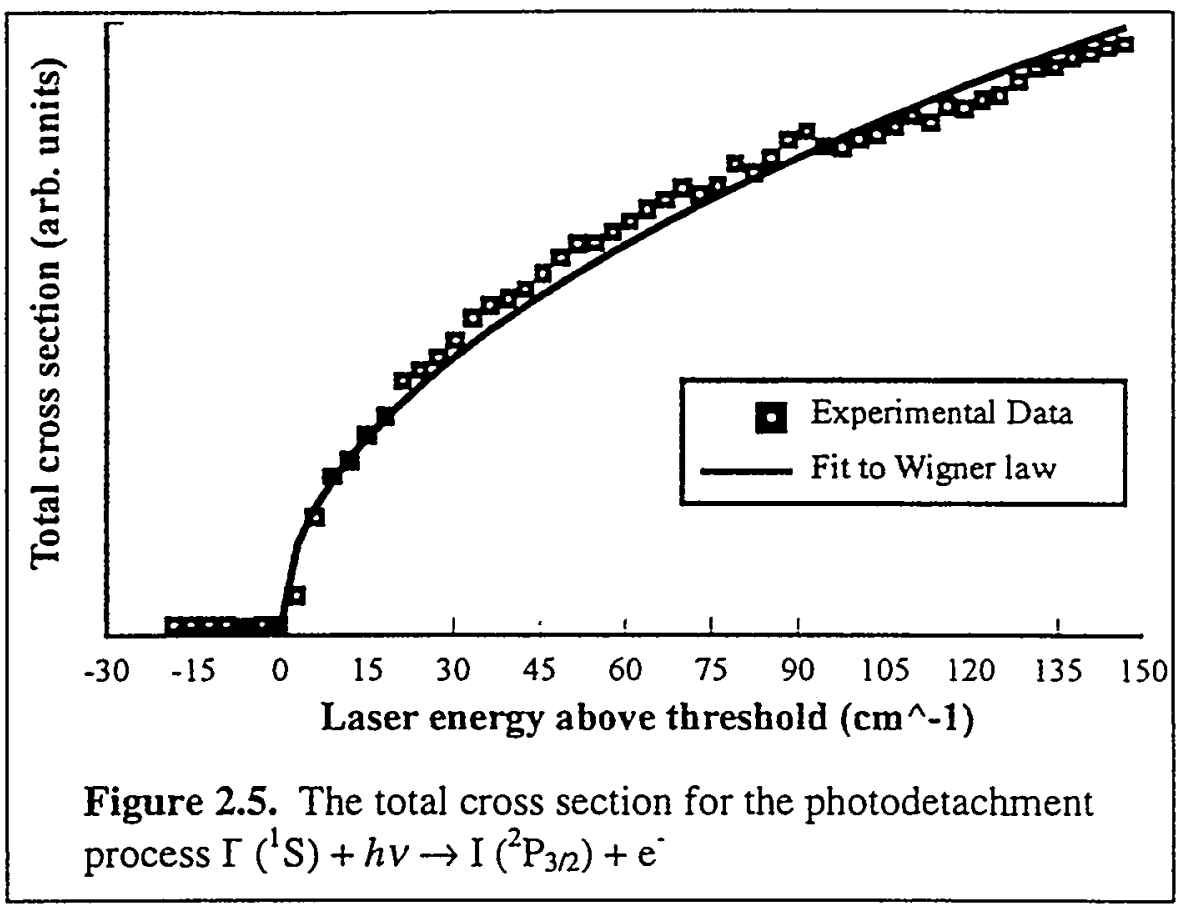

The total cross section was not measured at higher energies, so this result can only be considered as a lower bound on the range of validity of Wigner's law for the case of I: This result is of little significance for ZEKE spectroscopy, but is of some interest for the "partially discriminated threshold photodetachment" (PDTP) experiments on $\mathrm{Rg}_{\mathrm{n}} \mathrm{X}^{-}$ clusters discussed in chapter 4.

Another question worth raising with regard to Wigner's law is whether its range of validity changes as more solvent atoms are added to the halide chromophore of a cluster. Theoretical work by $\mathrm{O}^{\prime}$ Malley ${ }^{29}$ suggested a correction term to the Wigner law proportional to the polarizability of the neutral left behind by the departing electron. For s-wave detachment, O'Malley's formulation of the cross-section behavior is given in atomic units by

$$
\sigma \propto E^{1 / 2}\left[1-\frac{4}{3} \alpha_{d} E \ln (2 E)+O(E)\right]
$$


where $\alpha_{d}$ is the polarizability of the neutral after photodetachment. One might wonder from this if adding polarizable solvent atoms to the halide would influence the observed PDTP spectra. From our studies of $\mathrm{Ar}_{n} \mathrm{I}^{-}$and $\mathrm{Ar}_{n} \mathrm{Br}^{-}$clusters--to be discussed in Chapter 4--this appears not to be the case for Ar atoms. However, whether this effect may influence the observed detachment spectra of halide atoms clustered with more polarizable rare gases, such as the $\mathrm{Xe}_{\mathrm{n}}{ }^{-}$clusters recently studied in this group ${ }^{30}$ remains to be seen.

Wigner's law has been extended to understand the near-threshold behavior of molecular systems. ${ }^{31}$ Because the systems studied in this work may be thought of essentially as perturbed 'S halide atoms, we shall not concern ourselves with the details of this work. For an interesting discussion of deviations from the Wigner law for molecules with strong dipole moments, see the recent experimental results of Lineberger and co-workers on photodetachment of the $\mathrm{OH}^{-}$anion. ${ }^{32}$ This work is not of direct relevance here, where we are concerned with non-polar rare-gas solvents, but may be of some importance for the interpretation if the ZEKE spectra of clusters containing highly polar solvent molecules.

\subsection{The experimental apparatus}

The details of the design and operation of the experimental apparatus have been described previously, 33,34 and specific experimental details are given in Chapters 3 and 4 of this work. Therefore here we briefly summarize the operation of the apparatus, and then focus on recent improvements in its design.

The experimental apparatus is shown schematically in Figure 2.6. 


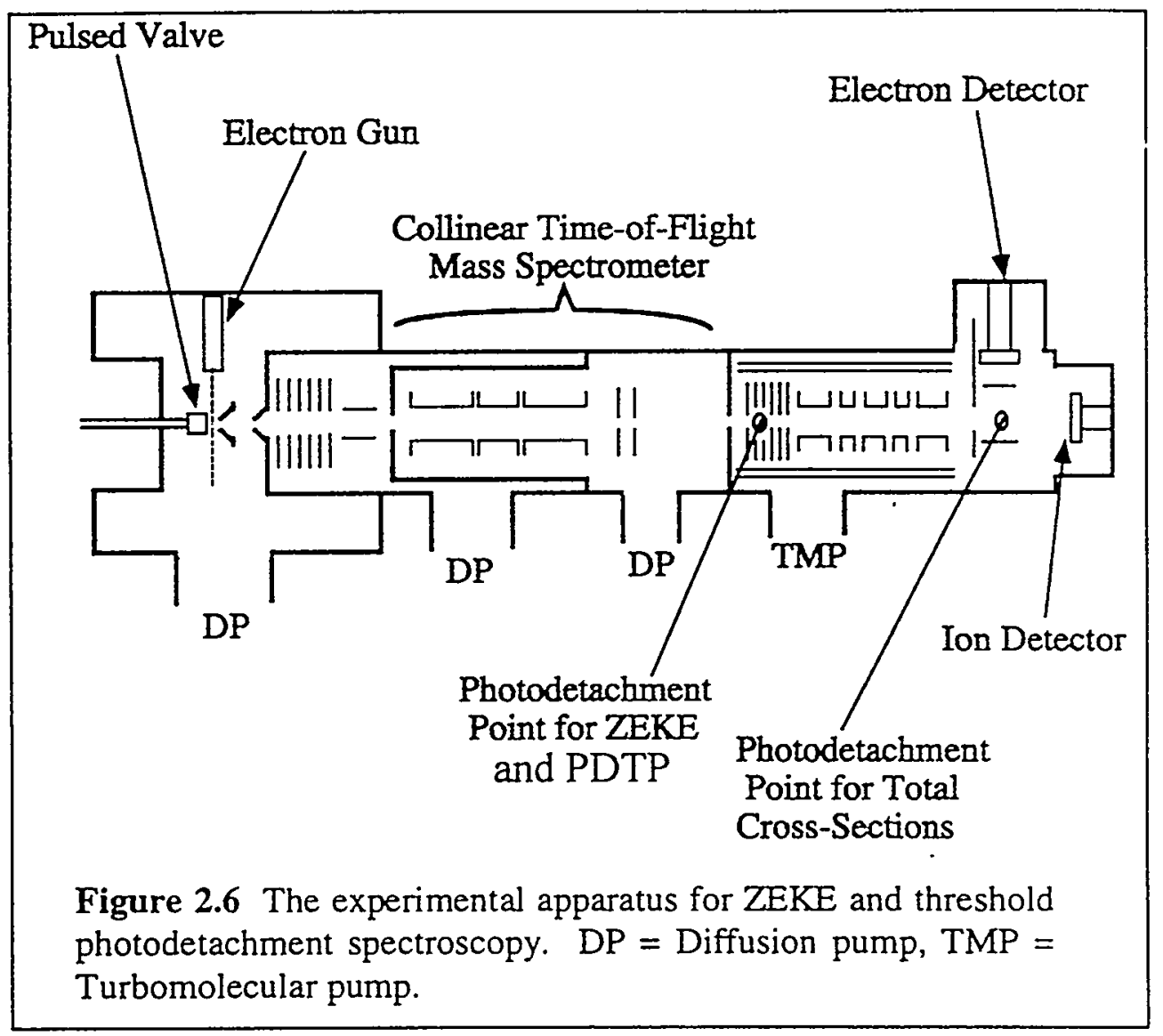

The cold anions are produced in a supersonic expansion from a pulsed valve crossed with a $1 \mathrm{keV}$ electron beam in the source chamber shown on the left-hand side of the diagram. The expansion passes through two skimmers and enters the first differentially pumped region, where the ions are accelerated to $1 \mathrm{keV}$. The second skimmer placed very close to the pulsed valve was found to increase production of large clusters, and this "double skimmer" setup is described in more detail in Section 2.2.1. The ions enter the second differentially pumped region and are separated according to mass using a Bakker-type (collinear) time-of-flight arrangement. ${ }^{35}$ Finally, the ions enter the detector region shown on the right hand side of the diagram where the mass-selected ion packet of interest is irradiated with a pulsed laser. The electrons are extracted by a weak electric field, and deflected $90^{\circ}$ upward to the microchannel plate electron detector. The ion signal is 
measured at the microchannel plate detector at the far end of the machine. Total cross sections, such as that shown in Figure 2.5, may be measured by detaching the ions at a point directly beneath the electron detector, so that effectively $100 \%$ of the detached electrons are detected.

When operating in partially discriminated threshold photodetachment (PDTP) mode, the electrons are intersected by the laser at a point approximately $0.5 \mathrm{~m}$ from the electron detector, and immediately extracted with a weak electric field (ca. 1 to $3 \mathrm{~V} / \mathrm{cm}$ ) applied to the extraction plates. In this mode, off-axis high-energy electrons are discriminated against. This mode of operation is equivalent to the "steradiancy detector" described by Spohr et al. ${ }^{36}$ When optimized for maximum electron signal, the resolution in this mode of operation is about $150 \mathrm{~cm}^{-1} .37$ However, we have found that by using very low extraction voltages and carefully tuning the electron einzel lens--pictured to the right of the electron extraction plates--to regulate the effective "aperture" size of the steradiancy detector, resolution on the order of $10 \mathrm{~cm}^{-1}$ may be achieved in PDTP mode. This enhanced resolution, however, comes at the expense of dramatically decreased electron signal.

To take the ZEKE spectrum of an anion, the anion is photodetached at the same point used for PDTP, however rather than extracting immediately, the electron extraction is delayed by $250-500$ ns. During this "waiting period," the higher energy electrons disperse. Thus the "steradiancy effect" for discriminating high energy electrons is greatly enhanced. Furthermore, during the delay, the electrons that are scattered along the beam axis have time to spread out, so that those with different kinetic energies experience different acceleration because of the differing amounts of time they spend in the 
extraction field. When the electrons are detected, a 30-120 ns wide gate is used to isolate those with initially zero kinetic energy in the ion beam frame of reference. This gated detection is what is meant by "temporal discrimination," however in the sense that the differences in arrival times of the electrons at the detector are due to the initial spreadingout of the on-axis scattered electrons, this may be also may be thought of as another form of spatial discrimination. In the original design of the ZEKE spectrometer the electrons experienced no electric field during the delay between photodetachment and extraction. Recently, however, we have found that by applying a very small (ca. $10 \mathrm{mV} / \mathrm{cm}$ ) uniform constant electric field to the extraction plates, with polarity opposite to the that of the extraction field, the ZEKE signal is greatly enhanced, with no loss of resolution. The details of this "electron deceleration field" will be described in Section 2.2.2.

\subsection{1 "Double skimmer" setup for large cluster production}

As mentioned above, it has been found that placing an additional skimmer very close to the opening of the pulsed valve greatly enhances the production of large van der Waals clusters. The skimmer used for this is manufactured by the Beam Dynamics Company, and is contoured, with a $2 \mathrm{~mm}$ orifice. It is placed $1 \mathrm{~mm}$ from the opening of the pulsed valve (General Valve Corp) which is used with a $0.5 \mathrm{~mm}$ or $0.8 \mathrm{~mm}$ orifice. This second skimmer is contained within the source chamber; that is, there is no differential pumping on either side of it. The opening of this skimmer is placed $2 \mathrm{~cm}$ from the opening of the conical skimmer which separates the source chamber from the first differentially pumped region. This setup is pictured in Figure 2.7. 


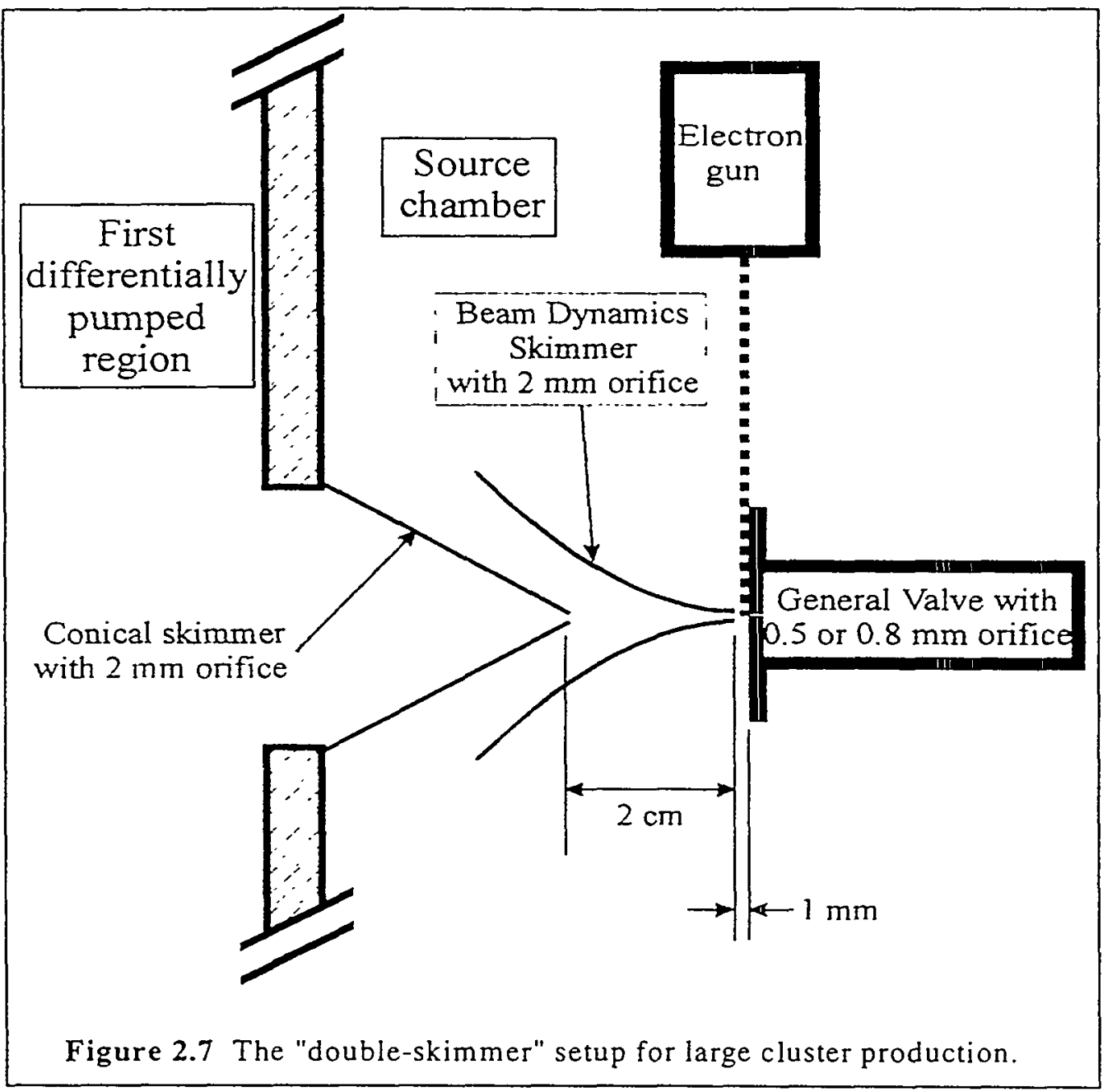

An example of the increased cluster production using this source is shown in Figure 2.8, which shows the mass spectrum of $\mathrm{Ar}_{\mathrm{n}} \mathrm{Cl}^{-}$clusters. The mass spectra with and without the second skimmer in place are shown in Figures 2.8(a) and 2.8(b), respectively. We see that without the second skimmer, the cluster with the greatest intensity is $\mathrm{ArCl}^{-}$ and the ion signal of the larger clusters decreases, until it becomes very difficult to observe clusters larger than $\mathrm{Ar}_{7} \mathrm{Cl}^{-}$. On the other hand, with the double skimmer setup, we can easily observe clusters up to $\mathrm{Ar}_{12} \mathrm{Cl}$. The ion signal for clusters with $n$ larger than 12 drops suddenly, possibly due to a solvent shell closing at this point ${ }^{38}$ however clusters up to $\mathrm{Ar}_{19} \mathrm{Cl}^{-}$are still plainly visible in the mass spectrum. This same enhancement in 
large cluster production has also been observed in the mass spectra of other systems studied in this laboratory, including $\mathrm{Xe}_{\mathrm{n}} \mathrm{I}^{-30}$ and $\mathrm{Cl}^{-}\left(\mathrm{N}_{2}\right)_{\mathrm{n}}{ }^{39}$.

The reason for the success of this scheme for producing large anion clusters is not certain. Relative to the knowledge about the formation of neutral clusters in supersonic expansions, ${ }^{40.41}$ little is known about the clustering processes that take place in this type of anion source. It is possible that the skimmer near the pulsed valve acts as a kind of "clustering channel" allowing more collisions to take place between the halide anions and rare-gas atoms in this confined space, so that larger clusters are formed. 

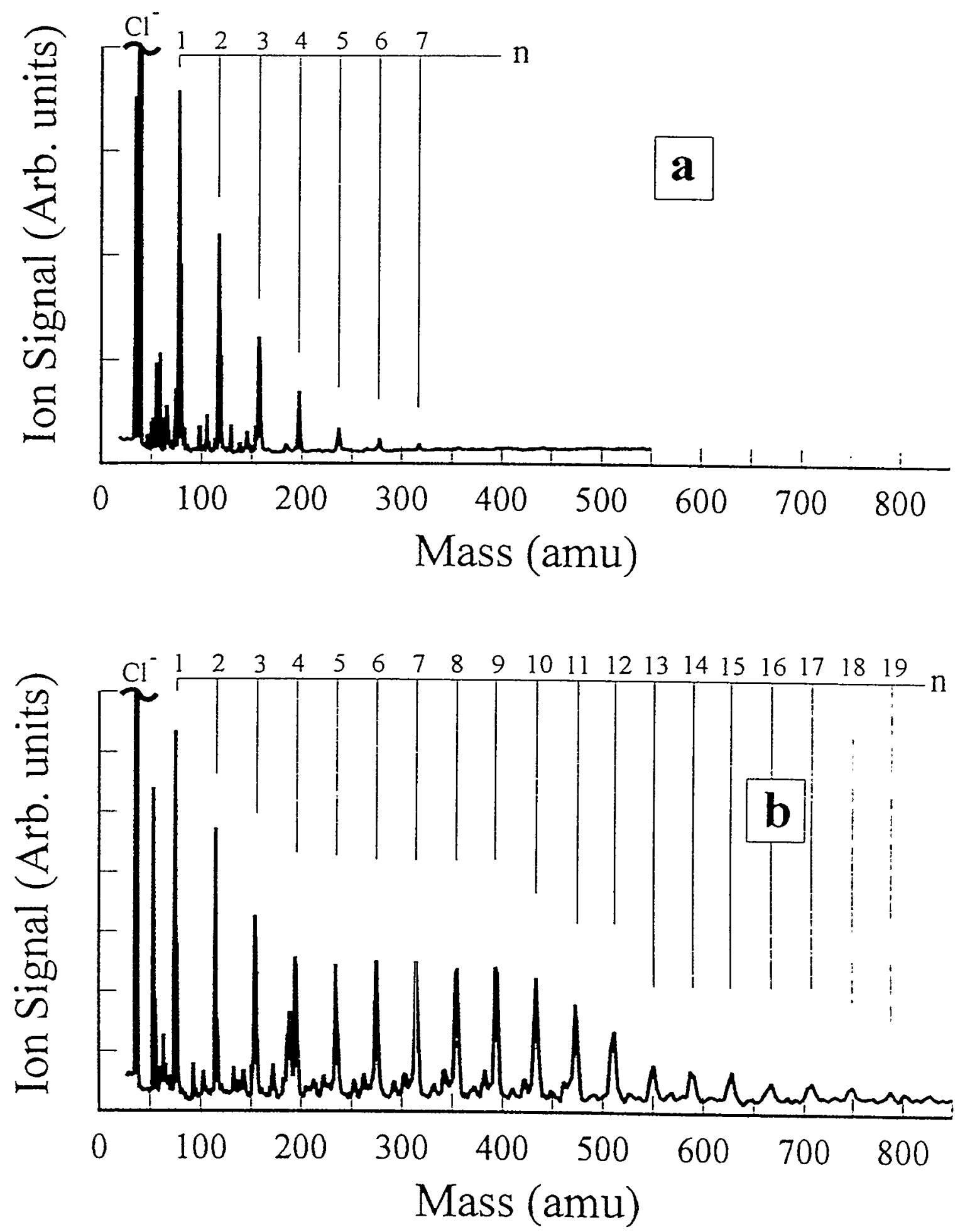

Figure 2.8. The mass spectra of $\mathrm{Ar}_{\mathrm{n}} \mathrm{Cl}$ : (a) Without the second skimmer near the pulsed valve. (b) With the double-skimmer setup shown in Figure 2.7. The impurity peak at $53 \mathrm{amu}$ is $\mathrm{Cl}^{-}\left(\mathrm{H}_{2} \mathrm{O}\right)$. 


\subsubsection{Electron deceleration field}

We mentioned above our finding that the application of a weak, positive electric field to the electron extraction plates results in enhancement of the observed ZEKE signal. Here we describe the details of the implementation of this scheme.

The extraction field is applied to of a series of seven molybdenum plates spaced 1 $\mathrm{cm}$ apart. Photodetachment takes place between the first two plates. The field is applied such that there is a potential gradient across the first four of the plates. The voltage for electron deceleration is applied at the same place. A schematic diagram of the circuit used to apply the deceleration voltage is shown in Figure 2.9.

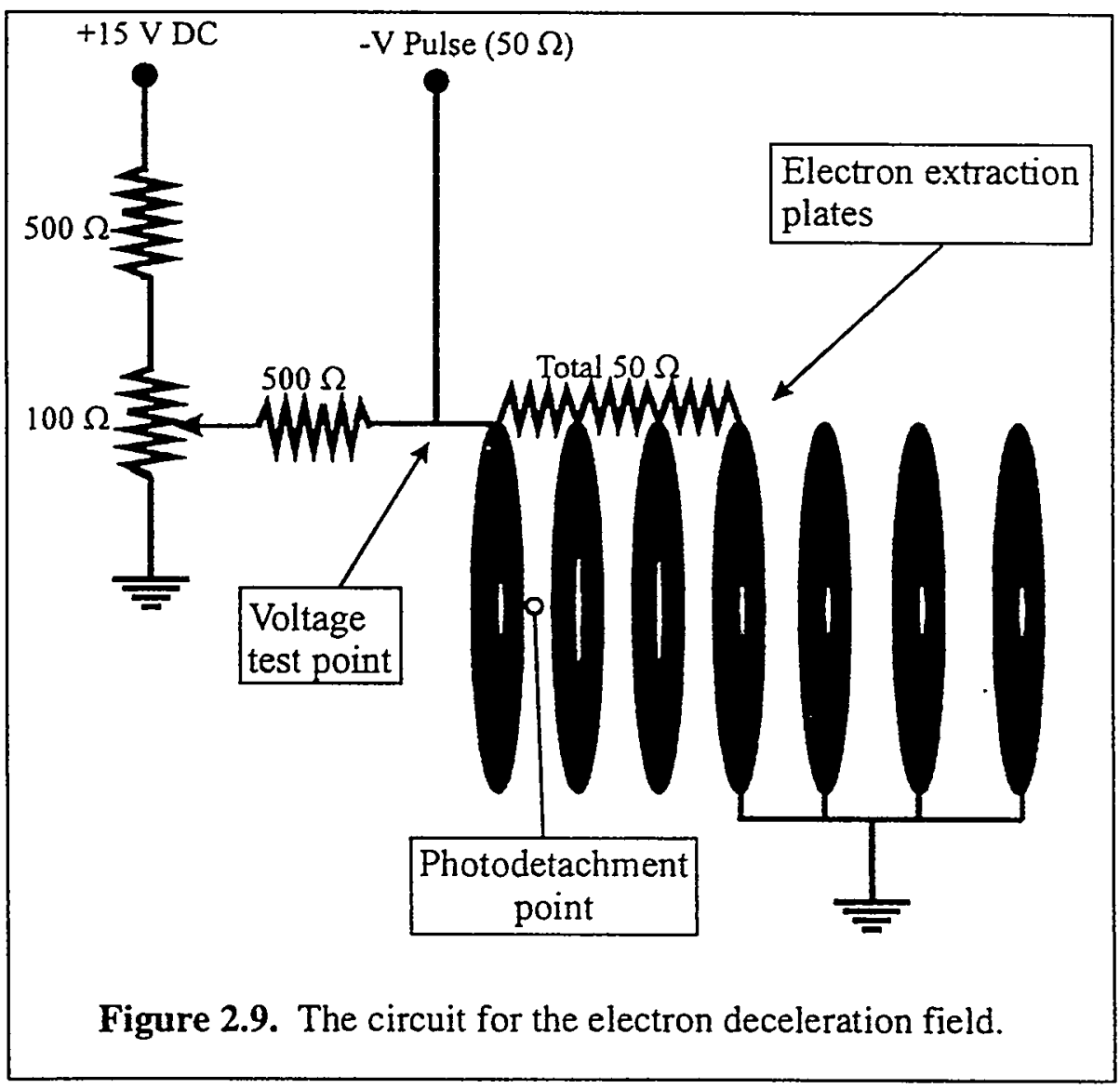

This is a simple voltage divider circuit, but a few points should be borne in mind in its use. The values shown for the resistances should not be changed too much, because if the 
impedance from the circuit it too high or low it will interfere with the pulsed extraction. The values of the resistances shown were chosen empirically to minimize interference with the extraction pulse. Also this circuit should have its own stable power supply. not used by any other device in the apparatus, to ensure stability and reproducibility of the voltage. Finally, when testing the voltage at the indicated test point with a voltmeter or oscilloscope, one should not leave the probe connected when the experiment is running, because the probe will disrupt the extraction pulse. The DC voltage should be measured with a high impedance probe, rather than with the $50 \Omega$ probe of an oscilloscope in order not to alter the voltage from the voltage divider.

An example of the improvement in signal with the electron deceleration field is shown in Figure 2.10. This figure shows the ZEKE spectrum of the $\mathrm{Cl}^{-}$atom (detaching to the ground ${ }^{2} \mathrm{P}_{3 / 2}$ state of $\mathrm{Cl}$, without [Fig. 2.10(a)] and with [Fig 2.10(b)] the electron deceleration field circuit in place. In both cases, the spectra are accumulated over 400 laser shots per point. The voltage measured at the test point with a high impedance probe was, $8 \mathrm{mV}$, corresponding to an electric field of about $3 \mathrm{mV} / \mathrm{cm}$. Both ordinates in the figure are calibrated to the absolute number of electrons collected. The resolution in both cases is $2 \mathrm{~cm}^{-1}$ FWHM, but we see a nearly three-fold increase in ZEKE signal when the electron deceleration circuit is used. It is found that with the deceleration field, much longer delay times can be employed. For example, when detaching the $\mathrm{Cl}^{-}$atom it was possible to see ZEKE signal at delay times of up to $1 \mu \mathrm{s}$. However, the resolution seemed to deteriorate at delay times above $500 \mathrm{~ns}$. We find that when using the deceleration field, it is necessary to use higher deceleration voltages when shorter delays are employed to achieve optimal results. 


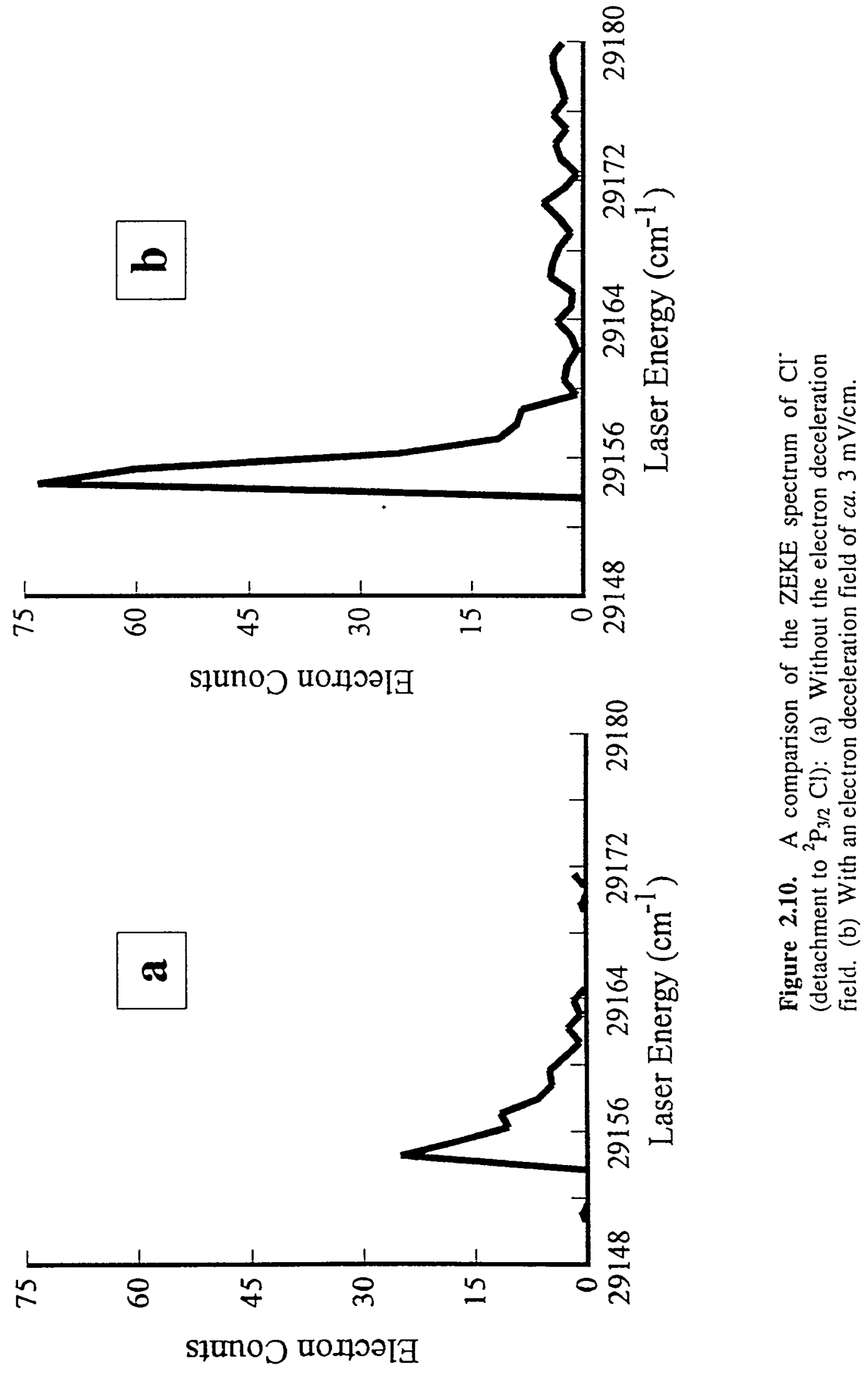


The reason for the improved ZEKE signal seen with this arrangement is not known with certainty. One possibility is that the deceleration field separates the detached electrons from the center of mass of the remaining undetached anions to a great enough extent that they are less subject to dispersal by the space charge of the remaining anions. It is also possible that the longer delay times are made possible by the deceleration of the electrons, which--especially light atoms like $\mathrm{Cl}^{-}$--would otherwise travel out of the extraction field within a few hundred ns. The increased electron signal may also be due to the fact that the decelerated electrons experience greater acceleration when the extraction field is applied, because they begin further up on the extraction potential gradient, and hence are better separated and collected with greater efficiency. 


\subsection{References for Chapter 2}

1 Z. Bacic and R. E. Miller, J. Phys. Chem. 100, 12945 (1996).

2 A. Weaver, Ph.D. Thesis, University of California, Berkeley, 1991.

3 D. W. Arnold, S. E. Bradforth, E. H. Kim, and D. M. Neumark, J. Chem. Phys. 102, 3493 (1995).

4 D. W. Arnold, S. E. Bradforth, E. H. Kim, and D. M. Neumark, J. Chem. Phys. 102, 3510 (1995).

5 B. J. Greenblatt, M. T. Zanni, and D. M. Neumark, Science 276, 1675 (1997).

${ }^{6}$ M. T. Zanni, T. Taylor, B. J. Greenblatt, and D. M. Neumark, (to be published).

7 I. Becker, G. Markovich, and O. Cheshnovsky, Phys. Rev. Lett. 79, 3391 (1997).

8 T. Tsukuda, M. A. Johnson, and T. Nagata, Chem. Phys. Lett. 268, 429 (1997).

9 S. T. Arnold, J. H. Hendricks, and K. H. Bowen, J. Chem. Phys. 102, 39 (1995).

10 G. Markovich, R. Giniger, M. Levin, and O. Cheshnovsky, J. Chem. Phys. 95, 9416 (1991).

11 G. Markovich, S. Pollack, R. Giniger, and O. Cheshnovsky, Z. Physik D 26, 98 (1993).

12 G. Markovich, S. Pollack, R. Giniger, and O. Cheshnovsky, J. Chem. Phys. 101, 9344 (1994).

13. G. Markovich, L. Perera, M. L. Berkowitz, and O. Cheshnovsky, J. Chem. Phys. 105, 2675 (1996).

14 K. Müller-Dethlefs, M. Sander, and E. W. Schlag, Z. Naturforsch. Teil A 39, 1089 (1984). 
15 K. Müller-Dethlefs, M. Sander, and E. W. Schlag, Chem. Phys. Lett. 12, 291 (1984).

16 K. Müller-Dethlefs, J. Electron Spectrosc. and Related Phenomena 75, 35 (1995).

17 P. Bellomo, D. Farre!ly, and T. Uzer, J. Phys. Chem. A 101, 8902 (1997).

18 T. N. Kitsopoulos, I. M. Waller, J. G. Loeser, and D. M. Neumark, Chem. Phys. Lett. 159,300 (1989).

19 G. Gantefor, D. M. Cox, and A. Kaldor, J. Chem. Phys. 94, 854 (1990).

20 G. Drechsler, C. Bassmann, U. Boesl, and E. W. Schlag, Z. Naturforsch. 49A, 1256 (1994).

21 C. Bassmann, U. Boesl, D. Yang, G. Drechsler, and E. W. Schlag, Intl. J. Mass Spec. Ion Proc. 159, 153 (1996).

22 E. P. Wigner, Phys. Rev. 73, 1002 (1948).

23 W. C. Lineberger and B. W. Woodward, Phys. Rev. Lett. 25, 424 (1970).

24 H. Hotop, T. A. Patterson, and W. C. Lineberger, Phys. Rev. A 8, 762 (1973).

25 D. Feldmann, Chem. Phys. Lett. 47, 338 (1977).

26 H. Hotop and W. C. Lineberger, J. Chem. Phys. 58, 2379 (1973).

27 K. R. Lykke, K. K. Murray, and W. C. Lineberger, Phys. Rev. A 43, 6104 (1991).

28 D. Calabrese, A. M. Covington, J. S. Thompson, R. W. Marawar, and J. W. Farley, Phys. Rev. A 54, 2797 (1996).

29 T. F. O'Malley, Phys. Rev. 137, A1668 (1964).

30 T. Lenzer, M. Furlanetto, N. Provinka, and D. M. Neumark, (to be published).

31 K. J. Reed, A. H. Zimmerman, H. C. Andersen, and J. I. Brauman, J. Chem. Phys. 64, 1368 (1976). 
32 J. R. Smith, J. B. Kim, and W. C. Lineberger, Phys. Rev. A 55, 2036 (1997).

33 T. N. Kitsopoulos, Ph.D. Thesis, University of California, 1991.

34 C. C. Arnold, Ph.D. Thesis, University of California, 1994.

35 M. B. Bakker, J. Phys. E 7, 364 (1974).

36 R. Spohr, P. M. Guyon, W. A. Chupka, and J. Berkowitz, Rev. Sci. Instrum. 42, 1872 (1971).

37 C. J. Chick, Y. Zhao, T. N. Kitsopoulos, and D. M. Neumark, J. Chem. Phys. 97, 6121 (1992).

38 T. Lenzer, I. Yourshaw, M. Furlanetto, and D. M. Neumark, (to be published).

39 I. Yourshaw, T. Lenzer, and D. M. Neumark, (unpublished results).

40 O. F. Hagena, Surface Science 106, 101 (1981).

41 S. DePaul, D. Pullman, and B. Friedrich, J. Phys. Chem. 97, 2167 (1993). 
Chapter 3. Zero electron kinetic energy (ZEKE) spectroscopy of the $\mathrm{KrBr}^{\circ}$, XeBr, and $\mathrm{KrCl}^{-}$anions*

\begin{abstract}
Three rare-gas halide $(\mathrm{RgX})$ anions, $\mathrm{KrBr}^{-}, \mathrm{XeBr}^{-}$, and $\mathrm{KrCl}^{-}$, and the corresponding neutral, open-shell van der Waals complexes are studied with anion zero electron kinetic energy (ZEKE) spectroscopy. The spectra for each system reveal well-resolved progressions in the low frequency vibrations of the anion and one or more of the three neutral electronic states accessed by photodetachment, providing a detailed spectroscopic probe of the $\mathrm{Rg}-\mathrm{X}^{*}$ and $\mathrm{Rg}-\mathrm{X}$ interaction potentials. In the case of $\mathrm{KrBr}$, transitions to all three of the "covalent" neutral electronic states (the $X \frac{1}{2}, I \frac{3}{2}$, and $I I \frac{1}{2}$ states) were observed. For $\mathrm{XeBr}^{-}$, transitions to the $X \frac{1}{2}$ and $I I \frac{1}{2}$ neutral states were observed. For $\mathrm{KrCl}$, only the $X \frac{1}{2}$ state could be studied. From our data, we construct model potentials for the anion and each observed neutral state, and these are compared with other experimental and theoretical potentials.
\end{abstract}

"Submitted to J. Chem. Phys. in slightly different form with co-authors Thomas Lenzer, Georg Reiser, and Daniel M. Neumark. 


\subsection{Introduction}

The characterization of the forces between weakly interacting species has attracted a great deal of experimental and theoretical attention in recent years. The interaction potentials between closed shell neutral species have been characterized in considerable detail as a result of this effort. 1,2 However, less is known about the interactions between open and closed shell atoms, or about those between ions and neutrals. In this work we describe new experimental results involving the latter two types of interactions. We report the results of studies of the rare gas-halide atom complexes $\mathrm{KrBr}, \mathrm{XeBr}^{*}$, and $\mathrm{KrCl}^{-}$using anion zero electron kinetic energy (ZEKE) spectroscopy. In these experiments we obtain spectroscopic information on both the neutral and negatively charged complex and derive accurate potentials for both the anion and neutral species. This work is a continuation of our earlier ZEKE studies of the ArBr, $\mathrm{KrI}$, and ArI complexes, ${ }^{3,4}$ and is part of an ongoing effort to obtain ZEKE spectra of the complete series of rare gas halides.

The rare gas halide $(\operatorname{RgX})$ species are of interest because the $\mathrm{Rg} \cdot \mathrm{X}^{-}$interaction potentials determine the transport properties of halide ions in rare gases; these are important in understanding plasmas and gas discharges. Prior to the work reported here, the only previous experimental results on the $\mathrm{KrBr}^{-}, \mathrm{XeBr}^{-}$and $\mathrm{KrCl}^{-}$anions came from ion mobility studies, 5.6 from which potentials can be obtained by iterative fitting or direct inversion. Interaction potentials had also been derived within the framework of theoretical ${ }^{7,8}$ and semi-empirical ${ }^{9-11}$ models. The work described here provides a direct spectroscopic probe of these species.

The rare gas-halogen $(\mathrm{RgX})$ complexes are important for their use in excimer lasers, in which lasing takes place between electronically excited, strongly bound charge transfer states and the repulsive wall of the weakly bound covalent ground states. 12 Excimer emission has also provided spectroscopic information on the charge-transfer and covalent states. In the cases of $\mathrm{KrBr}$ and $\mathrm{KrCl}$, emission from the $\mathrm{RgX}$ charge transfer 
states to the ground state (the $B \rightarrow X$ band) is broad and relatively unstructured, 12 as is typical of bound-free transitions. However, recent emission studies of the $B \rightarrow X$ band in $\mathrm{XeBr}$ reveal extensive vibrational structure. ${ }^{13,14}$ The covalent states of rare gas-halogen neutrals have also been probed in a series of scattering experiments. Information on the $\mathrm{RgX}$ species studied here comes from differential cross-section crossed molecular beam experiments of Lee and coworkers, ${ }^{15}$ which yielded potentials for the $\mathrm{KrBr}$ and $\mathrm{XeBr}$ complexes, and from integral cross section measurements by Aquilanti and co-workers 16 who have characterized the potential of $\mathrm{KrCl}$.

The neutral interactions are of interest also because they are simple examples of open shell-closed shell interactions. The two spin-orbit states of the ${ }^{2} \mathrm{P}$ halogen atom interact with the rare gas to give rise to three molecular electronic states. ${ }^{17,18}$ The lower ${ }^{2} \mathrm{P}_{3 / 2}$ state is split by the electrostatic interaction into two components, corresponding to $\Omega=1 / 2$ (the $X \frac{1}{2}$ state, in the notation used here) and $\Omega=3 / 2$ (the $I \frac{3}{2}$ state), where $\Omega$ is the projection of the total electronic angular momentum along the internuclear axis. The upper ${ }^{2} \mathrm{P}_{1 / 2}$ halogen state gives rise to the $I I \frac{1}{2}(\Omega=1 / 2)$ state in the complex.

Anion ZEKE spectroscopy of rare gas halides probes the van der Waals well region of the covalent states; this complements earlier studies of emission from excimer states. Our experiments also complement the scattering experiments because, whereas the scattering cross-sections contain information about the absolute values of the bond length and well depths of the complexes, the ZEKE spectra are sensitive only to the relative differences between the anion and neutral potentials. However, in the ZEKE spectra one can observe vibrationally-resolved photodetachment transitions to the various neutral electronic states, whereas in the crossed beam experiments the contributions of the $X \frac{1}{2}$ and $I \frac{3}{2}$ states to the experimental signal are not clearly separated and must be extracted by an appropriate data inversion procedure. Also, in the crossed beam experiments involving $\mathrm{Br}$ or I atoms, the $I I \frac{1}{2}$ electronic state arising from the upper ${ }^{2} \mathrm{P}_{1 / 2}$ spin-orbit state of the halogen atom is generally not probed because the population of this 
state is negligible. In the ZEKE experiments, well-resolved spectra of the $I I \frac{1}{2}$ states of the $\mathrm{KrBr}$ and $\mathrm{XeBr}$ systems are seen, and accurate potentials can be derived for these states for the first time.

The anion potentials derived here are a significant improvement over previously available potentials. While our ground state potentials for $\mathrm{KrBr}$ and $\mathrm{XeBr}$ are essentially the same as those derived from scattering and excimer emission experiments, our excited states potentials represent improvements over previous work, particularly for the $I I \frac{1}{2}$ state. In the case of $\mathrm{KrCl}$, our spectra confirm the neutral potentials previously deduced from the scattering experiments.

This article is organized as follows: In Section 3.2 we describe the experimental setup for anion production and ZEKE spectroscopy. In Section 3.3 we present the ZEKE spectra of $\mathrm{KrBr}^{-}, \mathrm{XeBr}^{-}$and $\mathrm{KrCl}^{-}$, and assign the observed electronic and vibrational structure. Section 3.4 deals with the construction of model potentials for fitting the vibrational structure and rotational contours of the ZEKE spectra. Finally, we compare our potentials with other experimental and theoretical results in Section 3.5.

\subsection{Experimental}

ZEKE spectroscopy was originally developed by Müller-Dethlefs et al. for photoionization of neutrals. ${ }^{19-21}$ It was first applied to the study of anions by Neumark and co-workers. ${ }^{22}$ The anion ZEKE apparatus used here has been described in detail elsewhere. ${ }^{23-25}$ A brief description follows.

$\mathrm{KrBr}^{-}$and $\mathrm{XeBr}$ anions are produced by expanding a mixture of $0.2 \% \mathrm{CF}_{2} \mathrm{ClBr} /$ $10-30 \% \mathrm{Kr}$ (or $\mathrm{Xe}$ ) / balance $\mathrm{He}$ into vacuum through a $0.5 \mathrm{~mm}$ aperture in a pulsed valve (General Valve Corp.). The expansion is crossed near the pulsed valve with a $1 \mathrm{keV}$ electron beam produced with a thoria-coated iridium filament (Electron Technology). Halide anions are produced by dissociative attachment and other secondary processes, and clusters form as the supersonic expansion cools. $\mathrm{KrCl}^{-}$anions are produced by 
passing the $\mathrm{Kr} / \mathrm{He}$ mixture over a reservoir containing $\mathrm{CCl}_{4}$ at room temperature. Backing pressures were typically 20-80 psi.

The anions pass through a skimmer into a differentially pumped region and are accelerated to $1 \mathrm{keV}$ into a $1 \mathrm{~m}$ collinear time-of-flight mass spectrometer. The $\mathrm{KrCl}^{-}$ results were obtained using an additional skimmer in the source chamber placed very close to the beam valve. ${ }^{26}$ The clusters separate according to mass, and the species of interest is irradiated with a pulse from an excimer-pumped dye laser (Lambda Physik) operating at a repetition rate of $30 \mathrm{~Hz}$. After a 200-500 ns delay, the electrons are extracted coaxially with the ion beam using a pulsed electric field and detected approximately $1 \mathrm{~m}$ away with a microchannel plate detector. The electrons are detected in a 35-100 ns gate, so that as the laser wavelength is scanned, only electrons with nearly zero kinetic energy relative to the anion packet are detected. The resulting spectral resolution is about $1-2 \mathrm{~cm}^{-1}$ for atomic anions. The peaks observed in this work are somewhat broader because of unresolved rotational structure.

In order to study the $X \frac{1}{2}$ and $I \frac{3}{2}$ states, DMQ laser dye was used for $\mathrm{KrBr}$ and $\mathrm{XeBr}$ and PTP dye was used for $\mathrm{KrCl}$. The laser pulse energy was about $20 \mathrm{~mJ} / \mathrm{pulse}$ for $\mathrm{KrBr}$ and $\mathrm{XeBr}$, and about 3-10 mJ/pulse for $\mathrm{KrCl}$. These spectra were averaged over 1000-2000 laser shots/point. For the II $\frac{1}{2}$ states of $\mathrm{KrBr}$ and $\mathrm{XeBr}$, light from Rhodamine 640 dye was frequency-doubled with a KDP crystal, yielding laser pulse energies of $c a .2-4 \mathrm{~mJ} / \mathrm{pulse}$. The electron signal was normalized to the ion signal and to the laser pulse energy. Spectra for the $I I \frac{1}{2}$ states were averaged over about 8000 laser shots per point. When using DMQ and PTP dyes, the laser wavelength was calibrated using a Fe-Ne hollow cathode lamp. An iodine cell was used to calibrate the fundamental wavelength when Rhodamine 640 dye was frequency-doubled. The spectra were smoothed with a 5-point, second order Savitzky-Golay algorithm, ${ }^{27}$ which had a negligible effect on the relative peak intensities. 


\subsection{Results}

\subsection{1. $\mathrm{KrBr}$}

The ZEKE spectra of $\mathrm{KrBr}^{-}$are shown in Figure 3.1. We observe two band systems, shown in Figures 3.1(a) and 3.1(b), separated by approximately the spin-orbit constant of $\mathrm{Br}\left(3685 \mathrm{~cm}^{-1}\right)$. The lower energy band system in Figure 3.1(a) results from transitions to the $X \frac{1}{2}$ and $I \frac{3}{2}$ states, and the higher energy system in Figure 3.1(b) is due to the $I I \frac{1}{2}$ state.

Assignment of the vibrational and electronic features in Figure 3.1 is facilitated by our earlier studies of the $\mathrm{ArI}, \mathrm{ArBr}^{-}$and $\mathrm{KrI}^{-}$spectra. ${ }^{3}$ Specifically, the anion vibrational frequencies are expected to be considerably larger than the neutral frequencies, and this enables one to distinguish among the three types of neutral $\leftarrow$ anion vibrational transitions ( $\left.v^{\prime}-v^{\prime \prime}\right)$ that contribute to the spectra: vibrational progressions in the neutral originating from a single anion vibrational level $v ", \Delta v=0$ sequence band transitions from a series of vibrational levels of the anion, and $\Delta v \neq 0$ hot band transitions from vibrationally excited anion levels.

Fig. 3.1(a) is dominated by one peak, labeled 1 , with a set of smaller peaks, $a_{1}, b_{1}$, and $c_{1}$, spaced by about $15 \mathrm{~cm}^{-1}$ toward lower energy. A second, weaker progression is seen at higher energies than peak 1 with a characteristic peak spacing of $20 \mathrm{~cm}^{-1}$. We assign peak 1 to the origin $(0-0)$ transition from the anion to the $X \frac{1}{2}$ state. Peaks $a_{1}, b_{1}$ and $c_{1}$ are assigned to $\Delta v=0$ sequence band transitions from vibrationally excited anion states, i.e. the $1-1,2-2$, and 3-3 transitions. The dominance of $\Delta v=0$ transitions shows that the anion geometry is very similar to the neutral $X \frac{1}{2}$ state geometry. 

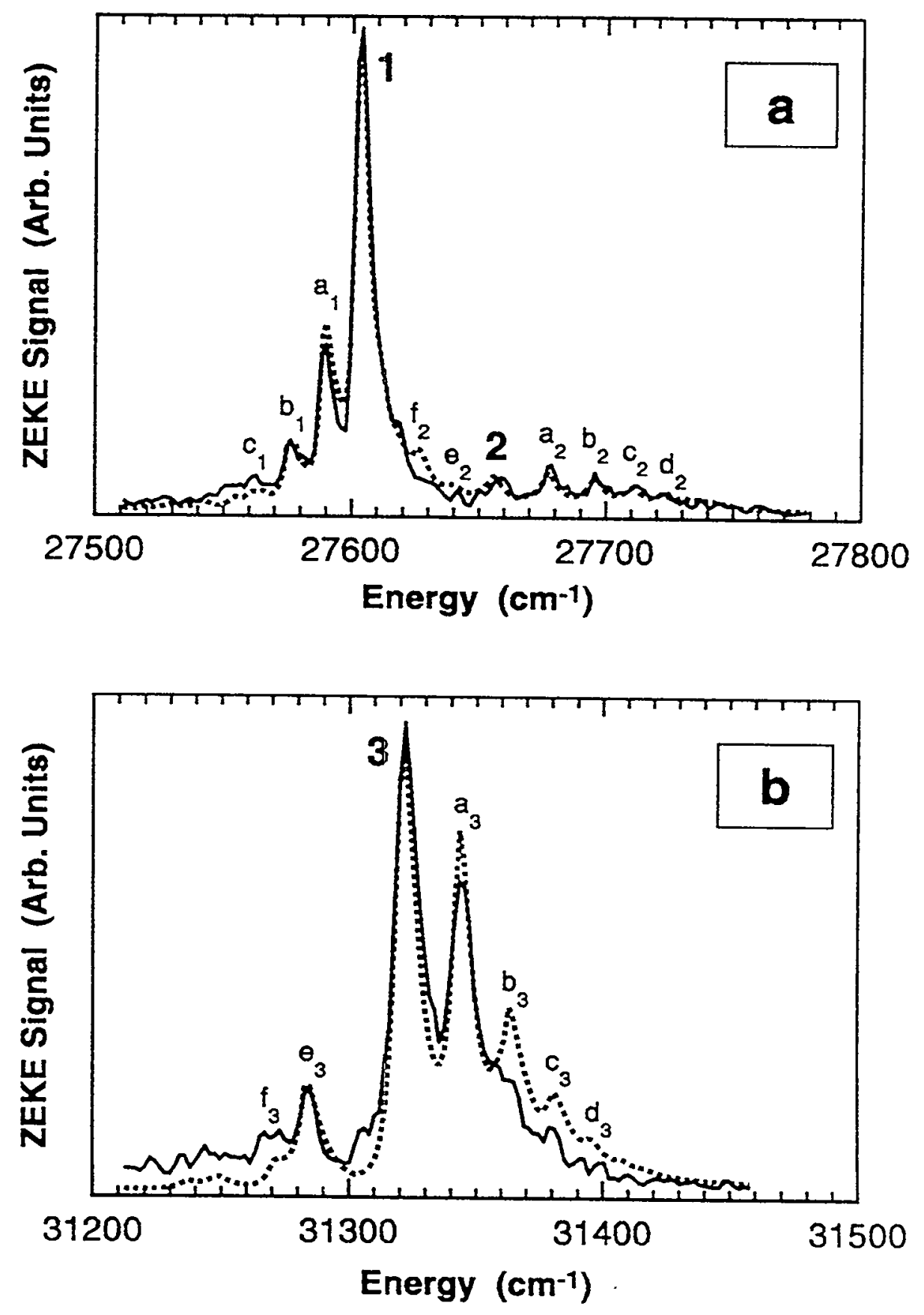

Figure 3.1. Experimental and simulated ZEKE spectra of $\mathrm{KrBr}$. The solid lines are the experimental spectra, and the dotted lines are the spectra calculated from the model potentials described in the text. (a) $X \frac{1}{2}$ and $I \frac{3}{2}$ states (halogen atom ${ }^{2} \mathrm{P}_{32}$ asymptote). (b) II $\frac{1}{2}$ state (halogen atom ${ }^{2} \mathrm{P}_{1 / 2}$ asymptote). 
Peak 2, the lowest energy member of the second progression, is assigned to the 0 0 transition to the $I \frac{3}{2}$ state. This assignment is made in part because it gives the best fit to a model potential (See Analysis section, below). Peaks $a_{2}, b_{2}, c_{2}$, and $d_{2}$ are assigned to the $\left(v^{\prime}-0\right)$ vibrational progression with $v^{\prime}=1-4$ originating from the anion $v^{\prime \prime}=0$ level. The extent of this progression indicates that the $I \frac{3}{2}$ state bond length is significantly shifted from the anion geometry. Peak $e_{2}$ is assigned to the 1-1 sequence band of the $I \frac{3}{2}$ state. Peak $\mathrm{f}_{2}, 40 \mathrm{~cm}^{-1}$ to the red of peak 2 is assigned to the $I \frac{3}{2} 0-1$ hot band transition, plus several overlapping bands from the $X \frac{1}{2}$ state.

In the II $\frac{1}{2}$ state spectrum, Fig. 3.1(b), we see the progression 3, $a_{3}, b_{3}, c_{3}$, and $d_{3}$, with a characteristic spacing of about $20 \mathrm{~cm}^{-1}$, and a smaller peak, $e_{3}, 37.2 \mathrm{~cm}^{-1}$ below peak 3. We assign peak 3 to the $0-0$ transition to the $I I \frac{1}{2}$ state, and the series $a_{3}, b_{3}, c_{3}$, $d_{3}$ to the $\left(v^{\prime}-0\right)$ progression with $v^{\prime}=1-4$. Peak $e_{3}$ corresponds to the $0-1$ hot band transition, and gives an accurate value for the anion vibrational frequency.

The complete set of peak positions and assignments is given in Table 3.1 . 
Table 3.1. Peak Assignments ( $\left.v_{-1}^{\prime}-v^{\prime \prime}\right)$ for KrBr ZEKE spectra (Figure 3.1). Energies are in $\mathrm{cm}^{-1}$.

\begin{tabular}{|c|c|c|c|c|}
\hline State & Peak & Position & $\begin{array}{l}\text { Relative } \\
\text { Energy }\end{array}$ & Assignment \\
\hline \multirow{4}{*}{$X 1 / 2$} & 1 & 27602.9 & 0 & $0 \leftarrow 0$ \\
\hline & $a_{1}$ & 27588.4 & -14.5 & $1 \leftarrow 1$ \\
\hline & $\mathrm{b}_{1}$ & 27576.2 & -26.7 & $2 \leftarrow 2$ \\
\hline & $c_{1}$ & 27561.0 & -41.9 & $3 \leftarrow 3$ \\
\hline \multirow{7}{*}{$I 3 / 2$} & 2 & 27657.0 & 0 & $0 \leftarrow 0$ \\
\hline & $a_{2}$ & 27678.4 & 21.4 & $1 \leftarrow 0$ \\
\hline & $b_{2}$ & 27695.3 & 38.3 & $2 \leftarrow 0$ \\
\hline & $c_{2}$ & 27710.7 & 53.7 & $3 \leftarrow 0$ \\
\hline & $\mathrm{d}_{2}$ & 27723.0 & 66.0 & $4 \leftarrow 0$ \\
\hline & $e_{2}$ & 27641.7 & -15.3 & $1 \leftarrow 1$ \\
\hline & $f_{2}$ & $\begin{array}{c}27619.0 \\
\text { (shoulder) }\end{array}$ & -38.0 & $0 \leftarrow 1$ \\
\hline \multirow{7}{*}{$I I I / 2$} & 3 & 31321.7 & 0 & $0 \leftarrow 0$ \\
\hline & $a_{3}$ & 31343.5 & 21.8 & $1 \leftarrow 0$ \\
\hline & $b_{3}$ & 31363.2 & 41.5 & $2 \leftarrow 0$ \\
\hline & $c_{3}$ & 31380.6 & 58.9 & $3 \leftarrow 0$ \\
\hline & $\mathrm{d}_{3}$ & 31398.3 & 76.6 & $4 \leftarrow 0$ \\
\hline & $e_{3}$ & 31284.5 & -37.2 & $0 \leftarrow 1$ \\
\hline & $\overline{f_{3}}$ & 31274.7 & -47.0 & $1 \leftarrow 2$ \\
\hline
\end{tabular}

\subsection{2. $\mathrm{XeBr}$}

The ZEKE spectra of $\mathrm{XeBr}$ are shown in Figures 3.2(a) and 3.2(b). Our assignment of the peaks proceeds in a fashion similar to the assignment of the $\mathrm{KrBr}$ spectrum. Again there are two band systems separated approximately by the Br spinorbit constant. The lower energy system in Figure 3.2(a) is dominated by a single peak, 1 , with a set of peaks $c_{1}, d_{1}$ and $e_{1}$ spaced at ca. $17 \mathrm{~cm}^{-1}$ intervals toward lower energy. We also observe a pair of small peaks, $a_{1}$ and $b_{1}, 26.5 \mathrm{~cm}^{-1}$ and $50.2 \mathrm{~cm}^{-1}$ to the blue of peak 1 , respectively. As above we assign peak 1 to the origin transition to the $X \frac{1}{2}$ state, and the peaks $c_{1}, d_{1}$ and $e_{1}$ to the sequence bands $1-1,2-2$ and $3-3$, respectively. Peaks $a_{1}$ 
and $b_{1}$ correspond to the 1-0 and 2-0 transitions, and are consistent within $2.5 \mathrm{~cm}^{-1}$ with the peak spacings calculated from the spectroscopic constants determined by Tellinghuisen and coworkers in their excimer emission study. ${ }^{14}$ As above, the dominance of the $0-0$ transition shows that the anion bond length is apparently quite close to that of the $X \frac{1}{2}$ state. However, in contrast to the $\mathrm{KrBr}^{-}$spectrum, transitions to the $I \frac{3}{2}$ state are not seen in Figure 3.2(a).

The more congested $I I \frac{1}{2}$ state spectrum in Figure 3.2(b) reveals two vibrational progressions. Peaks $3, a_{3}, b_{3}, c_{3}, d_{3}$ and $e_{3}$ are spaced by $20 \mathrm{~cm}^{-1}$ toward higher energy, peaks $3, \mathrm{f}_{3}$ and $\mathrm{g}_{3}$ are spaced by about $42 \mathrm{~cm}^{-1}$ toward lower energy. Based on this change in peak spacing, peaks $3-e_{3}$ are assigned to a progression arising from the ground anion vibrational state with the origin at peak 3 . Peaks $f_{3}$ and $g_{3}$ are assigned to the $0-1$ and $0-2$ hot band transitions, respectively. The $\mathrm{XeBr}$ peak positions and assignments are given in Table 3.2 .

Table 3.2. Peak assignments for XeBr ZEKE spectra (Figure 3.2). Energies are in $\mathrm{cm}^{-1}$.

\begin{tabular}{|c|c|c|c|c|}
\hline State & Peak & Position & Relative Energy & Assignment \\
\hline \multirow{5}{*}{$X 1 / 2$} & $\mathbf{1}$ & 27890.0 & 0 & $0 \leftarrow 0$ \\
\cline { 2 - 5 } & $\mathrm{a}_{1}$ & 27916.5 & 26.5 & $1 \leftarrow 0$ \\
\cline { 2 - 5 } & $\mathrm{b}_{1}$ & 27940.2 & 50.2 & $2 \leftarrow 0$ \\
\cline { 2 - 5 } & $\mathrm{c}_{1}$ & 27873.0 & -17.0 & $1 \leftarrow 1$ \\
\cline { 2 - 5 } & $\mathrm{d}_{1}$ & 27855.1 & -34.9 & $2 \leftarrow 2$ \\
\cline { 2 - 5 } & $\mathrm{e}_{1}$ & 27841.9 & -48.1 & $3 \leftarrow 3$ \\
\hline \multirow{5}{*}{$I I 1 / 2$} & 3 & 31623.6 & 0 & $0 \leftarrow 0$ \\
\cline { 2 - 5 } & $\mathrm{a}_{3}$ & 31647.6 & 24.0 & $1 \leftarrow 0$ \\
\cline { 2 - 5 } & $\mathrm{b}_{3}$ & 31667.1 & 43.5 & $2 \leftarrow 0$ \\
\cline { 2 - 5 } & $\mathrm{c}_{3}$ & 31687.5 & 63.9 & $3 \leftarrow 0$ \\
\hline & $\mathrm{d}_{3}$ & 31704.7 & 81.1 & $4 \leftarrow 0$ \\
\cline { 2 - 5 } & $\mathrm{e}_{3}$ & 31719.8 & 96.2 & $5 \leftarrow 0$ \\
\cline { 2 - 5 } & $\mathrm{f}_{3}$ & 31583.2 & -40.4 & $0 \leftarrow 1$ \\
\cline { 2 - 5 } & $\mathrm{g}_{3}$ & 31539.8 & -83.8 & $0 \leftarrow 2$ \\
\hline
\end{tabular}



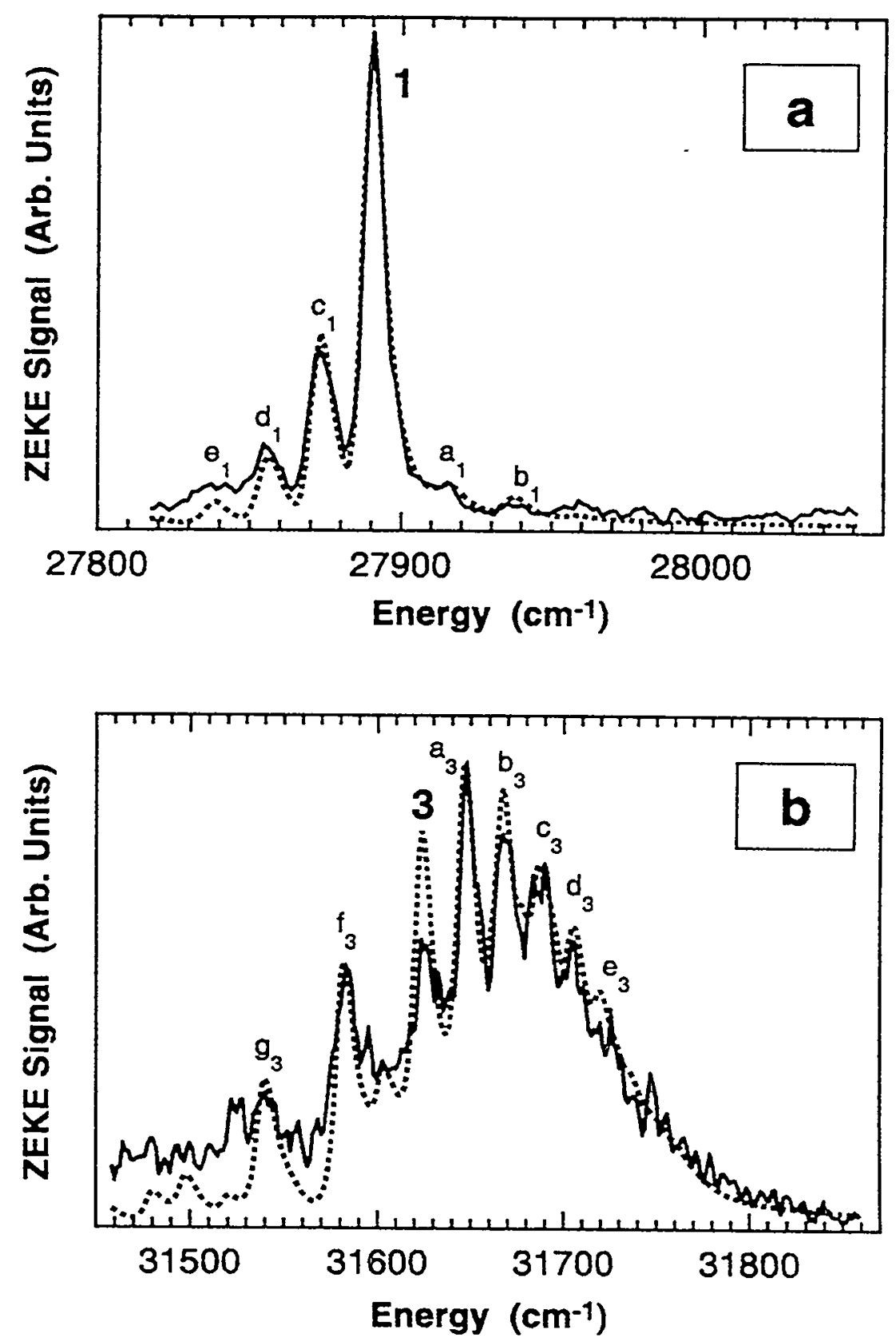

Figure 3.2. Experimental and simulated ZEKE spectra of $\mathrm{XeBr}^{-}$. The solid lines are the experimental spectra, and the dotted lines are the spectra calculated from the model potentials described in the text. (a) $X \frac{1}{2}$ state (halogen atom ${ }^{2} \mathrm{P}_{3 / 2}$ asymptote). The $I \frac{3}{2}$ state cannot be seen. (b) $I I \frac{1}{2}$ state (halogen atom ${ }^{2} \mathrm{P}_{1 / 2}$ asymptote). 


\subsection{3. $\mathrm{KrCl}$}

The ZEKE spectrum of $\mathrm{KrCl}$ is shown in Figure 3.3. The largest peak, labeled 1. is assigned to the origin transition to the $X \frac{1}{2}$ state. Peaks $1, c_{1}, d_{1}, e_{1}$ and $f_{1}$ are spaced approximately $26 \mathrm{~cm}^{-1}$ toward lower energy. The latter four peaks are assigned to $\Delta v=0$ sequence band transitions, with additional contributions from the hot band transitions listed in Table 3.3. Also, peaks $g_{1}$ and $h_{1}$ can be assigned to the overlapping hot band transitions given in Table 3.3. The partially resolved peaks $a_{1}$ and $b_{1}$ to the blue of 1 are assigned to the $\left(v^{\prime}-0\right)$ progression, yielding a frequency of $29 \mathrm{~cm}^{-1}$ for the $X \frac{1}{2}$ state. We were not able to observe the $I \frac{3}{2}$ state or the $I I \frac{1}{2}$ state for this system. The peak positions and assignments are shown in Table 3.3.

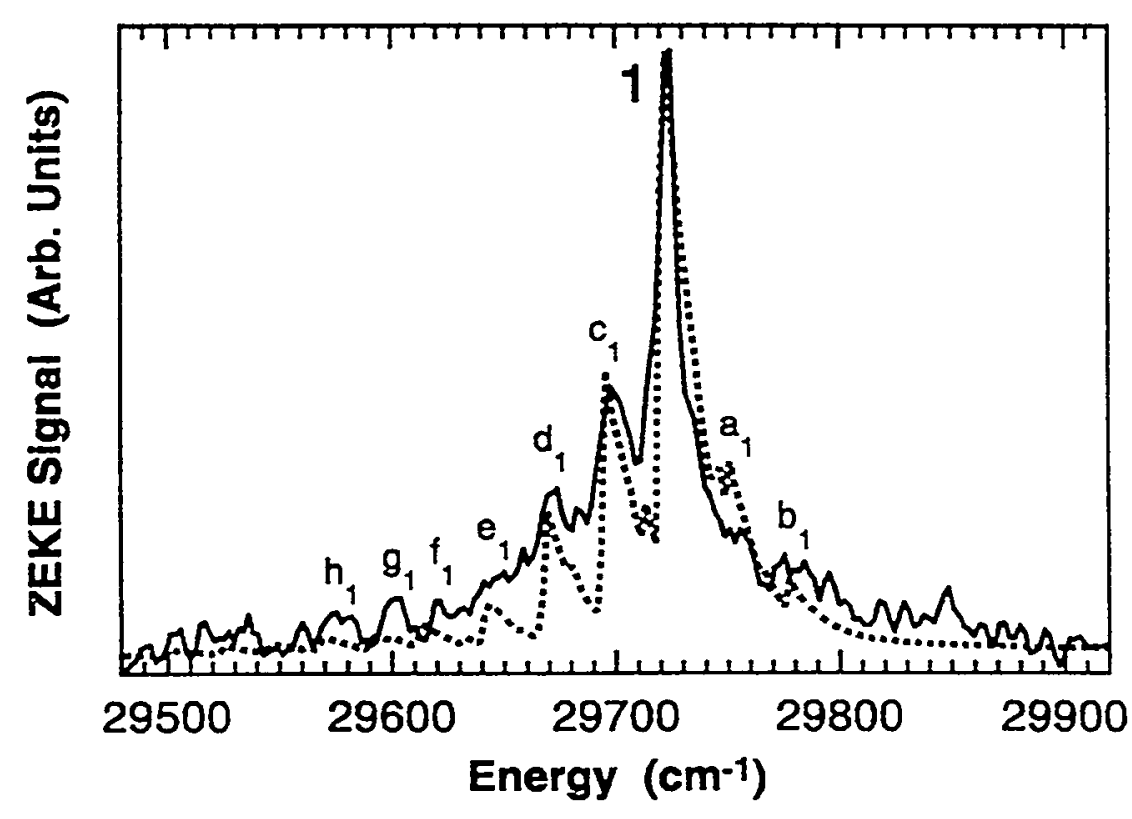

Figure 3.3. Experimental and simulated ZEKE spectrum of the $X \frac{1}{2}$ state (halogen atom ${ }^{2} \mathrm{P}_{3 / 2}$ asymptote) of $\mathrm{KrCl}^{-}$. The solid line is the experimental spectrum, and the dotted line is the spectrum calculated from the model potentials described in the text. 
Table 3.3. Peak assignments for $\mathrm{KrCl} \mathrm{ZEKE}$ spectrum (Figure 3.3). Energies are in $\mathrm{cm}^{-1}$. Assignments in parentheses contribute less than $20 \%$ of the total peak intensity. The assignment listed first contributes the most peak intensity.

\begin{tabular}{|c|c|c|c|c|}
\hline State & Peak & Position & $\begin{array}{c}\text { Relative } \\
\text { Energy }\end{array}$ & Assignment \\
\hline \multirow{9}{*}{$X 1 / 2$} & 1 & 29724.5 & 0 & $\begin{array}{c}0 \leftarrow 0 \\
(2 \leftarrow 1)\end{array}$ \\
\hline & $a_{1}$ & 29753.3 & 28.8 & $\begin{array}{l}1 \leftarrow 0 \\
3 \leftarrow 1\end{array}$ \\
\hline & $b_{1}$ & 29784.2 & 59.7 & $2 \leftarrow 0$ \\
\hline & $c_{1}$ & 29698.1 & -26.4 & $\begin{array}{c}1 \leftarrow 1 \\
(5 \leftarrow 3) \\
(3 \leftarrow 2)\end{array}$ \\
\hline & $\mathrm{d}_{\mathrm{l}}$ & 29673.9 & -50.6 & $\begin{array}{c}2 \leftarrow 2 \\
(0 \leftarrow 1)\end{array}$ \\
\hline & $e_{1}$ & 29645.4 & -79.1 & $3 \leftarrow 3$ \\
\hline & $f_{1}$ & 29621.3 & -103.2 & $\begin{array}{l}4 \leftarrow 4 \\
0 \leftarrow 2\end{array}$ \\
\hline & $g_{1}$ & 29601.6 & -122.9 & $\begin{array}{l}1 \leftarrow 3 \\
3 \leftarrow 4\end{array}$ \\
\hline & $\mathrm{h}_{1}$ & 29575.4 & -149.1 & $\begin{array}{l}2 \leftarrow 4 \\
4 \leftarrow 5\end{array}$ \\
\hline
\end{tabular}




\subsection{Analysis}

For each of the three species, the energy of peak 1 yields an accurate electron affinity: $27603(3) \mathrm{cm}^{-1}$ for $\mathrm{KrBr}, 27890$ (3) for $\mathrm{XeBr}$, and $29725(5) \mathrm{cm}^{-1}$ for $\mathrm{KrCl}$. These values are larger than the corresponding electron affinities of $\mathrm{Br}$ and $\mathrm{Cl}$, which are $27129.170 \mathrm{~cm}^{-1}$ and $29138.3 \mathrm{~cm}^{-1}$, respectively. ${ }^{28,29}$ The larger electron affinities for the complexes show that the $\mathrm{RgX}^{-}$dissociation energies are greater than the $\mathrm{RgX}$ dissociation energies, and that $\mathrm{XeBr}^{-}$is more strongly bound than $\mathrm{KrBr}^{-}$. Also, from the vibrational assignments in Tables 3.1-3.3 we directly obtain vibrational frequencies for the anion and neutral states.

To gain further insight into these complexes, we construct model potentials for the anion and neutral $\operatorname{RgX}$ complexes to simulate the experimental $\mathrm{ZEKE}$ spectra. The spectra are fit by choosing model anion and neutral potentials, and calculating the Franck-Condon factors, which, assuming a Boltzmann distribution of anion vibrational population, are used to produce a simulated spectrum to be compared with the experimental spectrum. The potential parameters and vibrational temperature are then adjusted in a trial-and-error fashion to produce the best agreement between the experimental and simulated spectra. The vibrational eigenvalues are calculated from the potentials using a discrete variable representation procedure ${ }^{30}$ based on a basis set of Morse potential eigenfunctions. ${ }^{31}$ See Appendix A for the details of this calculation.

We use the flexible, piecewise Morse-Morse-switching function-van der Waals (MMSV) potential form. This is the same potential form used by Lee and co-workers for the $\mathrm{RgX}$ neutral potentials ${ }^{15}$ and in our previous work. ${ }^{3}$ For the neutral, this potential has the reduced form, with $f(x)=V(R) / \varepsilon$ and $x=R / R_{m}$ :

$$
\begin{aligned}
f(x) & =e^{2 \beta_{1}(1-x)}-2 e^{\beta_{1}(1-x)}, & & 0<x \leq 1, \\
& =e^{2 \beta_{2}(1-x)}-2 e^{\beta_{2}(1-x)} \equiv M_{2}(x), & & 1<x \leq x_{1}, \\
& =S W(x) M_{2}(x)+[1-S W(x)] W(x), & & x_{1}<x<x_{2}, \\
& =-C_{6 r} x^{-6}-C_{8 r} x^{-8} \equiv W(x), & & x_{2} \leq x<\infty,
\end{aligned}
$$


where the switching function is given by

$$
S W(x)=\frac{1}{2}\left[\cos \frac{\pi\left(x-x_{1}\right)}{\left(x_{2}-x_{1}\right)}+1\right]
$$

and

$$
C_{6 r}=\frac{C_{6}}{\varepsilon R_{m}^{6}}, \quad C_{8 r}=\frac{C_{8}}{\varepsilon R_{m}^{8}}
$$

Here, $\varepsilon$ is the potential well depth and $R_{m}$ is the bond length. $C_{6}$ is the induced dipoleinduced dipole dispersion coefficient, and $C_{8}$ represents the induced dipole-induced quadrupole dispersion coefficient. Higher dispersion terms are neglected, as is the small induction term, varying as $R^{-8}$, arising from the halogen permanent quadrupole moment.

The anion potentials are of the same form, except that the dispersion terms are replaced by

$$
f(x)=-B_{4 r} x^{-4}-B_{6 r} x^{-6} \equiv W(x), \quad x_{2} \leq x<\infty
$$

with

$$
B_{4 r}=\frac{B_{4}}{\varepsilon R_{m}^{4}}, \quad B_{6 r}=\frac{B_{6}}{\varepsilon R_{m}^{6}} .
$$

and

$$
B_{4}=\frac{1}{2} q^{2} \alpha_{d}^{R g}, \quad B_{6}=\frac{1}{2} q^{2} \alpha_{q}^{R g}+C_{6}
$$

Here, $q$ is the halide charge and $B_{4}$ is the coefficient of the dominant term in the long range $\mathrm{RgX}$ potential, reflecting the dipole induced in the rare gas atom by the halide charge. The $B_{6}$ term arises from quadrupole induction and dipole dispersion terms. $\alpha_{d}{ }^{R g}$ and $\alpha_{q}{ }_{q}$ are the dipole and quadrupole polarizabilities of the rare gas, respectively.

The dispersion coefficients $C_{6}$ and $C_{\delta}$ are estimated using the formulas of Koutselos et al. 32 These formulas involve the dipole and quadrupole polarizabilities of each interacting atom, and an effective number of electrons, $N$, characteristic of each atom. $N$ is empirically determined from the like-atom $C_{6}$ coefficients. ${ }^{33-35}$ In the case of the halide atoms, the values of $N$ are assumed to be the same as those of the isoelectronic rare gas atoms. 
In calculating the dispersion coefficients for the neutral $\mathrm{RgX}$ complexes one must account for the open shell nature of the halogen atoms which results in anisotropic polarizabilities. The anisotropy of the dipole polarizability has been calculated for the $\mathrm{Cl}$ atom, neglecting spin-orbit effects, to be $14 \%$ relative to the average over all $M_{L}$ states. ${ }^{36}$ The halogen in the $\Sigma$ state of an $\operatorname{RgX}$ complex, with the unpaired electron oriented along the internuclear axis, thus has a smaller polarizability and smaller dispersion interaction than in the $\Pi$ state, where the unpaired electron is perpendicular to the axis. Bartolotti et al. ${ }^{37}$ have calculated the anisotropy of the quadrupole polarizability of the $\mathrm{Cl}$ atom. However, in this calculation, the value given for the quadrupole polarizability of the Ar atom is $18 \%$ higher than the accurate value of Thakkar et al. ${ }^{38}$ Therefore, the $\mathrm{Cl}$ quadrupole polarizabilities have been scaled down by this amount. This gives an anisotropy of $16 \%$ for $\alpha_{q}$. Because the anisotropy of $\alpha_{d}$ of $\mathrm{Br}$ has not been calculated, it was assumed to be the same as that of $\mathrm{Cl}$. Likewise, because calculations of $\alpha_{q}$ are not available for $\mathrm{Br}$, these were estimated using the "hydrogenic relationship" discussed by Sastri et al:39

$$
\alpha_{q} \equiv 1.570 \alpha_{d}^{1 / 2}
$$

The anisotropy of $\alpha_{q}$ was also assumed to be the same for $\mathrm{Br}$ as for $\mathrm{Cl}$. To find the dispersion coefficients for states including spin-orbit effects, we note that the $I \frac{3}{2}$ state has pure $\Pi$ character, while at long range the $X \frac{1}{2}$ state is a mixture of $2 / 3 \sum$ and $1 / 3 \Pi$ character, and the $I I \frac{1}{2}$ state has $1 / 3 \Sigma$ and $2 / 3 \Pi$ character. ${ }^{18}$ We assume that these mixing coefficients are constant for all regions of the potential where dispersion is the dominant interaction (i.e. $x>x_{2}$ in Eq. (1)).

The polarizabilities and effective numbers of electrons used here can be found in Table 3.4. The $C_{6}$ and $C_{8}$ coefficients for the various interactions are given with the other potential parameters, discussed below, in Tables 3.5-3.7. The $C_{6}$ values are fairly close to those of Lee and co-workers, 15 but the $C_{8}$ coefficients are in general larger because Lee and co-workers approximated $C_{8}$ with the values from the isoelectronic rare gas 
pairs. Because the ZEKE spectra are not sensitive to the very long range part of the potential, $B_{4}, B_{6}, C_{6}$ and $C_{8}$ were kept fixed at the calculated values during the fitting procedures.

Table 3.4. Dipole and quadrupole polarizabilities and effective numbers of electrons used to calculate dispersion and induction coefficients. In atomic units.

\begin{tabular}{|l|l|l|l|l|}
\hline Atom & $\begin{array}{l}\text { Corresponding } \\
\text { Neutral Spinless State }\end{array}$ & $\alpha_{\mathrm{d}}$ & $\alpha_{\mathrm{q}}$ & $N$ \\
\hline \multirow{3}{*}{$\mathrm{Cl}$} & $\Sigma$ & $13.3^{\mathrm{a}}$ & $72.0^{\mathrm{b}}$ & $4.2^{\mathrm{c}}$ \\
\cline { 2 - 5 } & $\Pi$ & $15.3^{\mathrm{a}}$ & $84.9^{\mathrm{b}}$ & $4.2^{\mathrm{c}}$ \\
\hline \multirow{3}{*}{$\mathrm{Br}$} & $\Sigma$ & $18.7^{\mathrm{d}}$ & $131^{\mathrm{e}}$ & $6.2^{\mathrm{c}}$ \\
\cline { 2 - 5 } & $\Pi$ & $21.5^{\mathrm{d}}$ & $154^{\mathrm{c}}$ & $6.2^{\mathrm{c}}$ \\
\hline $\mathrm{Cl}$ & --- & $28.1^{\mathrm{c}}$ & $\cdots$ & $5.404^{\mathrm{f}}$ \\
\hline $\mathrm{Br}$ & --- & $36.4^{\mathrm{c}}$ & --- & $6.309^{\mathrm{f}}$ \\
\hline $\mathrm{Kr}$ & $-\cdots$ & $16.79^{\mathrm{g}}$ & $99.296^{\mathrm{h}}$ & $6.309^{\mathrm{i}}$ \\
\hline $\mathrm{Xe}$ & --- & $27.16^{\mathrm{g}}$ & $223.29^{\mathrm{h}}$ & $7.253^{\mathrm{y}}$ \\
\hline
\end{tabular}

\section{References for Table 3.4}

(a) Ref. 36 .

(b) Values from Ref. 37 , scaled by a factor of 0.822 as explained in the text.

(c) Ref. 33.

(d) Derived from the spherically averaged value given in Ref. 33, assuming the same anisotropy for $\mathrm{Br}$ as for $\mathrm{Cl}$.

(e) Calculated from $\alpha_{d}$ of $\mathrm{Br}$ using the "hydrogenic relationship" $\alpha_{q} \cong 1.570 \alpha_{d}^{3 / 2}$ given in Ref. 39.

(f) Calculated from the $\mathrm{C}_{6}$ values of the corresponding isoelectronic rare gases from Ref. 34, using the Slater-Kirkwood formula (see, for example, Ref. 35).

(g) Ref. 34.

(h) Ref. 38

(i) Calculated from the $\mathrm{C}_{6}$ values from Ref. 34 . 
Since the ZEKE spectra do not give information about the absolute values of $\varepsilon$ and $R_{m}$, we have used results from previous experiments to guide our choice of these parameters. For $\mathrm{KrBr}$, we fix $R_{m}$ and $\varepsilon$ of the $X \frac{1}{2}$ state at the values determined in the scattering experiments of Lee and co-workers. ${ }^{15}$ For $\mathrm{KrCl}$ the values determined by Aquilanti and co-workers for the $X \frac{1}{2}$ states are used. ${ }^{16}$ For $\mathrm{XeBr}, R_{m}$ and $\varepsilon$ are taken from the $X \frac{1}{2}$ state potential of Tellinghuisen and coworkers, ${ }^{13,14}$ which they obtained by combining their vibrationally resolved $B \frac{1}{2} \rightarrow X \frac{1}{2}$ emission spectrum with the repulsive wall of the potential of Lee and co-workers. ${ }^{15}$ The emission spectrum independently provides a more precise well depth $\varepsilon$ than could be determined from the scattering experiments alone: Clevinger and Tellinghuisen cite an uncertainty of $0.8 \%$ for their value of $\varepsilon$, significantly more precise than the uncertainty cited for the scattering results $(-5 \%)$. However the uncertainty in $R_{m}$ is essentially the same as in Lee's potential $(-10 \%)$.

To determine $\varepsilon$ for the anions and the remaining electronic states we then use the relationships implied by Figure 3.4, on the following page, namely:

$$
\begin{aligned}
& \varepsilon_{a n}=v_{00}\left(X \frac{1}{2}\right)+\omega_{0}^{a n}+\varepsilon_{X}-\omega_{0}{ }^{\prime}-E A \\
& \varepsilon_{I}=\varepsilon_{X}-\Delta_{X \cdot I}-\omega_{0}{ }^{I}+\omega_{0} \\
& \varepsilon_{I I}=\varepsilon_{X}+\Delta_{s o}-\Delta_{X-I I}-\omega_{0}^{X}+\dot{\omega}_{0}^{I I}
\end{aligned}
$$

where $v_{00}\left(X \frac{1}{2}\right)$ is the origin of the $X \frac{1}{2}$ state, $\omega_{0}^{a n}, \omega_{0}^{X}$, etc. represent zero point energies, $E A$ is the electron affinity of the halogen atom, $\Delta_{X-l}$ is the $X \frac{1}{2}-I \frac{3}{2}$ state splitting (between $v=0$ levels), and $\Delta_{X-I I}$ is the $X \frac{1}{2}-I I \frac{1}{2}$ state splitting. 


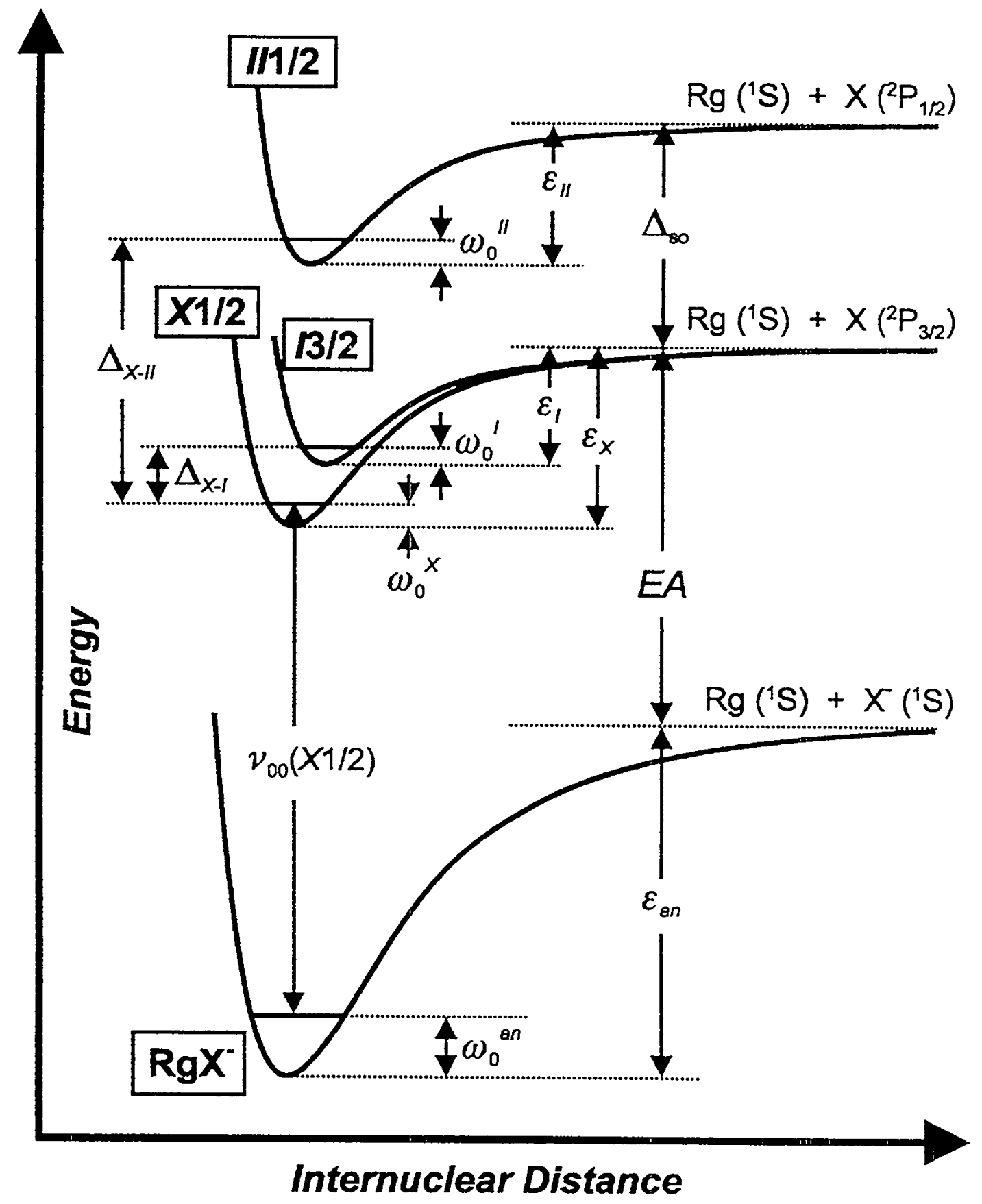

Figure 3.4. Schematic potential energy level diagram, showing the energetic relations among the atomic and molecular anion and neutral electronic states. 
Once $\varepsilon$ is fixed for the $I \frac{3}{2}, I I \frac{1}{2}$ and anion states, $R_{m}$ is found for these potentials by first adjusting $R_{m}$ of the anion to best reproduce the observed peak intensities of the $X \frac{1}{2}$ state portion of the spectrum. When $R_{m}$ is known for the anion, $R_{m}$ for the $X \frac{1}{2}$ and II $\frac{1}{2}$ states can then also be found by means of the Franck-Condon simulation.

For $\mathrm{KrBr}$ the initial values of the $X \frac{1}{2}$ and $I \frac{3}{2}$ state potential parameters $\beta_{1}, \beta_{2}, x_{1}$ and $x_{2}$ were taken to be the same as Lee's values. ${ }^{15}$ The $\beta_{1}$ parameter was kept fixed at the initial value because of the observation by Lee and co-workers that the slope of the repulsive part of the potential, with this $\beta_{1}$ values, agrees well with the slope determined from analysis of the excimer emission. ${ }^{15}$ The remaining parameters, $\beta_{2}, x_{1}$ and $x_{2}$ were then adjusted to reproduce the peak spacings seen in the ZEKE spectra.

In the case of $\mathrm{XeBr}$, the $\beta_{1}, \beta_{2}, x_{1}$ and $x_{2}$ parameters of the $X \frac{1}{2}$ state were adjusted to best fit the RKR turning points determined by Tellinghuisen and co-corkers. ${ }^{14}$ With this potential form, it was possible to reproduce the RKR turning point energies to within $3.5 \mathrm{~cm}^{-1}$. The vibrational spacings for the first nine levels of the resulting MMSV potential are within $0.2 \mathrm{~cm}^{-1}$ of those calculated from Tellinghuisen's spectroscopic constants, with the exception of the $v=0$ to $v=1$ spacing, which differed by $0.4 \mathrm{~cm}^{-1}$. This level of agreement was judged to be sufficient for the purposes of this work. Because of the accuracy of the Tellinghuisen potential, no adjustments were made to the $X \frac{1}{2}$ state MMSV parameters during the fitting procedure.

For $\mathrm{KrCl}$, the shape of the $X \frac{1}{2}$ state potential was estimated by choosing the MMSV parameters to reproduce the $X \frac{1}{2}$ state potential of Aquilanti and co-workers, ${ }^{16}$ who used a different representation of the potentials. This was not modified during the fitting because of the absence of sufficient detail in the ZEKE spectrum. Therefore, in the $\mathrm{KrCl}$ simulation only the anion parameters are adjusted.

Once the potentials are established by the Franck-Condon fitting procedure, a rotational simulation is performed to fit the observed asymmetric peak shapes. In this 
procedure, a set of rotational lines are calculated for each vibrational band, and these are convoluted with the asymmetric ZEKE instrumental line shape. The intensity of the ZEKE electron signal, $I(E)$, due to an individual line is represented by:

$$
\begin{aligned}
I(E) & =\frac{a\left(\frac{E-E_{0}}{\Gamma}\right)+b\left(\frac{E-E_{0}}{\Gamma}\right)^{3}}{1+c\left(\frac{E-E_{0}}{\Gamma}\right)^{2}+d\left(\frac{E-E_{0}}{\Gamma}\right)^{4}}, & & E \geq E_{0} \\
& =0, & & E<E_{0}
\end{aligned}
$$

with $a=4.3, b=0.19, c=4.2$, and $d=2.3$, and where $E-E_{0}$ is the energy above the threshold, $E_{0}$, of the line in $\mathrm{cm}^{-1}$, and $\Gamma$ is the full width at half maximum (FWHM) in $\mathrm{cm}^{-1}$. The line shape parameters are obtained by a non-linear least squares fit to the ZEKE spectra of $\mathrm{Br}^{-}$. This form differs from that used previously 3 and is a more accurate representation of the true ZEKE line shape. Readers are referred to our previous work ${ }^{3}$ and to Appendix B for further details of the rotational fitting procedure. As in previous work, the rotational temperature was assumed to be $40 \mathrm{~K}$.

The simulated spectra are shown as dotted lines superimposed on the experimental spectra in Figures 3.1-3.3. For the $\mathrm{KrBr}^{-}$and $\mathrm{XeBr}^{-}$spectra, the anion vibrational temperature for the $I I \frac{1}{2}$ state differs slightly from the lower energy state(s) because the spectra were taken with different source conditions. The best-fit potential parameters are given in Tables 3.5-3.7, and the potentials are plotted in Figure 3.5, on the following pages.

Fitting the $\mathrm{XeBr}$ and $\mathrm{KrCl}$ spectra is fairly straightforward, because the peak assignments are readily apparent by inspection of the spectra. However, for $\mathrm{KrBr}$ the fitting procedure is used as an aid in assigning spectral features, since not all of the assignments are obvious from the spectra. Specifically, although the assignments of the $X \frac{1}{2}$ state features are straightforward, the location of the origin of the $I \frac{3}{2}$ state is not obvious upon initial inspection. As mentioned in Section 3.3.1, peak 2 of Figure 3.1 (a) is assigned to the $I \frac{3}{2}$ origin because this allows the best fit with the model potential. Also, 
this assignment gives a value of the $X \frac{1}{2}-I \frac{3}{2}$ state splitting, $\Delta_{X-1}$, of $54.1 \mathrm{~cm}^{-1}$. This is somewhat larger than $\Delta_{X . I}$ for $\mathrm{ArBr}, 38.2 \mathrm{~cm}^{-1}, 3$ a result expected due to the stronger $\mathrm{Kr}$ $\mathrm{Br}$ interaction. On the other hand, if peak $\mathrm{e}_{2}$ were chosen as the $I \frac{3}{2}$ state origin, $\Delta_{X-I}$ would essentially be the same as in $\mathrm{ArBr}$, contrary to expectation. This further corroborates our choice of peak 2 as the $I \frac{3}{2}$ state origin.

The method for estimating the uncertainties of the potential parameters is discussed at length in our earlier work. ${ }^{3}$ Here, we present these estimated uncertainties along with the potential parameters in Tables 3.5-3.7. The anion and neutral potentials are plotted in Figure 3.5. It should be remembered that the uncertainties in $\varepsilon$ and $R_{m}$ are expected to be fairly rigorous, whereas the uncertainties given for the other potential parameters represent lower bounds on the true uncertainties, because a complete multivariate analysis of the correlations amongst these parameters was not performed. 
Table 3.5. MMSV potential parameters for $\mathrm{KrBr}$ and $\mathrm{KrBr}$, and zero point energies ( $\omega_{b}$ ) and fundamental vibrational frequencies $\left(v_{01}\right)$ calculated from the potentials. Term values $T_{o}$ are referenced to anion ground vibrational state. Estimated uncertainties are given in parentheses.

\begin{tabular}{|l|c|c|c|c|}
\hline & $X \mathbf{1} / \mathbf{2}$ & $I 3 / 2$ & $I I 1 / 2$ & Anion \\
\hline $\begin{array}{l}T_{v i b}(\mathrm{~K}) \text { of } \\
\text { anion }\end{array}$ & $68 .(4)$. & $68 .(4)$. & $45 .(3)$. & --- \\
\hline$T_{0}\left(\mathrm{~cm}^{-1}\right)$ & $27602.9(2.0)$ & $27657.0(4.0)$ & $31321.7(2.0)$ & 0 \\
\hline$\omega_{0}\left(\mathrm{~cm}^{-1}\right)$ & 12.5 & 11.7 & 12.4 & 19.2 \\
\hline$V_{01}\left(\mathrm{~cm}^{-1}\right)$ & 23.8 & 21.4 & 22.8 & 37.2 \\
\hline$\varepsilon(\mathrm{meV})$ & $19.9(1.0)$ & $13.1(0.9)$ & $15.7(1.0)$ & $79.5(1.0)$ \\
\hline$R_{m}(\AA)$ & $3.90(0.30)$ & $4.15(0.30)$ & $4.03(0.30)$ & $3.85(0.30)$ \\
\hline$\beta_{1}$ & $5.70(0.40)$ & $7.20(0.50)$ & $7.00(0.50)$ & $4.62(0.30)$ \\
\hline$\beta_{2}$ & $6.72(0.30)$ & $8.00(0.30)$ & $7.20(0.30)$ & $4.62(0.20)$ \\
\hline$x_{1}$ & $1.02(0.06)$ & $1.05(0.06)$ & $1.05(0.06)$ & $1.04(0.06)$ \\
\hline$x_{2}$ & $1.70(0.20)$ & $1.65(0.10)$ & $1.85(0.20)$ & $1.50(0.10)$ \\
\hline$C_{6}\left(\mathrm{eV} \cdot \AA^{6}\right)$ & $86.6(13.0)$ & $92.7(14.0)$ & $89.7(14.0)$ & $-\cdots$ \\
\hline$C_{8}\left(\mathrm{eV} \cdot \AA^{8}\right)$ & $740 .(230)$. & $801 .(250)$. & $770 .(240)$. & $\cdots$ \\
\hline$B_{4}\left(\mathrm{eV} \cdot \AA^{4}\right)$ & $\cdots---$ & --- & $\cdots$ & $17.91(2.70)$ \\
\hline$B_{6}\left(\mathrm{eV} \cdot \AA^{6}\right)$ & $\cdots--$ & $\cdots$ & $\cdots$ & $165 .(41)$. \\
\hline
\end{tabular}


Table 3.6. MMSV potential parameters for $\mathrm{XeBr}$ and $\mathrm{XeBr}$, and zero point energies $\left(\omega_{0}\right)$ and fundamental vibrational frequencies $\left(v_{01}\right)$ calculated from the potentials. Term values $T_{0}$ are referenced to anion ground vibrational state. Estimated uncertainties are given in parentheses.

\begin{tabular}{|l|l|l|l|}
\hline & $X 1 / 2$ & $I I 1 / 2$ & Anion \\
\hline $\begin{array}{l}T_{v i b}(\mathrm{~K}) \text { of } \\
\text { anion }\end{array}$ & $70 .(4)$. & $90 .(5)$. & -- \\
\hline$T_{0}\left(\mathrm{~cm}^{-1}\right)$ & $27890.0(2.0)$ & $31623.6(4.0)$ & 0 \\
\hline$\omega_{0}\left(\mathrm{~cm}^{-1}\right)$ & 12.3 & 12.6 & 21.3 \\
\hline$v_{01}\left(\mathrm{~cm}^{-1}\right)$ & 24.1 & 23.1 & 42.1 \\
\hline$\varepsilon(\mathrm{meV})$ & $31.53(0.25)$ & $25.52(0.74)$ & $126.92(0.50)$ \\
\hline$R_{m}(\AA)$ & $3.82(0.19)$ & $4.00(0.22)$ & $3.81(0.21)$ \\
\hline$\beta_{1}$ & $4.35(0.30)$ & $6.42(0.45)$ & $3.50(0.25)$ \\
\hline$\beta_{2}$ & $7.41(0.30)$ & $7.00(0.28)$ & $5.30(0.21)$ \\
\hline$x_{1}$ & $1.01(0.06)$ & $1.03(0.06)$ & $1.03(0.06)$ \\
\hline$x_{2}$ & $2.00(0.24)$ & $1.60(0.19)$ & $1.60(0.19)$ \\
\hline$C_{6}\left(\mathrm{eV} \cdot \AA^{6}\right)$ & $128 .(20)$. & $133 .(21)$. & ---- \\
\hline$C_{8}\left(\mathrm{eV} \cdot \AA^{8}\right)$ & $1260 .(400)$. & $1320 .(410)$. & --- \\
\hline$B_{4}\left(\mathrm{eV} \cdot \AA^{4}\right)$ & ---- & $\cdots-$ & $28.98(4.30)$ \\
\hline$B_{6}\left(\mathrm{eV} \cdot \AA^{6}\right)$ & $-\cdots--$ & $\cdots$ & $271 .(68)$. \\
\hline & & & \\
\hline
\end{tabular}


Table 3.7. MMSV potential parameters for $\mathrm{KrCl}$ and $\mathrm{KrCl}$, and zero point energies $\left(\omega_{5}\right)$ and fundamental vibrational frequencies $\left(v_{01}\right)$ calculated from the potentials. Term values $T_{0}$ are referenced to anion ground vibrational state. Estimated uncertainties are given in parentheses.

\begin{tabular}{|l|l|l|}
\hline & $X 1 / 2$ & Anion \\
\hline $\begin{array}{l}T_{v i b}(\mathrm{~K}) \text { of } \\
\text { anion }\end{array}$ & $210 .(10)$. & $-\cdots$ \\
\hline$T_{0}\left(\mathrm{~cm}^{-1}\right)$ & $29724.5(2.0)$ & 0 \\
\hline$\omega_{0}\left(\mathrm{~cm}^{-1}\right)$ & 16.0 & 29.3 \\
\hline$v_{01}\left(\mathrm{~cm}^{-1}\right)$ & 29.9 & 55.5 \\
\hline$\varepsilon\left(\mathrm{meV}^{-1}\right)$ & $22.01(1.00)$ & $95.7(1.0)$ \\
\hline$R_{m}(\AA)$ & $3.75(0.10)$ & $3.83(0.10)$ \\
\hline$\beta_{1}$ & $5.49(0.40)$ & $5.70(0.50)$ \\
\hline$\beta_{2}$ & $5.70(0.20)$ & $4.40(0.20)$ \\
\hline$x_{1}$ & $1.30(0.08)$ & $1.30(0.06)$ \\
\hline$x_{2}$ & $1.90(0.20)$ & $2.50(0.20)$ \\
\hline$C_{6}\left(\mathrm{eV} \cdot \AA^{6}\right)$ & $60.8(9.1)$ & $-\cdots$ \\
\hline$C_{8}\left(\mathrm{eV} \cdot \AA^{8}\right)$ & $473 .(71)$. & $-\cdots$ \\
\hline$B_{4}\left(\mathrm{eV} \cdot \AA^{4}\right)$ & $-\cdots$ & $17.91(2.70)$ \\
\hline$B_{6}\left(\mathrm{eV} \cdot \AA^{6}\right)$ & $-\cdots$ & $138 .(35)$. \\
\hline
\end{tabular}




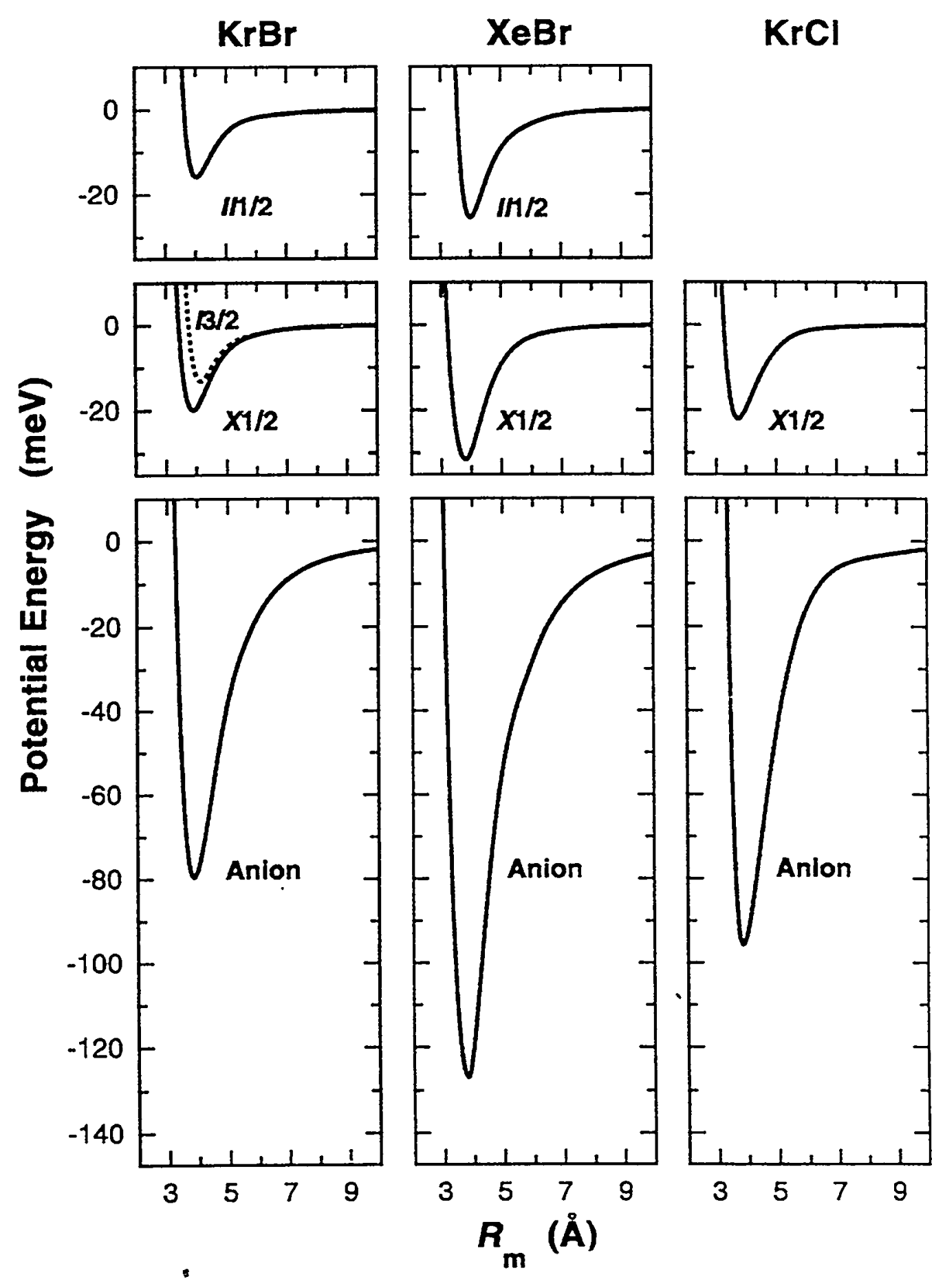

Figure 3.5. Plots of model potentials for the $\mathrm{RgX}$ anion and observed neutral states determined from the ZEKE spectra, using the MMSV potential parameters given in Tables 3.5-3.7. 


\subsection{Discussion}

In this section we discuss our results for the neutral and anion $\operatorname{RgX}$ potentials and compare them to previously published potentials. The neutral potentials presented here do not differ greatly from earlier potentials. For the $\operatorname{KrBr} X \frac{1}{2}$ state, of course, $\varepsilon$ and $R_{m}$ are the same as those from Lee's study, ${ }^{15}$ since these were not adjusted in our fitting procedure. Our values of $\beta_{2}, x_{1}$ and $x_{2}$ differ somewhat from Lee's values and result in an improved match with the vibrational spacings in the ZEKE spectra. As mentioned above, $\beta_{1}$ was not adjusted, in order to retain agreement with the repulsive wall slopes from emission studies. For the $I \frac{3}{2}$ state of $\mathrm{KrBr}$, the bond length is somewhat longer and the well depth a bit shallower than Lee's values. The difference in $R_{m}$ values is well within the stated $10 \%$ uncertainty, but the difference in $\varepsilon$ is just outside this range at $12 \%$.

Our $\mathrm{XeBr} X \frac{1}{2}$ state potential is more or less identical with that determined by Clevenger and Tellinghuisen, ${ }^{14}$ differing only because of the limitations of the MMSV potential form, and was not varied during the fit because of the much higher relative accuracy of the emission results. The few features of the $\mathrm{XeBr} X \frac{1}{2}$ state observed in the ZEKE spectrum (the progression $v^{\prime}=0,1,2 \leftarrow v^{\prime \prime}=0$, i.e., peaks $1, a_{1}$ and $b_{1}$ in Figure 2) are consistent with the emission results within our experimental uncertainty.

Our $X \frac{1}{2}$ state potential for $\mathrm{KrCl}$ is essentially identical to the integral crosssection potential, ${ }^{16}$ differing only in the choice of potential form. This is because the ZEKE spectra do not contain enough information to significantly improve on the potential obtained from the scattering experiments.

We obtain significantly more new information about the anion potentials. The trends in anion binding energies are similar to those seen in our preqvious study. ${ }^{3}$ The larger binding energy for $\mathrm{XeBr}^{-}$compared to $\mathrm{KrBr}^{-}$is due to the larger polarizability of $\mathrm{Xe}$, and the larger binding energy of $\mathrm{KrCl}^{-}$vs. $\mathrm{KrBr}^{-}$results from the smaller $R_{m}$ and stronger charge-polarizability attraction in $\mathrm{KrCl}^{-}$. For all three anions, the change in $R_{m}$ upon photodetachment to the $X \frac{1}{2}$ state is very small, even though the anion binding 
energy is considerably larger. Apparently the larger radius of the halide in the anions compensates for the stronger binding energy.

In Table 3.8 , on the following page, we compare the potential parameters $R_{m}$ and $\varepsilon$ of the anions from the present study with other values from the literature, all of which have been derived through less direct means. It can be seen that the literature values are quite scattered. In comparing our results with the results of Kirkpatrick and Viehland, ${ }^{6}$ who obtained potentials via direct inversion of ion mobility data, we find our well depths are systematically shallower and our bond lengths systematically longer. However, except for the $\mathrm{XeBr}^{-}$well depth, our values lie within or close to the $10 \%$ uncertainties cited by those authors. Similar discrepancies with other ZEKE potentials were explored in a recent paper by Kirkpatrick and Viehland, in which they used the ZEKE potentials ${ }^{3}$ of $\mathrm{ArI}^{-}$and $\mathrm{ArBr}^{-}$to simulate ion mobilities. ${ }^{40}$ They found that the ZEKE ArI potential satisfactorily reproduced the mobility data, despite significant differences in $R_{m}$ and $\varepsilon$ from the potentials obtained by direct inversion of mobility data. However, the agreement for $\mathrm{ArBr}^{-}$was not as good. The authors cite the relative insensitivity of the mobility data to well depth to explain these findings.

Potentials derived from the earlier mobility results of $\mathrm{McDaniel}$ and co-workers ${ }^{5}$ for $\mathrm{KrBr}^{-}$and $\mathrm{XeBr}^{-}$are slightly closer to ours, but show the same sign and order of magnitude deviations in $\varepsilon$ and $R_{m}$. The electron gas calculations of Waldman and Gordon $^{8}$ again give systematically larger well depths than ours, although the bond lengths are in reasonable agreement. 
Table 3.8. Comparison of ZEKE-determined anion potentials with literature potentials. Uncertainties are given in parentheses as reported in each work cited, if available.

\begin{tabular}{|c|c|c|c|c|c|c|}
\hline & \multicolumn{2}{|l|}{$\mathrm{KrBr}$} & \multicolumn{2}{|l|}{$\mathrm{XeBr}$} & \multicolumn{2}{|l|}{$\mathrm{KrCl}^{-}$} \\
\hline & $\varepsilon(\mathrm{meV})$ & $R_{m}(\AA)$ & $\varepsilon(\mathrm{meV})$ & $R_{m}(\AA)$ & $\varepsilon(\mathrm{meV})$ & $R_{m}(\AA)$ \\
\hline present work & $\begin{array}{l}79.5 \\
(1.0)\end{array}$ & $\begin{array}{l}3.85 \\
(0.30)\end{array}$ & $\begin{array}{l}126.92 \\
(0.50)\end{array}$ & $\begin{array}{l}3.81 \\
(0.21)\end{array}$ & $\begin{array}{l}95.7 \\
(1.0)\end{array}$ & $\begin{array}{l}3.83 \\
(0.10)\end{array}$ \\
\hline ion mobilities ${ }^{a}$ & 87.1 & 3.73 & 145 & 3.62 & $-\cdots$ & -- \\
\hline $\begin{array}{l}\text { ion } \\
\text { mobilities }\end{array}$ & $\begin{array}{l}88.7 \\
(8.9)\end{array}$ & 3.579 & $169(17)$ & 3.397 & $102(10)$ & 3.448 \\
\hline electron gas & 93 & 3.76 & 159 & 3.64 & $\ldots$ & -- \\
\hline electron gas ${ }^{d}$ & -- & $\overline{---}$ & -- & -- & 115 & 3.48 \\
\hline empirical ${ }^{e}$ & 98 & 3.99 & 142 & 4.10 & 105 & 3.85 \\
\hline empirical ${ }^{f}$ & 90.1 & 3.91 & 130 & 4.02 & 95.4 & 3.79 \\
\hline $\begin{array}{l}\text { semi- } \\
\text { empirical }\end{array}$ & 92.5 & 3.70 & 167 & 3.62 & 107 & 3.55 \\
\hline $\begin{array}{l}\text { semi- } \\
\text { empirical }\end{array}$ & 75.1 & 3.87 & 99.9 & 4.05 & $-\cdots$ & $\cdots$ \\
\hline $\begin{array}{l}\text { semi- } \\
\text { empirical }\end{array}$ & 85 & 3.79 & 146 & 3.74 & 108 & 3.53 \\
\hline
\end{tabular}

\section{References for Table 3.8}
(a) Ref. 5.
(b) Ref. 6.
(c) Ref. 8 .
(d) Ref. 7.
(e) Ref. 11.

(f) Empirical method of Ref. 11 modified as explained in the text.

(g) Ref. 32 .

(h) Ref. 9.

(i) Ref. 10 . 
The results of Pirani and coworkers in Table 3.8 are obtained by very simple formulas based on empirical polarizability correlations. ${ }^{11}$ Comparison to the ZEKE potentials show discrepancies greater than our experimental uncertainties, except for $R_{m}$ of $\mathrm{KrBr}$ and $\mathrm{KrCl}^{-}$. Because of the simplicity of this method, and its usefulness for predicting new potentials, it is of interest to "recalibrate" these polarizability correlation formulae using the current and earlier ZEKE results. Fitting the $\varepsilon$ and $R_{m}$ parameters of the current study, and also those of the previous work on $\mathrm{KrI}^{-}, \mathrm{ArBr}{ }^{-}$and $\mathrm{ArI}$ we obtain

$$
R_{m}=1.725 \frac{\alpha_{I}^{1 / 3}+\alpha_{B}^{1 / 3}}{\left[\alpha_{1} \alpha_{B}\left(1+\frac{1}{\rho}\right)\right]^{0.095}} \AA
$$

and

$$
\varepsilon=4380 \frac{\alpha_{B}}{R_{m}{ }^{4}}(1+\rho) \mathrm{meV}
$$

with

$$
\rho=\frac{\alpha_{1} \alpha_{B}}{\left[1+\left(2 \alpha_{1} / \alpha_{B}\right)^{3 / 3}\right] \alpha_{B}^{y / 2}}
$$

Here the anion and neutral polarizabilities, $\alpha_{l}$ and $\alpha_{B}$, are in $\AA^{3}$ and $R_{m}$ is in $\AA$. The numerical coefficients in Eqs. (3.12) and (3.13) differ somewhat from Pirani's values, 1.767 and 5200,11 which were obtained using $\varepsilon$ and $R_{m}$ for the $\mathrm{Li}^{+}-\mathrm{He}$ and $\mathrm{Li}^{+}-\mathrm{Ne}$ interaction potentials as references. The results using our parameters are given in Table VIII; a significant improvement is obtained, although agreement is certainly not perfect. Eqs. (3.12) and (3.13) should be useful in predicting other halide-rare gas interactions; this will be tested in ongoing studies of similar species.

Finally, we should remark on the apparent absence of the $I \frac{3}{2}$ state in the $\mathrm{XeBr}$ and $\mathrm{KrCl}^{-} \mathrm{ZEKE}$ spectra, and the much lower intensity of this state relative to the $X \frac{1}{2}$ state in the $\mathrm{KrBr}^{\circ} \mathrm{ZEKE}$ spectrum. Examination of these and our previous results on 
$\mathrm{ArI}^{-}, \mathrm{ArBr}^{-}$, and $\mathrm{KrI}^{-}$shows an overall trend in which the $I \frac{3}{2}$ transition is weaker for smaller halides and larger rare gas atoms. This may reflect variations in the transition moments, or perhaps the explanation lies in the differences between the s-wave partial detachment cross sections, since only those photoelectrons ejected with orbital angular momentum $l=0$ contribute to the ZEKE signal. ${ }^{22}$

\subsection{Concluding Remarks}

In this article, we have presented the $\mathrm{ZEKE}$ spectra of the $\mathrm{RgX}^{-}$complexes $\mathrm{KrBr}$, $\mathrm{XeBr}$, and $\mathrm{KrCl}$. We have obtained accurate electron affinities for these systems. Model anion and neutral potentials were constructed by Franck-Condon simulations of the spectra. In cases where comparison is possible, the neutral potentials are in reasonable agreement with the potentials from scattering experiments, with some minor adjustments in the well region for $\mathrm{KrBr}$ and $\mathrm{XeBr}$. The anion potentials constructed from the data are, we believe, the most accurate experimental determinations available for these systems so far.

We have recently obtained results for $\mathrm{Ar}_{n} \mathrm{Cl}^{-}$and $\mathrm{Xe}_{\mathrm{n}} \mathrm{I}^{-}$clusters. Analysis of these spectra will yield further insight into the pair potentials and many-body interactions that govern bonding and structure in these species.

\subsection{Acknowledgments}

This research is supported by the Air Force Office of Scientific Research under Grant No. F49620-97-1-0018. Georg Reiser and Thomas Lenzer thank the Deutsche Forschungsgemeinschaft for postdoctoral fellowships. 


\subsection{References for Chapter 3}

1 G. C. Maitland, M. Rigby, E. B. Smith, and W. A. Wakeham, Intermolecular Forces (Oxford University Press, Oxford, 1981).

2 R. A. Aziz, in Inert Gases, edited by M. L. Klein (Springer Verlag, 1984), pp. 5-86.

3 Y. Zhao, I. Yourshaw, G. Reiser, C. C. Arnold, and D. M. Neumark, J. Chem. Phys. 101, 6538 (1994).

4 I. Yourshaw, Y. Zhao, and D. M. Neumark, J. Chem. Phys. 105, 351 (1996).

5 D. R. Lamm, R. D. Chelf, J. R. Twist, F. B. Holleman, M. G. Thackston, F. L. Eisele, W. M. Pope, I. R. Gatland, and E. W. McDaniel, J. Chem. Phys. 79, 1965 (1983).

6 C. C. Kirkpatrick and L. A. Viehland, Chem. Phys. 98, 221 (1985).

7 Y. S. Kim and R. G. Gordon, J. Chem. Phys. 61, 1 (1974).

8 M. Waldman and R. G. Gordon, J. Chem. Phys. 71, 1325 (1979).

9 J. W. Wilson, J. H. Heinbockel, and R. A. Outlaw, J. Chem. Phys. 89, 929 (1988).

10 S. H. Patil, J. Chem. Phys. 89, 6357 (1988).

11 D. Cappelletti, G. Liuti, and F. Pirani, Chem. Phys. Lett. 183, 297 (1991).

12 P. J. Hay and W. R. Wadt, Annu. Rev. Phys. Chem. 30, 311 (1979).

13 J. O. Clevenger and J. Tellinghuisen, Chem. Phys. Lett. 231, 515 (1994).

14 J. O. Clevenger and J. Tellinghuisen, J. Chem. Phys. 103, 9611 (1995).

15 P. Casavecchia, G. He, R. K. Sparks, and Y. T. Lee, J. Chem. Phys. 75, 710 (1981).

16 V. Aquilanti, D. Cappelletti, V. Lorent, E. Luzzatti, and F. Pirani, J. Phys. Chem. 97, 2063 (1993).

17 V. Aquilanti, G. Liuti, F. Pirani, and F. Vecchiocattivi, J. Chem. Soc. Faraday Trans. 2 85,955 (1989).

18 H. Haberland, Z. Phys. A 307, 35 (1982).

19 K. Müller-Dethlefs, M. Sander, and E. W. Schlag, Z. Naturforsch. Teil A 39, 1089 (1984).

20 K. Müller-Dethlefs, M. Sander, and E. W. Schlag, Chem. Phys. Lett. 12, 291 (1984). 
21 K. Müller-Dethlefs and E. W. Schlag, Annu. Rev. Phys. Chem. 42, 109 (1991).

22 T. N. Kitsopoulos, I. M. Waller, J. G. Loeser, and D. M. Neumark, Chem. Phys. Lett. 159,300 (1989).

23 C. J. Chick, Y. Zhao, T. N. Kitsopoulos, and D. M. Neumark, J. Chem. Phys. 97, 6121 (1992).

24 T. N. Kitsopoulos, Ph.D. Thesis, University of California, 1991.

25 C. C. Arnold, Ph.D. Thesis, University of California, 1994.

26 T. Lenzer, I. Yourshaw, M. Furlanetto, and D. M. Neumark, to be published.

27 W. H. Press, S. A. Teukolsky, W. T. Vetterling, and B. P. Flannery, Numerical

Recipes, 2nd ed. (Cambridge University Press, Cambridge, 1992).

28 C. Blondel, P. Cacciani, C. Delsart, and R. Trainham, Phys. Rev. A 40, 3698 (1989).

29 R. Trainham, G. D. Fletcher, and D. J. Larson, J. Phys. B 20, L777 (1987).

30 J. C. Light, I. P. Hamilton, and J. V. Lill, J. Chem. Phys. 82, 1400 (1985).

31 E. M. Greenawalt and A. S. Dickinson, J. Mol. Spectrosc. 30, 427 (1969).

32 A. D. Koutselos, E. A. Mason, and L. A. Viehland, J. Chem. Phys. 93, 7125 (1990).

33 E. A. Mason and E. W. McDaniel, Transport Properties of Ions in Gases (Wiley, New York, 1988).

34 A. Kumar and W. J. Meath, Mol. Phys. 54, 823 (1985).

35 J. N. Wilson, J. Chem. Phys. 43, 2564 (1965).

36 K. Andersson and A. J. Sadlej, Phys. Rev. A 46, 2356 (1992).

37 L. J. Bartolotti, L. Ortiz, and Q. S. Xie, Int. J. Quant. Chem. 49, 449 (1994).

38 A. J. Thakkar, H. Hettema, and P. E. S. Wormer, J. Chem. Phys. 97, 3252 (1992).

39 M. V. K. Sastri, P. L. Narasimhulu, and K. D. Sen, J. Chem. Phys. 80, 584 (1984).

40 L. A. Viehland and C. C. Kirkpatrick, Chem. Phys. 202, 285 (1996). 
Chapter 4. Observation of many body effects in the zero electron kinetic energy (ZEKE) and threshold photodetachment spectra of $\operatorname{Ar}_{n} \mathrm{Br}^{\circ} \quad(n=2-9)$ and $\operatorname{Ar}_{n} \mathrm{I}^{-}$ $(n=2-19)^{*}$

\begin{abstract}
The anion zero electron kinetic energy (ZEKE) spectra of the van der Waals clusters $\mathrm{Ar}_{2.3} \mathrm{Br}^{-}$and $\mathrm{Ar}_{2.7} \mathrm{I}^{-}$have been measured, and partially discriminated threshold photodetachment (PDTP) experiments have been performed on $\mathrm{Ar}_{4.9} \mathrm{Br}^{-}$and $\mathrm{Ar}_{8.19}{ }^{\circ}$. Adiabatic electron affinities (EAs) have been determined from these results. The separation of the halogen ${ }^{2} \mathrm{P}_{32}$ asymptotic states was measured for the $\mathrm{Ar}_{2.3} \mathrm{Br}$ and $\mathrm{Ar}_{2.7 \mathrm{I}}$ neutral clusters, and the separations between the ${ }^{2} \mathrm{P}_{12}$ and ${ }^{2} \mathrm{P}_{32}$ asymptotic states was determined for $\mathrm{Ar}_{2-3} \mathrm{Br}$ and $\mathrm{Ar}_{2-3} \mathrm{I}$. Model potentials were constructed, using the pair potentials determined from previous work on the diatomic rare gas-halide atom complexes, as well as various non-additive terms, and the cluster minimum energy structures were determined using a simulated annealing procedure. A simple first-order degenerate perturbation theory model of the neutral cluster potentials was found to agree well with the ${ }^{2} \mathrm{P}_{32}$ asymptotic electronic state separations observed in the ZEKE spectra. The halogen spin-orbit splittings in the $\mathrm{Ar}_{2-3} \mathrm{Br}$ and $\mathrm{Ar}_{2-3} \mathrm{I}$ clusters were found to be slightly smaller than those of the free halogen atoms. The binding energies calculated from a model additive potentials were found to be inconsistent with the experimental electron affinities. Model potentials including many-body induction effects, three-body "exchange
\end{abstract}

* Originally published in slightly different form in J. Chem. Phys. 105, (1996), with coauthors Yuexing Zhao and Daniel M. Neumark. 
quadrupole" effects and a triple-dipole dispersion term were found to agree well with the experimental results. Many-body induction was found to be the dominant non-additive effect. The exchange quadrupole effect--i.e., the interaction of the exchange induced electron charge distribution distortion among argon atoms with the halide charge-- was also found to be important. 


\subsection{Introduction}

In most studies of weakly interacting atoms or molecules, pairwise additivity of the potentials is assumed. Given pair potentials, $V_{i j}$, between atoms $i$ and $j$, the pairwise additive approximation to the total potential of $N$ interacting atoms is

$$
V_{p a i r}=\sum_{i<j}^{N} V_{i j}\left(\left|\mathbf{r}_{i}-\mathbf{r}_{j}\right|\right)
$$

Here $\mathbf{r}_{i}$ and $\mathbf{r}_{j}$ represent the positions of atoms $i$ and $j$. If the atoms had closed valence shells, and if no deformation of the atomic charge distributions were induced by the interactions, then pairwise additivity would hold exactly. ${ }^{1}$ However, if the deformation of the charge distributions due to the interatomic interactions (e.g. dispersion, induction or exchange) is considered, the assumption of pairwise additivity breaks down.' This assumption can also break down if one of the atoms has an open valence shell. Then it is necessary to consider the electronic states of the open-shell atom which arise from the simultaneous presence of all the other atoms; the potential energy surfaces of these states cannot, in general, be obtained by simply adding the pair potentials in the sense of Equation (4.1). In either case it is necessary to extend Equation (4.1) to include nonadditive, or many-body, effects:

$$
\begin{aligned}
V_{\text {many-body }} & =V_{\text {pair }}+V_{\text {non-add }} \\
& =V_{\text {pair }}+\sum_{i<j<k}^{N} V_{i j k}\left(\mathbf{r}_{i}, \mathbf{r}_{j}, \mathbf{r}_{k}\right)+\ldots+\sum_{i<j<k \cdots z}^{N} V_{i j k z ;}\left(\mathbf{r}_{i}, \mathbf{r}_{j}, \mathbf{r}_{k} \cdots \mathbf{r}_{z}\right)
\end{aligned}
$$

Non-additive effects are believed to play a significant role in determining the properties of bulk matter. For example, the binding energies of rare gas solids ( $\mathrm{Ne}, \mathrm{Ar}, \mathrm{Kr}$ and $\mathrm{Xe}$ ) measured experimentally are about $7-10 \%$ smaller than the binding energies calculated from accurate pair potentials. ${ }^{2}$ However, there has been some controversy 
about the precise nature of the non-additive effects involved. ${ }^{2.3}$ Furthermore, it is in general difficult to extract detailed information about non-additive effects from measurements of bulk properties. ${ }^{3}$

Cluster studies represent an alternative approach to learning about non-additive effects. By probing the spectroscopy and/or energetics of a cluster as a function of its size, and comparing the results with predictions based on additive forces alone, one can obtain considerable insight into the various non-additive components of the interaction potential. ${ }^{3}$ To this end, we present in this paper the anion zero electron kinetic energy (ZEKE) spectra of the $\mathrm{Ar}_{2-3} \mathrm{Br}^{-}$and $\mathrm{Ar}_{2-7} \mathrm{I}^{-}$van der Waals clusters, and partially discriminated threshold photodetachment (PDTP) spectra of $\mathrm{Ar}_{4-9} \mathrm{Br}^{\circ}$ and $\mathrm{Ar}_{8-19} \mathrm{I}^{-}$. We also present the results of calculations with model potentials involving various non-additive terms, in an effort to understand the experimentally observed electron affinities (EAs) and electronic structure. Our results probe non-additive effects in both the cluster anion and the open-shell neutral cluster resulting from photodetachment. This work is an extension of our previous ZEKE study of the diatomic rare gas-halide atom complexes, ${ }^{4}$ and previous $\mathrm{ZEKE}$ work on the $\mathrm{ICO}_{2}$ complex. $^{5}$

In order to extract information on many-body effects from experimental studies of clusters, the pair potentials must be known more accurately than the magnitude of the many-body effect. Furthermore, the experiment must provide information about the "true" potential that can be compared with the results of calculations with model additive and non-additive potentials. This information may consist of spectroscopically measured vibrational frequencies, rotational constants, etc. In this case, accurate dynamical calculations are needed to extract this information from the model potentials for 
comparison with experiment. Alternately, some experiments allow a more direct measurement of the cluster binding energies, in which case comparison with model potentials is much more straightforward.

Non-additive effects can affect the rotational, vibrational, and electronic spectroscopy of a cluster. Much of the recent interest in this field has focused on high resolution spectroscopy of van der Waals clusters. For example, pure rotation spectra of $\mathrm{Ne}_{2} \mathrm{Kr}$ and $\mathrm{Ne}_{2} \mathrm{Xe}$ have been observed using fourier transform microwave spectroscopy. ${ }^{6}$ The structural information and nuclear hyperfine coupling constants determined from these spectra show evidence of non-additivity. There have also been a number of near and far infrared studies of molecular chromophores in rare gas clusters. ${ }^{3.7 .8}$ In many cases it is difficult to extract meaningful information about many-body forces from spectroscopic studies because the intermolecular pair potentials are often not well enough characterized, in that the uncertainty in the pair potentials is comparable to the magnitude of the manybody effects. There has, however, been recent experimental and theoretical progress in determining intermolecular pair potentials accurately enough to learn about three body interactions in the $\mathrm{Ar}_{2} \mathrm{HCl}^{3.8 .9 .}{ }^{10 \mathrm{a}} \mathrm{Ar}_{2} \mathrm{HF}^{7.9 .10 \mathrm{c}}$ and $\mathrm{Ar}_{2} \mathrm{DCl}^{3.10 \mathrm{~b}}$ systems. In work more closely related to the results presented here, the electronic spectroscopy of $A r_{1-4} \mathrm{Hg}$ has been studied with multi-photon ionization, ${ }^{11}$ and $\mathrm{Ar}_{n} \mathrm{Ba}$ clusters have been studied by laser induced fluorescence. ${ }^{12}$ Only the $\mathrm{Ar}_{1-4} \mathrm{Hg}$ study was mass-selective. From this work, progress has been made in identifying "non-additive" effects in the excited electronic state of these clusters with open-shell chromophores. ${ }^{11.12}$

It is challenging to extract information on non-additive effects from direct spectroscopic measurements such as those mentioned above. Even when mass selectivity 
can be obtained, non-trivial dynamical calculations are needed to extract the vibrational and rotational structure information from a many-body model potential in order to compare it with the experimental spectrum. It is desirable, therefore, to experimentally measure the binding energies ( $B E \mathrm{~s}$ ) of clusters, because $B E \mathrm{~s}$ can be readily obtained from many-body model potentials by simple methods. Moreover, there is generally an intuitive connection between a particular non-additive term and the cluster binding energy, in the sense that one can usually predict by inspection if the binding energy will increase or decrease when a given many-body term is added to a model potential. However, in most cases, $B E \mathrm{~s}$ of clusters cannot be directly obtained from experimental spectra. Exceptions include the pump-probe experiments of Janda and coworkers on $\mathrm{Ar}_{2-3} \mathrm{Cl}_{2}{ }^{13 a}$ and $\mathrm{HeBr}_{2}{ }^{13 b}$ and the stimulated emission pumping experiments on the carbazole-Ar system by Leutwyler and coworkers. ${ }^{14}$

Anion photoelectron spectroscopy (PES) of clusters has proved useful in providing more direct information about the relative binding energies of anion and neutral clusters. It also has the advantage of mass selectivity. Examples include the work of Markovich et al. on $\mathrm{X}^{-}\left(\mathrm{H}_{2} \mathrm{O}\right)_{\mathrm{n}}\left(\mathrm{X}^{-}=\mathrm{Cl}^{-}, \mathrm{Br}^{-}\right.$and $\left.\mathrm{I}^{-}\right),{ }^{15}$ Bowen and coworkers on $\mathrm{O}^{-} \mathrm{Ar}_{\mathrm{n}}{ }^{16}$ and Arnold et al. on $\mathrm{X}\left(\mathrm{CO}_{2}\right)_{\mathrm{n}}$ and $\mathrm{X}\left(\mathrm{N}_{2} \mathrm{O}\right)_{\mathrm{n}} \cdot{ }^{17}$ The theoretical calculations of Berkowitz et al. ${ }^{18}$ in conjunction with the PES spectra of Markovich et al. ${ }^{15}$ have demonstrated the importance of non-additive inductive effects in $\mathrm{Br}^{-}\left(\mathrm{H}_{2} \mathrm{O}\right)_{n}$ clusters.

However, there are two problems with trying to extract information on nonadditive forces from these studies. First, the pair potentials for the relevant neutral and ionic species are not in general known very accurately; this is particularly true for clusters involving molecular solvents. Secondly, the resolution of conventional anion PES is 
typically in the range of $80 \mathrm{~cm}^{-1}$ (Ref. 17)- $400 \mathrm{~cm}^{-1}$ (Ref. 15), depending on the type of energy analyzer used. Because of this limited resolution, anion PES experiments can only be sensitive to the largest non-additive effects, such as inductive effects in the anion clusters.

The anion $\mathrm{ZEKE}$ technique used in the present experiments on $\mathrm{Ar}_{n} \mathrm{Br}^{-}$and $\mathrm{Ar}_{n} \mathrm{I}^{-}$ combines the advantage of mass-selectivity with much higher resolution (ca. $2-3 \mathrm{~cm}^{-1}$ for atomic systems) than PES experiments. This resolution allows accurate measurement of electron affinities, as well as spectroscopic observation of the electronic structure of the neutral $\mathrm{Ar}_{\mathrm{n}} \mathrm{X}$ clusters. The $\mathrm{Ar}-\mathrm{X}^{-}$and $\mathrm{Ar}-\mathrm{X}$ pair potentials are known accurately from our previous work on the diatomic species. ${ }^{4}$ Thus, by employing simulated annealing procedures to determine the binding energies and neutral electronic structure from model potentials, we can directly compare our experimental results with the pairwise additive predictions, and explore the effects of various many-body corrections to the additive potentials. From this comparison, we can obtain a detailed picture of non-additive effects in $\mathrm{Ar}_{\mathrm{n}} \mathrm{X}^{*}$ and $\mathrm{A} r_{\mathrm{n}} \mathrm{X}$ clusters.

This paper is organized as follows. In Section 4.2 we briefly describe the experimental apparatus and techniques. In Section 4.3, we present the anion ZEKE and PDTP spectra, determine the experimental EAs, assign the electronic structure observed in the ZEKE spectra, and briefly discuss the observed vibrational structure. In Section 4.4 , we describe the methods and present the results of calculations of the cluster EAs and neutral electronic structure from model additive and non-additive potentials, and compare them with the experimental results. In Section 4.5, we summarize, considering what we 
can and cannot conclude about many-body interactions on the basis of our results, and suggest future avenues for experimental and theoretical research.

\subsection{Experiment}

Zero electron kinetic energy (ZEKE) spectroscopy was first developed for photoionization of neutrals by Müller-Dethlefs et $a .^{19}$ and applied to negative ion photodetachment by Neumark and co-workers. ${ }^{20}$ The experimental apparatus has been described in detail elsewhere. ${ }^{20}$ Briefly, $\mathrm{Ar}_{\mathrm{n}} \mathrm{X}^{-}$clusters are produced by expanding a mixture of approximately $0.1-0.5 \%$ Freon $\left(\mathrm{CF}_{3} \mathrm{I}\right.$ or $\mathrm{CF}_{2} \mathrm{ClBr}, \mathrm{PCR} \mathrm{Co}$.) in a ca. $25 \%$ argon' $75 \%$ helium mixture through a pulsed valve (General Valve Series 9) with a 0.5 mm diameter orifice. Backing pressures are typically 60-80 psi. The expansion is crossed with a $1 \mathrm{keV}$ electron beam. Halide anions are formed by dissociative attachment of low energy secondary electrons and undergo clustering in the continuum flow region of the free expansion. The molecular beam is collimated with a skimmer, accelerated to $1 \mathrm{keV}$, and mass-selected with a $1 \mathrm{~m}$ long collinear time-of-flight mass spectrometer. ${ }^{20 c .21}$ The mass selected ions then enter a differentially pumped detection region, and are irradiated with a pulse from an excimer pumped dye laser (Lambda Physik). For the ground states of $\mathrm{Ar}_{\mathrm{n}} \mathrm{I}, \mathrm{BBQ}, \mathrm{PBBO}$, Exalite 398, QUI and DMQ laser dyes (Exciton) were used. For the $\mathrm{AI}_{2-3} \mathrm{I}$ excited state scans rhodamine 610 dye was frequency doubled with a $\mathrm{KDP}$ crystal. For the $\mathrm{Ar}_{\mathrm{n}} \mathrm{Br}$ clusters, DMQ and PTP dyes were used for the ground states; rhodamine 640 was doubled with a KDP crystal for the excited state $\mathrm{Ar}_{2-3} \mathrm{Br}$ spectra. The power of the undoubled light was typically $7-20 \mathrm{~mJ}$ per pulse at the interaction region. The frequency doubled laser power was about $2 \mathrm{~mJ}$ per pulse. The laser wavelength was 
calibrated from $337-400 \mathrm{~nm}$ with the $\mathrm{Ne}$ lines observed by the optogalvanic effect in a FeNe hollow cathode lamp. The fundamental wavelength of the frequency doubled light was calibrated in the region $600-640 \mathrm{~nm}$ with an iodine absorption cell.

Two modes of electron detection were used in the present studies: the high resolution $\mathrm{ZEKE}$ mode, and the lower resolution partially discriminated threshold photodetachment (PDTP) mode. In the ZEKE mode, the photodetached electrons are extracted collinearly by a weak $(2-5 \mathrm{~V} / \mathrm{cm})$ electric field after a $300-500$ ns delay, and deflected to an off-axis microchannel detector. Detection is gated to provide temporal filtering. A series of apertures between the detachment point and detector provide spatial discrimination. This combination of spatial and temporal filtering discriminates against high energy electrons, so that as the laser wavelength is scanned, only photoelectrons with nearly zero kinetic energy are detected. The resolution of the instrument is about 2-3 $\mathrm{cm}^{-1}$ for atomic systems. ${ }^{20}$ However, in the spectra of molecules, the peaks are broadened by unresolved rotational structure. For the systems studied in this paper, the observed peaks were at least $8 \mathrm{~cm}^{-1}$ wide (FWHM).

In the PDTP mode ${ }^{20 b}$, there is no delay between the laser pulse and electron extraction, retaining only spatial filtering as in the "steradiancy detector" first described by Baer et al. ${ }^{22}$ This results in some discrimination against electrons with energies greater than about $150 \mathrm{~cm}^{-1}$, and leads to peaks about $200 \mathrm{~cm}^{-1}$ wide in the present case. However, the thresholds, and hence the electron affinities, can be determined more accurately than this, to within approximately $\pm 50 \mathrm{~cm}^{-1}$. Because nearly all of the electrons are collected, this mode of operation has the advantage of much higher sensitivity than the ZEKE mode. 
The ZEKE spectra were averaged over several thousand laser shots per point taken in several separate scans. The PDTP spectra were averaged over 300-1000 laser shots per point. All spectra were normalized to the ion signal and laser power.

No obvious "magic numbers" were seen in the mass spectra. The ion signal smoothly decreased in intensity with increasing cluster size in the mass spectra of both the $\mathrm{Ar}_{\mathrm{n}} \mathrm{Br}^{-}$and $\mathrm{Ar}_{\mathrm{n}} \mathrm{I}^{-}$clusters.

\subsection{Results}

\subsection{1. $\mathrm{Ar}_{2-3} \mathrm{I}^{-}, \mathrm{Ar}_{2-3} \mathrm{Br}^{-}$}

The ZEKE spectra of $\mathrm{Ar}_{2} \mathrm{Br}^{-}$and $\mathrm{Ar}_{3} \mathrm{Br}^{-}$are shown in Figure 4.1, along with the spectrum of the diatomic ArBr- complex, reproduced from Reference 4. ZEKE spectra of $\mathrm{ArI}^{-}, \mathrm{Ar}_{2} \mathrm{I}^{-}$and $\mathrm{Ar}_{3} \mathrm{I}^{-}$are displayed in Figure 4.2. All the spectra have two sets of features, separated by approximately the spin orbit splitting of the halogen atoms: $3685 \mathrm{~cm}^{-1}$ for $\mathrm{Br}$ and $7603.15 \mathrm{~cm}^{-1}$ for $\mathrm{I}^{23}$ We assign the lower energy set of features to electronic states arising from the ground ${ }^{2} \mathrm{P}_{3 / 2}$ state of the halogen atom, and the higher energy features to ${ }^{2} \mathrm{P}_{1 \mathrm{n}}$ asymptotic states. The ground state manifolds of the $\mathrm{Ar}_{2-3} \mathrm{I}$ clusters are dominated by two sharp, intense peaks, labeled $X$ and $I$, separated by about $40-65 \mathrm{~cm}^{-1}$. In the $\mathrm{Ar}_{2-3} \mathrm{Br}$ spectra, both features are also present, but peak $I$ is less intense and distinct than in the $A r_{n} I$ spectra. 

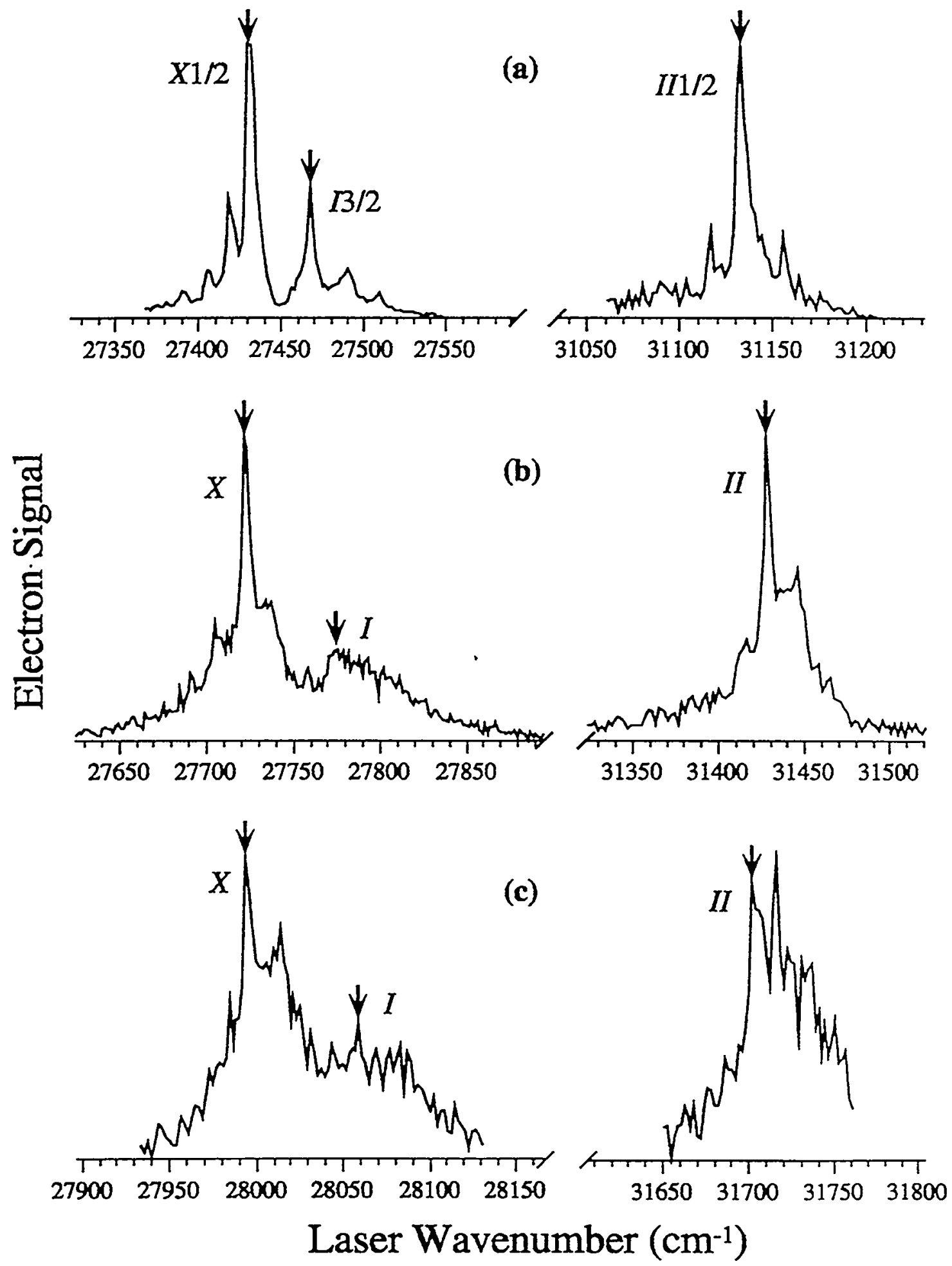

Figure 4.1. Zero electron kinetic energy (ZEKE) spectra of (a) $\mathrm{ArBr}^{-}$, (b) $\mathrm{Ar}_{2} \mathrm{Br}^{\circ}$, and (c) $\mathrm{Ar}_{3} \mathrm{Br}$. The arrows indicate the neutral electronic state origins. 


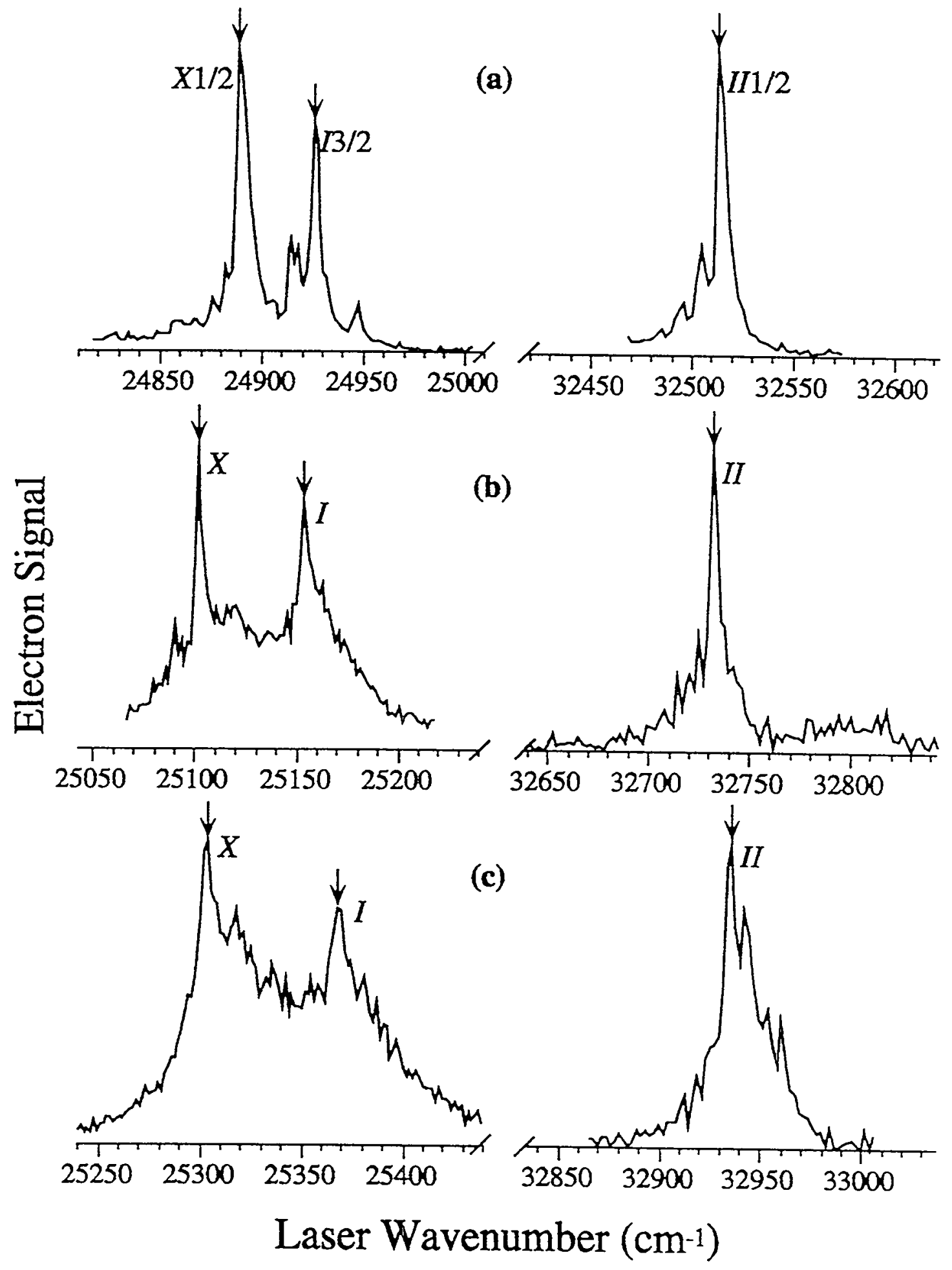

Figure 4.2. $\mathrm{ZEKE}$ spectra of (a) $\mathrm{ArI}^{-}$, (b) $\mathrm{Ar}_{2} \mathrm{I}^{-}$, and (c) $\mathrm{Ar}_{3} \mathrm{I}^{-}$. The arrows indicate the neutral electronic state origins. 
In the previous work on the diatomic species ${ }^{4}$ the corresponding features were assigned to the origins of the two electronic states that correlate to the halogen ${ }^{2} \mathrm{P}_{32}$ asymptote, referred to as the $X \frac{1}{2}\left(j_{a}=\frac{1}{2}, \Omega=\frac{1}{2}\right)$ and $I \frac{3}{2}\left(j_{a}=\frac{3}{2}, \Omega=\frac{3}{2}\right)$ states, in Hund's case (c) notation. ${ }^{24}$ The feature labeled $I I \frac{1}{2}$ in the diatomic spectra was assigned to the origin of the $I I \frac{1}{2}$ state $\left(j_{a}=\frac{3}{2}, \Omega=\frac{1}{2}\right)$, which correlates to the halogen ${ }^{2} \mathrm{P}_{1 / 2}$ asymptote. We expect an analogous set of three doubly degenerate electronic states to be present in the polyatomic clusters. The lower ${ }^{2} \mathrm{P}_{3 n}$ halogen state is split into two doubly degenerate states by the weak interaction with the argon atoms. We refer to these states as the $X$ and $I$ states, by analogy with the diatomic case, dropping the $\Omega$ designation, as this is no longer a good quantum number in the polyatomic case. Note that here the " $X$ state" always refers to the lowest energy state at the equilibrium geometry regardless of the symmetry of the cluster.

The $X$ and $I I$ state origins are blue shifted relative to the corresponding atomic lines by several hundred $\mathrm{cm}^{-1}$. The blue shift increases as the number of argon atoms increases. This demonstrates that the anionic clusters are more strongly bound than the neutral species.

In the $\mathrm{Ar}_{2} \mathrm{Br}^{*}$ spectrum [Figure 4.1(b)] we see some partially resolved peaks to the red and blue of the $X$ state origin. There is also a long "tail" to the blue of the $I$ state origin. We attribute all these features to transitions to or from vibrationally excited states. Based on our previous interpretation of the diatomic $\mathrm{ArBr}^{-}$spectrum, ${ }^{4}$ it is likely that the features to the red of the $X$ state origin are due to hot-band or sequence band transitions from vibrationally excited anion states. Likewise, the features to the blue of the $X$ state 
origin may be transitions to vibrationally excited neutral ground states and/or hot-band transitions to the $I$ state. The vibrational progressions are not as well resolved as in the diatomic spectra. The observed structure is probably due to many overlapping transitions involving more than one vibrational mode. This spectral congestion appears to be more severe to the blue of the $I$ state origin, possibly indicating a larger geometry change between the $I$ state and the anion than between the $X$ state and the anion, as was seen in $\mathrm{ArBr}^{-4}$ The peaks to the red and blue of the $I I$ state origin may similarly be understood as sequence band or hot-band transitions, and transitions to vibrationally excited neutral states, respectively.

The spectrum of $\mathrm{Ar}_{3} \mathrm{Br}^{-}$[Figure 4.1(c)] appears even more congested. There is a distinct peak $20 \mathrm{~cm}^{-1}$ to the blue of the $X$ state origin, in addition to numerous poorly resolved features. Again there appears to be an extended unresolved progression to the blue of the $I$ state origin. The $I I$ state has two prominent peaks, separated by $14 \mathrm{~cm}^{-1}$, plus some other indistinct peaks to the blue. It is not clear which of the two peaks is in fact the II state origin.

In the case of $\mathrm{Ar}_{2} \mathrm{I}$ [Figure 4.2(b)], the vibrational structure is somewhat less well resolved than in $\mathrm{Ar}_{2} \mathrm{Br}^{-}$. There is a clear feature $11 \mathrm{~cm}^{-1}$ to the red of the $X$ state origin, as well as some poorly resolved structure between the origins of the $X$ and I states. There is a tail to the blue of the $I$ state origin. The spectrum of the $I I$ state is rather sparse, with some peaks $10-30 \mathrm{~cm}^{-1}$ to the red of the origin due to sequence or hot bands, and a slight shoulder to the blue. The lack of any extended progression indicates that the anion-II state transition is quite vertical. 
The spectrum of $\mathrm{Ar}_{3} \mathrm{I}^{-}$[Figure 4.2(c)] shows clearer vibrational resolution than $\mathrm{Ar}_{2} \mathrm{I}$. There are two peaks spaced by 8 and $32 \mathrm{~cm}^{-1}$ from the $X$ state origin. However, the sequence band structure to the red of the origin is not resolved. The II state of $\mathrm{Ar}_{3} \mathrm{I}$ displays three distinct peaks 7,17 and $24 \mathrm{~cm}^{-1}$ to the blue of the origin, as well as some less distinct sequence band structure to the red.

The partially resolved vibrational structure seen in these spectra is of considerble interest and will be considered further in future publications. In this paper, we are primarily concerned with the accurate electron affinities and state splittings yielded by these spectra.

\subsection{2. $\mathrm{Ar}_{4.7} \mathrm{I}^{-}$}

The ZEKE spectra of the $\mathrm{Ar}_{4-7} \mathrm{I}^{\circ}$ clusters are shown in Figure 3. For these clusters we studied only the lower $\left({ }^{2} \mathrm{P}_{32}\right.$ asymptotic) states. In the $\mathrm{Ar}_{4} \mathrm{I}$ spectrum [Figure 4.3(a)] the origins of the $X$ and $I$ electronic states are distinct. In the $\mathrm{Ar}_{5} \mathrm{I}$ spectrum [Figure 4.3(b)] the peak corresponding to the $X$ state origin is quite broad, and the $I$ state appears as an unresolved shoulder. The $I$ state also appears relatively less intense than in the $\mathrm{Ar}_{1-1} \mathrm{I}$ spectra. Based on the profile of this shoulder, we can only estimate the position of the $I$ state origin to $\pm 20 \mathrm{~cm}^{-1}$. 


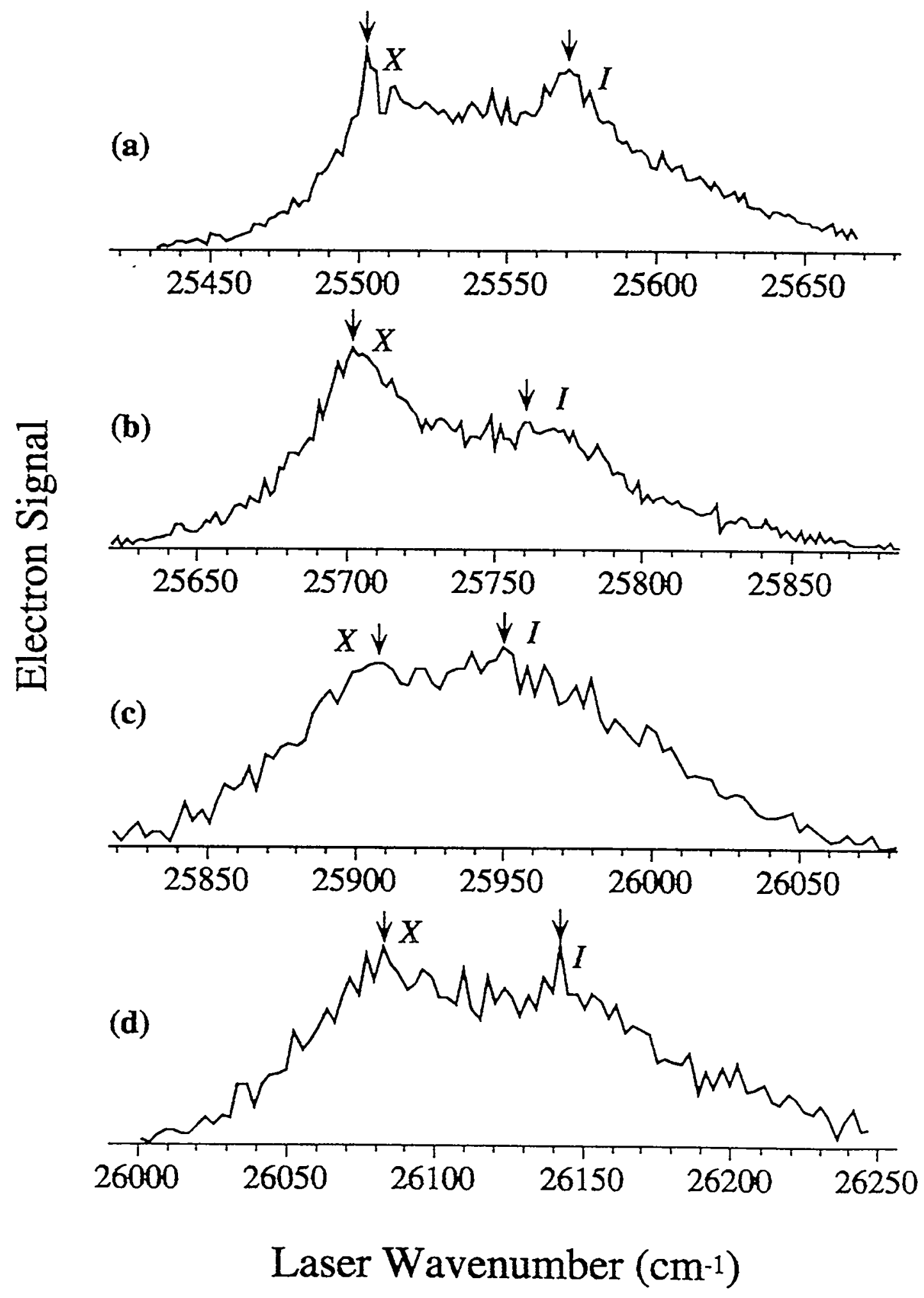

Figure 4.3. ZZEKE spectra of (a) $\mathrm{Ar}_{4} \mathrm{I}^{-}$, (b) $\mathrm{Ar}_{5} \mathrm{I}^{\circ}$, (c) $\mathrm{Ar}_{6} \mathrm{I}^{\circ}$, and (d) $\mathrm{Ar}_{7} \mathrm{I}^{-}$. The arrows indicate the neutral electronic state origins. 
The spectra of $\mathrm{Ar}_{6} \mathrm{I}$ and $\mathrm{Ar}_{7} \mathrm{I}$ [Figures 4.3(c) and 4.3(d)] are more congested. The positions of the $X$ and $I$ state origins can be estimated, as indicated by the arrows in the figures, but it is not possible to discern any reproducible vibrational structure. As we go from $\operatorname{Ar}_{4} I$ to $\operatorname{Ar}_{5} I$, the separation between the $X$ and $I$ states appears to decrease. Although the exact splitting is difficult to discern from the $\mathrm{Ar}_{6} \mathrm{I}^{-}$spectrum, it appears in this case that the $X-I$ splitting again decreases somewhat from $\mathrm{Ar}_{5} I$. However, the splitting appears to increase again in the $\mathrm{Ar}_{7} \mathrm{I}$ spectrum, in which the two states are better resolved than in the $\mathrm{Ar}_{6} \mathrm{I}$ spectrum.

We attempted to observe ZEKE spectra of $\mathrm{Ar}_{8} \mathrm{I}^{*}$ and larger clusters, but obtained only unstructured spectra with no reproducible features.

\subsubsection{Partially Discriminated Threshold Photodetachment Spectra}

Because of the increasing spectral congestion with increasing cluster size and the difficulty of producing sufficient quantities of large clusters with our source, it was not possible to perform the ZEKE experiment on clusters with $\mathrm{n}>7$ in the case of $A r_{n} \mathrm{I}$, and $\mathrm{n}>3$ for $\mathrm{Ar}_{n} \mathrm{Br}^{\circ}$. In the PDTP mode of operation it is possible to work with much smaller quantities of anions, because nearly all of the photoelectrons near the detachment threshold are collected. Therefore, only the PDTP experiment was performed for $\mathrm{Ar}_{4.9} \mathrm{Br}^{*}$ and $\mathrm{Ar}_{8-19} \mathrm{I}^{\circ}$.

The PDTP spectra of $\mathrm{Ar}_{4.9} \mathrm{Br}^{-}$are shown in Figure 4.4, and those of $\mathrm{Ar}_{8.19} \mathrm{I}^{-}$in Figure 4.5 on the following pages. 


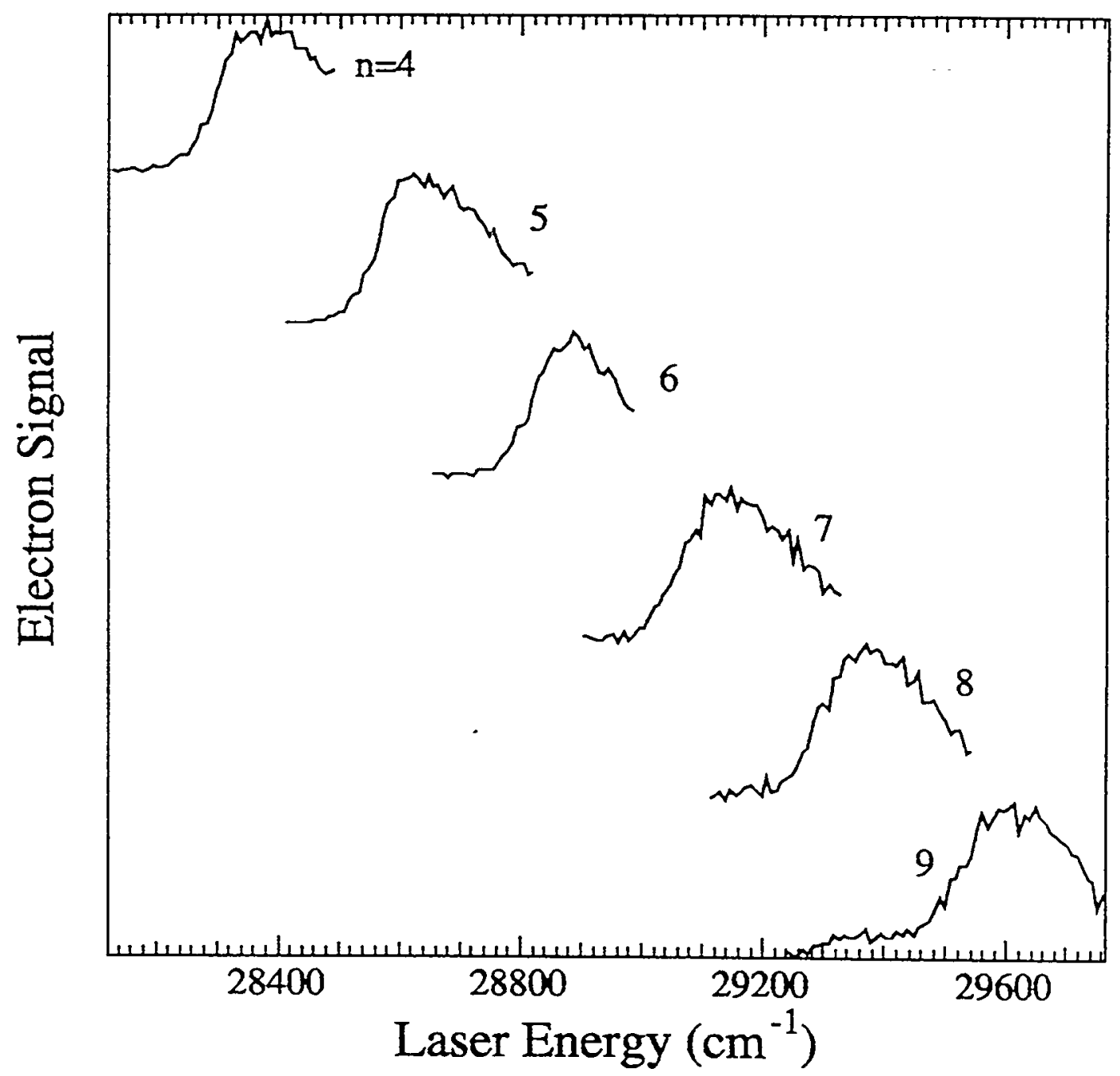

Figure 4.4. Partially discriminated threshold photodetachment (PDTP) spectra of $\mathrm{Ar}_{4.9} \mathrm{Br}^{-}$ 


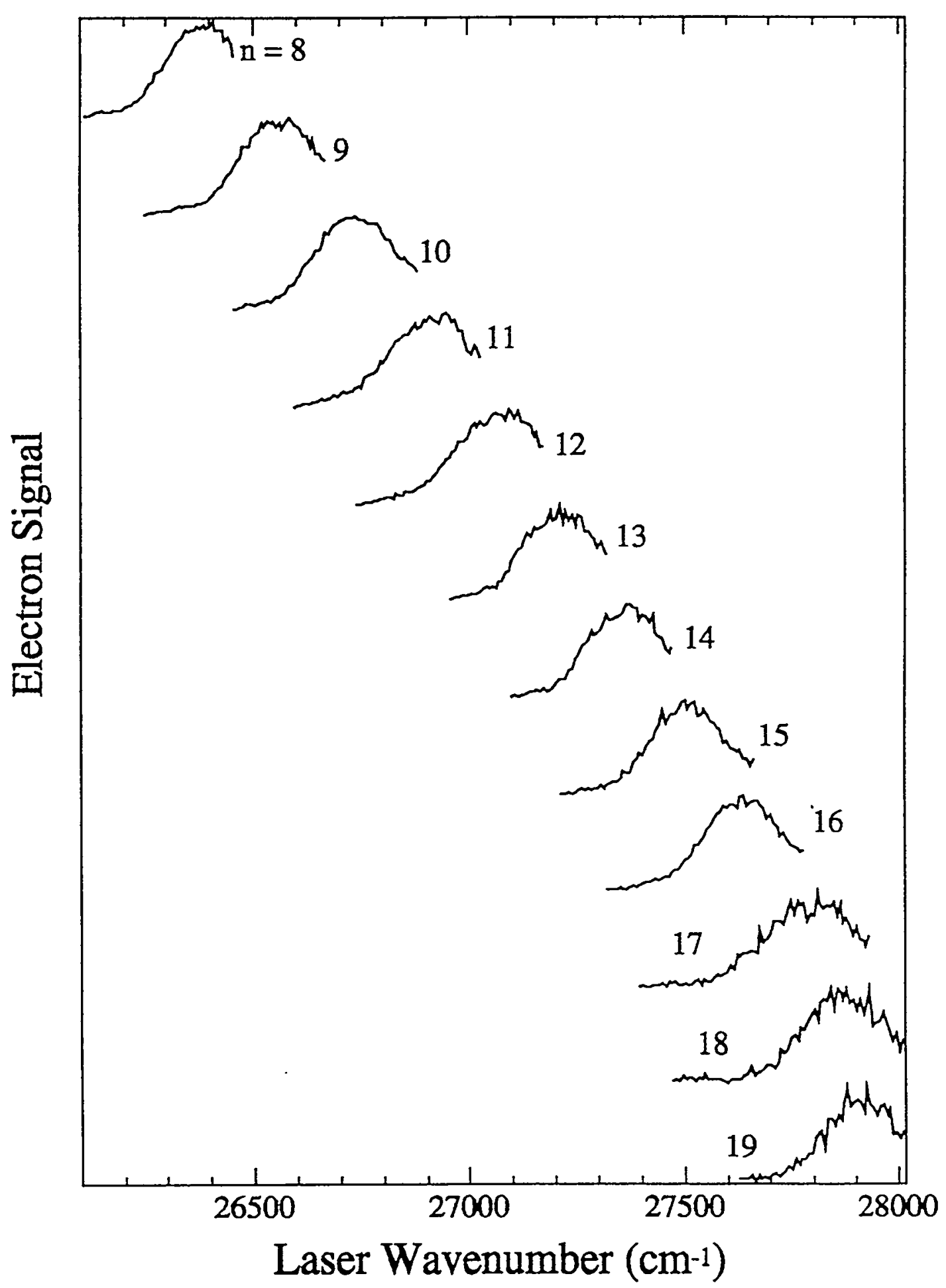

Figure 4.5. PDTP spectra of $\mathrm{Ar}_{8-19} \mathrm{I}$. 
The peaks are all about $200 \mathrm{~cm}^{-1}$ wide (FWHM), with a rising edge of about $100 \mathrm{~cm}^{-1}$. In order to estimate the adiabatic EAs from these thresholds, we compared the PDTP and ZEKE spectra of $\mathrm{Ar}_{5} \mathrm{I}^{-}$. The adiabatic $E A$ of $\mathrm{Ar}_{5} \mathrm{I}^{-}$from the $\mathrm{ZEKE}$ spectrum corresponds to the $25 \%$ point on the rising edge of the PDTP threshold. Because the shape of the PDTP spectra is relatively unchanging for the larger clusters, we estimated the adiabatic $E A$ s of the larger $\mathrm{Ar}_{\mathrm{n}} \mathrm{Br}^{-}$and $\mathrm{Ar}_{\mathrm{n}} \mathrm{I}^{-}$clusters from the $25 \%$ point of the partially discriminated cross sections. A reasonable estimate of the uncertainties in the EAs determined in this way is $\pm 50 \mathrm{~cm}^{-1}$, corresponding approximately to the width of the rising edge of the PDTP thresholds.

The adiabatic electron affinities determined from the ZEKE and PDTP spectra of $\mathrm{Ar}_{\mathrm{n}} \mathrm{Br}$ and $\mathrm{Ar}_{\mathrm{n}} \mathrm{I}$ are shown in Tables 4.1 and 4.2, respectively. The origins of the $I$ and $I I$ states, when observed, are also shown, as well as the neutral electronic state splittings $\Delta_{X-1}$ and $\Delta_{x \cdot I l}$. The stated uncertainties in the results obtained from ZEKE spectra were determined by considering the width and reproducibility of the peaks, as well as the scan step size. 
Table 3.1. Experimental adiabatic electron affinities, excited state origins, and electronic state splittings for $\mathrm{Ar}_{\mathrm{n}} \mathrm{Br}$. All energies are in $\mathrm{cm}^{-1}$. Uncertainties are in parentheses.

\begin{tabular}{|c|c|c|c|c|c|}
\hline $\mathrm{n}$ & $E A(X$ State Origin) & $I$ State Origin & $\Delta_{X-I}$ & $I I$ State Origin & $\Delta_{x \cdot I I}$ \\
\hline 0 & $27129.2^{\mathrm{a}}$ & - & - & $30814^{\mathrm{a} . \mathrm{b}}$ & $3685^{\mathrm{b}}$ \\
\hline 1 & $27429.6(3.0)$ & $27467.4(1.6)$ & $37.8(2.3)$ & $31132.3(1.6)$ & $3702.7(3.4)$ \\
\hline 2 & $27722.4(3.0)$ & $27775.5(5.0)$ & $53.1(5.5)$ & $31427.8(2.2)$ & $3705.4(3.7)$ \\
\hline 3 & $27994.6(3.0)$ & $28059.4(1.6)$ & $64.8(2.3)$ & $31702.8(2.2)$ & $3708.2(3.7)$ \\
\hline 4 & $28266(50)$ & - & - & - & - \\
\hline 5 & $28532(50)$ & - & - & - & - \\
\hline 6 & $28778(50)$ & - & - & - & - \\
\hline 7 & $29029(50)$ & - & - & - & - \\
\hline 8 & $29258(50)$ & - & - & - & - \\
\hline 9 & $29491(50)$ & - & - & - & - \\
\hline
\end{tabular}

Table 4.2. Experimental adiabatic electronic affinities, excited state origins, and electronic state splittings for $A_{n} I$.

\begin{tabular}{|c|c|c|c|c|c|}
\hline $\mathrm{n}$ & $\begin{array}{c}E A(X \text { State } \\
\text { Origin) }\end{array}$ & $I$ State Origin & $\Delta_{X-I}$ & $I I$ State Origin & $\Delta_{X \cdot I I}$ \\
\hline 0 & $24673.3^{\mathrm{a}}$ & - & - & $32276.5 \mathrm{a}, \mathrm{b}$ & $7603.15^{\mathrm{b}}$ \\
\hline 1 & $24888.3(3.0)$ & $24925.5(1.5)$ & $37.2(2.2)$ & $32512.6(2.2)$ & $\begin{array}{c}7624.3 \\
(3.7)\end{array}$ \\
\hline 2 & $25100.9(3.0)$ & $25152.9(3.0)$ & $52.0(3.4)$ & $32731.2(2.2)$ & $\begin{array}{c}7630.3 \\
(3.7)\end{array}$ \\
\hline 3 & $25303.0(3.0)$ & $25368.0(4.5)$ & $65.0(5.1)$ & $32936.4(2.2)$ & $\begin{array}{c}7633.4 \\
(3.7)\end{array}$ \\
\hline 4 & $25502.2(3.0)$ & $25571(10)$ & $69(10)$ & - & - \\
\hline 5 & $25702(10)$ & $25762(10)$ & $60(14)$ & - & - \\
\hline 6 & $25907(15)$ & $25950(15)$ & $43(21)$ & - & - \\
\hline 7 & $26083(15)$ & $26163(10)$ & $60(18)$ & - & - \\
\hline 8 & $26247(50)$ & - & - & - & - \\
\hline 9 & $26413(50)$ & - & - & - & - \\
\hline 10 & $26582(50)$ & - & - & - & - \\
\hline 11 & $26753(50)$ & - & - & - & - \\
\hline 12 & $26904(50)$ & - & - & - & - \\
\hline 13 & $27079(50)$ & - & - & - & - \\
\hline 14 & $27226(50)$ & - & - & - & - \\
\hline 15 & $27375(50)$ & - & - & - & - \\
\hline 16 & $27488(50)$ & - & - & - & - \\
\hline 17 & $27617(50)$ & - & - & - & - \\
\hline 18 & $27717(50)$ & - & - & - & - \\
\hline 19 & $27794(50)$ & - & & - & - \\
\hline
\end{tabular}

${ }^{a}$ H. Hotop and W.C. Lineberger, J. Phys. Chem. Ref. Data 14, 731 (1985). 
${ }^{b}$ C.E. Moore, Atomic Energy Levels, v. I, Circ. Natl. Bur. Std. 467 (1949). 


\subsection{Analysis and Discussion}

Our goal in this section is to compare the experimentally observed electron affinities and electronic structure with predictions from model potentials. The schematic energy level diagram shown in Figure 6.6 on the next page relates the experimental observables, i.e. the adiabatic EAs and neutral electronic state splittings, to the model anion and neutral potentials. The observed $X-I$ state splitting is then calculated from the model potential using

$$
\Delta_{X-I}=\varepsilon_{X}-\omega_{0}^{X}-\varepsilon_{1}+\omega_{0}^{I},
$$

where $\varepsilon_{X}$ and $\varepsilon_{l}$ are the classical binding energies, and $\omega_{0}^{X}$ and $\omega_{0}^{I}$ are the zero point energies of the $X$ and $I$ states, respectively. Similarly, the $X-I I$ state splitting is given by

$$
\Delta_{X-l l}=\varepsilon_{X}-\omega_{0}^{X}-\varepsilon_{l l}+\omega_{0}^{l \prime}+\Delta
$$

where $\Delta$ is the atomic spin-orbit splitting. Using similar notation for the anion binding energy and zero point energy, the calculated adiabatic $E A$ of a cluster is given by

$$
E A\left(\mathrm{Ar}_{\mathrm{n}} \mathrm{Br}\right)=E A(\mathrm{Br})+\varepsilon_{a}-\omega_{0}^{a}-\varepsilon_{X}+\omega_{0}^{X}
$$

where $E A(\mathrm{Br})$ is the electron affinity of a bare bromine atom $\left(3.3636 \mathrm{eV}^{25}\right)$. An analogous equation holds for $\mathrm{Ar}_{\mathrm{n}} \mathrm{I}$ clusters, with $E A(\mathrm{I})=3.0591 \mathrm{eV}^{26}$

Notice from Equations (4.3) and (4.4) that a comparison of the neutral potentials with the experimental electronic state splittings is possible without any knowledge of the anion potential. Thus to some extent the neutral and anion potentials can be compared with experiment independently of each other. 


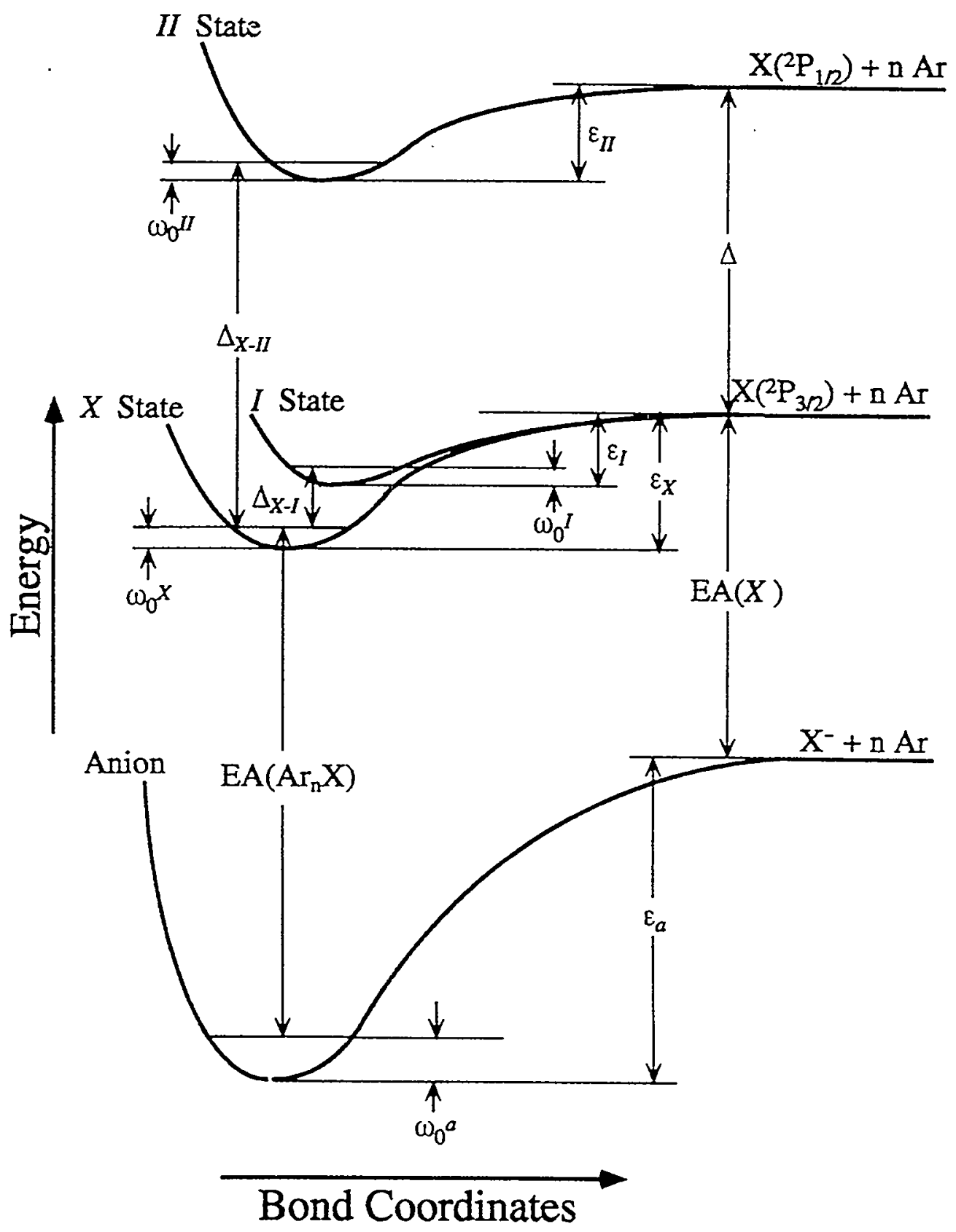

Figure 4.6. Schematic energy level diagram of the $\mathrm{Ar}_{\mathrm{n}} \mathrm{X}^{-}$anion and $A r_{0} \mathrm{X}$ neutral electronic states. 
In order to use (4.3)-(4.5), we need model potential functions, and methods to determine the minimum energy cluster geometries and to calculate the zero point energies. These are described below. We demonstrate that the experimentally observed cluster properties are not consistent with pairwise additive potentials, and then consider various non-additive corrections to the potentials.

\subsubsection{Pair Potentials}

The pair potential of the $\mathrm{ArBr}$ neutral has been determined in scattering experiments by Lee et al. ${ }^{27}$ The scattering experiments characterized only the neutral $X \frac{1}{2}$ and $I \frac{3}{2}$ state potentials. Our previous $\mathrm{ZEKE}$ results ${ }^{4}$ on the $\mathrm{ArBr}$ complex provided further refinement of Lee's potential, as well as information on the neutral $I I \frac{1}{2}$ state and anion potentials. In the case of ArI, scattering results are not available, so the ZEKE spectrum is the onlye source of information on the ArI diatomic potentials.

The neutral Ar-X potentials are of the Morse-Morse-switching function-van der Waals (MMSV) form. The reduced form of this potential, with $x=r / r_{m}$ and $f(x)=V(r) / \varepsilon$ is

$$
\begin{aligned}
f(x) & =e^{2 \beta_{1}(1-x)}-2 e^{\beta_{1}(1-x)}, & & 0<x \leq 1, \\
& =e^{2 \beta_{2}(1-x)}-2 e^{\beta_{2}(1-x)} \equiv M_{2}(x), & & 1<x \leq x_{1}, \\
& =S W(x) M_{2}(x)+[1-S W(x)] W(x), & & x_{1}<x<x_{2}, \\
& =-C_{6 r} x^{-6}-C_{8 r} x^{-8} \equiv W(x), & & x_{2} \leq x<\infty,
\end{aligned}
$$

where $\varepsilon$ is the well depth, $r_{m}$ is the bond length, and the switching function is given by

$$
S W(x)=\frac{1}{2}\left[\cos \frac{\pi\left(x-x_{1}\right)}{\left(x_{2}-x_{1}\right)}+1\right],
$$


The reduced dimensionless coefficients $C_{6 r}$ and $C_{8 r}$ are related to the usual dispersion coefficients $C_{6}$ and $C_{8}$ by

$$
C_{6 r}=\frac{C_{6}}{\varepsilon r_{m}^{6}}, \quad C_{8 r}=\frac{C_{8}}{\varepsilon r_{m}^{8}} .
$$

The anion potentials have the same form, except that the van der Waals portion is replaced by a function including charge-induced dipole $\left(r^{-4}\right)$, and charge-induced quadrupole and dispersion $\left(r^{-6}\right)$ terms:

$$
f(x)=-B_{4 r} x^{-4}-B_{6 r} x^{-6} \equiv W(x), \quad x_{2} \leq x<\infty,
$$

with

$$
B_{4 r}=\frac{B_{4}}{\varepsilon r_{m}^{4}}, \quad B_{6 r}=\frac{B_{6}}{\varepsilon r_{m}^{6}}
$$

Further details about the construction of the $\mathrm{Ar}-\mathrm{Br}$ and $\mathrm{Ar}-\mathrm{I}$ pair potentials are given in Reference 4. The MMSV potential parameters used in this work are given in Table 4.3, below.

Table 4.3. MMSV pair potential parameters of argon halides.

\begin{tabular}{|c|c|c|c|c|c|c|c|c|}
\cline { 2 - 9 } \multicolumn{1}{c|}{} & \multicolumn{4}{c|}{ ArI } & \multicolumn{4}{c|}{ ArBr } \\
\cline { 2 - 9 } \multicolumn{1}{c|}{} & $X_{\frac{1}{2}}$ & $I_{\frac{3}{2}}$ & $I_{\frac{1}{2}}$ & Anion & $X_{\frac{1}{2}}$ & $I_{\frac{3}{2}}$ & $I_{\frac{1}{2}}$ & Anion \\
\hline$\varepsilon(\mathrm{meV})$ & 18.8 & 13.9 & 16.0 & 45.8 & 16.5 & 11.5 & 14.0 & 54.4 \\
\hline$r_{m}(\AA)$ & 3.95 & 4.18 & 4.11 & 4.07 & 3.73 & 3.94 & 3.89 & 3.78 \\
\hline$\beta_{1}$ & 7.15 & 7.25 & 6.90 & 5.70 & 6.80 & 7.72 & 6.70 & 5.10 \\
\hline$\beta_{2}$ & 6.18 & 6.30 & 6.40 & 4.45 & 6.50 & 7.10 & 6.35 & 4.45 \\
\hline$x_{1}$ & 1.01 & 1.04 & 1.04 & 1.08 & 1.02 & 1.012 & 1.01 & 1.065 \\
\hline$x_{2}$ & 1.62 & 1.62 & 1.64 & 1.62 & 1.59 & 1.63 & 1.58 & 1.66 \\
\hline$C_{6}\left(\mathrm{eV} \cdot \AA^{6}\right)$ & 98.4 & 98.4 & 98.4 & - & 65.2 & 70.2 & 68.8 & - \\
\hline$C_{8}\left(\mathrm{eV} \cdot \AA^{8}\right)$ & 715 & 715 & 715 & - & 379 & 379 & 379 & - \\
\hline$B_{4}\left(\mathrm{eV} \cdot \AA^{4}\right)$ & - & - & - & 12.8 & - & - & - & 12.5 \\
\hline$B_{6}\left(\mathrm{eV} \cdot \AA^{6}\right)$ & - & - & - & 162 & - & - & - & 120.5 \\
\hline
\end{tabular}


Some of the parameters used here have been modified slightly from those published previously. ${ }^{4}$ The reason for this is that in the previous work, the well depths of the three neutral electronic states and the anion were related to each other using the relationships implied by Figure 4.6, with the zero point energies used in these relations assumed to be equal to half of the observed vibrational fundamental frequencies. A slightly more accurate procedure, used to obtain the parameters in Table 4.3 , is to calculate the actual zero point energies from the model potentials. The well depth parameters are then iteratively adjusted in order to satisfy Equations. (4.3)- (4.5), as well as to fit the observed spectra. The well depths obtained in this way differ from those given previously by no more than $2-3 \mathrm{~cm}^{-1}$, which is within the uncertainties stated in Reference 4.

It is important here to consider the uncertainties in the pair potential parameters. In the case of the $\mathrm{ArBr}$ potentials, the scattering experiments provide information on the absolute values of the well depths and bond lengths for the $X \frac{1}{2}$ state. On the other hand, the ZEKE spectra, although quite sensitive to the relative bond lengths and well depths between the anion and neutral states, are not very sensitive to the absolute values of these parameters. Therefore, the $r_{m}$ and $\varepsilon$ parameters for the $X \frac{1}{2}$ state of $\mathrm{ArBr}$ were fixed at the values of Lee $e t$ al. $^{27}$ The parameters for the anion and remaining neutral states were then adjusted to be consistent with the relations implied by Fig. 4.6, as well as to reproduce the ZEKE spectrum. The uncertainties in $r_{m}$ and $\varepsilon$ stated in Reference 27 are $\pm 0.2 \AA$ and $\pm 9 \mathrm{~cm}^{-1}$, respectively, so the absolute uncertainties of $r_{m}$ and $\varepsilon$ for the anion and the other neutral states are of about the same order. However, the relative uncertainties in $r_{m}$ and $\varepsilon$ between the anion and neutral are significantly smaller than this. 
For example, because the uncertainty in the $E A$ obtained from the $\mathbb{Z E K E}$ spectrum is \pm 3 $\mathrm{cm}^{-1}$, the difference $\varepsilon_{a}-\varepsilon_{X}$ is known with about this same uncertainty. (See Equation 2). Similarly, the relative uncertainties in $r_{m}$ are found to be about $1-2 \%(0.04-0.08 \AA$ ), based on the fit to the ZEKE spectra.

For ArI, for which scattering experiments have not been performed, modified versions of the polarizability correlation formulae of Pirani et $a l^{28}$ were used to estimate $r_{m}$ and $\varepsilon$ for the $I I \frac{1}{2}$ state of ArI, as described in Reference 4. Then the remaining neutral and anion potentials were adjusted to fit the ZEKE spectrum. The estimated absolute uncertainties in $r_{m}$ and $\varepsilon$ of ArI are $\pm 18 \mathrm{~cm}^{-1}$ for $\varepsilon$, and $\pm 0.2 \AA$ for $r_{m}$. However, the same considerations about the relative uncertainties among the anion and neutral states also apply for ArI. The relative uncertainties in $\varepsilon$ and $r_{m}$ are $\pm 3 \mathrm{~cm}^{-1}$ and $\pm 0.04-0.08 \AA$, respectively.

To model the Ar-Ar pair interaction, the accurate Hartree-Fock Dispersion (HFDB2) potential of Aziz and Slaman ${ }^{29}$ was used. For this potential $r_{m}=3.7565 \AA$ and $\varepsilon=$ $99.5465 \mathrm{~cm}^{-1}$. For the detailed form and other parameters of this well-known potential, see Reference 29.

The pairwise additive approximations to the $\mathrm{Ar}_{n} \mathrm{Br}^{-}$and $\mathrm{Ar}_{n} \mathrm{I}^{-}$binding energies were found by minimizing the additive potentials, using the simulated annealing procedure to be described below, from:

$$
\varepsilon_{a}=\min \left(V_{A r X}+V_{A r A r}\right)
$$

with $V_{A r X}=\sum_{i} V_{i 0}\left(\left|\mathbf{r}_{i}-\mathbf{r}_{0}\right|\right)$, and $V_{A r A r}=\sum_{i<j} V_{i j}\left(\left|\mathbf{r}_{i}-\mathbf{r}_{j}\right|\right)$, where the sums run over the Ar atoms, $\mathbf{r}_{i}$ is an Ar atom position, and $\mathbf{r}_{0}$ is the halide position. The calculation of the 
neutral potentials is more complex because of the open shell nature of the halogen atom, and is discussed in Section 4.4.5, below.

\subsubsection{Simulated Annealing Method}

We use a simple molecular dynamics simulated annealing procedure to determine the minimum energy cluster geometries. The simulated annealing program used here was adapted from a molecular dynamics program written by $\mathrm{Li}$ and Martens. ${ }^{30}$ The procedure used is as follows:

(1) Random initial atomic positions are generated. The initial positions lie within a 6-15 $\AA$ box, depending on the size of the cluster, and are subject to the constraint that no two atoms may be closer than a certain cutoff distance, usually $3.5 \AA$. The latter condition ensures that the cluster starts out in an attractive region of the potential surface so that dissociation does not occur.

(2) The classical equations of motion are solved for about 5 ps, using a Gear predictor-corrector algorithm started with a 16 step Runge-Kutta algorithm ${ }^{30}$. The step size is $5 \mathrm{fs}$.

(3) Kinetic energy is removed by rescaling the atomic velocities. When starting with random positions, the kinetic energy is removed very quickly, so that the velocities and kinetic energies are essentially reset to zero with each rescaling. This rapid quenching was found to be necessary to prevent evaporation.

(4) Steps (2) and (3) are repeated until a minimum is found. This typically requires $100-250 \mathrm{ps.}$ 
(5) Beginning with the minimum configuration found by the above procedure, kinetic energy is added, constrained so that the translational energy of the cluster centerof-mass and its angular momentum are zero. To prevent evaporation, the initial kinetic energy was usually set to not more than $25-33 \%$ of the total well depth. Then steps (2) and (3) are repeated, but with kinetic energy removed much more gradually, by rescaling the velocities by a factor ${ }^{31}$

$$
\left[1+\frac{\tau_{\text {scale }}}{\tau_{\text {const }}}\left(\frac{K E_{\text {targ }}}{K E_{\text {avg }}}-1\right)\right]^{1 / 2}
$$

every 5 ps. Here $\tau_{\text {scale }}$ is the time between rescalings, $\tau_{\text {const }}$ is a time constant--typically 50 or 100 ps-- $K E_{\text {avg }}$ is the average kinetic energy, and $K E_{\text {targ }}$ is a target kinetic energy, set to a very small value in order to find a minimum. The entire procedure typically requires 510 ns.

(5) Finally, the minimum energy configuration is located more precisely using a simple gradient minimization routine. ${ }^{32}$

The entire annealing procedure was repeated 5-20 times for each cluster to ensure that the global minimum was found. In this process, low-lying local minima were often also found. In order to locate higher lying local minima, an interval of 250 fs or less between rescaiing steps is used in steps (2)- (4), to prevent equilibration of the cluster as kinetic energy is removed.

For further details about simulated annealing and the computer program used to implement it, see Appendix C. 


\subsubsection{Zero Point Energy Calculation}

Once the minimum energy configurations and classical binding energies are found, it is necessary to know the zero point energies in order to use Equations (4.3)- (4.5). The model potentials are analytical functions of the nuclear Cartesian coordinates, allowing the zero point energies to be estimated by the following procedure. The normal coordinates of the clusters were found in terms of linear combinations of Cartesian displacement coordinates, using standard techniques. ${ }^{33}$ Then each of the $3 N-6$ singlemode vibrational Schrödinger equations was solved using a simple one-dimensional discrete variable representation (DVR) procedure..$^{5.34,35.36}$ In this way, the anharmonicity of the potential is approximately accounted for, although interactions between normal modes are neglected. The total zero point energy was then obtained by adding up the single mode values. The zero point calculation was limited to the portion of the potential in the vicinity of the minimum structure, so that any splittings due to tunneling are not reproduced.

For details of the computer program used to calculate the zero point energies, refer to Appendix C, Sections C5 and C6.

\subsubsection{Anion Minimum Energy Geometries}

The minimum energy geometries found using pairwise additive potentials for $\mathrm{Ar}_{2.19} \mathrm{I}^{\circ}$ are shown in Figure 4.7. Similar structures were found for $\mathrm{Ar}_{2.9} \mathrm{Br}$. The calculated anion binding energies and zero point energies are given in Tables 4.4 and 4.5, on the following pages. 

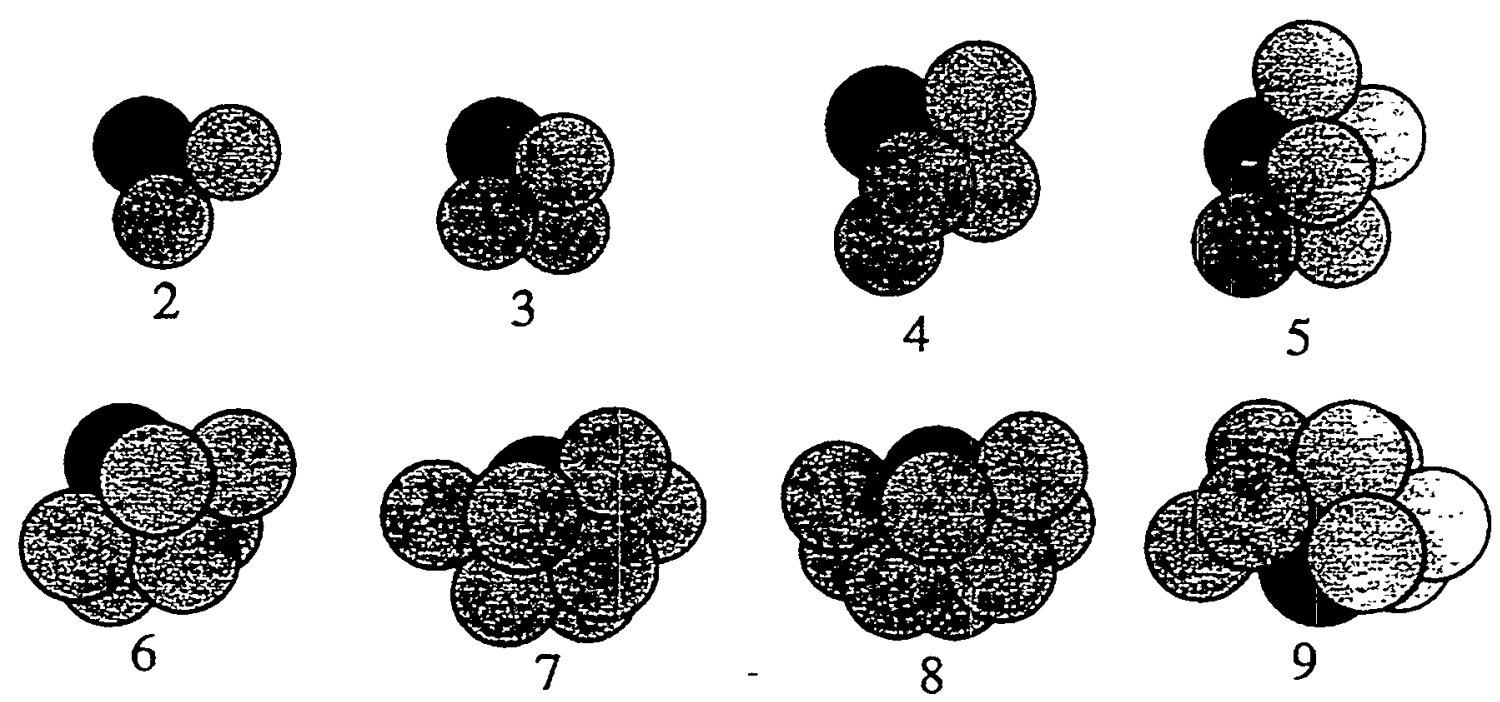

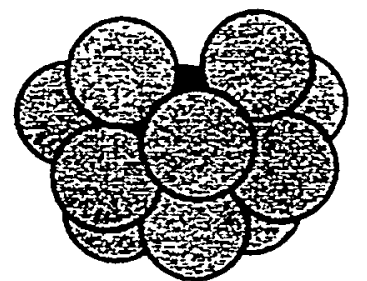

10

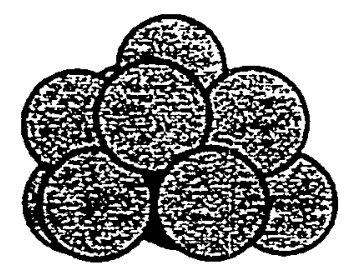

11

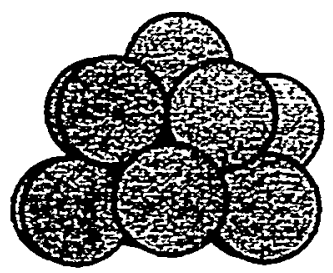

12

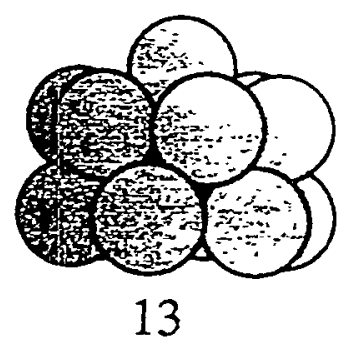

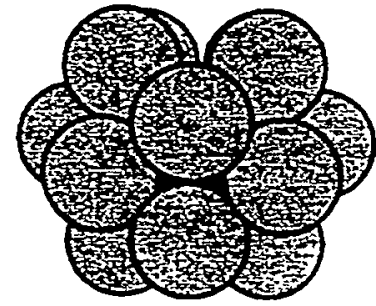

14

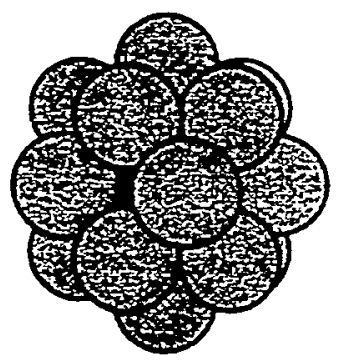

17

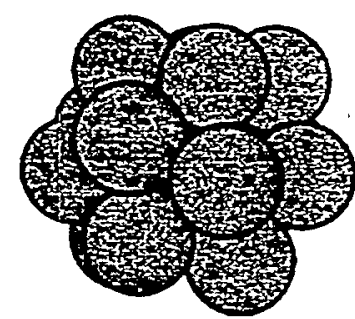

15

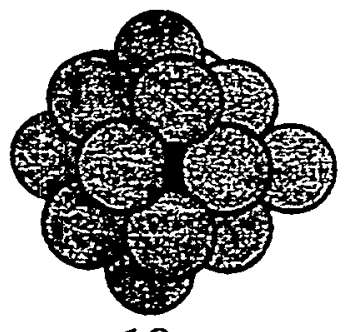

18
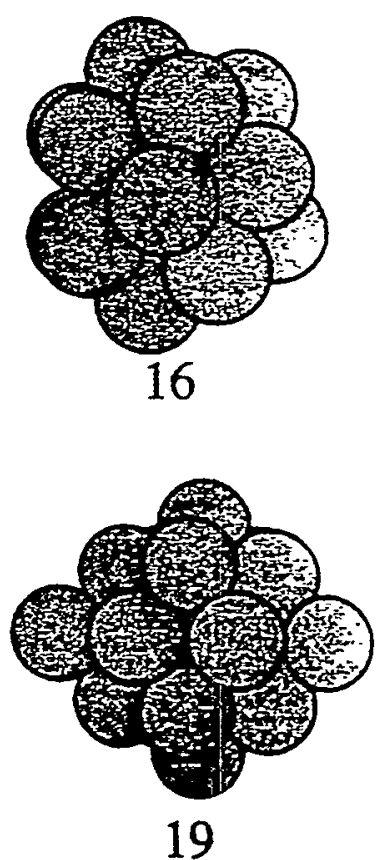

Figure 4.7. Minimum energy structures of $\mathrm{Ar}_{2-19} \mathrm{I}$ clusters found using pairwise additive potentials. 
Table 4.4. Results of calculations with pairwise additive $\mathrm{Ar}_{n} \mathrm{Br}^{\circ}$ anion potentials, and "matrix-additive" $\mathrm{Ar}_{n} \mathrm{Br}$ neutral potentials. All energies are in $\mathrm{cm}^{-1}$.

\begin{tabular}{|c|c|c|c|c|c|c|c|c|c|c|c|}
\hline $\mathrm{n}$ & $\varepsilon_{a}$ & $\varepsilon_{x}$ & $\varepsilon_{I}$ & $\varepsilon_{I I}$ & $\omega_{0}^{a}$ & $\omega_{0}^{X}$ & $\omega_{0}^{I}$ & $\omega_{0}^{I I}$ & $\Delta_{X-I}$ & $\Delta_{x-I I}$ & $E A_{\text {add }}$ \\
\hline 0 & 0 & 0 & 0 & 0 & 0 & 0 & 0 & 0 & 0 & 3685 & 27129.2 \\
\hline 1 & 438.8 & 133.1 & 92.8 & 112.9 & 20.7 & 15.6 & 13.5 & 13.4 & 38.2 & 3703.0 & 27429.8 \\
\hline 2 & 977.1 & 349.4 & 295.4 & 319.6 & 56.8 & 42.3 & 45.0 & 44.6 & 56.6 & 3717.1 & 27742.3 \\
\hline 3 & 1614.9 & 662.6 & 599.9 & 628.7 & 107.4 & 85.1 & 89.6 & 88.6 & 67.3 & 3722.4 & 28059.2 \\
\hline 4 & 2260.0 & 977.3 & 916.6 & 945.0 & 157.8 & 127.6 & 135.2 & 132.9 & 68.4 & 3722.6 & 28382 \\
\hline 5 & 2911.3 & 1293.6 & 1243.7 & 1267.3 & 207.5 & 168.7 & 181.1 & 176.4 & 62.3 & 3719.0 & 28708 \\
\hline 6 & 3659.7 & 1696.2 & 1677.2 & 1686.0 & 267.5 & 211.7 & 248.5 & 230.0 & 55.8 & 3713.6 & 29037 \\
\hline 7 & 4318.6 & 2042.9 & 2001.2 & 2014.8 & 315.5 & 268.9 & 289.7 & 272.9 & 62.5 & 3717.1 & 29358 \\
\hline 8 & 5069.8 & 2465.0 & 2413.9 & 2432.4 & 371.1 & 315.8 & 337.1 & 323.6 & 72.4 & 3725.4 & 29679 \\
\hline 9 & 5815.2 & 2880.0 & 2807.1 & 2837.7 & 421.3 & 360.3 & 375.7 & 370.1 & 88.2 & 3737.1 & 30003 \\
\hline
\end{tabular}

Table 4.5. Results of calculations with pairwise additive $\mathrm{Ar}_{n}{ }^{-}$anion potentials, and "matrix-additive" $A r_{n} I$ neutral potentials. All energies are in $\mathrm{cm}^{-1}$.

\begin{tabular}{|c|c|c|c|c|c|c|c|c|c|c|c|}
\hline $\mathrm{n}$ & $\varepsilon_{a}$ & $\varepsilon_{X}$ & $\varepsilon_{I}$ & $\varepsilon_{I I}$ & $\omega_{0}^{a}$ & $\omega_{0}^{X}$ & $\omega_{0}^{I}$ & $\omega_{0}^{I I}$ & $\Delta_{X . I}$ & $\Delta_{X-I I}$ & $E A_{\text {add }}$ \\
\hline 0 & 0 & 0 & 0 & 0 & 0 & 0 & 0 & 0 & 0 & 7603.15 & 24673.3 \\
\hline 1 & 369.4 & 151.6 & 112.1 & 129.0 & 17.3 & 14.6 & 12.3 & 13.2 & 37.2 & 7624.4 & 24888.3 \\
\hline 2 & 838.4 & 385.4 & 332.0 & 355.5 & 51.0 & 41.2 & 42.4 & 42.4 & 54.5 & 7634.3 & 25116.5 \\
\hline 3 & 1406.8 & 718.1 & 653.1 & 682.5 & 98.4 & 83.2 & 85.8 & 85.4 & 67.6 & 7640.9 & 25346.8 \\
\hline 4 & 1982.0 & 1051.5 & 986.0 & 1016.3 & 145.8 & 125.1 & 130.1 & 128.7 & 70.5 & 7642.0 & 25583 \\
\hline 5 & 2562.3 & 1385.6 & 1328.5 & 1355.1 & 192.7 & 165.8 & 174.9 & 171.3 & 66.2 & 7639.2 & 25823 \\
\hline 6 & 3229.2 & 1799.2 & 1767.6 & 1782.7 & 249.4 & 208.3 & 233.9 & 222.5 & 57.3 & 7633.8 & 26062 \\
\hline 7 & 3815.2 & 2151.3 & 2112.5 & 2126.9 & 295.3 & 257.8 & 279.6 & 264.3 & 60.6 & 7634.0 & 26300 \\
\hline 8 & 4469.2 & 2569.3 & - & - & 343.5 & 300.4 & - & - & - & - & 26530 \\
\hline 9 & 5094.8 & 2963.2 & - & - & 379.9 & 334.7 & - & - & - & - & 26760 \\
\hline 10 & 5731.8 & 3369.0 & - & - & 436.9 & 390.0 & - & - & - & - & 26990 \\
\hline 11 & 6366.8 & 3764.8 & - & - & 476.1 & 428.1 & - & - & - & - & 27227 \\
\hline 12 & 7044.3 & 4199.3 & - & - & 539.6 & 483.9 & - & - & - & - & 27463 \\
\hline 13 & 7796.7 & 4706.4 & - & - & 586.8 & 530.4 & - & - & - & - & 27707 \\
\hline 14 & 8519.3 & 5159.0 & - & - & 645.1 & 572.8 & - & - & - & - & 27961 \\
\hline 15 & 9280.6 & 5686.6 & - & - & 710.5 & 641.6 & - & - & - & - & 28198 \\
\hline 16 & 9914.3 & 6143.3 & - & - & 777.5 & 693.7 & - & - & - & - & 28360 \\
\hline 17 & 10561.3 & 6589.0 & - & - & 850.4 & 770.0 & - & - & - & - & 28565 \\
\hline 18 & 11102.2 & 7061.3 & - & - & 913.0 & 804.9 & - & - & - & - & 28606 \\
\hline 19 & 11648.0 & 7498.0 & - & - & 979.3 & 880.8 & - & - & - & - & 28725 \\
\hline
\end{tabular}


For $\mathrm{Ar}_{2-3} \mathrm{X}^{-}(\mathrm{X}=\mathrm{Br}$ or $\mathrm{I})$, there is only one minimum, in which all atoms are in contact with each other. Linear $\left(\operatorname{Ar}_{2} \mathrm{X}\right)$ or planar $\left(\mathrm{Ar}_{3} \mathrm{X}\right)$ geometries are not stable with additive potentials. Furthermore, the $X-I$ state splittings expected for linear and planar geometries are not consistent with those observed experimentally.

In the minimum energy structures of larger clusters $\left(\mathrm{Ar}_{n} \mathrm{I}^{*} 4 \leq n \leq 17\right.$, and $\mathrm{Ar}_{n} \mathrm{Br}$ $4 \leq n \leq 9)$, all the Ar atoms contact the central halide atom. This type of structure is energetically favorable because each Ar- $\mathrm{X}^{\circ}$ "bond" is about four times stronger than an Ar-Ar "bond." For $\mathrm{Ar}_{4} \mathrm{X}$; , one local minimum isomer is seen $\left(C_{3 v}\right.$ point group) which has one $\mathrm{Ar}$ atom in contact with the other three argons but not with the halide. In $\mathrm{Ar}_{4} \mathrm{I}$; its energy is about $200 \mathrm{~cm}^{-1}$ higher than that of the global minimum. The analogous $\mathrm{Ar}_{4} \mathrm{Br}$. isomer lies $256 \mathrm{~cm}^{-1}$ above the global minimum. These energy differences correspond approximately to one $\operatorname{Ar}-\mathrm{X}^{-}$"bond". $\operatorname{Ar}_{5} \mathrm{X}^{\mathrm{X}}$ has two local minima with approximately the same separations from the global minimum as in the $\mathrm{Ar}_{4} \mathrm{X}^{-}$clusters.

The clusters with $6 \leq n \leq 17$ show two types of local minima. In one type, the Ar atoms are all in contact with the halide--as in the global minimum--but have fewer Ar-Ar "bonds." These typically differ in energy from the global minimum by approximately the magnitude of an Ar-Ar "bond," i.e. about $100 \mathrm{~cm}^{-1}$. The other type, seen already for $\mathrm{n}<6$, are structures in which one or more Ar atoms are not in direct contact with the halide. This type of isomer usually differs in energy from the global minimum by approximately the energy of one or more Ar-X" "bonds."

For $\operatorname{Ar}_{n} \mathrm{I}$, rare gas atoms continue to fit around the halide without significant crowding up to $n=15$. At $n=16$ there is some crowding, so that the Ar-I contribution to the potential is reduced. $\mathrm{Ar}_{17} \mathrm{I}$ " constitutes a "closed" solvent shell (at $0 \mathrm{~K}$ ). It consists of a 
capped pentagonal bipyramid structure $\left(D_{5 h}\right)$, with the axial $A r$ atoms significantly further from the halide than the others. Subsequent Ar atoms are added outside the first solvent shell. In the case of $\mathrm{Ar}_{n} \mathrm{Br}^{-}$, we did not observe the closing of the solvent shell since we did not perform calculations for $n \gg 9$.

\subsubsection{Neutral Open-Shell Potentials}

Because of the anisotropy of the open-shell halogen atom in the neutral clusters, the potentials cannot in general be obtained by simply adding the $\mathrm{Ar}-\mathrm{X}$ pair potentials. This is clear from the observed spectra. For example, in the diatomic ArI molecule an X-I splitting of $37 \mathrm{~cm}^{-1}$ is observed. ${ }^{4}$ If the potentials were simply additive, one would predict an $X-I$ splitting of $74 \mathrm{~cm}^{-1}$ for $A_{2} I$. The observed $\Delta_{X \cdot l}$ in $A_{r_{2}} I$ is $52 \mathrm{~cm}^{-1}$. The simple additive prediction is well outside experimental uncertainty.

This "non-additivity" of the open-shell potentials has been discussed by Lawrence and Apkarian, ${ }^{37}$ whose explanation we follow here. The non-additivity can most easily be understood if we momentarily neglect the effect of spin-orbit coupling. In this case there are two electronic states of the diatomic complex corresponding to the two possible orientations of the singly occupied halogen p-orbital relative to the argon atom..$^{24.38} \mathrm{~A}^{2} \Sigma$ state arises when the singly occupied p-orbital lies along the internuclear axis, and a doubly degenerate ${ }^{2} \Pi$ state corresponds to the singly occupied p-orbital lying perpendicular to the internuclear axis. However, if the cluster contains additional Ar atoms, $\Lambda$ is no longer a good quantum number if the polyatomic cluster is not linear. Consider, for example, the case of $\mathrm{Ar}_{2} \mathrm{I}$. The singly occupied halogen p-orbital will not, in general, lie either parallel or perpendicular to either of the Ar-I internuclear axes. 
Therefore the Ar-I interaction potentials in $\mathrm{Ar}_{2} \mathrm{I}$ will not be the same as the potentials of either the ${ }^{2} \Sigma$ or ${ }^{2} \Pi$ diatomic states, but--in the first approximation--may be considered to be linear combinations of the diatomic potentials. Thus, in order to obtain the potentials of $\mathrm{Ar}_{2} \mathrm{I}$, and larger open-shell clusters, from the diatomic potentials, our concept of pairwise additivity must be extended to include this mixing of the diatomic electronic states. We describe how this is done in more detail below.

A simple first-order perturbation theory treatment of the interaction of an openshell atom with several closed-shell (rare gas) atoms in terms of the diatomic potentials has been developed by various workers..$^{39,40,41,42}$ These methods have been used to study open-shell atoms in rare gas matrices, clusters, and on surfaces. ${ }^{12.37 .43,44.45}$ Our implementation here most closely resembles that of Lawrence and Apkarian, ${ }^{37}$ who studied the emission spectra of I atoms in $\mathrm{Xe}$ and $\mathrm{Kr}$ matrices. The theory is briefly as follows.

The $A r_{n}-X$ interaction is modeled by an effective potential depending on the rare gas coordinates and on the coordinates of the "hole" in the singly occupied halogen porbital in an arbitrary space-fixed frame:

$$
H=\sum_{k} V_{A r_{n} X}\left(\mathbf{r}, \mathbf{R}_{k}\right)+H_{s o}
$$

Here, the sum is over the rare gas atoms, $\mathbf{r}$ is the coordinate of the "hole," $\mathbf{R}_{k}$ are the rare gas coordinates relative to the halogen nucleus, and $H_{s o}$ is the spin-orbit interaction Hamiltonian.

The potential $V_{A r_{k} x}$ is then expanded in Legendre polynomials in $\hat{\mathbf{r}} \cdot \hat{\mathbf{R}}_{k}$. We are ultimately interested in the matrix elements of $H^{\prime}$ in a p-orbital basis, and only the first two even terms of the expansion contribute to these. Hence, we write 


$$
H^{\prime}=\sum_{k}\left[V_{0}\left(r, R_{k}\right)+V_{2}\left(r, R_{k}\right) P_{2}\left(\hat{\mathbf{r}} \cdot \hat{\mathbf{R}}_{k}\right)\right]+H_{s o}
$$

In the diatomic case (one $\mathrm{Ar}$ atom), the expectation values of these two expansion coefficients, $V_{0}(R)$ and $V_{2}(R)$, can be shown, using the relations given by Haberland ${ }^{24}$ and Aquilanti et al. ${ }^{38}$ to be related to the spectroscopic diatom potentials by

and

$$
\begin{aligned}
& V_{0}(R)=\frac{1}{3}\left[V_{x \frac{1}{2}}(R)+V_{t \frac{1}{2}}(R)+V_{l t \frac{1}{2}}(R)\right] \\
& V_{2}(R)=\frac{5}{3}\left[V_{x_{\frac{1}{2}}}(R)+V_{l t \frac{1}{2}}(R)-2 V_{t \frac{1}{2}}(R)\right] .
\end{aligned}
$$

Here, the zero for each potential is set at the potential asymptote. $\left({ }^{2} \mathrm{P}_{1 \Omega}\right.$ for $V_{13 \Omega}$, and ${ }^{2} \mathrm{P}_{3 \Omega}$ for $V_{X 12}$ and $\left.V_{l 11 \Omega}\right)$. In deriving these equations it is assumed that the spin-orbit constant, $\Delta$, is independent of $R$.

With some effort, one can show that for a cluster with many Ar atoms, the perturbation Hamiltonian $\mathrm{H}^{\prime}$ is given by a $6 \times 6$ matrix: ${ }^{37.42}$

$$
\mathrm{H}=\sum_{k} V_{0}\left(R_{k}\right) \cdot \mathbf{1}+V_{2}\left(R_{k}\right) \cdot \mathbf{M}\left(\mathbf{R}_{k}\right)
$$

where $\mathbf{M}\left(\mathbf{R}_{k}\right)$ is a $6 \times 6$ Hermitian matrix involving the argon atom coordinates. The detailed form of the matrix $\mathbf{H}^{\prime}$ has been given, in the $\left|J, m_{J}\right\rangle$ basis, by Lawrence and Apkarian. ${ }^{37}$ Diagonalization of $\mathbf{H}^{\prime}$ yields three doubly degenerate eigenvalues, corresponding to the potentials of the $X, I$ and $I I$ states.

In our implementation, an analytical form for the eigenvalues was found using the Maple V program. This allowed the eigenvalues to be calculated approximately 10 times faster than by numerical diagonalization and saved considerable computer time. The potentials, $V_{A r_{a} X}$, are then referred to their own asymptotes by adding $\frac{1}{3} \Delta$ to the $X$ and $I$ 
state potentials, and subtracting $\frac{2}{3} \Delta$ from the $I I$ state potential. The total potential of the cluster is then obtained by adding the Ar-Ar potentials in a pairwise fashion. The well depths are found by minimizing these potentials using the simulated annealing and gradient minimization procedures described in Section 4.4.2. For the $X$ state, for example,

$$
\varepsilon_{X}=\min \left(V_{A r_{n} X}+V_{A r A r}\right)
$$

with $V_{A r A r}$ the same as in Equation (4.11).

There are several assumptions implicit in this treatment of the open shell potentials. First, the basis set is limited to p-orbitals; excited orbitals of the halogen or rare gas atoms are not included. Thus, many-body effects due to polarization of the halogen atom or charge-transfer are neglected. Also, we assume that the spin-orbit constant, $\Delta$, is independent of the internuclear separations, as well as independent of the number of rare gas atoms in the cluster. To verify the former assumption, $\Delta$ was calculated as a function of $R$ for $\mathrm{ArBr}$ and $\mathrm{ArI}$, using the relations given by Haberland ${ }^{24}$ and Aquilanti et al. ${ }^{38}$, and the three diatomic potential energy curves determined from the ZEKE spectra. The calculated $\Delta$ does not vary more than $1 \mathrm{meV}(0.1 \%)$ for ArI and not by more than $5 \mathrm{meV}$ (1\%) for $\mathrm{ArBr}$ for $R$ greater than the zero crossing point. The assumption that $\Delta$ is independent of the number of argon atoms is more questionable, as we will see below.

The above method of calculating the adiabatic potential surfaces was used directly in the simulated annealing procedure for the smaller clusters $(n \leq 6)$. For the larger clusters, the annealing was first performed using the anion potentials described above, and then the system was allowed to relax (to optimize the geometry) on each of the neutral surfaces. In most cases, the anion and neutral have approximately the same global minimum 
configurations. There are some exceptions. For instance, the global minimum isomer of the $\mathrm{Ar}_{5} \mathrm{Br}^{-}$anion has all five of the $\mathrm{Ar}$ atoms in contact with the $\mathrm{Br}^{-}$atom, but this geometry corresponds to a local minimum of the neutral $X$ state surface. In such cases, the neutral minimum corresponding to the anion global minimum was always used to compute the "adiabatic" EAs and neutral electronic state splittings.

The results of the calculation of the neutral binding energies, zero point energies, $\Delta_{x-l,}$, and $\Delta_{X-l l}$ are presented in Tables 4.4 and 4.5 , for $\mathrm{Ar}_{2.9} \mathrm{Br}$ and $\mathrm{Ar}_{2-19} \mathrm{I}$, respectively. It is interesting to note that for all $\mathrm{n}>1$, the zero point energy of the $I$ state is greater than that of the $X$ state, contrary to intuition. This seems to be due to the steep repulsive wall of the $I \frac{1}{2}$ diatomic state, which causes the antisymmetric modes to be more steeply curved in the $I$ than in the $X$ state. The result is an increase in $\Delta_{X \cdot I}$ over what would be calculated if the zero point energies were neglected.

We can compare the $X-I$ splittings calculated using Equation (4.3) with the experimental results without reference to the anion potential. This comparison is shown in Figure 4.8. In the cases where the two states are well resolved, the agreement with experiment is quite satisfactory.

For $\mathrm{n}=2$ and 3 the splitting between the $X$ and $I I$ states may also be compared with experiment using Equation (4.4), as shown in Figure 4.9. 
(a)

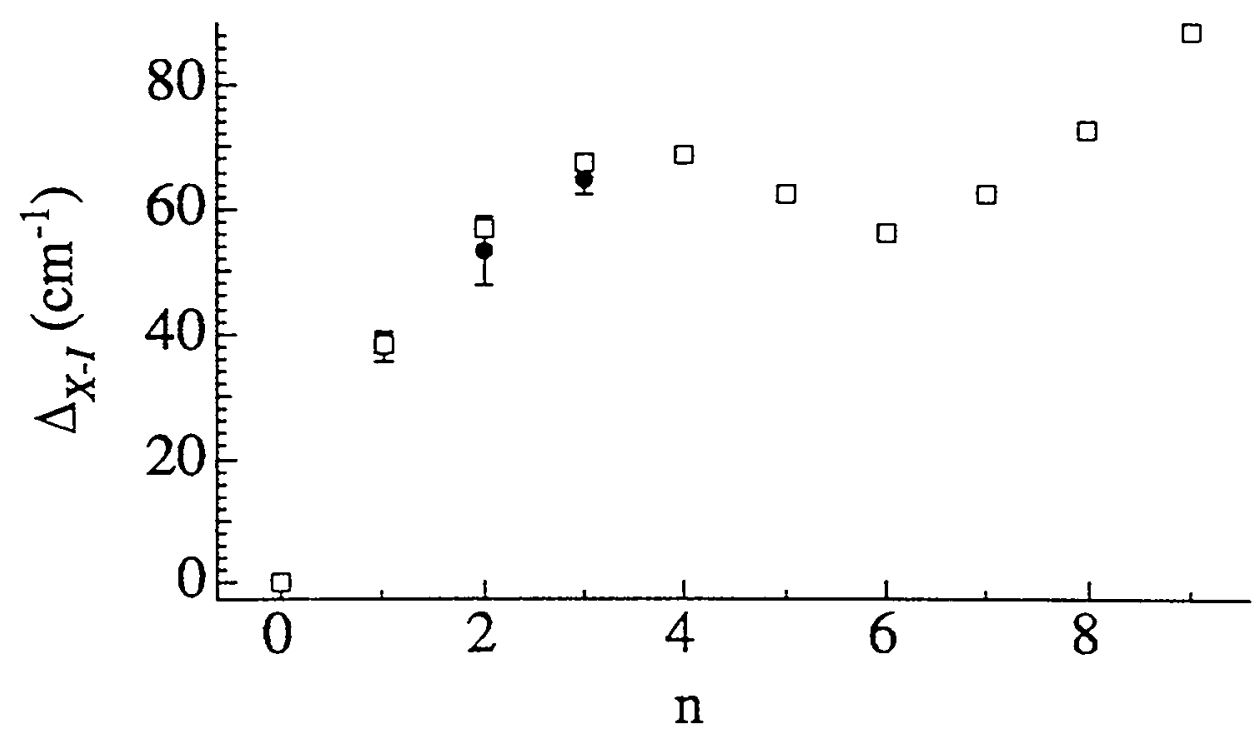

(b)

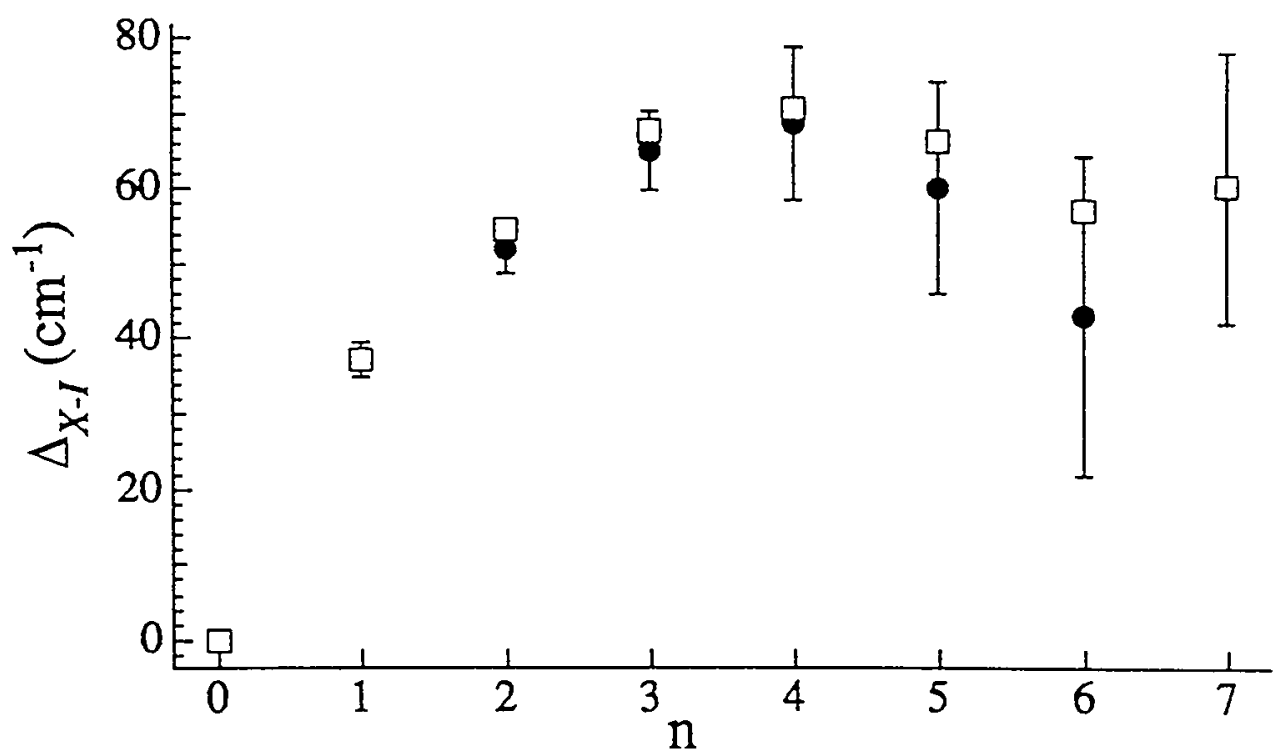

Figure 4.8. Comparison of experimental and calculated $X-I$ state splittings for (a) $\mathrm{Ar}_{\mathrm{n}} \mathrm{Br}$ and (b) $A r_{n} I$. Solid circles: experimental. Open squares: calculated as described in Section 4.4.5. 
(a)

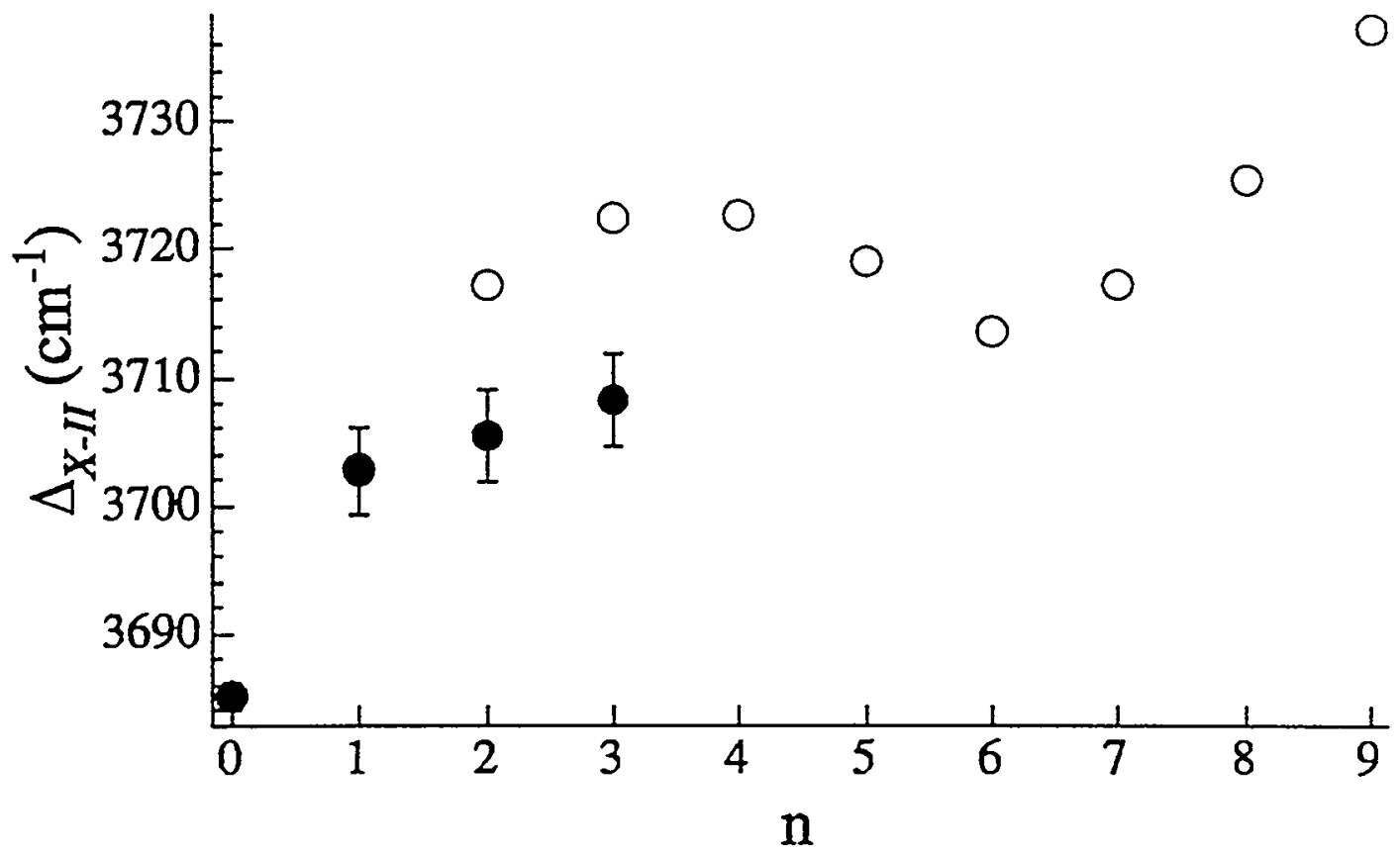

(b)

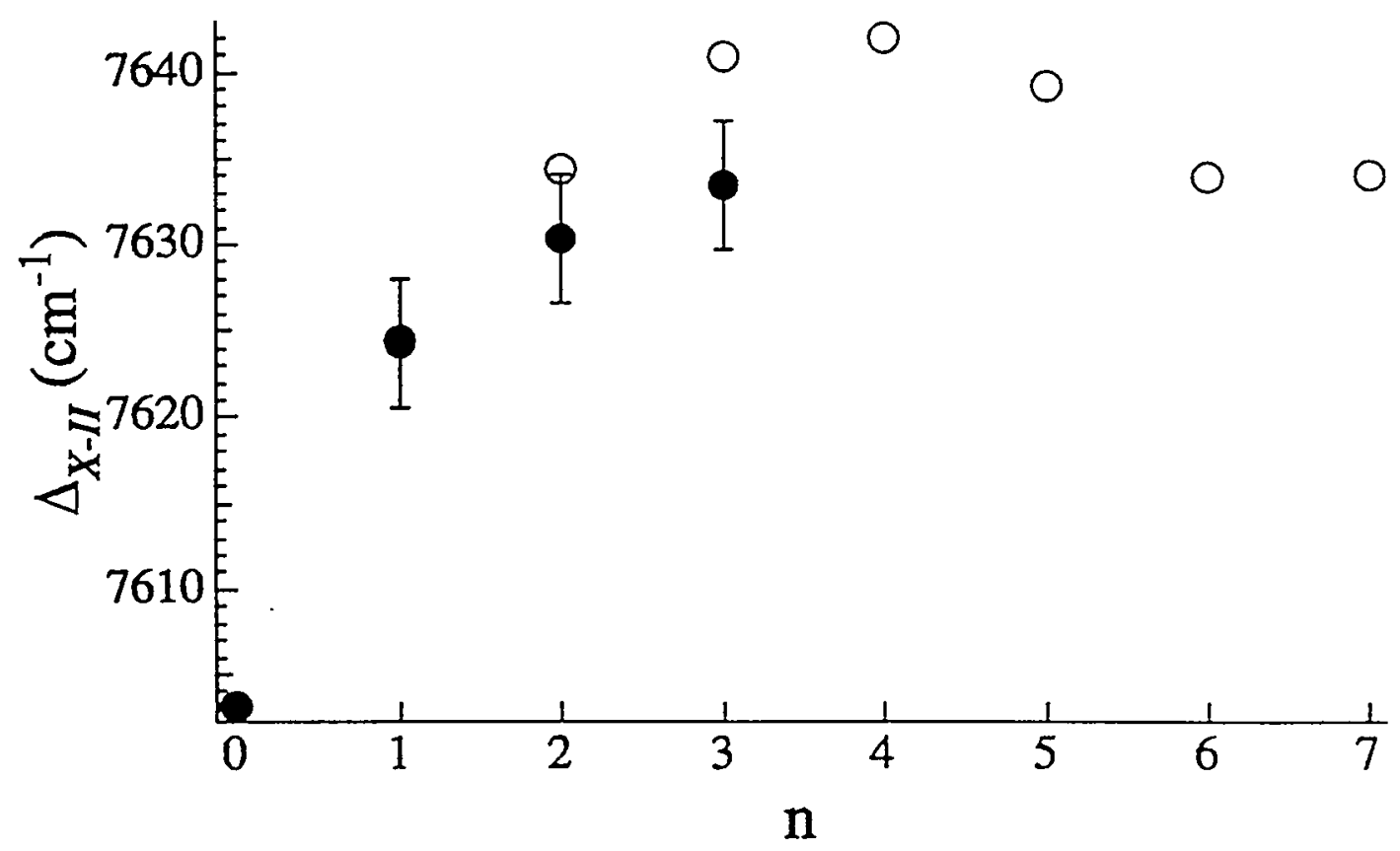

Figure 4.9. Comparison of experimental and calculated $X$-II state splittings for (a) $\mathrm{Ar}_{n} \mathrm{Br}$, and (b) $\mathrm{Ar}_{n} \mathrm{I}$. Solid circles: experimental. Open circles: calculated as described in Section 4.4.5. 
For both $\mathrm{Ar}_{2-3} \mathrm{Br}$ [Figure 4.9(a)] and $\mathrm{Ar}_{2-3} \mathrm{I}$ [Figure 4.9(b)], the theoretical $\Delta_{x-I I}$ is greater than the experimental value by about $5-15 \mathrm{~cm}^{-1}$. The agreement is somewhat worse for $\mathrm{Ar}_{2-3} \mathrm{Br}$ than for $\mathrm{Ar}_{2-3} \mathrm{I}$. This discrepancy could mean that the atomic spin-orbit splitting, $\Delta$, is not independent of the number of Ar atoms, as was assumed above. It is known that the spin-orbit splitting of atoms in rare gas matrices is different from that of the free atoms. For example, Lawrence and Apkarian found that the I atom spin-orbit splitting is decreased by about $3 \%$ or $5 \%$ in $\mathrm{Xe}$ or $\mathrm{Kr}$ matrices, respectively. ${ }^{37}$ We observe a smaller decrease of $\Delta$ in the small clusters studied here: about $0.06 \%-0.1 \%$ in $\mathrm{Ar}_{2.3} \mathrm{I}$ and $0.3 \%$ $0.4 \%$ in $\mathrm{Ar}_{2-3} \mathrm{Br}$.

Generally speaking, the open shell interactions described in this section are nonadditive, in the sense that they are of the form of the additional terms in Eq. 4.2. However, they are not true many-body effects because they can be obtained directly from the pair potentials, and do not introduce additional interactions between the Ar atoms in contrast to the effects described in Section 4.4.7, below. As pointed out by Sando and coworkers, ${ }^{42}$ the open-shell potentials in the $n>1$ clusters can be considered to be additive as matrices rather than as scalars, and we will refer to these interactions as "matrix additive" effects in the rest of this discussion.

\subsubsection{Electron Affinities Calculated from Additive Potentials}

The adiabatic EAs calculated from Equation (4.5) using the additive anion potentials [Equation (4.11)] and "matrix additive" neutral potentials [Equation (4.17)] are given in Tables 4.4 and 4.5. These are compared with the experimental EAs in Figures 4.10 and 4.11. 
(a)

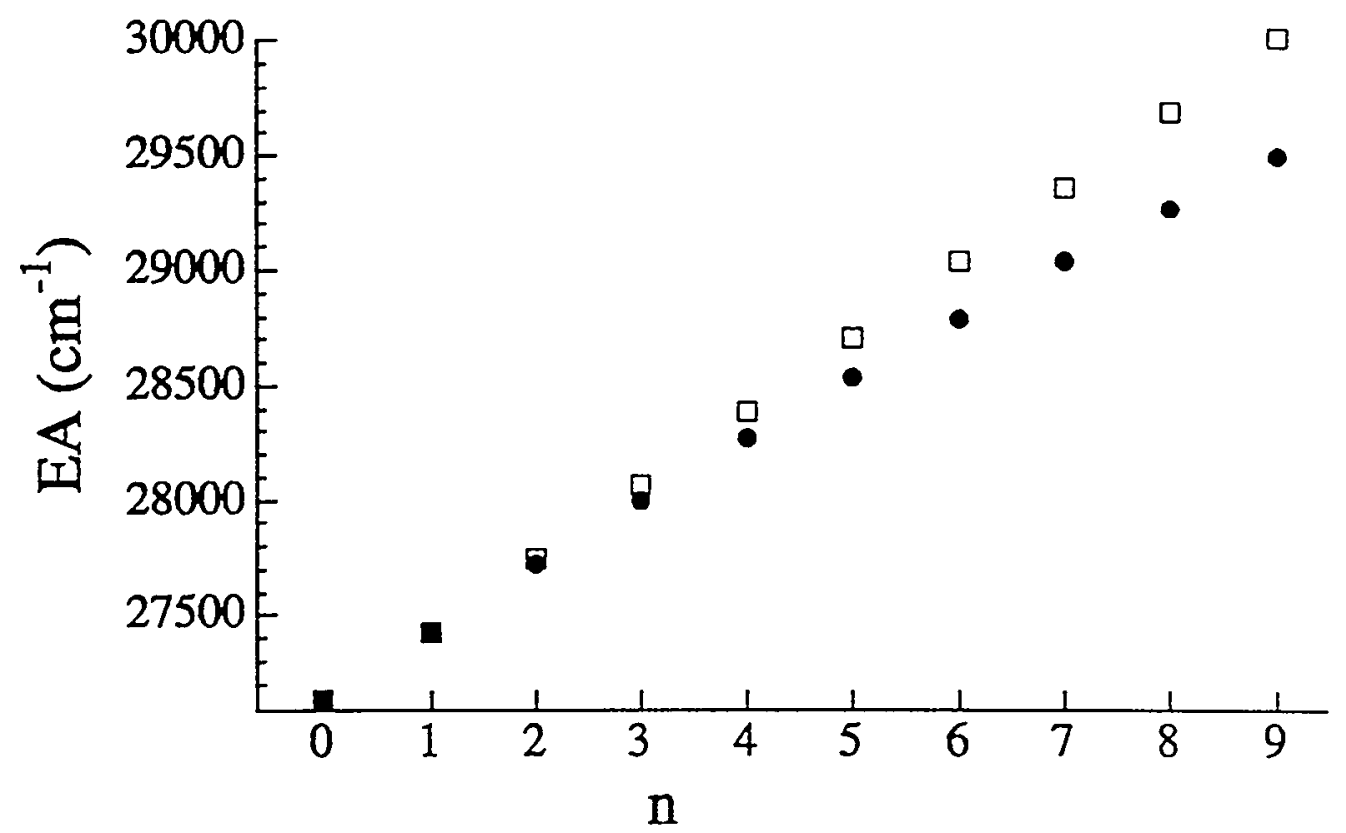

(b)
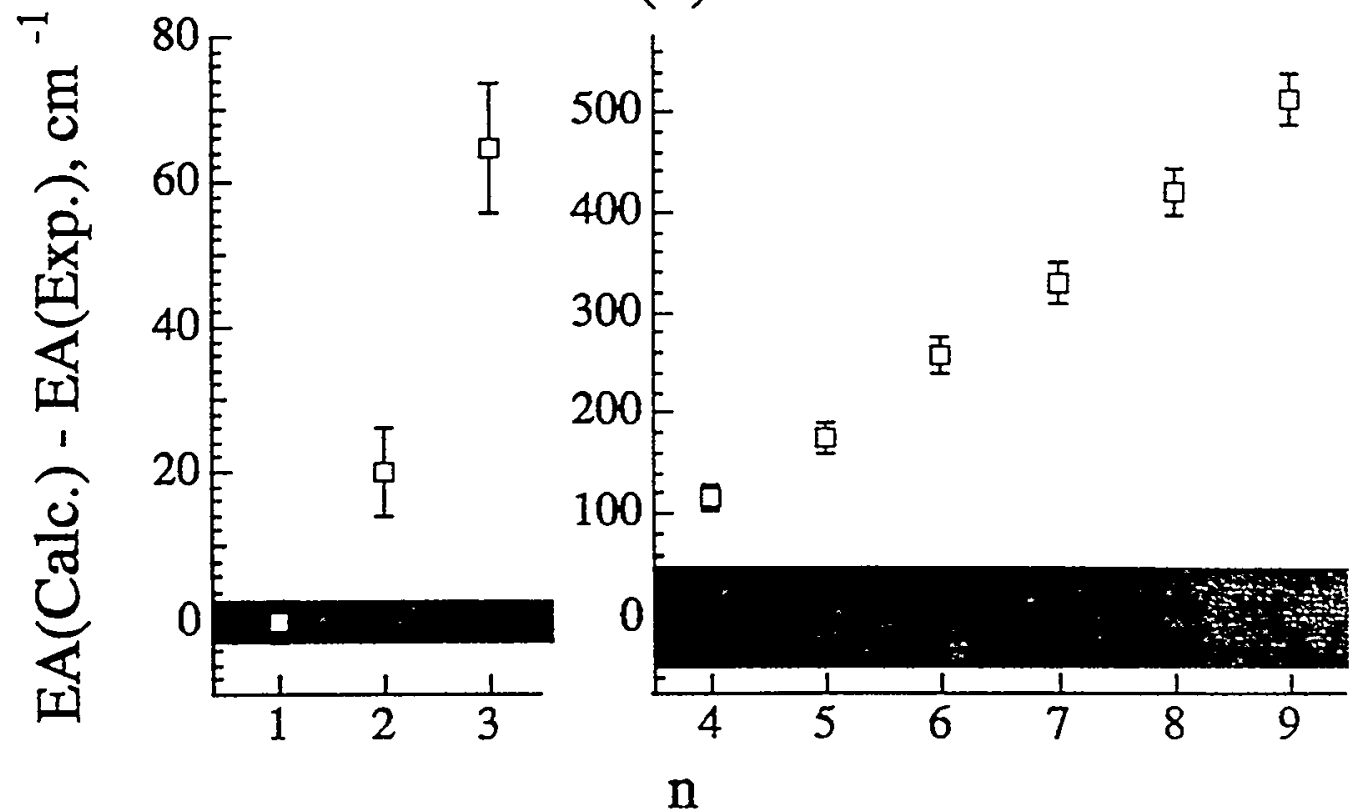

Figure 4.10. Comparison of experimental $\mathrm{Ar}_{n} \mathrm{Br}$ electron affinities $(E A s)$ with those calculated from the pairwise additive model. (a) $E A$ as a function of $\mathrm{n}$. Solid circles: experimental $E A$ s. Open squares: additive calculation. (b) Difference between calculated and experimental $E A s$ as a function of $\mathrm{n}$. The shaded region represents the experimental uncertainty. The error bars represent the uncertainty in the calculated $E A s$. 
(a)

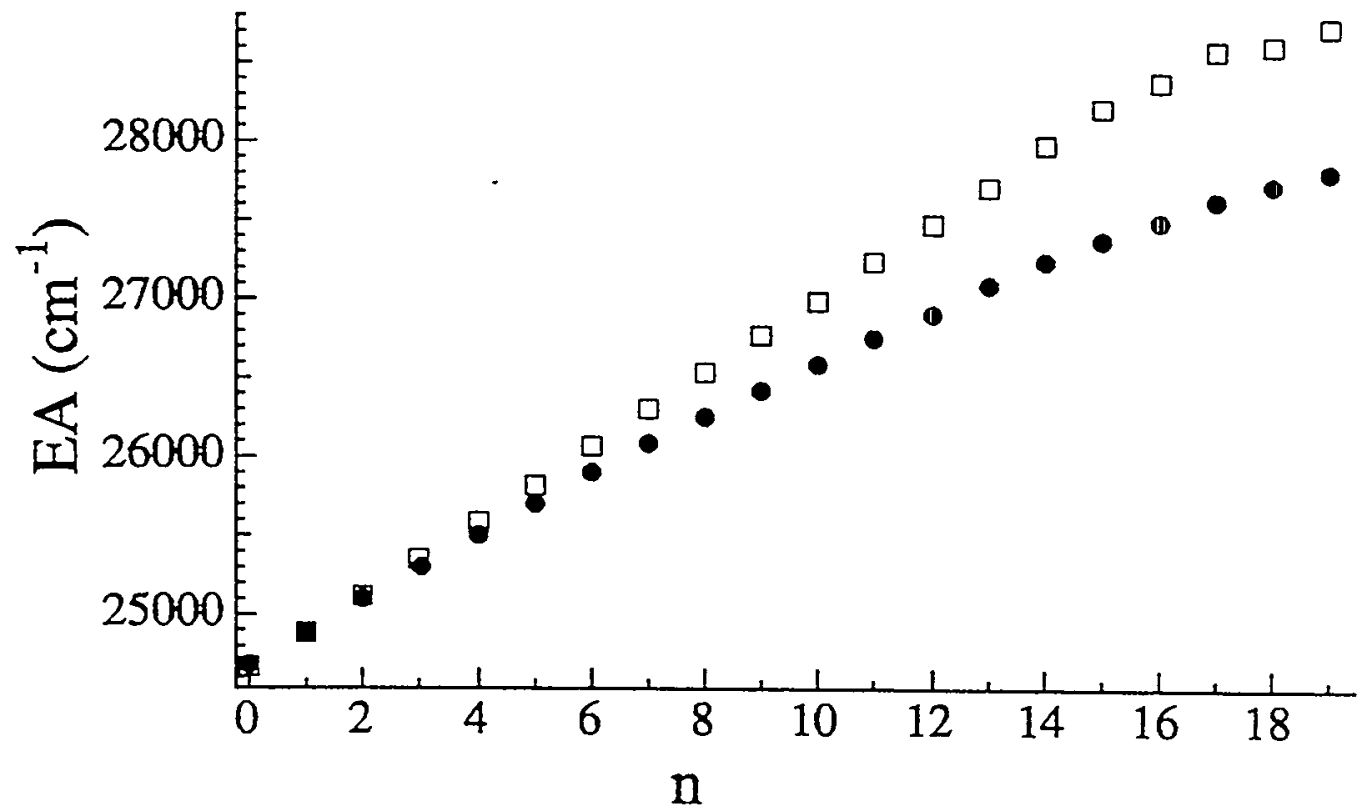

(b)
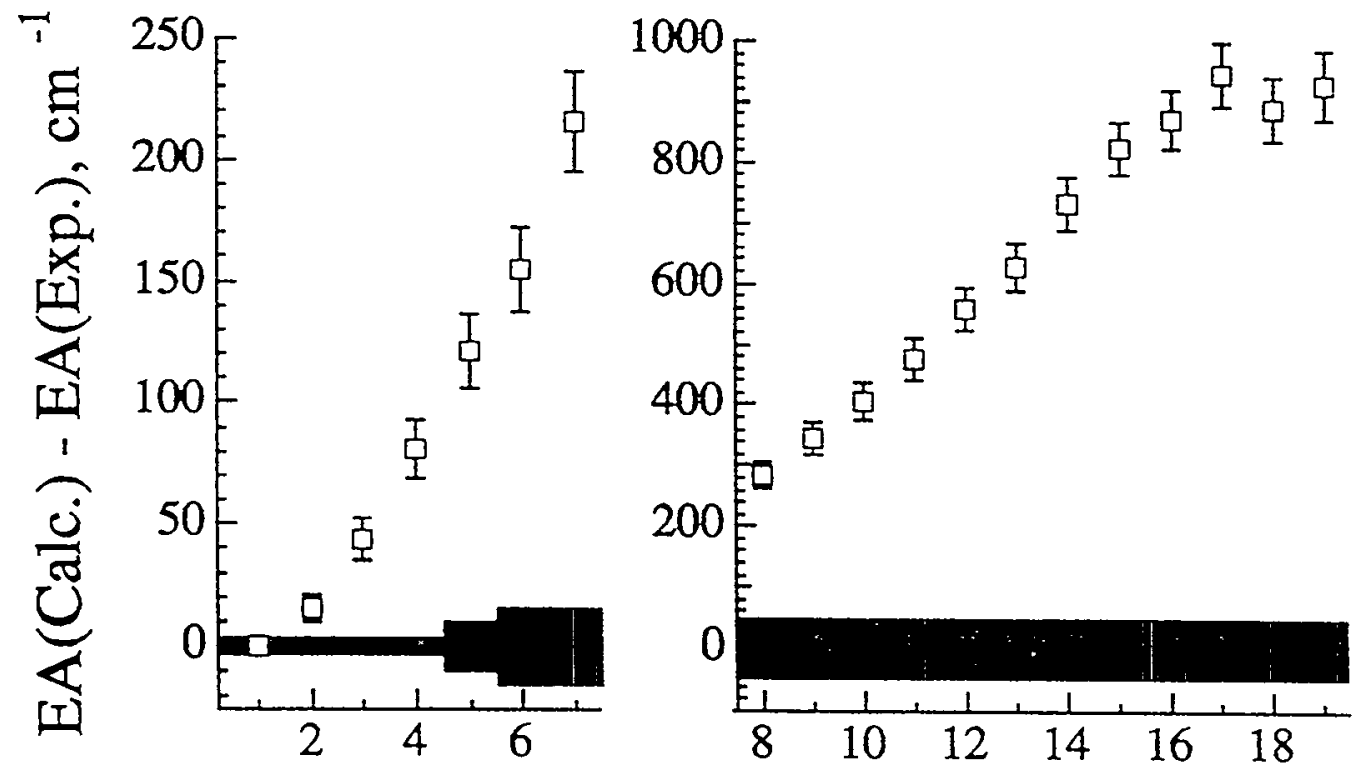

n

Figure 4.11. Comparison of experimental $A r_{n} I E A$ s with those calculated from the pairwise additive model. (a) $E A$ as a function of $n$. Solid circles: experimental $E A$ s. Open squares: additive calculation. (b) Difference between calculated and experimental $E A$ s plotted as a function of $\mathrm{n}$. The shaded region represents the experimental uncertainty. The error bars represent the uncertainties in the calculated $E A s$. 
First, notice that in both $\mathrm{Ar}_{2-9} \mathrm{Br}$ and $\mathrm{Ar}_{2-19} \mathrm{I}$ the calculated $E A s$ are significantly larger than the experimental results. For $\mathrm{Ar}_{17-19} \mathrm{I}$, the calculated $E A \mathrm{~s}$ are almost $1000 \mathrm{~cm}^{-1}$ larger than the experimental values. Furthermore, the calculated $E A s$ for $n \leq 17$ are nearly linear as a function of $\mathrm{n}$. There is a slight positive curvature due to the nonadditivity of the neutral $X$ state, and for $n>17$ the plot becomes flat in the case of $A_{n} I^{*}$. On the other hand, the experimental EAs display a significant negative curvature when plotted versus $\mathrm{n}$. In $A r_{n} \mathrm{I}$, the flattening out at $\mathrm{n}=17$ is not observed. Clearly the model potentials, as described so far, are not consistent with experiment.

Before we consider many-body effects in the anion, let us first rule out other possible explanations for this inconsistency. We first consider the propagation of the uncertainties in the pair potentials. The theoretical error bars shown in Figures 4.10(b) and 4.11 (b) were estimated by assuming an uncertainty of $\pm 3 \mathrm{~cm}^{-1}$ in the quantity $\varepsilon_{a}-\varepsilon_{X}$ for the pair potentials, as discussed above, and multiplying this by the number of $\mathrm{Ar}-\mathrm{X}$ nearest neighbors. The uncertainty in the Ar-Ar potential, and that due to "relaxation" of the geometry is neglected. The shaded areas in the Figures represent the experimental uncertainties. The theoretical and experimental uncertainty regions show no overlap for $\mathrm{n}>2$. If a much more conservative estimate of the uncertainties is desired, we can consider the individual uncertainties in the diatomic well depths, i.e. $9 \mathrm{~cm}^{-1}$ for $\mathrm{ArBr}$ and $\mathrm{ArBr}$, and $18 \mathrm{~cm}^{-1}$ for ArI and ArI. Even in this case, the experimental and theoretical error ranges overlap only for $\mathrm{Ar}_{2} \mathrm{Br}$ and $\mathrm{Ar}_{2-3} \mathrm{I}$.

Furthermore, because the trends in the size dependence of the observed EAs are so different from those of the calculated $E A$, it does not seem possible to modify the pair 
potentials so as to simultaneously account for all the experimental EAs. Any modification of the pair potentials would result in the same more or less linear trend in theoretical EAs.

One might also ask whether the population of local minima affects the trends in the experimental EAs. We can rule this out for $\mathrm{Ar}_{2-3} \mathrm{X}$, for which there is only one possible minimum geometry. For $\mathrm{n}=4$ and 5 the only local minima give calculated $E A$ s much lower than the experimental result. For some of the larger clusters there may be local minima that would be consistent with the experimental EAs. However, we know from the diatomic spectra ${ }^{4}$ that the vibrational temperatures in the beam are on the order of $50 \mathrm{~K}$. In light of this, a significant population of larger clusters occupying local minima several hundred $\mathrm{cm}^{-1}$ above the global minimum seems unlikely. For this reason, and because it is not possible to account for the observed $E A$ s of the small clusters with alternate minima, it is very unlikely that population of local minima could be the sole explanation for the observed trends in the EAs.

Next we consider various non-additive terms in the potentials.

\subsubsection{Many-Body Interactions}

Non-additive (or many-body) interactions fall into three categories: those present in both the anion and the neutral, those unique to the neutral, and those unique to the anion. Many-body interactions present in both anion and neutral include dispersion (Axilrod-Teller) and exchange interactions. Interactions unique to the neutral include the many-body effects due to the open shell nature of the halogen atom, which have already been discussed in Section 4.4.5. Many-body effects unique to the anion are those involving the charge on the halide atom. These include non-additive induction effects, and 
the interaction of the halide charge with multipole moments caused by exchange and dispersion interactions between pairs of argon atoms.

Since the experimental observable, the $E A$, depends on the difference between the anion and neutral potentials [see Equation (4.5)], we expect the many-body effects unique to the anion to be the most important in explaining the observed trends.

We will consider each non-additive effect in turn, incorporating it into our simulated annealing procedure to test its effect on cluster energetics at the minimum energy geometry.

\subsubsection{Triple-Dipole Interaction}

The leading term in the non-additive dispersion energy, the triple-dipole interaction, was first derived by Axilrod and $\operatorname{Teller}^{46}$, and independently by Muto. ${ }^{47}$ The form of the triple-dipole potential is, for three atoms $i, j$ and $k$,

$$
V_{d d d}=C_{9} \frac{\left(3 \cos \theta_{i} \cos \theta_{j} \cos \theta_{k}+1\right)}{R_{i j}^{3} R_{j k}^{3} R_{i k}^{3}},
$$

where $\theta_{i}$ is the interior angle $\angle j i k, R_{i j}$ is the internuclear distance between atom $i$ and atom $j$, and $C_{9}$ is a constant depending only on the identities of the three atoms. $C_{9}$ can be calculated using semi-empirical methods ${ }^{48}$, or by fitting to $a b$ initio calculations. ${ }^{49}$ However, because such results are not available for the $\mathrm{Ar}_{n} \mathrm{X}$ or $\mathrm{Ar}_{n} \mathrm{X}^{-}$systems considered here, we use the approximation to $C_{9}$ discussed by various authors, ${ }^{48 \mathrm{~b} .50}$

$$
C_{9}=\frac{3}{2} \alpha_{i} \alpha_{j} \alpha_{k} \frac{\eta_{i} \eta_{j} \eta_{k}\left(\eta_{i}+\eta_{j}+\eta_{k}\right)}{\left(\eta_{i}+\eta_{j}\right)\left(\eta_{j}+\eta_{k}\right)\left(\eta_{i}+\eta_{k}\right)}
$$


where $\alpha_{i}$ and $\eta_{i}$ are, respectively, the dipole polarizability and average excitation energy of atom $i$.

A simple approximation to $\eta_{i}$ is, in atomic units, ${ }^{50.51}$

$$
\eta_{i}=\left(\frac{N_{i}}{\alpha_{i}}\right)^{\frac{1}{2}}
$$

Here, $N_{i}$ is an effective number of electrons for a given atom. Substituting (4.20) into (4.19) gives a three-body analogue of the Slater-Kirkwood formula ${ }^{51}$ for the $C_{6}$ dispersion coefficient. In Koutselos and Mason's treatment ${ }^{50 a}$, which we follow here, $N_{i}$ is treated as an empirical parameter determined from the corresponding $C_{6}$ two-body dispersion coefficient for like atoms. Furthermore, the values of $N_{i}$ for the halide anions for which the $C_{6}$ coefficients are not known are assumed to be the same as those of the corresponding isoelectronic rare gases. Some theoretical and empirical justification of the approximations involved in this approach is given by Koutselos and Mason, who estimate an uncertainty of $5 \%-10 \%$ for $C_{9}$ coefficients determined in this way. ${ }^{50 a}$ The parameters $N$ and $\alpha$ as well as the values of $C_{9}$ calculated from (4.19) and (4.20) are given in Table 4.6. It should be noted that Equations (4.18) and (4.19) are, strictly speaking, valid only for atoms in S-states. ${ }^{466.50 a}$ In extending their use to P-state halogens we are implicitly neglecting the anisotropy of the halogen atom polarizability. 
Table 4.6. Atomic dipole and quadrupole polarizabilities, effective numbers of electrons, and $C_{9}$ coeffecients for Ar-Ar-X interactions.

\begin{tabular}{|c|c|c|c|c|}
\hline Atom & $\alpha\left(\mathrm{a}_{0}{ }^{3}\right)$ & $C\left(\mathrm{a}_{0}^{\mathrm{S}}\right)$ & $N$ & $C_{9}\left(\mathrm{eV} \cdot \AA^{\mathrm{g}}\right)$ \\
\hline $\mathrm{Ar}$ & $11.08^{\mathrm{a}}$ & $27.11^{\mathrm{d}}$ & $5.90^{\mathrm{c}}$ & - \\
\hline $\mathrm{Br}$ & $35.2^{\mathrm{b}}$ & $164^{\mathrm{d}}$ & $6.70^{\mathrm{c}}$ & 127 \\
\hline $\mathrm{Br}$ & $20.6^{\mathrm{c}}$ & - & $6.2^{\mathrm{c}}$ & 83 \\
\hline $\mathrm{I}$ & $52.7^{\mathrm{b}}$ & $254^{\mathrm{d}}$ & $7.79^{\mathrm{c}}$ & 179 \\
\hline $\mathrm{I}$ & $36.1^{\mathrm{c}}$ & - & $6.5^{\mathrm{c}}$ & 129 \\
\hline
\end{tabular}

\section{References for Table 4.6}

${ }^{2}$ R.R. Teachout and R.T. Pack, At. Data 3, 195 (1971).

${ }^{\mathrm{b}}$ H. Coker, J. Phys. Chem. 80, 2078 (1976).

${ }^{c}$ Handbook of Chemistry and Physics, 74th ed. (CRC, Boca Raton, 1994), pp.10-198.

${ }^{d}$ M.V.K. Sastri, P.L. Narasimhulu and K.D. Sen, J. Chem. Phys. 80, 584 (1984). Note that we use Buckingham's definition [Adv. Chem. Phys. 12, 107 (1967)] of the quadrupole polarizability, $C$, which is equal to half of the quadrupole polarizability, $\alpha_{q}$, used by Sastri et al. [See E.A. Gislason and M.S. Rajan, Chem. Phys. Lett. 50. 251 (1977) and references therein for information on the various quadrupole polarizability conventions.]

${ }^{c}$ E.A. Mason and E.W. McDaniel, Transport Properties of Ions in Gases (Wiley, New York, 1988), pp. 533-4. 
The triple-dipole interaction is repulsive for near equilateral geometries. In the case of $\mathrm{Ar}_{2} \mathrm{I}^{-}, V_{d d d}$ at the equilibrium geometry is $+8.1 \mathrm{~cm}^{-1}$, and $6.3 \mathrm{~cm}^{-1}$ for $\mathrm{Ar}_{2} \mathrm{I}$. For $\mathrm{Ar}_{2} \mathrm{Br}^{-}$and $\mathrm{Ar}_{2} \mathrm{Br}$, the results are $9.0 \mathrm{~cm}^{-1}$ and $5.7 \mathrm{~cm}^{-1}$, respectively. The larger values for the anionic clusters are mainly due to their greater polarizabilities. The net result, then, is a decrease in the calculated $E A$ by about $2-3 \mathrm{~cm}^{-1}$ compared with the additive potentials. This effect is of the same order as the experimental uncertainty, but may be more significant for larger clusters. In the calculations below on clusters with $n \geq 3$, only the Ar-Ar-X triple-dipole interactions are included. The Ar-Ar-Ar interactions are neglected, because we expect their energies to be nearly equal in the anion and neutral.

It has been shown that higher-multipole three-body dispersion terms, such as the dipole-dipole-quadrupole ( $V_{d d q}$ ) potential, may also contribute substantially to the threebody dispersion energy. ${ }^{2}$ To ascertain their importance here, we used the formulae of Koutselos and Mason ${ }^{50 a}$ for the higher multipole coefficients, and the geometrical factors given by Bell ${ }^{52}$ to estimate $V_{d d q}$ for $\mathrm{Ar}_{2} \mathrm{I}^{-}$and $\mathrm{Ar}_{2} \mathrm{I}$. At the equilibrium geometries of the clusters determined with additive potentials, we obtain approximately $4 \mathrm{~cm}^{-1}$ for $\mathrm{Ar}_{2} \mathrm{I}^{-}$and $3 \mathrm{~cm}^{-1}$ for $\mathrm{Ar}_{2} \mathrm{I}$. The resulting $1 \mathrm{~cm}^{-1}$ shift in the $E A$ is smaller than the experimental uncertainty. Therefore, $V_{d d q}$ and all higher multipole three-body dispersion terms were neglected in subsequent calculations.

\subsubsection{Three-Body Exchange}

The second type of three-body interaction that occurs in both anion and neutral clusters is the three-body exchange interaction. This is caused by the exchange induced 
electron charge distortion on a pair of atoms, which alters the pair's exchange interaction with a third atom. This effect is difficult to model without recourse to $a b$ initio calculations, and has been the subject of some controversy. ${ }^{2.3}$ As far as we are aware, such calculations are not available for the $\mathrm{Ar}_{n} \mathrm{Br} / \mathrm{Ar}_{n} \mathrm{Br}^{*}$ or $\mathrm{Ar}_{n} \mathrm{HAr}_{n} I^{-}$systems studied here. However, we can get an idea of the magnitude of this effect from an $a b$ initio calculation on $\mathrm{Ar}_{3}$ by Chalasinski et al. ${ }^{49}$ For equilateral $\mathrm{Ar}_{3}$ at internuclear separations close to the equilibrium $\mathrm{Ar}_{2}$ bond length, they find the sum of first and second order exchange threebody energies to be $-1.5 \mathrm{~cm}^{-1}$, or about $42 \%$ of the third order dispersion non-additive energy $\left(+3.6 \mathrm{~cm}^{-1}\right)$, and of opposite sign. If we assume the exchange nonadditivity is a similar percentage of the dispersion nonadditivity in the $\mathrm{Ar}_{n} \mathrm{Br} / \mathrm{Ar}_{n} \mathrm{Br} r^{-}$and $\mathrm{Ar}_{n} \mathrm{I} \mathrm{Ar}_{n} \mathrm{I}^{-}$ systems, we would anticipate a $2-4 \mathrm{~cm}^{-1}$ negative contribution to the binding energies, and an approximately $1 \mathrm{~cm}^{-1}$ difference between anion and neutral three-body exchange energies. Because this effect is expected to be small compared with our experimental uncertainties, and due to the practical difficulty of accurately modeling it, it will be neglected here.

\subsubsection{Induction Non-Additivity}

The anion pair potentials are dominated by induction. Likewise, we expect a rather large non-additive effect to arise from the interaction between multipole moments induced in the rare gas atoms by the halide charge. In addition, there is non-additivity due to the polarization of the halide atom itself. Because these effects are entirely absent in the neutral clusters (if we neglect the relatively small inductive effects due to the permanent 
quadrupole moment of the neutral halogen), we expect the induction non-additivity to have a large effect on the $E A$.

A model for treating non-additive effects in systems of polarizable particles, furst developed by Vesely ${ }^{53}$, has been extended and used extensively by various workers in computer simulations of solvated ions ${ }^{54}$ and electrons, ${ }^{55}$ polar liquids, ${ }^{56}$ and ionic clusters. $^{18.56 d .57}$ Our adaptation of this model is as follows.

Each atom is characterized by a point charge (halide only) and point dipole and quadrupole polarizabilities (halide and rare gases), located at the nucleus. We assume that the induced dipole of an atom depends linearly on the electric field produced by the charges and multipoles of the other atoms via the dipole polarizability, $\alpha$. We neglect the cubic dependence on the electric field due to the hyperpolarizability $\gamma$, and all higher terms. Likewise, we consider only quadrupoles induced by the field gradient due to the other atoms, characterized by the quadrupole polarizability $C$, neglecting the smaller contribution quadratic in the electric field via the dipole-quadrupole hyperpolarizability $B,^{58}$ and higher terms.

We also neglect the damping of the polarizabilities and charges at short range due to exchange or charge-transfer. Such effects are believed to be significant in the case of hydrogen bonding ${ }^{56 a}$ and in anions in ionic crystals. ${ }^{59}$ However, they are probably less important in the weakly bound clusters considered here.

With these assumptions, the electric field at atom $i$ is given by ${ }^{60}$

$$
E_{\alpha}^{(i)}=\sum_{j \neq i}\left(-T_{\alpha}^{(i j)} q_{j}+T_{\alpha \beta}^{(i j)} \mu_{\beta}^{(j)}-\frac{1}{3} T_{\alpha \beta \gamma}^{(i j)} \Theta_{\beta \gamma}^{(j)}\right),
$$

and the electric field gradient is ${ }^{60}$ 


$$
E_{\alpha \beta}^{(i)}=\sum_{j \neq i}\left(-T_{\alpha \beta}^{(i j)} q_{j}+T_{\alpha \beta \gamma}^{(i j)} \mu_{\gamma}^{(j)}-\frac{1}{3} T_{\alpha \beta \gamma \delta}^{(i j)} \Theta_{\gamma \delta}^{(j)}\right) .
$$

Here, following the notation of Buckingham, ${ }^{60}$ the subscripts $\alpha, \beta, \gamma$ and $\delta$ stand for any of the Cartesian components of a vector or tensor, and repeated Greek subscripts imply summation over the three components. The permanent electric charge is represented by $q_{t}$ ( -1 for the halide and 0 for the rare gases), and $\mu_{\alpha}^{(i)}$ and $\Theta_{\alpha \beta}^{(i)}$ are components of the induced dipole and quadrupole moments, respectively, at atom $i$. We use Buckingham's definition of the quadrupole moment as a traceless tensor. ${ }^{60}$ The multipole interaction tensors are defined by $T_{\alpha \beta \ldots .}^{(i j)}=\nabla_{\alpha} \nabla_{\beta} \cdots \nabla_{v}\left(1 / R_{i j}\right)$, where $\mathbf{R}_{i j}$ is the vector from atom $j$ to atom $i$. The induced dipole at atom $i$ is then given by ${ }^{60}$

$$
\mu_{\alpha}^{(i)}=\alpha_{i} E_{\alpha}^{(i)},
$$

and the induced quadrupole is ${ }^{60}$

$$
\Theta_{\alpha \beta}^{(i)}=C_{i} E_{\alpha \beta}^{(i)},
$$

where $\alpha_{i}$ and $C_{i}$ are the dipole and quadrupole polarizabilities, respectively, of atom $i$. The values of $\alpha$ and $C$ used here are given in Table 4.6.

At each time step in the simulated annealing procedure, the induced moments are calculated iteratively from Equations (4.21)-(4.24). At the first time step, the field and field gradient due to the halide permanent charge are initially calculated from (4.21) and (4.22). Then the induced moments are found from (4.23) and (4.24), and substituted back into (4.21) and (4.22). The process is repeated until the magnitudes of the induced moments do not change by more than one part in $10^{-10}$ with successive iterations. It is found that the moments converge about twice as fast if the individual moments are 
immediately substituted into (4.21) and (4.22) for subsequent calculations during a given iteration, rather than "saved" until the next iteration. For subsequent $\mathrm{MD}$ time steps, the algorithm is initiated with the induced moments saved from the previous MD step. This saves some computer time.

The total induction energy is then given by

$$
V_{\text {ind.toral }}=V_{q \mu}+V_{q \theta}+V_{\mu \mu}+V_{\mu \Theta}+V_{\Theta \Theta}+V_{\text {self }}
$$

where the first five terms on the right hand side are the charge-dipole, charge-quadrupole, dipole-dipole, dipole-quadrupole and quadrupole-quadrupole interaction energies. The final term is the energy required to create the induced dipoles and quadrupoles, given by $^{61.62}$

$$
V_{\text {self }}=\sum_{i}\left(\frac{\mu_{\alpha}^{(i)} \mu_{\alpha}^{(i)}}{2 \alpha_{i}}+\frac{\Theta_{\alpha \beta}^{(i)} \Theta_{\alpha \beta}^{(i)}}{6 C_{i}}\right)
$$

where the sum runs over all atoms. By using (4.21)-(4.24) for one of each of the dipoles and quadrupoles in (4.26) and substituting the explicit expressions for the interaction energies $^{60}$ and (4.26) into (4.25), one can show that (4.25) simplifies to

$$
V_{\text {ind, total }}=\frac{1}{2} V_{q \mu}+\frac{1}{2} V_{q \Theta}=\sum_{i} \sum_{j \neq i} q_{i}\left(-\frac{1}{2} T_{\alpha}^{(i j)} \mu_{\alpha}^{(j)}+\frac{1}{6} T_{\alpha \beta}^{(i j)} \Theta_{\alpha \beta}^{(j)}\right)
$$

This equation gives the total induction energy of the cluster. However, part of this energy is already implicitly included in the Ar- $\mathrm{X}^{*}$ pair potential. In order to extract the nonadditive portion, we calculate the induction energy for each $\mathrm{Ar}-\mathrm{X}^{-}$pair, neglecting the other Ar atoms in the cluster, using the same iterative method. The sum of the pair induction energies is then subtracted from (4.27) to give the non-additive induction energy: 


$$
V_{\text {ind }}=V_{\text {ind, }, \text { otal }}-V_{\text {ind, pair }} \text {. }
$$

In practice, due to the computational "expense" of this iterative calculation, a simpler model was employed for the initial simulated annealing procedure. In the simpler model, the interaction energy between dipoles directly induced in the rare gas atoms by the halide charges is calculated. ${ }^{63}$ The minimum energy geometries found with the simpler model were then optimized using the full iteratively calculated induction model described above. This simple induction model is described in detail in Appendix C.

The results of the calculation for $\mathrm{Ar}_{2} \mathrm{I}^{-}$and $\mathrm{Ar}_{2} \mathrm{Br}^{-}$show that the non-additive induction effect is indeed quite large. For $\mathrm{Ar}_{2} \mathrm{Br}$, for example, $V_{\text {ind }}$ is $35.3 \mathrm{~cm}^{-1}$. The result for $\mathrm{Ar}_{2} \mathrm{I}^{-}$is somewhat smaller, because of the larger $\mathrm{Ar}-\mathrm{X}^{*}$ internuclear distance. The nonadditive induction energy is always found to be positive, showing that it is dominated by the repulsion between adjacent induced multipoles on the Ar atoms. The dipole term of (4.27) contributes $32.7 \mathrm{~cm}^{-1}$ to the total in $\mathrm{Ar}_{2} \mathrm{Br}^{-}$, and the quadrupole term contributes 2.6 $\mathrm{cm}^{-1}$. Thus, it does appear necessary to include the induced quadrupole effect, usually neglected in this type of simulation, for accurate calculation of the binding energies. The results for the larger clusters are discussed below.

\subsubsection{Exchange and Dispersion Multipoles}

As first described by Dick and Overhauser, ${ }^{64}$ the exchange repulsion between two closed shell atoms produces a buildup of negative charge near the nuclei and a depletion of electron density between the nuclei. At large distances from the pair of atoms this distortion of the electron clouds is equivalent to a set of multipole moments, as discussed by Jansen. ${ }^{65}$ If the atoms are identical, the first non-vanishing moment is a quadrupole. 
There is also a quadrupole, of opposite sign, arising from the dispersion interaction between two atoms. At the usual van der Waals distances, the dispersion contribution is somewhat smaller than the exchange contribution. ${ }^{66}$

In the case of $\mathrm{Ar}_{n} \mathrm{Br}^{-}$and $\mathrm{Ar}_{n} I$, a three-body effect then arises from the interaction of the halide charge with the $\mathrm{Ar}_{2}$ exchange/dispersion multipoles. This is another type of many-body interaction that is present in the anionic but not in the neutral clusters, and is therefore expected to have a significant effect on the $E A$. As with induction, we expect the interaction of the permanent quadrupole of the neutral halogen atom with the exchange/dispersion moments to be negligible.

In their studies of the $\mathrm{Ar}_{2}-\mathrm{HCl},-\mathrm{DCl}$, and - $\mathrm{HF}$ systems, Hutson and coworkers ${ }^{10}$ have found that the interaction of the exchange/dispersion quadrupole of the $\mathrm{Ar}_{2}$ unit with the permanent multipoles of the HX molecule is quite important. This work was mainly concerned with the interpretation of the vibration-rotation spectra ${ }^{7.8}$ of the clusters. However they also found the contribution to the binding energy to be significant. Chalasinski et al. ${ }^{67}$ have found these conclusions about the importance of the exchange quadrupole effect on the $\mathrm{Ar}_{2} \mathrm{HX}$ potential energy surfaces to be qualitatively consistent with their $a b$ initio calculations. In recent work more closely related to our own, Burcl et al. have extracted information about the exchange multipole energy from $a b$ initio calculations on $\mathrm{Ar}_{2} \mathrm{Cl}^{-68}$ These authors calculated this effect to be $-12.8 \mathrm{~cm}^{-1}$ near the equilibrium geometry of $\mathrm{Ar}_{2} \mathrm{Cl}$. In this light we expect the exchange/dispersion multipole contribution to the non-additive binding energies of our $\mathrm{Ar}_{n} \mathrm{Br}^{-}$and $\mathrm{Ar}_{n}{ }^{-}$clusters also to be quite significant. 
Jansen derived a simple expression for the exchange quadrupole using an effective one electron model for the atomic charge distributions. ${ }^{65}$ In this approach, the electronic charge density of an atom is approximated by a single Gaussian function,

$$
\rho_{i}(\mathbf{r})=-\frac{|e| \beta^{3}}{\pi^{3 / 2}} e^{-\beta^{2}\left|\mathbf{R}_{i}-r\right|^{2}},
$$

where $\beta$ is the Gaussian range parameter, $\mathbf{R}_{i}$ is the position of the nucleus of atom $i$, and $\mathbf{r}$ is the position of the effective electron. Then the atomic wave function is defined as

$$
\varphi_{i}(\mathbf{r})=\left|\frac{\rho_{i}(\mathbf{r})}{e}\right|^{1 / 2}
$$

The zero-order wavefunction of a pair of atoms, $i$ and $j$, is taken to be the antisymmetrized product of the two atomic wavefunctions (normalized to 2):

$$
\Psi_{i j}^{0}\left(\mathbf{r}, \mathbf{r}^{\prime}\right)=\frac{1}{\left(1-S_{i j}^{2}\right)^{1 / 2}}\left[\varphi_{i}(\mathbf{r}) \varphi_{j}\left(\mathbf{r}^{\prime}\right)-\varphi_{i}\left(\mathbf{r}^{\prime}\right) \varphi_{j}(\mathbf{r})\right] .
$$

Here $\mathbf{r}$ and $\mathbf{r}^{\prime}$ are the positions of the two electrons, and $S_{i j}$ is the overlap integral, which for like atoms with Gaussian wavefunctions (4.30) is given by

$$
S_{i j}^{2}=\exp \left(-\beta^{2} R_{i j}^{2} / 2\right),
$$

where $R_{i j}$ is the internuclear separation between the atoms. Then, taking the expectation value of the quadrupole moment operator with wavefunction (4.31), a simple expression for the cylindrically symmetric exchange quadrupole is found: ${ }^{65}$

$$
\Theta_{e x}\left(R_{i j}\right)=-\frac{|e| R_{i j}^{2}}{2}\left(\frac{S_{i j}^{2}}{1-S_{i j}^{2}}\right) .
$$

In Jansen's original treatment, the range parameter $\beta$ was estimated from the long range dispersion interactions, and assumed to be valid for short range exchange interactions. 
This method of estimating $\beta$ is now believed to significantly overestimate the exchange quadrupole $^{69.70}$ An approach that has been used to improve the accuracy of the model is to fit the one-electron functional form for the quantity of interest to the results of accurate $a b$ initio calculations, to arrive at a more reasonable value of $\beta .^{10,69}$ Here, we shall use the value $\beta=0.936 \AA^{-1}$, derived in this way by Hutson and coworkers ${ }^{10 c}$ from an SCF calculation of the quadrupole moment of $\mathrm{Ar}_{2}$.

The problem now arises of how to calculate the interaction energy of the exchange quadrupole with the halide charge. The simplest way is to represent the exchange charge distribution with a point quadrupole, calculated from (4.32), located at the midpoint between the two Ar atoms. The energy is then obtained from the standard expression for a charge-quadrupole interaction. ${ }^{60}$ However, because the typical halide- $\mathrm{Ar}_{2}$ distances in the clusters are on the same order as the Ar-Ar distance, the point quadrupole representation overestimates the magnitude of the interaction. The point quadrupole representation was used by Hutson et al. in their work on $\mathrm{Ar}_{2}-\mathrm{HCl}$ and $-\mathrm{DCl}$, and was found by them to somewhat overcorrect the pairwise additive potential. ${ }^{10 a, b}$ In more recent work on $\mathrm{Ar}_{2}-\mathrm{HF}$, Ernesti and Hutson ${ }^{10 c}$ proposed a distributed dipole representation: $\mathrm{The}^{\mathrm{Ar}} \mathrm{r}_{2}$ exchange charge distribution is represented by opposed point dipole moments at the two Ar nuclei, parallel to the internuclear axis, with magnitudes chosen to give the same overall quadrupole moment as (4.32). Ernesti and Hutson found the distributed dipole representation superior to the point quadrupole representation for $\mathrm{Ar}_{2}-\mathrm{HF}$, but noted that it somewhat underestimated the electric field of the true charge distribution $^{10 c}$ 
The difficulty with both of these approaches arises from the use of a multipole representation at short range. Therefore, it seems logical to attempt a more direct calculation of the interaction of the exchange charge distribution with the halide charge. To do this, we form an effective charge density--the part of the charge density that contributes to the exchange quadrupole--by subtracting the atomic charge densities (4.29) from the charge density of the antisymmetrized wavefunction (4.31):

$$
\begin{aligned}
\rho_{e f f}(\mathbf{r}) & =-|e| \int\left|\Psi_{i j}^{0}\left(\mathbf{r}, \mathbf{r}^{\prime}\right)\right|^{2} d \mathbf{r}^{\prime}-\rho_{i}(\mathbf{r})-\rho_{j}(\mathbf{r}) \\
& =-\frac{|e| S_{i j}^{2}}{1-S_{i j}^{2}}\left(\frac{\beta}{\pi^{1 / 2}}\right)^{3}\left[e^{-\beta^{2}\left|\mathbf{R}_{i}-\mathbf{r}\right|^{2}}+e^{-\beta^{2}\left|\mathbf{R}_{j}-\mathbf{r}\right|^{2}}-2 e^{-\beta^{2}\left|\mathbf{R}_{c}-\mathbf{r}\right|^{2}}\right]
\end{aligned}
$$

Here, $\mathbf{R}_{C}=\frac{1}{2}\left(\mathbf{R}_{i}+\mathbf{R}_{j}\right)$ is the midpoint between the two Ar nuclei. We see that the effective charge density is the sum of two negative Gaussian charge distributions located at the nuclei, and a positive Gaussian distribution, twice as large, at $\mathbf{R}_{C}{ }^{64}$ If we approximate the halide with a point charge at $\mathbf{R}_{0}$, the Coulomb interaction energy is then found to be $\mathrm{e}^{71}$

$$
V_{e c}=\sum_{i<j} \frac{e^{2} S_{i j}^{2}}{1-S_{i j}^{2}}\left[\frac{\operatorname{erf}\left(\beta R_{i 0}\right)}{R_{i 0}}+\frac{\operatorname{erf}\left(\beta R_{j 0}\right)}{R_{j 0}}-2 \frac{\operatorname{erf}\left(\beta R_{C 0}\right)}{R_{C 0}}\right]
$$

where $R_{i 0}, R_{j 0}$ and $R_{C 0}$ are the distances of the halide from the Ar nuclei and the midpoint between the nuclei, respectively, and $i$ and $j$ run over the Ar atoms. The error functions in (4.34) can be easily evaluated using standard subroutines. ${ }^{72}$ In the limit $\beta R \rightarrow \infty, \operatorname{erf}(\beta R) \rightarrow 1$. So at long range, (4.34) is equivalent to the Coulomb interaction of the halide charge with negative point charges $\delta=-|e| S_{i j}^{2} /\left(1-S_{i j}^{2}\right)$ at the Ar nuclei, and 
a positive point charge, $+2|\delta|$, at $\mathbf{R}_{C} \cdot{ }^{65}$ In order to prevent non-physical behavior of Equation (4.34) for small values of $R_{C O}$ (near linear geometries), $V_{e c}$ is cut off for $\mathrm{Ar}$ - $\mathrm{Ar}$ separations greater then a certain value, typically $6.5 \AA$.

We should discuss the approximations implicit in (4.34). First, the nuclear charges are, in effect, approximated by Gaussian distributions with the same $\beta$ parameter as the atomic electron densities. Thus, effects of nuclear de-shielding are not included in (4.34). Second, the approximation of the halide by a point charge will underestimate the extent of overlap effects and hence tend to slightly overestimate the magnitude of the interaction energy. This deficiency could be corrected if more were known about the charge densities of the halide atoms. Finally, and most importantly, we are still working within the Gaussian one-electron approximation. A single Gaussian function is known to be a rather poor approximation to the true electron density of an atom. ${ }^{70}$ This problem could be overcome by using a more accurate model of the $\mathrm{Ar}_{2}$ charge distribution, such as the result of an $a b$ initio calculation with Gaussian type basis functions. The method of Gaussian multipoles developed by Wheatley ${ }^{71}$ could then be used to calculate the Coulomb energy. Despite the limitations of the present model, we nonetheless expect (4.34) to give a more accurate value of $V_{e c}$ than either the point quadrupole or distributed dipole representations.

The three models of $V_{e c}$ are compared for $\mathrm{Ar}_{2} \mathrm{X}^{-}$in Figure 4.12. In the Figure, the $\mathrm{Ar}-\mathrm{Ar}$ distance is held constant at the equilibrium value of the $\mathrm{Ar}_{2}$ molecule, and the $\mathrm{Ar}-\mathrm{Ar}$ axis is kept perpendicular to $\mathbf{R}_{C 0}$, as the halide- $A r_{2}$ distance is varied. It can be seen that at large separations, the three models approach each other, as expected. However, at separations near the equilibrium structures of $\mathrm{Ar}_{2} \mathrm{I}^{-}$and $\mathrm{Ar}_{2} \mathrm{Br}$, the differences among the 
three models are quite significant. For example, at $R_{C O}=3.61 \AA$, corresponding to $\mathrm{Ar}_{2} \mathrm{I}$, Equation (4.34) gives $V_{e c}=-15.0 \mathrm{~cm}^{-1}$, compared with $-18.0 \mathrm{~cm}^{-1}$ for the point quadrupole model, and $-12.6 \mathrm{~cm}^{-1}$ for the distributed dipole representation. The differences among the three models at the equilibrium $R_{C 0}$ of $\mathrm{Ar}_{2} \mathrm{Br}^{-}(3.28 \AA$ ) are even more pronounced. We conclude that at the interatomic distances considered here, it is important to use an accurate representation of the exchange charge distribution to calculate $V_{\text {ec. }}$ In the remainder of this work, we shall use (4.34) for $V_{\text {ec. }}$.

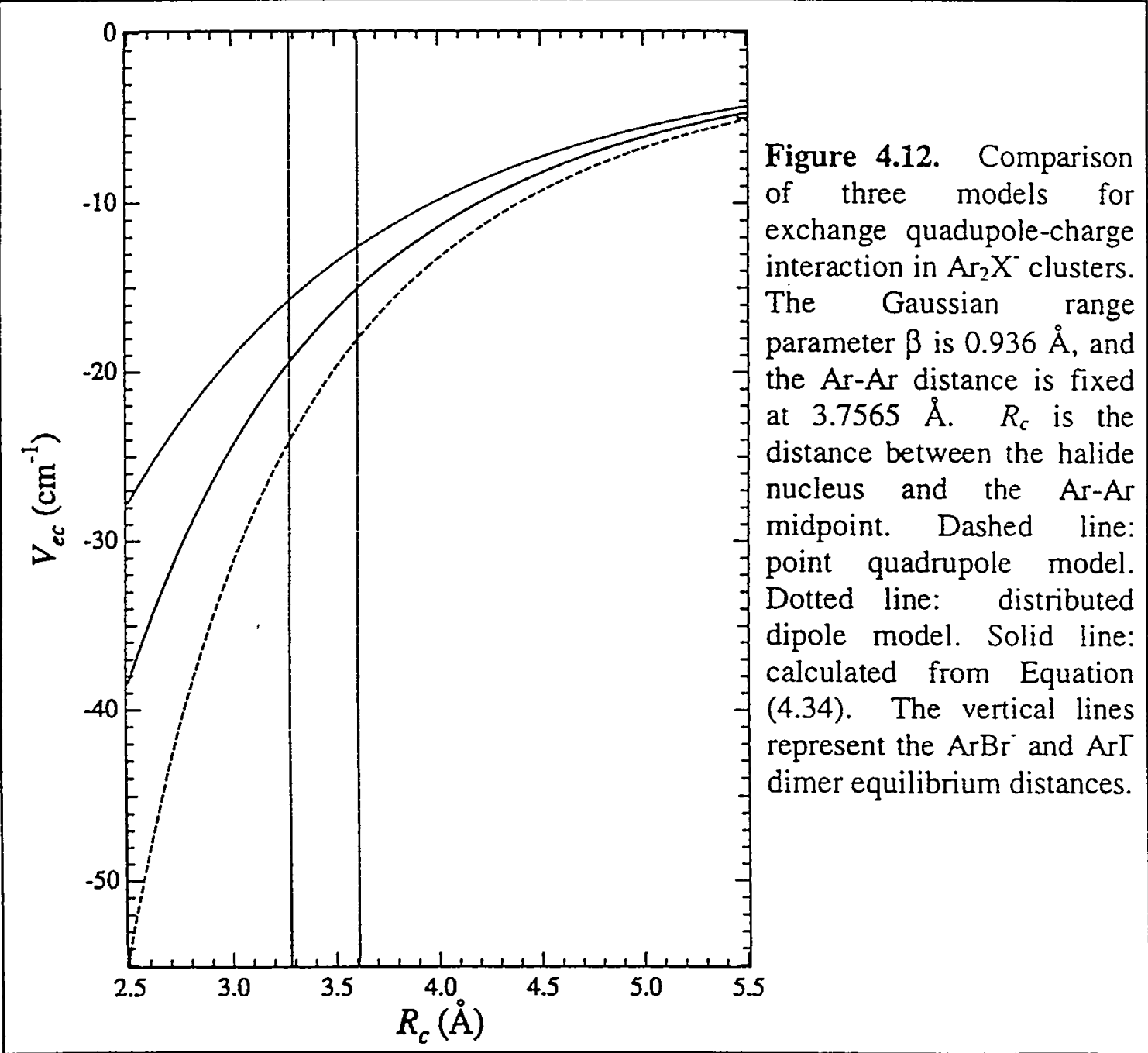


We also need to consider the multipole moments induced in the rare gas atoms by dispersion. Hunt ${ }^{73}$ has developed a model for the dispersion induced dipole and quadrupole moments in terms of atomic polarizabilities and dispersion coefficients. The average dipole moment induced on atom $i$ by the dispersion interaction with other like atoms is given by:

$$
\mu^{i, d i s p}=C_{\mu} \sum_{j \neq i} \frac{\hat{\mathbf{R}}_{i j}}{R_{i j}^{7}}, \text { with } C_{\mu}=\frac{3}{2} \frac{C_{6} B}{\alpha}
$$

where $\hat{\mathbf{R}}_{i j}$ is the unit vector pointing from atom $j$ to atom $i, C_{6}$ is the $\mathrm{Ar}-\mathrm{Ar}$ dispersion coefficient, $\alpha$ is the dipole polarizability, and $B$ is the dipole-quadrupole hyperpolarizability. The components of the dispersion induced quadrupole moment on atom $i$ are given by:

$$
\Theta_{\alpha \beta}^{i, d i s p}=-C_{\Theta} \sum_{j \neq i} \frac{T_{\alpha \beta}^{(i j)}}{2 R_{i j}^{3}}, \text { with } C_{\Theta}=\frac{1}{4} \frac{C_{6} B}{\alpha}
$$

where $T_{\alpha \beta}^{(i j)}=\nabla_{\alpha} \nabla_{\beta}\left(1 / R_{i j}\right)$. For example, in the special case of two atoms lying on the Z-axis, the quadrupoles have cylindrical symmetry, with $\Theta_{z z}^{i \text { disp }}=-C_{\Theta} / R_{i j}^{6}$, and $\Theta_{x x}^{i . d i s p}=\Theta_{y y}^{i, d i s p}=-\frac{1}{2} \Theta_{z z}^{i, d i s p}$. Following Emesti and Hutson ${ }^{10 c}$ the values of $C_{\mu}$ and $C_{\Theta}$ were found using the $C_{6}$ constant from the Aziz HFDIDl potential, ${ }^{29}$ and the ratio $B / \alpha$ from the calculation of Maroulis and Bishop. ${ }^{74}$ We obtain $C_{\mu}=1252 e a_{0}^{8}$ and $C_{\theta}=208.6$ $e a_{0}^{8}$. The total dispersion induced dipoles and quadrupoles are calculated from (4.35) and

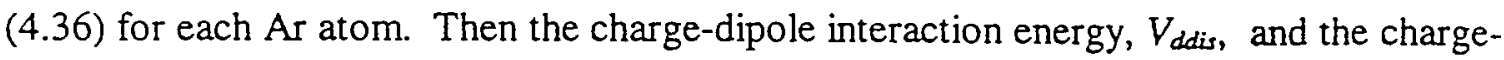
quadrupole energy, $V_{\text {qdis, }}$, are computed from the standard electrostatic formulae. ${ }^{60} \mathrm{We}$ denote the total charge-dispersion multipole energy by $V_{\text {mdis }}=V_{\text {ddis }}+V_{\text {qdis. }}$ 
We should note that this calculation is carried out independently of the nonadditive induction energy calculation described in the previous section (4.4.7.3). Therefore, interactions between the electrostatically induced multipoles and the exchange/dispersion induced multipoles have been neglected. This is reasonable because the exchange/dispersion multipoles are about an order of magnitude smaller than the charge induced multipoles, and, therefore, the interactions of the exchange/dispersion multipoles with the charge are much larger than their interactions with the charge induced multipoles.

The charge-dipole energy, $V_{\text {ddis }}$, is generally positive and about $30 \%$ as large as $V_{e c}$. This proportion is qualitatively consistent with the calculations of Lacey and Byers Brown ${ }^{66}$ and with the results of Emesti and Hutson. ${ }^{10 c} V_{q d i s}$ is negative, and only about $5 \%$ as large as $V_{d d i s .}$ For example, in $\mathrm{Ar}_{2} \mathrm{Br}$, the dispersion dipole energy is $+6.0 \mathrm{~cm}^{-1}$, and the dispersion quadrupole energy is $-0.3 \mathrm{~cm}^{-1}$. This may be compared with the exchange charge energy of $-20.3 \mathrm{~cm}^{-1}$. Thus we see that the dispersion dipole makes a nonnegligible contribution to the non-additive energy, whereas the dispersion quadrupole could be neglected without any significant loss of accuracy.

Complete results for the larger clusters are discussed in the next section.

\subsubsection{Electron Affinities Calculated with Many-Body Potentials}

In order to assess the importance of the various many-body effects mentioned above, we re-optimized the minimum energy geometries found from the simulated annealing procedure with the additive potentials, successively adding the many-body terms, in order of their relative magnitudes. 
The first non-additive term considered was the multipole induction energy; the anion potential was then found from

$$
\varepsilon_{a}=\min \left(V_{A r X}+V_{A r A r}+V_{i n d}\right)
$$

where the right hand terms are the pairwise additive argon-halide and argon-argon potentials, and the many-body multipole induction potential. The neutral potential was identical to that used in the calculation in Section 4.4.6:

$$
\varepsilon_{x}=\min \left(V_{A r_{x} X}+V_{A r A r}\right)
$$

where $V_{A_{a} X}$ is the "matrix additive" $X$ state potential described in Section 4.4.5. We refer to the electron affinities calculated from (4.37a), (4.38a) and (4.5) as $E A_{\text {ind. }}$.

We next considered the effect of addition of the exchange-charge and multipole dispersion energies. The anion binding energies are then

$$
\varepsilon_{a}=\min \left(V_{A r X}+V_{A r A r}+V_{\text {trd }}+V_{e c}+V_{m d s}\right) \text {, }
$$

and the neutral binding energies still given by (4.38a). The electron affinities calculated from (4.37b), (4.38a) and (4.5) are referred to as $E A_{\text {ind }+e c+m d i s .}$

Finally, the Axilrod-Teller term was included in both the anion and neutral potentials to give

$$
\begin{aligned}
& \varepsilon_{a}=\min \left(V_{A r X}+V_{A r A r}+V_{\text {ind }}+V_{e c}+V_{\text {mdis }}+V_{a t}^{\text {anion }}\right), \\
& \varepsilon_{X}=\min \left(V_{A r_{n} X}+V_{A r A r}+V_{a t}^{\text {neural }}\right) .
\end{aligned}
$$

The EAs calculated from (4.37c), (4.38c) and (4.5) are referred to as $E A_{\text {ind }+e c+m d i s+a t .}$ The binding energies calculated from (4.37c) and (4.38c) and their components are shown graphically in Figures 4.13 and 4.14. The anion and neutral binding energies calculated from (4.37a), (4.37b) and (4.38a), and from (4.37c) and (4.38c) are given, along with the 
corresponding zero-point energies, in Tables 4.7 and 4.8. The theoretical electron affinities $E A_{\text {ind }}, E A_{\text {ind+ec+mdis }}$ and $E A_{\text {ind }+e c+m d i s+a t}$ are given in Tables 4.9 and 4.10. The deviations of the theoretical EAs from the experimental values are shown in Figures 4.15 and 4.16 . 


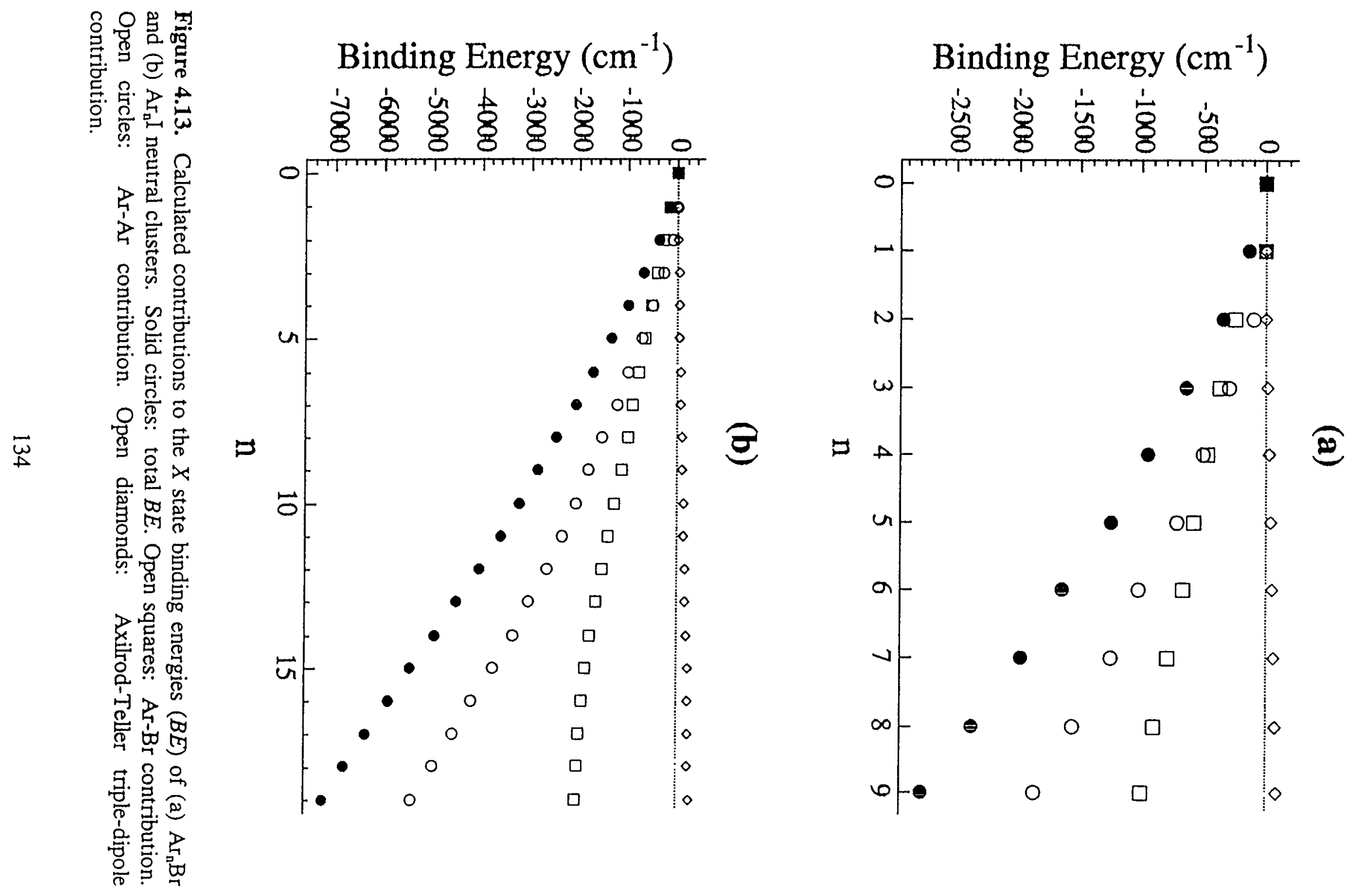


(a)

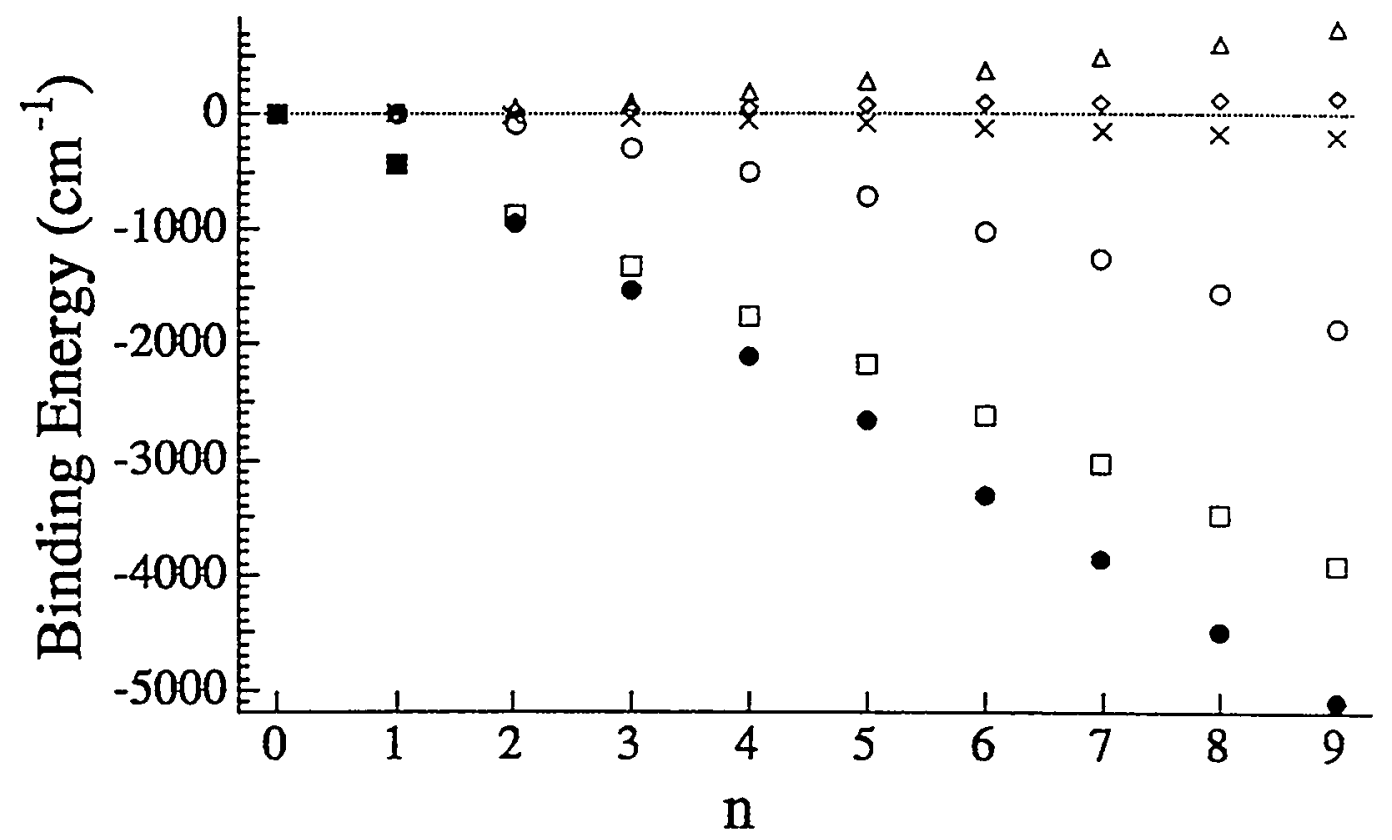

(b)

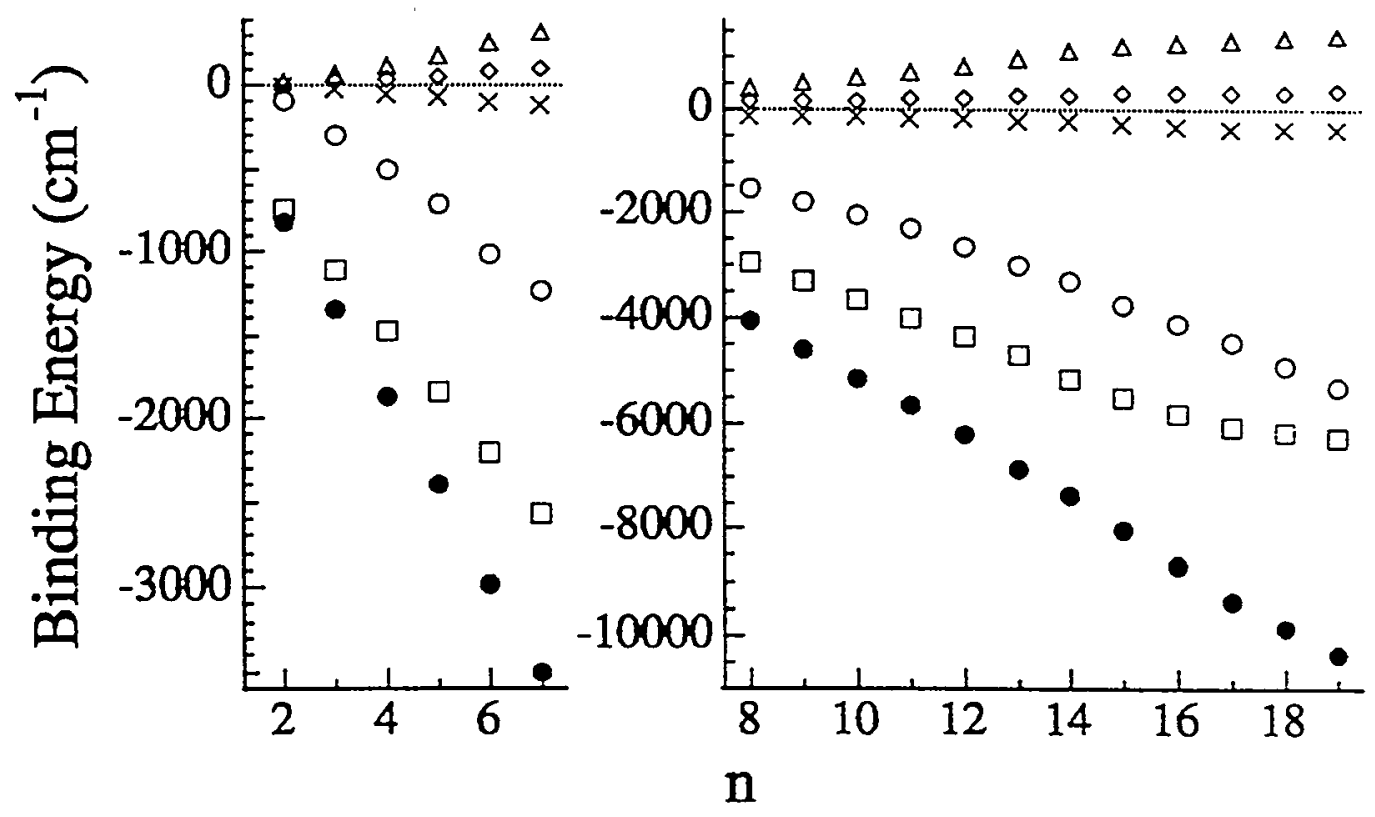

Figure 4.14. Calculated contributions to the binding energies of (a) $\mathrm{Ar}_{n} \mathrm{Br}^{\circ}$ and (b) $\mathrm{Ar}_{\mathrm{n}} \mathrm{I}^{-}$ anions. Solid circles: total $B E$. Open squares: $\mathrm{Ar}-\mathrm{Br}$ contribution. Open circles: $\mathrm{Ar}-\mathrm{Ar}$ contribution. Open triangles: non-additive induction. Crosses: exchange charge and multipole dispersion. Open diamonds: triple-dipole dispersion. 
(a)
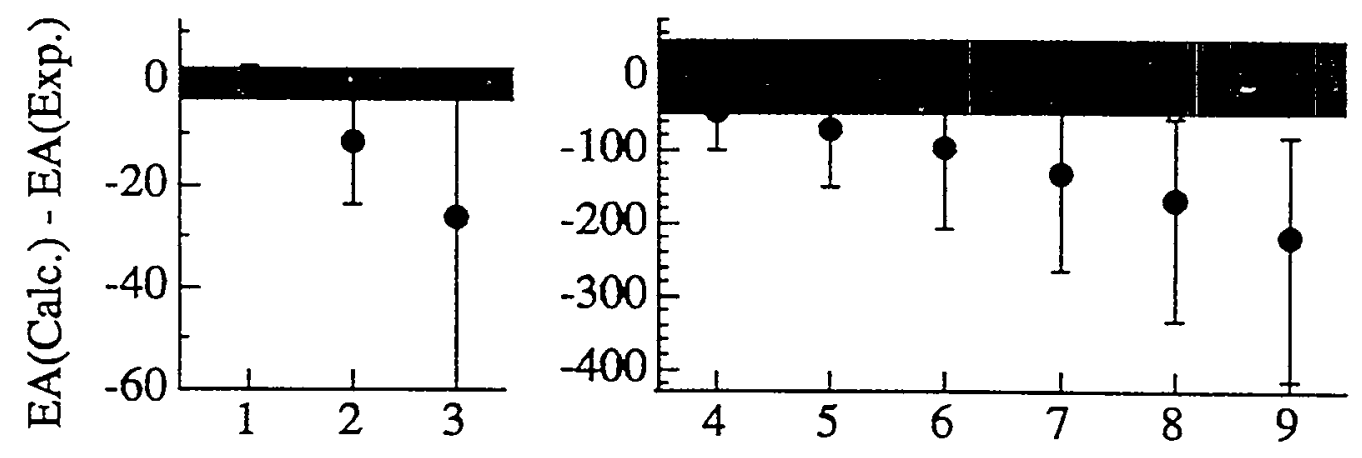

$\mathrm{n}$

(b)
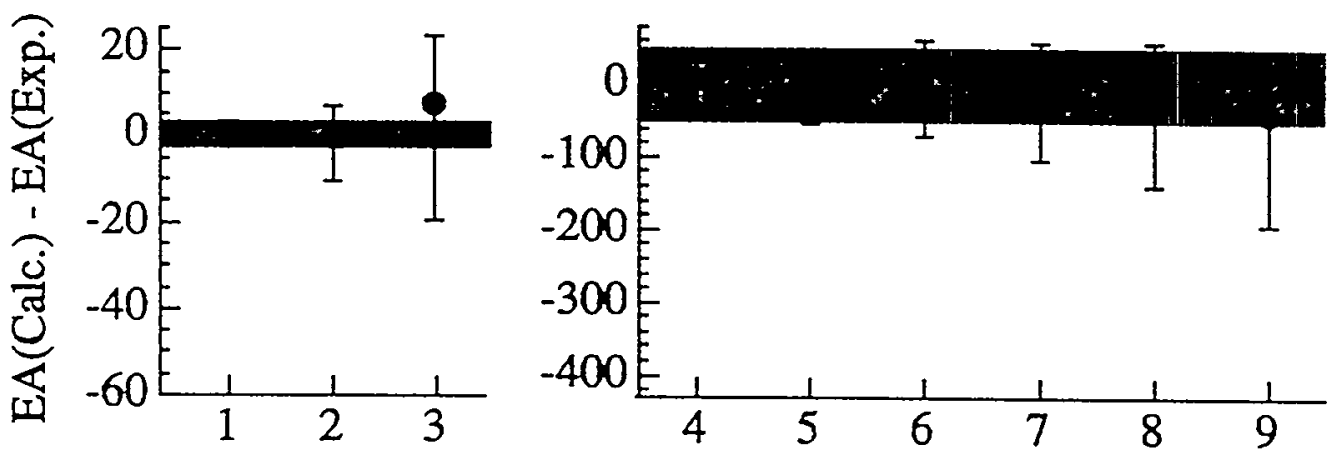

$\mathrm{n}$

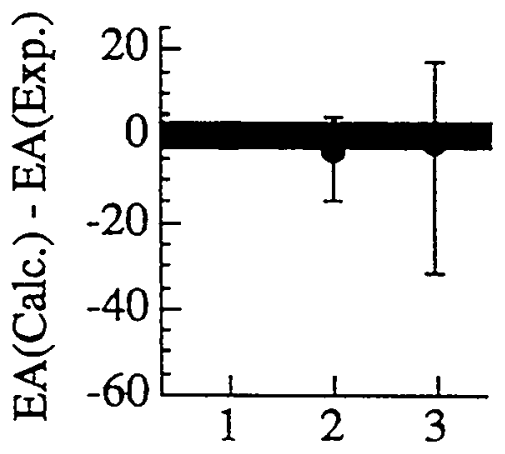

(c)

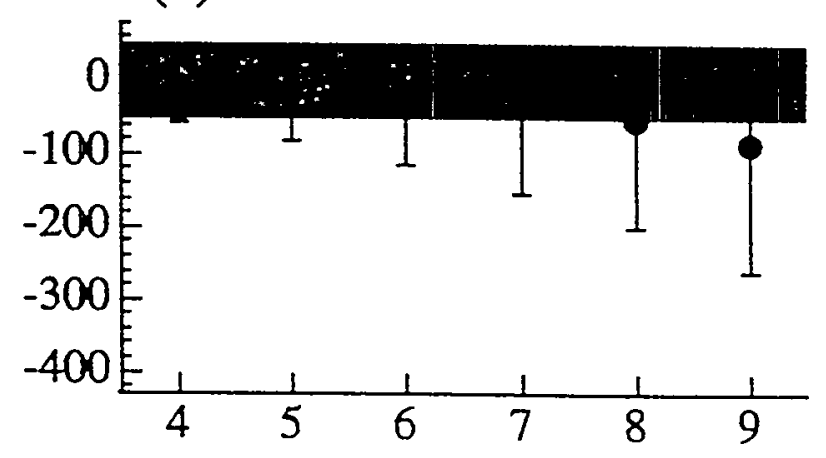

n

Figure 4.15. Differences between calculated and experimental $E A$ s for $\mathrm{Ar}_{\mathrm{n}} \mathrm{Br}$, plotted as a function of n, including various three body terms: (a) $E A_{\text {ind }}-E A_{\text {exp }}$, (b) $E A_{\text {ind }+e c+m d i s}$ $E A_{\text {expt, }}$ (c) $E A_{\text {ind }+ \text { ec+mdit }+a t}-E A_{\text {expl. }}$. The shaded region represents the experimental uncertainty. The error bars represent the uncertainty in the calculated $E A$ s. 
(a)
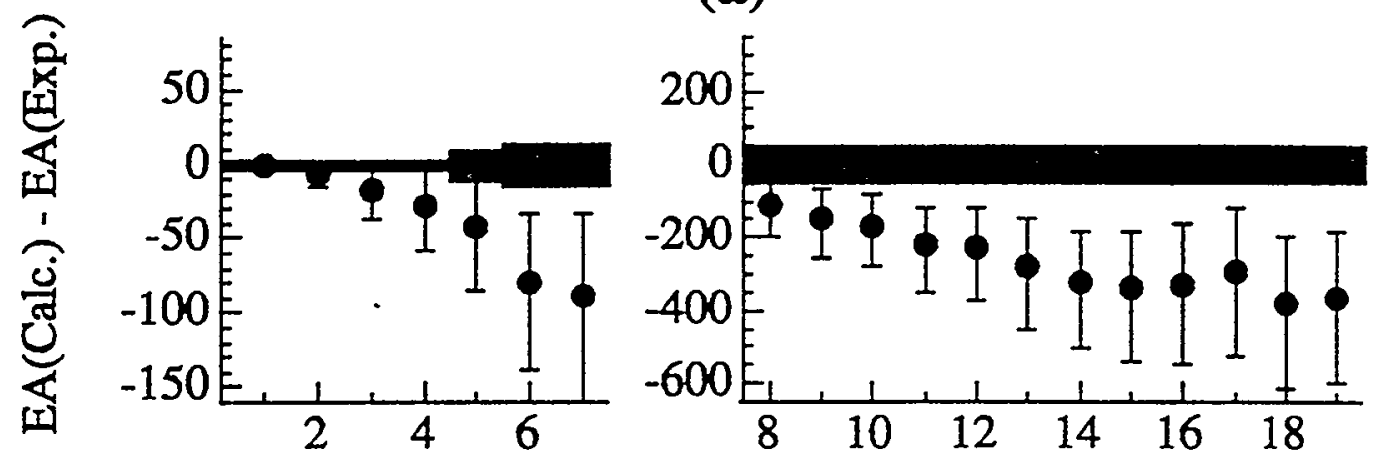

n

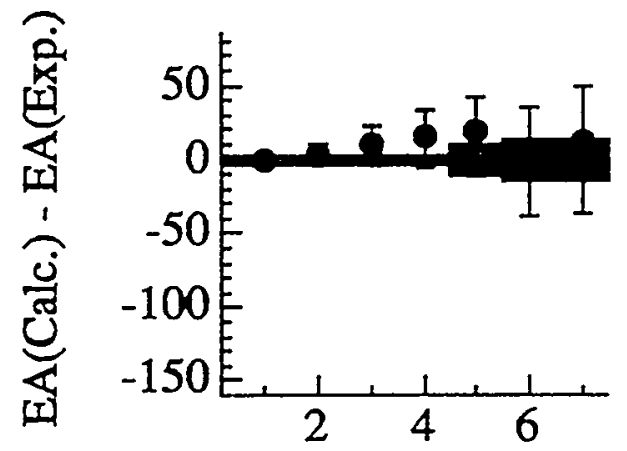

(b)

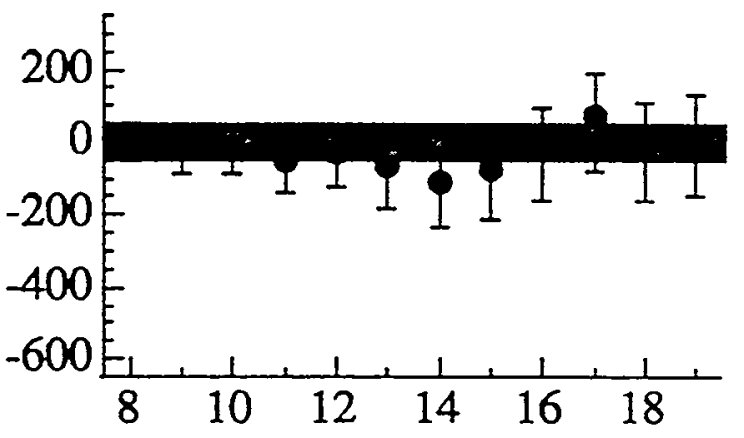

$\mathbf{n}$

(c)
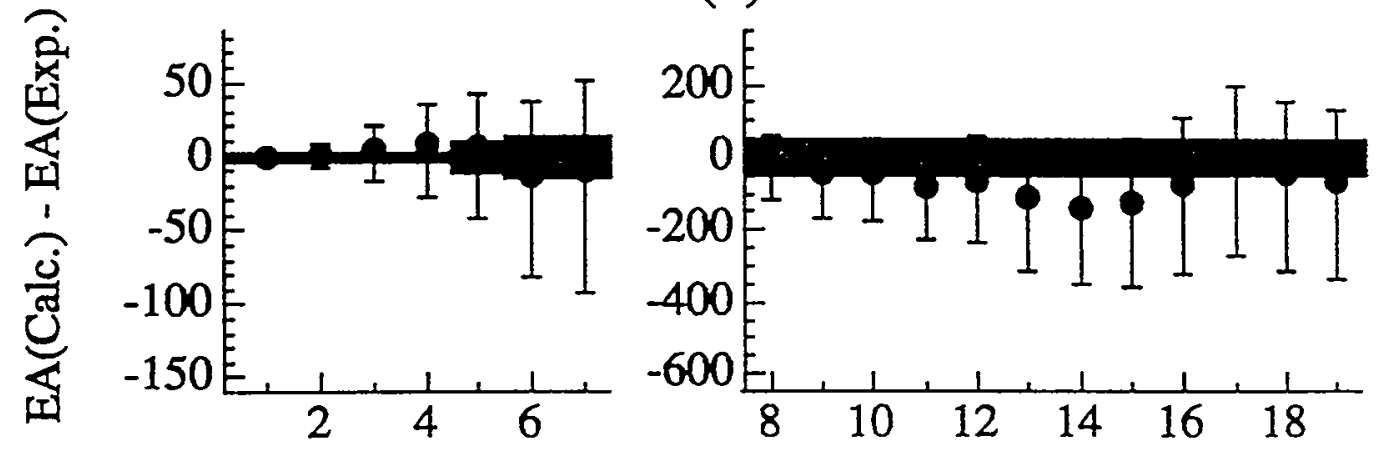

n

Figure 4.16. Differences between calculated and experimental $E A s$ for $A r_{n} I$, plotted as a function of n, including various three body terms: (a) $E A_{\text {ind }}-E A_{\text {expl, }}$, (b) $E A_{\text {ind }+ \text { ec }+m d i s}$ $E A_{\text {expt }}$, (c) $E A_{\text {ind }+e c+m d i t+a t}-E A_{\text {expt }}$. The shaded region represents the experimental uncertainty. The error bars represent the uncertainty in the calculated $E A$ s. 
Table 4.7. Calculated $\mathrm{Ar}_{n} \mathrm{Br}^{-}$anion and $\mathrm{Ar}_{n} \mathrm{Br}$ neutral $X$ state binding energies and zero point energies, including non-additive terms. IND: non-additive induction. EC: exchange charge. MDIS: dispersion multipole. AT: Axilrod-Teller triple-dipole dispersion. Energies are in $\mathrm{cm}^{-1}$.

\begin{tabular}{|c|c|c|c|c|c|c|c|c|}
\hline \multirow{2}{*}{$\mathrm{n}$} & \multicolumn{3}{|c|}{ IND } & \multicolumn{2}{c|}{ IND+EC+MDIS } & \multicolumn{2}{c|}{$\begin{array}{c}\text { IND+EC+MDIS } \\
+ \text { AT (anion) }\end{array}$} & \multicolumn{2}{c|}{ AT (neutral) } \\
\cline { 2 - 9 } & $\varepsilon_{a}$ & $\omega_{0}^{a}$ & $\varepsilon_{a}$ & $\omega_{0}^{a}$ & $\varepsilon_{a}$ & $\omega_{0}^{a}$ & $\varepsilon_{x}$ & $\omega_{0}^{x}$ \\
\hline 1 & 438.8 & 20.7 & 438.8 & 20.7 & 438.8 & 20.7 & 133.1 & 15.6 \\
\hline 2 & 942.4 & 53.7 & 955.9 & 56.1 & 946.8 & 55.0 & 343.7 & 41.7 \\
\hline 3 & 1515.3 & 98.5 & 1555.7 & 105.1 & 1528.9 & 102.3 & 646.1 & 83.3 \\
\hline 4 & 2081.3 & 142.6 & 2148.4 & 153.2 & 2103.9 & 148.8 & 950.0 & 124.7 \\
\hline 5 & 2640.9 & 185.0 & 2734.3 & 199.0 & 2672.3 & 193.5 & 1255.5 & 164.7 \\
\hline 6 & 3273.3 & 236.7 & 3402.4 & 255.2 & 3315.3 & 247.6 & 1643.6 & 207.8 \\
\hline 7 & 3819.9 & 278.3 & 3974.4 & 300.4 & 3871.5 & 291.8 & 1978.7 & 262.0 \\
\hline 8 & 4434.4 & 326.4 & 4620.2 & 351.6 & 4494.4 & 341.1 & 2385.1 & 307.4 \\
\hline 9 & 5029.0 & 367.2 & 5238.7 & 395.2 & 5091.0 & 381.9 & 2783.7 & 349.6 \\
\hline
\end{tabular}

Table 4.8. Calculated $A r_{n}{ }^{-}$anion and ${ }^{A} r_{n} I$ neutral $X$ state binding energies and zero point energies, including non-additive terms. IND: non-additive induction. EC: exchange charge. MDIS: dispersion multipole. AT: Axilrod-Teller triple-dipole dispersion. Energies are in $\mathrm{cm}^{-1}$.

\begin{tabular}{|c|c|c|c|c|c|c|c|c|}
\hline \multirow{2}{*}{$\mathrm{n}$} & \multicolumn{3}{|c|}{ IND } & \multicolumn{2}{l|}{ IND+EC+MDIS } & \multicolumn{2}{c|}{$\begin{array}{c}\text { ND+EC+MDIS } \\
+ \text { AT (anion) }\end{array}$} & \multicolumn{2}{c|}{ AT (neutral) } \\
\cline { 2 - 9 } & $\varepsilon_{a}$ & $\omega_{0}^{a}$ & $\varepsilon_{a}$ & $\omega_{0}^{a}$ & $\varepsilon_{a}$ & $\omega_{0}^{a}$ & $\varepsilon_{x}$ & $\omega_{0}^{x}$ \\
\hline 1 & 369.4 & 17.3 & 369.4 & 17.3 & 369.4 & 17.3 & 151.6 & 14.6 \\
\hline 2 & 814.6 & 48.4 & 825.2 & 50.4 & 817.0 & 49.2 & 379.0 & 40.6 \\
\hline 3 & 1338.4 & 90.2 & 1369.9 & 96.0 & 1345.7 & 92.6 & 699.7 & 81.4 \\
\hline 4 & 1859.9 & 132.3 & 1912.3 & 141.5 & 1871.5 & 136.4 & 1020.8 & 122.1 \\
\hline 5 & 2378.0 & 171.8 & 2451.2 & 184.0 & 2393.7 & 177.7 & 1342.6 & 161.6 \\
\hline 6 & 2967.4 & 221.9 & 3066.3 & 239.4 & 2985.9 & 229.2 & 1740.5 & 203.9 \\
\hline 7 & 3476.4 & 261.5 & 3595.8 & 280.9 & 3499.6 & 270.5 & 2080.2 & 251.2 \\
\hline 8 & 4040.0 & 306.9 & 4179.8 & 330.7 & 4062.8 & 316.2 & 2482.0 & 292.6 \\
\hline 9 & 4567.5 & 344.5 & 4721.2 & 366.2 & 4585.0 & 352.0 & 2859.1 & 325.1 \\
\hline 10 & 5102.2 & 386.5 & 5285.1 & 415.0 & 5131.7 & 398.9 & 3246.9 & 378.1 \\
\hline 11 & 5623.9 & 423.4 & 5821.0 & 456.8 & 5647.2 & 436.2 & 3627.1 & 415.7 \\
\hline 12 & 6197.5 & 475.9 & 6427.8 & 510.4 & 6233.0 & 488.9 & 4047.9 & 470.3 \\
\hline 13 & 6818.0 & 516.8 & 7068.8 & 554.8 & 6847.5 & 528.7 & 4533.9 & 513.4 \\
\hline 14 & 7363.8 & 547.9 & 7625.0 & 593.4 & 7371.8 & 557.4 & 4957.0 & 554.8 \\
\hline 15 & 8021.4 & 610.6 & 8322.8 & 649.3 & 8038.4 & 617.9 & 5465.2 & 619.4 \\
\hline 16 & 8615.8 & 683.1 & 8973.2 & 725.1 & 8673.8 & 694.6 & 5908.9 & 667.5 \\
\hline 17 & 9232.3 & 763.9 & 9647.9 & 808.9 & 9336.7 & 781.2 & 6361.9 & 747.4 \\
\hline 18 & 9737.0 & 818.1 & 10159.9 & 864.8 & 9840.0 & 836.3 & 6810.4 & 802.8 \\
\hline
\end{tabular}




\begin{tabular}{|l|l|l|l|l|l|l|l|l|}
\hline 19 & 10247.0 & 872.0 & 10676.5 & 921.2 & 10347.4 & 891.3 & 7254.8 & 855.9 \\
\hline
\end{tabular}

Table 4.9. $\mathrm{Ar}_{\mathrm{n}} \mathrm{Br}$ electron affinities calculated with various non-additive terms. $E A_{\text {ind }}$ : non-additive induction term only. $E A_{\text {ind }+e c+m d i s}$ : non-additive induction, exchange charge, and multipole dispersion terms. $E A_{\text {ind }+e c+m d i s+a t:}$ induction, exchange charge, multipole dispersion, and triple-dipole dispersion terms. Energies are in $\mathrm{cm}^{-1}$.

\begin{tabular}{|c|c|c|c|}
\hline $\mathrm{n}$ & $E A_{\text {ind }}$ & $E A_{\text {ind }+e c+m d i s}$ & $E A_{\text {ind }+e c+m d i s+a t}$ \\
\hline 0 & 27129.2 & 27129.2 & 27129.2 \\
\hline 1 & 27429.8 & 27429.8 & 27429.8 \\
\hline 2 & 27710.8 & 27721.9 & 27718.9 \\
\hline 3 & 27968.5 & 28002.2 & 27993.0 \\
\hline 4 & 28218 & 28275 & 28259 \\
\hline 5 & 28460 & 28540 & 28517 \\
\hline 6 & 28681 & 28792 & 28761 \\
\hline 7 & 28897 & 29029 & 28992 \\
\hline 8 & 29088 & 29249 & 29205 \\
\hline 9 & 29271 & 29453 & 29404 \\
\hline
\end{tabular}


Table 4.10. $A r_{n} I$ electron affinities calculated with various non-additive terms. $E A_{\text {ind }}$ : non-additive induction term only. $E A_{\text {ind }+e c+m d i s}$ : non-additive induction, exchange charge, and multipole dispersion terms. $E A_{\text {ind }+e c+m d i s+a t:}$ induction, exchange charge, multipole dispersion, and triple-dipole dispersion terms. Energies are in $\mathrm{cm}^{-1}$.

\begin{tabular}{|c|c|c|c|}
\hline $\mathrm{n}$ & $E A_{\text {ind }}$ & $E A_{\text {ind }+e c+m d i s}$ & $E A_{\text {ind +ec+mdis+at }}$ \\
\hline 0 & 24673.3 & 24673.3 & 24673.3 \\
\hline 1 & 24888.3 & 24888.3 & 24888.3 \\
\hline 2 & 25095.4 & 25103.9 & 25102.6 \\
\hline 3 & 25286.6 & 25312.3 & 25308.1 \\
\hline 4 & 25474 & 25518 & 25510 \\
\hline 5 & 25660 & 25721 & 25708 \\
\hline 6 & 25828 & 25909 & 25893 \\
\hline 7 & 25995 & 26095 & 26073 \\
\hline 8 & 26138 & 26254 & 26231 \\
\hline 9 & 26268 & 26400 & 26372 \\
\hline 10 & 26410 & 26565 & 26537 \\
\hline 11 & 26537 & 26701 & 26673 \\
\hline 12 & 26680 & 26875 & 26840 \\
\hline 13 & 26798 & 27011 & 26972 \\
\hline 14 & 26903 & 27119 & 27086 \\
\hline 15 & 27039 & 27302 & 27248 \\
\hline 16 & 27156 & 27472 & 27411 \\
\hline 17 & 27323 & 27693 & 27614 \\
\hline 18 & 27336 & 27712 & 27669 \\
\hline 19 & 27431 & 27811 & 27731 \\
\hline & & & \\
\hline
\end{tabular}


It is important to note that the many-body terms $V_{i n d}, V_{e c}, V_{m d i s}$, and $V_{a t}^{\text {anton }}$ all depend on the absolute values of the Ar-X distances. Therefore, we must consider the uncertainties in these terms due to the absolute uncertainty in $R_{m}$ in the pair potentials, which, as mentioned in Section 4.4.1, is $\pm 0.2 \AA$ for both $\mathrm{ArBr}^{-}$and ArI'. In order to estimate the uncertainties in the many-body terms, we calculated the changes in these terms in the $\mathrm{Ar}_{2} \mathrm{Br}^{-}$and $\mathrm{Ar}_{2} \mathrm{I}^{-}$systems with the $\mathrm{Ar}$ - $\mathrm{Ar}$ distance fixed at the $\mathrm{Ar}_{2}$ equilibrium value, as the $\operatorname{Ar}-X^{-}$distances were varied over $\pm 0.2 \AA$ about the $\operatorname{Ar} X^{-}$equilibrium values. To estimate these uncertainties in the larger clusters, the $\mathrm{Ar}_{2} \mathrm{X}$ uncertainties were multiplied by the number of nearest neighbor Ar-Ar pairs in contact with the halide. The uncertainties introduced into the calculated EAs were found to be significantly larger than those due to the uncertainty in $\varepsilon_{a}-\varepsilon_{X}$ in the pair potentials. These uncertainties are displayed as error bars in Figures 4.15 and 4.16.

The addition of the many-body induction term significantly decreases the $E A$ compared with the additive calculation. [Compare Figures 4.15(a) and 4.16(a) with Figures 4.10 and 4.11.] $E A_{\text {ind }}$ is closer to the experimental values than $E A_{\text {add }}$, but is somewhat overcorrected. This is clearest in the $\mathrm{Ar}_{n} \mathrm{I}$ clusters, in which $E A_{\text {tru }}$ lies well below the experimental uncertainty region (shaded areas in Figures 4.15 and 4.16) for $\mathrm{n}=6,7$ and 9-19. Thus, the $A r_{n} I$ results clearly indicate the need for additional nonadditive terms. In the $\mathrm{Ar}_{\mathrm{n}} \mathrm{Br}$ clusters, the experimental and theoretical $E A$ uncertainty regions overlap except for $\mathrm{n}=8$ and 9 , but the $E A_{\text {ind }}$ values are all systematically lower than the experimental $E A s$, again suggesting that induction effects alone decrease the electron affinity by too much. 
Inclusion of $V_{e c}$ and $V_{m d i s}$ in the calculation brings the theoretical EAs closer to the experimental results. [See Figures 4.15(b) and 4.16(b).] In the case of $\mathrm{Ar}_{n} \mathrm{Br}$, $E A_{\text {ind+ectmdis }}$ lies within the experimental error bars in all cases except for $\mathrm{Ar}_{3} \mathrm{Br}$, which is overcorrected by about $9 \mathrm{~cm}^{-1}$. But even in this case, the model potential and experimental uncertainty regions overlap. For the $\operatorname{Ar}_{\mathrm{n}} \mathrm{I}$ clusters, $E A_{\text {ind }+e c+m d i s}$ is overcorrected by $3.8-20.1 \mathrm{~cm}^{-1}$ for $\mathrm{n}=2-5$, which is outside the experimental error bars (the shaded region in Figures 4.15 and 4.16 ). For $6 \leq n \leq 19, E A_{\text {ind }+e c+m d i s}$ lies within experimental uncertainties except for $n=13-15$ and $n=17$. However, as in the $\mathrm{Ar}_{\mathrm{p}} \mathrm{Br}$ clusters, the theoretical and experimental uncertainty ranges overlap in all cases for $A r_{n} I$.

Inclusion of the Axilrod-Teller term brings the theoretical EAs closer to experiment for the smaller clusters, but overcorrects somewhat for some of the larger clusters. [See Figures 4.15(c) and 4.16(c).] Now for the $A r_{n} B r$ clusters $E A_{\text {ind }+e c+m d t s+a t}$ lies within the experimental uncertainties for all cases except $n=9$. For $A r_{24} I$, addition of the Axilrod-Teller term brings the theoretical $E A$ closer to the experimental result, but is still a few wavenumbers above the experimental error bars. For $\mathrm{n}=5-10,17$ and 18 , the theoretical result is within experimental uncertainties, but lies below the uncertainty region for $n=11-16$ and 19. But, again, the theoretical and experimental error bars overlap in all cases. Thus, inclusion of the triple-dipole term appears to help somewhat for the smaller clusters, but, because of the uncertainties in $V_{i n d}$ and $V_{e c}+V_{m d i s}$, it is not possible to draw definite conclusions about the importance of the Axilrod-Teller term from the present results. To do so would require more precise knowledge of $r_{m}$ in the pair potentials.

\subsection{Conclusions}


We have obtained experimental electron affinities and electronic structure information from the ZEKE and PDTP spectra of $\mathrm{Ar}_{2-9} \mathrm{Br}^{-}$and $\mathrm{Ar}_{2-19} \mathrm{I}^{-}$. We have compared these with electronic state splittings and EAs calculated from both pairwise additive and non-additive model potentials. The following conclusions can be drawn from this work.

(1) The first-order degenerate perturbation theory treatment of the open-shell neutral potentials described in Section 4.4 .5 is accurate enough to account for the $X-I$ electronic state splittings observed in $\mathrm{Ar}_{2-3} \mathrm{Br}$ and $\mathrm{Ar}_{2-7} \mathrm{I}$, within experimental uncertainties. (See Figure 4.8). However, this model somewhat overestimates the $X-I I$ state splittings for $\mathrm{Ar}_{2.3} \mathrm{Br}$ and $\mathrm{Ar}_{2-3} \mathrm{I}$, possibly indicating that the spin-orbit splitting decreases as $\mathrm{Ar}$ atoms are added around the halogen. (See Figure 4.9).

(2) A pairwise additive model of the anion potentials is completely inadequate to account for the experimentally measured EAs. (See Figures 4.10 and 4.11). Non-additive effects in the anion are clearly very important.

(3) The many-body induction effect is the most important non-additive effect in the anion potential. Inclusion of $V_{\text {ind }}$ accounts for most of the discrepancy between the additive and experimental $E A$ s, but somewhat overcorrects, especially in the case of $A r_{n} I$. This result is consistent with the work of Berkowitz et at. ${ }^{18}$ who found a non-additive inductive effect to be very important to model the experimental $B E S$ of $\mathrm{Br}^{-}\left(\mathrm{H}_{2} \mathrm{O}\right)_{\mathrm{n}}$ clusters. We also note that, although not explicitly discussed by Bowen and coworkers in their paper on $\mathrm{Ar}_{\mathrm{n}} \mathrm{O}^{-}$clusters, ${ }^{16}$ it seems likely that the non-additive induction effect may in large part account for the non-linearity of the binding energies as a function of $n$ observed by them for $n<12$. (See Figure 4 of Reference 16.) 
(4) The exchange/dispersion multipole term also plays an important role. In both the $\mathrm{Ar}_{2-9} \mathrm{Br}^{\circ}$ and $\mathrm{Ar}_{2-19} \mathrm{I}^{\circ}$ clusters inclusion of the $V_{e c}$ and $V_{\text {mdis }}$ terms in the $E A$ calculation brings the calculated EAs within the experimental error bars, when the uncertainty in the size of the induction effect due to the uncertainty of the pair potential bond length is taken into account.

(5) Inclusion of the triple-dipole dispersion effect in the anion and neutral potentials appears to slightly improve the agreement with experiment for the smaller clusters $\left(\mathrm{Ar}_{2.5} \mathrm{Br}^{-}\right.$and $\left.\mathrm{Ar}_{2-5} \mathrm{I}^{\circ}\right)$, but makes the fit slightly worse for the larger clusters. However, due to the uncertainties in $r_{m}$ in the Ar- $\mathrm{X}^{-}$pair potentials, nothing conclusive can be said about the role of the triple-dipole dispersion effect in these clusters on the basis of our results. Furthermore, we cannot draw any conclusions about the role of three-body exchange effects or higher-order multipole dispersion terms from the present work.

Overall, this type of detailed energetic study of many-body effects is complementary to studies of non-additive effects via high resolution spectroscopy. In this type of experiment we are able to directly measure the difference between anion and neutral binding energies, allowing a direct comparison of experimental observables with model potentials including non-additive effects. However, due to limited resolution and uncertainties in the pair potentials this experiment is not sensitive to the most subtle nonadditive effects, such as the triple-dipole dispersion energy. This is in contrast to the high resolution spectroscopic studies of the $\mathrm{Ar}_{2}-\mathrm{HX}$ systems, ${ }^{3.7-10}$ which provide precise values of molecular constants. Comparison of such results with non-additive model potentials is more difficult, but can in principle provide more precise information on non-additive effects. 
Further theoretical work needs to be done to interpret the vibrational structure observed in the smaller clusters $\left(\mathrm{Ar}_{2-3} \mathrm{Br}^{-}\right.$and $\left.\mathrm{Ar}_{2-3} \mathrm{I}^{-}\right)$studied here. These spectra present an opportunity to test the various methods of dynamical calculations that have been developed for weakly bound clusters, ${ }^{75}$ and such studies would be welcome.

In the future, we hope to observe the $\mathrm{ZEKE}$ spectrum of $\mathrm{Ar}_{2} \mathrm{Cl}^{-}$. This would allow direct comparison with the recently published ab initio study of this system by Burcl et $a l .{ }^{68}$ Also, the $\mathrm{Ar}_{2} \mathrm{Cl}$ neutral cluster would present a more tractable problem for $a b$ initio theorists than the larger halogen containing clusters studied in the present work.

\subsection{Acknowledgments}

This research is supported by the Air Force Office of Scientific Research under Contract No. F49620-94-1-0115, and by a Department of Defense Augmentation Award for Science and Engineering Research Training under Contract No. F49620-94-1-0412. We also wish to thank R. B. Gerber, P. Jungwirth, A. Krylov, C. C. Martens and Z. Li for helpful discussions and for providing molecular dynamics programs. 


\subsection{References for Chapter 4}

${ }^{1}$ H. Margenau, and N. R. Kestner, Theory of Intermolecular Forces (Pergamon Press, Oxford, 1969), Ch. 5.

${ }^{2}$ W.J. Meath, and R.A. Aziz, Mol. Phys. 52, 225 (1984).

${ }^{3}$ M.J. Elrod, and R.J. Saykally, Chem. Rev. 94, 1975 (1994), and references therein.

${ }^{4}$ Y. Zhao, I. Yourshaw, G. Reiser, C.C. Arnold, and D.M. Neumark, J. Chem. Phys. 101, 6538 (1994).

${ }^{5}$ Y. Zhao, C.C. Arnold, and D.M. Neumark, J. Chem. Soc. Faraday Trans. 89, 1449 (1993).

${ }^{6}$ Y. Xu, W. Jäger, and M. C. L. Gerry, J. Chem. Phys. 100, 4171 (1994).

${ }^{7}$ M.A. Suhm, and D.J. Nesbitt, Chem. Soc. Rev. 24, 45 (1995); A. McIlroy, R. Lascola, C.M. Lovejoy, and D.J. Nesbitt, J. Phys. Chem. 95, 2636 (1991).

${ }^{8}$ M.J. Elrod, J.G. Loeser, and R.J. Saykally, J. Chem. Phys. 98, 5352 (1993).

${ }^{9}$ M. M. Szczesniak, G. Chalasinski, and P. Piecuch, J. Chem. Phys. 99, 6732 (1993); S. M. Cybulski, M. M. Szczesniak, and G. Chalasinski, J. Chem. Phys. 101, 10708 (1994). ${ }^{10}$ (a) A.R. Cooper, and J.M. Hutson, J. Chem. Phys. 98, 5337 (1992); (b) M.J. Elrod, R.J. Saykally, A.R. Cooper, and J.M. Hutson, Mol. Phys. 81, 579 (1994); (c) A. Ernesti, and J.M. Hutson, Phys. Rev. A 51, 239 (1995).

"S. Martrenchard-Barra, C. Jouvet, C. Lardeux-Dedonder, and D. Solgadi, J. Chem. Phys. 98, 5281 (1993). 
${ }^{12}$ J.P. Visticot, P. de Pujo, J.M. Mestdagh, A. Lallement, J. Berlande, O. Sublemontier, P. Meynadier, and J. Cuvellier, J. Chem. Phys. 100, 158 (1994).

${ }^{13}$ (a) C.R. Bieler, D.D. Evard, and K.C. Janda, J. Phys. Chem. 94, 7452 (199C); (b) D.G.

Jahn, S.G. Clement, and K.C. Janda, J. Chem. Phys. 101, 283 (1994).

${ }^{14}$ T. Bürgi, T. Droz, and S. Leutwyler, Chem. Phys. Lett. 225, 351 (1994).

${ }^{15}$ G. Markovich, S. Pollack, R. Giniger, and O. Cheshnovsky, J. Chem. Phys. 101, 9344 (1994).

${ }^{16}$ S.T. Arnold, J.H. Hendricks, and K.H. Bowen, J. Chem. Phys. 102, 39 (1995).

${ }^{17}$ D.W. Arnold, S.E. Bradforth, E.H. Kim, and D.M. Neumark, J. Chem. Phys. 102, 3510 (1995); 97, 9468 (1992).

${ }^{18}$ L.S. Sremaniak, L. Perera, and M.L. Berkowitz, Chem. Phys. Lett. 218, 377 (1994).

${ }^{19}$ K. Müller-Dethlefs, M. Sander, and E.W. Schlag, Z. Naturforsch. Teil A 39, 1089 (1984); Chem. Phys. Lett. 12, 291 (1984); K. Müller-Dethlefs, and E.W. Schlag, Ann. Rev. Phys. Chem. 42, 109 (1991).

${ }^{20}$ (a) T.N. Kitsopoulos, I.M. Waller, J.G. Loeser, and D.M. Neumark, Chem. Phys. Lett. 159, 300 (1989); (b) C.J. Chick, Y. Zhao, T.N. Kitsopoulos, and D.M. Neumark, J. Chem. Phys. 97, 6121 (1992); (c) T.N. Kitsopoulos, Ph.D. Thesis, University of California, Berkeley, 1991; (d) C.C. Arnold, Ph.D. Thesis, University of California, Berkeley, 1994.

${ }^{21}$ J.M.B. Bakker, J. Phys. E 6, 785 (1973).

${ }^{22}$ T. Baer, W.B. Peatman, and E.W. Schlag, Chem. Phys. Lett. 4, 243 (1969); R. Spohr, P.M. Guyon, W.A. Chupka, and J. Berkowitz, Rev. Sci. Instrum. 42, 1872 (1971). 
${ }^{23}$ C. E. Moore, Atomic Energy Levels, v. I, Circ. Natl. Bur. Std. 467 (1949).

${ }^{24}$ H. Haberland, Z. Phys A. 307, 35 (1982).

${ }^{a}$ C. Blondel, P. Cacciani, C. Delsart and R. Trainham, Phys. Rev. A 40, 3698 (1989).

${ }^{b}$ C.E. Moore, Atomic Energy Levels, v. I, Circ. Natl. Bur. Std. 467 (1949).

${ }^{25}$ C. Blondel, P. Cacciani, C. Delsart, and R. Trainham, Phys. Rev. A 40, 3698 (1989).

${ }^{26}$ H. Hotop, and W.C. Lineberger, J. Phys. Chem. Ref. Data 14, 731 (1985).

${ }^{27}$ P. Casavecchia, G. He, R.K. Sparks, and Y.T. Lee, J. Chem. Phys. 75, 710 (1981).

${ }^{28}$ (a) G. Liuti, and F. Pirani, Chem. Phys. Lett. 122, 245 (1985); (b) D. Cappelletti, G.

Liuti, and F. Pirani, Chem. Phys. Lett. 183, 297 (1991); (c) R. Cambi, D. Cappelletti, G.

Liuti, and F. Pirani, J. Chem. Phys. 95, 1852 (1991).

29 R.A. Aziz, and M.J. Slaman, Mol. Phys. 58, 679 (1986). A more accurate Ar-Ar potential has recently been published--the HFDID1 potential of Aziz, which was fit to more recent spectroscopic data [R.A. Aziz, J. Chem. Phys. 99, 4518 (1993)]. However it was felt that the simpler HFD-B2 potential is sufficiently accurate for the purposes of this work.

${ }^{30}$ Z. Li, A. Borrmann, and C.C. Martens, J. Chem. Phys. 97, 7234 (1992); A. Borrmann, Z. Li, and C.C. Martens J. Chem. Phys. 98, 8514 (1993).

${ }^{31}$ M. P. Allen, and D. J. Tildesley, Computer Simulation of Liquids (Oxford Univ. Press, Oxford, 1987), p. 231.

${ }^{32}$ P.R. Bevington, Data Reduction and Error Analysis for the Physical Sciences (McGraw-Hill, New York, 1969), Ch. 11. 
${ }^{33}$ E.B. Wilson, J.C. Decius, and P.C. Cross, Molecular Vibrations (Dover, New York, 1955).

${ }^{34}$ D.O. Harris, G.G. Engerholm, and W.D. Gwinn, J. Chem. Phys. 43, 1515 (1965).

${ }^{35}$ J.C. Light, I.P. Hamilton, and J.V. Lill, J. Chem. Phys. 82, 1400 (1985).

${ }^{36}$ R.B. Metz, Ph.D. Thesis, University of California, Berkeley, 1991.

37 W.G. Lawrence, and V.A. Apkarian, J. Chem. Phys. 101, 1820 (1994). Note the misprint in Eqn. Al of this paper: The matrix element $\left\langle\frac{1}{2},-\frac{1}{2}|H| \frac{3}{2}, \frac{1}{2}\right\rangle$ should read $\frac{1}{3} V_{01}-\frac{2}{3} V_{-10}$, in the notation of this reference.

${ }^{38}$ V. Aquilanti, G. Liuti, F. Pirani, and F. Vecchiocattivi, J. Chem. Soc. Faraday Trans. 2 85,955 (1989).

${ }^{39}$ W.E. Baylis, J. Phys. B 10, LA77 (1977).

${ }^{40}$ D. L. Huestis, and N. E. Schlotter, J. Chem. Phys. 69, 3100 (1978).

${ }^{41}$ F.O. Ellison, J. Am. Chem. Soc. 85, 3540 (1963); J.C. Tully, J. Chem. Phys. 58, 1396 (1973).

42 (a) K.M. Sando, G.J. Erickson, and R.C. Binning Jr., J. Phys. B 12, 2697 (1979); (b)

G.J. Erickson, and K.M. Sando, Phys. Rev. A 22, 1500 (1980).

${ }^{43}$ L.C. Balling, and J.J. Wright, J. Chem. Phys. 79, 2941 (1983).

${ }^{44}$ J.A. Boatz, and M.E. Fajardo, J. Chem. Phys. 101, 3472 (1994).

${ }^{45}$ (a) A.I. Krylov, R.B. Gerber, and V.A. Apkarian, Chem. Phys. 189, 261 (1994); (b) A.I. Krylov, and R.B. Gerber, Chem. Phys. Lett. 231, 395 (1994).

${ }^{46}$ B.M. Axilrod, and E. Teller, J. Chem. Phys. 11, 299 (1943); B.M. Axilrod, J. Chem. Phys. 19, 719 (1951). 
47 Y. Muto, Proc. Phys-Math. Soc. Japan 17, 629 (1943).

${ }^{48}$ (a) K.T. Tang, J.M. Norbeck, and P.R. Certain, J. Chem. Phys. 64, 3063 (1976); (b)

D.J. Margoliash, T.R. Proctor, G.D. Zeiss, and W.J. Meath, Mol. Phys. 35, 747 (1978);

(c) A. Kumar, and W.J. Meath, Mol. Phys. 54, 823 (1985).

${ }^{49}$ G. Chalasinski, M.M. Szczesniak, and S.M. Cybulski, J. Chem. Phys. 92, 2481 (1990).

${ }^{50}$ (a) A.D. Koutselos, and E.A. Mason, J. Chem. Phys. 85, 2159 (1986); (b) H.L. Kramer, and D.R. Herschbach, J. Chem. Phys. 53, 2792 (1970); (c) K.T. Tang, Phys. Rev. 177, 108 (1969).

${ }^{51}$ J.C. Slater, and J.G. Kirkwood, Phys. Rev. 37, 682 (1931); K.S. Pitzer, Adv. Chem. Phys. 2, 59 (1959); J.N. Wilson, J. Chem. Phys. 43, 2564 (1965).

52 R. J. Bell, J. Phys. B 3, 751 (1970).

${ }^{53}$ F.J. Vesely, J. Comput. Phys. 24, 361 (1977).

${ }^{54}$ E.L. Pollock, and B.J. Alder, Phys. Rev. Lett. 41, 903 (1978).

${ }^{55}$ P. Stampfli, J. Chem. Phys. 101, 6024 (1994).

${ }^{56}$ For example, water: (a) F.H. Stillinger, and C.W. David, J. Chem. Phys. 69, 1473 (1978); (b) P. Barnes, J.L. Finney, J.D. Nicholas, and J.E. Quinn, Nature 282, 459 (1979);

(c) P. Ahlström, A. Wallqvist, S. Engström, and B. Jönsson, Mol. Phys. 68, 563 (1989);

(d) J. Caldwell, L.X. Dang, and P.A. Kollman, J. Am. Chem. Soc. 112, 9144 (1990);

acetone: (e) P. Jedlovszky, and G. Pálinkás, Mol Phys. 84, 217 (1995).

${ }^{57}$ For example, (a) Xe ${ }_{n}^{++}$: J.G. Gay, and B.J. Berne, Phys. Rev. Lett. 49, 194 (1982); (b) $\mathrm{Na}^{+}\left(\mathrm{H}_{2} \mathrm{O}\right)_{\mathrm{n}}$ : P. Perez, W.K. Lee, and E.W. Prohofsky, J. Chem. Phys., 79, 388 (1983); (c) $\mathrm{X}\left(\mathrm{H}_{2} \mathrm{O}\right)_{\mathrm{n}}(\mathrm{X}=$ halide and alkali ions): S. Sung, and P.C. Jordan, J. Chem. Phys. 85, 4045 
(1986); (d) $\mathrm{X}_{\mathrm{n}} \mathrm{Br}_{2}{ }^{-}\left(\mathrm{X}=\mathrm{Ar}, \mathrm{CO}_{2}\right)$ : L. Perera, and F.G. Amar, J. Chem. Phys. 90, 7354 (1989); (e) $\left(\mathrm{H}_{2} \mathrm{O}\right)_{\mathrm{n}} \mathrm{X}\left(\mathrm{X}=\mathrm{Na}^{+}, \mathrm{Cl}\right)$ : L. Perera, and M.L. Berkowitz, J. Chem. Phys. 95 , 1954 (1991); 99, 4236 (1993).

${ }^{58}$ The contribution of the term in $B$ to the induced quadrupole moment is estimated to be approximately $20 \%$ of the $C$ contribution, and of opposite sign, where we have used the values of $B$ for Ar from Ref. 74 .

${ }^{59}$ U.C. Dikshit, and M. Kumar, Physica Status Solidi B 165, 599 (1991).

${ }^{60}$ A.D. Buckingham, Adv. Chem. Phys. 12, 107 (1967).

${ }^{61}$ C.J.F. Böttcher, Theory of Electric Polarization, $2^{\text {nd }}$ Ed., Vol. I (Elsevier, Amsterdam, 1973).

${ }^{62}$ J. Applequist, Chem. Phys. 85, 279 (1984); 190, 153 (1995).

${ }^{63}$ R.L. Asher, D.A. Micha, and P.J. Brucat, J. Chem. Phys. 96, 7683 (1992).

${ }^{64}$ B.G. Dick, Jr., and A.W. Overhauser, Phys. Rev. 112, 90 (1958).

${ }^{65}$ L. Jansen, Adv. Quantum Chem. 2, 119 (1965).

${ }^{66}$ A.J. Lacey, and W. Byers Brown, Mol. Phys. 27, 1013 (1974).

${ }^{67}$ (a) M.M. Szczesniak, G. Chalasinski, and P. Piecuch, J. Chem. Phys. 99, 6732 (1993);

(b) S.M. Cybulski, M.M. Szczesniak, and G. Chalasinski, J. Chem. Phys. 101, 10708 (1994).

${ }^{68}$ R. Burcl, S.M. Cybulski, M.M. Szczesniak, and G. Chalasinski, J. Chem. Phys. 103, 299 (1995).

${ }^{69}$ B. Guillot, R.D. Mountain, and G. Birnbaum, Mol. Phys. 64, 747 (1988).

${ }^{70}$ W. Kolos, and A. Les, Int. J. Quantum Chem. 6, 1101 (1972). 
${ }^{71}$ R.J. Wheatley, Mol. Phys. 79, 597 (1993); J. Comput. Chem. 15, 1187 (1994).

${ }^{72}$ W.H. Press, S.A. Teukolsky, W.T. Vetterling, and B.P. Flannery, Numerical Recipes, $2^{\text {nd }}$ Ed. (Cambridge Univ. Press, Cambridge, 1992), p. 213.

${ }^{73}$ K.L.C. Hunt, Chem. Phys. Lett. 70, 336 (1980).

${ }^{74}$ G. Maroulis, and D.M. Bishop, J. Phys. B. 18, 4675 (1985).

${ }^{75}$ See, for example, (a) the vibrational SCF method: T.R. Horn, R.B. Gerber, and M.A. Ratner, J. Chem. Phys. 91, 1813 (1989); T.R. Horn, R.B. Gerber, J.J. Valentini, and M.A. Ratner, J. Chem. Phys. 94, 6728 (1991), (b) DVR method: R.M. Whitnell, and J.C. Light, J. Chem. Phys. 90, 1774 (1989); D.M. Leitner, J.D. Doll, and R.M. Whitnell, J. Chem. Phys. 94, 6644 (1991), (c) basis set expansion methods: A.R. Cooper, S. Jain, and J.M. Hutson, J. Chem. Phys. 98, 2160 (1993). 
Appendix A. Program for calculating vibrational states and Franck-Condon factors for highly anharmonic one-dimensional potentials based on the eigenfunctions of the Morse potential using the DVR method

In this Appendix we describe the computer program used to calculate the vibrational eigenvalues, eigenfunctions and Franck-Condon factors for simulating the rare-gas halide diatomic spectra described in Chapters 3 and 4. The program uses a matrix method (the discrete variable representation, or DVR, method) to solve the Hamiltonian, with the eigenfunctions of a Morse oscillator as a basis set. The use of a Morse eigenfunction basis set has advantages over the more commonly used oscillator basis set when one is dealing with highly anharmonic potentials, such as those of the van der Waals complexes of interest here.

\section{A1. Brief description of the DVR method}

In the DVR method, or in any matrix method, one first sets up the Schrödinger equation in a matrix representation

$$
\mathrm{H} \cdot \mathrm{c}=E \mathbf{c},
$$

where $\mathbf{H}=\mathbf{T}+\mathbf{V}$ is the Hamiltonian, $\mathbf{T}$ is the kinetic energy matrix and $\mathbf{V}$ is the potential energy matrix. The problem then is to solve for the eigenvalues $E_{n}$ and eigenvectors $\mathbf{c}_{n}$.

To this one chooses a set of basis functions $\left|\phi_{n}\right\rangle$ which are solutions of

$$
H_{0}\left|\phi_{0}\right\rangle=E_{n}^{0}\left|\phi_{n}\right\rangle
$$

where

$$
H_{0}=T+V_{0} \text {. }
$$


$V_{0}$ is a reference potential, for which the eigenfunctions, $\left|\phi_{n}\right\rangle$, and the eigenvalues, $E_{n}^{0}$, are known.

Introducing the "difference potential," $\Delta V=V-V_{0}$, which is the difference between the potential of interest and the reference potential, the Hamiltonian becomes $H=H_{0}+\Delta V$, and the matrix elements of $\mathbf{H}$ are

$$
\begin{aligned}
\left\langle\phi_{n}|H| \phi_{m}\right\rangle & =\left\langle\phi_{n}\left|H_{0}\right| \phi_{m}\right\rangle+\left\langle\phi_{n}|\Delta V| \phi_{m}\right\rangle \\
& =E_{n}^{0}+\left\langle\phi_{n}|\Delta V| \phi_{m}\right\rangle
\end{aligned} .
$$

The Schrödinger equation then becomes

$$
\left(\mathbf{E}^{0}+\Delta \mathbf{V}\right) \cdot \mathbf{c}=E \mathbf{c}
$$

In order to calculate the matrix elements of the difference potential, we then use the "transformation method" of Harris, Engerholm and Gwinn, ${ }^{1}$ commonly known as the HEG method. The first step in this method is to find the matrix elements, $\left\langle\phi_{n}|X| \phi_{m}\right\rangle$, of the position operator, $X$, in our basis; i.e., we find the transformation matrix $\mathrm{T}$ such that

$$
\mathbf{T}^{-1} \cdot \mathbf{X} \cdot \mathbf{T}=\operatorname{diag}\left(R_{l}\right)
$$

The eigenvalues, $R_{i}$, of $\mathrm{X}$ are the DVR points. It is then a simple matter to calculate the $\Delta \mathbf{V}$ in the DVR basis, because the potential (and difference potential) are diagonal in the position representation, so that

$$
\Delta \mathrm{V}_{\mathrm{DVR}}=\left[\begin{array}{cccc}
\Delta V\left(R_{0}\right) & & & 0 \\
& \Delta V\left(R_{1}\right) & & \\
& & \ddots & \\
0 & & & \Delta V\left(R_{N}\right)
\end{array}\right] .
$$

In order to solve Eq. (A6), we must then transform $\mathbf{E}^{0}$ into the DVR basis using the transformation matrices from (A7) 


$$
\mathrm{E}_{\mathrm{DVR}}^{0}=\mathrm{T}^{-1} \cdot \mathrm{E}^{0} \cdot \mathrm{T} .
$$

It can be show $\mathrm{n}^{2}$ that the errors introduced in this approach are minimized if the matrix $\mathbf{X}$ is tridiagonal, as it is in the harmonic oscillator basis. For further details and listing of computer code for implementing the DVR method using a harmonic oscillator basis, see the dissertation of R. B. Metz. ${ }^{3}$

Once the eigenvectors $\mathrm{c}_{n}$ and the eigenvalues $E_{n}$ are found by diagonalizing Eq. (A6) it is a simple matter to simulate the vibrational stick spectrum. The anion and neutral eigenvectors are calculated in the same basis, so the Franck-Condon factors (FCFs) are found from

$$
\operatorname{FCF}\left(v_{n}^{a n} \leftarrow v_{m}^{\text {neut }}\right)=\mathrm{c}_{n}^{-1} \cdot \mathbf{c}_{m} .
$$

The peak spacings are found from the differences in the anion and neutral eigenvalues, and then the FCFs are multiplied by a Boltzmann factor for an assumed anion vibrational temperature.

Functions of $R$ are quite easy to calculate in the DVR basis. For example we find the rotational constant for a given vibrational level from

$$
B_{v}=\frac{\hbar^{2}}{2 \mu} \cdot \frac{1}{\left\langle v\left|R^{2}\right| v\right\rangle}=\frac{\hbar^{2}}{2 \mu} \cdot \frac{1}{\mathbf{c}_{v}^{-1} \cdot \mathbf{R} \cdot \mathbf{R} \cdot \mathbf{c}_{v}},
$$

where $\mathrm{R}$ is the diagonal matrix of DVR points $\operatorname{diag}\left(R_{i}\right)$.

\section{A2. How to implement DVR using Morse basis functions}

The implementation of the DVR method with a harmonic oscillator basis is straightforward, because the matrix elements of the position operator have a simple analytical form. ${ }^{3}$ The eigenfunctions of the Morse oscillator are more complicated, and 
no analytical form exists for $\left\langle\phi_{n}|X| \phi_{m}\right\rangle$. However, because the eigenfunctions of the Morse oscillator are a closer approximation to the eigenfunctions of the eigenfunctions of a diatomic van der Waals molecule, it is worthwhile investigating the problem of using the Morse eigenfunctions in the DVR scheme because faster convergence may be expected. This problem was considered, and solved, by Greenawalt and Dickinson in 1969. ${ }^{4}$ Here we describe their approach to the problem, and our implementation.

The Morse potential 5 is given by

$$
V(R)=D_{e}\left(1-\exp \left(-a\left(R-R_{e}\right)\right)\right)^{2}
$$

where $D_{e}$ is the well depth, $R_{e}$ is the bond length, and $a$ is a parameter that determines shape of the potential. Note that in this Appendix, we define the parameter $a$ in the Morse potential such that it has units of inverse length, whereas the $\beta$ parameter of the Morse portion of the MMSV potential [See Eq. (2.1)] is unitless. We also define the quantity

$$
t=\frac{\left(2 \mu D_{c}\right)^{1 / 2}}{\hbar a}
$$

where $\mu$ is the reduced mass. The number of eigenvalues of the Morse potential is the number of integers from zero to $t-\frac{1}{2}$. The eigenfunctions of the Morse potential are then given by

$$
\phi_{n}(y)=N_{n}^{-1 / 2} y^{i-n-\frac{1}{2}} \exp \left(-\frac{y}{2}\right) L_{2 t-n-1}^{2 t-2 n-1}(y)
$$

with

$$
y=2 t \exp \left(-a\left(R-R_{e}\right)\right)
$$

where the $L_{n}^{m}(y)$ are the Laguerre polynomials, and $N_{n}$ is a normalization factor given by 


$$
N_{n}=\frac{\Gamma(n+1) \Gamma(2 t-n) \Gamma(2 t-2 n-1)}{a \Gamma(2 t-n)}
$$

Because no analytical form exists for $\left\langle\phi_{n}|X| \phi_{m}\right\rangle$ in the Morse basis, we use a generalization of the HEG transformation method, ${ }^{6}$ as follows. First we choose a function, $u(R)$, such that the elements $\left\langle\phi_{n}|u(R)| \phi_{m}\right\rangle$ constitute a tridiagonal matrix. Then we diagonalize the matrix $\mathbf{u}$ to find the transformation matrix $\boldsymbol{T}$ such that $T^{-1} \cdot \mathbf{u} \cdot \mathbf{T}=\operatorname{diag}\left(\lambda_{i}\right)$. The DVR points are then found by inverting the function $\lambda_{1}=u\left(R_{t}\right)$, and the rest of the calculation proceeds as above from Eq. (A8). Greenawalt and Dickenson ${ }^{4}$ showed that for the Morse basis, the function

$$
u(R)=\frac{1}{y}=\frac{1}{2 t \exp \left(-a\left(R-R_{e}\right)\right)}
$$

gives a tridiagonal matrix, with elements

$$
\left\langle\phi_{n}|u(R)| \phi_{m}\right\rangle=\delta_{n, m} \frac{t}{2(t-n)(t-n-1)}+\delta_{n, m-1}\left(\frac{N_{m}}{N_{n}}\right)^{1 / 2}, \quad n \leq m
$$

Note that it is not necessary to calculate the Laguerre polynomials in Eq. (A14) in order to find the matrix elements from Eq. (A18). Also note that the ratio of the two normalization constants in (A18) can be simplified so that one need not evaluate the gamma functions in Eq. (A16). Therefore computation of the matrix elements of $\mathrm{X}$ in the Morse basis is not significantly more time-consuming than in the harmonic oscillator basis. 


\section{A3. Choosing the parameters for the Morse basis}

In this section we describe general guidelines for choosing the optimal Morse basis set parameters that we have found effective in our calculations with the $\operatorname{RgX}$ anion and neutral MMSV potentials. Note that these are only guidelines, and some trial and error may be necessary to find the best basis set parameters for other potential forms.

First, we set $R_{e}$ of the Morse potential equal to the bond length of the smaller of the neutral or anion MMSV potentials. Next we choose the desired number of basis functions, $N$. Twenty-five to 75 basis functions were found to be sufficient for most purposes. We then choose the parameter $t$ [which is roughly equal to the total number of bound vibrational states of the Morse potential; see Eq. (A13)] so that the shape of the Morse eigenfunctions include functions with shapes similar to those expected for the range of vibrational levels of the model potential we wish to calculate. To do this, we set

$$
\frac{t}{N}=\frac{\text { Estimated total number of MMSV eigenvalues }}{\text { Number of MMSV eigenvalues to calculate }} .
$$

The total number of MMSV eigenvalues is estimated by using $\left(\beta_{1}+\beta_{2}\right) /\left(2 R_{m}\right)$ in place of $a$ in Eq. (A13)--where $\beta_{1}, \beta_{2}$, and $R_{m}$ are the MMSV parameters as defined in Eq. (2.1.)-- along with the well depth of the MMSV potential, and $\mu$ calculated from $R_{m}$ of the MMSV potential. If one chooses $t$ by trial and error instead, as is sometimes necessary, one must remember that the quantity $t-\frac{1}{2}$ must be larger than the number of basis functions, $N$. Next one chooses the Morse potential force constant

$$
k=\left.\frac{d^{2} V(R)}{d R^{2}}\right|_{R_{e}}=2 a D_{c} .
$$


Finally, using these values for $t$ and $k$, one calculates $a$ and $D_{e}$ from Eqs. (A13) and (A20).

As written, the program automatically computes the Morse basis set parameters based on the desired number of eigenvalues to calculate and the number of basis functions used according to the above considerations if one uses the MMSV potential form. If one uses a different potential form, one must provide the Morse parameters $R_{m}$, $a$, and $D_{e}$ in the input file to the program.

\section{A4. Comparison of Morse and harmonic oscillator basis set convergence for a} highly anharmonic potential

In this section we test the performance of the Morse DVR program by comparing its convergence behavior with a harmonic oscillator based DVR program for the MMSV potential for the $I \frac{3}{2}$ state of KrI. This potential has a well depth of $16.7 \mathrm{meV}$ and supports 18 bound vibrational states. See Ref. 7 for further details of the KrI potential. The program was tested by calculating the eigenvalues using both the Morse and harmonic basis sets, increasing the basis set size, $N$, by 5 with each calculation. The calculation was performed wit The results for $v=1,5,10$ and 15 are shown in Figure Al on the following page. The ordinate of each graph is the relative difference between the eigenvalue calculated with a given number of basis functions, and that calculated with 5 fewer basis functions. 

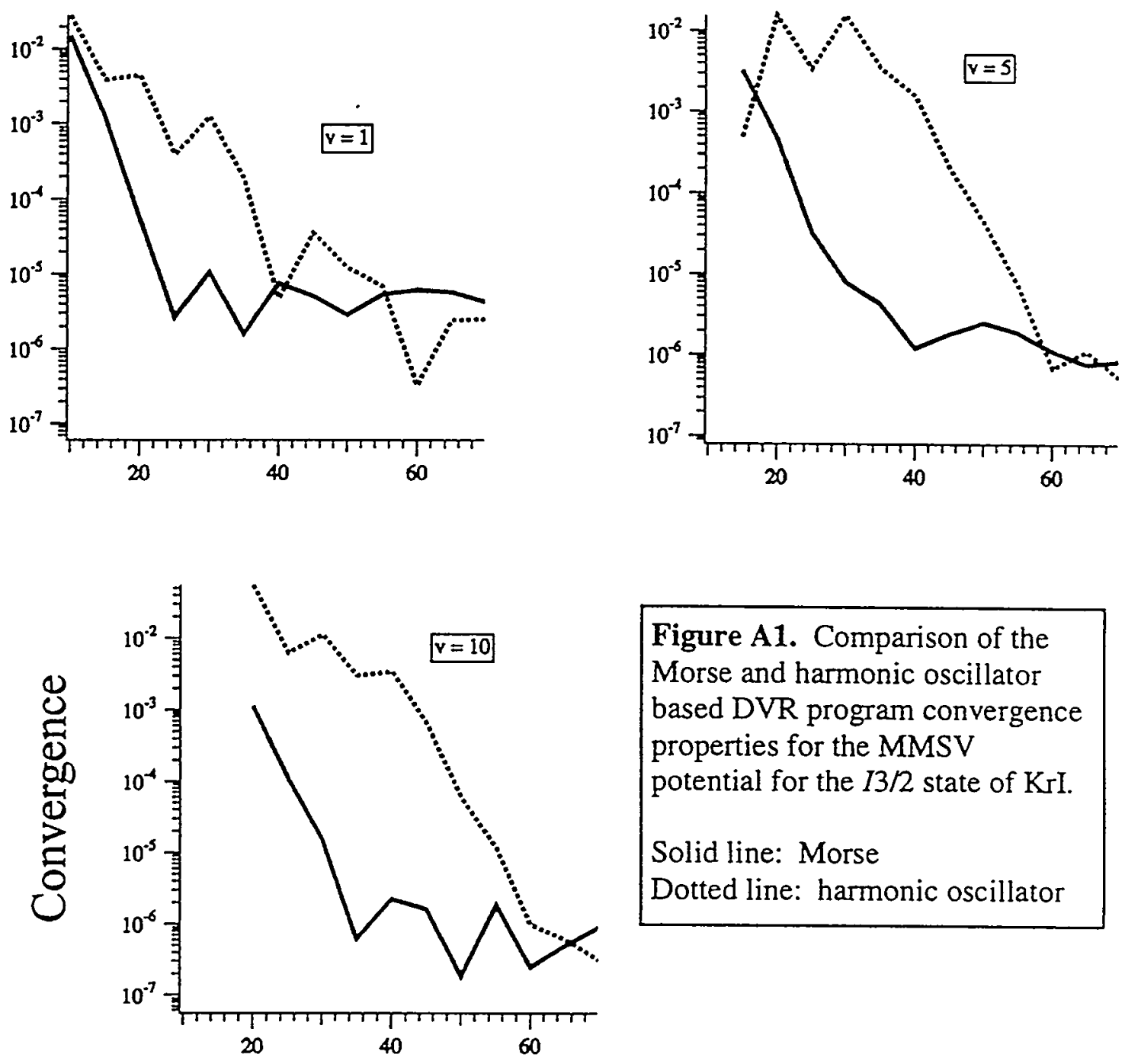

Figure A1. Comparison of the Morse and harmonic oscillator based DVR program convergence properties for the MMSV potential for the $13 / 2$ state of $\mathrm{KrI}$. Solid line: Morse Dotted line: harmonic oscillator

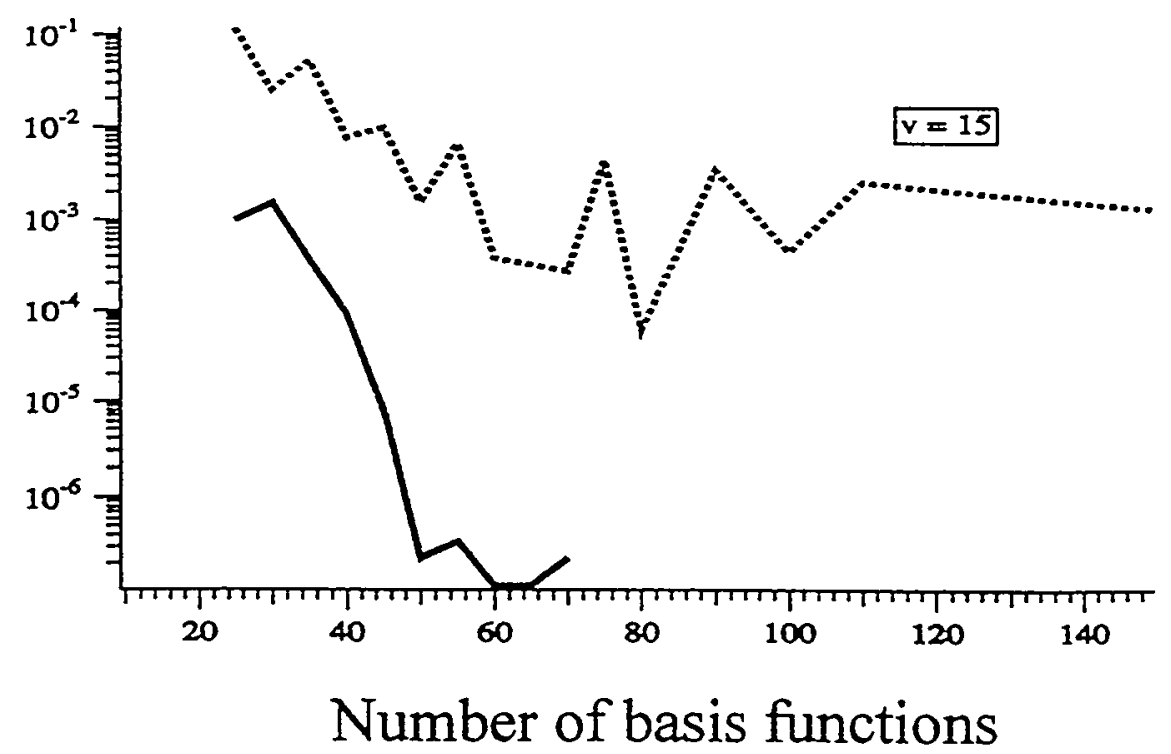


This number gives an indication of the convergence of the program. Also, the exponent of the ordinate is an approximation to the number of significant figures of the calculated eigenvalues. We see that for $v=1,5$ and 10 , the Morse program converges with 25-35 basis functions, whereas, the harmonic oscillator program requires $40-60$ basis functions to converge. In the case of $v=15$, the Morse program converges with 50 basis functions, but the harmonic oscillator program does not converge with up to 150 basis functions, demonstrating the inadequacy of the harmonic oscillator model for treating high vibrational levels of anharmonic oscillators.

In general, then, it is found that the Morse program requires nearly a factor of 2 fewer basis functions than the harmonic oscillator program for satisfactory performance with anharmonic potentials. This is made more significant when one considers that the speed of the "rate limiting step" in the calculation, solution for the eigenvalues and eigenvectors of Eq. (A6), is proportional to the third power of the number of basis functions. ${ }^{8}$ Therefore the use of the Morse basis offers an approximately eight fold increase in computing speed over the harmonic oscillator basis.

\section{A5. Documentation of the Morse DVR program "idvr": example of the $X \frac{1}{2}$ state of} XeBr

In this section we demonstrate the how to use the interactive Morse DVR program "idvr" for fitting diatomic ZEKE spectra. 


\section{A5.1. The input files}

A sample input file for fitting the $\mathrm{XeBr} X \frac{1}{2}$ state spectrum is shown below. Note that the line numbers in boldface are for reference only and are not included in the actual input file. The file is named "xebr_x_dvrin."

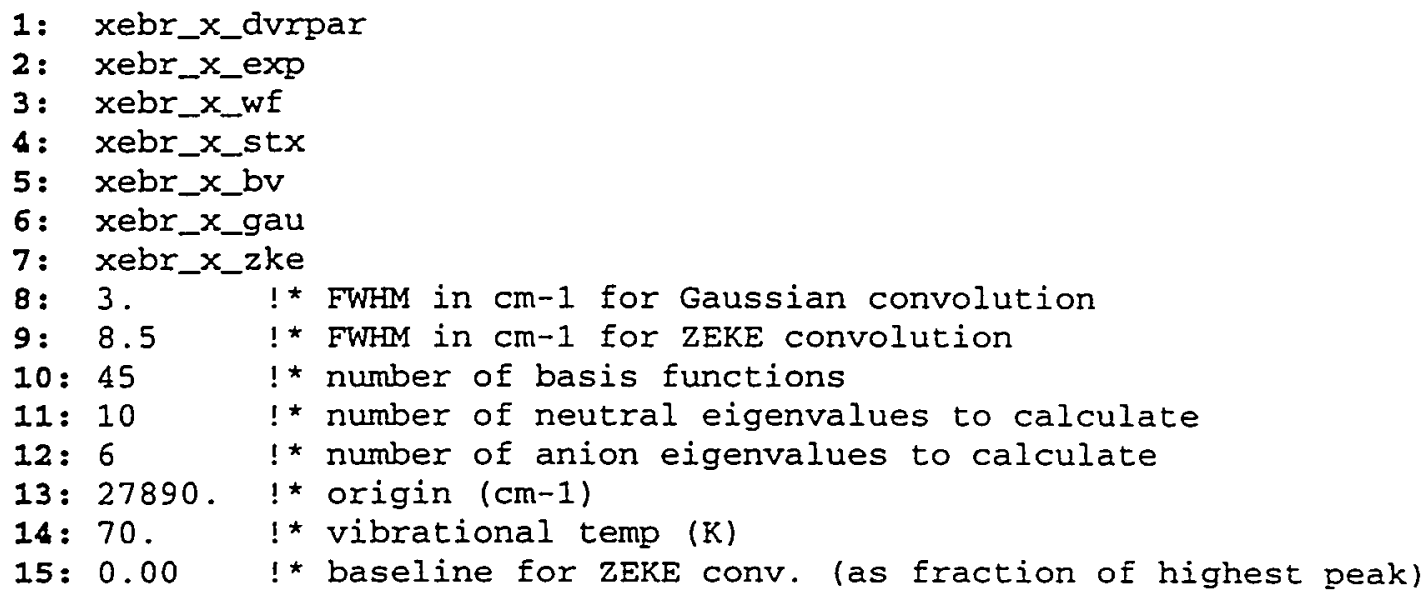

The first 7 lines are the names of files used by the program. Lines 1 and 2 are input files which are not modified by the program. Line 1 is the name of a file which contains the potential and basis set parameters. (See below for a sample parameter file.) Line 2 is the name of the file containing the experimental spectrum that is being fit. This file must be in wavenumber format. It is used in order to calculate the chi-square goodness of fit function for comparison with the model spectrum. Lines 3-7 list the names of the output files. Line 3 names the file to save the DVR points and eigenvectors for the anion and neutral. The eigenvectors should be examined to ensure proper convergence: for each vibrational level, the eigenvector should be close to 0 at the smallest and largest DVR points. If this is not the case, it is necessary to either adjust the basis set parameters or use a larger number of basis functions. Line 4 is the name of the file to save the vibrational stick spectrum in wavenumber format. Line 5 is the name of the file to save 
the rotational constants. The rotational constants are listed in this file in the same order as the vibrational sticks, and correspond to the rotational constants for the neutral vibrational level corresponding to each vibrational stick. Line $\mathbf{6}$ is the output file for a spectrum consisting of the vibrational sticks convoluted a Gaussian function with the FWHM (full width at half maximum) given in line 8. Line 7 is the output file for the stick spectrum convoluted with the asymmetric ZEKE line shape, with the FWHM given in line 9. See Eq. (4.11) of Chapter 4 for the form of the ZEKE line shape. Both of the convoluted spectra are output in wavenumber format. Line 10 is the number of basis functions to use in the calculation. Lines 11 and 12, respectively, are the number of neutral and anion eigenvalues one wishes to calculate. Line 13 is the position of the vibrational origin, in wavenumbers. Line 14 is the anion vibrational temperature. Line 15 is a baseline, expressed as a fraction of the maximum peak, which may be added to the spectrum convoluted with the ZEKE line shape.

The parameter file "xebr_x_dvrpar" is listed below. Again, the boldface line numbers to not appear in the actual file.

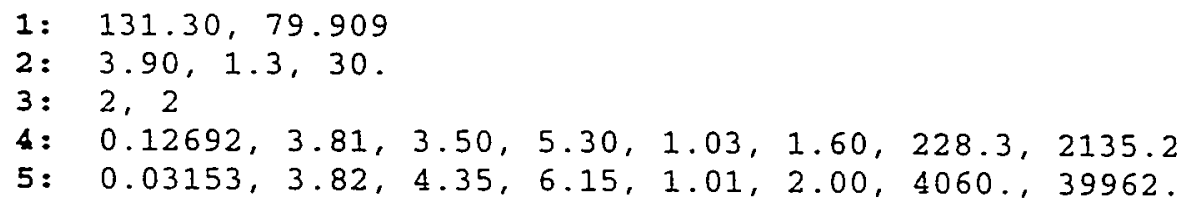

Line 1 lists the atomic masses in amu. Line 2 lists the Morse basis parameters $R_{e}, a$, and t. If one uses the MMSV potential form, these parameters are not used by the program, but are calculated directly from the MMSV potential parameters. See Section A3, above, for a discussion of the choice of the basis parameters. Line 3 contains integers which specify which potential form to use for the neutral and anion. See the source code listing 
of the subroutine "poten" in Section A6.6, below, for a listing of the available potential forms, and their associated parameters. Lines 4 and $\mathbf{5}$ list the potential parameters of the for the neutral and anion potentials, respectively. In this example the potentials are of the MMSV form. The order and units of the MMSV parameters in each line are:

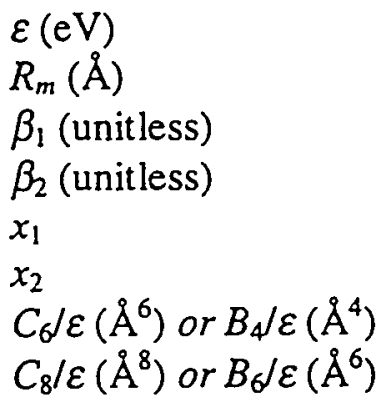

Note that the values of the dispersion coefficients input to the program are divided by the well depth, so the numbers shown in the sample input file differ from the parameters given in Table 3.4 of Chapter 4. Note also that because of this quirk in the program one must change the dispersion inputs whenever one changes the well depth. Refer to the source code listing in Section A6.6 for the order and units of the parameters for other potential forms.

\section{A5.2. Running the program}

An example of running the Morse DVR program "idvr" using the input files shown in the previous section is given below. Because this is an interactive program the input and output files names are input in response to prompts from the program rather than using pipes in the customary Unix style. The user input is shown in boldface.

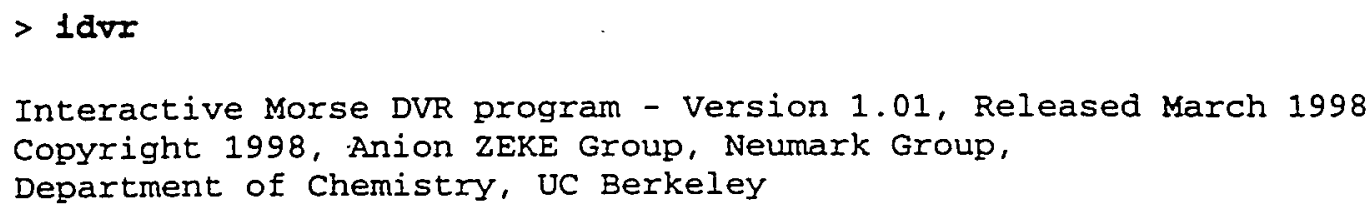




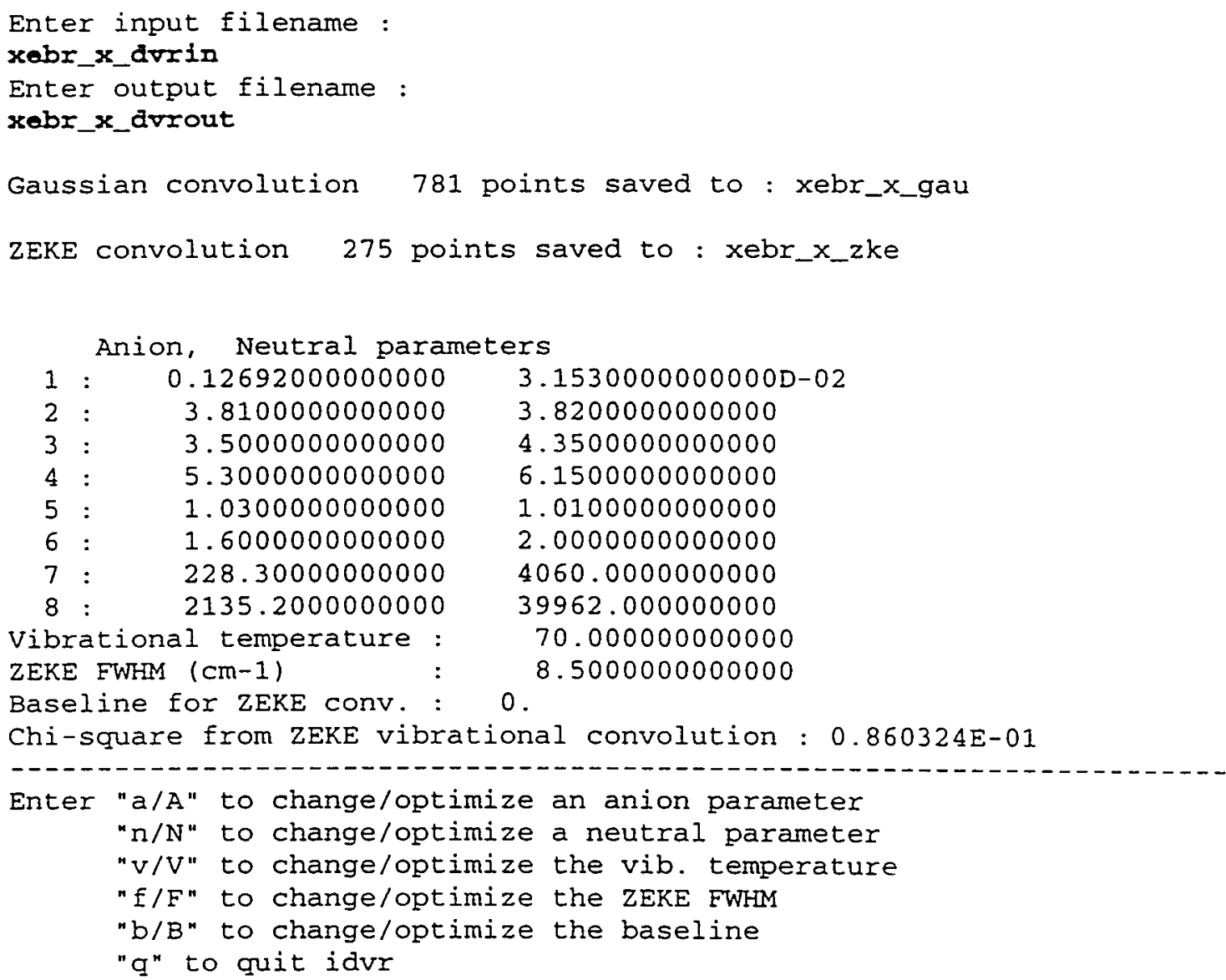

At this point, the user may examine the convoluted spectra, and the output file "xebr_x_dvrout," which lists the eigenvalues and Franck-Condon factors. If the fit is not satisfactory, one can modify the parameters as often as desired before exiting the program. Note that the modified parameters are not saved in the parameter file.

\section{A5.3. Outline of the program}

In the table below we list the subroutines and functions that are used by the "idvr" interactive Morse DVR fitting program sorted according to which file they reside in. 
Table A1. Subroutines and functions used by the "idvr" program.

\begin{tabular}{|c|c|c|}
\hline $\begin{array}{l}\text { Source } \\
\text { File }\end{array}$ & $\begin{array}{l}\text { Subroutine } \\
\text { or Function }\end{array}$ & Description \\
\hline \multirow[t]{10}{*}{ idvr.f } & idvr & main program \\
\hline & umat & sets up the $\mathbf{u}$ matrix using Eq. (A18) \\
\hline & rcalc & calculates the DVR points $R_{i}$ \\
\hline & initpars & reads in the potential parameters \\
\hline & diffpot & calculates the difference potential matrix [Eq. (A8)] \\
\hline & morseig & calculates the matrix $\mathrm{E}_{\mathrm{DVR}}^{0}$ from Eq. (A9) \\
\hline & hcalc & sets up the total Hamiltonian matrix $\mathbf{H}$ \\
\hline & setbasis & $\begin{array}{l}\text { chooses the Morse basis set parameters according to the } \\
\text { considerations of Section A3 }\end{array}$ \\
\hline & calcfcf & calculates the Franck-Condon factors \\
\hline & bvcalc & $\begin{array}{l}\text { calculates rotational constants for individual vibrational } \\
\text { levels from Eq. (A11) }\end{array}$ \\
\hline rsb.f & $\mathrm{rsb}$ & $\begin{array}{l}\text { EISPACK subroutine for computing the eigenvalues and } \\
\text { eigenvectors of a real (double-precision) symmetric band } \\
\text { matrix }\end{array}$ \\
\hline rs.f & rs & $\begin{array}{l}\text { EISPACK subroutine for computing the eigenvalues and } \\
\text { eigenvectors of a real (double-precision) symmetric } \\
\text { matrix }\end{array}$ \\
\hline matrix.f & showarr4 & subroutine to print out a matrix \\
\hline \multirow[t]{7}{*}{ poten2.f } & poten & calculates the potential for a specified potential form \\
\hline & $\mathrm{ms} 4$ & Maitland-Smith $n-4$ anion potential function \\
\hline & anmmsv & anion MMSV potential function \\
\hline & morse & Morse potential function \\
\hline & bucky & Buckingham exp-n potential function \\
\hline & anmmsvus & unscaled anion MMSV potential function \\
\hline & $\mathrm{ms} 6$ & Maitland-Smith $n-6$ neutral potential function \\
\hline
\end{tabular}




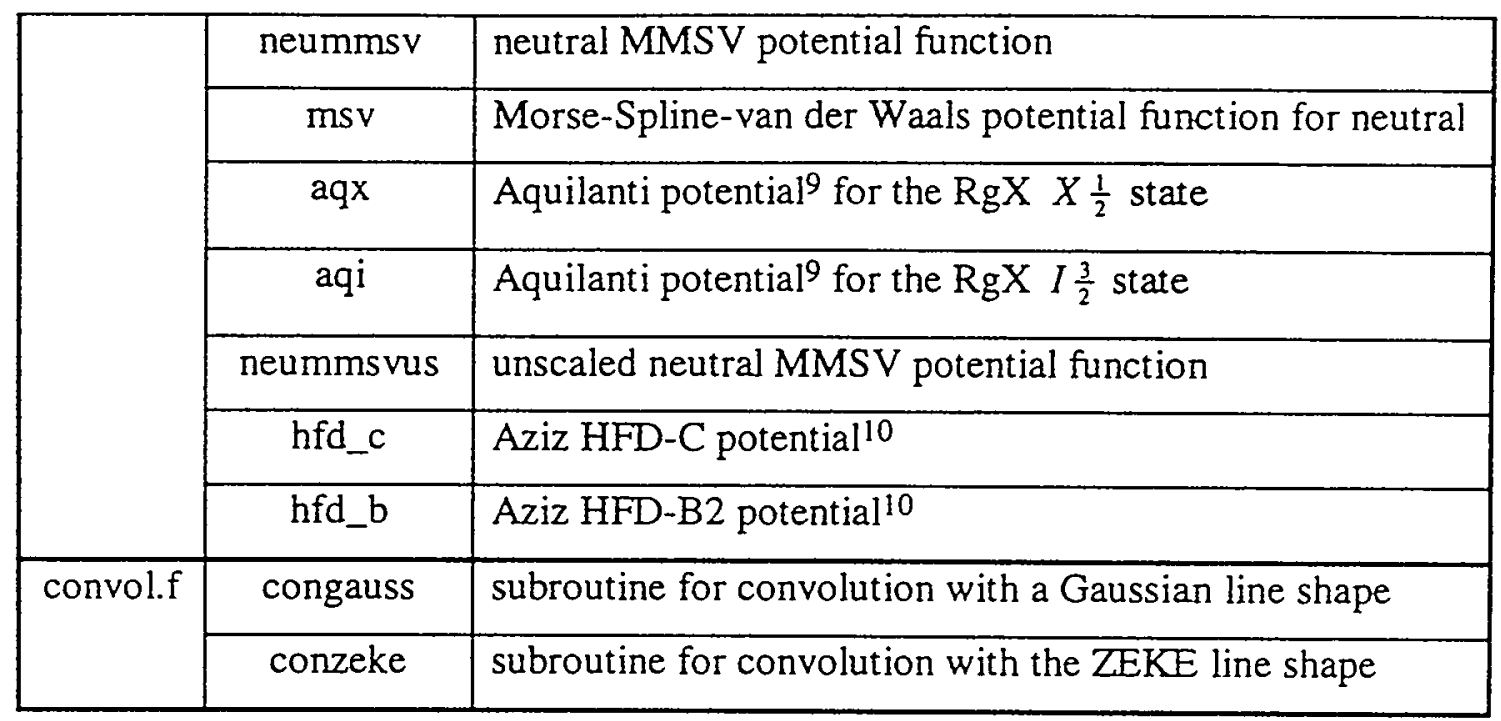

\section{A6. Source code for the Morse DVR program "idvr.f"}

In this section we list the complete source code of the "idvr" program and relevant subroutines and functions. The "rsb" and "rs" subroutines are the standard EISPACK functions for matrix diagonalization; ${ }^{11}$ hence the only the headers are listed for these.

\section{A6.1. File "makeidvr"}

The makefile used for recompiling the Morse DVR program is shown below. To recompile the program one enters "make - $f$ makeidvr".

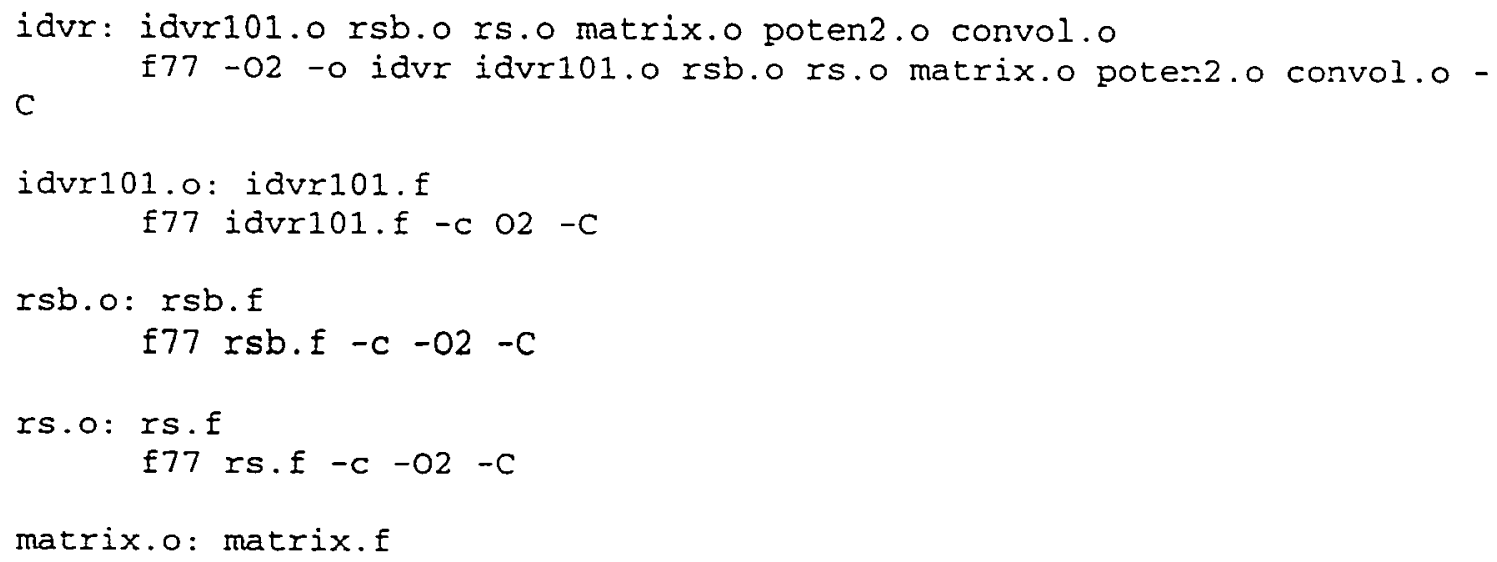


f77 matrix.f $-\mathrm{c}-\mathrm{O} 2-\mathrm{c}$

poten2.o: poten2. 王

f77 poten2. $f-c-02-C$

convol.o: convol.

f77 convol. $\mathrm{f}-\mathrm{C}-\mathrm{O} 2-\mathrm{C}$

\section{A6.2. File "idvr101.f"}

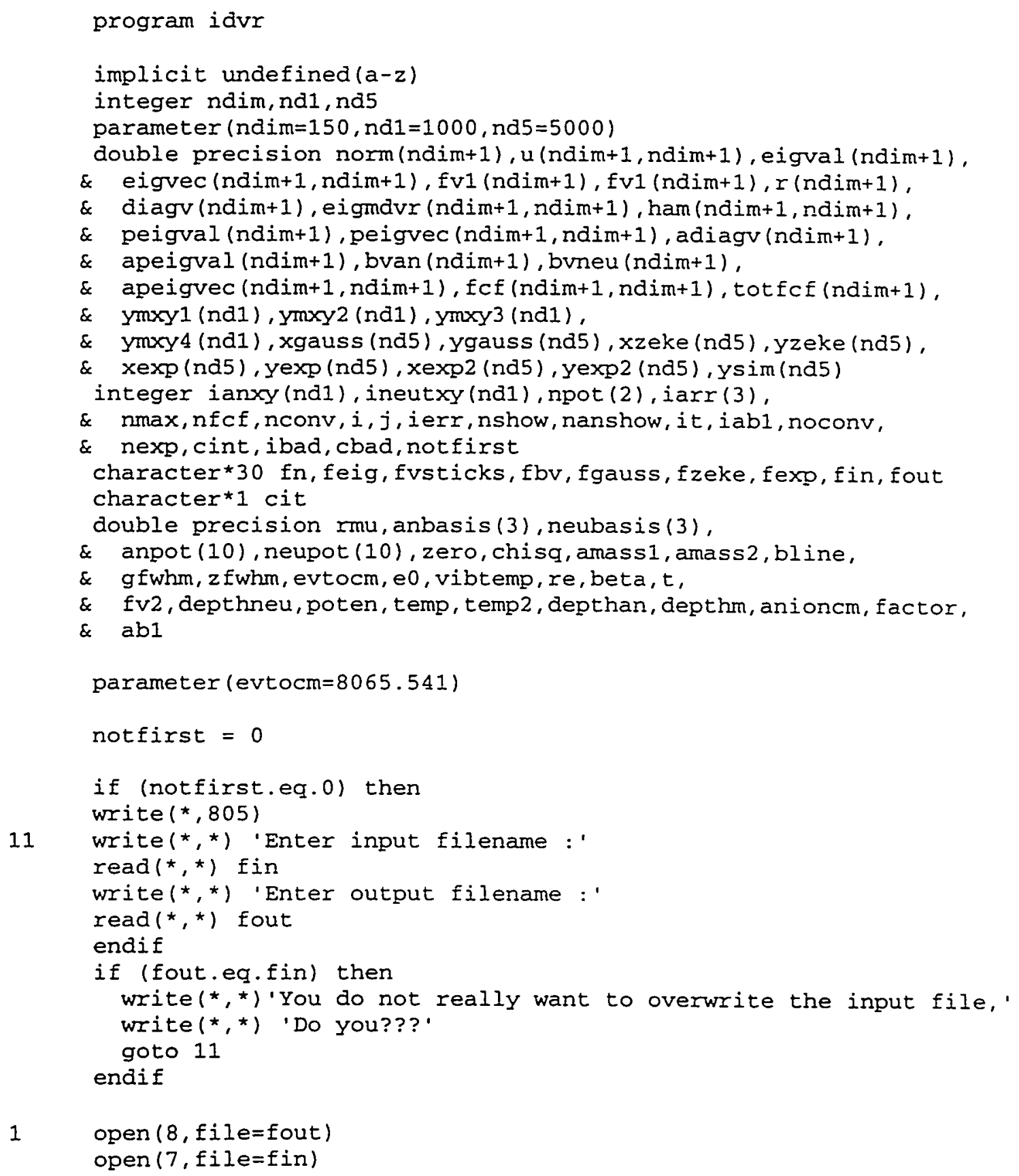




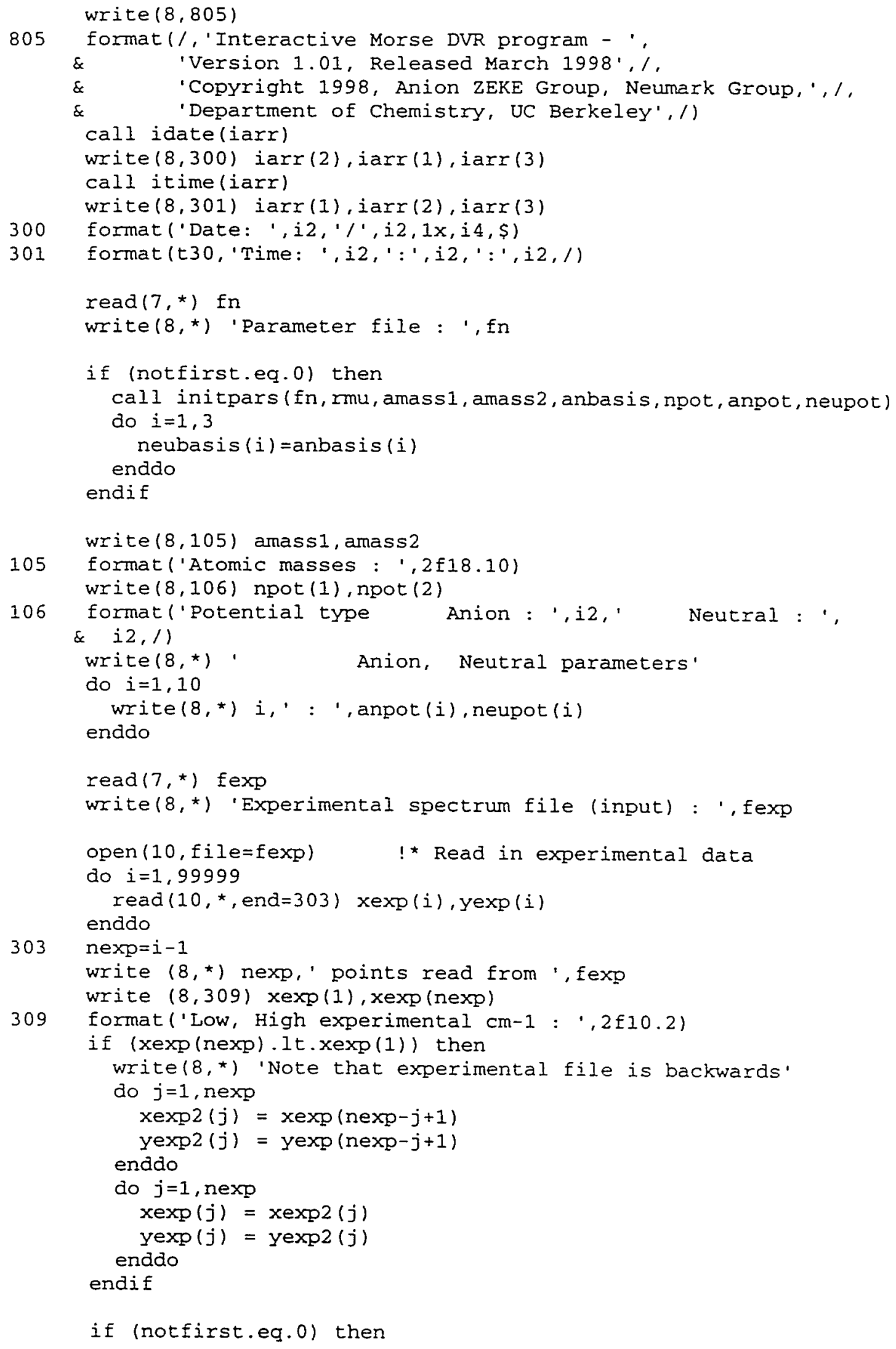




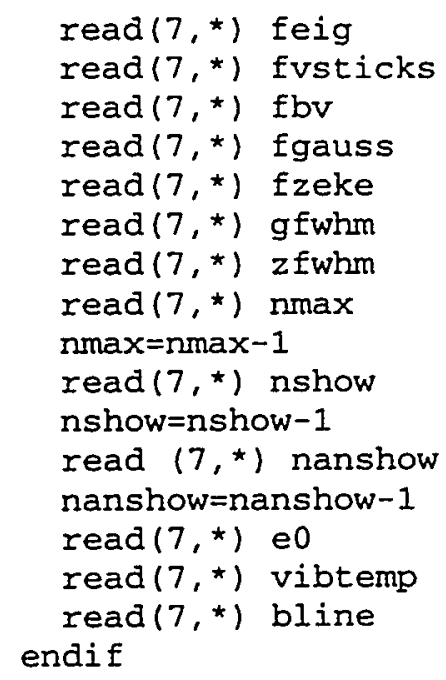


format (' Depth $=$ ', f10.6,' eV')

write $(8, \star)$

call umat (nmax, $t$, ndim, norm, $u$ )

call rsb(ndim+1, nmax+1, 2, u, eigval, 1, eigvec, fv1, iv2, ierr)

if (ierr.ne.0) then

write $(*, *) \cdot * \star *$ ierr=', ierr,' from $r s b '$

endif

call rcalc(nmax, ndim, re, $t$, beta, eigval, r)

call diffpot (ndim, nmax, rmu, neubasis, 2 , npot (2), neupot, $\&$ $r$, diagv, depthneu)

open (2, file=feig)

write $(2, *)$

write $(2, *)$ 'DVR points (Angsroms, cm-1):'

write $(2, *)$

write $(2,345)$

345 format ( $2 x$, 'r(i)', $5 x$, 'anion', $4 x$, 'neutral')

do $400 i=0$, nmax

write $(2,350) \simeq(i+1),(\operatorname{poten}(1, \operatorname{npot}(1), \operatorname{anpot}, r(i+1))+$

$\& \quad$ anpot (1))*evtocm, (poten $(2, \operatorname{npot}(2), \operatorname{neupot}, r(i+1))+$

$\&$ depthneu) ${ }^{\star}$ evtocm

400

continue

350 format ( $\mp 8.4,1 x, \mp 8.2,1 x, f 8.2$ )

call morseig (ndim, nmax, rmu, neubasis, eigvec, eigmdvr)

call hcalc (ndim, nmax, eigmdvr, diagv, ham)

call rs (ndim+1, nmax+1, ham, peigval, 1, peigvec, fv1, fv2, ierr)

call bvcalc (ndim, nmax, nshow, rmu, $r$, peigvec, bvneu)

write $(8, *)$ ' Neutral vibrational eigenvalues and rotational'

write $(8, *)$ ' constants (in $\mathrm{cm}-1$ ) : '

write $(8, \star)$ '

write $(8,245)$

245 format (15x, 'Total', 6x, 'Spacing', 6x,'B(v)')

write $(8, *)$ ' '

temp $2=0$.

do $i=0$, nshow

zero $=($ peigval $(1)) \star 8065.541$

temp $=($ peigval $(i+1)) \star 8065.541$

write $(8,250) i$, temp-zero, temp-temp2, bvneu $(i+1)$

temp2 = temp

endio

format $(1 x, ' v=', i 2,4 x, f 9.2,4 x, £ 8.2,4 x, £ 9.5)$

write $(2, *)$

write $(2, *)$ 'Neutral eigenfunctions:'

call showarr4 (2, ndim+1, peigvec, nmax +1 , nshow +1 )

$\star$

* Anion calculation--using same basis

call diffpot (ndim, nmax, rmu, neubasis, 1, npot (1), anpot, 


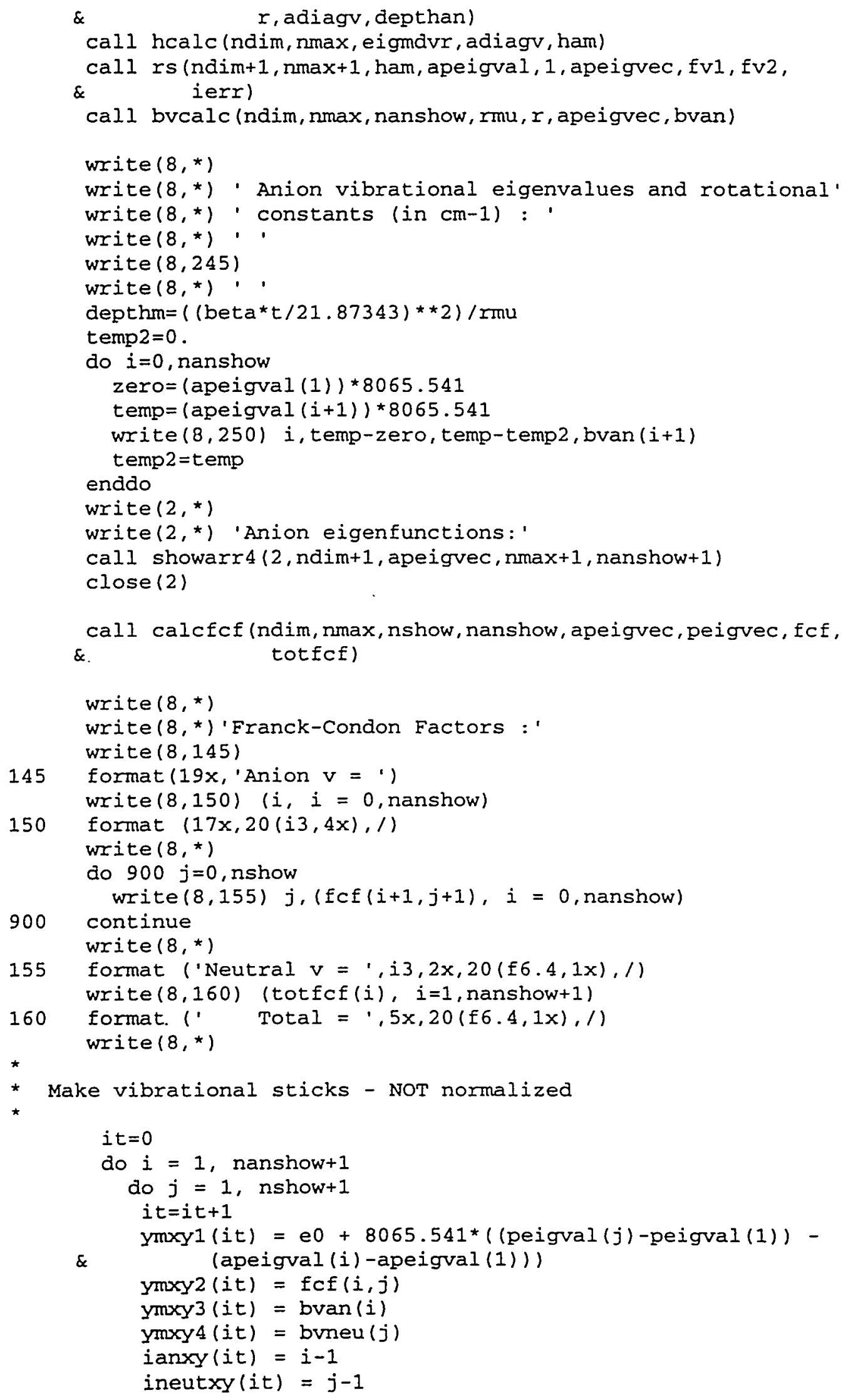




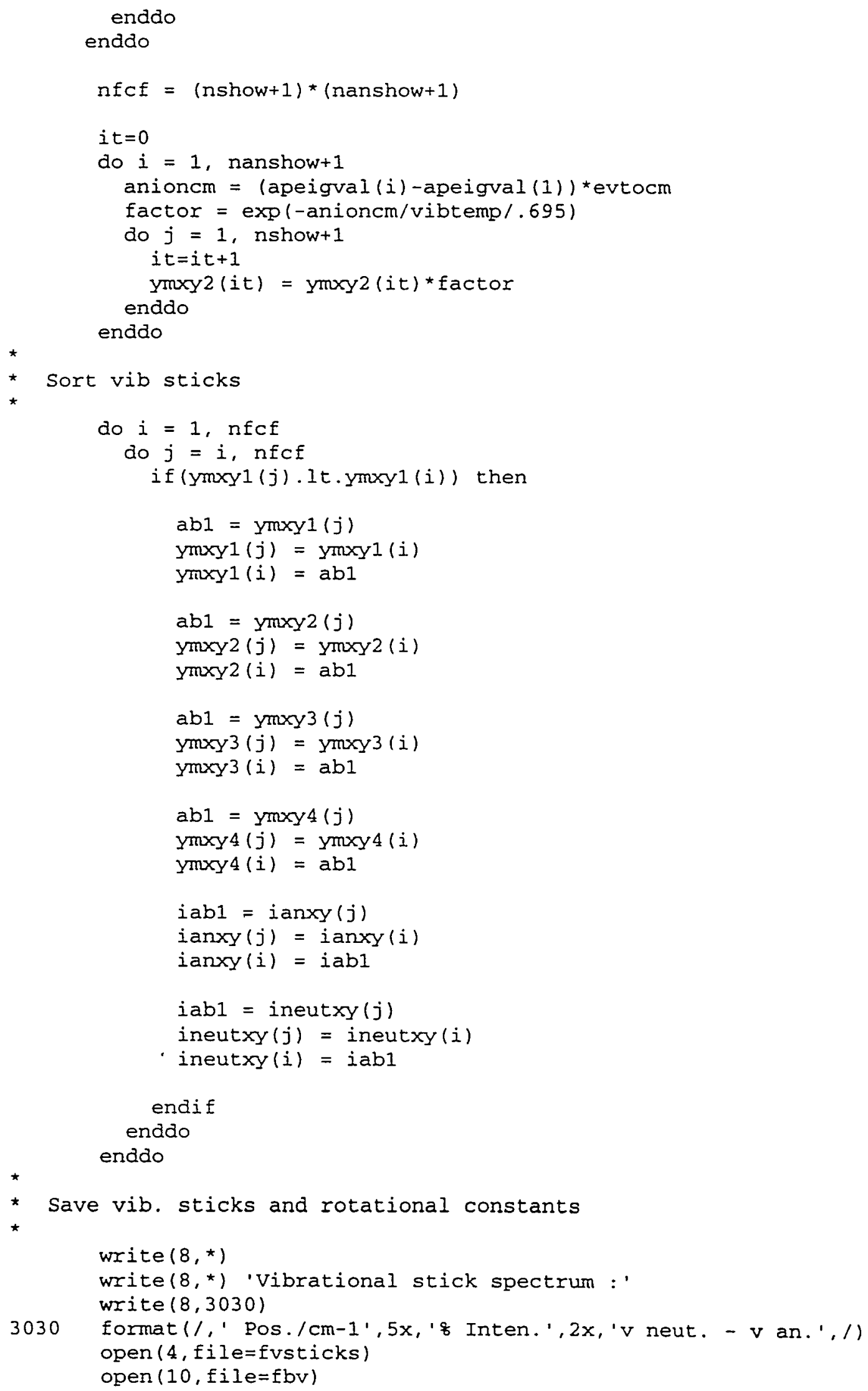




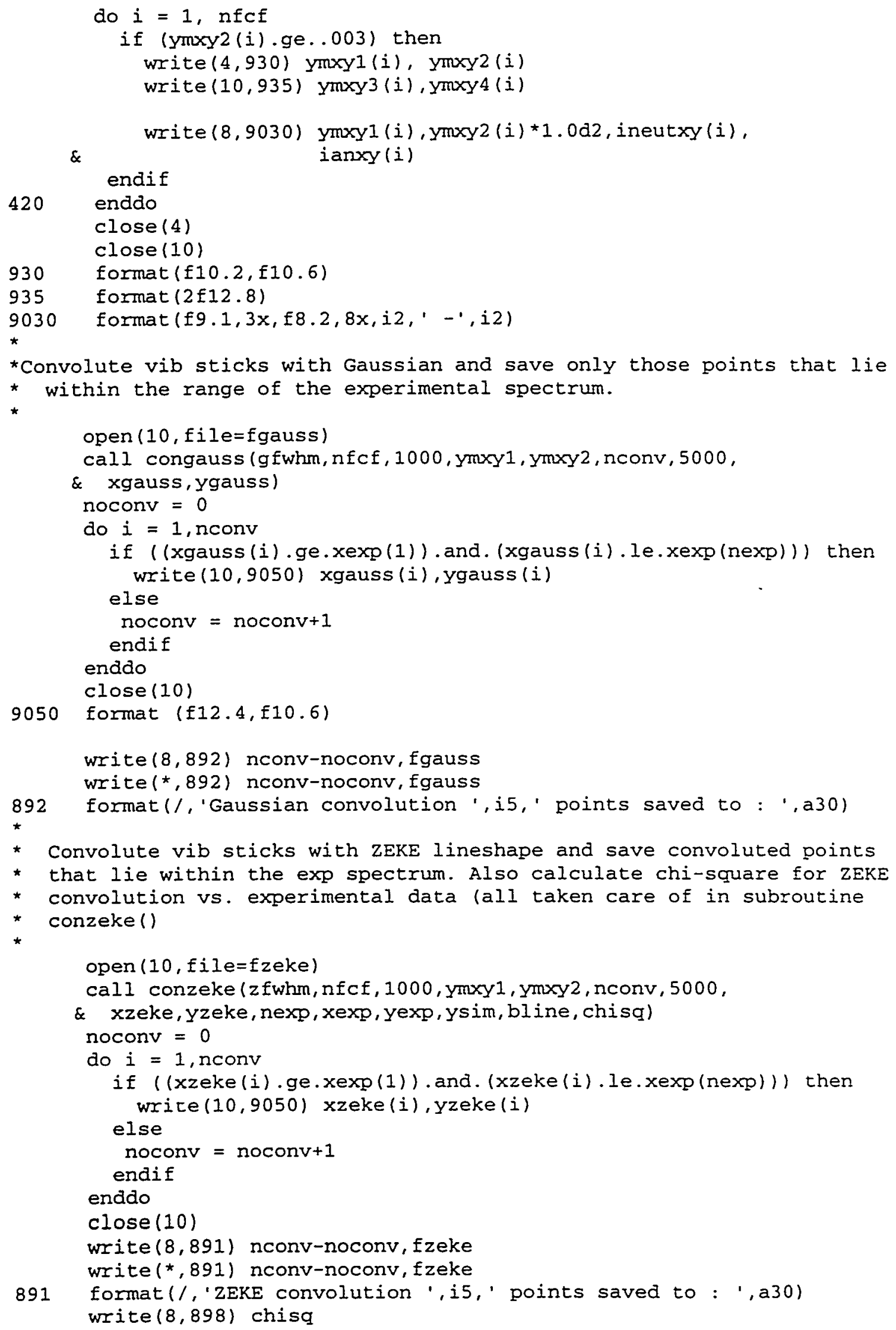




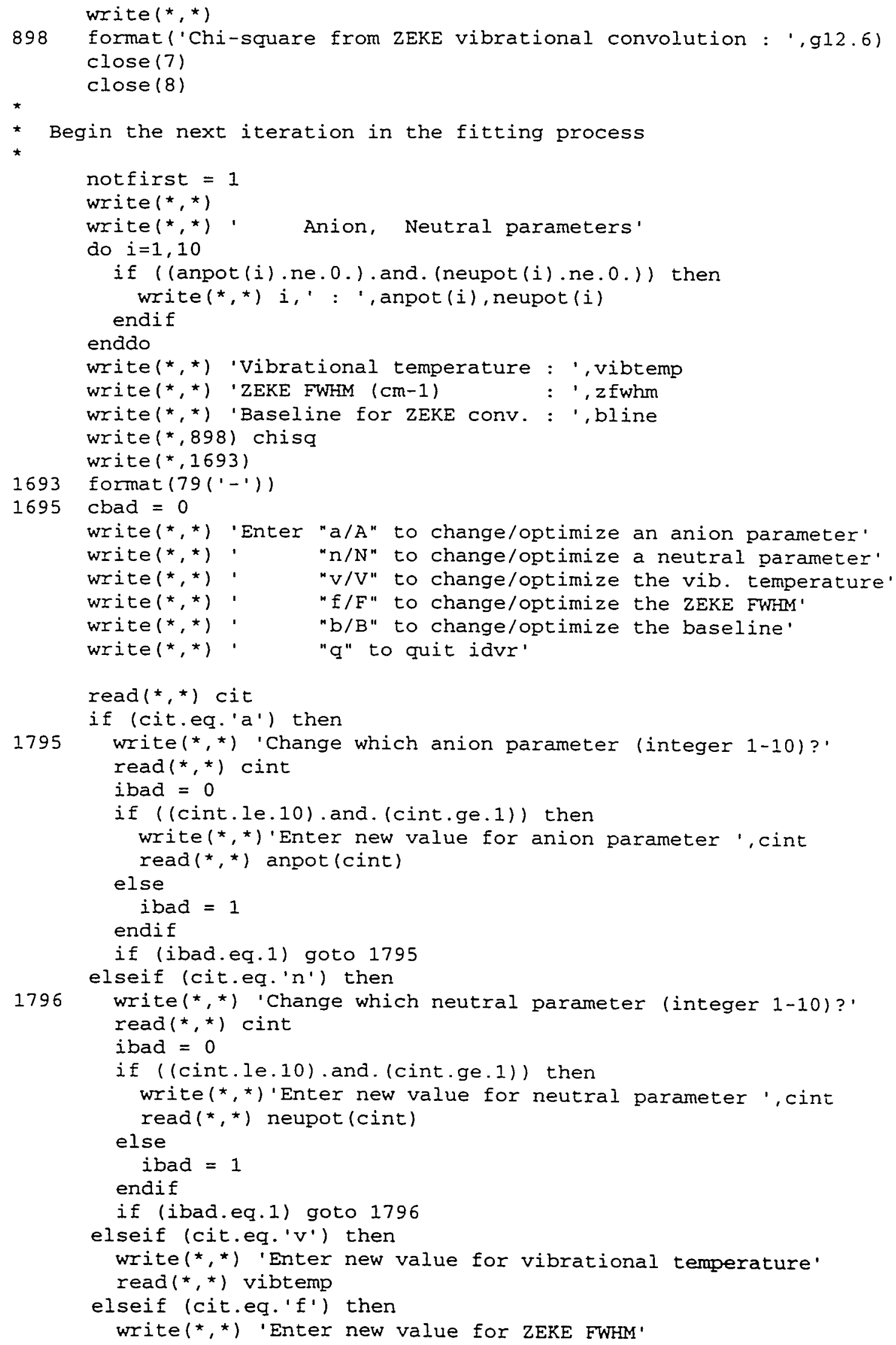




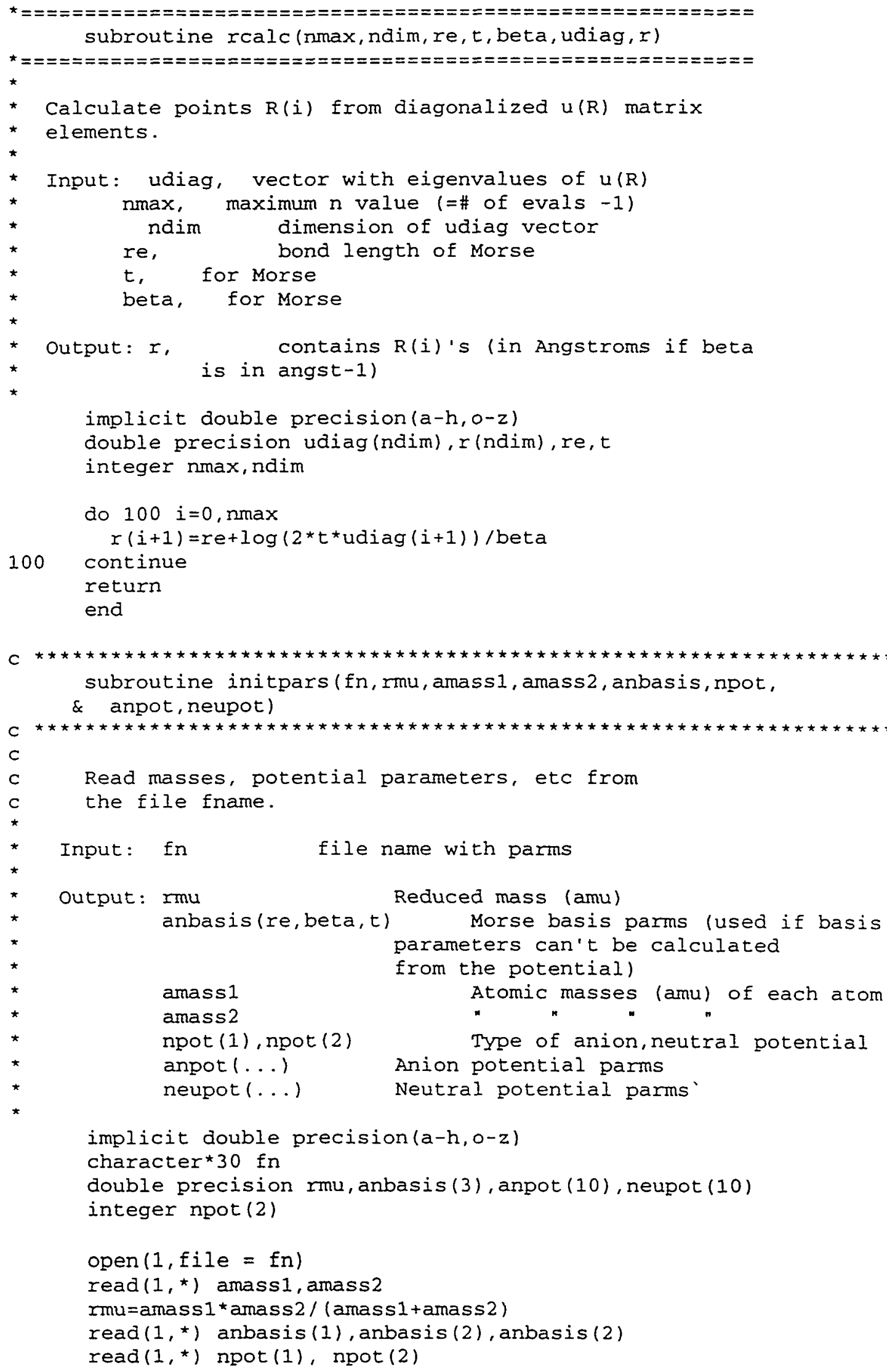

Read masses, potential parameters, etc from

the file fname. 


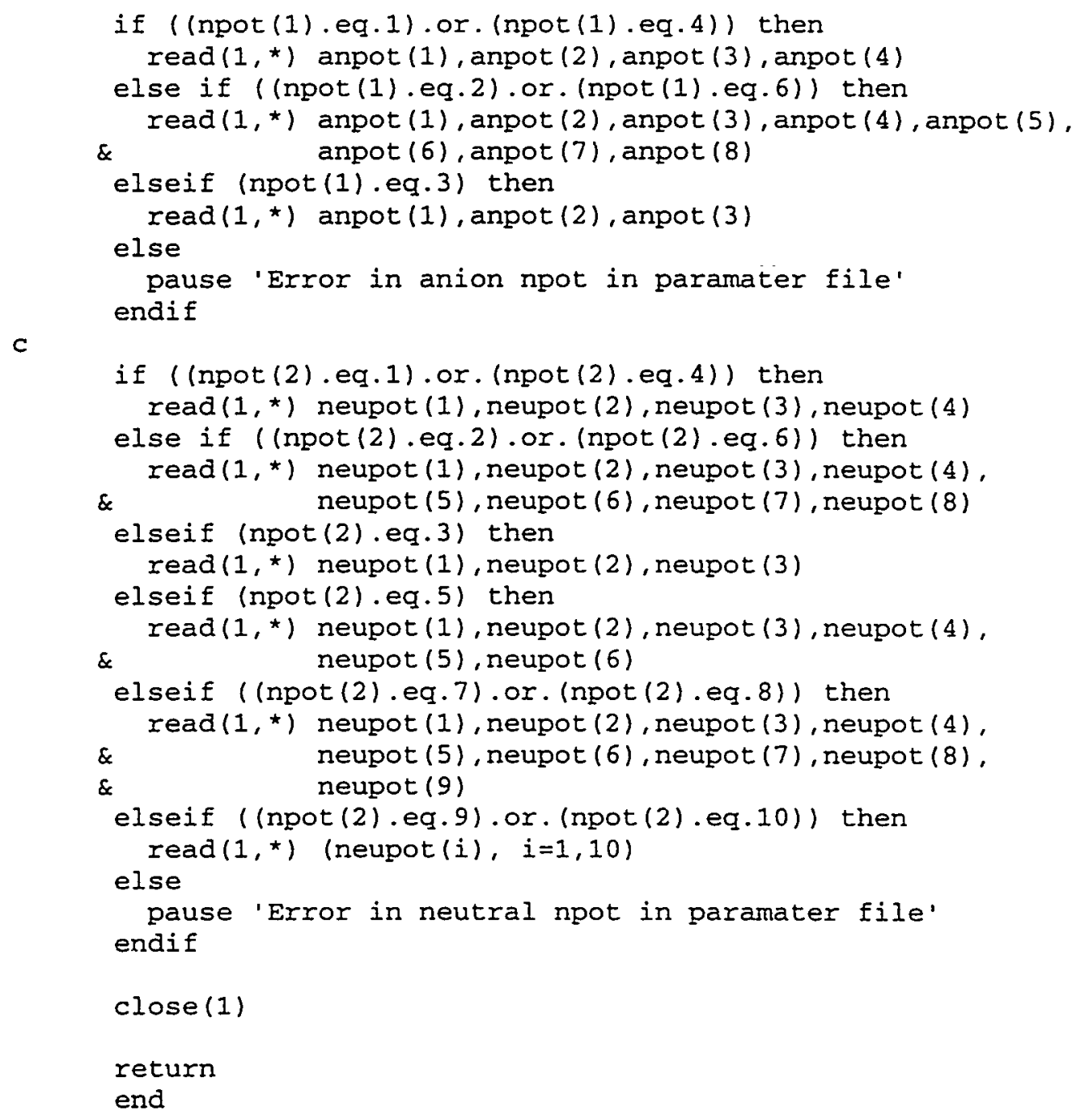




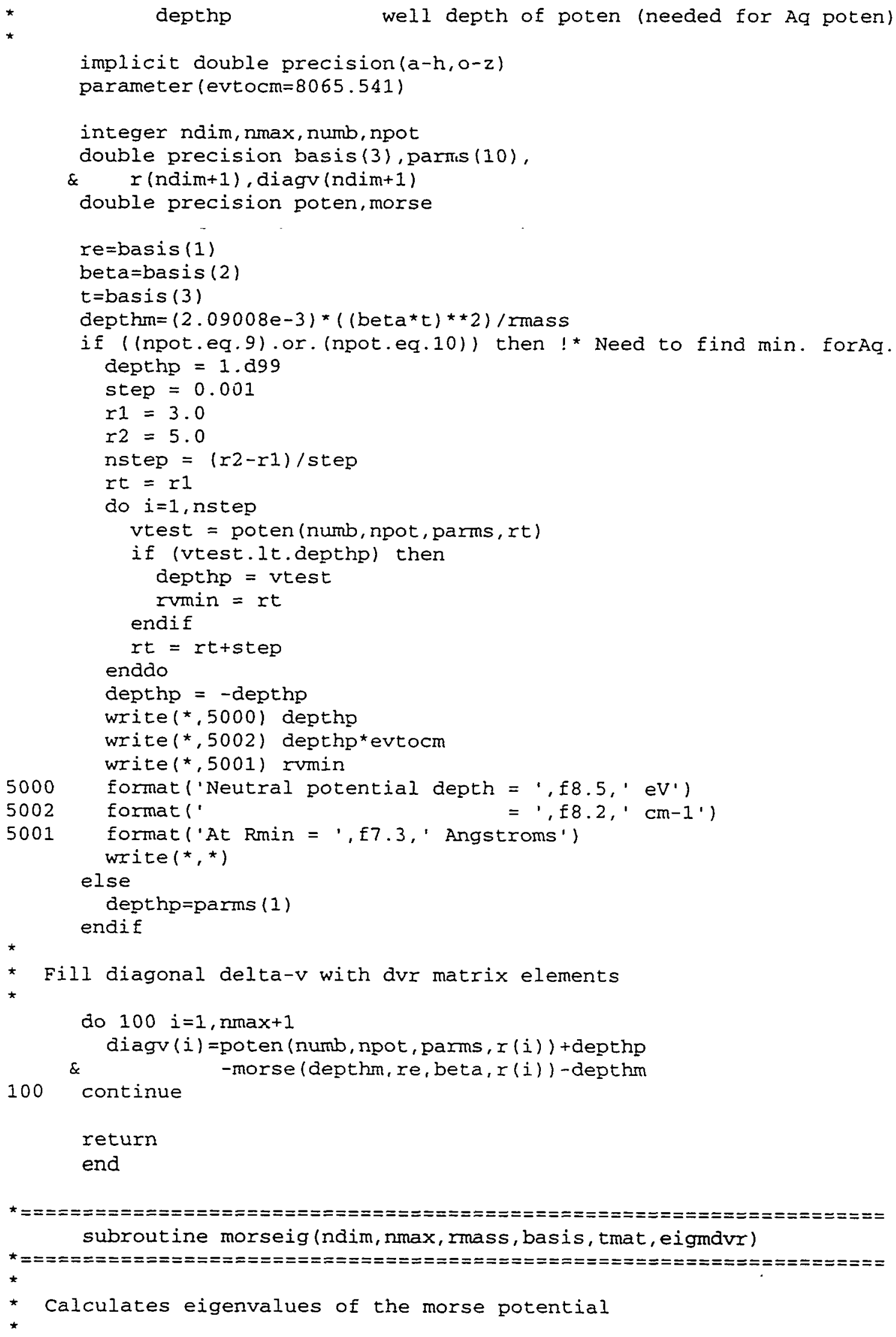




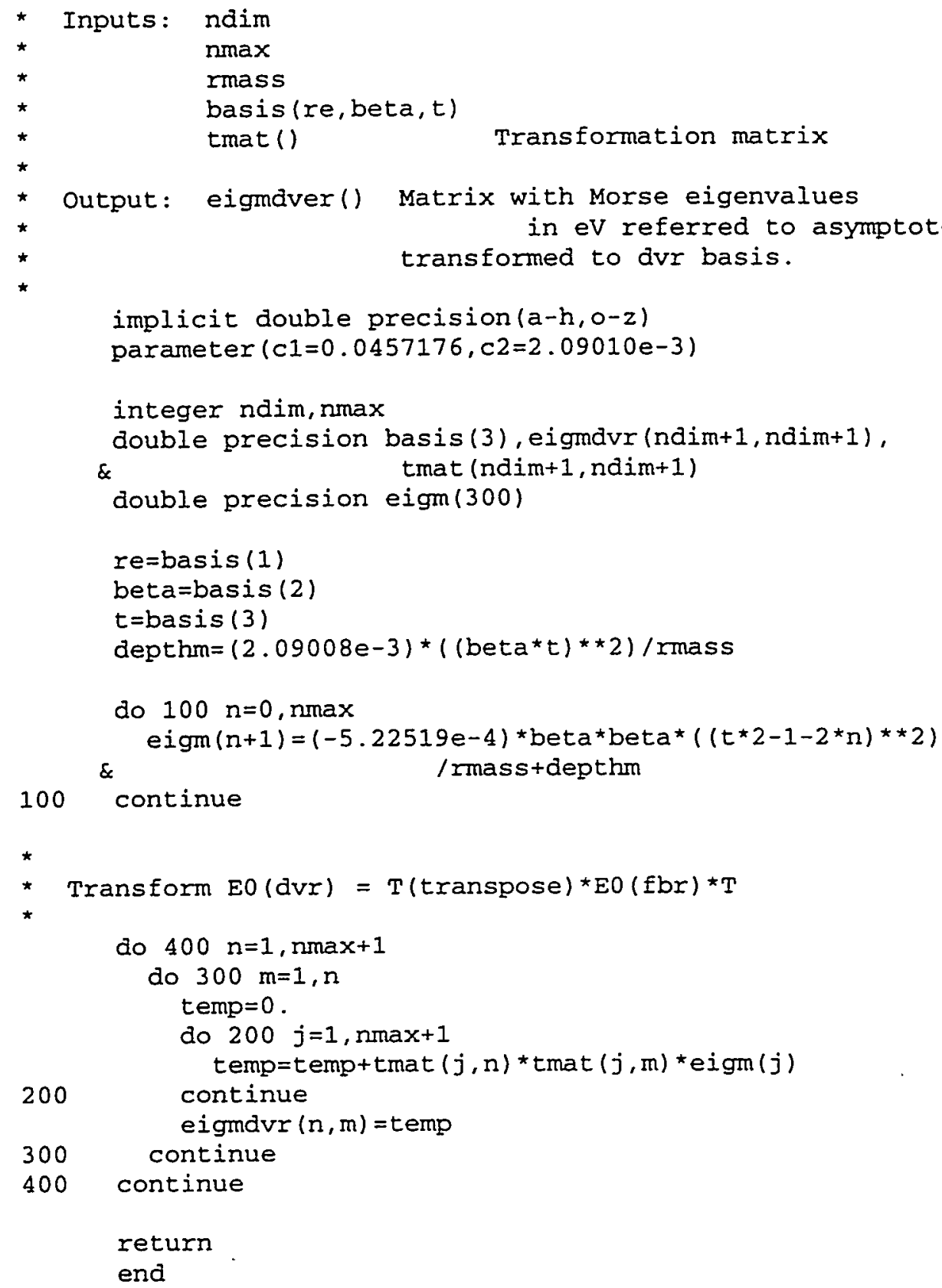




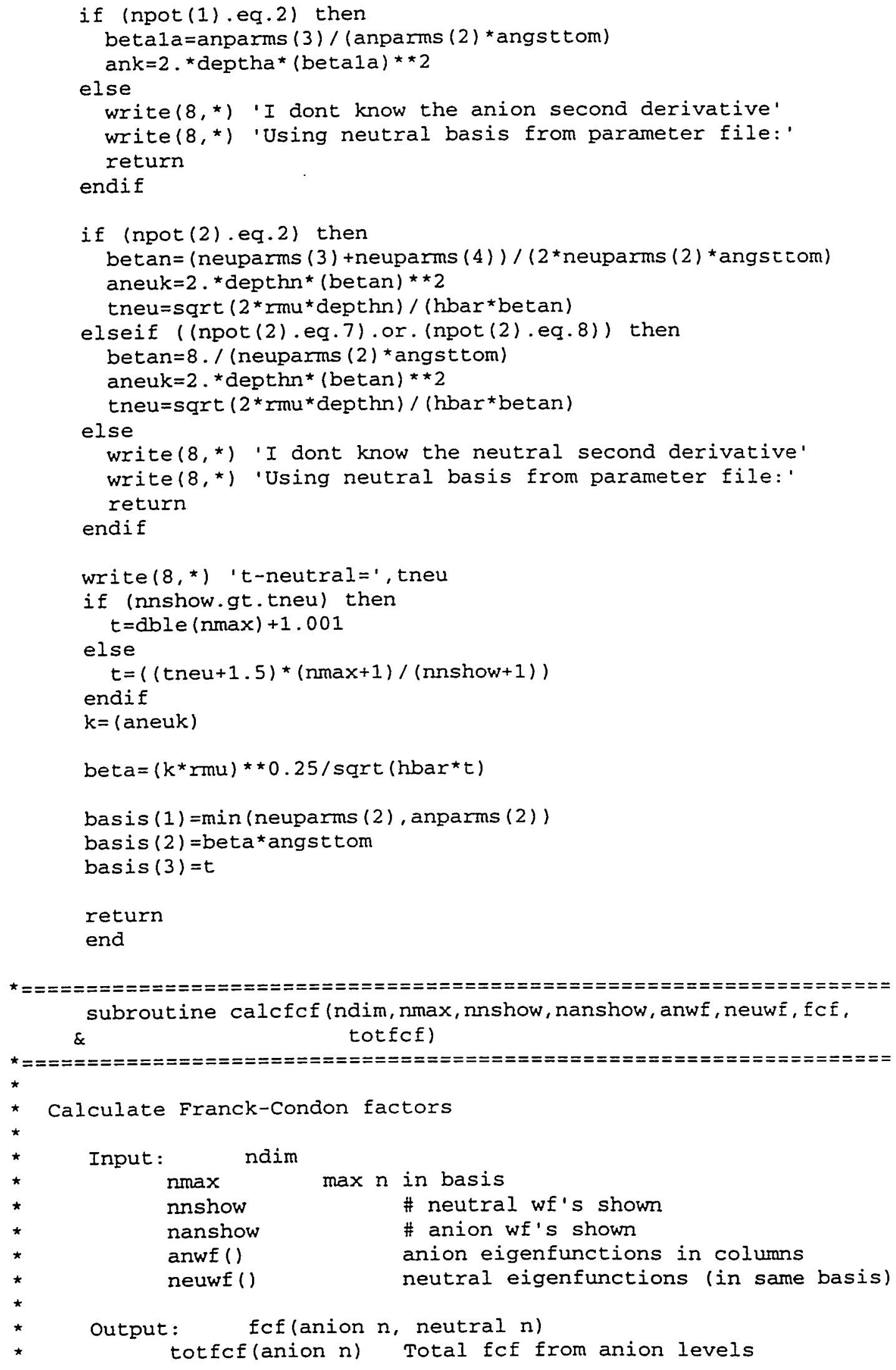


implicit double precision $(a-h, o-z)$

integer ndim, nmax, nnshow, nanshow

double precision anwf(ndim+1, ndim+1), neuwf (ndim+1, ndim+1), fcf (ndim+1, ndim+1), totfcf (ndim+1)

do $100 i=1$, nanshow 1

ftot $=0$.

do $200 j=1$, nnshow +1

ftemp $=0$.

do $300 \mathrm{n}=1, n \max +1$

ftemp $=f \operatorname{temp}+\operatorname{anwf}(n, i) *$ neuwf $(n, j)$

300 continue $f c f(i, j)=f$ temp $f_{\text {temp }}$

$f t o t=f t o t+f c f(i, j)$

200 continue

100 continue

totfcf $(i)=f t o t$

return

end

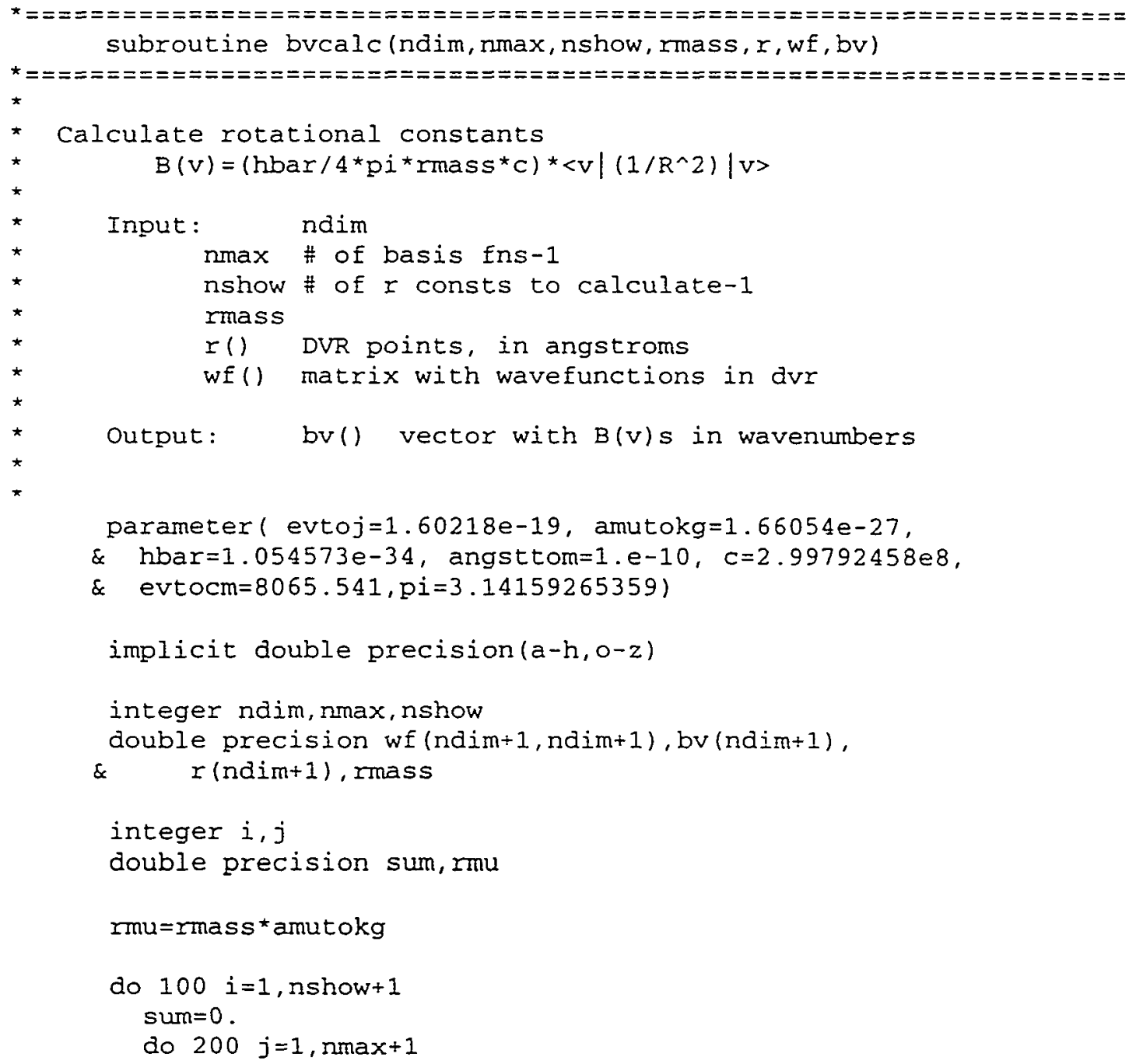




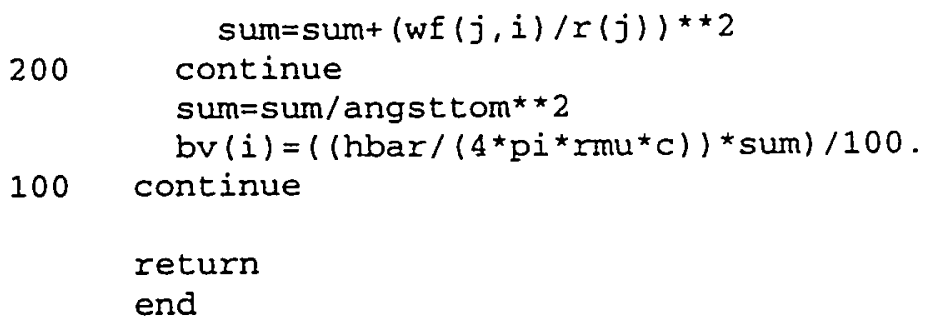

\section{A6.3. File "rsb.f'11}

subroutine $r s b(\mathrm{~nm}, \mathrm{n}, \mathrm{mb}, \mathrm{a}, \mathrm{w}, \operatorname{matz}, z, \mathrm{fv} 1, \mathrm{fv} 2$, ierr)

C

integer $\mathrm{n}, \mathrm{mb}, \mathrm{nm}$, ierr, matz

double precision $a(n m, m b), w(n), z(n m, n), f v 1(n), f v 2(n)$

logical tf

this subroutine calls the recommended sequence of subroutines from the eigensystem subroutine package (eispack) to find the eigenvalues and eigenvectors (if desired) of a real symmetric band matrix.

on input

$\mathrm{nm}$ must be set to the row dimension of the two-dimensional array parameters as declared in the calling program dimension statement.

$\mathrm{n}$ is the order of the matrix a.

$\mathrm{mb}$ is the half band width of the matrix, defined as tire number of adjacent diagonals, including the principal diagonal, required to specify the non-zero portion of the lower triangle of the matrix.

a contains the lower triangle of the real symetric band matrix. its lowest subdiagonal is stored in the last $n+1-m b$ positions of the first column, its next subdiagonal in the last $n+2-m b$ positions of the second colum, further subdiagonals similarly, and finally its principal diagonal in the $n$ positions of the last column. contents of storages not part of the matrix are arbitrary.

matz is an integer variable set equal to zero if only eigenvalues are desired. otherwise it is set to any non-zero integer for both eigenvalues and eigenvectors.

on output

$w$ contains the eigenvalues in ascending order.

$z$ contains the eigenvectors if matz is not zero. 
ierr is an integer output variable set equal to an error completion code described in the documentation for tqliat and tql2. the normal completion code is zero.

Ev1 and fv2 are temporary storage arrays.

questions and comments should be directed to burton s. garbow, mathematics and computer science div, argonne national laboratory

this version dated august 1983.

\section{A6.4. File "rs.f"11}

subroutine $r s(n m, n, a, w, \operatorname{mat} z, z, f v 1, f v 2, i e r r)$

integer $n, \mathrm{~nm}$, ierr, matz

double precision $a(n m, n), w(n), z(n m, n), f v 1(n), f v 2(n)$

this subroutine calls the recommended sequence of subroutines from the eigensystem subroutine package (eispack) to find the eigenvalues and eigenvectors (if desired)

of a real symmetric matrix.

on input

$\mathrm{nm}$ must be set to the row dimension of the two-dimensional array parameters as declared in the calling program dimension statement.

$n$ is the order of the matrix a.

a contains the real symetric matrix.

matz is an integer variable set equal to zero if only eigenvalues are desired. otherwise it is set to any non-zero integer for both eigenvalues and eigenvectors.

on output

$w$ contains the eigenvalues in ascending order.

$z$ contains the eigenvectors if matz is not zero.

ierr is an integer output variable set equal to an error completion code described in the documentation for tqlrat and tql2. the normal completion code is zero.

fv1 and fv2 are temporary storage arrays.

questions and comments should be directed to burton s. garbow, mathematics and computer science div, argonne national laboratory this version dated august 1983. 


\section{A6.5. File "matrix.f"}

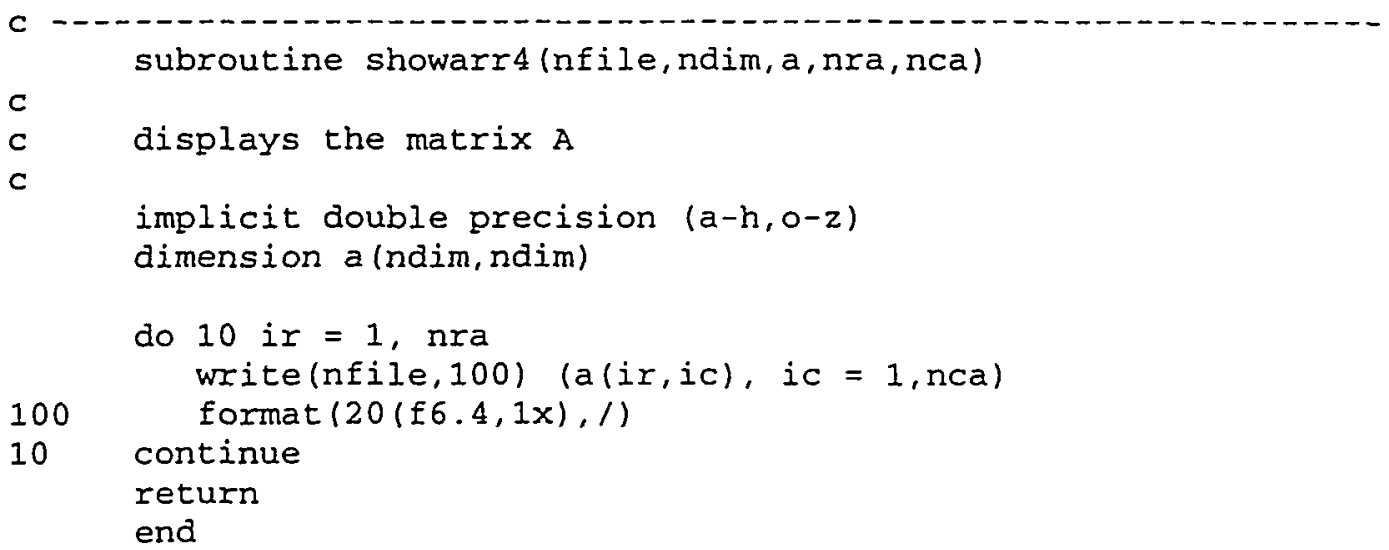

\section{A6.6. File "poten2.f"}

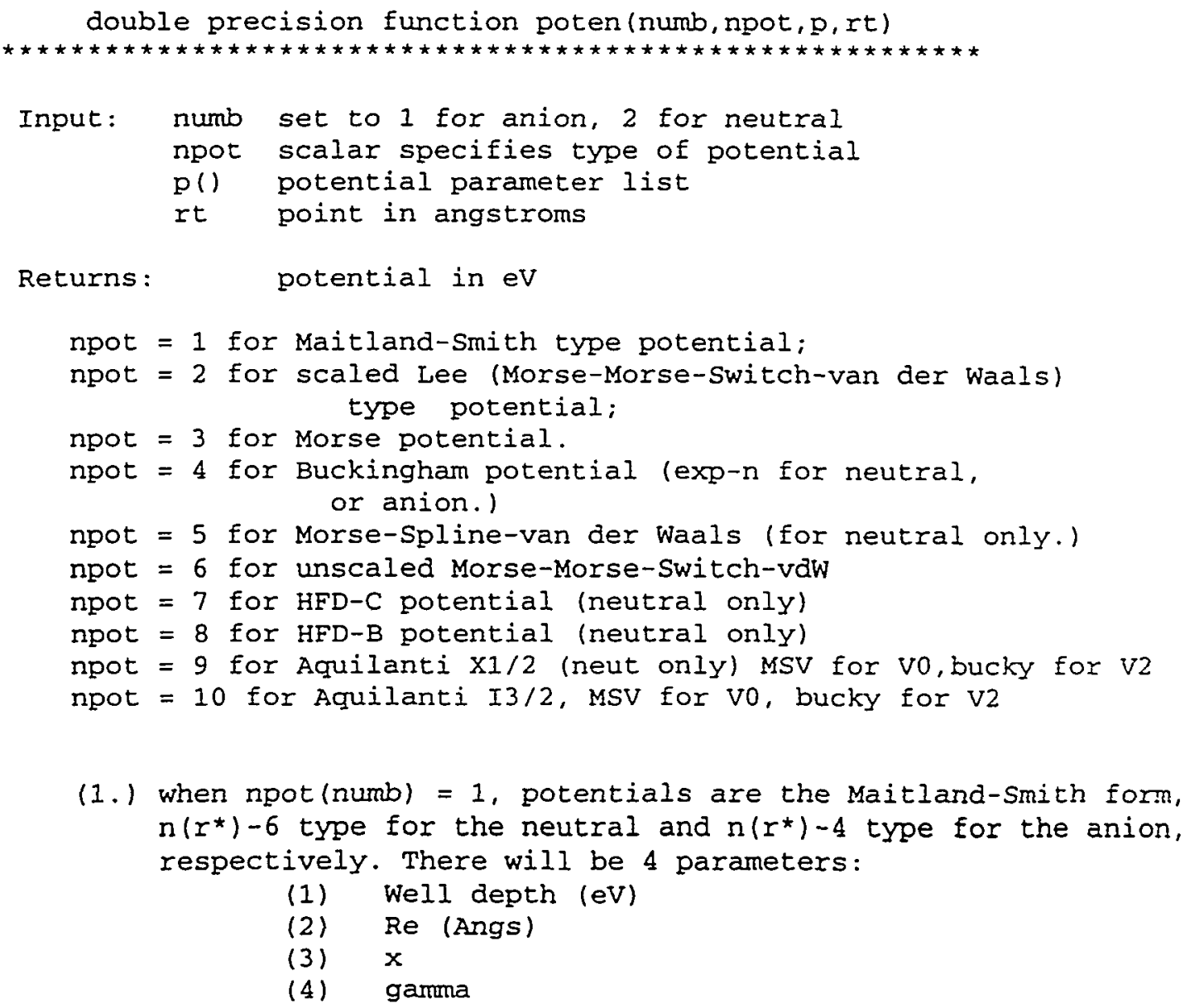


(2.) when npot (numb) $=2$, potentials are the Lee form, i.e., the Morse-Morse-Switch-van der Waals form, there will be eight parameters for each potential:

(1) Well depth (eV)

(2) $\operatorname{Re}$ (Angst.)

(3) Beta1 (unitless)

(4) Beta2 (unitless)

(5) $x 1$

(6) $\times 2$

(7) anion $C 4 /$ well depth, or neutral $c 6 /$ well depth

(8) anion $\mathrm{C} 6 /$ well depth, or neutral $\mathrm{C} 8 /$ well depth (units Angs^ $(-n), n=4,6$ or 8 )

(3.) when npot (numb) $=3$, potentials take on the Morse potentiai form, there will be three parameters for each potential.

(1) depth (eV)

(2) $\mathrm{Re}$ (Angst)

(3) Beta (Angst-1)

(4.) npot (numb) $=4$ : Buckingham $(\exp -n)$ potential:

(1) Well depth

(2) $\operatorname{Re}$

(3) beta

(4) $\mathrm{xm}$

(5.) npot (2)=5: Morse-Spline-van der Waals (Aquilanti)--Neutral 6 parameters

(1) Depth (eV)

(2) Rmin (Angst)

(3) Beta (Angst-1) -- Unscaled, different from Aqui.

(4) $\times 1$ (unitless)

(5) $\times 2$ (unitless)

(6) $\left.\mathrm{CO}\left(\mathrm{eV}^{\star} \text { Angst^}\right)^{\wedge}\right)$

(6.) npot (numb) = 6: Unscaled Morse-Morse-Switch-vdw --Same parameters as scaled MMSV, but betas have angst- 1 units, and $C n$ have $e^{\star}$ Angs $^{\wedge}(-n)$ units lobtained from scaled beta and $\mathrm{cn}$ by dividing by $R e$ or multiplying by well depth, respectively)

(7.) $\operatorname{npot}(2)=7$ : HFD-C potential
(1) Depth (ev)
(2) $\operatorname{Re}$ (Angst)
(3) Alpha
(4) gamma
(5) A
(6) C6 (unitless)
(7) $\mathrm{C8}$
(8) $\mathrm{C} 10$
(9) $\mathrm{D}$

(8.) $\operatorname{npot}(2)=8$ : HFD-B potential

(1) Depth (eV)
(2) Re (Angst)
(3) Alpha
(4) beta
(5) A




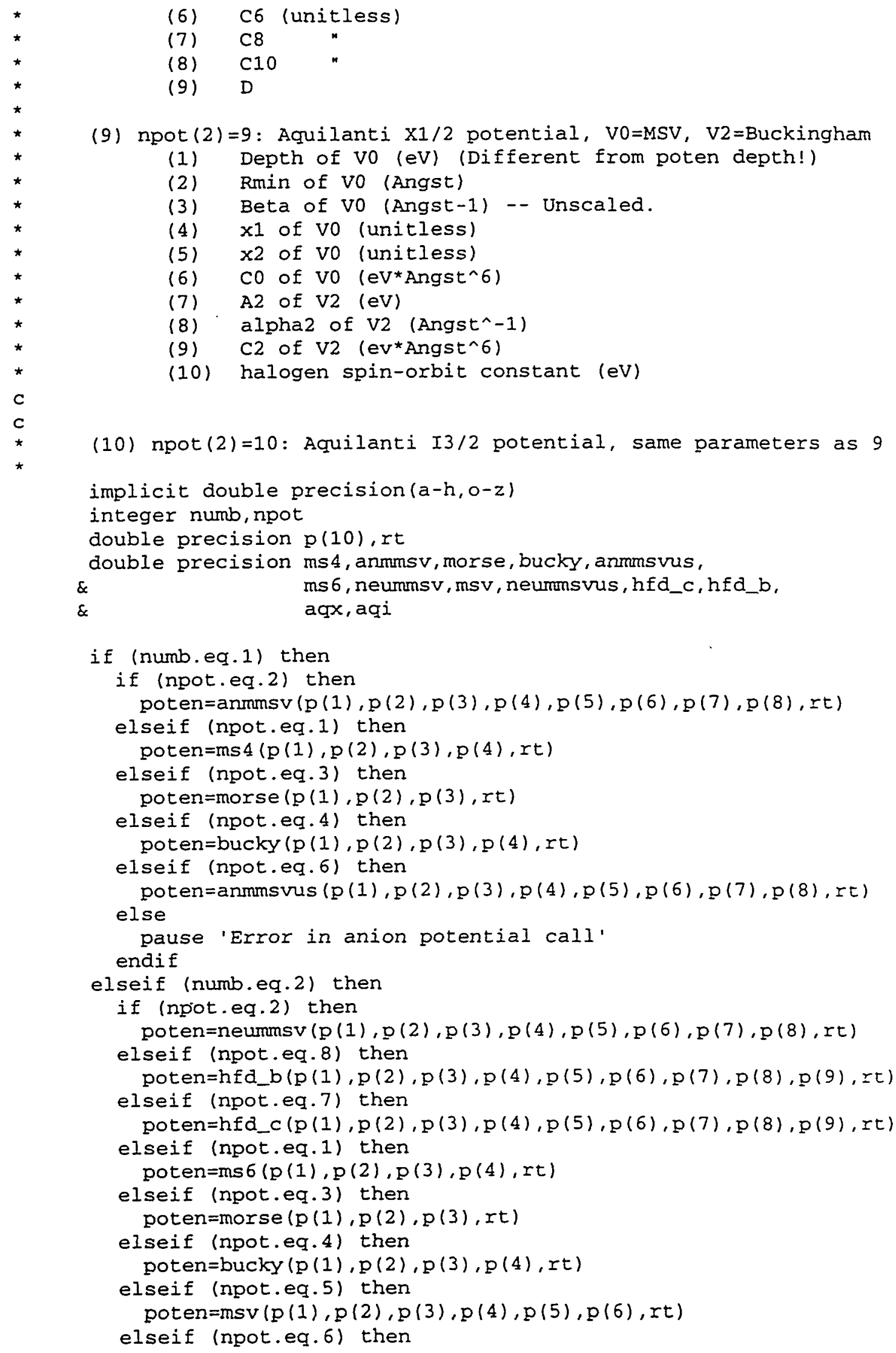


poten $=$ neummsvus $(p(1), p(2), p(3), p(4), p(5), p(6), p(7), p(8), x t)$

elseif (npot.eq.9) then

poten=agx $(p(1), p(2), p(3), p(4), p(5), p(6), p(7), p(8), p(9)$,

$\&$ $p(10), r t)$

elseif (npot.eq.10) then

$\&$

poten=aqi $(p(1), p(2), p(3), p(4), p(5), p(6), p(7), p(8), p(9)$,

else $\mathrm{p}(10), r t)$

pause 'Error in neutral potential call'

endif

endif

return

end

double precision function ms4(depth1, rminl, xm1, xlamdal, rt)

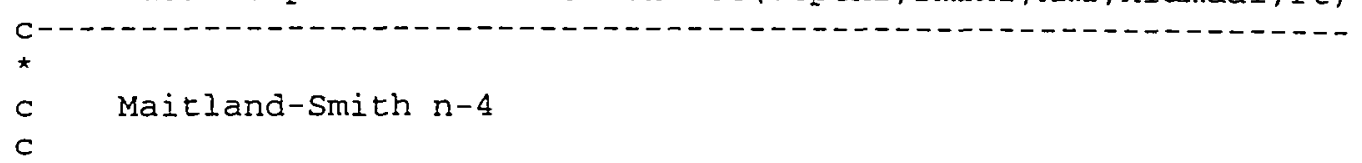

implicit double precision $(a-h, 0-z)$

$x t=r t / r \min 1$

$\operatorname{rtn}=x \operatorname{cm} 1+x \operatorname{landa} 1 *(x t-1$.

ms $4=\operatorname{depth} 1 /(\operatorname{rtn}-4) *.(4 . *(1 . / x t) * \star r t n-$

$\&$ $r \operatorname{tn} *(1 . / x t) \star \star 4)$

return

end

double precision function anmmsv(depth1, rminl, pmbetal1,

\& pmbeta21, xrstar11, xrstar21, c4vdw1, c6vdw1, rt)

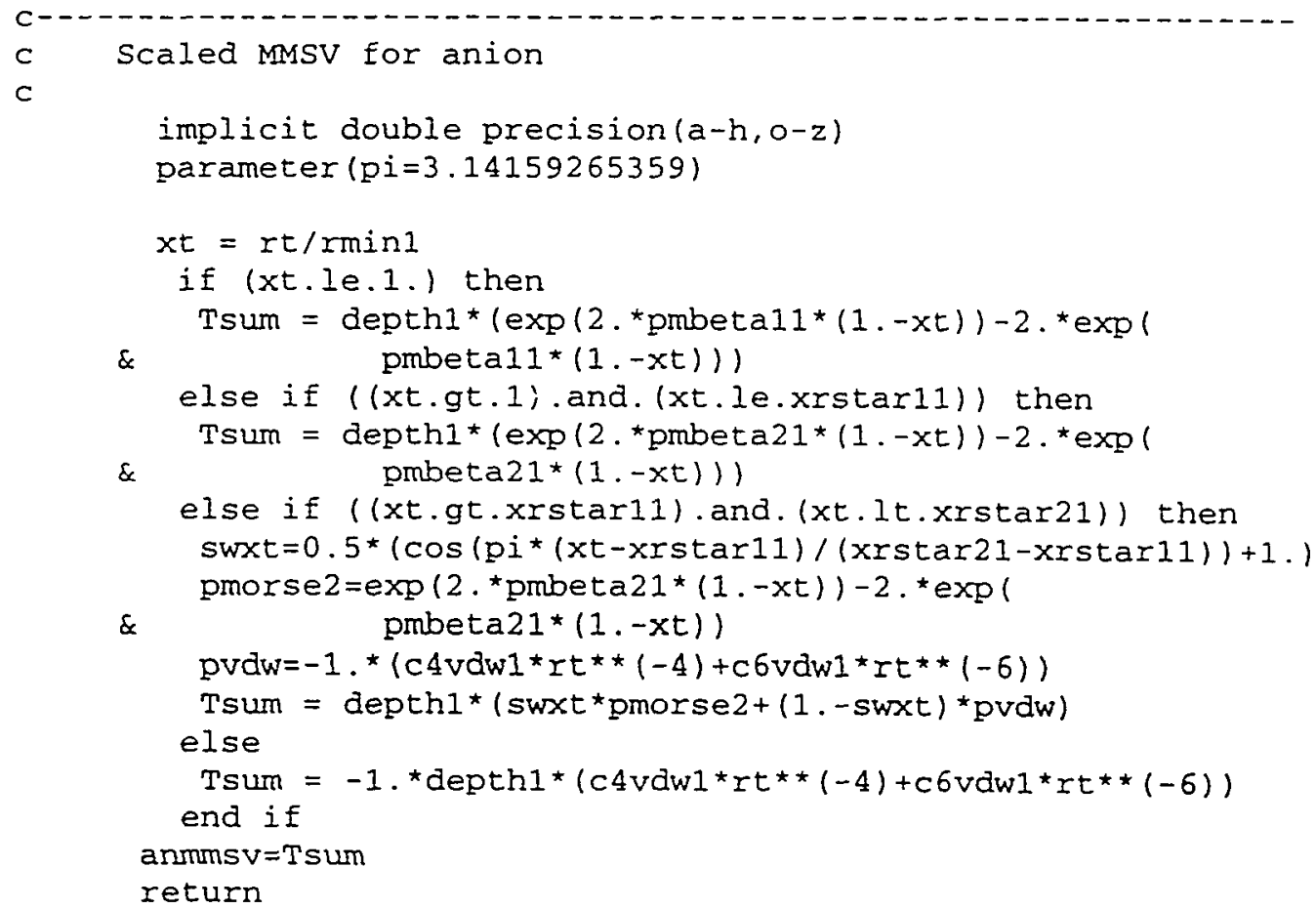


double precision function aqx(depth, rmin, beta, $x 1, \times 2$, \& c0,a2, alpha2, c2, soconst, rt)

* X1/2 State Aquilanti, JPC v.97, p.2063

implicit double precision $(a-h, 0-z)$ double precision msv

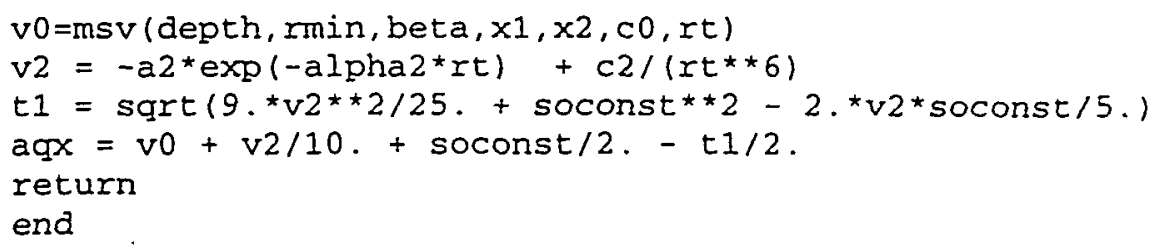

* I3/2 State Aquilanti

implicit double precision $(a-h, o-z)$

double precision msv

v0 $=m s v($ depth, rmin, beta, $x 1, x 2, c 0, r t)$

$v 2=-a 2^{\star} \exp \left(-a l p h a 2^{\star} r t\right)+c 2 /(r t \star \star 6)$

aqi $=\mathrm{v} 0-\mathrm{v} 2 / 5$. 
return

end

double precision function neumsvus (depth2, rmin2, pmbeta12,

\& pmbeta22, xrstar12, xrstar22,c6vdw2,c8vdw2,rt)

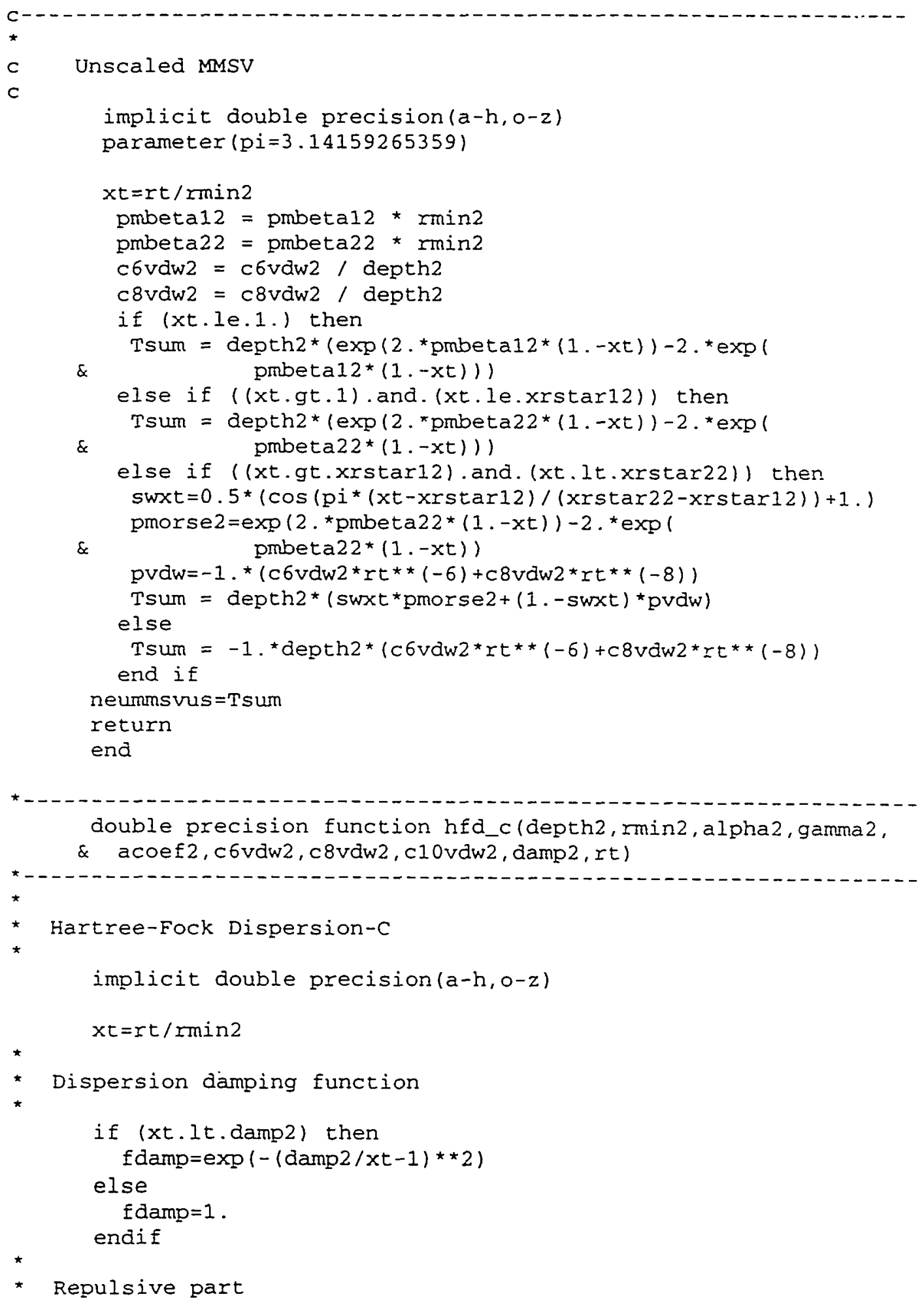




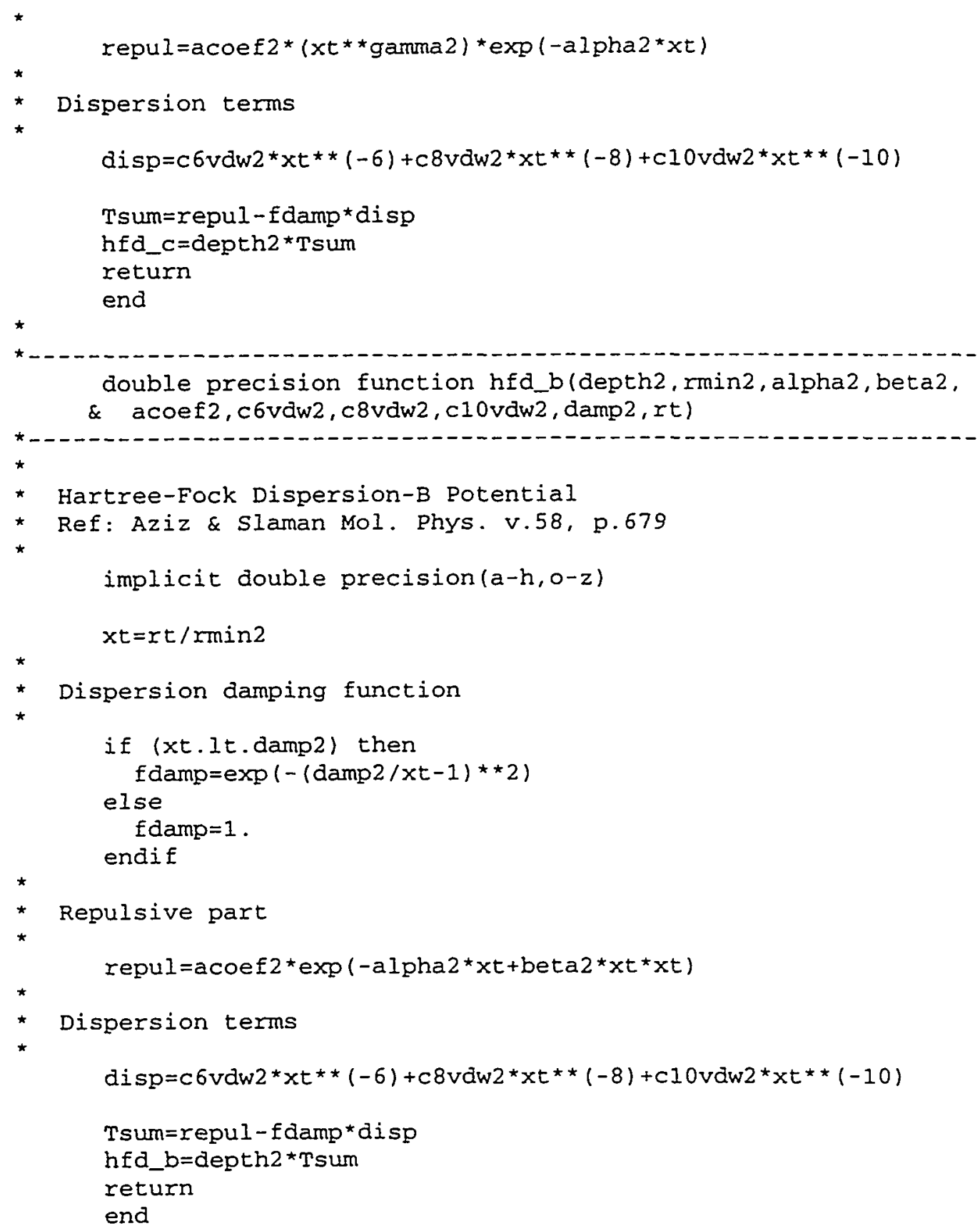

\section{A6.7. File "convol.f"}

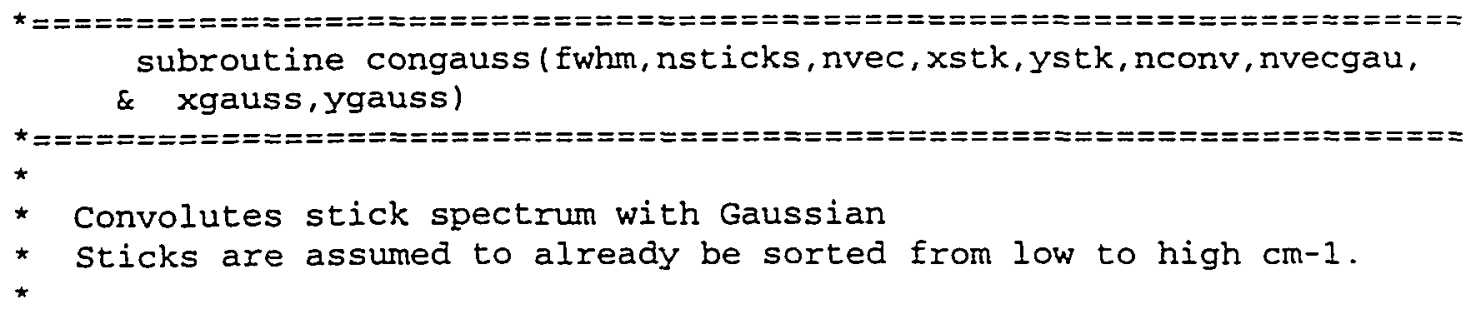




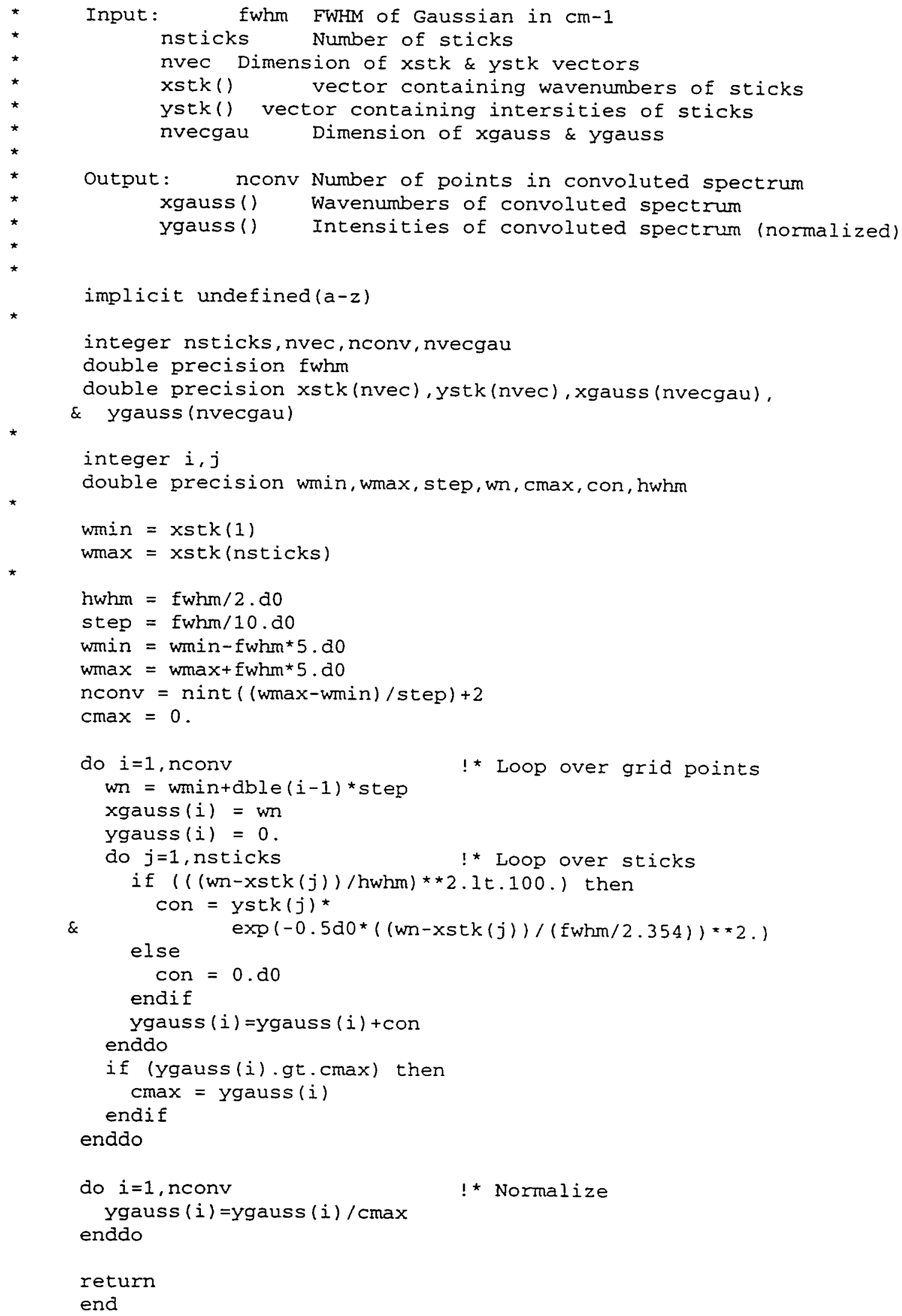




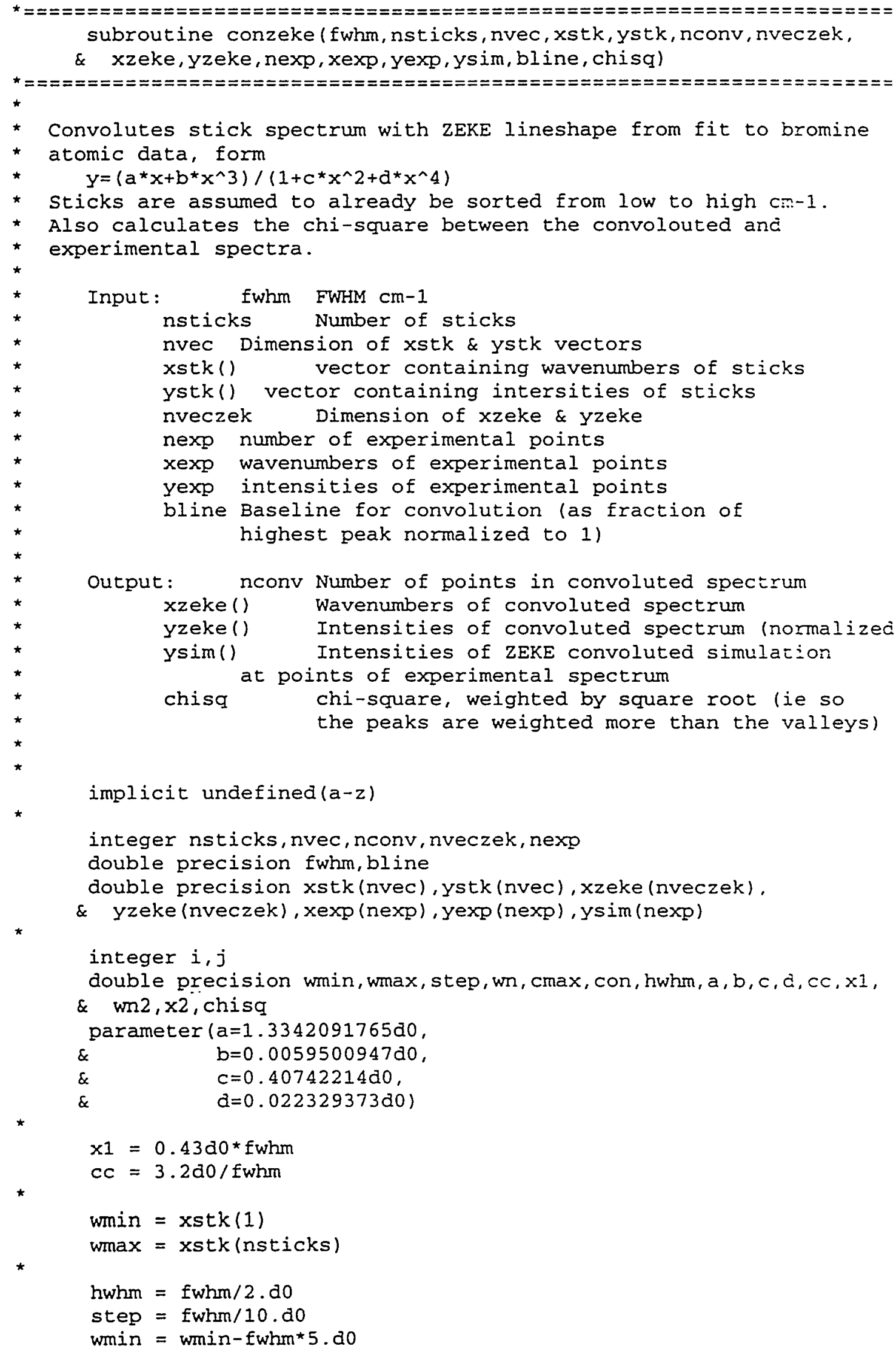

implicit undefined $(a-z)$

integer nsticks, nvec, nconv, nveczek, nexp

double precision fwhm,bline

double precision xstk (nvec), ystk (nvec), xzeke (nveczek).

$\star$ \& $y z e k e(n v e c z e k), x \exp (n \exp ), y \exp (n \exp ), y s i m(n \exp )$

integer $i, j$

double precision wmin, wmax, step, wn, cmax, con, hwhm, a,b,c, $, c c, x 1$, \& wn $2, \times 2$, chisq

parameter $(a=1.3342091765 d 0$,

\& $\quad b=0.0059500947 d 0$ 。

$\& \quad c=0.40742214 \mathrm{do}$,

\& $\quad d=0.022329373 d 0$

*

$\mathrm{x} 1=0.43 \mathrm{~d} 0{ }^{\star} \mathrm{fwhm}$

$\mathrm{cc}=3.2 \mathrm{~d} 0 / \mathrm{fwhm}$

*

wmin $=\operatorname{xstk}(1)$

wmax $=x s t k$ (nsticks)

$\mathrm{hwhm}=\mathrm{fwhm} / 2 \cdot \mathrm{do}$

step $=\mathrm{fwhm} / 10 \cdot \mathrm{do}$

wmin $=w m i n-f w h m \star 5 . d 0$ 


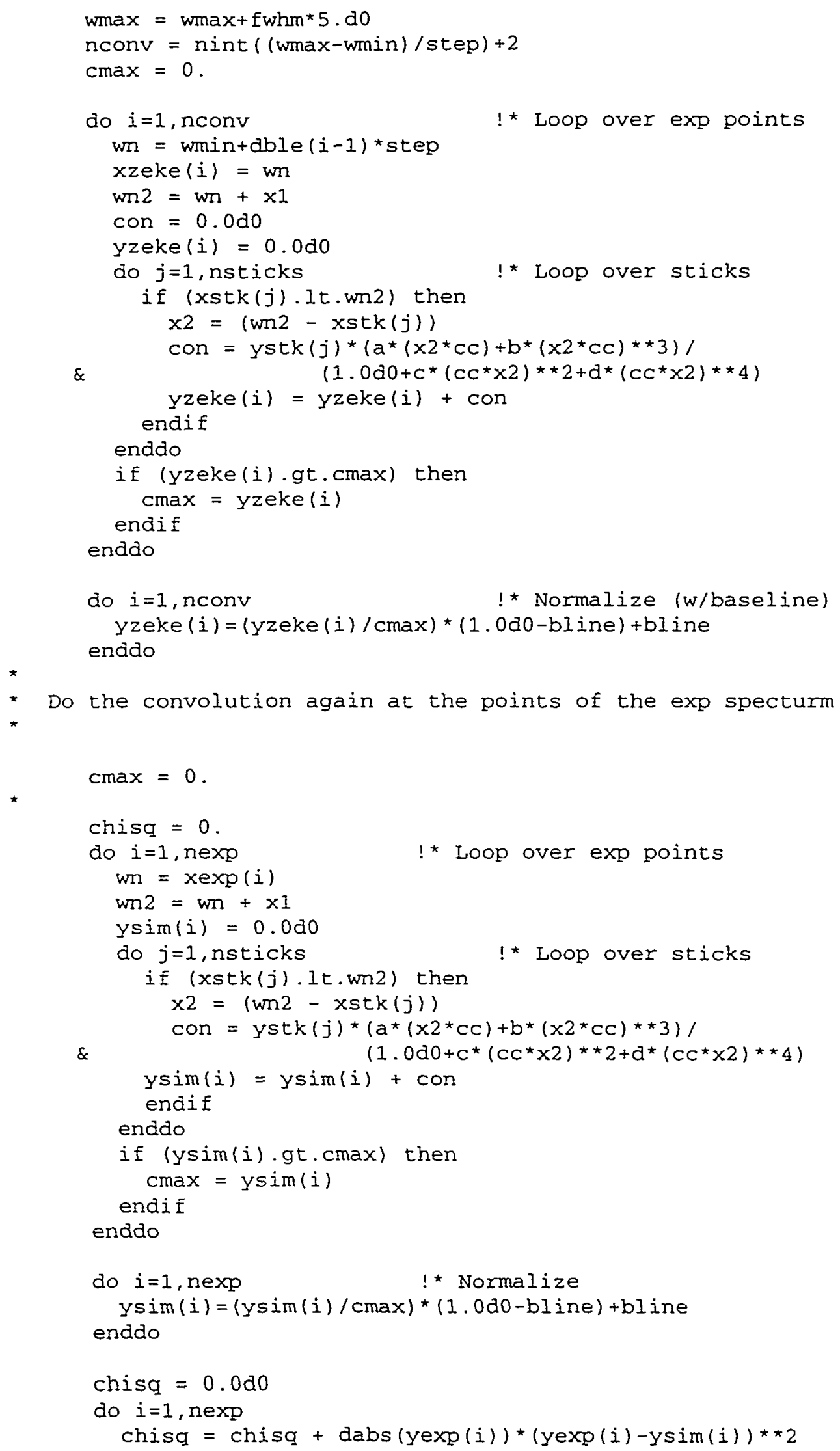


enddo

return

end 


\section{A7. References for Appendix A}

${ }^{1}$ D. O. Harris, G. G. Engerholm, and W. D. Gwinn, J. Chem. Phys. 43, 1515 (1965).

2 J. C. Light, I. P. Hamilton, and J. V. Lill, J. Chem. Phys. 82, 1400 (1985).

3 R. B. Metz, Ph.D. Thesis, University of California, 1991.

4 E. M. Greenawalt and A. S. Dickinson, J. Mol. Spectrosc. 30, 427 (1969).

5 P. M. Morse, Phys. Rev. 34, 57 (1929).

6 A. S. Dickenson and P. R. Certain, J. Chem. Phys. 49, 4209 (1968).

7 Y Zhao, I. Yourshaw, G. Reiser, C. C. Annold, and D. M. Neumark, J. Chem. Phys. 101,6538 (1994).

8 W. H. Press, S. A. Teukolsky, W. T. Vetterling, and B. P. Flannery, Numerical Recipes, 2nd ed. (Cambridge University Press, Cambridge, 1992).

9 V. Aquilanti, D. Cappelletti, V. Lorent, E. Luzzatti, and F. Pirani, J. Phys. Chem. 97, 2063 (1993).

10 R. A. Aziz and M. J. Slaman, Mol. Phys. 58, 679 (1986).

11 The EISPACK source code may be obtained by email from netlib@research.att.com or by FTP from the server "research.att.com" using the login name "netlib." 


\section{Appendix B. Program for fitting rovibronic transitions in $\mathrm{RgX}^{*}$ photodetachment.}

In this appendix we describe the computer program used to simulate the rotational profiles of the line shapes observed in the ZEKE spectra of the diatomic RgX' complexes. First we review the derivation of the selection rules and rotational line strength factors for photoelectron transitions of Hund's case (c) molecules. We then describe in detail the use of the rotational fitting program.

\section{B1. Line strength factors for photodetachment of Hund's case (c) molecules."}

As mentioned in Chapter 3, the peak shapes of the ZEKE spectra are asymmetric and change from band to band. In addition, the peak widths vary with source conditions. We explain the peak shape and width in terms of the asymmetric ZEKE experimental peak shape combined with the effect of the unresolved rotational structure associated with each vibrational transition. Both effects are discussed in this section, with the main focus on how one treats the rotational contribution to the peak widths.

The ejected electron has spin $s=1 / 2$ and orbital angular momentum $l=0$ (only $s$ wave photodetachment is observed in anion ZEKE experiments, as discussed in Chapter 1). Hence, the anion $\rightarrow$ neutral rotational selection rules are $\Delta J= \pm \frac{1}{2}, \pm \frac{3}{2}$. Since individual rotational lines are not resolved in our spectra, several assumptions are required to simulate these transitions. For calculating the energies of the rotational transitions in each band, equilibrium rotational constants $B_{e}$ are assumed, using the $R_{m}$ values from the potentials obtained in the vibrational analysis described in chapter 2 .

\footnotetext{
- Most of this section was originally published in slightly revised form in J. Chem. Phys. 101, 6538 (1994), with co-authors Y. Zhao, G. Reiser, C. C. Arnold, and D. M. Neumark.
} 
We now derive expressions for the relative intensities of the rotational transitions in each band. Xie and Zare ${ }^{1,2}$ derived an expression for the photoionization probability of a diatomic molecule conforming to Hund's case (b) in terms of a generalized rotational line strength factor and the reduced multipole moments. Here, we adapt this approach to a Hund's case (c) molecule. A diagram of the Hund's case (c) angular momentum scheme is presented for reference in Figure B1. The results of this treatment are valid for photodetachment as well as photoionization. Although we do not resolve individual rotations in the spectra presented in this work, the results presented below should be useful for future investigations in which rotational structure is resolved. Moreover, the final expressions obtained are quite simple, and result in a very satisfactory fitting of the peak shapes.

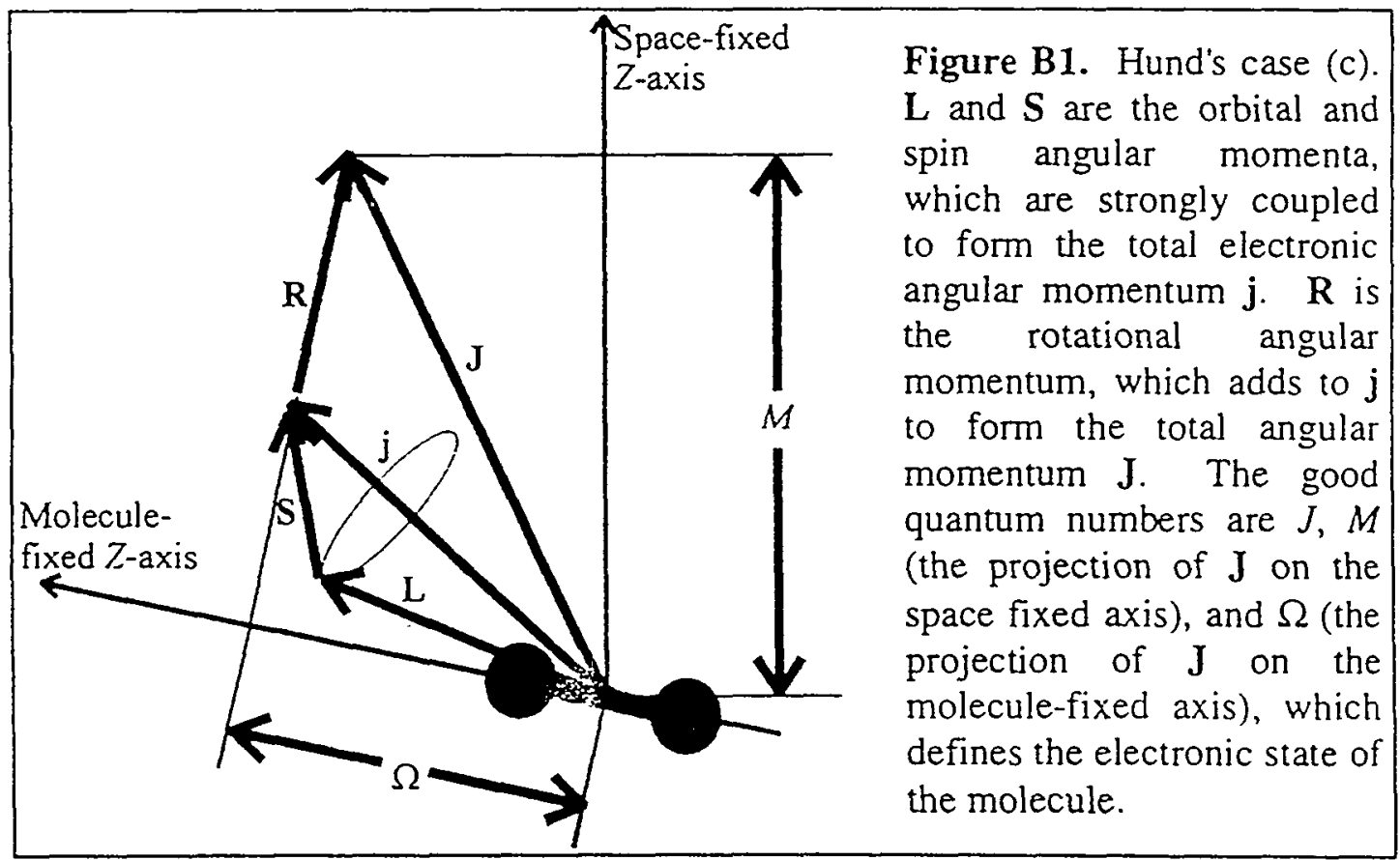

The eigenstates of a case (c) molecule are represented by

$$
|n J \Omega M v\rangle=|J \Omega M\rangle|n \Omega\rangle|v\rangle
$$


where the angular momentum quantum numbers are defined in Table $B 1 ; v$ is the vibrational quantum number, and $n$ represents the remaining quantum numbers.

Table B1. Quantum number nomenclature for rotational analysis.

\begin{tabular}{|c|c|c|l|}
\hline $\begin{array}{c}\text { Angular } \\
\text { Momentum }\end{array}$ & $\begin{array}{c}\text { Molecule-Fixed } \\
\text { Projection }\end{array}$ & $\begin{array}{c}\text { Space-Fixed } \\
\text { Projection }\end{array}$ & \multicolumn{1}{|c|}{ Description } \\
\hline$J$ & $\Omega$ & $M$ & $\begin{array}{l}\text { Total angular momentum of } \\
\text { neutral }\end{array}$ \\
\hline$J^{-}$ & $\Omega^{-}$ & $M^{-}$ & $\begin{array}{l}\text { Total angular momentum of } \\
\text { anion }\end{array}$ \\
\hline$j$ & $\omega$ & $m$ & $\begin{array}{l}\text { Total photoelectron angular } \\
\text { momentum (orbital + spin) }\end{array}$ \\
\hline 1 & $\mu$ & $\mu_{0}$ & Angular momentum of photon \\
\hline$k$ & $q$ & $p$ & $\begin{array}{l}\text { Vector sum of photon and } \\
\text { photoelectron angular } \\
\text { momenta }\end{array}$ \\
\hline
\end{tabular}

On the right side of Equation B1 we have assumed the Born-Oppenheimer approximation and independence of rotational from vibrational degrees of freedom to separate the eigenstate into rotational, electronic, and vibrational parts. The detached electron is assumed to be well approximated asymptotically by a partial wave expansion in a spherically symmetric potential. ${ }^{3}$ The eigenstate of a given electron partial wave in the coupled representation appropriate to case (c) is $|j m\rangle$, where $j$ is the total (orbital and spin) angular momentum of the electron partial wave, and $m$ is its projection in the spacefixed frame. The electronic dipole operator transforms as a spherical tensor, $T\left(1, \mu_{0}\right)$, under rotation. The photodetachment probability for a given electron angular momentum from rotational state $J$ of the anion to state $J$ of the neutral is then (neglecting constants and the vibrational eigenvectors) 


$$
P_{j}\left(J^{-}, J\right) \propto \sum_{m} \sum_{M^{\prime}, M^{-}} \mid\left\langle J \Omega M\left|\left\langle n \Omega\left|\left\langle j m\left|T\left(1, \mu_{0}\right)\right| n^{-} \Omega^{-}\right\rangle\right| J^{-} \Omega^{-} M^{-}\right\rangle\right|^{2} .\right.
$$

It is necessary to get the electron and photon wavefunctions into the moleculefixed frame, so that we can separate nuclear and electron coordinates. To do this we transform the dipole operator and electron eigenstates to the molecule fixed frame using the Wigner rotation matrices as defined by $\mathrm{Zare}^{4}$ as follows:

$$
\begin{aligned}
T\left(1, \mu_{0}\right) & =\sum_{\mu} D_{\mu_{0} \mu}^{\prime^{*}}(R) T(1, \mu) & \text { (photon) } \\
\langle j m) & =\sum_{\omega}\left[D_{m \omega}^{\prime^{*}}(R)\right]^{+}\langle j \omega| & \text { (electron) }
\end{aligned}
$$

where $R$ stands for the Euler angles $\phi, \theta$, and $\chi$, and the angular momentum quantum numbers are defined in Table B1.

The rotational eigenfunctions are

$$
\begin{aligned}
\left|J^{-} \Omega^{-} M^{-}\right\rangle & =\left(\frac{2 J^{-}+1}{8 \pi^{2}}\right)^{1 / 2} D_{M^{-} \Omega^{-}}^{J^{-}}(R), \\
\langle J \Omega M| & =\left(\frac{2 J+1}{8 \pi^{2}}\right)^{1 / 2}\left[D_{M \Omega}^{J^{-}}(R)\right]^{+} .
\end{aligned}
$$

Substituting Equations B3 and B4 into Eq. B2 gives

$$
\begin{aligned}
P_{j}\left(J^{-}, J\right) \propto & \frac{(2 J+1)\left(2 J^{-}+1\right)}{\left(8 \pi^{2}\right)^{2}} \\
& \times \sum_{m} \sum_{M, M^{-}} \mid \sum_{\mu, \omega}\left(\int D_{M \Omega}^{J}(R) D_{m \omega}^{j}(R) D_{-\mu_{0}-\mu}^{1}(R) D_{-M^{-}-\Omega^{-}}^{j^{-}}(R) d R\right) \\
& \times\left\langle n \Omega\left|\left\langle j \omega|T(1, \mu)| n^{-} \Omega^{-}\right\rangle\right|^{2},\right.
\end{aligned}
$$

where all the dependence on angular nuclear coordinates is contained in the integral over the four rotation matrices. 
To evaluate the integral over the four rotation matrices, we expand those from the detached electron and dipole operator in a Clebsch-Gordon series ${ }^{4}$

$$
D_{m \omega}^{j}(R) D_{-\mu_{0}-\mu}^{1}(R)=\sum_{k}(2 k+1)\left(\begin{array}{ccc}
j & 1 & k \\
m & -\mu_{0} & p
\end{array}\right)\left(\begin{array}{ccc}
j & 1 & k \\
\omega & -\mu & q
\end{array}\right) D_{-p-q}^{k}(R)
$$

with

$$
\begin{aligned}
& k=1+j, \cdots,|1-j|, \\
& p=\mu_{0}-m, \\
& q=\mu-\omega .
\end{aligned}
$$

In Equation (B6) we have coupled the electron and photon angular momenta, where $k$ is the vector sum of the photon electron angular momenta, and $p$ and $q$ are the space- and molecule-fixed projections of this quantity, respectively (see Table B1). Substituting this into Equation (B5) we obtain an integral over three rotation matrices ${ }^{4}$

$$
\int D_{M \Omega}^{J}(R) D_{-p-q}^{k}(R) D_{-M^{-}-\Omega^{-}}^{J^{-}}(R) d R=8 \pi^{2}\left(\begin{array}{ccc}
J^{-} & k & J \\
M^{-} & p & -M
\end{array}\right)\left(\begin{array}{ccc}
J^{-} & k & J \\
\Omega^{-} & q & -\Omega
\end{array}\right) .
$$

Using Eqs. (B6) and (B7) in Eq. (B5) we obtain

$$
\begin{aligned}
& P_{j}\left(J^{-}, J\right) \propto(2 J+1)\left(2 J^{-}+1\right) \\
& \quad \times \sum_{m} \sum_{M, M^{-}} \mid \sum_{k}(2 k+1)\left(\begin{array}{ccc}
j & 1 & k \\
m & -\mu_{0} & p
\end{array}\right)\left(\begin{array}{ccc}
J^{-} & k & J \\
M^{-} & p & -M
\end{array}\right)\left(\begin{array}{ccc}
J^{-} & k & J \\
\Omega & \Omega-\Omega^{-} & -\Omega
\end{array}\right) \\
& \quad \times \sum_{\mu, \omega}\left(\begin{array}{ccc}
j & 1 & k \\
\omega & -\mu & q
\end{array}\right)\left\langle n \Omega\left|\left\langle j \omega|T(1, \mu)| n^{-} \Omega^{-}\right\rangle\right|^{2} .\right.
\end{aligned}
$$

The condition $q=\Omega-\Omega^{-}$(i.e., $\mu+\Omega^{-}=\omega+\Omega$ ) determines the electronic selection rules.

The $M$ and $M^{-}$dependent terms in the square of the sum over $k$ for a given $k^{\prime}$ and $k^{\prime \prime}$ give rise to sums 


$$
\sum_{M, M^{-}}\left(\begin{array}{ccc}
J^{-} & k^{\prime} & J \\
M^{-} & p & -M
\end{array}\right)\left(\begin{array}{ccc}
J^{-} & k^{\prime \prime} & J \\
M^{-} & p & -M
\end{array}\right)=\frac{1}{2 k^{\prime}+1} \delta_{k^{\prime} \cdot k^{-}}
$$

so that the cross terms vanish, leaving

$$
P_{j}\left(J^{-}, J\right) \propto \sum_{k} S_{k}\left(J^{-}, J\right)\left|\mu_{j}(k, q)\right|^{2}
$$

Here, following Xie and Zare, 1,2 we have defined the rotational line strength factor ${ }^{*}$

$$
S_{k}\left(J^{-}, J\right)=\left.(2 J+1)\left(2 J^{-}+1\right)\left(\begin{array}{ccc}
J^{-} & k & J \\
\Omega^{-} & \Omega-\Omega^{-} & -\Omega
\end{array}\right)\right|^{2}
$$

which contains all the $J$ and $J^{-}$dependence, and a generalized multipole transition moment

$$
\left|\mu_{j}(k, q)\right|^{2}=(2 k+1) \mid \sum_{\mu, \omega}\left(\begin{array}{ccc}
j & 1 & k \\
\omega & -\mu & q
\end{array}\right)\left\langle n \Omega\left|\left\langle j \omega|T(1, \mu)| n^{-} \Omega^{-}\right\rangle\right|^{2} .\right.
$$

The phrase "generalized multipole transition moment" refers to the fact that this term involves both the incoming photon and ejected electron angular momenta, in contrast to the familiar "dipole transition moment" in photo absorption processes which involves only the photon.

In anion ZEKE spectroscopy, $j=\frac{1}{2}$ (because $l=0$ for the detached electrons that are detected) and Eq. (B10) becomes

$$
P_{1 / 2}\left(J^{-}, J\right) \propto C_{1 / 2} S_{1 / 2}\left(J^{-}, J\right)+S_{3 / 2}\left(J^{-}, J\right)
$$

The expression for the rotational line strength factor may be compared with the familiar expression for the Hönl-London factors for the intensity of rotational transitions in the photoabsortion process $J^{\prime \prime}, K^{\prime \prime} \rightarrow J^{\prime}, K^{\prime}$ of a rigid rotor: ${ }^{4}$

$$
S\left(J^{\prime} K^{\prime}, J^{\prime \prime} K^{\prime \prime}\right)=\left(2 J^{\prime}+1\right)\left(2 J^{\prime \prime}+1\right)\left(\begin{array}{ccc}
J^{\prime} & 1 & J^{\prime \prime} \\
-K^{\prime} & K^{\prime}-K^{\prime \prime} & K^{\prime \prime}
\end{array}\right)^{2} .
$$


with

$$
C_{1 / 2}=\left|\frac{\mu_{1 / 2}\left(\frac{1}{2}, q\right)}{\mu_{1 / 2}\left(\frac{1}{2}, q\right)}\right|^{2} .
$$

For $s$-wave detachment, $\Delta J=J^{-}-J= \pm \frac{1}{2}, \pm \frac{3}{2}$. Expressions for the line strength factors for transitions from $\Omega^{-}=0$ to $\Omega=\frac{1}{2}$ ( $X \frac{1}{2}$ and $I I \frac{1}{2}$ states) and to $\Omega=\frac{3}{2}$ ( $I \frac{3}{2}$ state) for each of the allowed branches are given in Table B2. Note that $S_{1 / 2}\left(J^{-}, J\right)$ vanishes for the $\Delta J= \pm \frac{3}{2}$ branches of the transitions to $\Omega=\frac{1}{2}$ states and for all branches of transitions to the $\Omega=\frac{3}{2}$ state.

Table B2. Rotational line strengths for transitions to the three neutral states

\begin{tabular}{|c|c|c|c|c|}
\hline \multirow{2}{*}{$\Delta \mathbf{J}$} & \multicolumn{2}{|c|}{$S_{1 / 2}\left(J^{-}, J\right)$} & \multicolumn{2}{c|}{$S_{3 / 2}\left(J^{-}, J\right)$} \\
\cline { 2 - 5 } & $I \frac{3}{2}$ & $X \frac{1}{2}$ or $I I \frac{1}{2}$ & $I \frac{3}{2}$ & $X \frac{1}{2}$ or $I I \frac{1}{2}$ \\
\hline$+\frac{1}{2}$ & 0 & $J^{-}+1$ & $\frac{3\left(J^{-}+1\right)\left(J^{-}+2\right)}{2\left(2 J^{-}+3\right)}$ & $\frac{J^{-}\left(J^{-}+1\right)}{2\left(2 J^{-}+3\right)}$ \\
\hline$-\frac{1}{2}$ & 0 & $J^{-}$ & $\frac{3 J^{-}\left(J^{-}-1\right)}{2\left(2 J^{-}-1\right)}$ & $\frac{J^{-}\left(J^{-}+1\right)}{2\left(2 J^{-}-1\right)}$ \\
\hline$+\frac{3}{2}$ & 0 & 0 & $\frac{\left(J^{-}+2\right)\left(J^{-}+3\right)}{2\left(2 J^{-}+3\right)}$ & $\frac{3\left(J^{-}+1\right)\left(J^{-}+2\right)}{2\left(2 J^{-}+3\right)}$ \\
\hline$-\frac{3}{2}$ & 0 & 0 & $\frac{\left(J^{-}-1\right)\left(J^{-}-2\right)}{2\left(2 J^{-}-1\right)}$ & $\frac{3 J^{-}\left(J^{-}-1\right)}{2\left(2 J^{-}-1\right)}$ \\
\hline
\end{tabular}

One can calculate a rotational stick spectrum by multiplying Eq. (B13) by a Boltzmann factor, treating the coefficient $C_{1 / 2}$ (for the $\Omega=\frac{1}{2}$ neutral states) as an adjustable parameter. To obtain realistic peak shapes, the rotational lines must be 
convoluted with the empirically determined ZEKE instrumental line shape. Refer back to Chapter 3 [Eq. (3.11)] for the detailed form of the ZEKE line shape.

\section{B2. Documentation of the rotational fitting program "rfit": example of the XeBr} $X \frac{1}{2}$ state

This section describes the computer program used to fit the rotational profiles of the $\mathrm{RgX} \mathrm{ZEKE}$ spectra. The program as input uses the vibrational stick spectrum generated by the fitting program "idvr" (described in Appendix A) to general the final rotational spectrum convoluted with the ZEKE line shape. The available variable parameters in the rotational fitting procedure are the rotational temperature, the ZEKE FWHM (full width at half maximum), the parameter $C_{1 / 2}$ discussed above [see Eqs. (B13) and (B14)], the baseline, an offset for the origin, and the maximum peak intensity. Note that the input vibrational sticks are not modified in any way: the program "rfit" only fits the above mentioned above. The program uses a simple gradient minimization algorithm ${ }^{5}$ to perform the optimization.

\section{B2.1. The input files}

An example of the input file for the $X \frac{1}{2}$ state of $\mathrm{XeBr}$ is shown below. The line numbers in boldface are only for reference, and are not included in the actual input file. The file is named "xebr_x_rfin." The comments after the "!*" symbol may be included after numerical input lines, but not after text (file name) input lines.

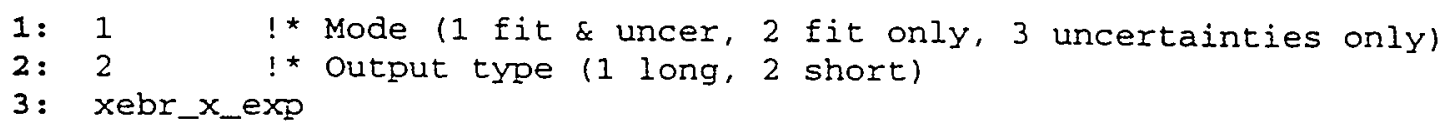




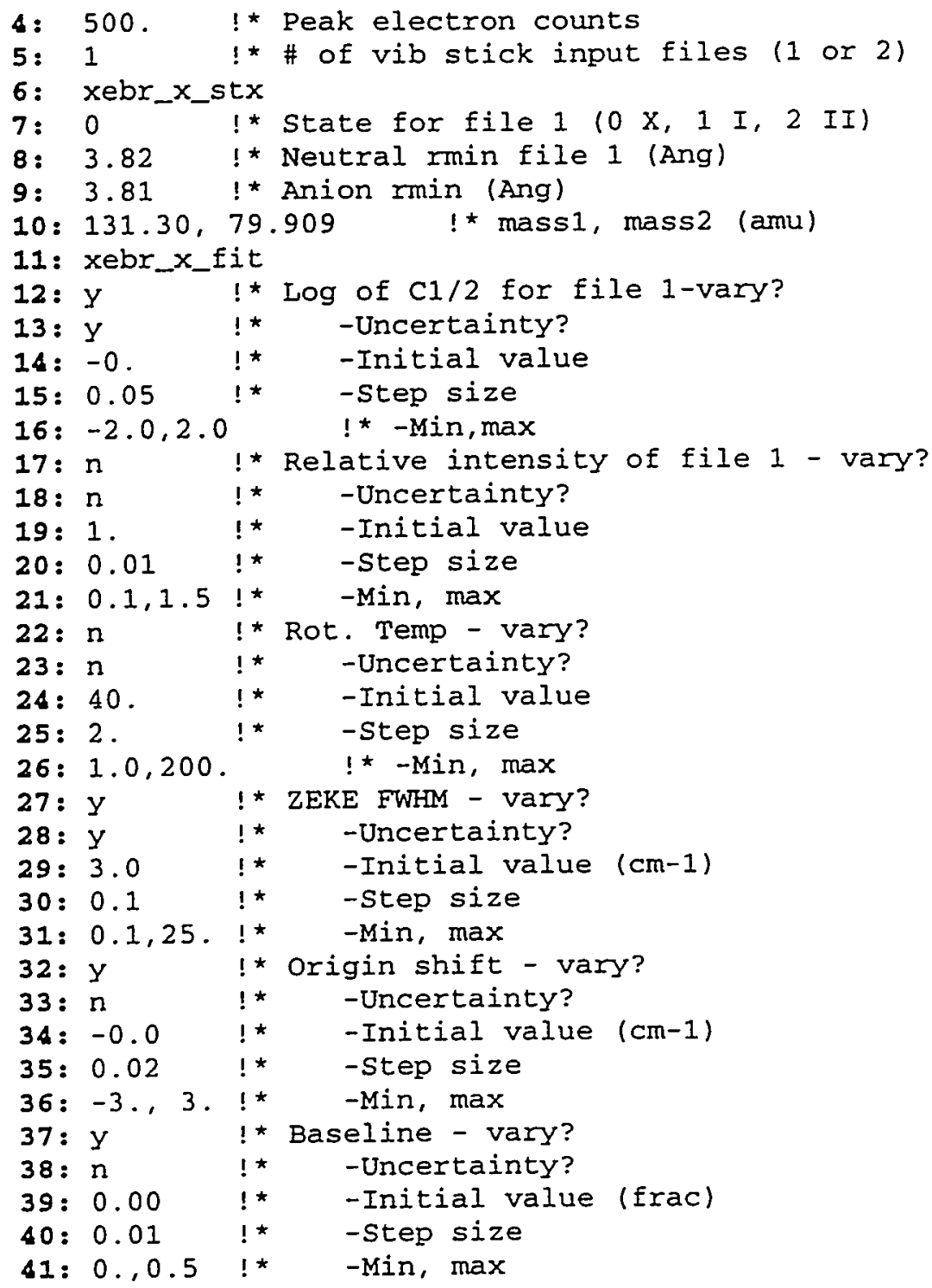

The first line is an integer which tells the program whether to find uncertainties (standard deviations) during the fit or not. If line 1 is set to 1 then both the fit is performed and uncertainties are found. If line 2 is set to 2 , only the fit is performed, and if it is set to 3 only uncertainties are found, using the initial values given for the fitting parameters. The program performs a full multivariate analysis of the parameters in finding the uncertainties, so this can be quite time-consuming, and it is often desirable to run in mode 2 when quick results are needed. Line 2 is an integer: 1 for long form 
output, i.e. each step in the gradient minimization is shown, 2 for short form output, where only the final result of the fit are shown, and the intermediate steps during the process of finding the standard deviations.

Line 3 contains the name of the experimental spectrum, which must be in wavenumber format. If uncertainties are to be calculated, line 4 should contain an estimate of the number of electron counts at peak in the experimental spectrum. This number is used in the calculation of the absolute value of $\chi^{2}$ (Ref. 5). When uncertainties are to be calculated, the standard deviation is defined by a change in a given parameter, with re-optimization of the other parameters, such that $\chi^{2}$ is increased by one. ${ }^{5}$ If uncertainties are not calculated, the absolute value of $\chi^{2}$ is not important.

Line $\mathbf{5}$ indicates the number of vibrational stick spectra to be read in (either 1 or 2). In this example only one file is read in. However, one may read in, for example. both the vibrational stick spectra for the $X \frac{1}{2}$ and $I \frac{3}{2}$ electronic states for simultaneous fitting with the same experimental spectrum. If two vibrational stick files were specified, the input file would have to contain additional lines to specify fitting parameters for the second electronic state; for details see the listing of the source code in Section B3 below. Line 6 contains the file name of the vibrational stick spectrum for the first (and in this example, only) electronic state. Line 7 is an integer which specifies the case (c) electronic state: 1 for the $X \frac{1}{2}$ state, 2 for the $I \frac{3}{2}$ state, or 3 for the $I I \frac{1}{2}$ state. This input determines the form of the rotational line strength factors, as shown in Table B2. Lines 8 and 9 specify the neutral and anion bond lengths, respectively, in $\AA$. Line 10 lists the atomic masses in amu. This information is used to calculate $B_{e}$. 
Line 11 specifies the name of the file to which the final rotational spectrum, convoluted with the ZEKE line shape, is saved. This spectrum is in wavenumber format, with the same number and spacing of points as the experimental spectrum.

The remaining line, 12-41, are grouped into sets of five lines for each parameter in the fit. The first line of each set is set to " $y$ " if the parameter is to be adjusted during the fit, or to " $n$ " if it is to be fixed at its initial value. The second line of the set is set to " $y$ " if one wishes to find the standard deviation of a given parameter or " $n$ " if not. Note that line 1 of the input file must be set to 1 or 3 for any uncertainties to be computed. The third line of each set specifies the initial value of the parameter. The fourth line specifies the initial step size for the gradient minimization. The step size is adjusted as the fit progresses. The fifth line lists the minimum and maximum allowable values of the parameters. An explanation of each parameter and is given below:

Lines 12-16: base $10 \log$ of $C_{\mathrm{Y} / 2}$ [see Eqs. (B13) and (B14)]

Lines 17-21: intensity of the highest peak of the convoluted spectrum relative to the experimental spectrum. If two electronic states are included in the fit, the intensity of one may be fixed and the other allowed to vary in order to determine the relative transition strengths.

Lines 22-26: the rotational temperature of the anion in Kelvin. A Boltzmann distribution of anion rotational states is assumed.

Lines 27-31: the ZEKE FWHM in $\mathrm{cm}^{-1}$

Lines 32-36: the shift in the rotational origin, in $\mathrm{cm}^{-1}$

Lines 37-41: the baseline added to the convoluted spectrum, as a fraction of the maximum peak height. 


\section{B2.2. Running the program}

One runs the program in the usual Unix fashion, by piping in the input file. If one is calculating standard deviations as well as performing a fit it is usually desirable to run the program in the background, as in the following example using the input file "xebr_x_rfin" discussed above:

$>$ nice +19 sfit < xebr_x_rfin > xebr_x_Ifout \&

$>$

The output file "xebr_x_rfout" then contains the optimized parameters and standard deviations, and the convoluted spectrum is saved to the file "xebr_x_fit" named in the input file.

\section{B2.3. Outline of the program}

The program "rfit" is contained in only one file, "rfit.f." There is no makefile; the program is recompiled with a command such as:

$>£ 77-0$-0 Ifit Ifit.f $-C$

The subroutines and functions used by the program are listed in Table B3. 
Table B3. Subroutines and functions used by the rotational fitting program "rfit".

\begin{tabular}{|c|l|}
\hline $\begin{array}{c}\text { Subroutine or } \\
\text { Function }\end{array}$ & main program \\
\hline rfit & general-purpose subroutine implementing the gradient \\
optimization method
\end{tabular}




\section{B3. Source code for the rotational fitting program "rfit"}

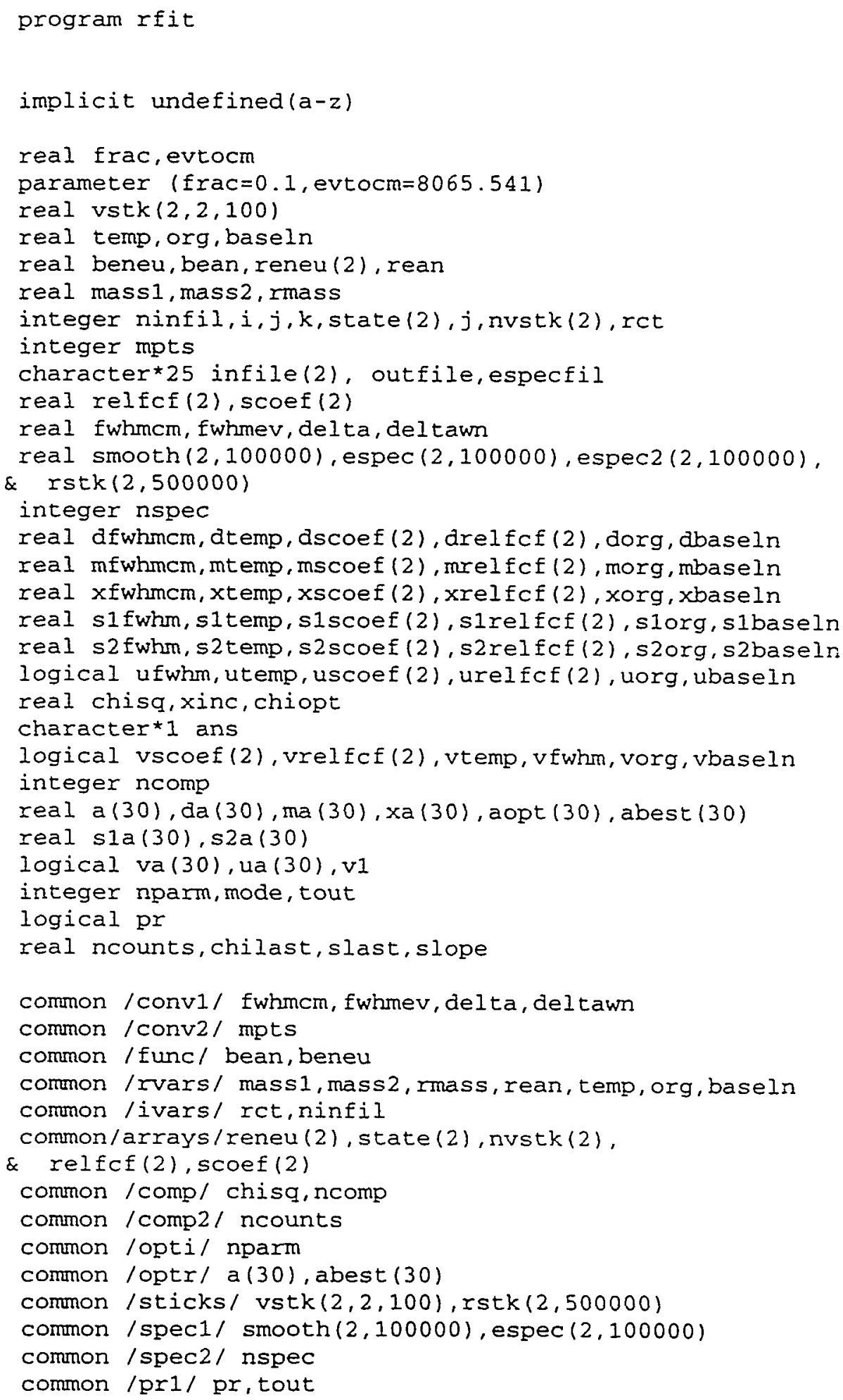

* Read in constants and fitting parameters 


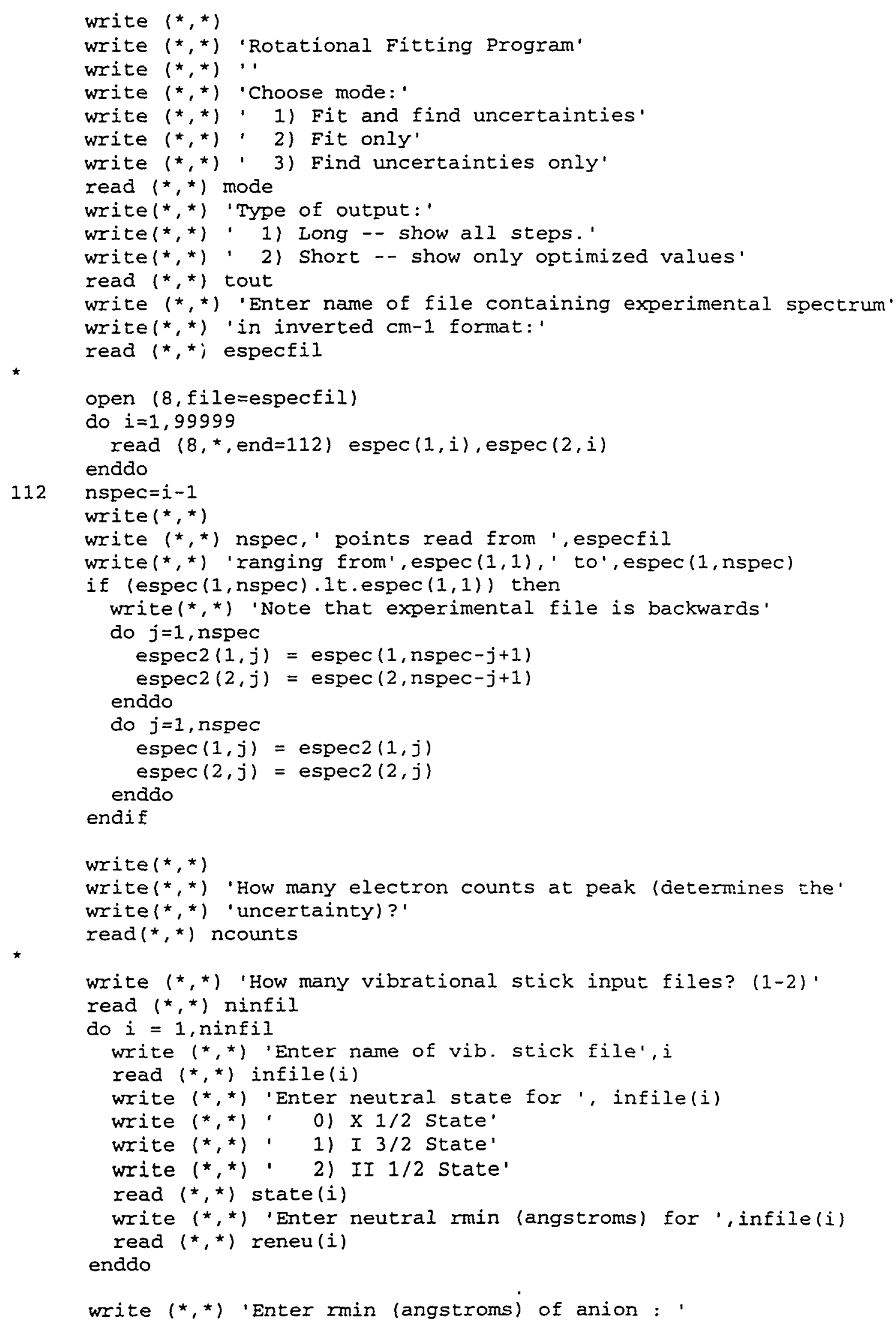




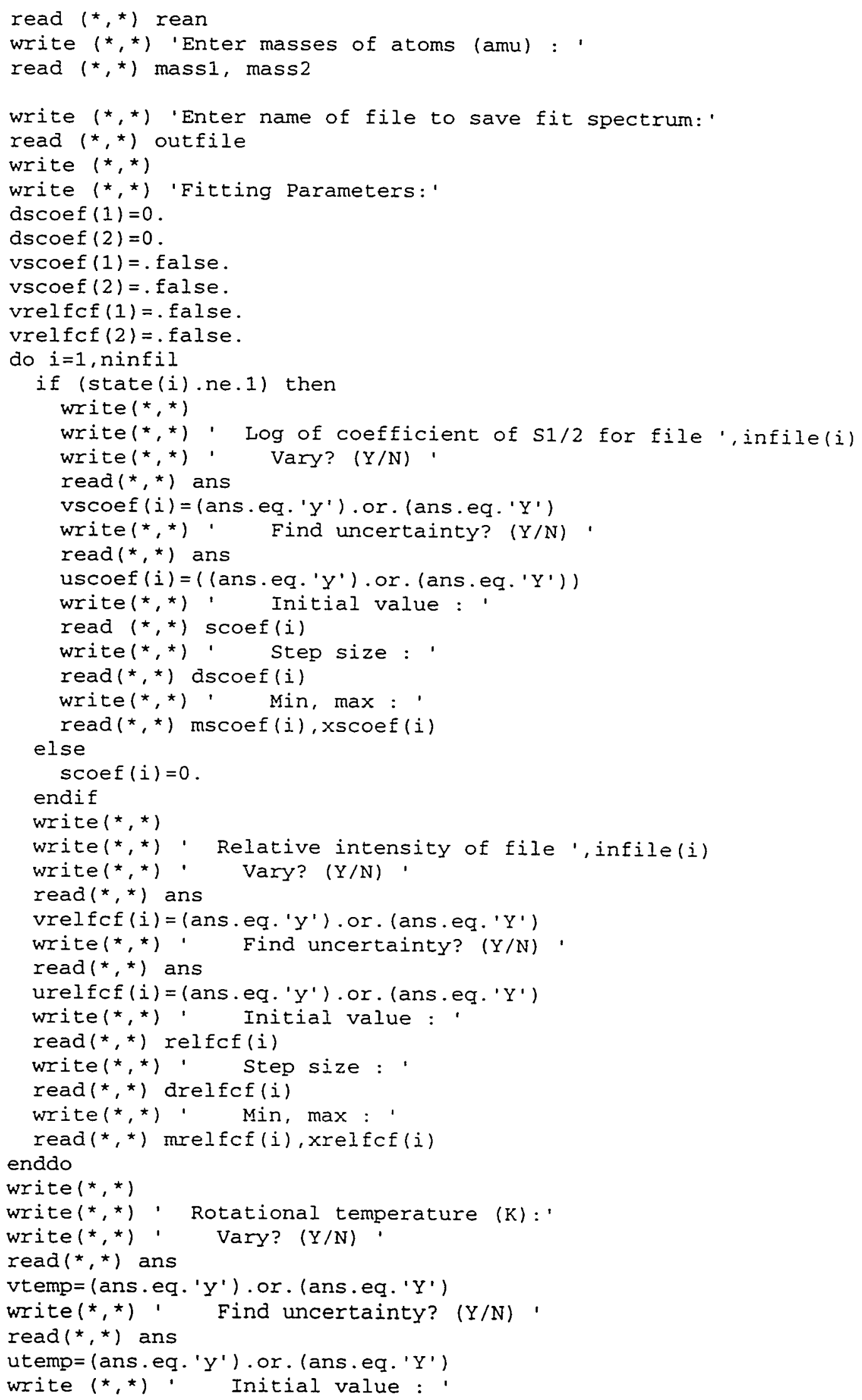




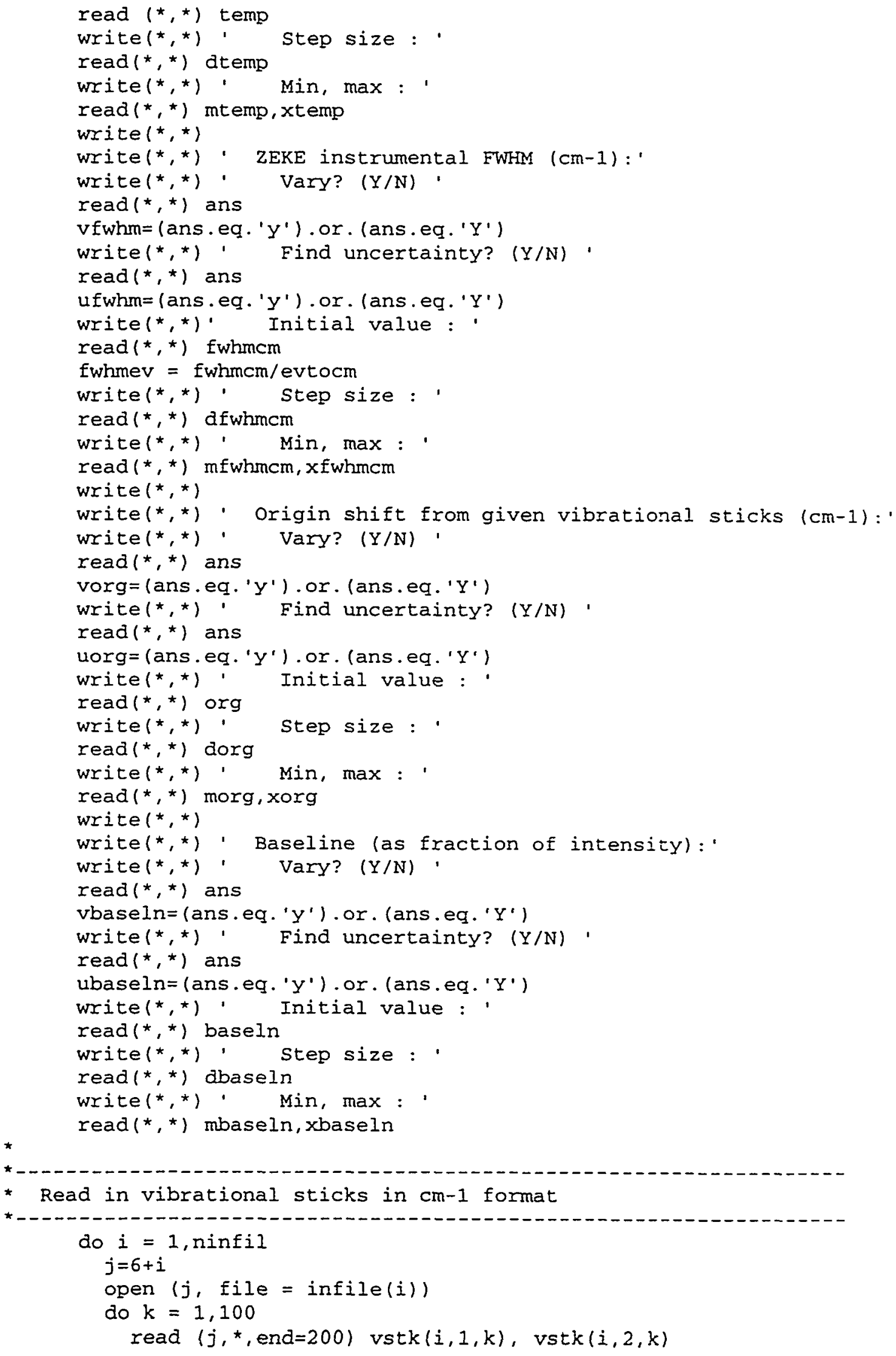




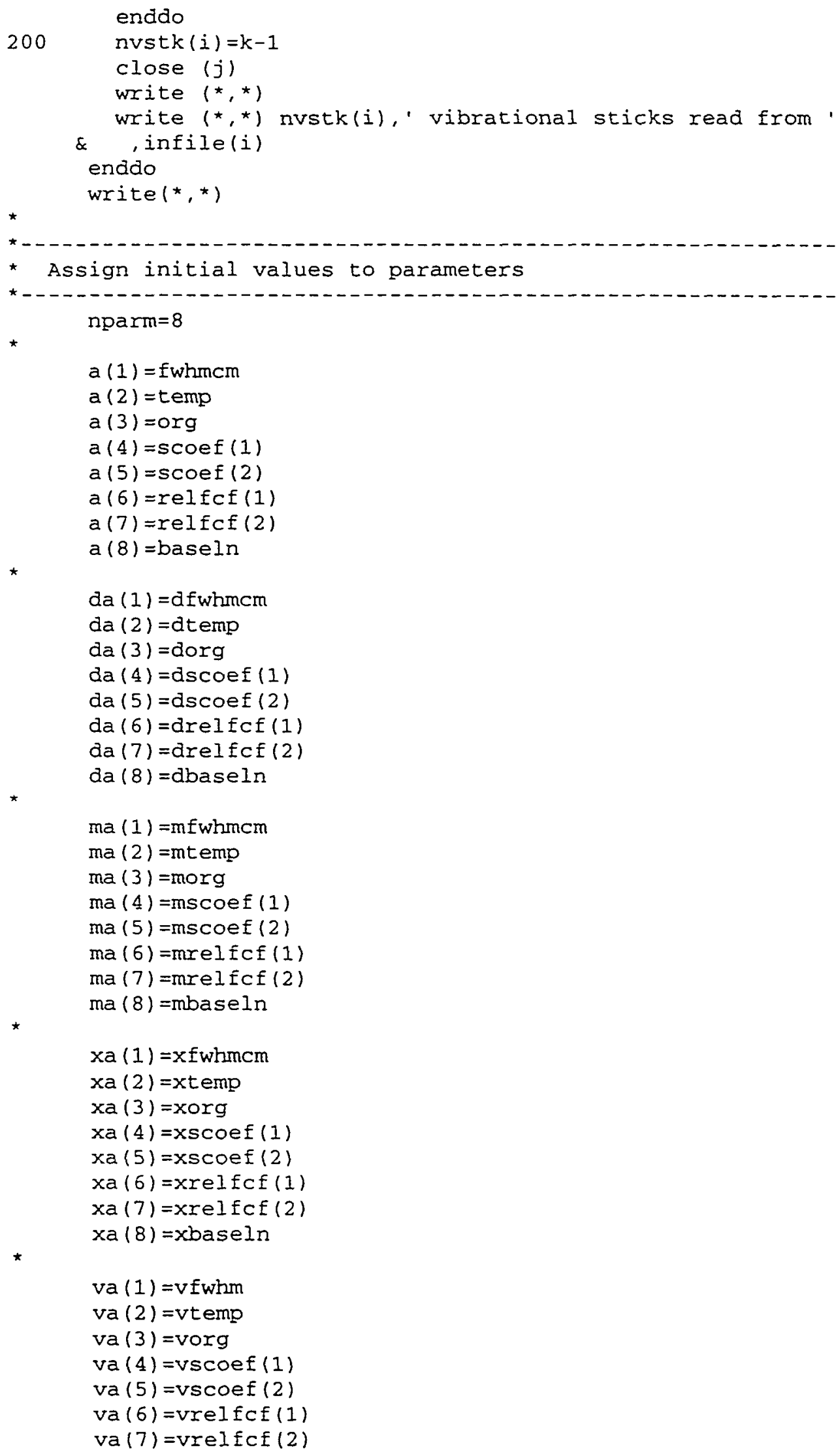




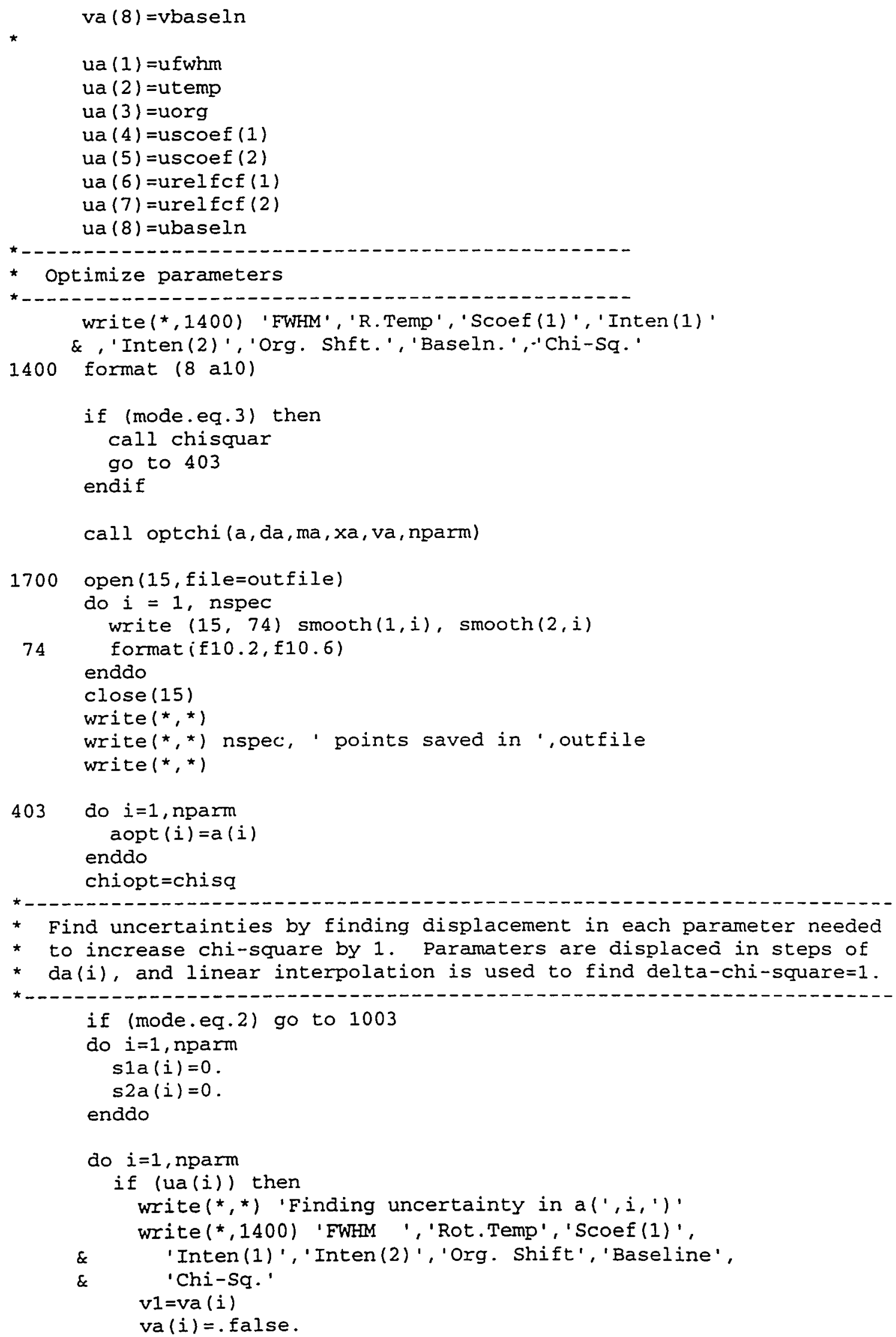




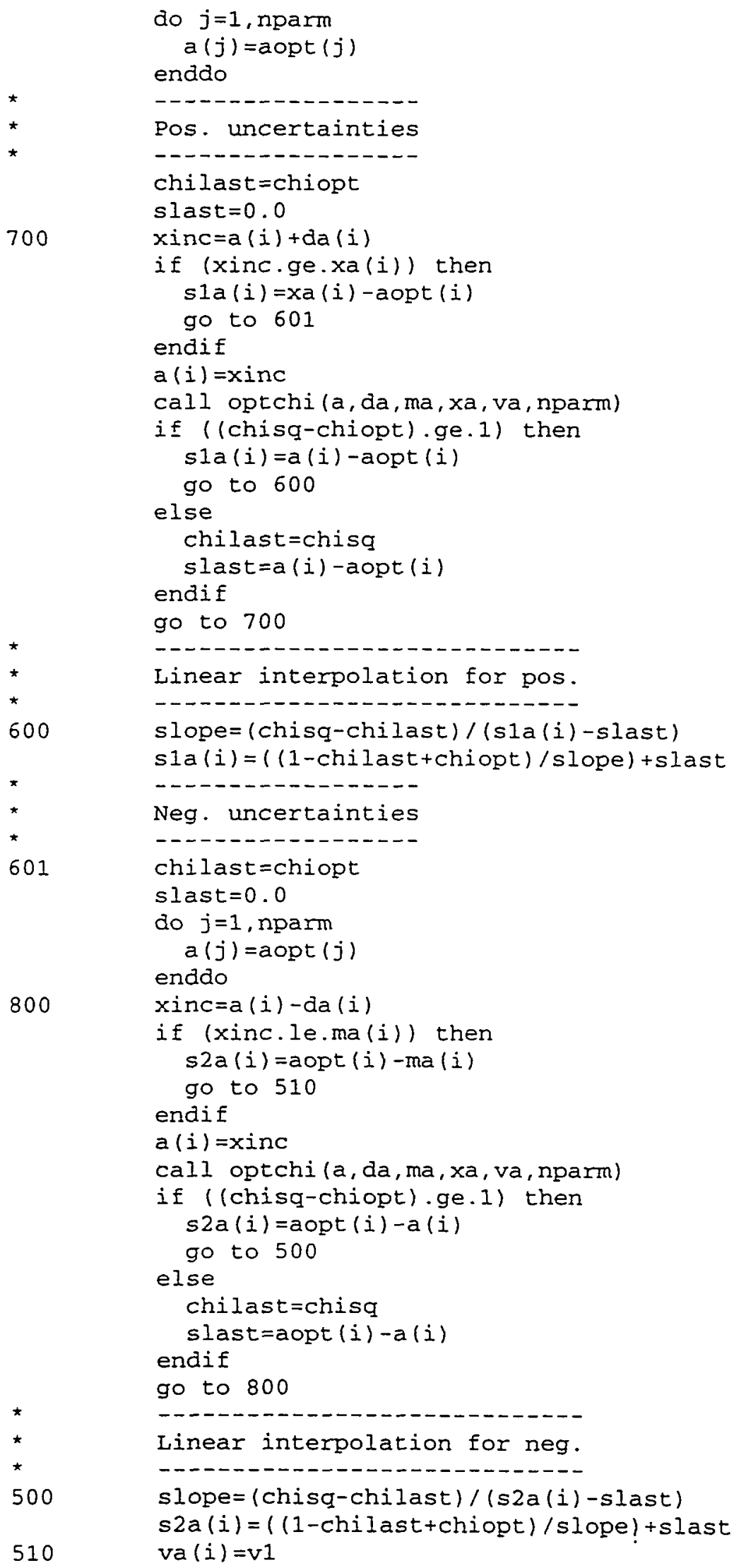




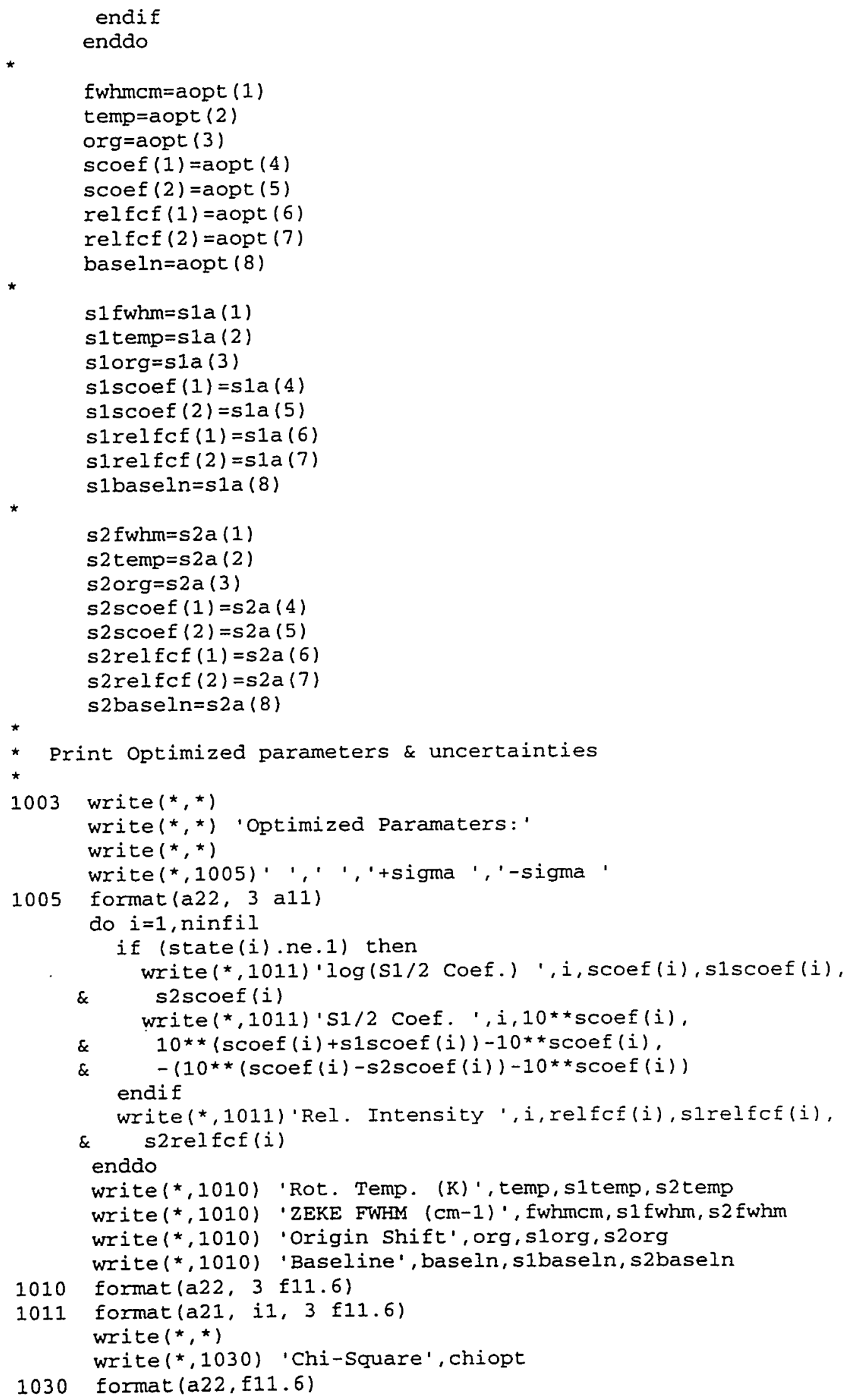


write $\left(*,{ }^{\star}\right)$

end

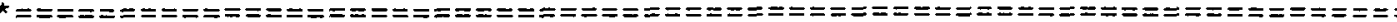

* Subroutine to find optimal parameters, given starting parameter

* values, a; parameters to vary, va; min and max parm values, ma,

$\star x a$.

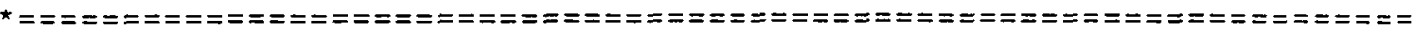

subroutine optchi (a, da, ma, xa, va, nparm)

parameter ( $\mathrm{Erac}=0.1$, niter $=20)$

integer nparm, rct, ninfil

real a (30), da (30), ma (30), xa (30)

real ga (30)

real abest $(30)$

logical va(30), vany,pr

real chisq, chibest, chinow, chiprev, gnorm, xinc

integer $i$, count, ncomp, tout

common /comp/ chisq, ncomp

cormon /ivars/ rct,ninfil

common /pri/pr,tout

common /spec2/ nspec

* Find gradient of Chi-Square

pr $=$.true.

call chisquar

if (tout.eq.2) then

pr $=$. false.

endif

chinow=chisq

chibest $=$ chisq

do $i=1$, nparm

abest $(i)=a(i)$

enddo

count $=1$

vany $=$. false.

do $i=1$, nparm

if (va(i)) then

vany $=$.true

endif

enddo

if (.not.vany) then

return

endif

1500 do $i=1, n p a r m$

ga $(i)=0$

enddo

pr $=$. false.

do $i=1$, nparm

if (.not.va(i)) go to 300 


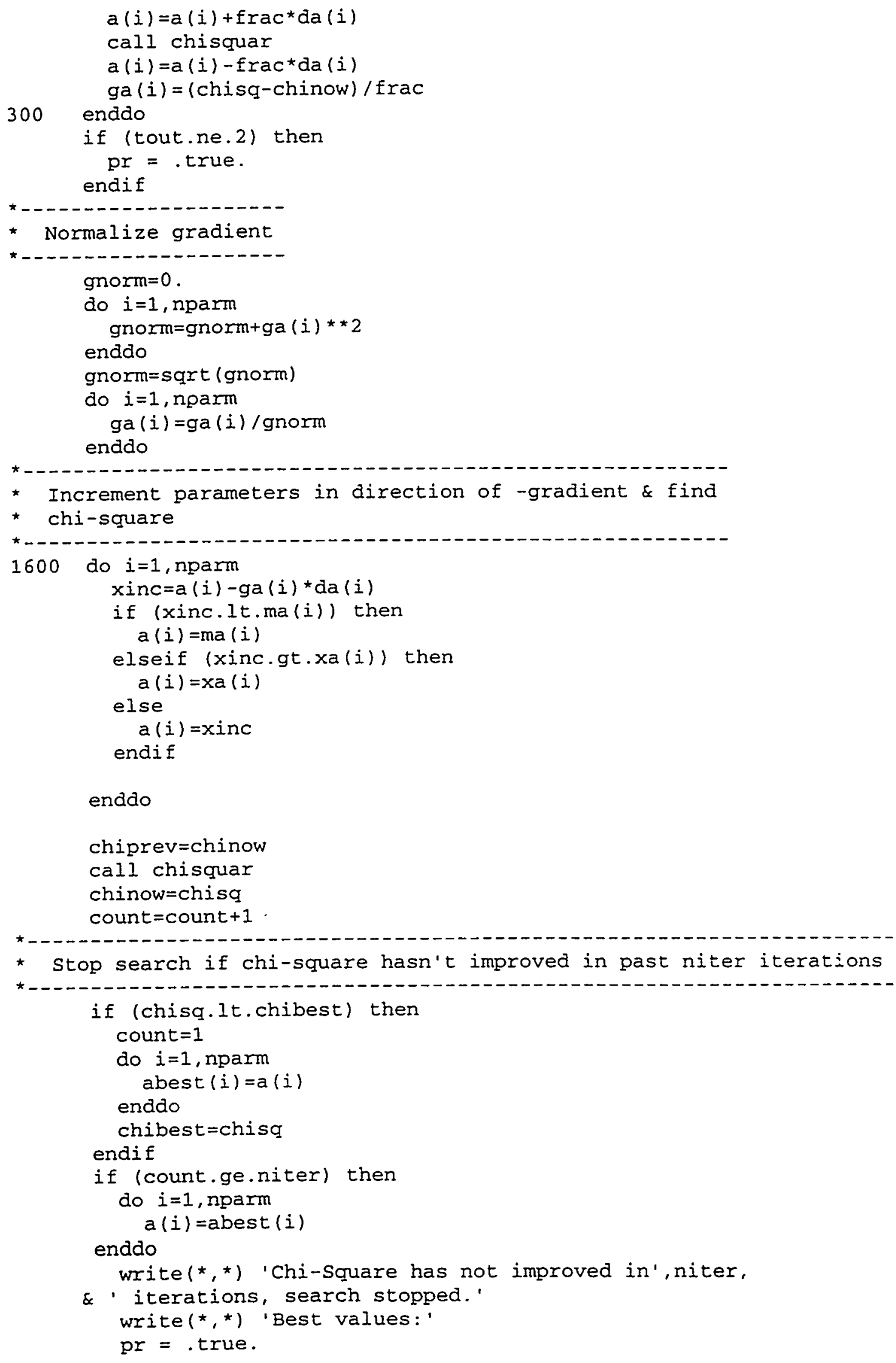


call chisquar

return

endif

* Find gradient again if chi-square has increased, otherwise

* continue to increment parameters along same gradient.

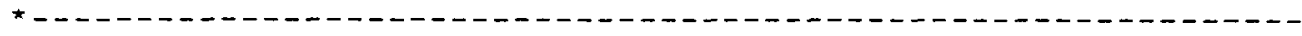

if (chinow.gt.chiprev) go to 1500

go to 1600

end

*

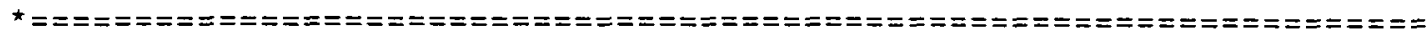

* Subroutine to find chi-square from parameter list a (nparm).

* $a(1)=$ fwhmcm, $a(2)=$ rot. temp, $a(3)=$ origin shift

$\star \quad a(4)=\operatorname{scoef}(1), a(5)=\operatorname{scoef}(2), a(6)=$ rel inten(1),

* $a(7)=$ rel inten $(2), a(8)=$ baseline.

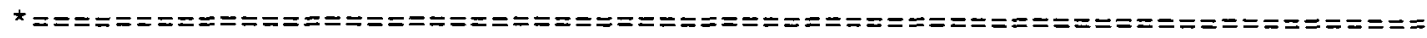
subroutine chisquar

integer nparm

real a $(30)$, abest $(30)$

parameter (evtocm $=8065.541$ )

real vstk $(2,2,100), \operatorname{rstk}(2,500000)$

real temp, org

real reneu( 2 )

integer state(2), nvstk(2), rct, ninfil

integer mpts

real relfcf (2), scoef (2)

real fwhmcm, fwhmev

real $\operatorname{smooth}(2,100000)$, espec $(2,100000)$

integer nspec

real chisq

integer ncomp

logical pr

common /convi/ fwhmcm, fwhmev, delta, deltawn

common /conv2/ mpts

comnon /func/ bean, beneu

comron /rvars/ mass1, mass2, rmass, rean, temp, org, baseln

common /ivars/ rct, ninfil

common/arrays/reneu(2), state (2), nvstk (2),

$\& \quad r e l f c f(2), \operatorname{scoef}(2)$

common /comp/ chisq, ncomp

common/opti/nparm

common/optr/a $(30)$, abest (30)

common /sticks/vstk(2,2,100), rstk $(2,500000)$

common /spec1/ smooth(2,100000), espec $(2,100000)$

common /spec2/ nspec

common /pr1/ pr, tout

fwhmcm $=a(1)$

fwhmev $=$ fwhmcm/evtocm

temp=a (2)

org $=a(3)$

$\operatorname{scoef}(1)=a(4)$

$\operatorname{scoef}(2)=a(5)$ 
relfcf $(1)=a(6)$

relfcf $(2)=a(7)$

baseln $=a(8)$

call rsticks (vstk, rstk, NR)

call conzeke (rstk, rct, espec, nspec, fwhmcm, relfcf (1), baseln,

\& smooth, NR, NS)

call cp2 (espec, smooth, nspec)

if (pr) then

write(*,1405) fwhmcm, temp, scoef(1), relfcf(1),

\& relfcf $(2)$, org, baseln, chisq

endif

1405 format ( $8 \mathrm{~g} 10.4$ )

return

end

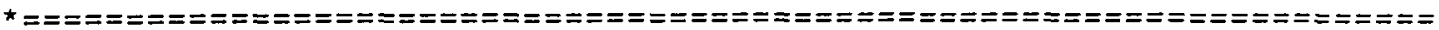

* Calculate rotational sticks-Note in this subroutine, scoef()

* is assumed to actually be $\log (\operatorname{scoef}())$. And $s c()$ is the real coef.

* This is different from the rsticks. $f$ program.

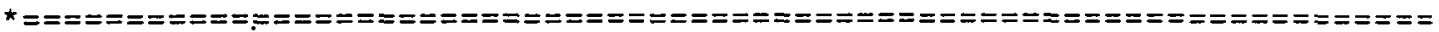
subroutine rsticks (vstk, rstk, NR)

$\star$

* Common variables

$\star$

parameter (evtocm $=8065.541$ )

common /func/ bean, beneu

common /rvars/ mass1,mass2, rmass, rean, temp, org, basein

common /ivars/ ret, ninfil

common/arrays/reneu (2), state (2), nvstk (2),

\& $\operatorname{relfcf}(2), \operatorname{scoef}(2)$

*

real bean, beneu

real massl, mass2, rmass, rean, temp, baseln

integer rct,ninfil

real reneu(2), relfcf(2), scoef (2)

integer state(2), nvstk(2)

*

* Variables local to rsticks subroutine:

$\star$

real omneu, vorg, vfcf, maxstk, jan, jneu, linstr, sc (2)

integer $i, k, r c(2)$

real vstk $(2,2,100), \operatorname{rstk}(2,500000)$, rstk2 $(2,500000)$

*_...

do $i=1$, ninfil

$\operatorname{sc}(i)=10 *$ scoef $(i)$

enddo

rmass $=\operatorname{mass} 1 * \operatorname{mass} 2 /(\operatorname{mass} 1+\operatorname{mass} 2)$

bean $=$ be (rean, rmass)

rct $=1$

do $i=1$, ninfil

beneu $=$ be (reneu $(i)$, rmass $)$

if (state (i).eq.1) then

omneu $=1.5$ 


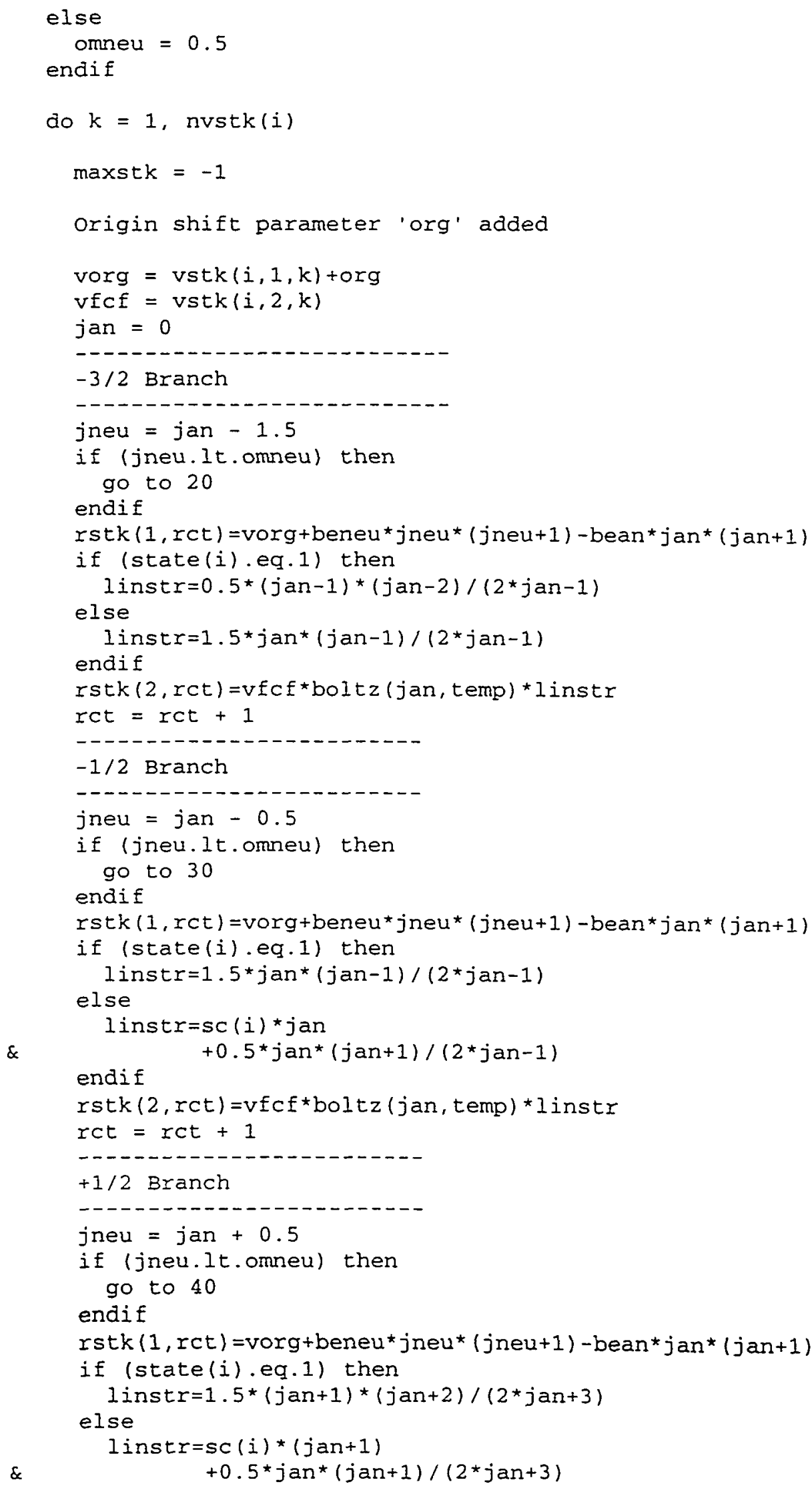




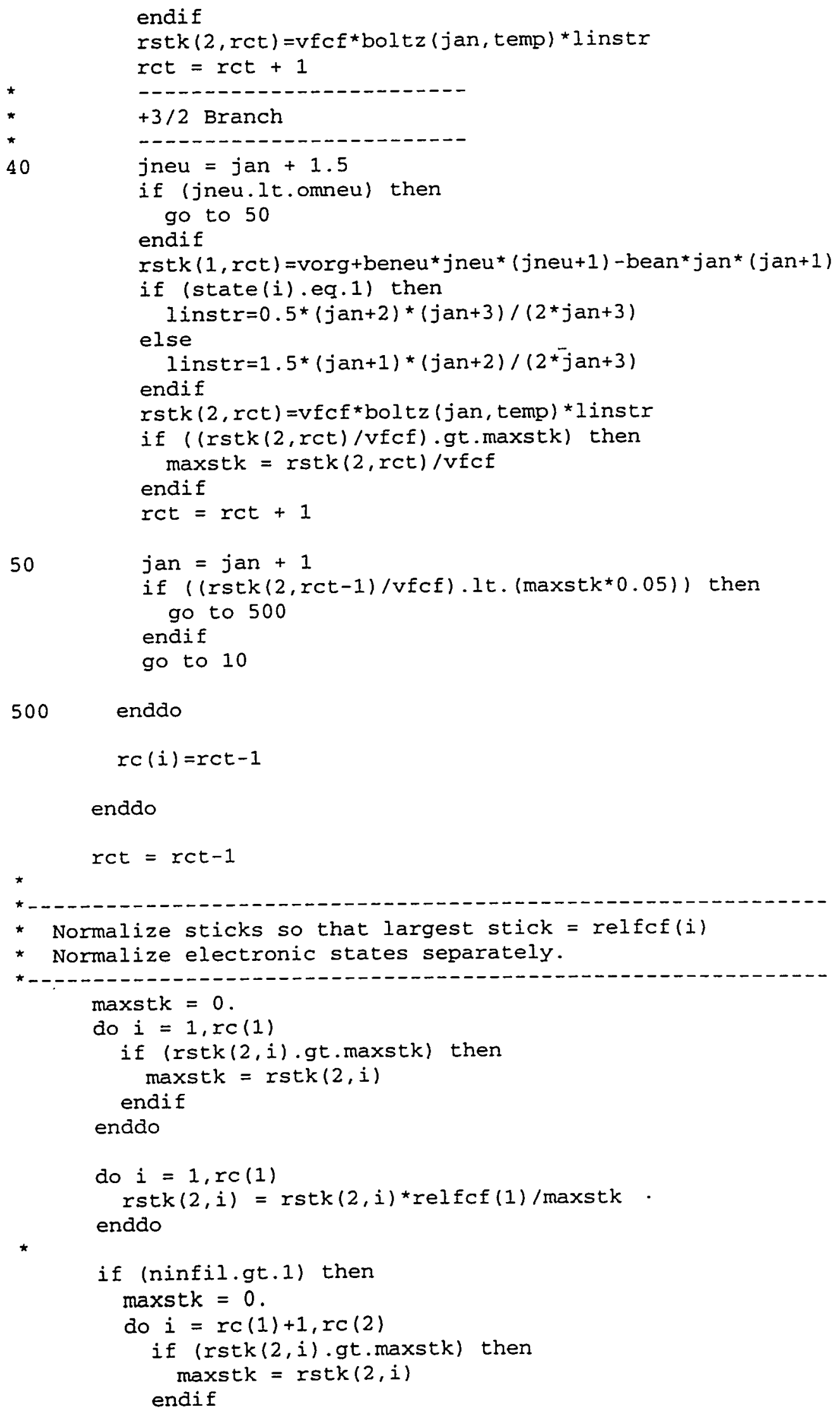




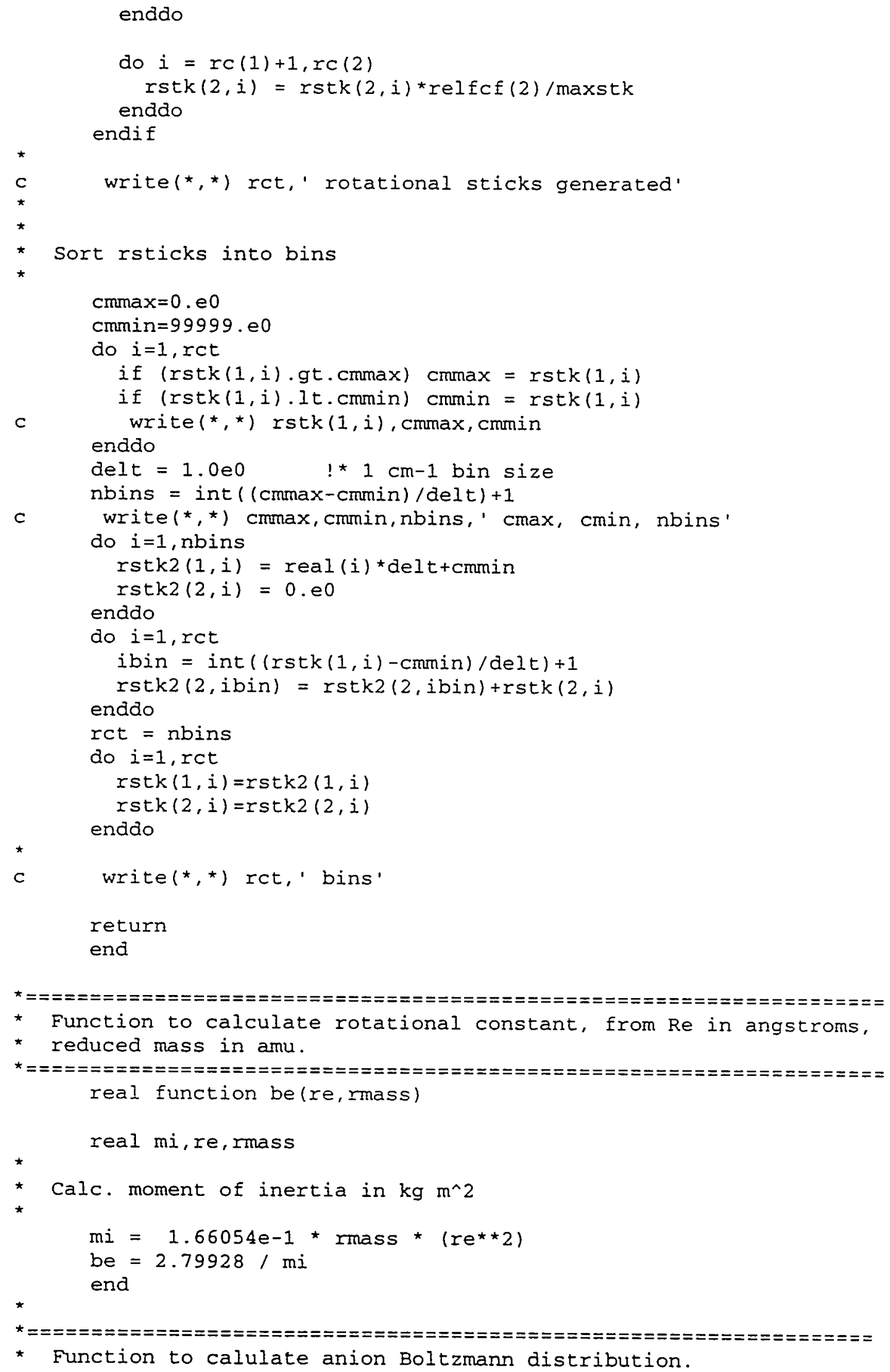$$
\text { * Calc. moment of inertia in } \mathrm{kg} \mathrm{m}^{\wedge} 2
$$$$
\mathrm{mi}=1.66054 \mathrm{e}-1 \text { * rmass } *(r e * * 2)
$$$$
\text { be }=2.79928 / \mathrm{mi}
$$$$
\text { end }
$$ 
* Note that line strength already includes degeneracy.

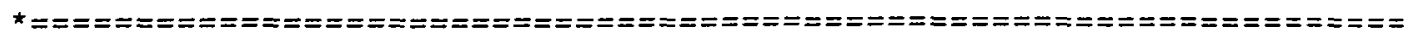
real function boltz (jan, temp)

*

* Convert temp in $\mathrm{K}$ to $\mathrm{cm}-1$

$\star$

common /func/ bean, beneu

real jan, temp, tempk, er

real bean, beneu

tempk $=0.69504 *$ temp

er $=\operatorname{bean}^{\star} j a n *(j a n+1)$

bolt $z=2.71828 * *(-$ er $/$ tempk $)$

return

end

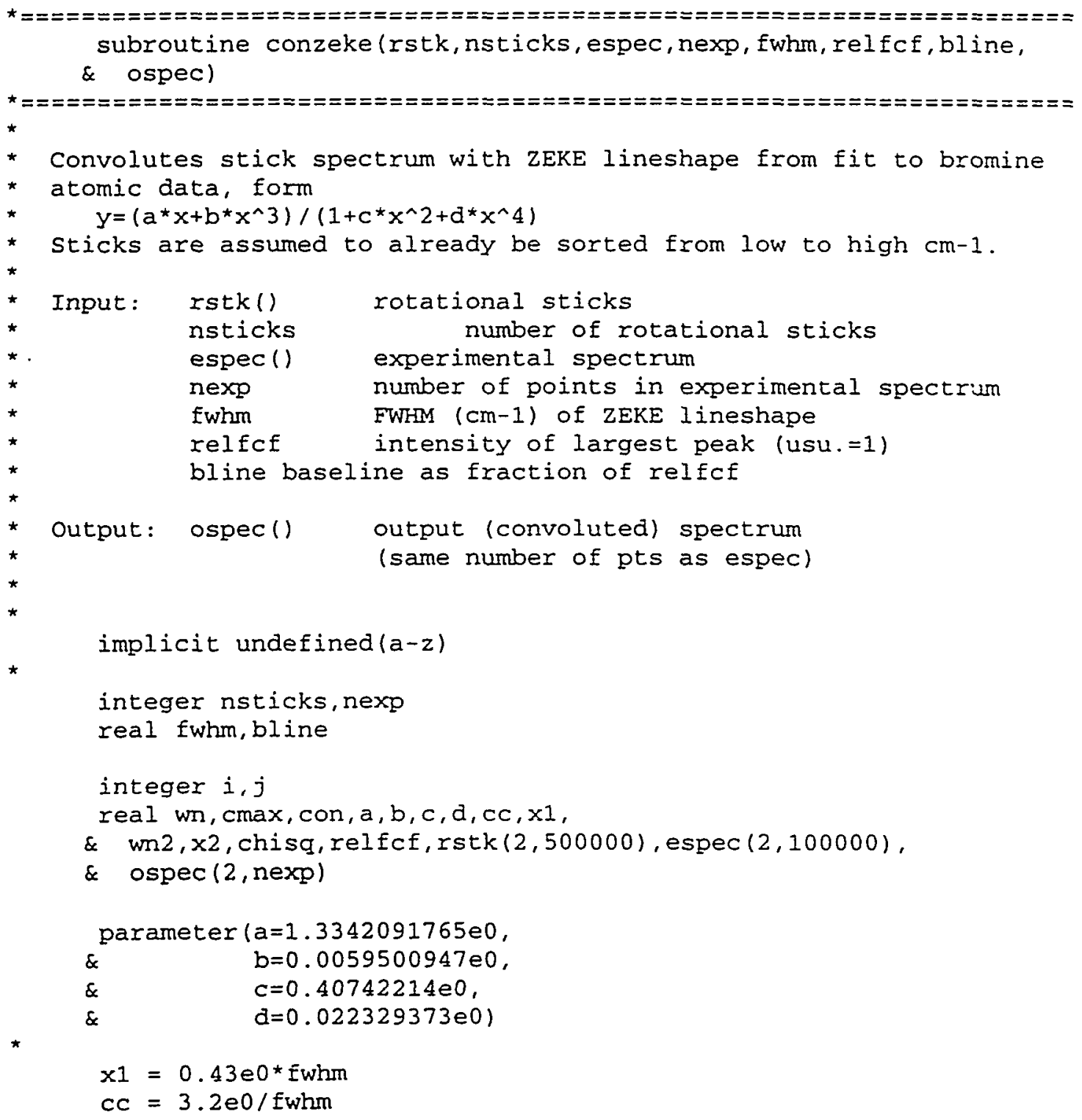




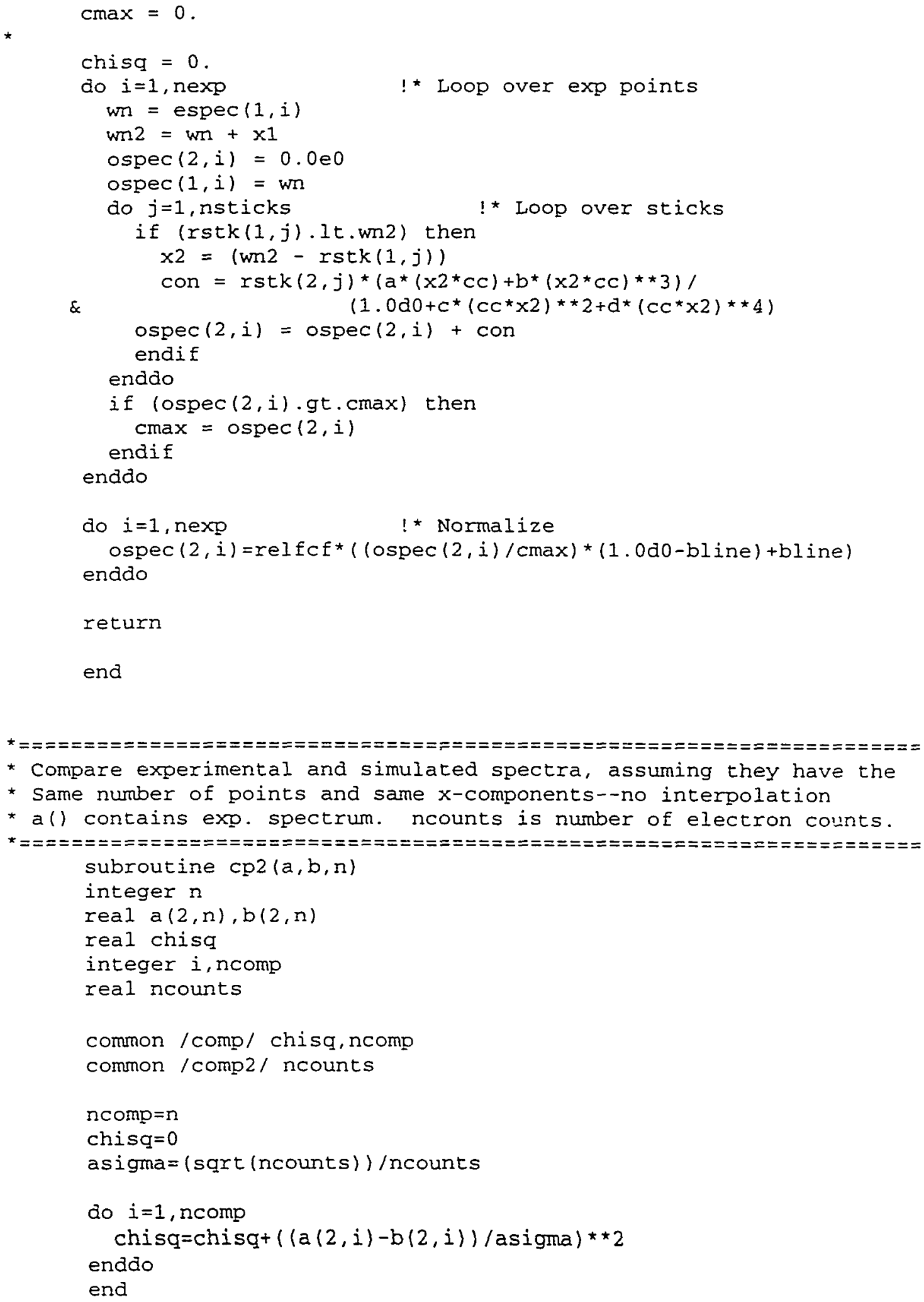


B4. References for Appendix B

1 J. Xie and R. N. Zare, J. Chem. Phys. 93, 3033 (1990).

2 J. Xie and R. N. Zare, J. Chem. Phys. 97, 2891 (1992).

3 A. D. Buckingham, B. J. Orr, and J. M. Sichel, Philos. Trans. R. Soc. London, Ser. A 268, 147 (1970).

4 R. N. Zare, Angular Momentum (Wiley, New York, 1988).

5 P. R. Bevington, Data Reduction and Error Analysis for the Physical Sciences (McGraw-Hill, New York, 1969). 


\section{Appendix C. Simulated Annealing}

\section{C1. Introduction}

One problem we face in trying to understand the energetics of a cluster containing many atoms or molecules is determining the global minimum equilibrium geometry. Especially in the case of the weakly bound clusters considered in this work, the number of possible isomers increases dramatically as one adds more atoms. For example a cluster of 13 identical Lennard-Jones atoms has 988 stable, distinct geometric isomers.' It is also known that for large clusters the number of local minima increases exponentially with the number of atoms. ${ }^{2}$ Often there are many local minima located close in energy to the global minimum energy geometry, and this makes the problem of finding the global minimum non-trivial.

\section{C2. Brief survey of methods of finding global minima of clusters}

If one had an arbitrarily fast computer, the problem of finding the global minimum would simple. One could simply calculate the cluster potential at all points on as small a grid as required to locate the minimum. In this "brute force" approach, it would be necessary to calculate the potential points on a $3 N-6$ dimensional grid ( $N$ being the number of atoms in a polyatomic cluster). If a 100 point grid were used, this would amount to $10^{6}, 10^{12}$ and $10^{18}$ calculations of the potential for 3,4 and 5 atom clusters, respectively. Hence, we can see that with currently available computers, such a "brute force" calculation is impractical for clusters with more than 3 or 4 atoms. In the case of some small clusters, this approach could be speeded up by making assumptions about the 
symmetry of the equilibrium geometry. However this is not a generally used technique for large clusters and will not be considered further.

Many algorithms are available for finding the minimum of a mathematical function when initial values of the independent variables are assumed. ${ }^{3}, 4$ These include gradient minimization, ${ }^{4}$ the simplex method, ${ }^{3}$ and conjugate gradient methods. ${ }^{3}$ If the internuclear coordinates of the cluster are taken to be the independent variables and the potential energy the function, then any of these methods may be used to efficiently find a minimum on the potential energy surface. The problem with all of these techniques when used by themselves, however, is that they cannot distinguish between local and global minima. For a given initial configuration of atoms, the minimization algorithm may become trapped in a local minimum.

One approach that may be used to try to locate the global minimum energy structure is to apply the minimization algorithm repeatedly, with many different--possibly randomly chosen--starting geometries, and with luck and persistence the global minimum may be stumbled upon in this way. This is a viable approach, but it suffers from the drawback that it is hard to determine if one has found the true global minimum. Also it is not a very systematic approach, relying as it does on random or arbitrarily chosen points on the potential energy surface.

Another quite elegant method for finding the global optimum of a system was first developed and applied to problems in computer design by Kirkpartick et al. ${ }^{5}$ This is the method of simulated annealing, which was based on the Metropolis Monte-Carlo algorithm $^{6}$ originally developed as a general-purpose tool for characterizing the 
properties of large collections of interacting molecules at a given temperature, i.e., a way of simulating a canonical ensemble of particles at equilibrium.

The original Metropolis Monte Carlo method ${ }^{6}$ may be outlined as follows:

(1) An arbitrary initial configuration of the system is chosen.

(2) A random displacement is made in the system.

(3) The change in the energy, $\Delta E$, of the system is calculated.

(4) If $\Delta E<0$, the move is accepted, the system is reset with the new configuration, and the simulation continues again with step 2 .

(5) If $\Delta E>0$, the move is allowed with a probability of $\exp (-\Delta E / k T)$, where $k$ is the Boltzmann constant and $T$ is the temperature. In practice, this is implemented by choosing a random number, $\xi$, between 0 and 1 , and allowing the move if $\xi<\exp (-$ $\triangle E / k T)$.

Metropolis et al. showed that this if one averages any property of interest over each step in the simulation, one may calculate accurate values of thermodynamic properties, the accuracy being limited only by the duration of the simulation (and of course by the accuracy of the model interaction potential between the particles).

To illustrate how the Metropolis algorithm is applied to global optimization, we begin by following the discussion in Press et al. ${ }^{3}$ of the simulated annealing method applied to the "travelling salesperson" problem. In this problem, a salesperson must travel to a large number of cities, visiting no city more than once, and the cost of traveling between the cities is proportional to the distance between the cities. The problem then consists of finding the order of cities that minimizes the cost. This is a 
problem in discrete combinatorial mathematics. According to Kirkpatrick et al..$^{5}$ there is no known algorithm for finding an exact solution to this problem for which computation time does not scale exponentially with the number of cities visited. This is consistent with our above discussion of the scaling of the 'brute force' approach for finding the global minimum energy of a cluster. However, according to Press et al., "simulated annealing has effectively 'solved' the famous traveling salesman problem..." 3 The difficulty in this problem lies in the fact that there are many possible near-solutions-solutions close in cost to the optimal solution, as is the case in the cluster problem. The simulated annealing algorithm for a discrete combinatorial problem such as this is:

(1) Choose an initial ordering (permutation) of the set of cities.

(2) Calculate the "cost function" or "objective function," which we will also call $E$, which in this case is the sum of the distances between the cities taken in order.

(3) Rearrange the ordering of the cities.

(4) As in the Metropolis algorithm, evaluate $E$ for the new permutation and keep the move if $E$ is reduced, or, if $E$ is increased, keep the move with a probability proportional to $\exp (-E / k T)$. In this specific example, of course, $k$ no longer has the same value as the Boltzmann constant, but may be considered a scaling factor between the cost function $E$ and an effective "temperature," $T$.

(5) Run the Metropolis algorithm for a large number of iterations, to allow the system to "equilibrate."

(6) Gradually reduce the effective temperature, $T$, according to an "annealing schedule," until the system becomes "frozen," i.e., there is no further change in the arrangement of order of visiting the cities. 
Press et al. ${ }^{3}$ provide a computer program to explicitly treat the traveling salesperson problem by simulated annealing, as well as an excellent introduction to the concepts of simulated annealing and other optimization methods.

The beauty of the simulated annealing algorithm as shown in this example is that, unlike the direct minimization procedures mentioned above the algorithm is much less likely to become trapped in local minima. The reason for this is that the system of interest is allowed to explore a portion of phase space at each stage in the annealing schedule, and moreover, "uphill" moves are allowed, according to the Boltzmann distribution. Thus, even if the system finds itself within a local optimum during the procedure, it has a chance to "escape" to find the global optimum.

Kirkpatrick et al. confined their discussion to discrete systems, and simulated annealing has since been used extensively in VLSI design and for other discrete combinatorial problems. The implementation of a simulated annealing algorithm becomes more complicated in the case of a problem involving continuous variables. A large part of the problem lies in the optimal choice of step sizes and directions. This problem has been discussed at length by Vanderbilt and Louie. ${ }^{7}$

The problem of finding the global minimum energy of a cluster is, of course, a problem involving continuous variables. Monte Carlo approaches have been successfully used to such problems; see, for example, Ref. 8. Another approach is also used for physical systems, in which the molecular dynamics (MD) methods are used to simulate the actual motions of the atoms and molecules in the system. Various methods are used to adjust the system in order to produce a distribution kinetic energies corresponding to a 
certain temperature. These methods are described in more detail in the following sections. Then, as in Monte Carlo methods, the temperature is reduced according to an annealing schedule, until the system freezes at the global minimum configuration.

The molecular dynamics approach to simulated annealing has the advantage of intuitive simplicity, and a closer resemblance to the actual behavior of a physical system. Thus it is the method of choice if one wishes to study such things as the dynamics of cluster formation. However, if one wishes to simply find equilibrium geometries, the molecular dynamics method would seem to be more computationally time-consuming than the Monte-Carlo approach. This is because to perform a $\mathrm{MD}$ simulation one must calculate forces as well as potentials in order to solve the equations of motion. The MD approach was used for the current studies because easily-adapted pre-existing $\mathrm{MD}$ programs were available, not because of considerations of computational efficiency. To our knowledge no systematic study has been made of the relative computational efficiency of the Monte Carlo and MD simulated annealing methods.

\section{C3. Molecular dynamics simulated annealing}

In this section we describe our approach to simulated annealing, which is based on a molecular dynamics program obtained from Prof. C. C. Martens (UC Irvine). First we review the basics of constant-energy (microcanonical) molecular dynamics simulation methods. We then discuss how to implement constant-temperature molecular dynamics (to simulate a canonical ensemble). Finally we discuss a modification of the latter method for simulated annealing. 


\section{C3.1 Microcanonical molecular dynamics}

The basic problem that we will deal with here is how to simulate the motions of atoms which interact according to potential energy functions. Our treatment is drawn mainly from the book Computer Simulation of Liquids, by M. P. Allen and D. J. Tildesley, ${ }^{9}$ which discusses these issues in much more depth, and is an essential resource for anyone interested in molecular dynamics simulations. We assume that the BornOppenheimer approximation is valid, and treat the atoms as points interacting according to classical mechanics. The problem then becomes how to solve the equations of motion of a set of $i$ atoms with masses $m_{i}$ and momenta $\mathbf{p}_{i}$ at positions $\mathbf{r}_{i}$ under the influence of the potential $V\left(\mathbf{r}_{i}\right)$ due to interactions with the other atoms. The equations of motion can then be represented by: 9

$$
\begin{aligned}
& \frac{d}{d t} \mathbf{r}_{i}=\frac{\mathbf{p}_{i}}{m_{i}} \\
& \frac{d}{d t} \mathbf{p}_{i}=-\nabla V\left(\mathbf{r}_{i}\right)=\mathbf{f}_{i}
\end{aligned}
$$

where $f_{t}$ is the force on atom $i$.

Of the many algorithms available for solving the equations of motion, the most commonly used methods in molecular dynamics computations are the Gear predictorcorrector algorithm, which solves the set of first order differential equations given above, and the Verlet algorithm, which treats the problem in terms of a single set of second order differential equations. Both of these are finite difference methods, i.e. methods which start from an initial configuration and propagate the equations forward in time. For a discussion of the relative merits of these and other methods, see Allen and Tildesley. 9 Well-documented computer code to implement these algorithms as described 
in Allen and Tildesley's book may be obtained from the website: http://toucan.phy.bris.ac.uk/AllenTildesley/home.html. The program used in this work employs the Gear predictor-corrector algorithm. Hence we confine our discussion to a brief description of this method.

As the name implies, the Gear algorithm takes place in two steps, a prediction step, and a correction step. In the first step, the configuration of the atoms are estimated from the previous configuration by a truncated Taylor expansion around the time, $t$. The program used in this work is a six-value Gear algorithm provided by the group of Prof. C. C. Martens. ${ }^{10}$ We show here the first step of this algorithm, using the notation of Allen and Tildesley:

$$
\begin{aligned}
& \mathbf{r}^{p}(t+\delta t)=\mathbf{r}(t)+\delta t \mathbf{v}(t)+\frac{1}{2} \delta t^{2} \mathrm{a}(t)+\frac{1}{6} \delta t^{3} \mathbf{b}(t)+\frac{1}{24} \delta t^{4} \mathrm{c}(t) \frac{1}{120} \delta t^{5} \mathrm{~d}(t) \\
& \mathbf{v}^{p}(t+\delta t)=\mathbf{v}(t)+\delta t \mathrm{a}(t)+\frac{1}{2} \delta t^{2} \mathbf{b}(t)+\frac{1}{6} \delta t^{3} \mathrm{c}(t)+\frac{1}{24} \delta t^{4} \mathrm{~d}(t) \\
& \mathbf{a}^{p}(t+\delta t)=\mathbf{a}(t)+\delta \mathrm{b}(t)+\frac{1}{2} \delta t^{2} \mathrm{c}(t)+\frac{1}{6} \delta t^{3} \mathrm{~d}(t) \\
& \mathbf{b}^{p}(t+\delta t)=\mathbf{b}(t)+\delta t \mathbf{c}(t)+\frac{1}{2} \delta t^{2} \mathrm{~d}(t) \\
& \mathbf{c}^{p}(t+\delta t)=\mathbf{c}(t)+\delta t \mathrm{~d}(t) \\
& \mathrm{d}^{p}(t+\delta t)=\mathrm{d}(t)
\end{aligned}
$$

Here, $\mathbf{r}, \mathbf{v}$, and a stand for the set of positions, velocities and accelerations of all of the atoms, $\mathbf{b}$ and $\mathbf{c}$ stand for the first and second time derivatives, respectively, of the acceleration, and $\delta t$ stands for the time step. The superscript $p$ indicates that these are predicted values.

The equations of motion are introduced in the second, "corrector," step. In this step the forces are calculated from Eqs. (C1) and (C2) from the predicted positions $\mathbf{r}$ at time $t+\delta t$. Then the "correct" accelerations $\mathbf{a}^{c}$ are calculated from Equation (C3). The error in the predicted accelerations are then estimated from 


$$
\Delta \mathbf{a}(t+\delta t)=\mathbf{a}^{c}(t+\delta t)-\mathbf{a}^{p}(t+\delta t)
$$

Finally, the initially predicted values are corrected using this estimated enor in the acceleration:

$$
\begin{aligned}
& \mathbf{r}^{c}(t+\delta t)=\mathbf{r}^{p}(t+\delta t)+c_{0} \Delta \mathbf{a}(t+\delta t) \\
& \mathbf{v}^{c}(t+\delta t)=\mathbf{v}^{p}(t+\delta t)+c_{1} \Delta \mathbf{a}(t+\delta t) \\
& \mathbf{a}^{c}(t+\delta t)=\mathbf{a}^{p}(t+\delta t)+c_{2} \Delta \mathbf{a}(t+\delta t) \\
& \mathbf{b}^{c}(t+\delta t)=\mathbf{b}^{p}(t+\delta t)+c_{3} \Delta \mathbf{a}(t+\delta t) \\
& \mathbf{c}^{c}(t+\delta t)=\mathbf{c}^{p}(t+\delta t)+c_{4} \Delta \mathbf{a}(t+\delta t) \\
& \mathbf{d}^{c}(t+\delta t)=\mathbf{d}^{p}(t+\delta t)+c_{5} \Delta \mathbf{a}(t+\delta t)
\end{aligned}
$$

The coefficients $c_{0}, \ldots, c_{4}$ are chosen to optimize the stability and accuracy of the algorithm, and suggested coefficients given by Gear.

\section{C3.2. Constant temperature molecular dynamics}

The method in the above section is used to simulate atoms in the microcanonical ensemble, i.e., with total energy conserved. The temperature of a cluster simulated in this way is not a well defined quantity, but follows a distribution, as the kinetic energy is not constant during the simulation. One method that has been used to simulate a canonical ensemble of atoms is to periodically rescale the velocities of all the particles by a factor:

$$
\chi=\sqrt{\frac{T_{\text {targ }}}{T_{\text {avg }}}}
$$

where $T_{\text {rarg }}$ is the desired temperature of the system and $T_{\text {avg }}$ is the average kinetic temperature of the system calculated from:

$$
T_{\text {avg }}=\frac{2}{3 N k}\langle K E\rangle
$$


where $N$ is the total number of atoms, $k$ is Boltzmann's constant, and $\langle K E\rangle$ is the average kinetic energy from the preceding time interval. This and other methods of constant temperature $\mathrm{MD}$ are described by Allen and Tildesley. ${ }^{9}$.

\section{C3.3. Using the rescaling of velocities for simulated annealing}

Our implementation of simulated annealing using molecular dynamics was suggested by modification of the above constant-temperature $\mathrm{MD}$ algorithms described above in which the velocities are rescaled at each time step by a factor involving a time constant that governs the relaxation rate towards the desired "constant" temperature. ${ }^{9}$ This suggested a method of setting the annealing schedule where the average kinetic energy of the cluster is decreased by rescaling the velocities of the atoms by the factor

$$
\chi=\sqrt{1+\frac{\Delta t}{t_{\text {const }}}\left(\frac{T_{\text {targ }}}{T_{\text {avg }}}-1\right)},
$$

where $t_{\text {const }}$ is the time constant, and $\Delta t$ is an interval of a number of time steps--in our application about 100-1000 time steps were used for $\Delta t$. The target temperature is then set to a number very small number, and the cluster is allowed to relax to this temperature. Whether or not this type of annealing schedule is optimal is open to debate, as many other schedules are possible.

\section{C4. Documentation of the simulated annealing program "amain.f"}

In this section we discuss in detail our implementation of the simulated annealing problem as applied to the $\mathrm{Rg}_{\mathrm{n}} \mathrm{X}$ systems, including examples of how to run the program, 
as well as a discussion of the construction of the program for those who wish to modify

it.

\section{C4.1 The input file}

In order to run the simulated annealing program one must supply a parameter file containing the pair potential parameters, flags to indicate which many body forces to include, and so on. A sample parameter file is given below, followed by a discussion of each of its components. This example is for the $\mathrm{Ar}_{6} \mathrm{I}^{-}$anion without any many-body forces included, titled "in 1" :

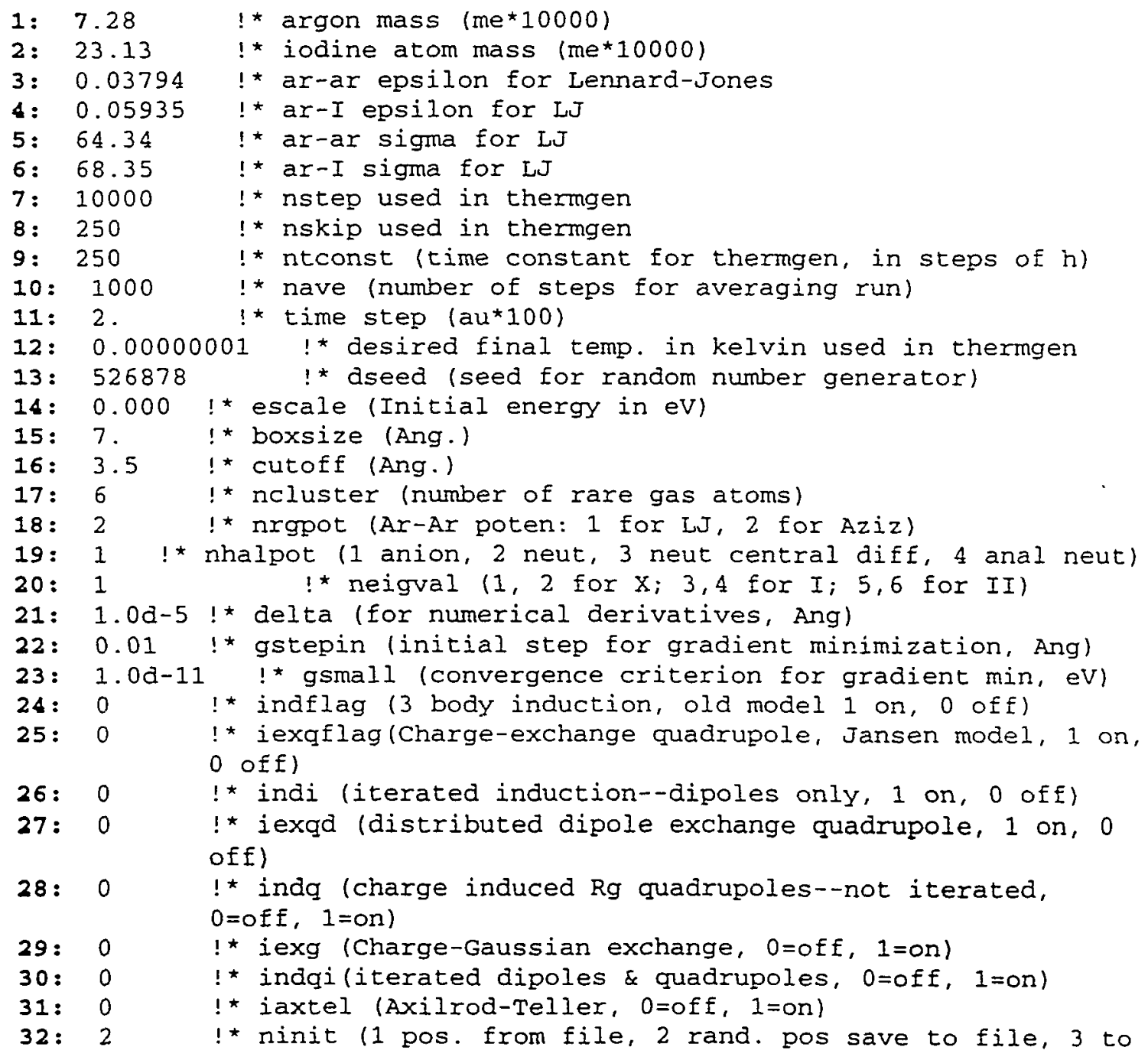




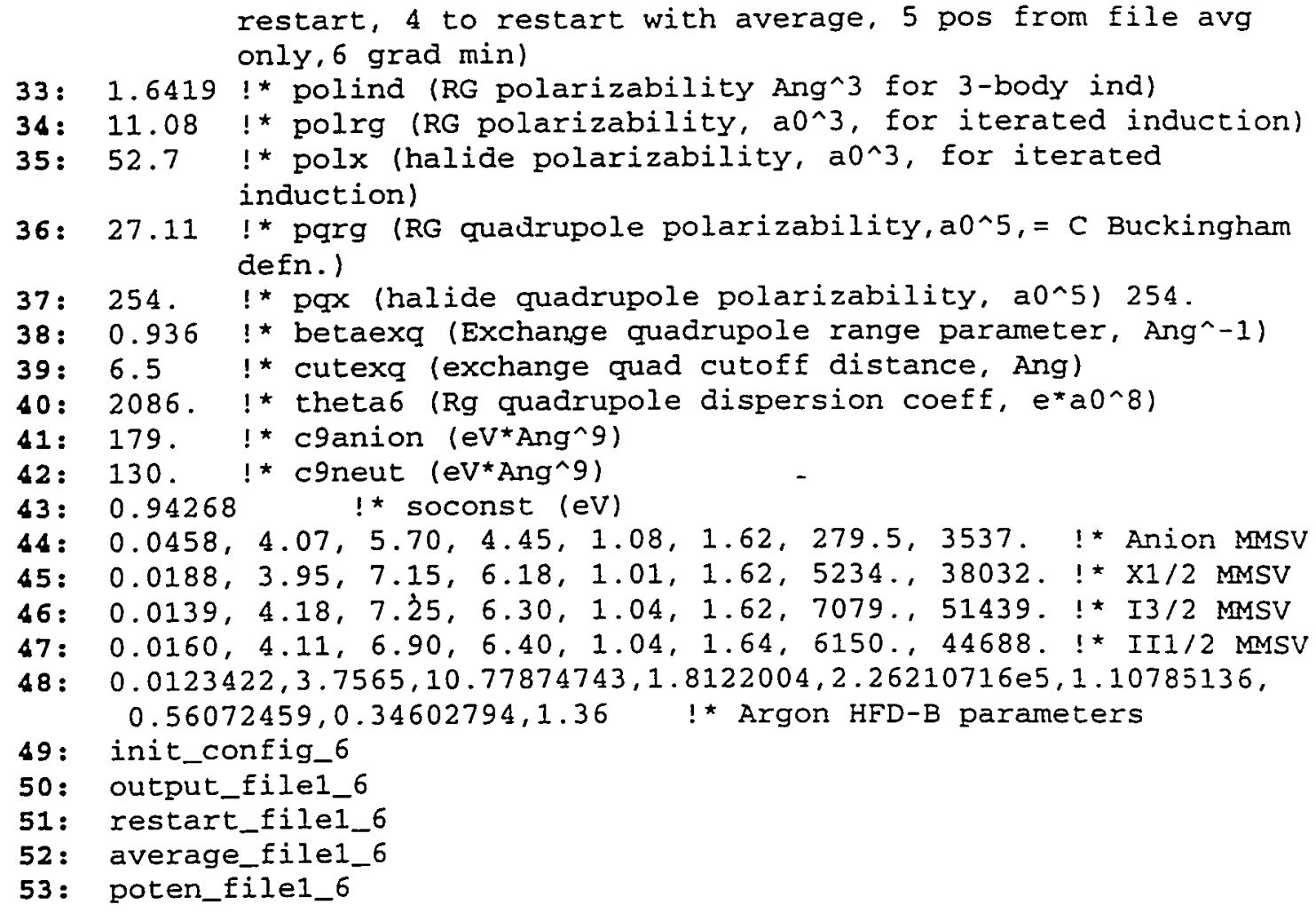

Note that the line numbers in boldface are not included in the actual file, but are shown here for reference purposes. All 53 lines of the file must be present, regardless of whether an anion or neutral simulation is being performed, or which many-body potential options are in use. The comments after the "!*" symbols are ignored by the program; in some cases the variable name used in the program is given in this comment field, as well as a brief explanation of its meaning. Note that comments are not allowed after lines containing file names (lines $49-53$ ), and will cause the program to crash.

Lines 1 and 2 are the masses of the rare gas and halide, respectively, in units of $10000 \mathrm{~m}_{e}$, where $\mathrm{m}_{\mathrm{e}}$ is the atomic unit of mass (i.e., the electron mass). Lines $3-4$ are Lennard Jones parameters, which were not used in the present application. One does have the option to use the Lennard Jones parameters for the Ar-Ar potential, however one may not use the many-body forces with this option. 
Lines 7-14 provide information about the timescale of the simulation and the annealing schedule. The basic time step of the simulation is set by Line 8 , in $100 \tau_{0}$ (where $\tau_{0}$ is the atomic unit of time, equal to approximately $0.024 \mathrm{fs}$ ). Thus the value of 2 in the sample input file corresponds to a time step of about $5 \mathrm{fs}$. Line 7, nstep is the total number of $\mathrm{MD}$ steps in the simulation. Line 8 , nskip is the number of MD time steps between energy rescalings. Line 9 , ntconst, is the time constant, in units of number of MD steps, that determines the factor by which the energies are rescaled after each nskip number of time steps, by the equation (See Chapter 4, Section 4.4.2):

$$
f a c=\sqrt{1+\frac{\text { nskip }}{\text { ntconst }}\left(\frac{T_{\text {rarg }}}{T_{\text {avg }}}-1\right)}
$$

Typically, when starting with a random configuration of atoms, we set ntconst equal to nskip, so that the rescaling factor is close to 0 (because for SA we normally set $\left.K E_{\text {targ }}<<K E_{\text {avg }}\right)$ and energy is removed as fast as possible from the cluster. When the simulated annealing run, the cluster is allowed to run, and the average positions of the atoms are determined during this "averaging run." Line 10 , nave, indicates the number of time steps to perform this averaging. Line $\mathbf{1 2}$ is sets the desired final temperature for the simulation, which is used to directly calculate $K E_{\text {targ }}$ in the above equation. When the program is used for simulated annealing this line should be set as arbitrarily close to 0 as possible. In other applications, i.e., $\mathrm{MD}$ simulation of a system at a non-zero temperatures, this could be set to higher temperatures, or one could modify the program so that this variable could be used to set the annealing schedule. Line 13 , dseed, is the seed for the random number generator, and may be any integer. Note that the sequence of "random" numbers produced by the program is entirely determined by the initial choice 
of seed. Thus if one does not change dseed one will produce identical initial configurations of atoms, and if all the other parameters for the anneal are the same, one will arrive at identical final configurations. Therefore it is essential to change dseed every time one wishes to start with a "fresh" initial configuration. Line 14, escale, is the initial total kinetic energy, in $\mathrm{eV}$, given to the atoms. This energy is distributed randomly among all the atoms. In general, for the first step when the atom positions are randomly chosen with a box, escale is set to 0 , so that the initial kinetic energy of the atoms is determined only by the potential of the other atoms. If escale is set to a larger value, the atoms tend to evaporate out of the box.

Lines 15-17 determine the initial placement of the atoms in a box when the flag ninit (line 32) is set to 2 . When this flag is set, the atoms are placed randomly within a cubic box with the side dimension boxsize (line 15) given in Angstroms. The initial configuration of the atoms is not allowed to contain any pairs closer together than cutof $f$ (line 16). The value shown, $3.5 \AA$, was found to be useful so that the initial configuration does not contain atoms in highly repulsive regions of the potential surface, which would lead to rapid evaporation. Line 17 gives the number of rare gas atoms (the number of halide atoms is always assumed to be one). This initial configuration is saved to the file named in line 49, here called "init_config_6." The format of this file is explained in detail below.

Lines 18-21 are integer inputs that tell the program what kinds of pair potentials to use. Line 18 nrgpot may be set to 1 to use the Lennard-Jones form for the Rg-Rg interaction, or to 2 to use the more accurate Hartree-Fock Dispersion (HFD-B2) form. ${ }^{11}$ The parameters for the Ar-Ar HFD-B2 potential are given in line 48, listed in the same 
order as in Ref. ${ }^{11}$. In all of the simulations in this work, the HFD-B2 form was used for the Ar-Ar pair potential. Line 19, nhalpot, indicates whether to calculate the anion or neutral potential. If nhalpot is set to 1 , the anion $\mathrm{Rg}_{\mathrm{n}} \mathrm{X}^{-}$potential is used, with the anion MMSV parameters given in line 44 and many body terms as described below. If nhalpot is set to 2,3 or 4 , the neutral $\mathrm{Rg}_{\mathrm{n}} \mathrm{X}$ potential is calculated according to the method outlined in Chapter 4. If nhalpot is set to 2 or 3, the neutral eigenvalues are calculated by numerical diagonalization of the $6 \times 6$ matrix Eqn. (4.16) of Chapter 4. Line 21 specifies the displacement, delta, in $\AA$, to be used for calculation of the forces from the potentials. For nhalpot equal to 2 , the forces are calculated by finite difference, whereas if nhalpot $=3$, the forces are calculated by central difference, which in principle should give more accurate values of the forces. ${ }^{3}$ However the calculation runs somewhat slower with nhalpot $=3$, and the precise values of the forces are not extremely significant for simulated annealing applications, so it is not recommended to run in the mode. When nhalpot is set to 4, the neutral eigenvalues are found analytically, resulting in an approximate tenfold increase in speed. Therefore, this is the recommended mode of operation for calculation of neutral potentials. The numerical mode of calculation may be used as a check on the analytical calculation.

Line 32, ninit, specifies the mode of operation of the program. These modes are explained below. When ninit is equal to:

1. The initial positions and momenta of the atoms are read in from the file named in line 49 , here called "init_config_6," the final configuration is save in the file named in line 51, here "restart_filel_6" the configuration after the annealing run 
is save in the file named in line 52 , here "average_file $1 \_6, "$ and the final total potential is saved in the file on line 53 , here named "poten_file 1_6;"

2. The atoms are placed at random in a box, and if escale is non-zero, are given initial kinetic energies totaling escale. This initial configuration is save in the file named in line 49, and the output files are saved as described above for ninit $=1$;

3. The program is run with the initial configuration taken from the file named in line 51 ("restart_file1_6"), and the final configuration is saved to this same file; the "average" and "poten" files are also updated after running in this mode, but the "init_config" file is not modified; this mode of operation is used if one wishes to "restart" the simulation from the point where the previous simulation ended.;

4. The program is run as if ninit $=3$, above, except that the initial configuration is read in from line 52, here "average_file 1_6;" this mode is useful for rapid cooling of a cluster when it is near a minimum configuration;

5. In this mode, the initial positions and momenta are read in from the file named in line 49 ("init_config_6"), and only the averaging run is performed, the results of which are saved in "average_file1_6" (line 52); the simulated annealing procedure is not performed in this mode; this mode is useful to get an idea of the standard deviations in atomic positions and potential energy of a given configuration;

6. In this mode, the initial positions are read in from the file specified in line 49 , and a gradient minimization procedure is performed; the final potential is saved in the 
file named in line $\mathbf{5 3}$, but the final configuration is not saved. This mode is used to quickly optimize the potential when one is very near the minimum.

In all modes of operation, an output file, named in line $\mathbf{5 0}$ is produced, which contains information about the cluster geometry, potential, etc., and is described in detail below.

Lines 24-31 are flags which tum on or off various many-body interactions. Except for the Axilrod-Teller interaction (line 31), these flags are only applicable to the anionic clusters and are ignored by the program when a neutral cluster is being simulated.

Lines $24,26,28$ and 30 specify various modes of implementation of the induction effects described in Chapter 4. When line 30, indqi, is set to 1 , the full iterative procedure including induced dipoles and quadrupoles is performed for finding the nonadditive energy. This mode was used to obtain the results presented in Chapter 4 . The other flags may be set to implement simpler (and faster running) models of the nonadditive induction energy. If line 26 , indi, is set to 1 , the iterated induction procedure is performed neglecting the quadrupole moments of the atoms. The inclusion of quadrupole induction effects is an extension of the method as originally introduced ${ }^{12}$ and as usually implemented for simulations of polar solvents, as for example by Jedlovzky et al.,13 which normally stop at the dipole term. However, it seems that the quadrupole nonadditive portion contributes significantly to the non-additive energy in the case of highly polarizable anions and moderately polarizable rare gases such as Ar. It is probable that simulations involving highly polarizable rare gases, such as those of the $\mathrm{Xe}_{\mathrm{n}} \mathrm{I}^{-}$clusters that are currently underway in the Neumark group, the quadrupole induction effect should be quite significant. 
When indflag is set (line 24) a simpler model of the many body interaction is used. In this case, we only consider the dipoles induced in the rare gases by the halide charge, and the pair interactions between these induced dipoles. Then for an $\mathrm{Rg}_{n} \mathrm{X}^{-}$ cluster, the non-additive induction energy is approximated by

$$
V_{i n d}=\sum_{i<j} \frac{1}{R_{i j}^{3}}\left[\mu_{i} \cdot \mu_{j}-\frac{3\left(\mu_{i} \cdot \mathbf{R}_{i j}\right)\left(\mu_{i} \cdot \mathbf{R}_{i j}\right)}{R_{i j}^{2}}\right]
$$

where $\mathbf{R}_{i j}$ is the vector from rare gas atom $i$ to rare gas atom $j, \mu_{i}$ is the dipole moment induced by the halide charge on rare gas atom $i$, and the sum runs over all pairs of rare gas atoms. The induced dipole moments are found from

$$
\mu_{i}=\frac{q \alpha_{i}}{R_{i}^{3}} \mathbf{R}_{i}
$$

where $q$ is the halide charge, $\alpha_{i}$ is the rare gas dipole polarizability, and $\mathbf{R}_{i}$ is the vector from the rare gas to the halide. This induction model may be considered a first order approximation to the full iterative solution of Eqn. (4.23) of Chapter 4. For an example of the application of this model with simulated annealing see the work of Asher et al. ${ }^{14}$ on metal-argon clusters.

When indq, line 28 , is set to 1 the non-additive energy due to induced quadrupole moments is included in a non-iterative manner similar to the dipole induction model described above. When indq is set, the quadrupole moments induced in each rare gas atom by the halide charge are calculated, and the pair interactions of these quadrupoles are calculated, as well as the interactions of the quadrupoles with the dipoles calculated iteratively. For the formulas for the quadrupole-quadrupole and dipole-dipole interactions see Buckingham. ${ }^{15}$ In order for this to function properly, indi (line 26) 
must also be set to 1 . If indi is not set, the dipole-quadrupole interactions will not be included and the overall effect of the quadrupole will be severely underestimated.

The flags 25, 27 and 29 control which of the three models described in Chapter 4 for the "exchange quadrupole" effect is used. If iexqflag (line 25) is set to 1 , the nonadditive exchange quadrupole energy is calculated from the interaction of the halide charge with the cylindrically symmetric quadrupole moments located at the midpoints between the nuclei of two rare gas atoms, $\Theta_{e x}^{(i j)}$, calculated from Equation 32 of Chapter 4. The total charge-exchange quadrupole energy for the cluster is then given by:

$$
V_{c c}(\text { point quadrupole })=\frac{q \Theta_{e x}^{(i j)}}{R_{c 0}^{3}}\left(\frac{3}{2} \cos ^{2} \phi-\frac{1}{2}\right)
$$

where $\mathbf{R}_{c 0}$ is the vector from the $\mathrm{Rg}-\mathrm{Rg}$ midpoint to the halide nucleus, $\phi$ is the angle between the quadrupole and $\mathbf{R}_{c 0}$. When line 27 (iexqd) is set to 1 , the distributed dipole model of the exchange quadrupole introduced by Hutson et al. ${ }^{16}$ is used. When line 29 (iexg), the "Gaussian exchange-charge," model developed in Chapter 4 is employed, and the non-additive energy is found from Equation 34 of Chapter 4.

Line 31, iaxtel, is set to 1 to turn on the Axilrod-Teller three body dispersion interaction [see Eqn. (4.18) of Chapter 4]. This interaction may be used for both the anion or neutral calculation.

Lines 33-43 of the input file contain atomic data and interaction constants used in the calculation of the many-body potentials. These are listed below in the same notation as Chapter 4:

Line $33 \quad$ polind $\quad \alpha(\mathrm{Rg}) \quad$ Dipole polarizability of the rare gas atom in $\AA^{3}$ 


\begin{tabular}{|c|c|c|c|}
\hline Line 34 & polrg & $\alpha(\mathrm{Rg})$ & Dipole polarizability of the rare gas atom in $a_{0}{ }^{3}$ \\
\hline Line 35 & polx & $\alpha\left(\mathrm{X}^{-}\right)$ & Dipole polarizability of the halide anion in $a_{0}{ }^{3}$ \\
\hline Line 36 & pqrg & $C(\mathrm{Rg})^{*}$ & Quadrupole polarizability of the rare gas in $a_{0}{ }^{5}$. \\
\hline Line 37 & pqx & $C\left(\mathrm{X}^{*}\right)^{*}$ & Quadrupole polarizability of the halide anion in $a_{0}^{5}$. \\
\hline Line 38 & $\begin{array}{l}\text { betae } \\
\text { exchange }\end{array}$ & & Gaussian range parameter in $\AA^{-1}$ \\
\hline
\end{tabular}
Line 39 cutexg A distance in $\AA$ which is used as the cutoff point for the
exchange-charge calculation, to prevent non-physical behavior of Equation 34 of Chapter 4.
Line 40 theta6 $C_{\theta}$ Coefficient for calculating the dispersion induced quadrupole moment on a rare gas atom [see Eqn. (4.36), Chapter 4]

Line 41 c9anion $\quad C_{9}\left(X^{-}-\mathrm{Rg}-\mathrm{Rg}\right)$ Axilrod-Teller coefficient for halide anion-

rare gas three-body dispersion interaction in eV. $\AA^{9}$.
Line 42 c9neut $C_{9}(\mathrm{X}-\mathrm{Rg}-\mathrm{Rg})$ Axilrod-Teller coefficient for halogen

neutral-rare gas three-body dispersion interaction in $\mathrm{eV} \cdot \AA^{9}$.
Line 43
soconst
$\Delta \quad$ Halogen spin-orbit constant in $\mathrm{eV}$.

\footnotetext{
- Note that the definition of the quadrupole polarizability here is that of Buckingham, ${ }^{15}$ which differs from other definitions (e.g. this is not the same $\alpha_{q}$ used in Chapter 3: see the footnote to Table VI of Chapter 4).
} 
The MMSV pair potential parameters for the $\mathrm{RgX}^{-}$anion are given in line 44, and the MMSV parameters for the neutral $X 1 / 2,13 / 2$, and $I I 1 / 2$ states are given in lines 45, 46 and 47 respectively. The order and units of the MMSV parameters in each line the same as those in the input file for the Morse DVR program described in Appendix A (See Section A5.1).

\section{C4.2 The atomic configuration files}

This section describes the format of the files which describe the configurations of atoms, which are named in lines 49,51 and 52 of the input file. A configuration of $N$ rare gas atoms and one halogen atom would be represented in the following format:

$\begin{array}{llllllll}1 & X_{1} & Y_{1} & Z_{1} & & P_{X}^{(1)} & P_{Y}^{(1)} & P_{Z}^{(1)} \\ N & & & & \vdots & & & \\ N+1 & X_{N} & Y_{N} & Z_{N} & & P_{X}^{(N)} & P_{Y}^{(N)} & P_{Z}^{(N)} \\ X_{\text {hal }} & Y_{\text {hal }} & Z_{\text {hal }} & & P_{X}^{(h a l)} & P_{Y}^{(h a l)} & P_{Z}^{(h a l)}\end{array}$

The first $N$ lines of the file give the rare gas configuration, and the final line is the halide configuration. The first column is an integer index. $X_{N}, Y_{N}$ and $Z_{N}$ are the Cartesian coordinates in units of $a_{0} / 10 . P_{X}^{(N)}$, etc. are the corresponding components of the momentum vector, with units $1000 \mathrm{~m}_{e} \mathrm{a}_{0} / \tau_{0}$.

\section{C4.3 Running the program: example of $\mathrm{Ar}_{6} \mathrm{I}^{-}$}

To show how the simulated program is used we will show how to run the program using the input file "in1" shown in section C4.1. This input file is set up to find a minimum energy configuration of a $\mathrm{Ar}_{6} \mathrm{I}^{-}$cluster with no many body interaction, starting 
from a random configuration of atoms. Below, we present the actual computer session.

The user input is shown in boldface:

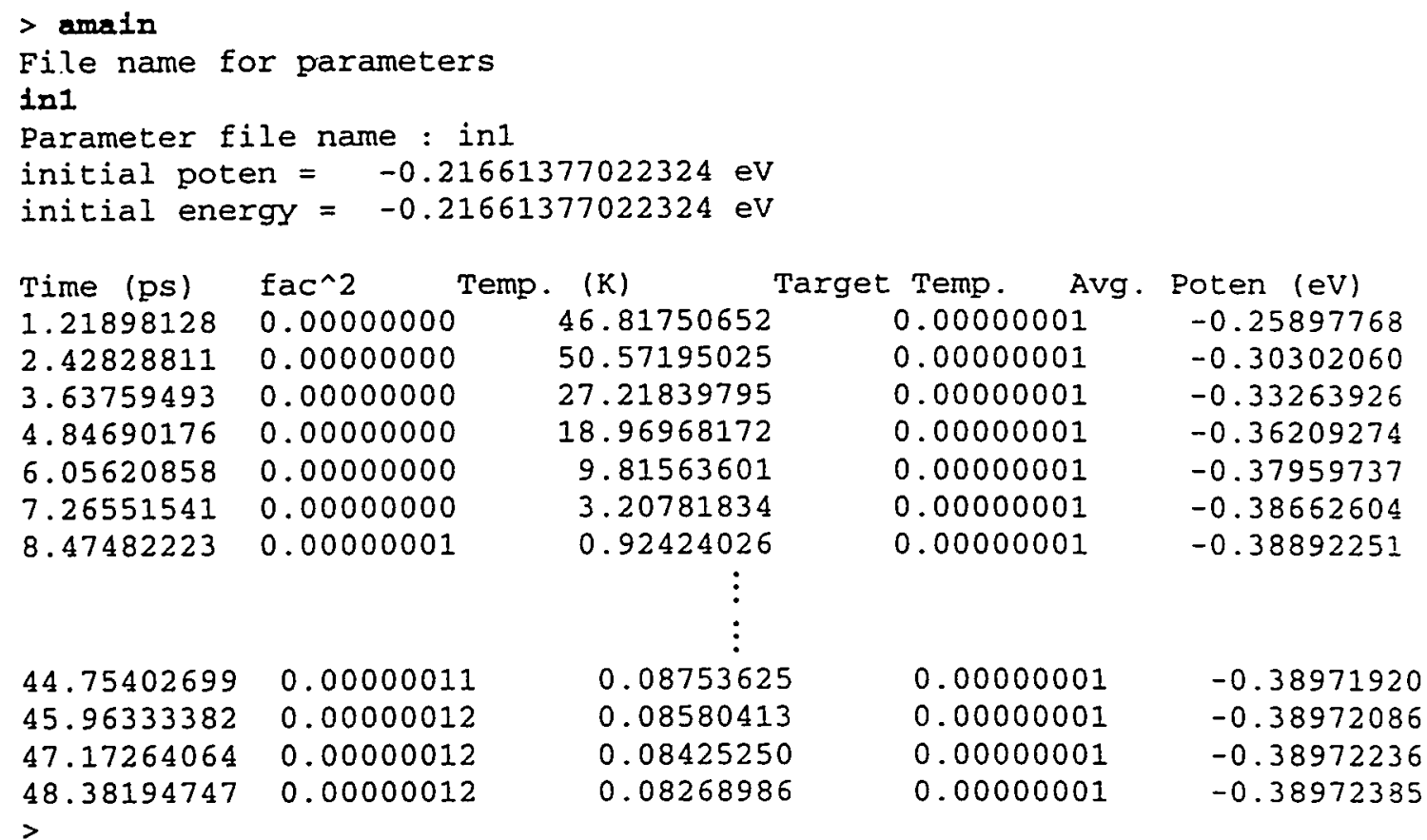

Here we see that because the factor for rescaling the velocities is practically zero the cluster cools quite rapidly. We can then examine the $\mathrm{Rg}-\mathrm{X}$ and $\mathrm{Rg}-\mathrm{Rg}$ bond distances given in the output file "output_file1_6," to ascertain the structure of this cluster. Alternately, one could plot the data from the averaged configuration file "average_file1_6," with a three-dimensional plotting program. We find that this configuration has the six argons all about $4 \AA$ from the iodine ion, and arranged in a warped trapezoid-like arrangement, as shown in figure $\mathrm{C} 1$. 


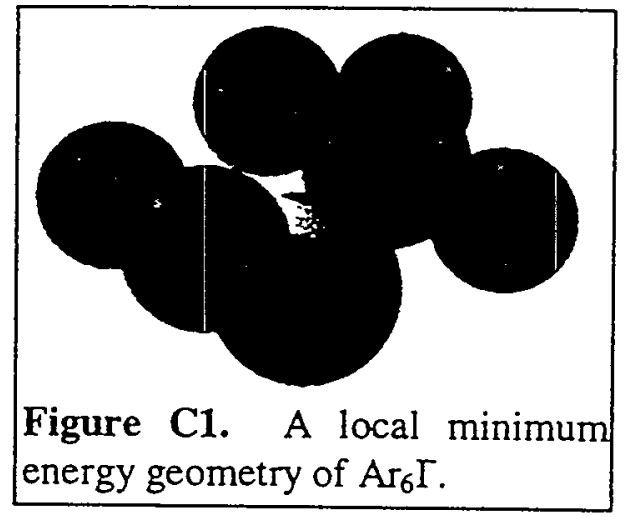

\section{C4.3.1 Gradient minimization when near a local minimum}

To illustrate the use of the gradient minimization procedure for optimizing the binding energy, we modify the input "in1" to "in2," showing below only the lines of "in2" that are different from "in $1: "$

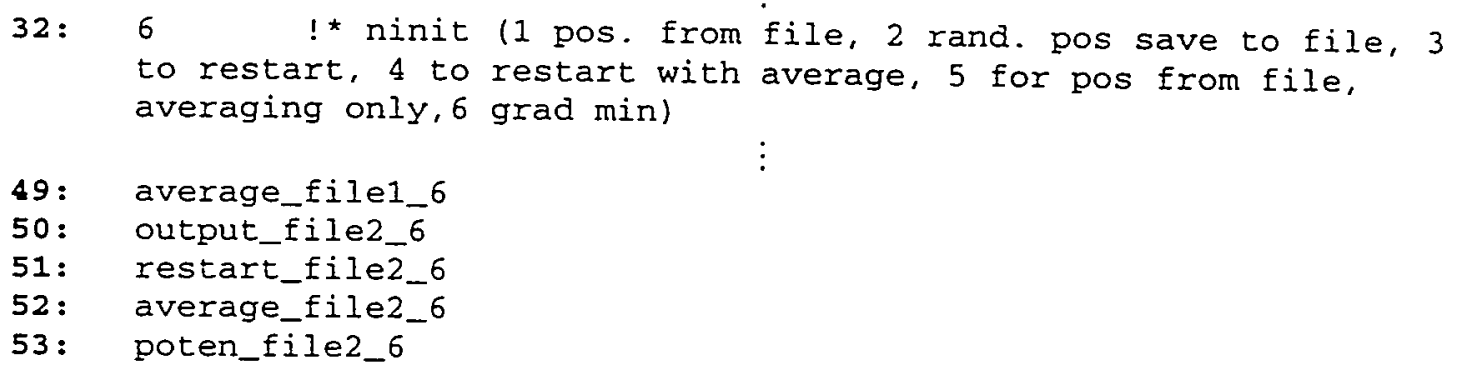

Here we have changed the file name in line 49 to "average_file 1_6," which contains the configuration output from the first run of the program, and set the flag ninit to 6 for gradient minimization. We perform the minimization as follows:

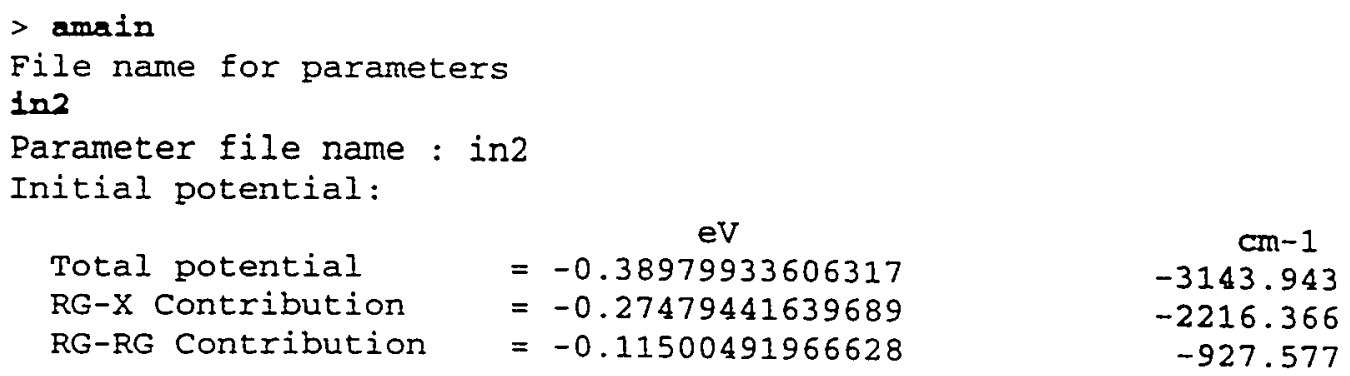


Potential after gradient minimization:

$\mathrm{eV}$

Total potential $=-0.38980032561780$

$\mathrm{RG}-\mathrm{X}$ Contribution $\quad=-0.27479155778784$

RG-RG Contribution $\quad=-0.11500876782996$ $\mathrm{cm}-1$

$-3143.951$

$-2216.343$

$-927.608$

We see that because the cluster is already quite cold, the gradient minimization in this case refines the minimum energy only by about $0.03 \mathrm{~cm}^{-1}$.

\section{C4.3.2 Reheating and annealing}

Now that we have minimum energy configuration of the $\mathrm{Ar}_{6} \mathrm{I}$, we must perform the simulated annealing procedure to try to determine if this is this is the global minimum. To do this we make the following changes to the input file, and rename it "in3:"

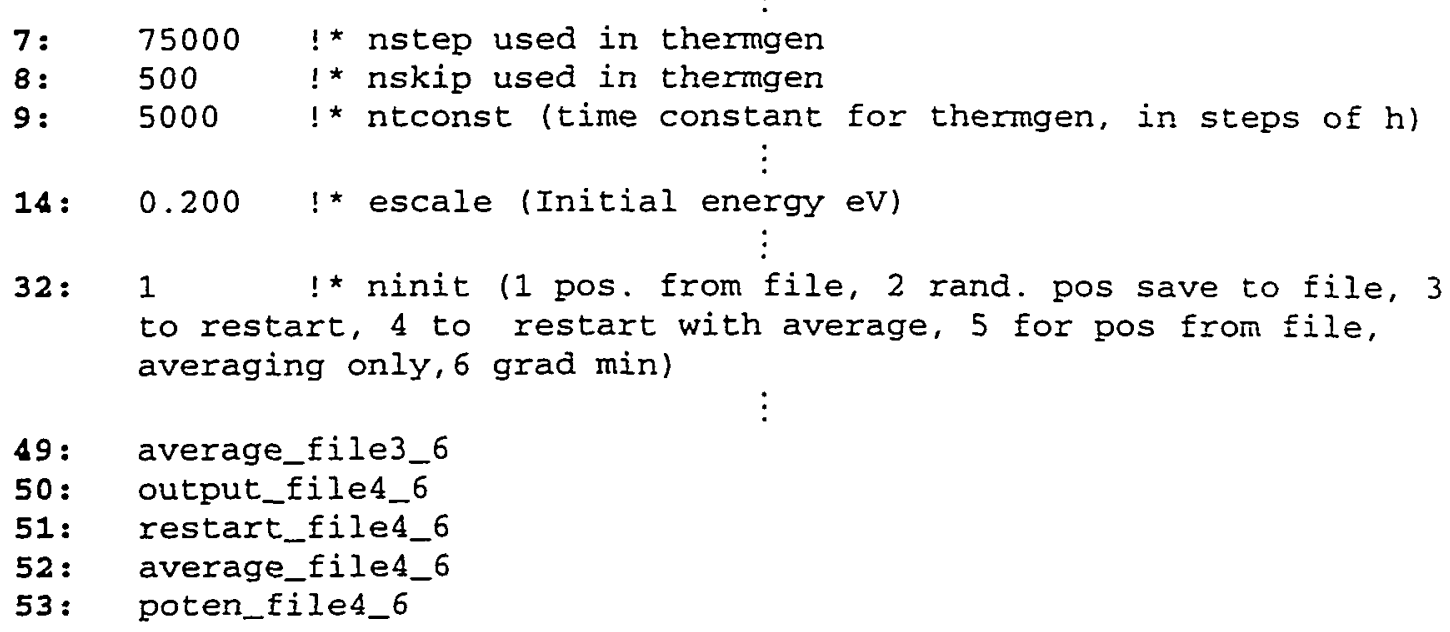

Here we have changed nstep so that the total duration of the SA run will be about $360 \mathrm{ps}$, and increased nskip by a factor of 2 , so that the cluster will have more time to equilibrate between rescalings. Most importantly, the time constant ntconst has been set to be 10 times as large as nskip, so that the kinetic energies will be rescaled by a 
factor of 0.9 and that the cooling will take place slowly. The cluster is heated initially by changing escale, the initial kinetic energy, to $200 \mathrm{meV}$, which will be distributed randomly to the atoms in the cluster. Experience has shown that in general one should not set escale to more than about one half of the potential of the minimum energy of the cluster (here ca. $400 \mathrm{meV}$ ), in order to prevent evaporation of atoms. The starting point is the configuration output from the gradient minimization in the file "average_file3_6," which file name is no place in line 49 of the input file. The flag ninit is changed to 1 to start the program with this initial configuration (plus the added kinetic energy). We now do the anneal:

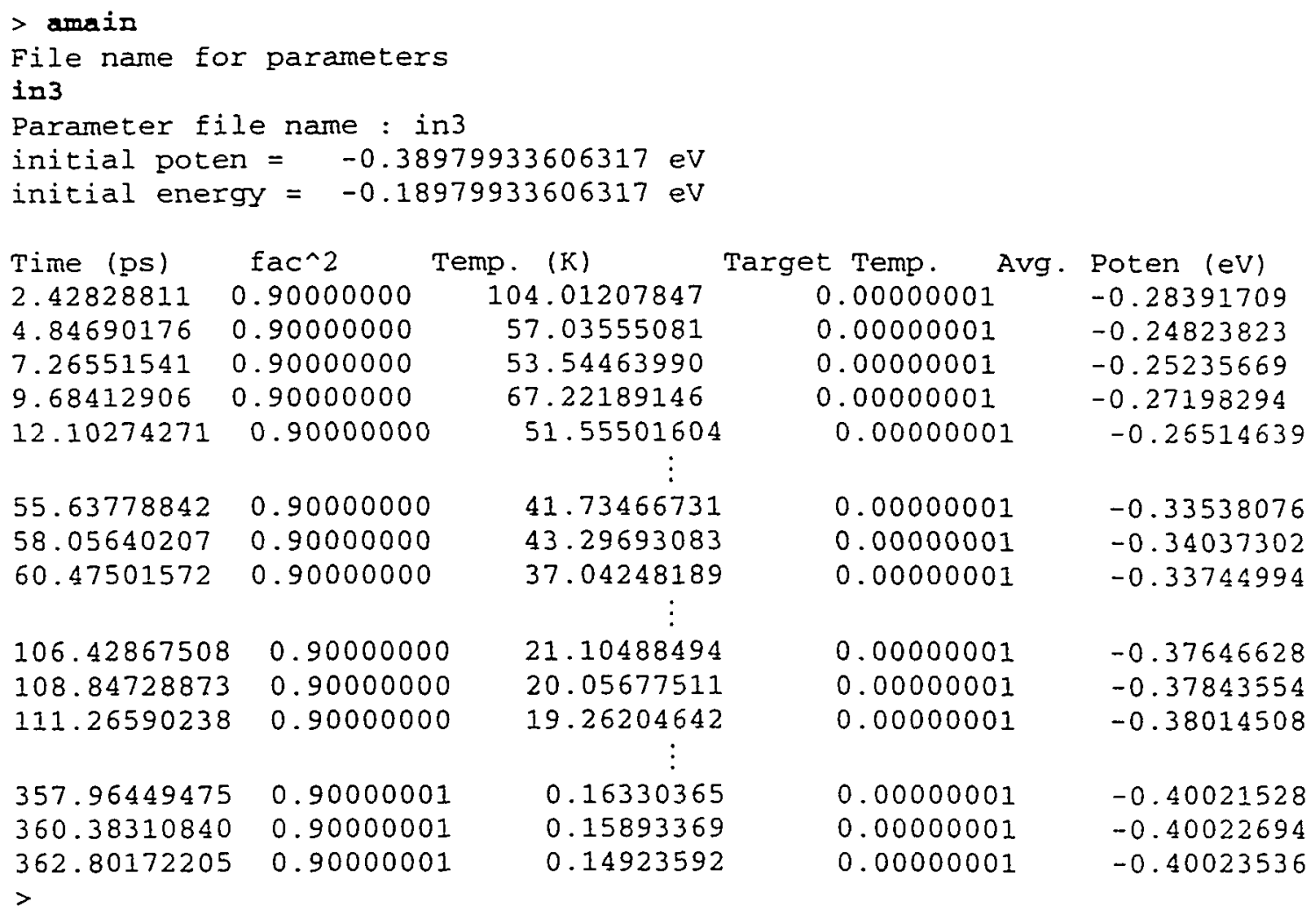

We see that at the end of the annealing procedure, the potential energy is lower than that of the previously located minimum, indicating that we have found a different 
minimum on the potential energy surface. Examining the output files we find that this is indeed a different isomer of $\mathrm{Ar}_{6} \mathrm{I}^{-}$with the structure pictured in Figure C2.

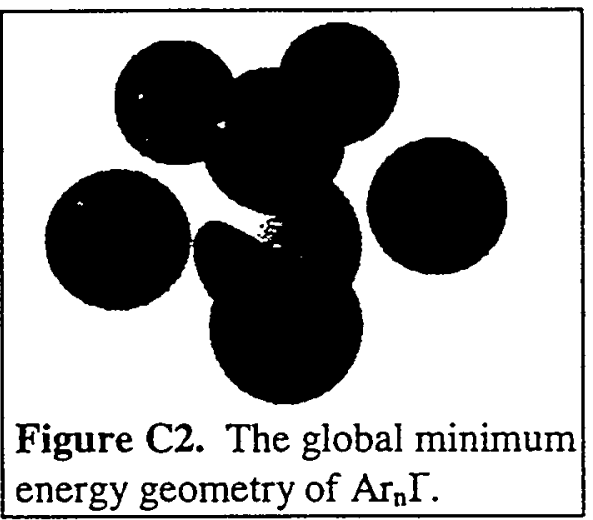

This is the configuration that we recognize as the global minimum energy geometry from Figure 7 of Chapter 4 . In this structure, as in the previously located minimum, all of the Ar atoms are in "contact" with the $\mathrm{I}^{-}$atom. However, we see that in this structure, the number of Ar-Ar nearest neighbor "bonds" is 10 , compared to 9 for the isomer pictured in Figure C1. Furthermore, the energies of these structures differ by $85 \mathrm{~cm}^{-1}$, which is reasonably close to the Ar-Ar dimer bond energy (about $100 \mathrm{~cm}^{-1}$ ).

We know from previous experience that this is the global minimum of $\operatorname{Ar}_{6} \mathrm{I}$. However, with an unknown system, a greater number of annealing runs must be performed to ensure that the global minimum has been found. It is not possible to give an exact answer to the question of how many times one must repeat the annealing process, because, as noted above, simulated annealing is not an exact method. The number of runs necessary will increase with the complexity of the system studied. In general, one should perform the process enough times to gain an understanding of the various categories of local minima for a given system and their relative energies. 


\section{C4.4. Outline of the program}

The subroutines and functions used by the simulated annealing program are presented in Table $\mathrm{Cl}$ organized according to which file they reside in.

Table C1. Files, subroutines and functions used by the simulated annealing program

\begin{tabular}{|c|c|c|}
\hline File & $\begin{array}{l}\text { Subroutine } \\
\text { or Function }\end{array}$ & Description \\
\hline param.file & & $\begin{array}{l}\text { Contains constants and common blocks used by the } \\
\text { program }\end{array}$ \\
\hline amain.f & & Main program; reads input and writes output file \\
\hline aderiv.f & deriv & $\begin{array}{l}\text { Function to calculate the forces on each atom and } \\
\text { potential of the cluster for a given atomic configuration }\end{array}$ \\
\hline \multirow[t]{3}{*}{ initzandiat.f } & initpos & Generates random initial positions for the atoms \\
\hline & initzangdiat & $\begin{array}{l}\text { Removes CM translation and sets overall angular } \\
\text { momentum of cluster to zero }\end{array}$ \\
\hline & ggubs & Random number generator \\
\hline thermgen.f & thermgen & $\begin{array}{l}\text { Propagates the MD equations of motion according to } \\
\text { the scheme described above. }\end{array}$ \\
\hline \multirow[t]{4}{*}{ pforce.f } & anmmsv & Function for anion MMSV pair potential and force \\
\hline & hfd_b & Function for HFD-B2 (Rg-Rg) pair potential and force \\
\hline & pfind & $\begin{array}{l}\text { Calculates three-body induction potential and forces } \\
\text { from Eq. }(\mathrm{ClO})\end{array}$ \\
\hline & pfexq & $\begin{array}{l}\text { Calculates the three-body "exchange-quadrupole" } \\
\text { potential and forces from Eq. (C12) }\end{array}$ \\
\hline
\end{tabular}




\begin{tabular}{|c|c|c|}
\hline & porb & $\begin{array}{l}\text { Calculates neutral many-body eigenfunctions } \\
\text { numerically. }\end{array}$ \\
\hline & meummsv & Calculates neutral MMSV pair potential \\
\hline & porba & Calculates neutral many-body eigenvalues analytically \\
\hline & veqd & $\begin{array}{l}\text { Calculated exchange charge effect using distributed } \\
\text { dipole model }\end{array}$ \\
\hline & vindi & $\begin{array}{l}\text { Calculates many-body dipole induction potential using } \\
\text { the iterative method. }\end{array}$ \\
\hline & vindq & $\begin{array}{l}\text { Calculates induced quadrupole-induced quadrupole and } \\
\text { induced quadrupole-induced dipole potential; for use in } \\
\text { conjunction with the subroutine "vindi" }\end{array}$ \\
\hline & vinddq & $\begin{array}{l}\text { Calculates many-body dipole and quadrupole potential, } \\
\text { using the iterative method described in Chapter } 4 .\end{array}$ \\
\hline & exg & $\begin{array}{l}\text { Charge exchange calculation of three body potential } \\
\text { from Eq. (4.34) }\end{array}$ \\
\hline & erf & Error function adapted from Ref. ${ }^{3}$ \\
\hline & vat & Calculates three-body Axilrod-Teller potential \\
\hline averages.f & averages & $\begin{array}{l}\text { Calculates average parameters during a constant energy } \\
\mathrm{MD} \text { run }\end{array}$ \\
\hline ch.f & $\mathrm{ch}$ & $\begin{array}{l}\text { EISPACK subroutine for finding the eigenvalues and } \\
\text { eigenvectors of a complex Hermitian matrix }\end{array}$ \\
\hline
\end{tabular}




\section{C5. Source code for the simulated annealing program.}

\section{C5.1. File "Makefile"}

The makefile used for recompiling the simulated annealing program is shown below. To recompile the program one enters simply "make" because the makefile has the default name. The executable code will be save in the subdirectory "RUN" with the makefile as shown.

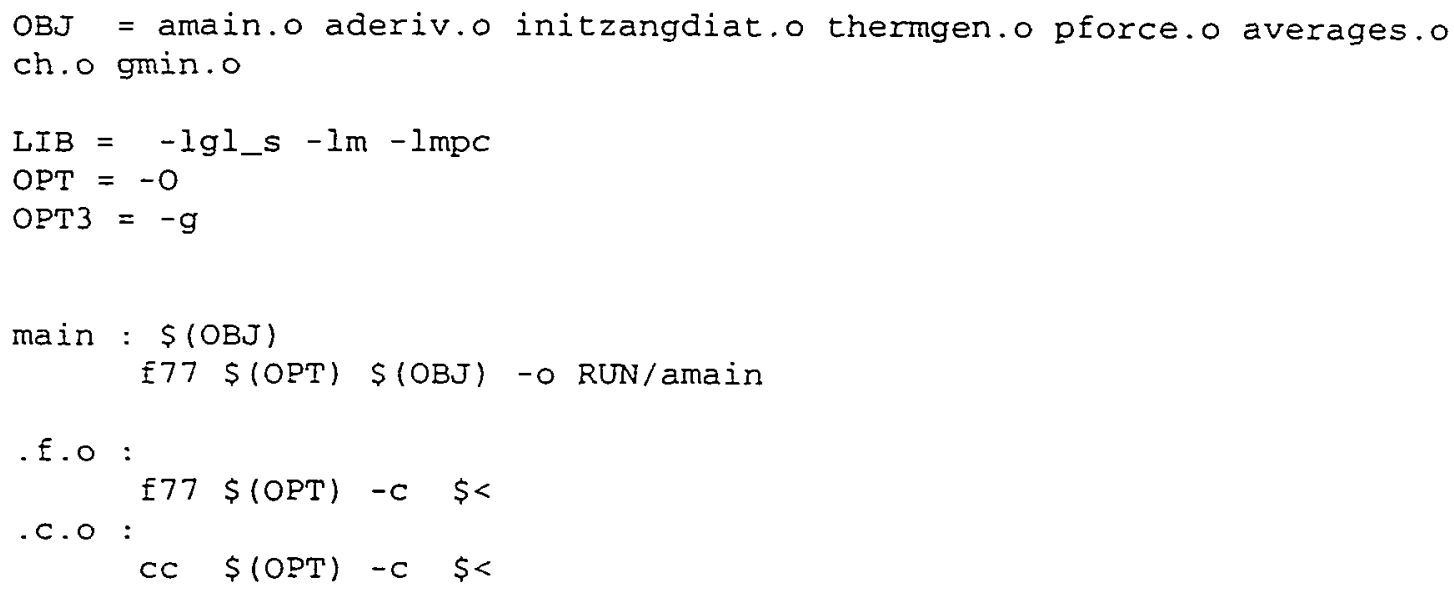

\section{C5.2. File "param.file"}

C

IMPLICIT double precision (A-H,O-Z)

C PARAMETER (ncluster=19, nmolec=1, natmax = ncluster+nmolec)

c PARAMETER ( $N Q=6, N D I M=N Q * N a t m a x, N E Q M=N D I M, N E Q=N D I M$ )

parameter ( $\mathrm{nCl}=25, \mathrm{nmolec}=1$, natmaxc $=\mathrm{ncl}+\mathrm{nmolec}$ )

parameter ( $N Q=6, N D I M C=N Q^{\star}$ natmaxc, NEQMC=NDIMC, NEQC=NDIMC)

PARAMETER (ECONV $=2196 . \mathrm{d0}$, DCONV $=0.0529 \mathrm{d0}$ )

parameter ( $\mathrm{p} i=3.1415926535898 \mathrm{do}, \mathrm{a} 0=0.529177249 \mathrm{do}$,

\& harev $=27.2113961 d 0$, evtocm $=8065.5410 \mathrm{do}$ )

parameter (evtoj=1.60217733d-19, amutokg=1.6605402d-27,

\& hztocm $=3.335640952 \mathrm{~d}-11$, hbarev $=6.5821220 \mathrm{~d}-16$,

\& hbar $=1.05457266 \mathrm{~d}-34$ )

$c$

common /nc/ncluster, natmax, NDIM, NEQM, NEQ

common /param/rmc, rm1, eec, eeint, sigc, sigint, nrgpot

common /param2/nhalpot, neigval, delta

common /atoms/poten, rgrgpot, rgxpot, cddpot, exqpot, vindit, vexqd,

\& Igdrgq, rgqq, rgqxd, viq, cdpot, cqpot, cdqpot, gexpot, daispot .

\& gdispot, gextot, atpot 
common /atoms1/rgxXpot, rgxIpot, rgxIIpot

common /atoms $2 /$ potfin, tempfin

common / threeb/indflag, iexgflag, indi, iexqd, indq, indqi, iexg,

\& iaxtel

c ncluster $=$ number of rare gas atoms

$c$ nmolec $=$ number of halide atoms

c Constants from Cohen \& Taylor, Rev. Mod. Phys. Vol. 59, No. 4

c hbarev units $\mathrm{eV}^{\star} \mathrm{s}$

c hbar units $J^{*} S$

\section{C5.3. File "amain.f"}

* Simulated annealing program by Zhiming Li and Prof. CC Martens (UC Irvine)

* Modified and extended by Ivan Yourshaw

*

include 'param.file'

common /anion/ $\mathrm{p}(10), \mathrm{q}(10)$

common /neutral/ px(10),p1(10),p2(10), soconst

common/nonadpar/ polind, betaexq, cutexg,polrg, polx, thetas,

\& pqrg,pqx,c9at

common/box/boxsize, cutoff

C

DIMENSION YO (neqme), $d x$ (ncl), dy (ncl), dz (ncl), dist (ncl),

$\& \quad$ ang $(\mathrm{ncl}, \mathrm{ncl}), \mathrm{yave}(\mathrm{neqc}), \operatorname{rgx}(2, \mathrm{ncl}), \operatorname{rgrg}(2, \mathrm{ncl}, \mathrm{ncl})$,

\& $\quad \operatorname{dip}($ natmaxc), qin (natmaxc)

double precision $\mathrm{p}(10), \mathrm{q}(10), \mathrm{p} x(10), \mathrm{p} 1(10), \mathrm{p} 2(10)$

character 30 aparam, initcond, outcond, restart, avrestart, potfile

character ${ }^{8} 80$ comment 1 , comment 2

$\mathrm{C}$

integer iarr(3)

c OPEN FILES

$c$

write $(*, *)$ 'File name for parameters'

read $(*, *)$ aparam

write $(*, *)$ 'Parameter file name: ',aparam

open ( 7 , file=aparam)

read $(7, *)$ rmc

read $(7, *) \operatorname{rm} 1$

$\operatorname{read}(7, *)$ eec

read $(7, *)$ eeint

$\operatorname{read}(7, *)$ sigc

$\operatorname{read}(7, *)$ sigint

c

$\operatorname{READ}(7, *)$ nstepT

$\operatorname{READ}(7, *)$ nskipT

read $(7, *)$ ntconst

read $(7, *)$ nave

!* Number of steps for averaging

$\operatorname{READ}(7, *) \mathrm{h}$

c

$\operatorname{READ}(7, *)$ ekelvin

$\operatorname{READ}(7, *)$ dseed 


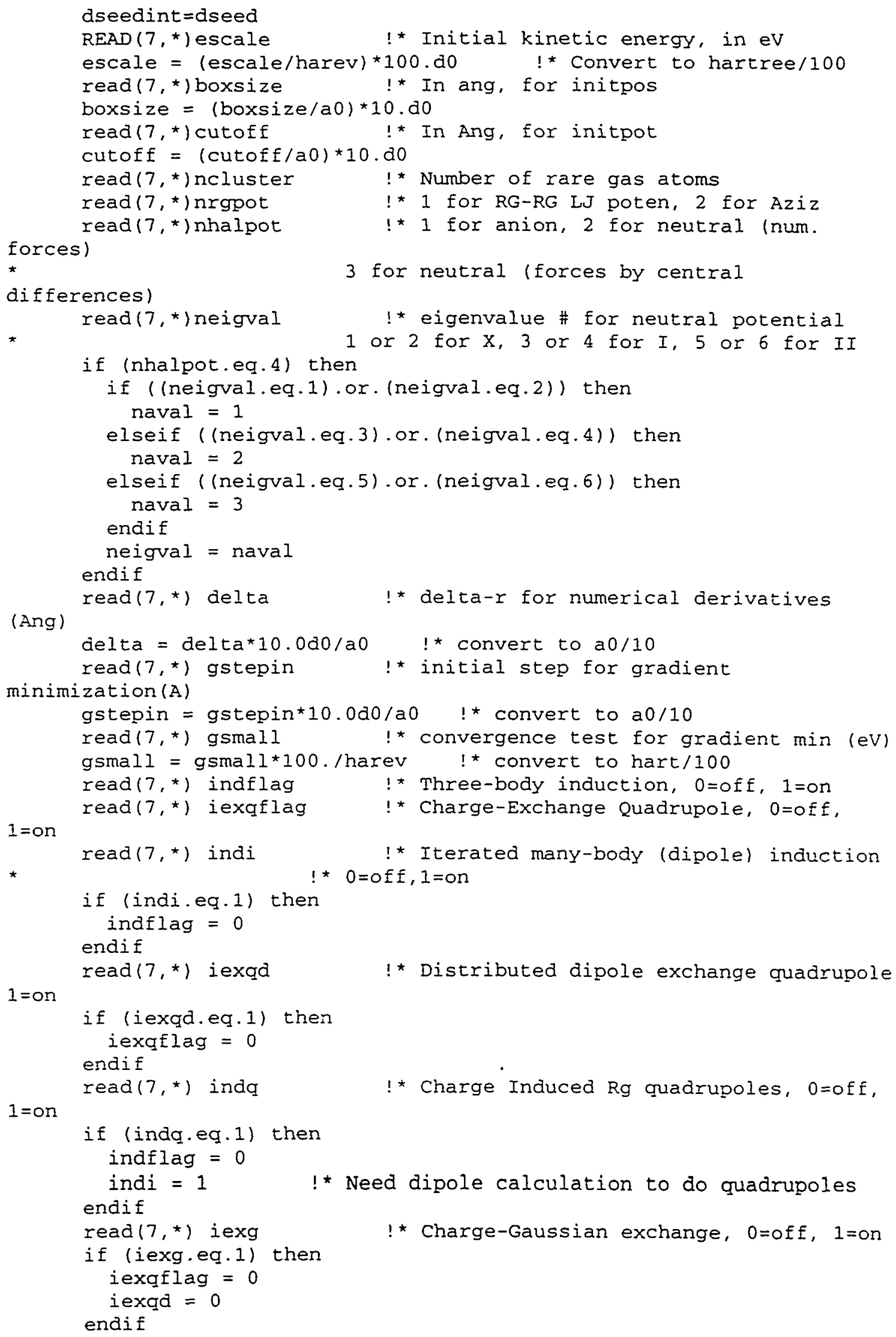




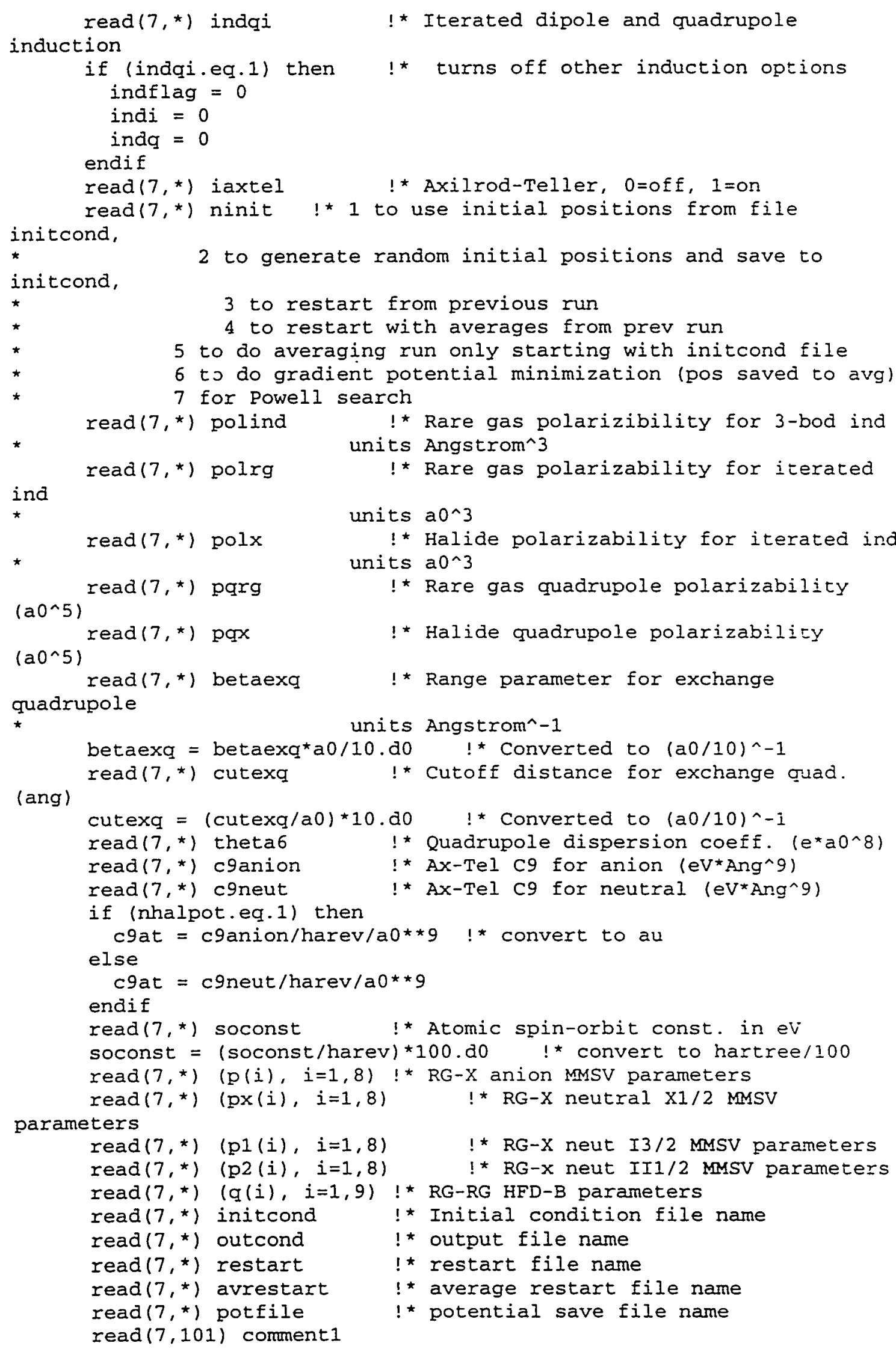




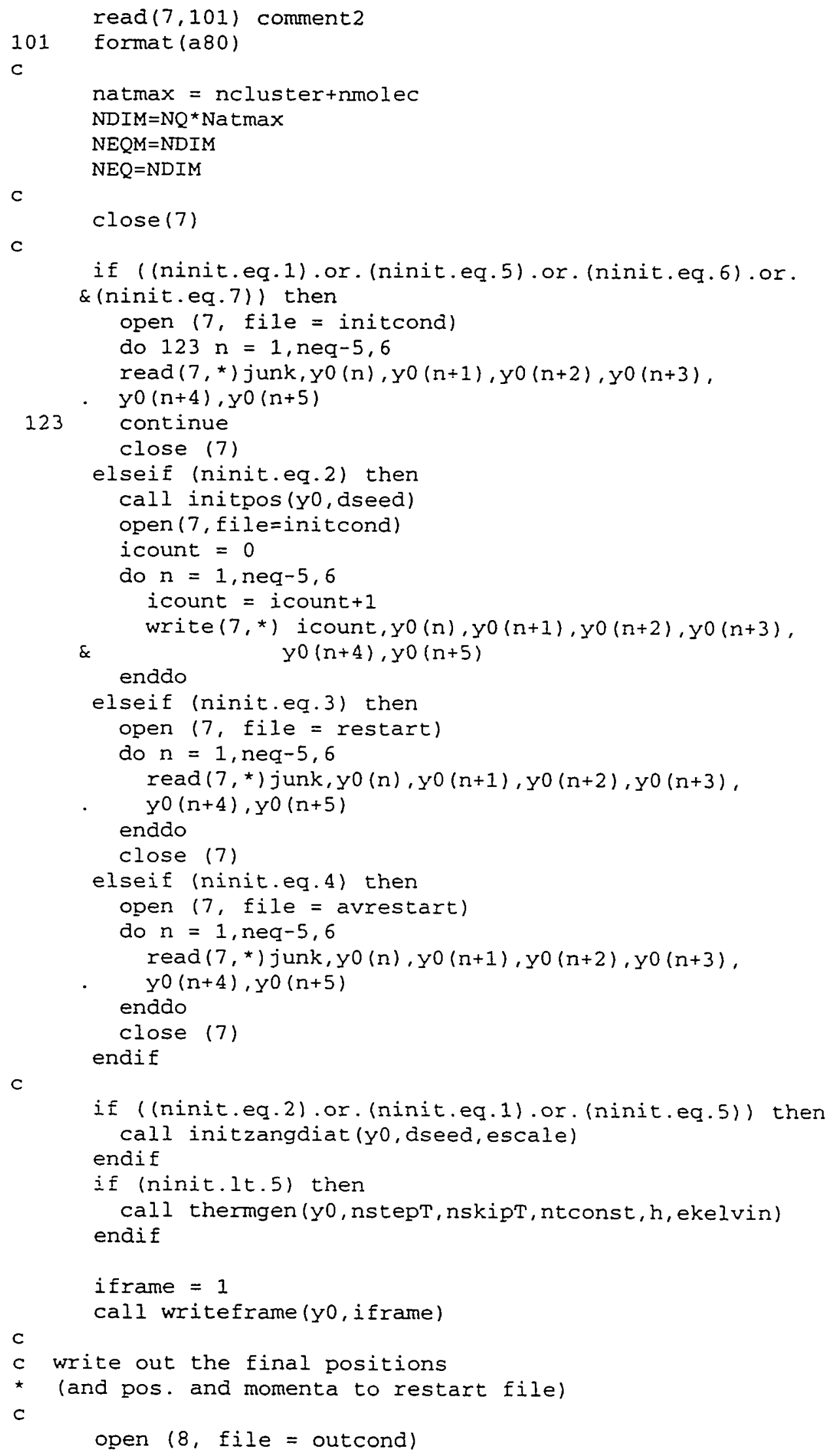




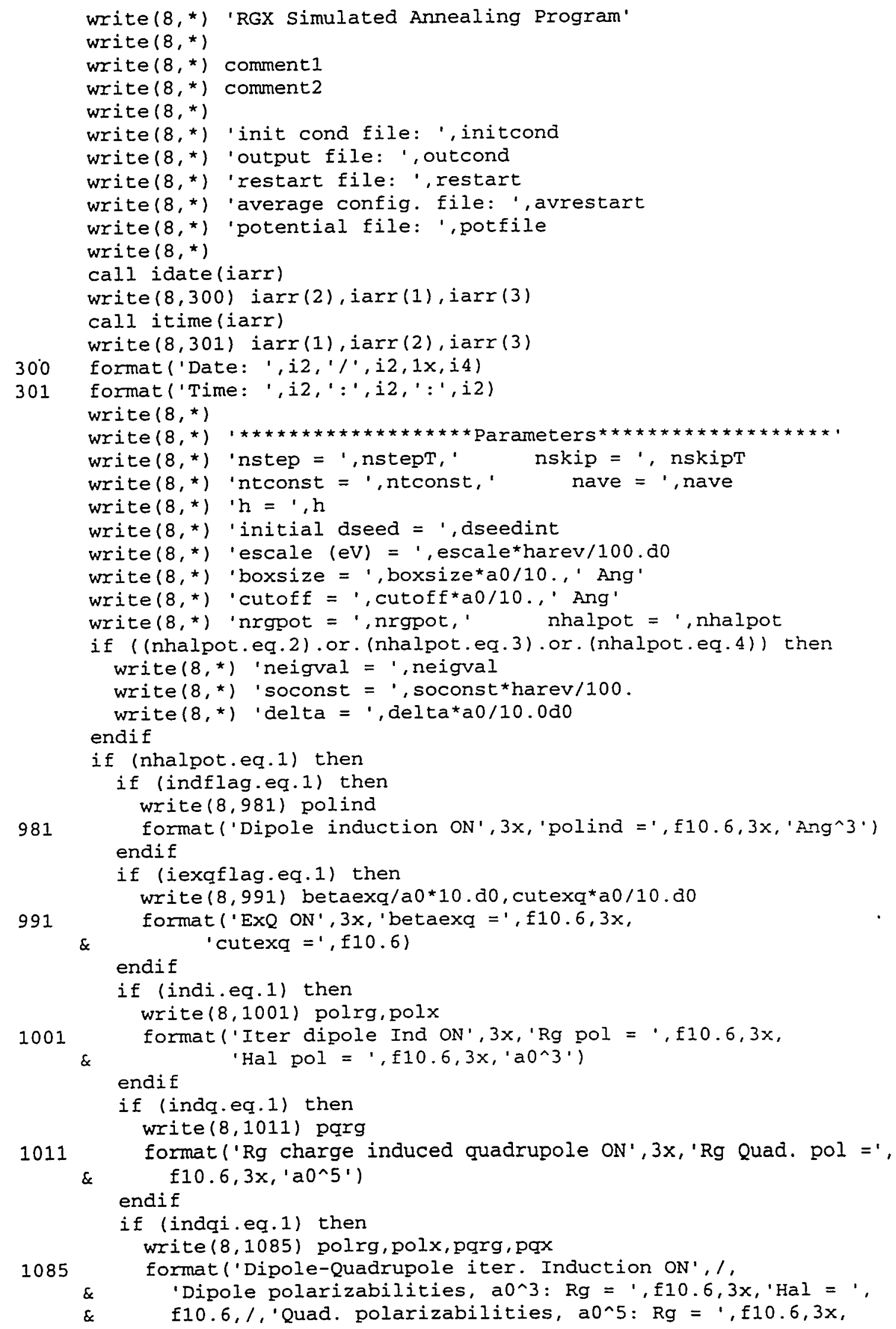




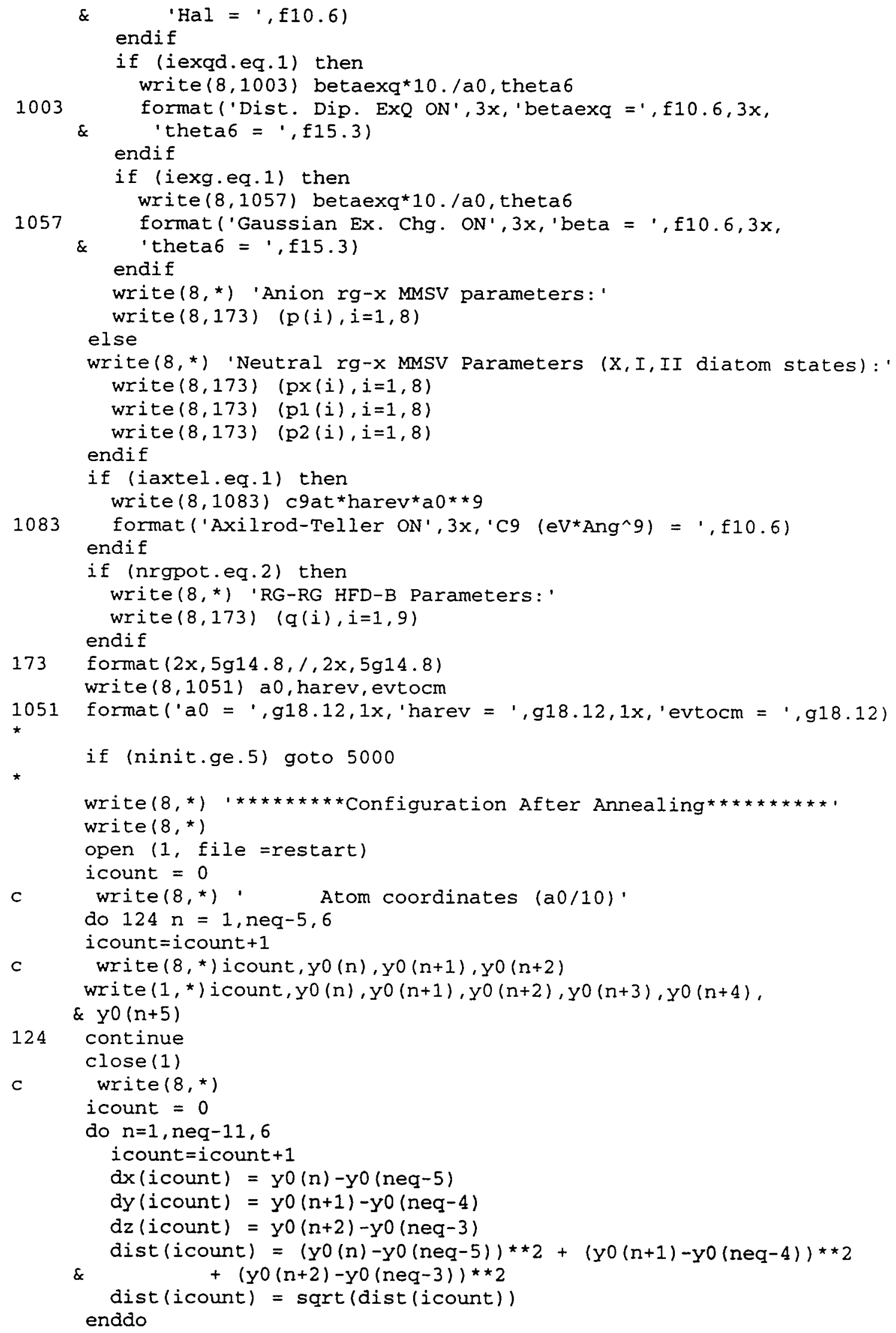




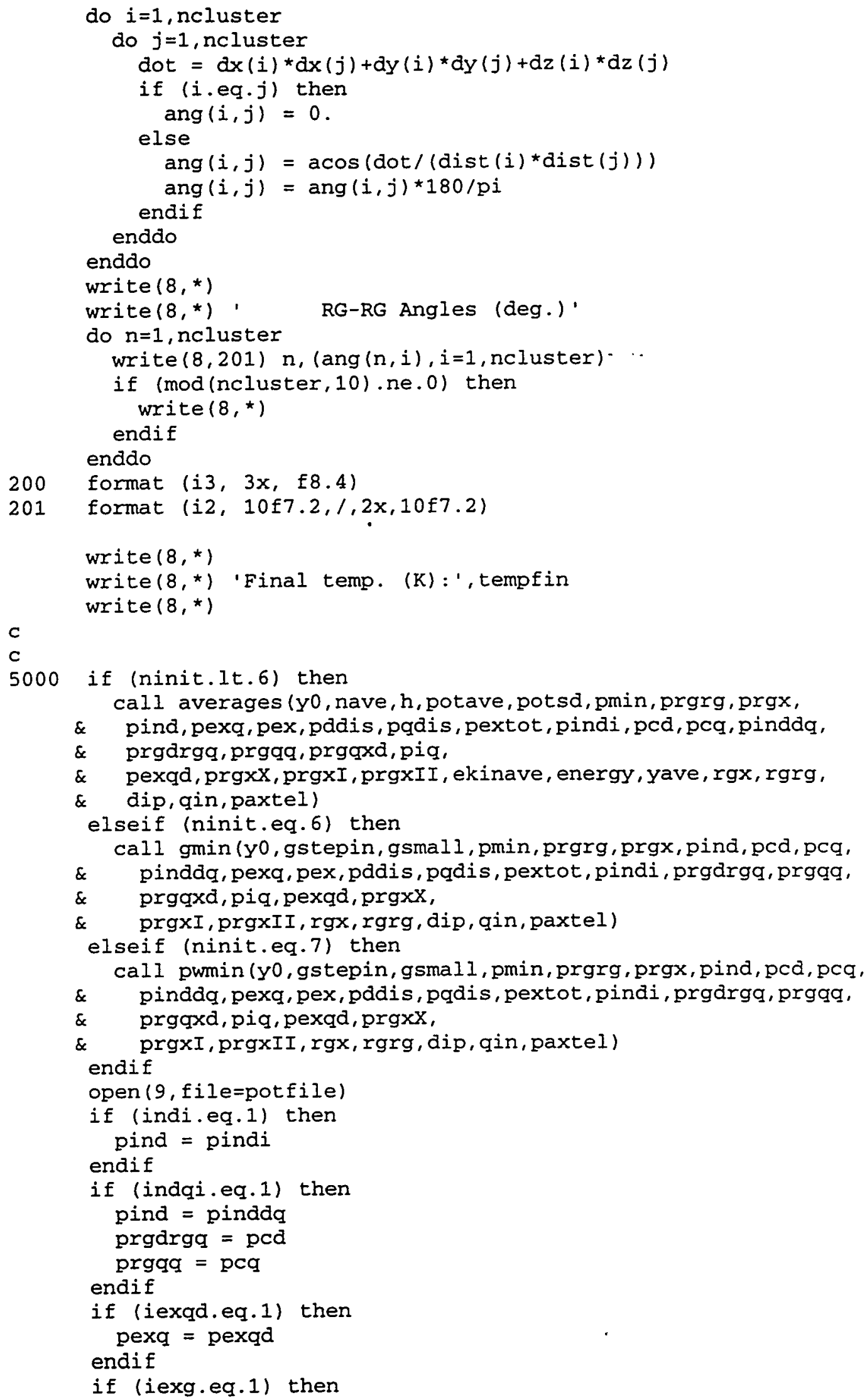




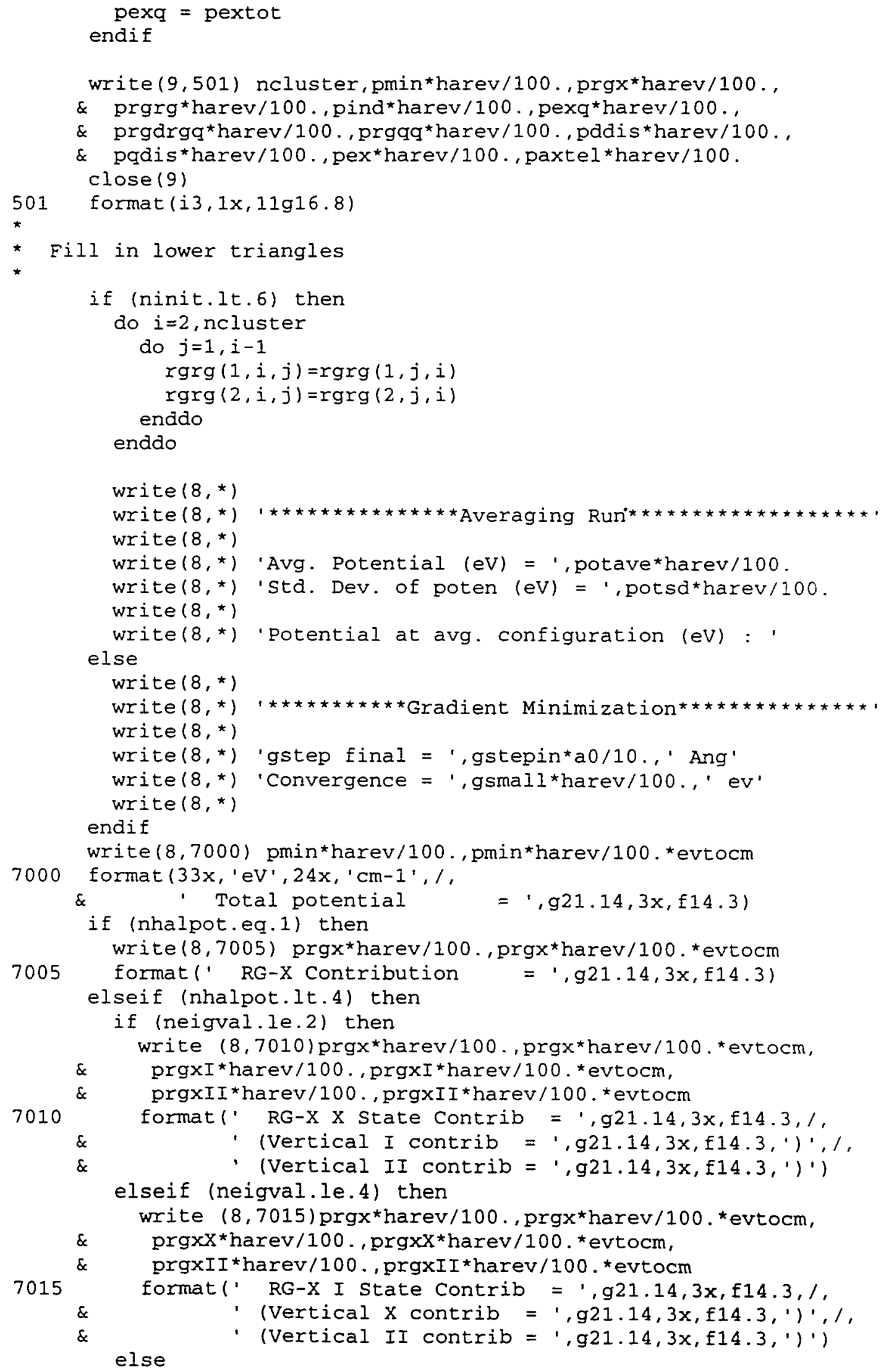


write $(8,7020)$ prgx harev/100.,prgx*harev/100.*evtocm,

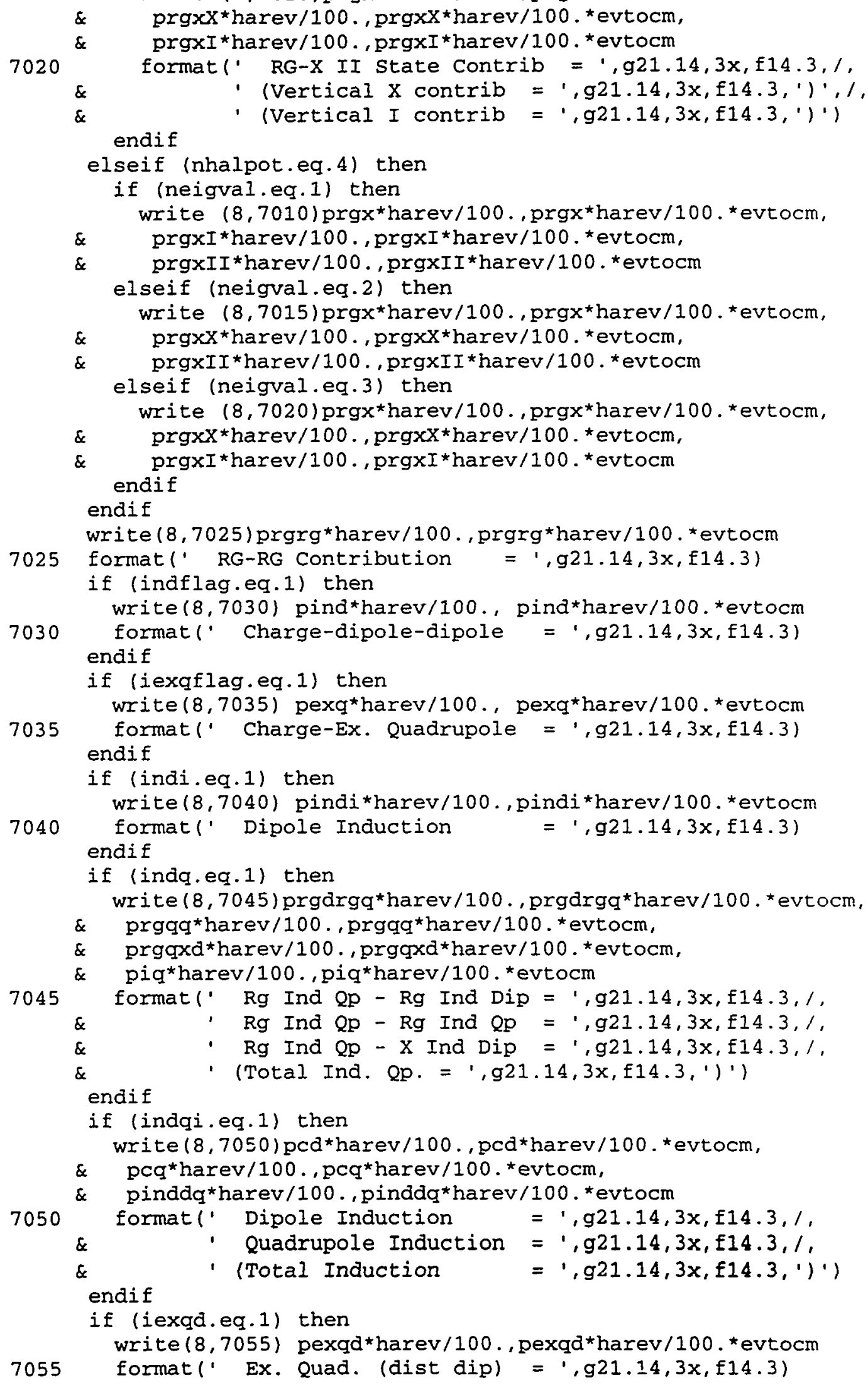




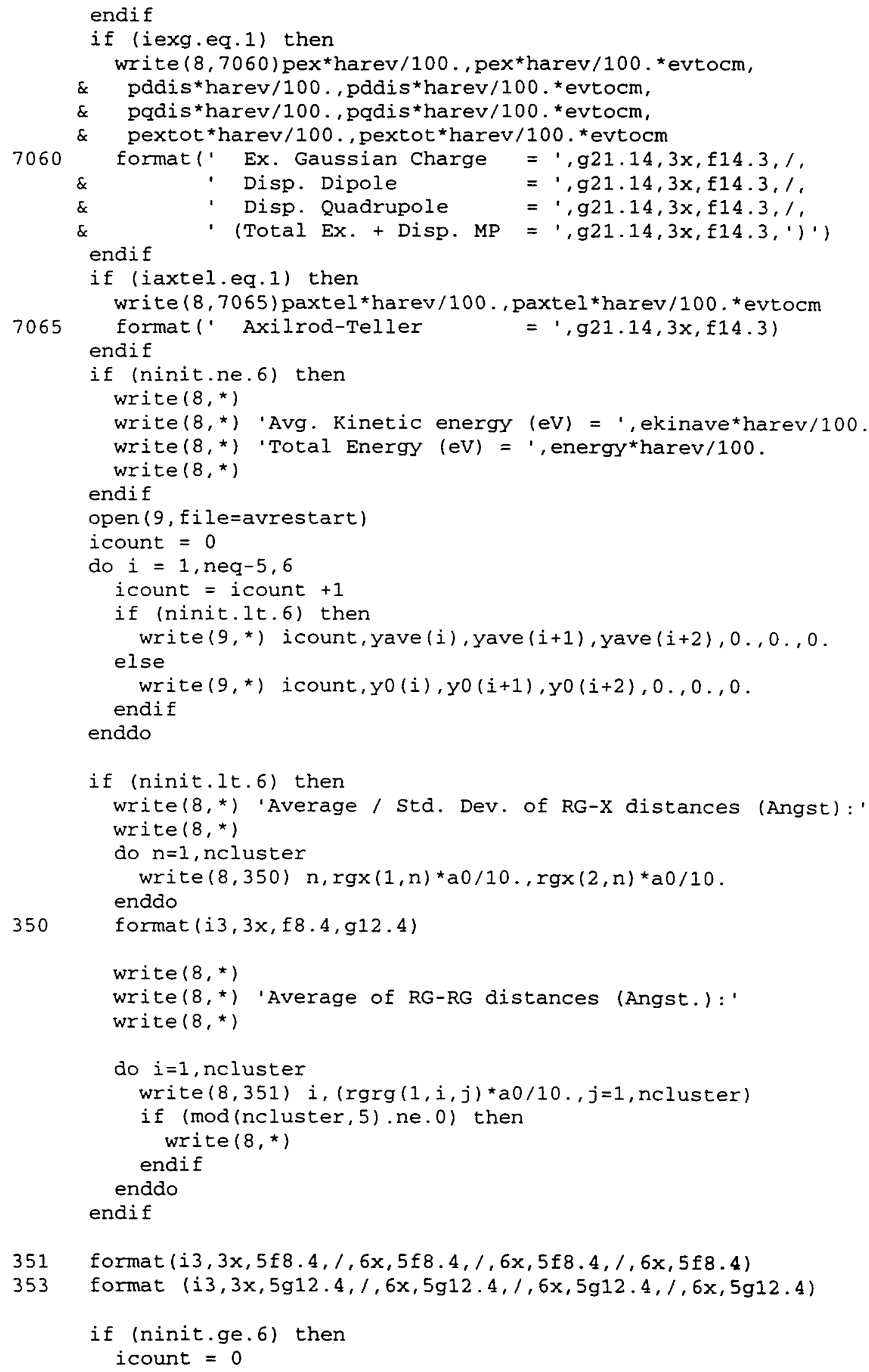




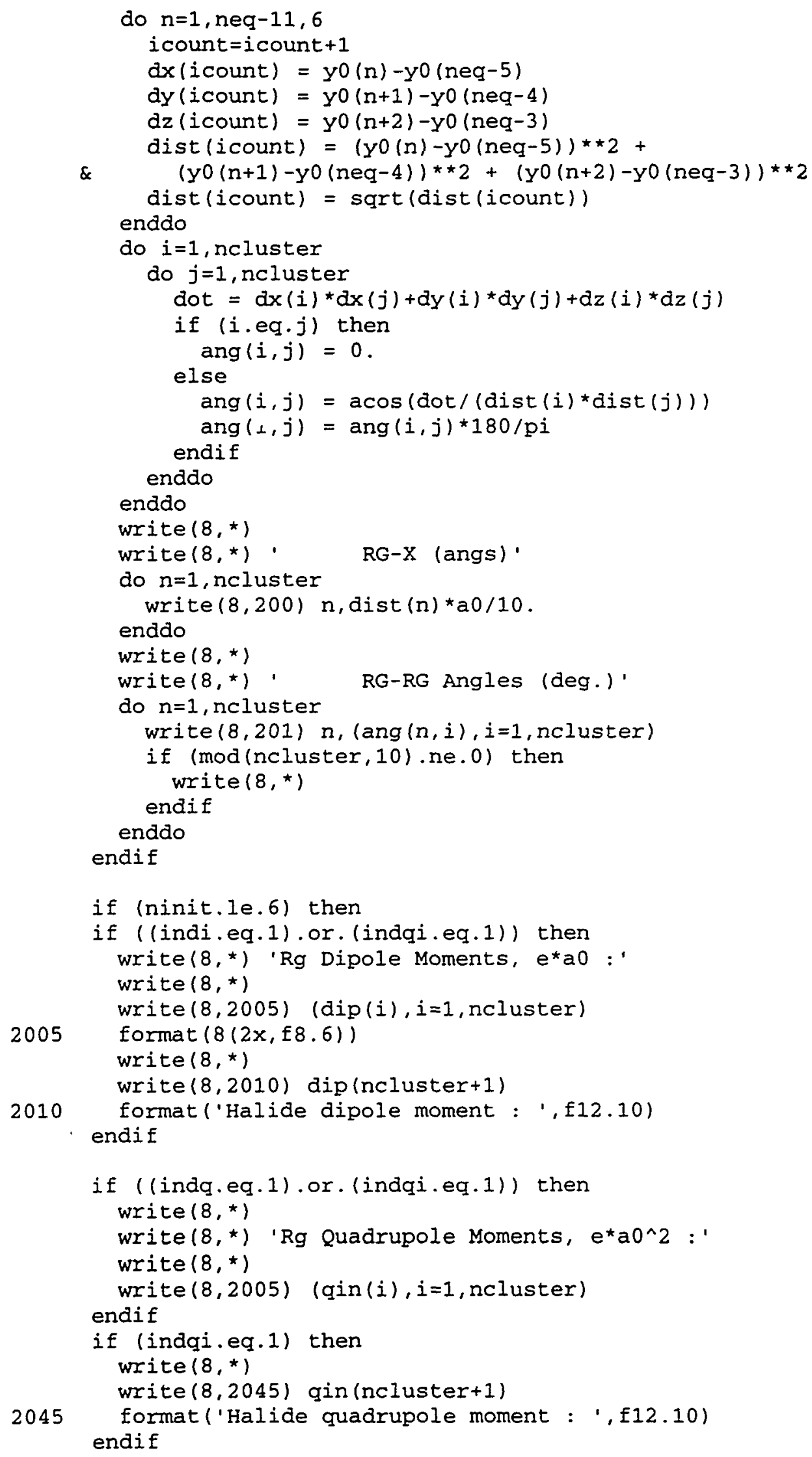


endif

close (8)

stop

end

\section{C5.4. File "aderiv.f"}

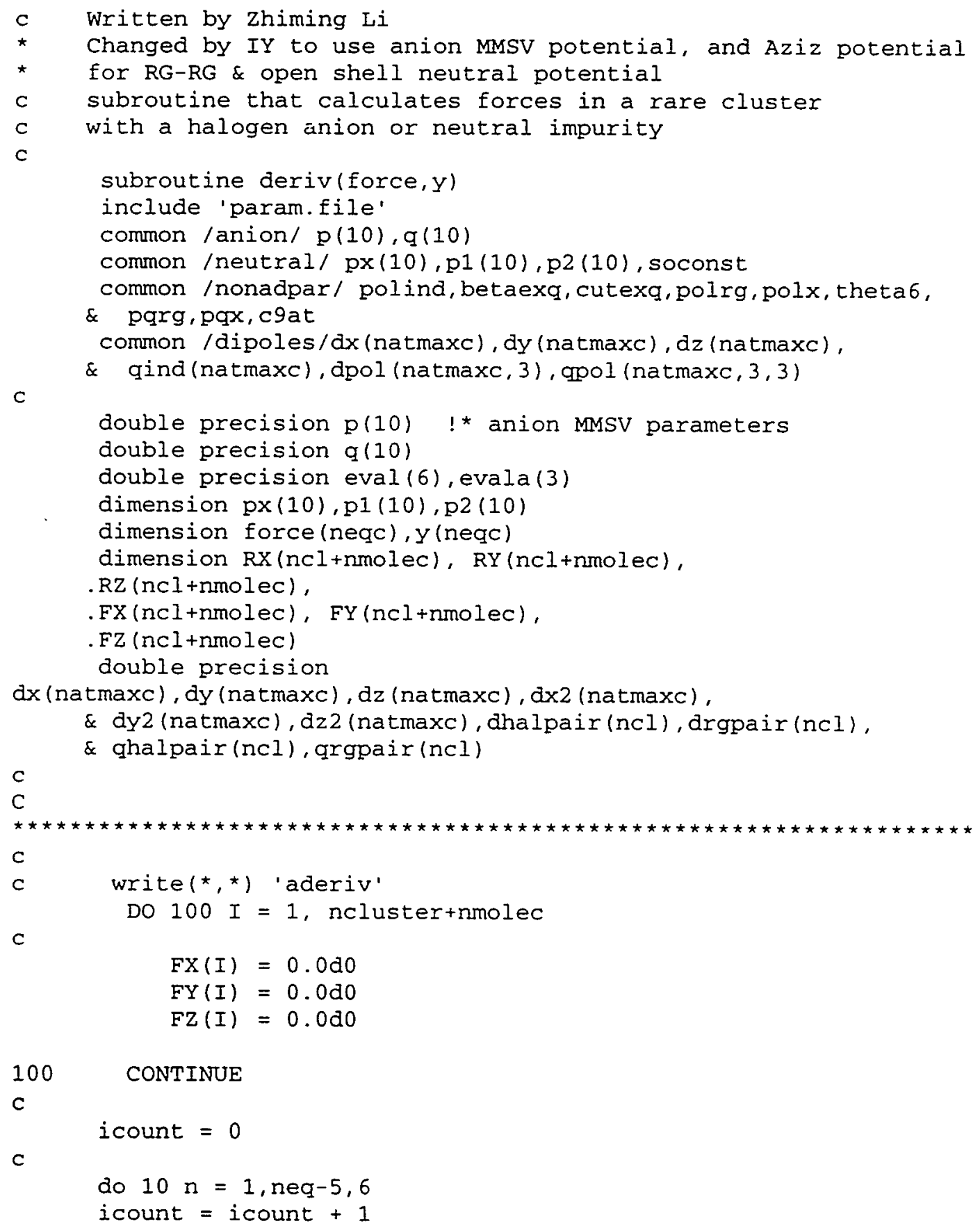




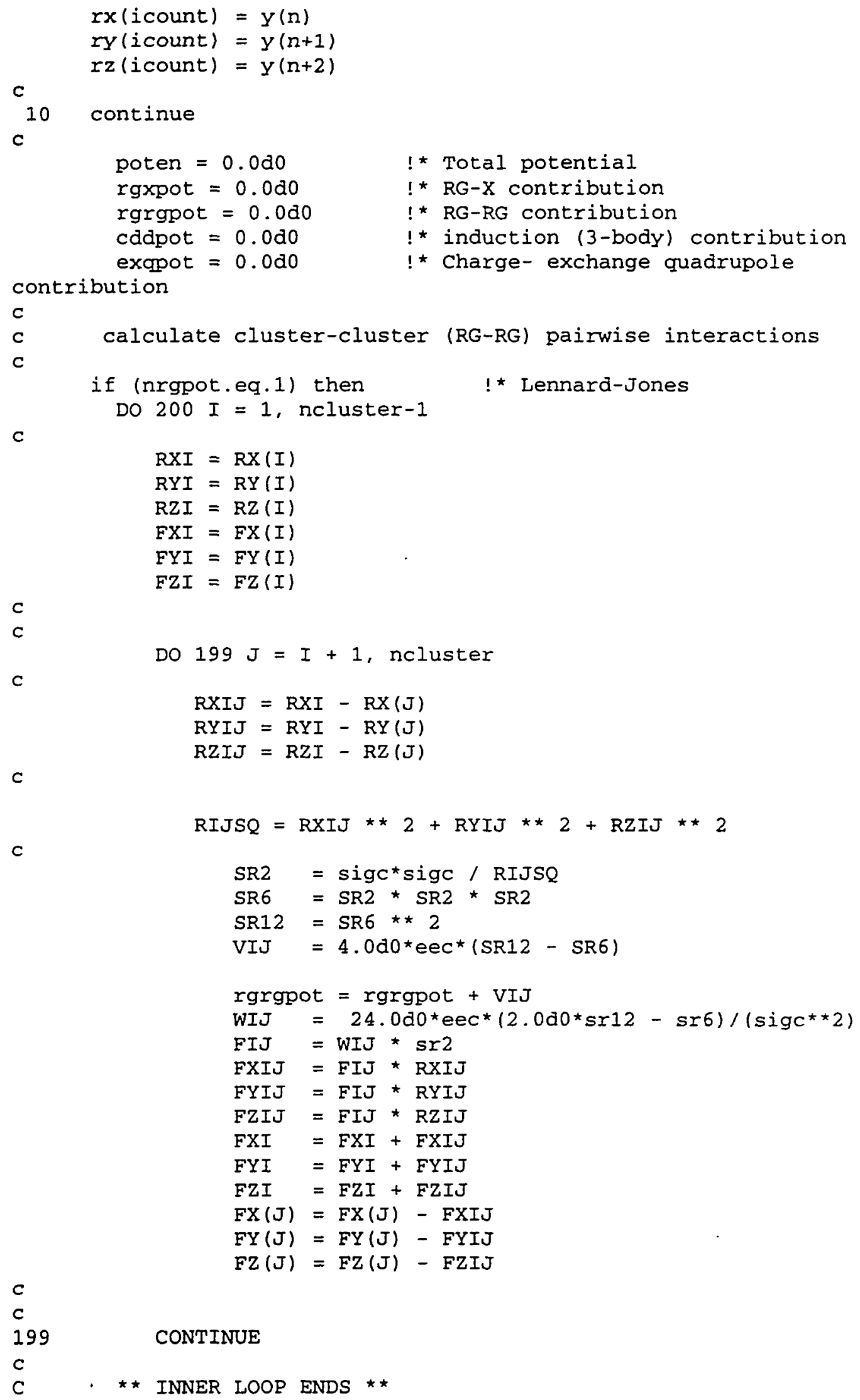

$c$

c

C

DO $199 \mathrm{~J}=\mathrm{I}+1$, ncluster

C

$$
\begin{aligned}
& \text { RXIJ }=\text { RXI }-R X(J) \\
& \text { RYIJ }=\text { RYI - RY (J) }
\end{aligned}
$$$$
R Z I J=R Z I-R Z(J)
$$

C

$$
\text { RIJSQ }=\text { RXIJ } \star \star 2+R Y I J \star * 2+R Z I J \star * 2
$$

C 


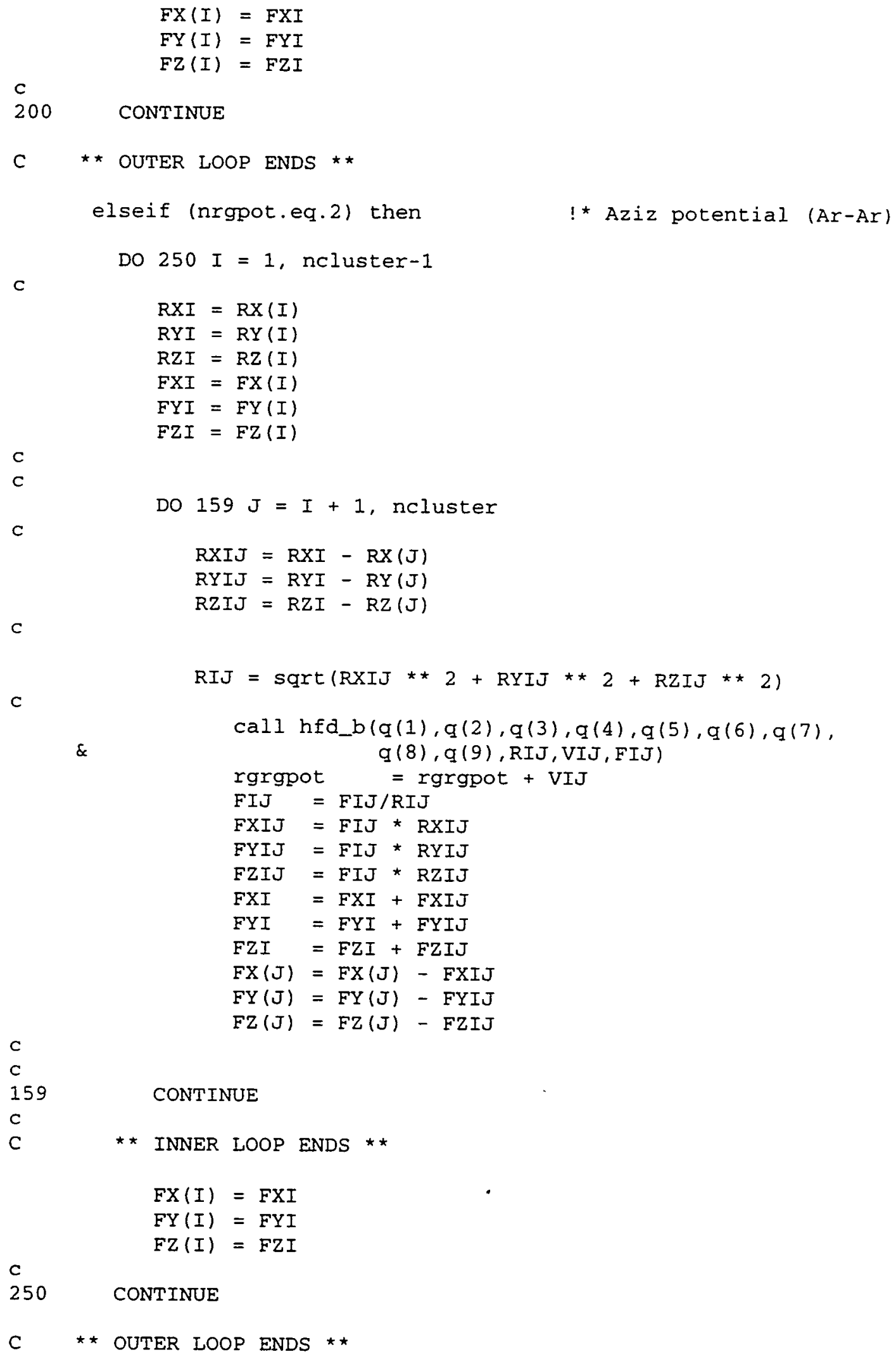

C

250 CONTINUE

C * * OUTER LOOP ENDS * * 


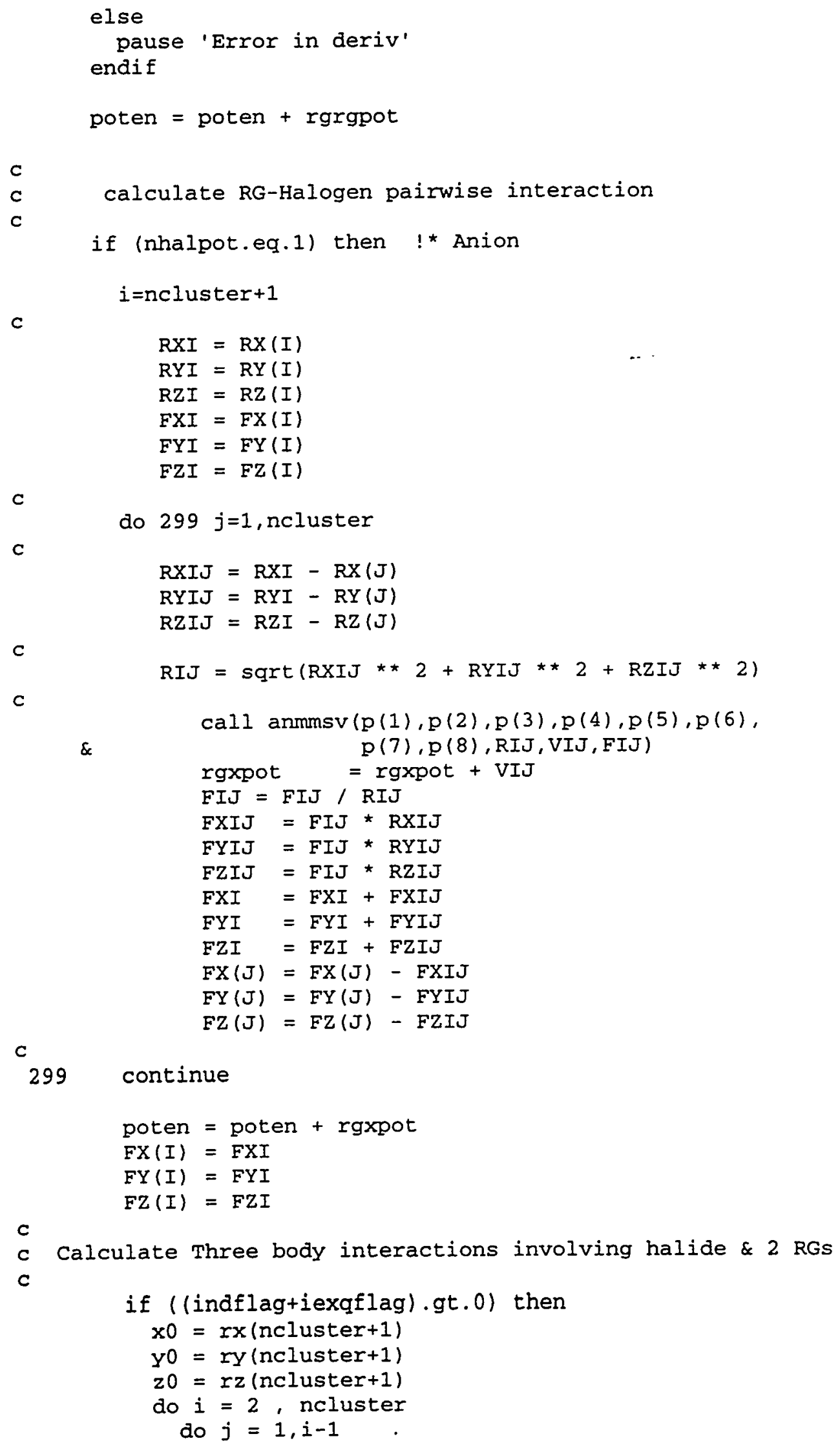

c

C Calculate Three body interactions involving halide \& 2 RGs

c 


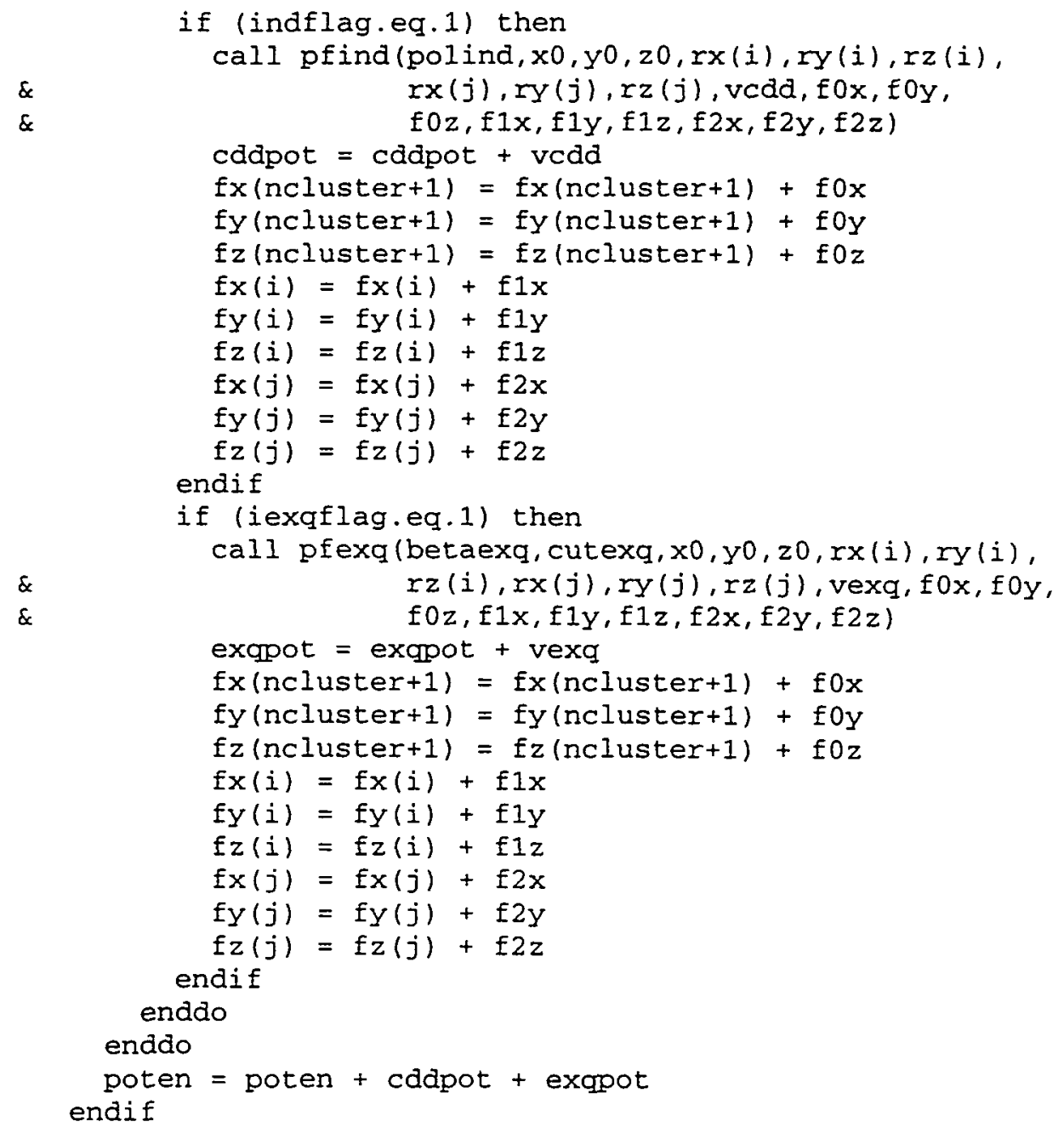

* Many body induction (by iteration) and new exchange quadrupole model,

* \& quadrupole induction

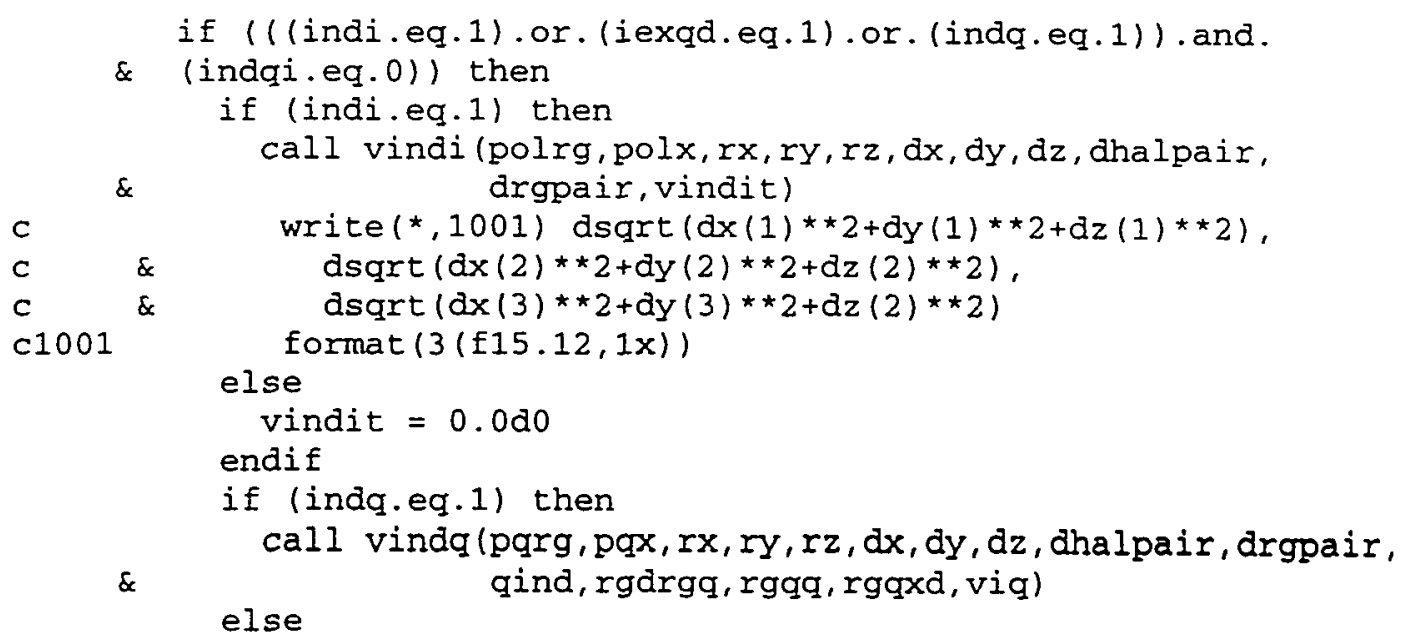




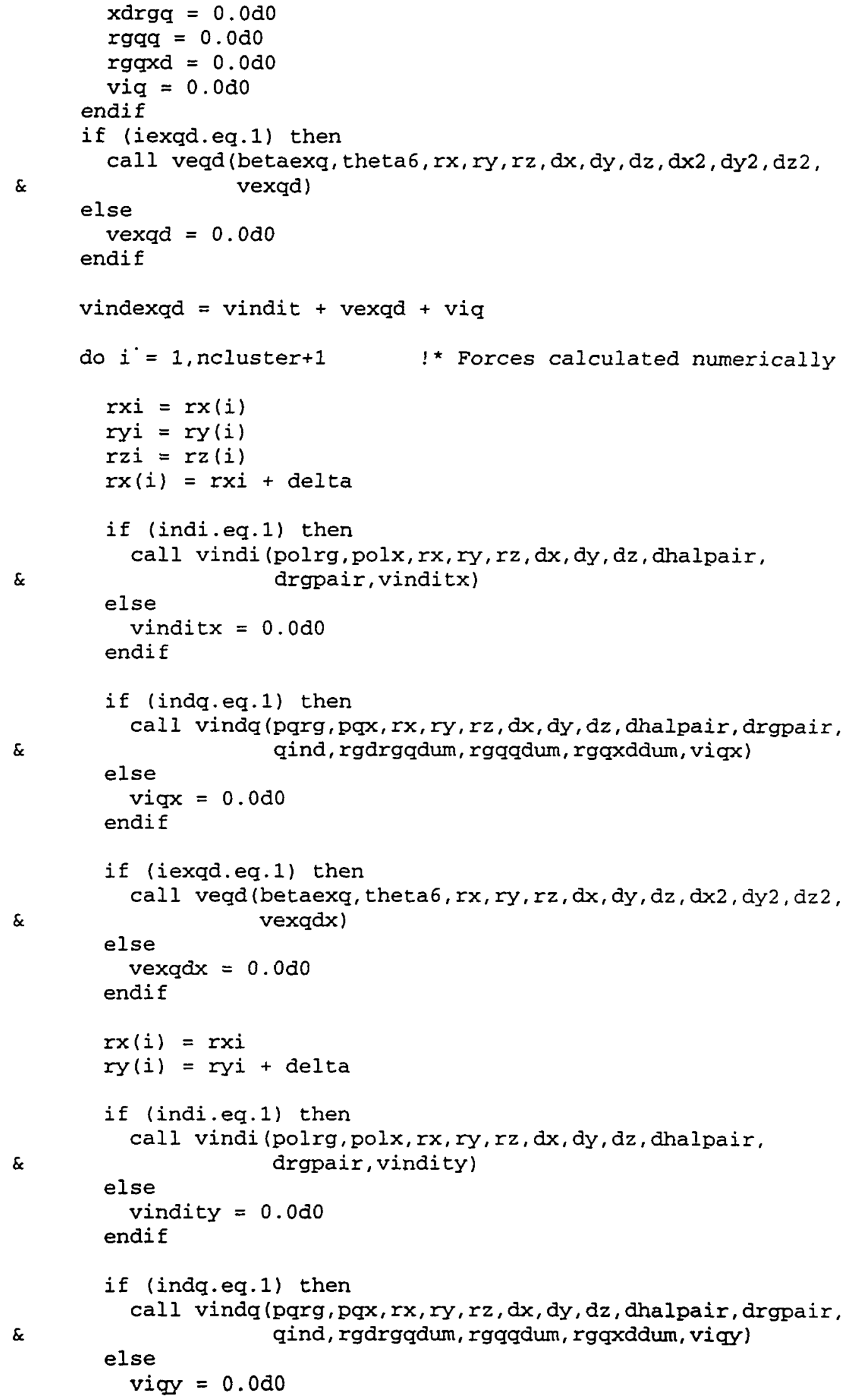


endif

if (iexgd.eq.1) then

call veqd (betaexq, theta $6, r x, r y, r z, d x, d y, d z, d x 2, d y 2, d z 2$,

$\&$ vexqdy)

else

vexqdy $=0.0 d 0$

endif

$r y(i)=r y i$

$r z(i)=r z i+\operatorname{delta}$

if (indi.eq.1) then

call vindi (polrg, polx, rx, ry, rz, dx, dy, dz, dhalpair.

$\&$

else drgpair, vinditz)

$$
\text { vinditz }=0.0 \mathrm{~d} 0
$$

endif

if (indq.eq.1) then

call vindq (pqrg, pqx, rx, ry, rz,dx,dy,dz, dhalpair,drgpair,

$\&$ qind, rgdrgqdum, rgqgdum, rgqxddum, viqz )

else

vigz $=0.0 \mathrm{~d} 0$

endif

if (iexqd.eq.1) then

call veqd (betaexq, theta $6, r x, r y, r z, d x, d y, d z, d x 2, d y 2, d z 2$,

$\&$ vexgdz)

else

vexgdz $=0.0 d 0$

endif

$r z(i)=r z i$

$f x(i)=f x(i)-($ vinditx+vexqdx+viqx-vindexqd)/delta

$f_{y}(i)=f_{y}(i)-(v i n d i t y+v e x q d y+v i q y-v i n d e x q d) / d e l t a$

$f z(i)=f z(i)-($ vinditz+vexqdz+viqz-vindexqd)/delta

enddo

poten $=$ poten + vindexqd

endif

* Iterated dipoles and quadrupoles, Gaussian Exchange \& Axilrod-Teller

if ((indqi.eq.1).or.(iexg.eq.1).or.(iaxtel.eq.1)) then

if (indqi.eq.1) then

call vinddq (polrg,polx, pqrg, pqx, rx, ry,rz, dpol, dhalpair,

$\&$ drgpair, qhalpair, qrgpair, qpol, cdpot, cqpot, cdqpot)

else

cdpot $=0$.

cqpot $=0$.

cdqpot $=0$.

endif 
if (iexqd.eg.1) then

call veqd (betaexq, theta $6, r x, r y, r z, d x, d y, d z, d x 2, d y 2, d z 2$,

$\&$ vexqd)

elseif (iexg.eq.1) then

call exg (betaexg, theta $6, r x, r y, r z$, gexpot, ddispot, qdispot,

$\&$ gextot)

vexqd $=0.0 \mathrm{~d} 0$

else

vexqd $=0$.

gexpot $=0$.

ddispot $=0$.

qdispot $=0$.

gextot $=0$.

endif

if (iaxtel.eq.1) then

call vat (c9at, rx, ry, rz, atpot)

else

atpot $=0$.

endif

vindexqd $=$ cdqpot + vexqd + gextot + atpot

do $i=1$, ncluster+1 $\quad$ * Forces calculated numerically

$r \times i=r \times(i)$

$r y i=r y(i)$

$r z i=r z(i)$

$r x(i)=r x i+\operatorname{delta}$

if (indqi.eq.1) then call vinddq (polrg, polx, pgrg, pqx, rx, ry, rz, dpol, dhalpair ,

$\&$ drgpair, qhalpair, qrgpair, gpol, cddum, cqdum, cdqpotx)

else

cdppotx $=0$.

endif

if (iexqd.eq.1) then

call veqd (betaexq, theta $6, r x, r y, r z, d x, d y, d z, d x 2, d y 2, d z 2$,

$\&$ vexqdx)

elseif (iexg.eq.1) then

call exg (betaexq, theta $6, r x, r y, r z$, gexdum, ddisdum, qdisdum,

$\&$ gextotx)

vexqdx $=0.0 d 0$

else

gextotx $=0$.

vexgdx $=0.0 d 0$

endif

if (iaxtel.eq.1) then

call vat (c9at, rx, ry, rz, atpot $x$ )

else

atpotx $=0$.

endif

$\operatorname{rx}(i)=\mathrm{rxi}$

ry $(i)=r y i+$ delta 
if (indqi.eq.1) then

call vinddg (polrg, polx,pqrg,pqx, rx, ry, rz, dpol, dhalpair, drgpair, qhalpair, grgpair, qpol, cddum, cqdum, cdqpoty)

else cdqpoty $=0$.

endif

if (iexgd.eq.1) then call vegd (betaexq, theta $6, r x, r y, r z, d x, d y, d z, d x 2, d y 2, d z 2$, vexqdy)

elseif (iexg.eq.1) then

call exg (betaexq, theta $6, r x, r y, r z$, gexdum, ddisdum, qdisdum, 


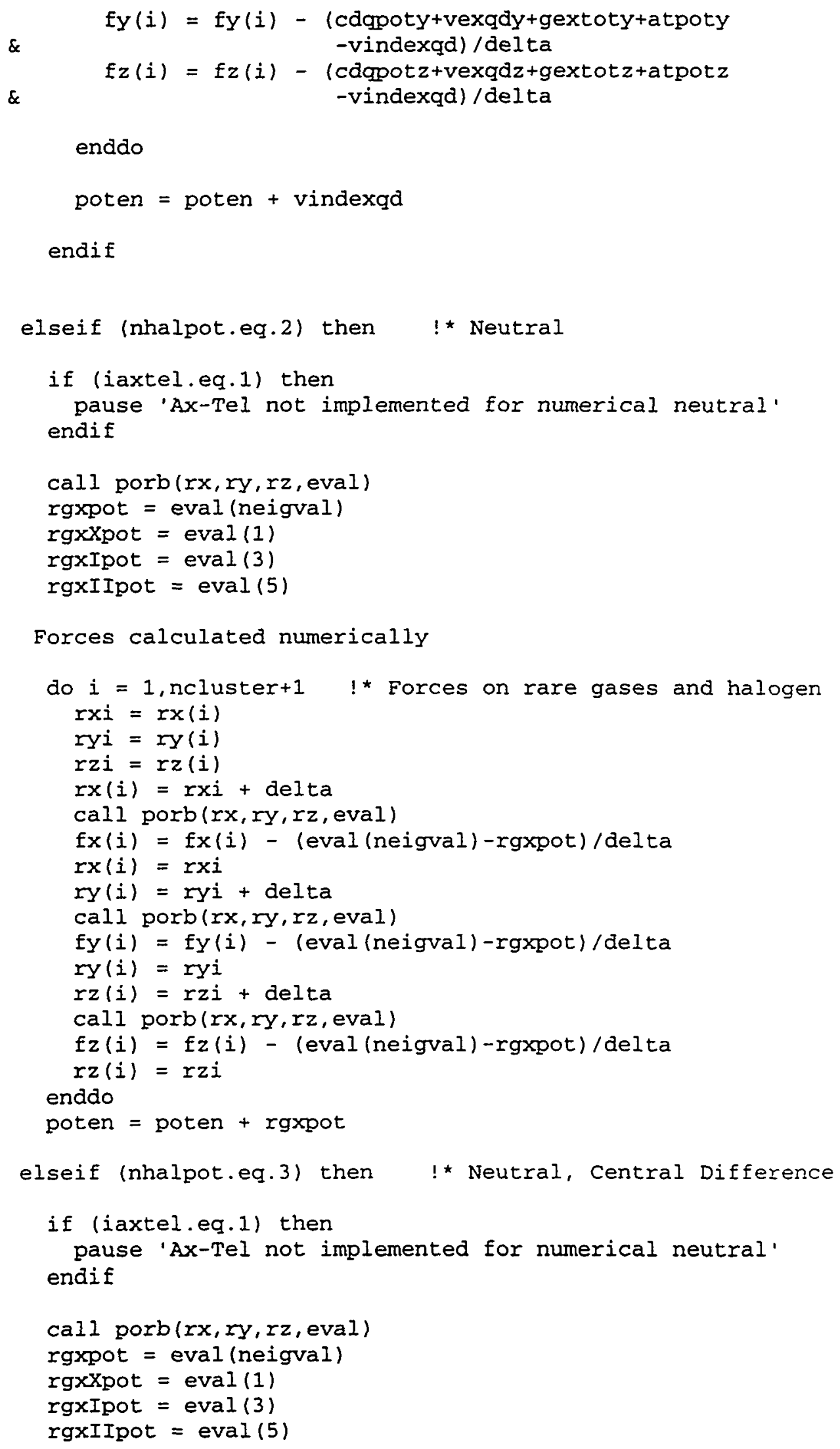

enddo

poten $=$ poten + vindexgd

endif

elseif (nhalpot.eq.2) then !* Neutral

if (iaxtel.eq.1) then

pause 'Ax-Tel not implemented for numerical neutral' endif

call porb (rx, ry, rz, eval)

rgxpot = eval (neigval)

rgxXpot $=$ eval $(1)$

rgxipot $=$ eval $(3)$

rgxIIpot $=\operatorname{eval}(5)$

Forces calculated numerically

do $i=1$, ncluster+1 $!$ * Forces on rare gases and halogen

$r x i=r x(i)$

$r y i=r y(i)$

$r z i=r z(i)$

$r x(i)=r x i+$ delta

call porb(rx, ry,rz, eval)

$\mathrm{fx}(i)=f x(i)-($ eval (neigval) - rgxpot)/delta

$r x(i)=r x i$

$r y(i)=r y i+$ delta

call porb(rx,ry,rz, eval)

$f_{y}(i)=f_{y}(i)-($ eval (neigval) - rgxpot) $/$ delta

$r y(i)=r y i$

$r z(i)=r z i+$ delta

call porb(rx, ry, rz, eval)

$f z(i)=f z(i)-($ eval (neigval) - rgxpot) $/$ delta

$r z(i)=r z i$

enddo

poten $=$ poten + rgxpot

elseif (nhalpot.eq.3) then !* Neutral, Central Difference

if (iaxtel.eg.1) then

pause 'Ax-Tel not implemented for numerical neutral' endif

call porb (rx, ry, rz, eval)

rgxpot = eval (neigval)

rgxxpot $=$ eval (1)

rgxIpot $=$ eval (3)

rgxIIpot $=$ eval $(5)$ 


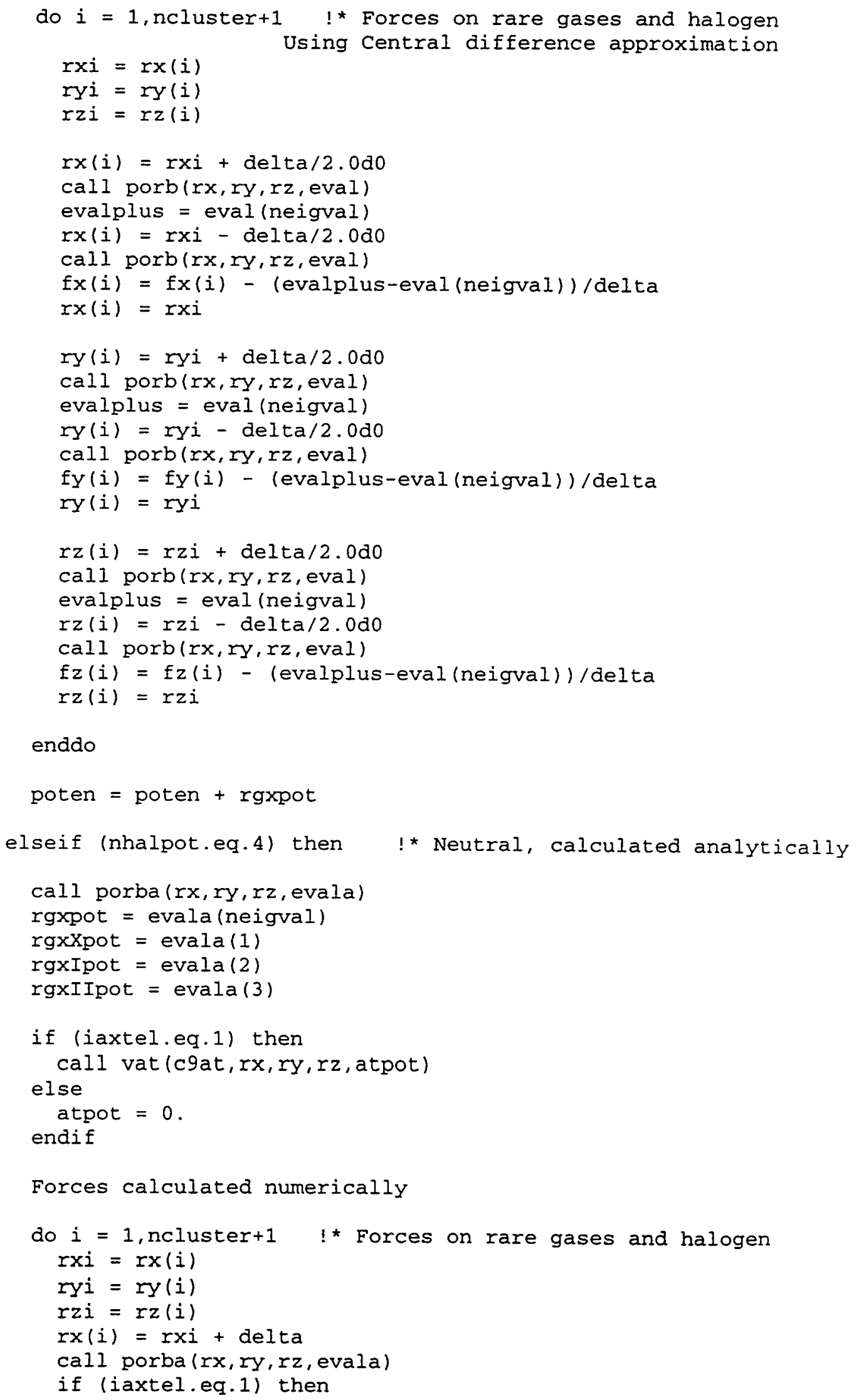




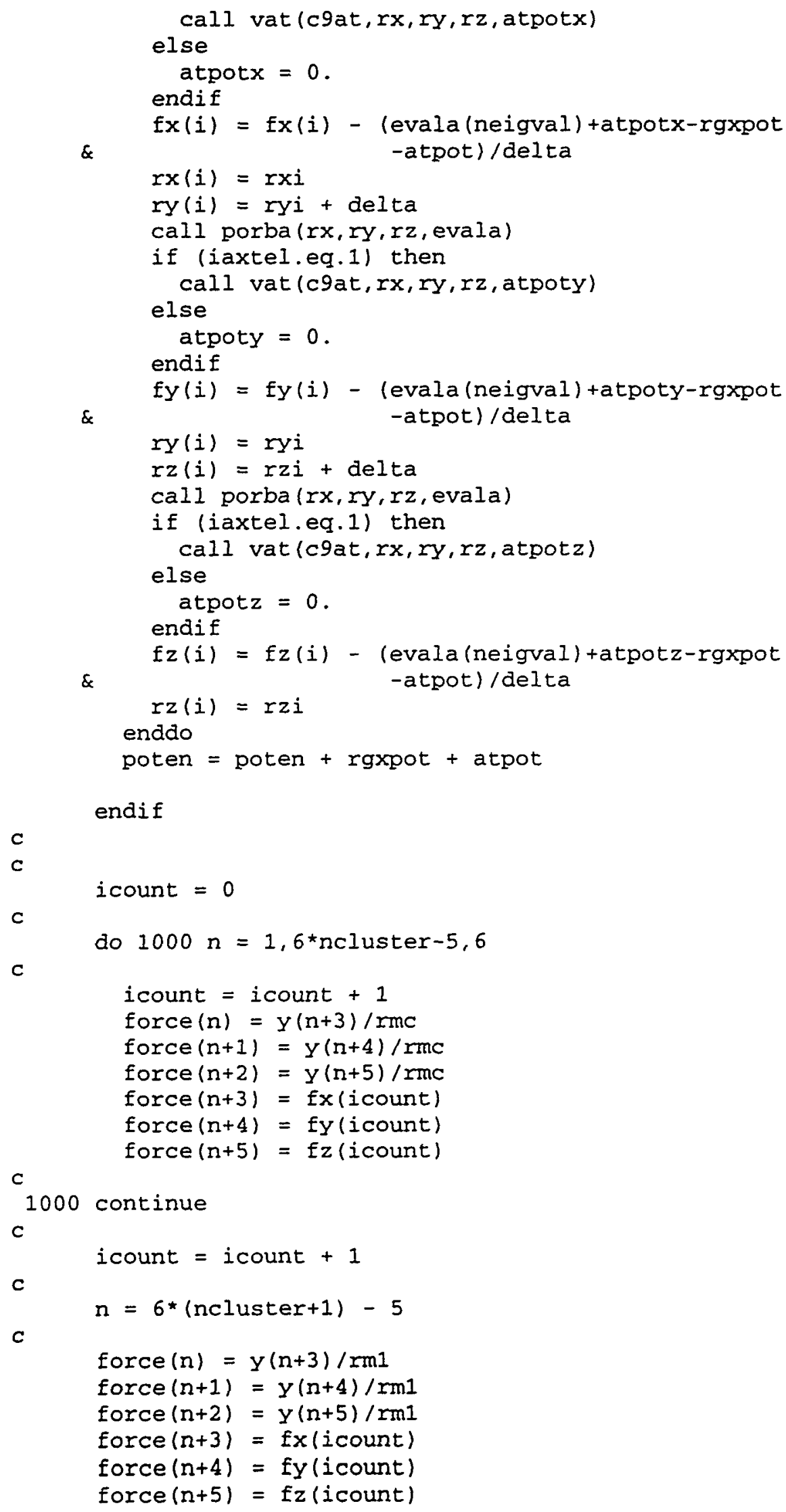


$c$

RETURN

END

$\mathrm{C}$

subroutine kinetic (y, ekin)

include 'param.file'

c

dimension $y$ (negmc)

ekin $=0.0 \mathrm{do}$

c

do $100 \mathrm{n}=1,6^{*}$ ncluster $-5,6$

ekin $=$ ekin

100

. $+(y(n+3) \star \star 2+y(n+4) \star \star 2+y(n+5) \star \star 2) /(2.0 \mathrm{~d} 0 \star \mathrm{rmc})$

c continue

$n=6^{*}$ (ncluster +1$)-5$

ekin $=$ ekin $+(y(n+3) * \star 2+y(n+4) \star \star 2+y(n+5) * \star 2) /(2.0 d 0 * n m 1)$

return

end

C5.5. File "initzangdiat.f"

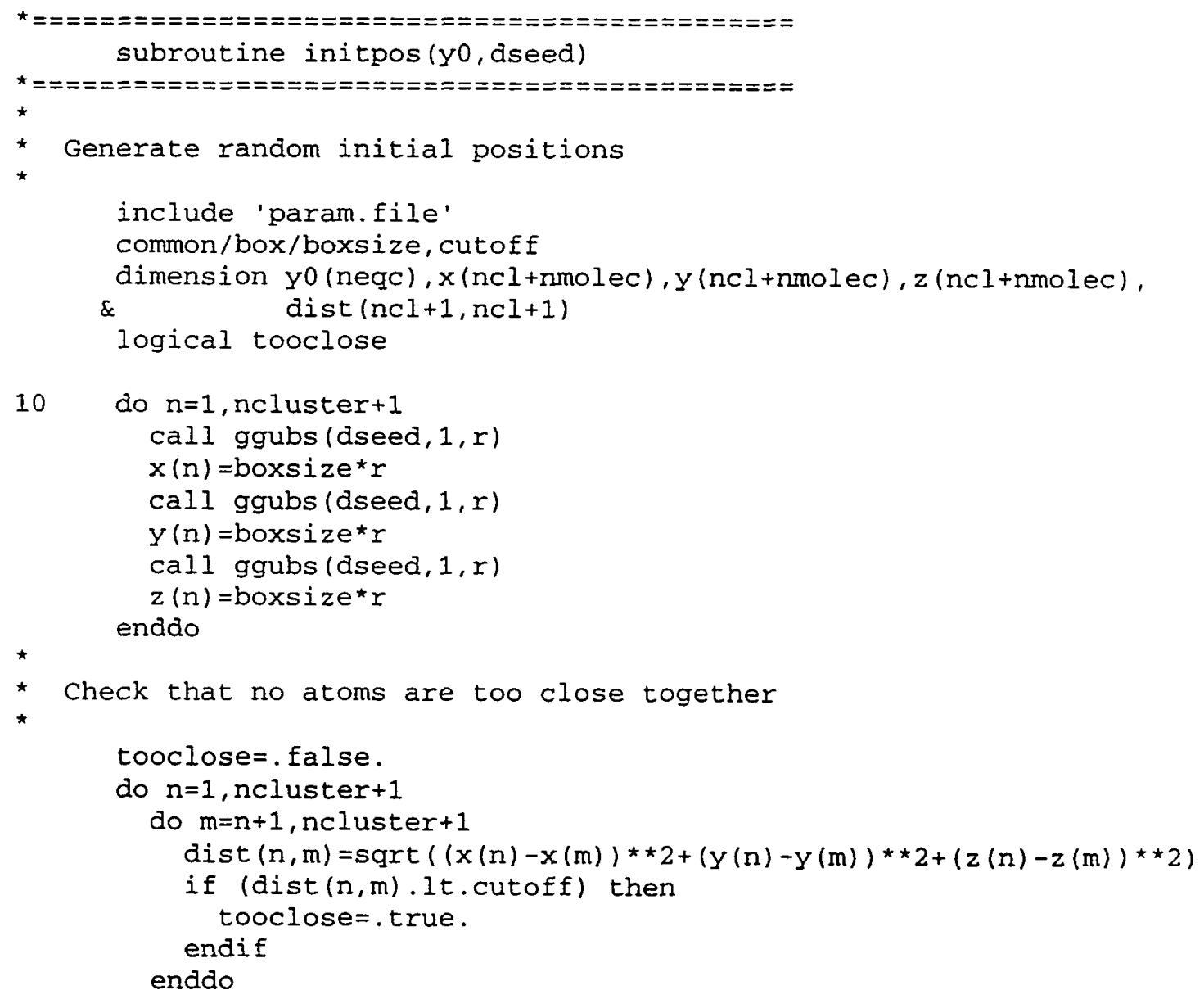




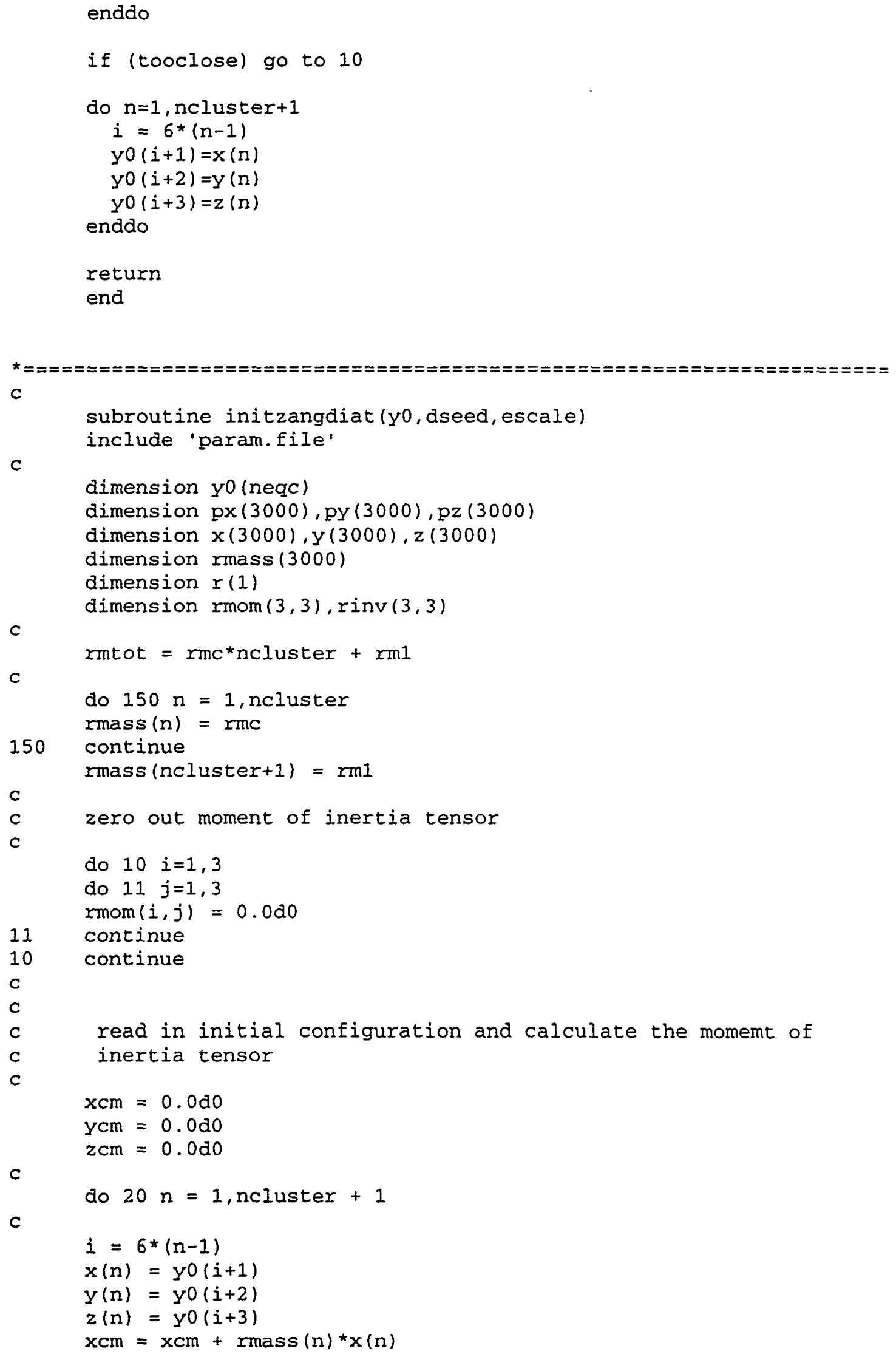




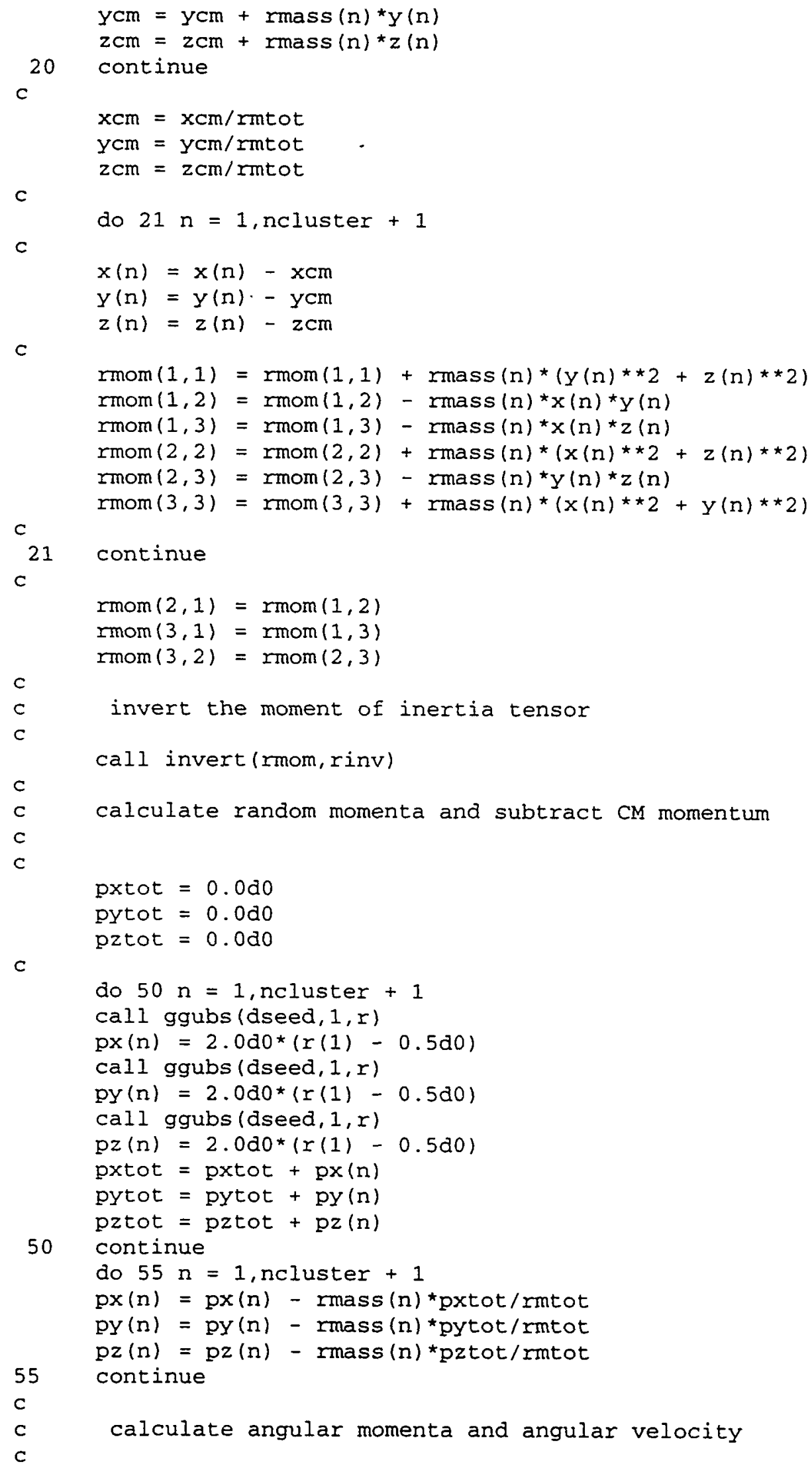




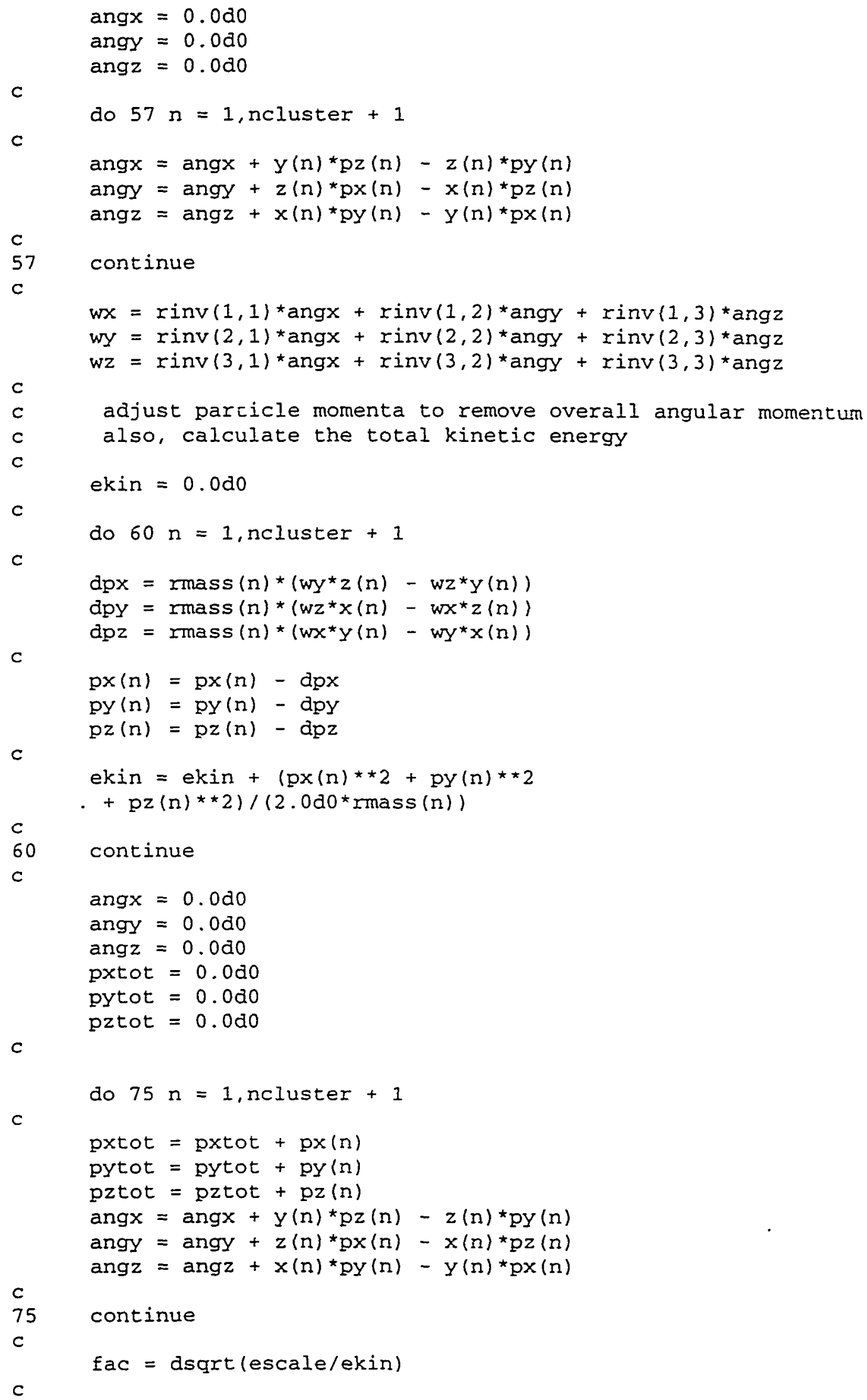

C 


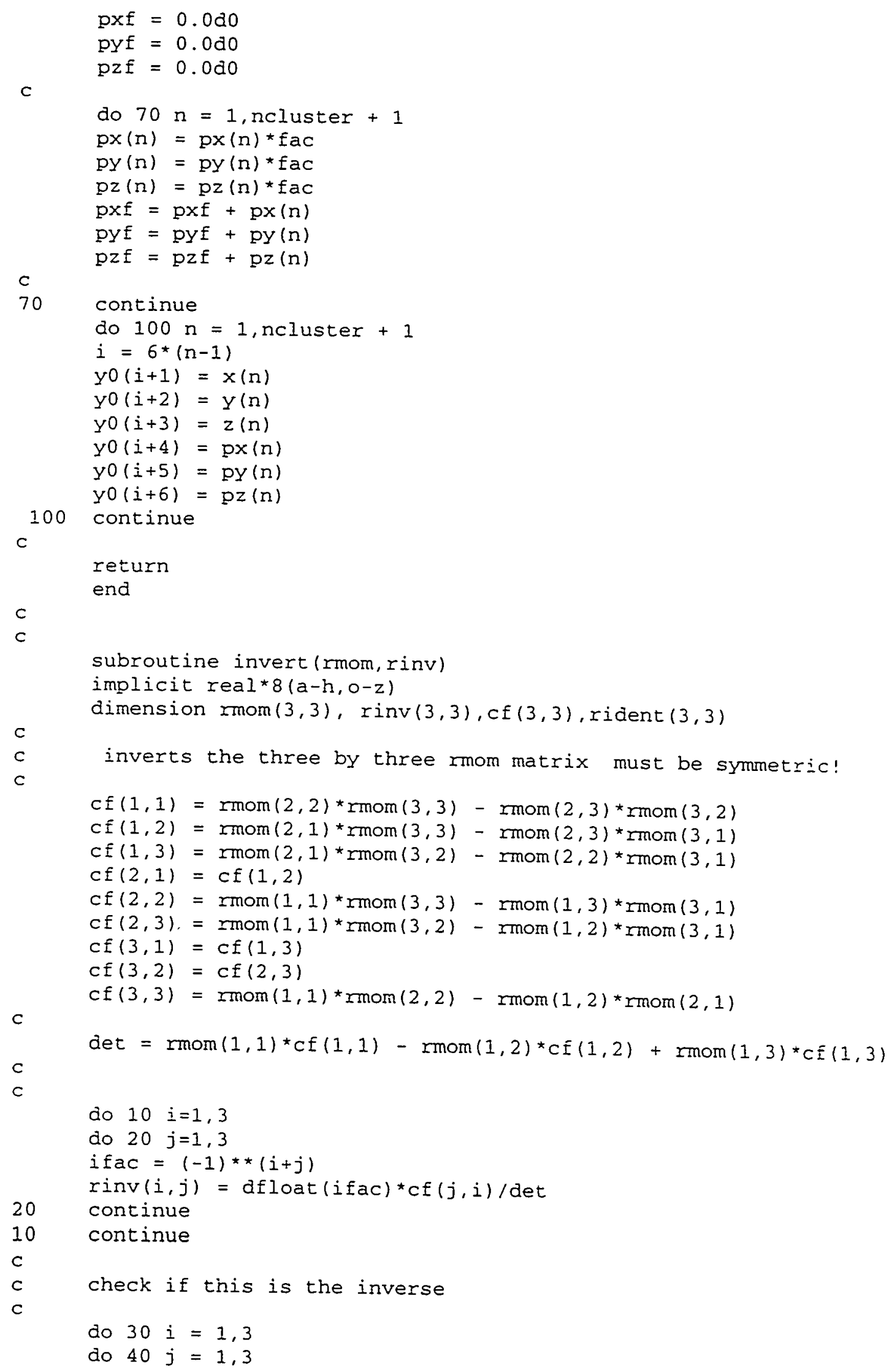




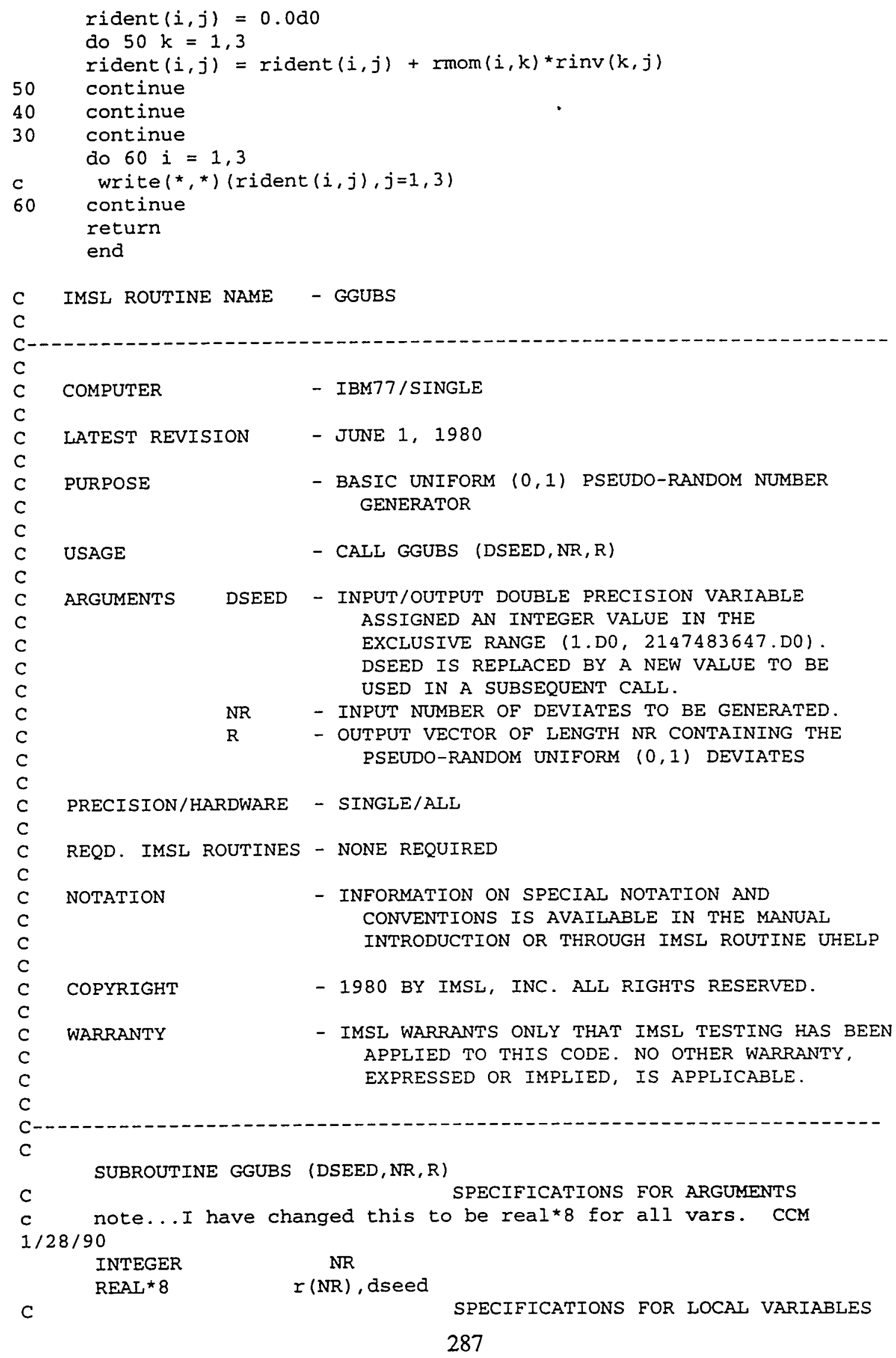




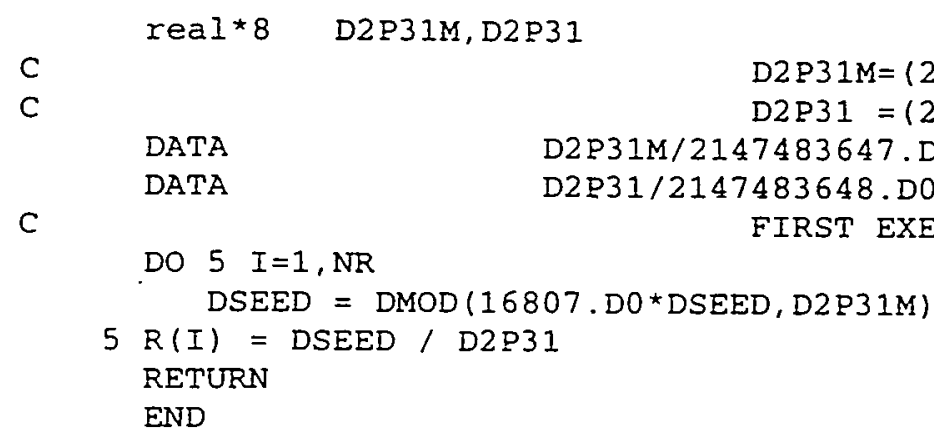

\section{C5.6. File "thermgen.f"}

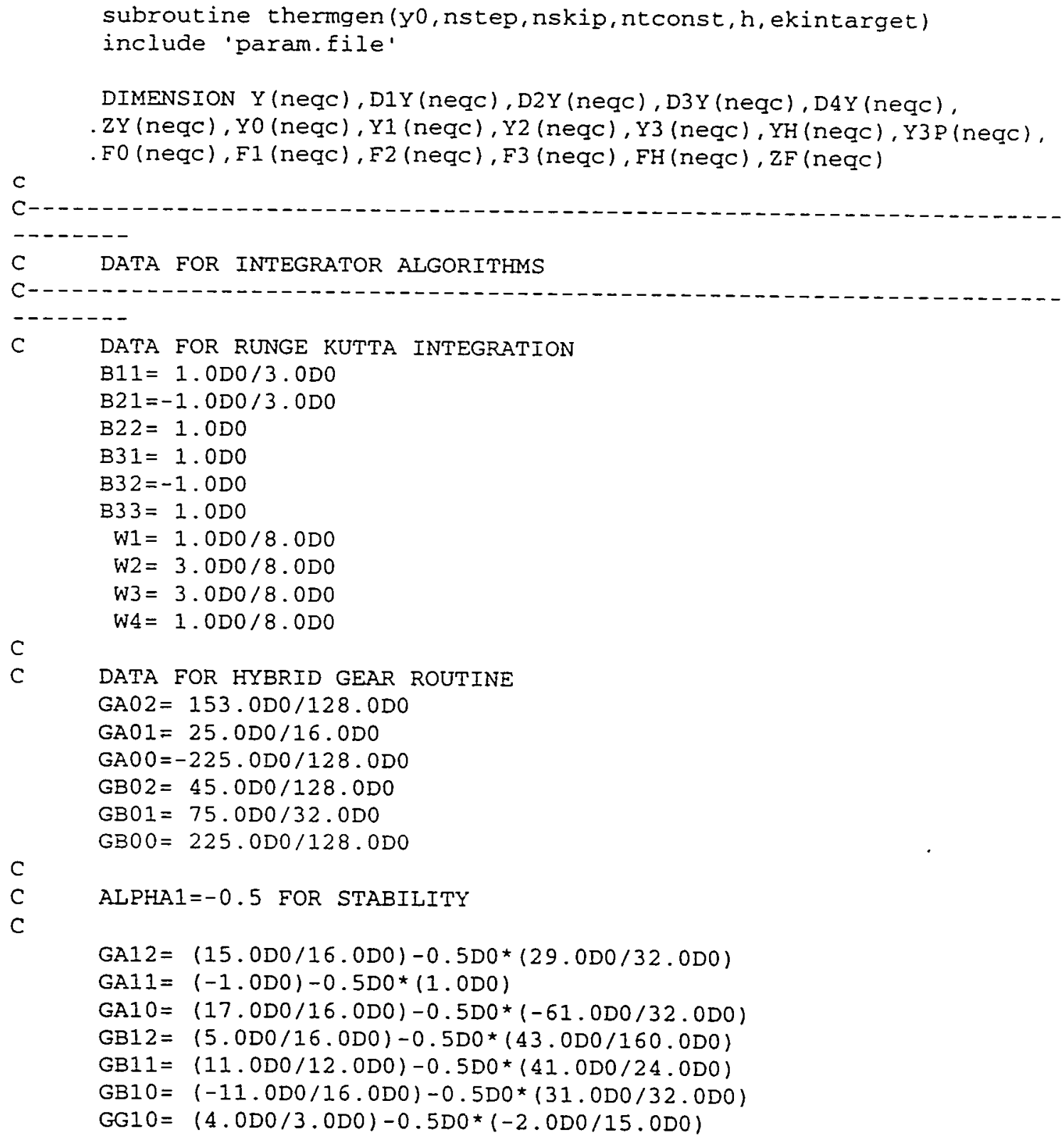




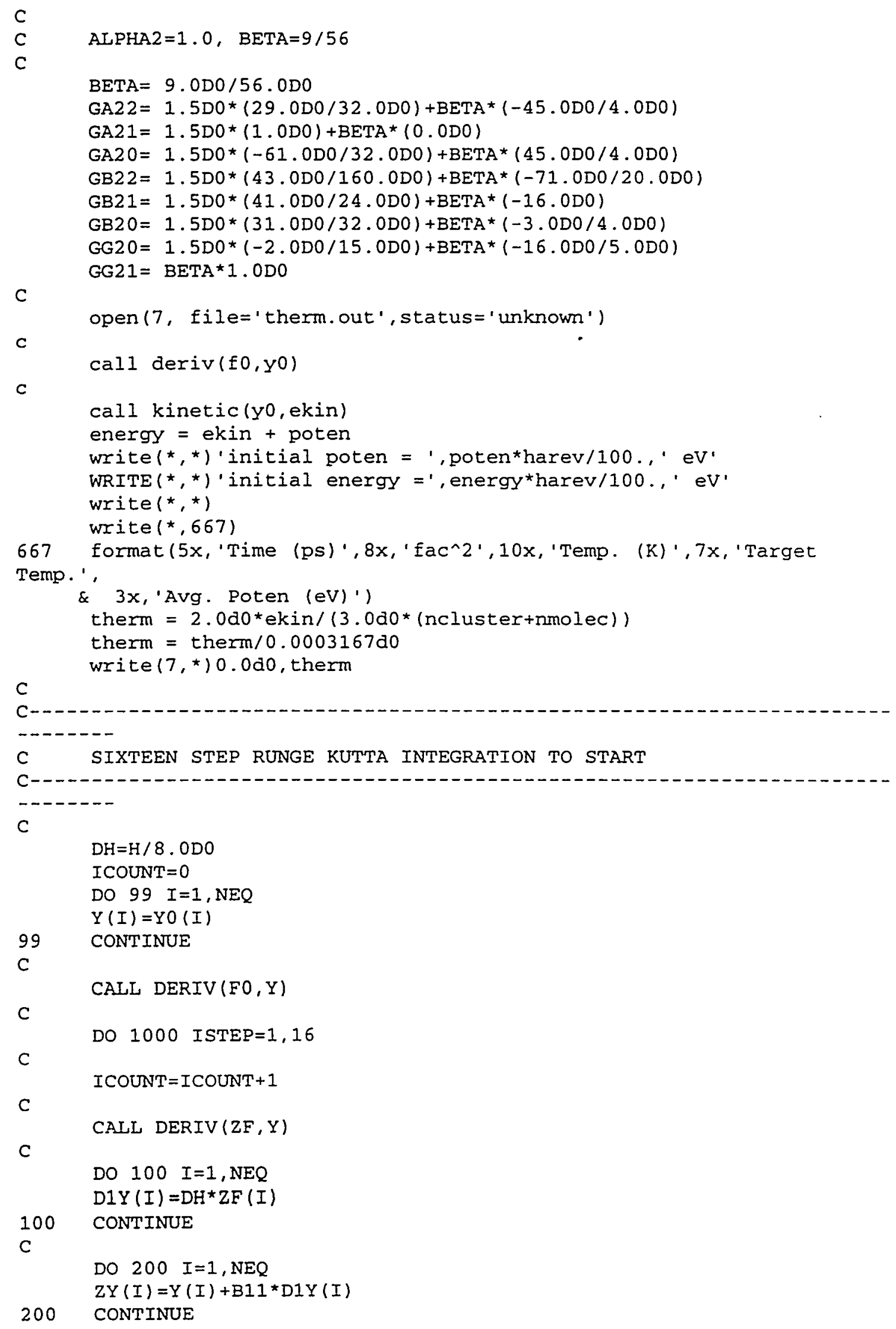




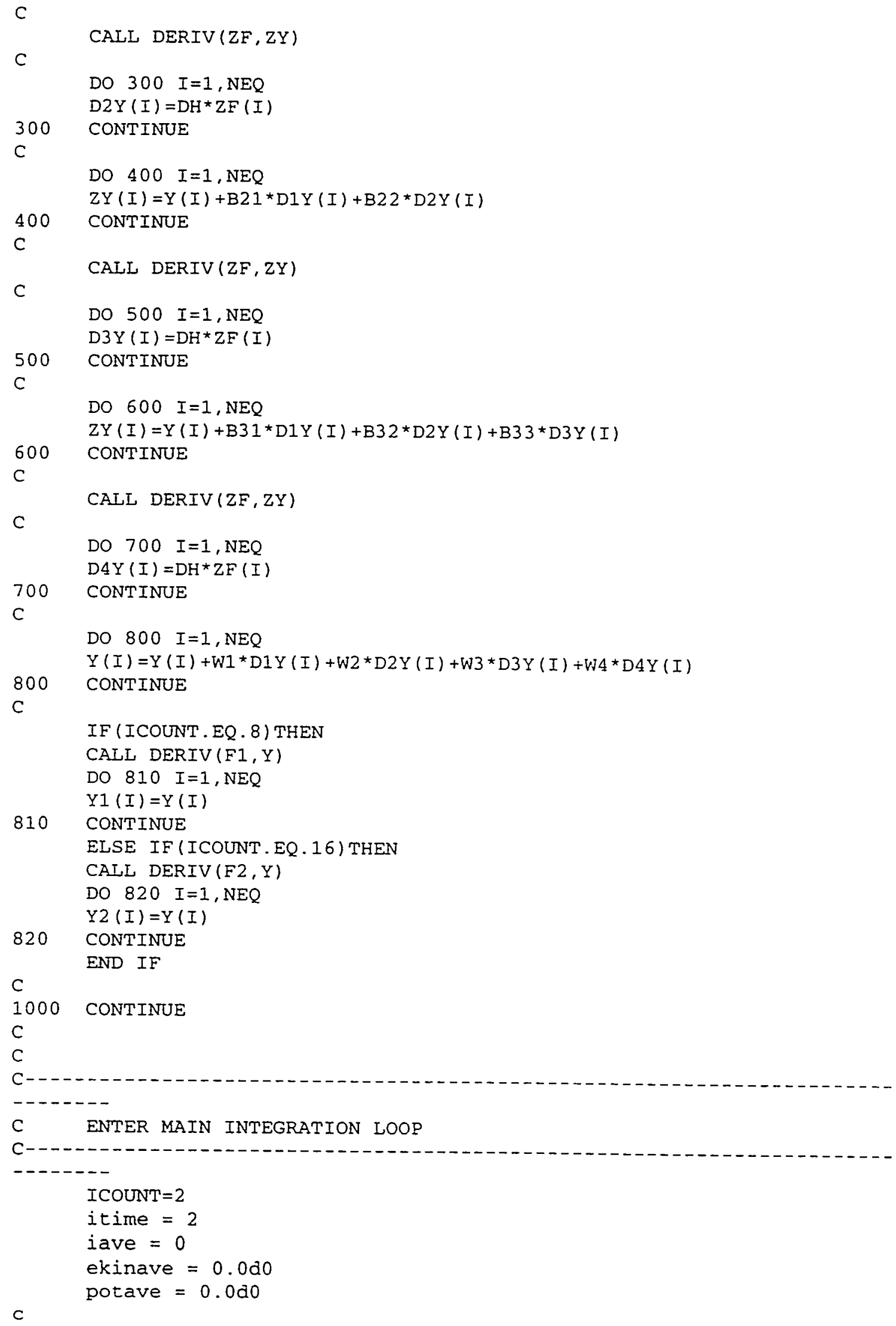




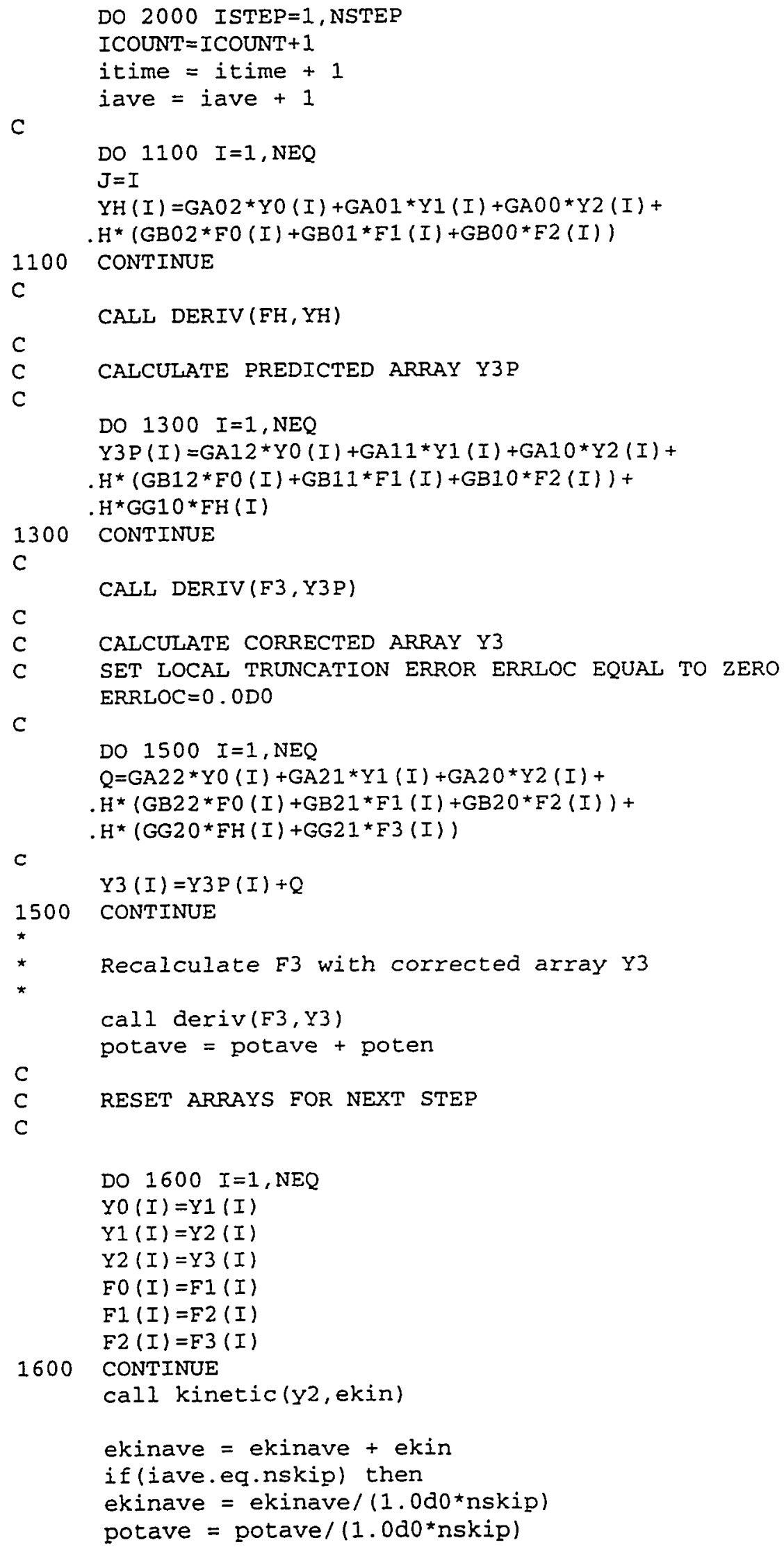




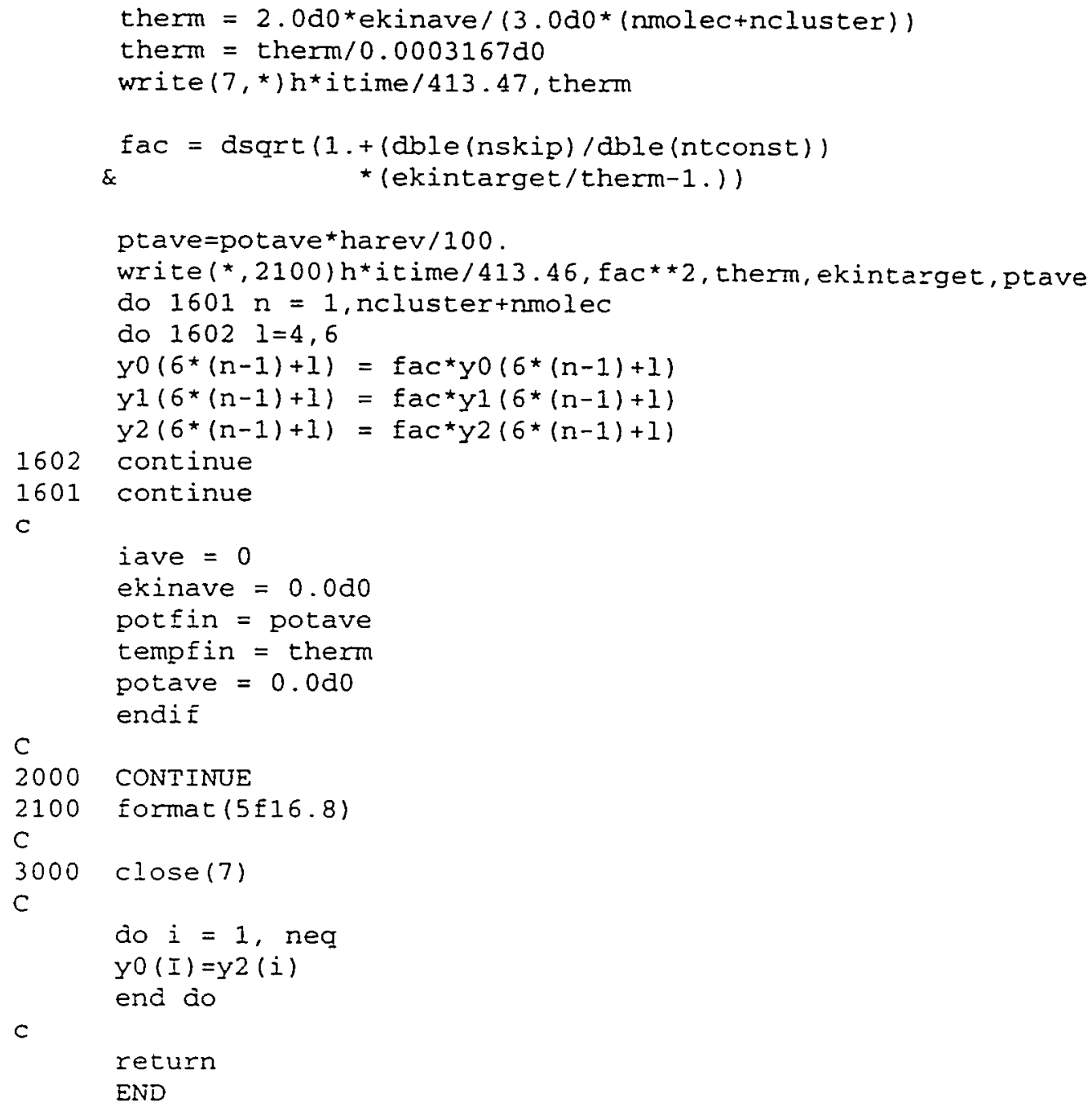

\section{C5.7. File "pforce.f"}

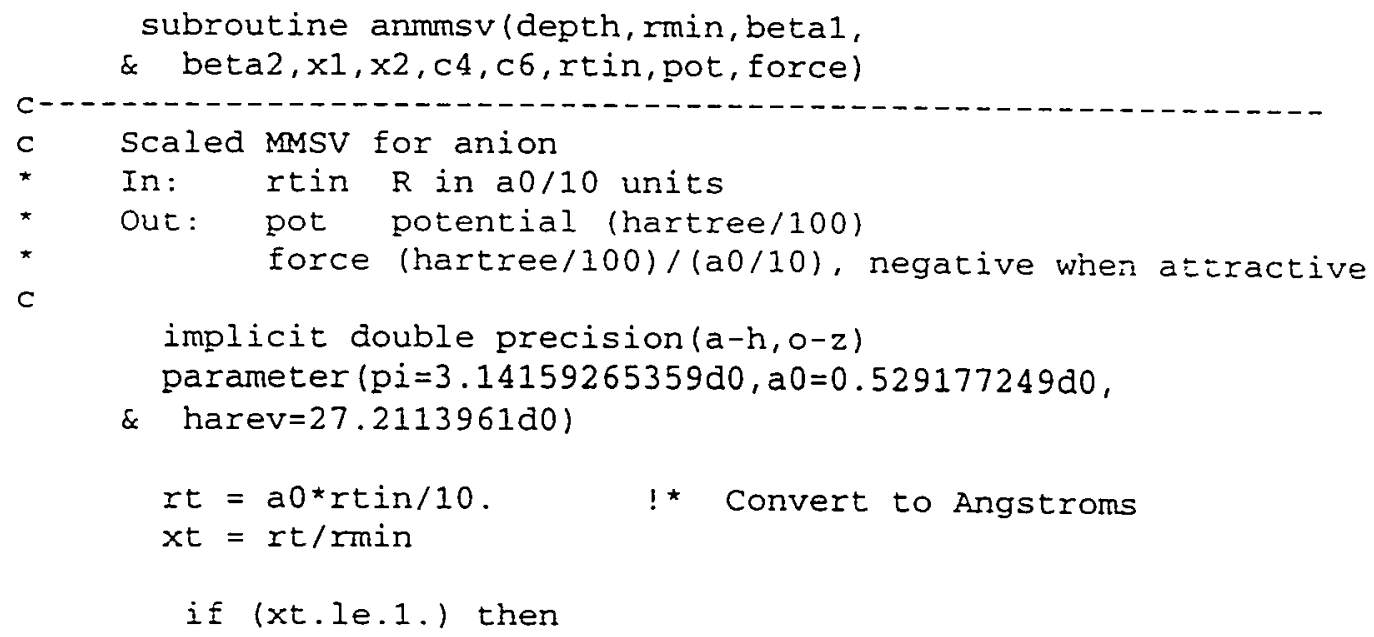




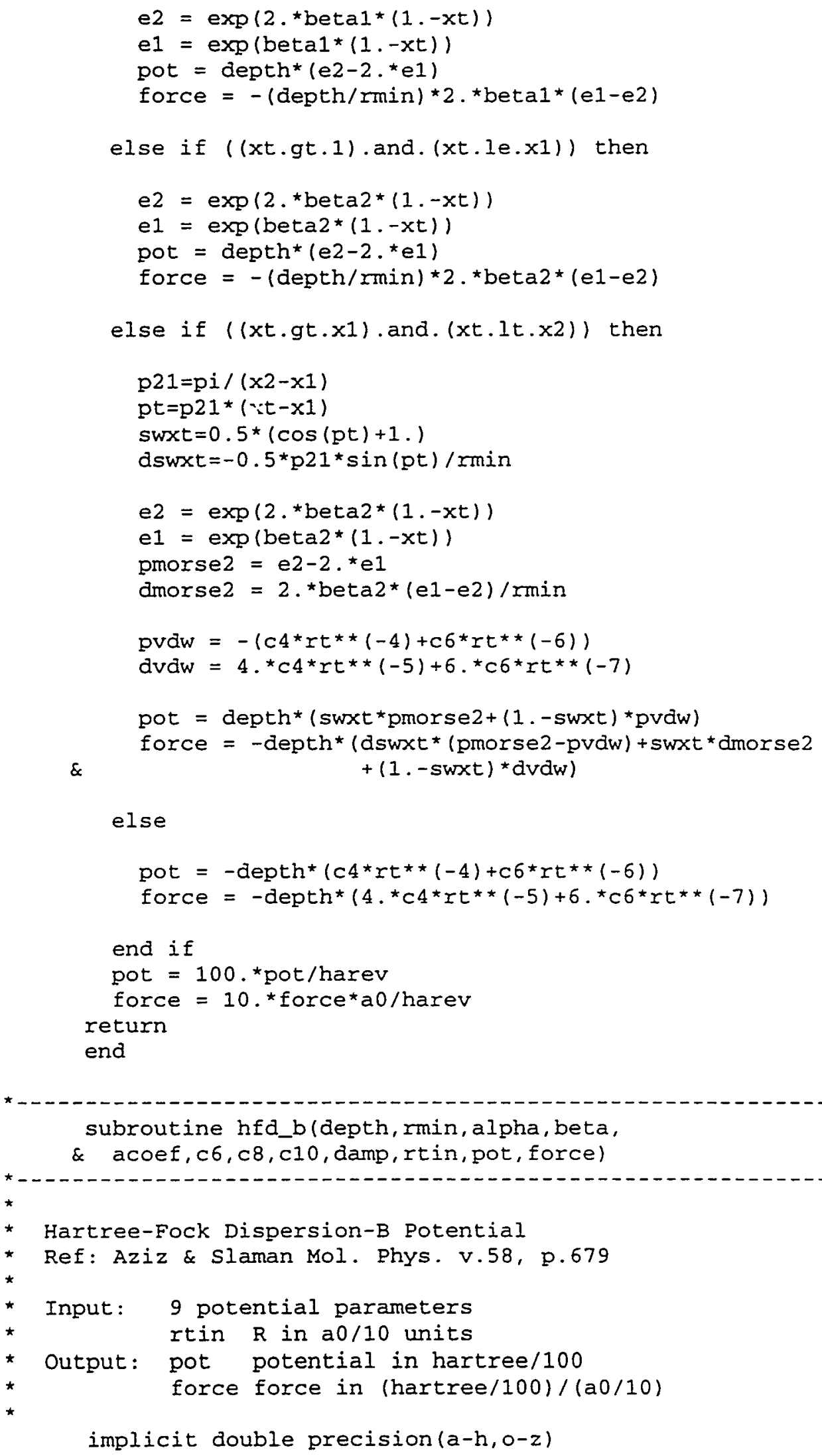




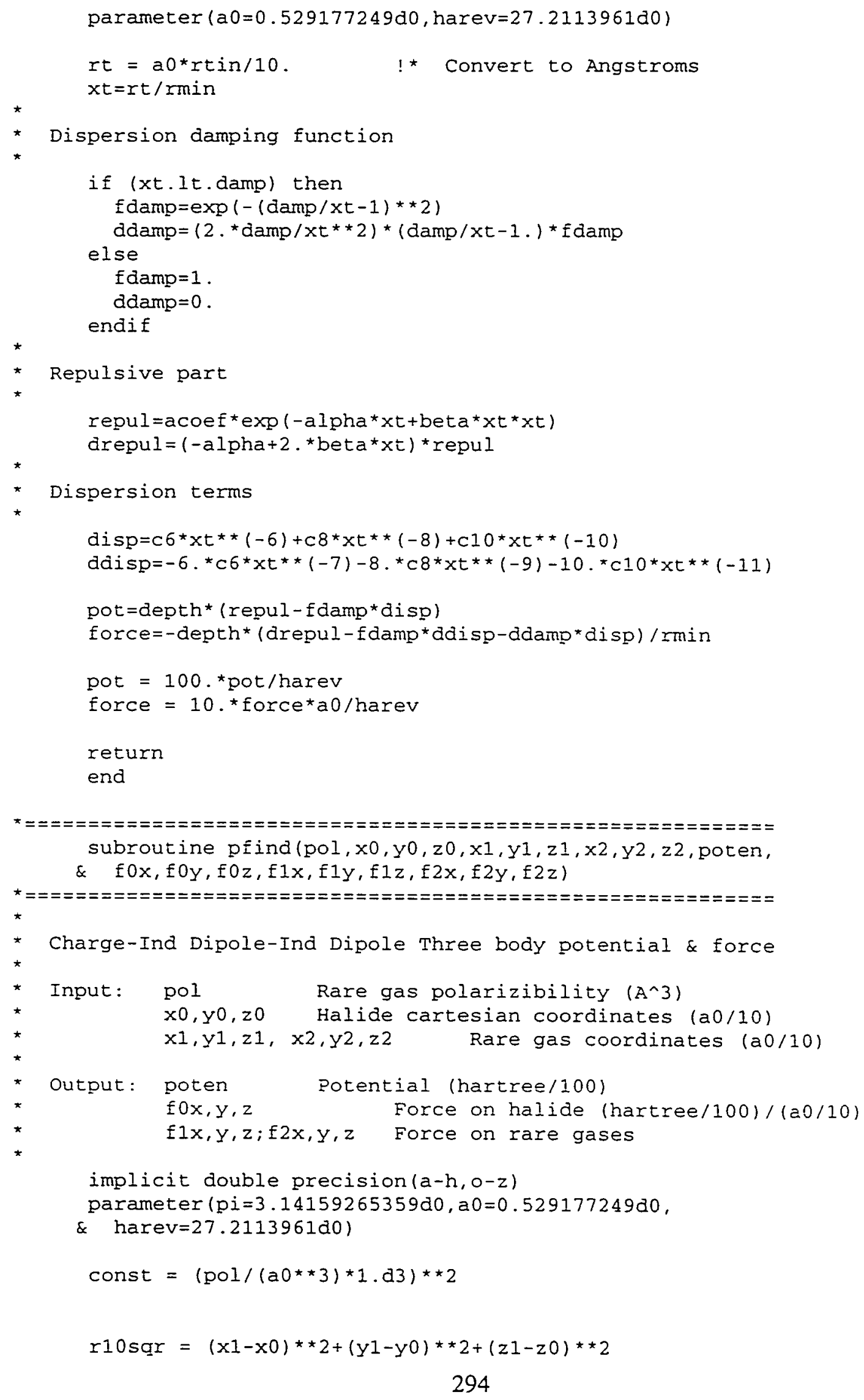




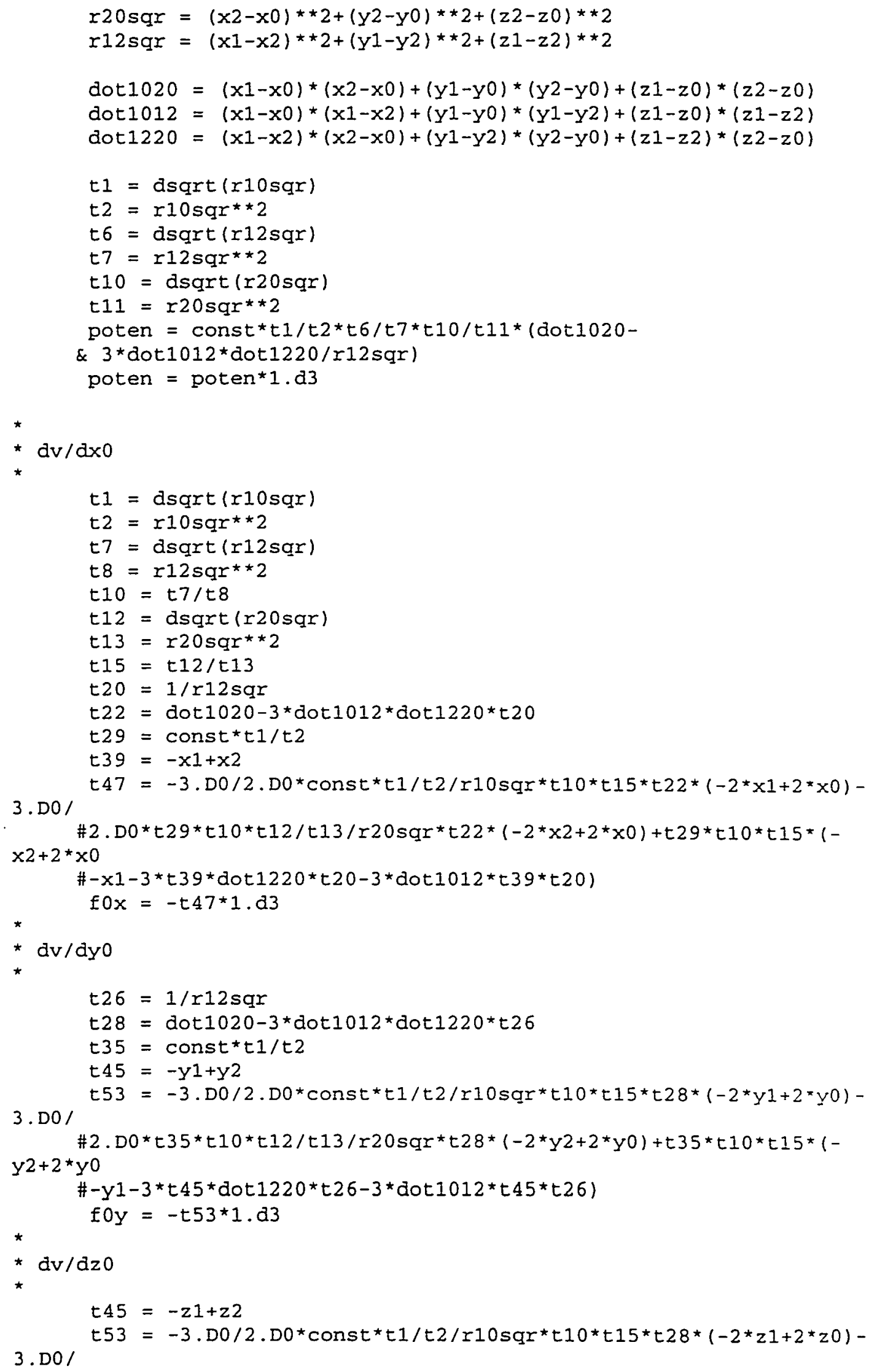




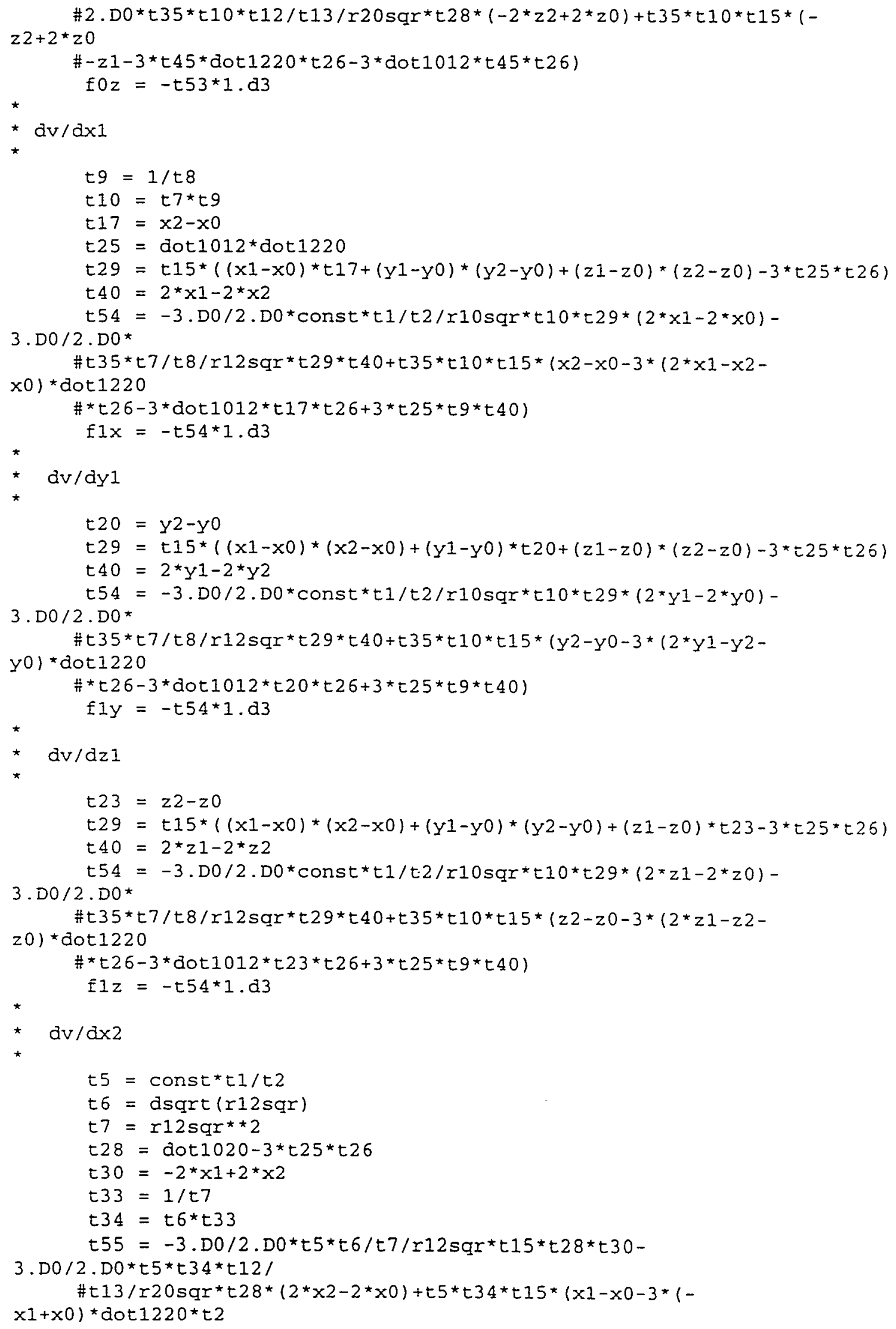




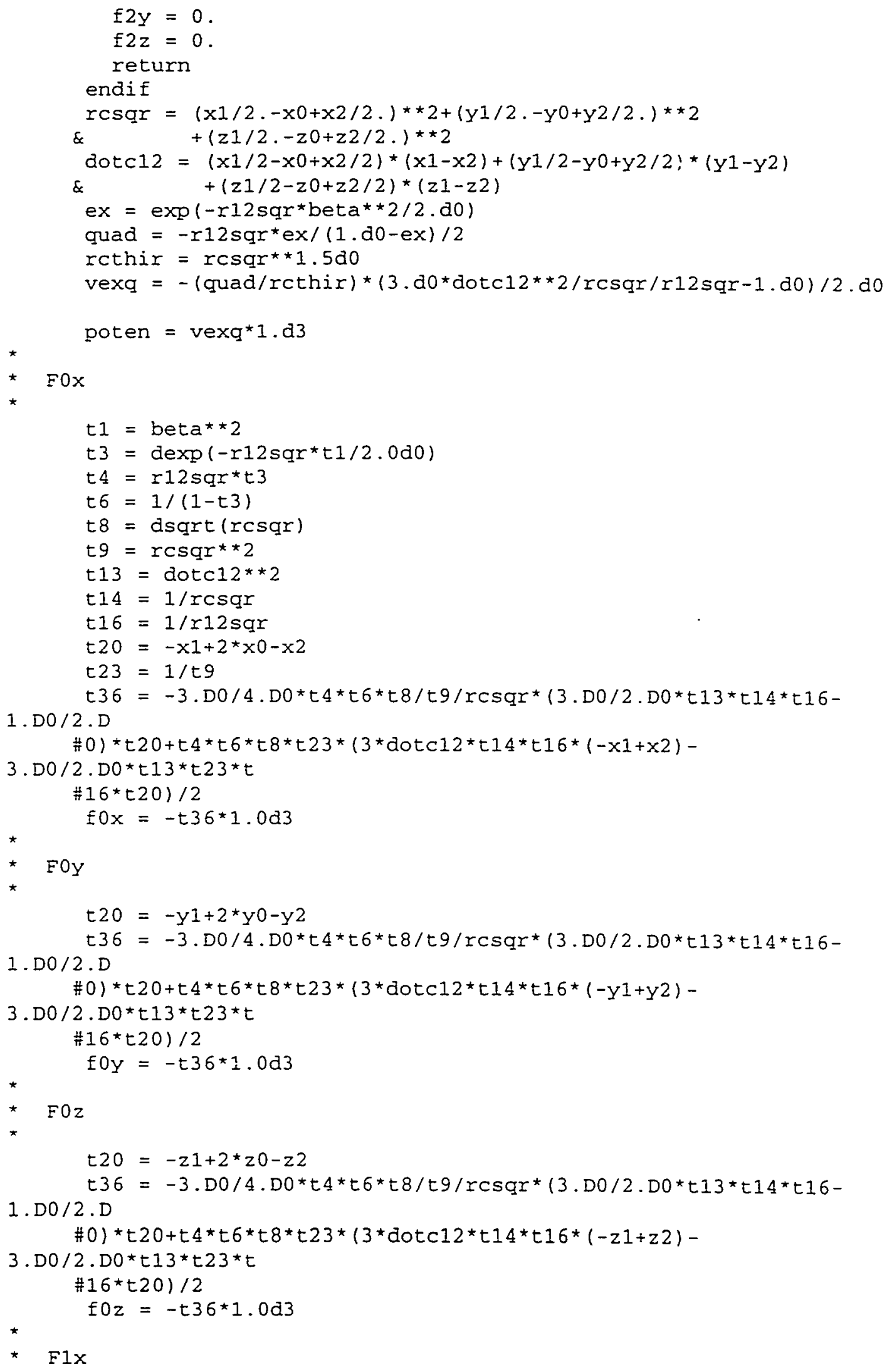




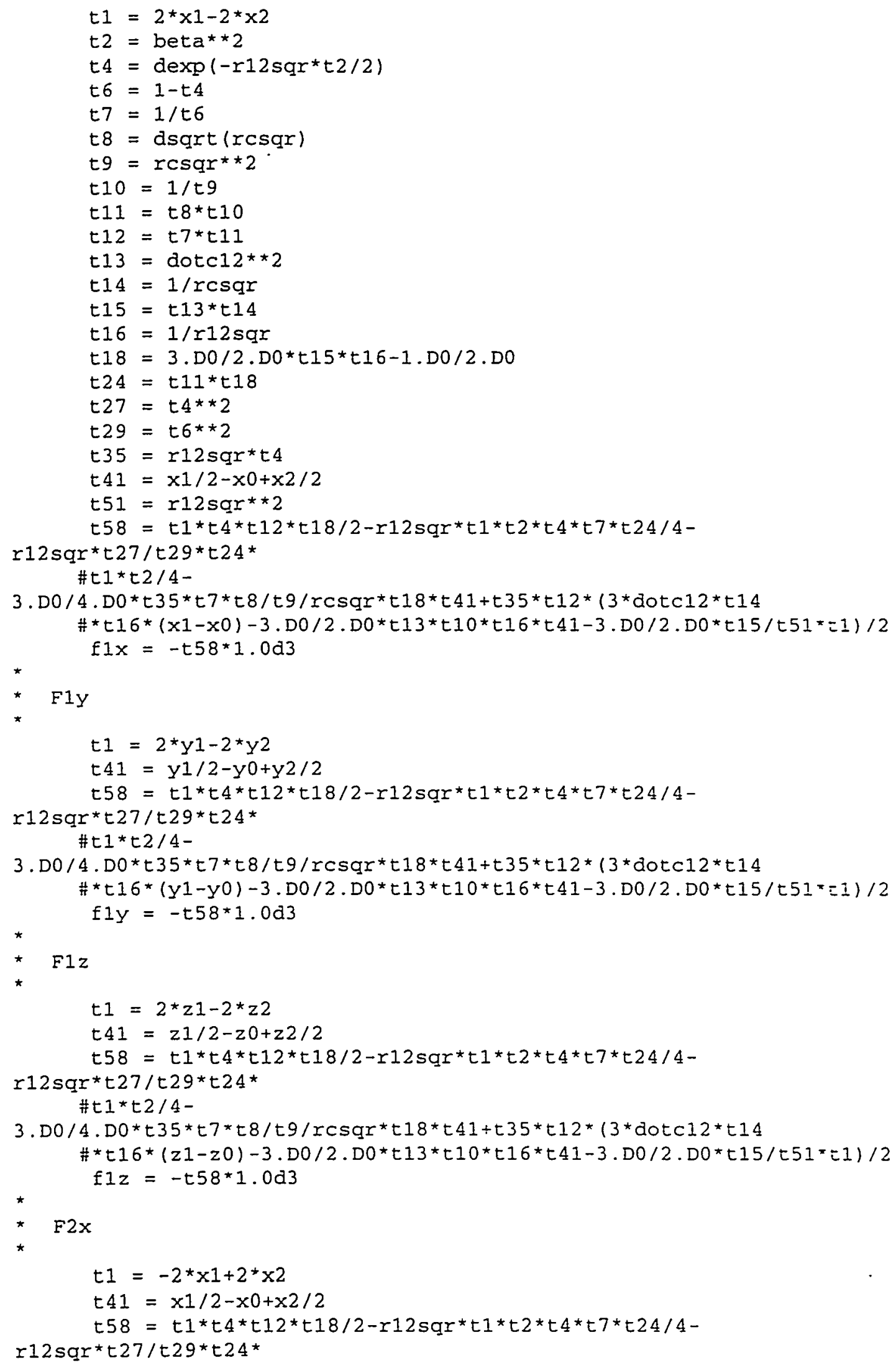


integer ncluster, $i, j, k$, ierr

double precision $\mathrm{qx}(\mathrm{ncl}+1), q y(\mathrm{ncl}+1), \mathrm{qz}(\mathrm{ncl}+1)$,

\& $\mathrm{px}(10), \mathrm{p} 1(10), \mathrm{p} 2(10)$

double precision vr $(-1: 1,-1: 1)$, vi $(-1: 1,-1: 1)$,

$\& \operatorname{vjr}(6,6), \operatorname{vji}(6,6), \operatorname{eval}(6), \operatorname{evecr}(6,6), \operatorname{evec} i(6,6), \operatorname{fv} 1(6)$,

$\& \operatorname{fv} 2(6), \operatorname{fm} 1(2,6)$

double precision rneumms, rt, soconst

double precision sr 2 , sr 3 , xhal, yhal, zhal,

\& $\quad x k, y k, z k, v 0 k, v 2 k, c 1, r s q, v x 0, v i 0, v i i 0$

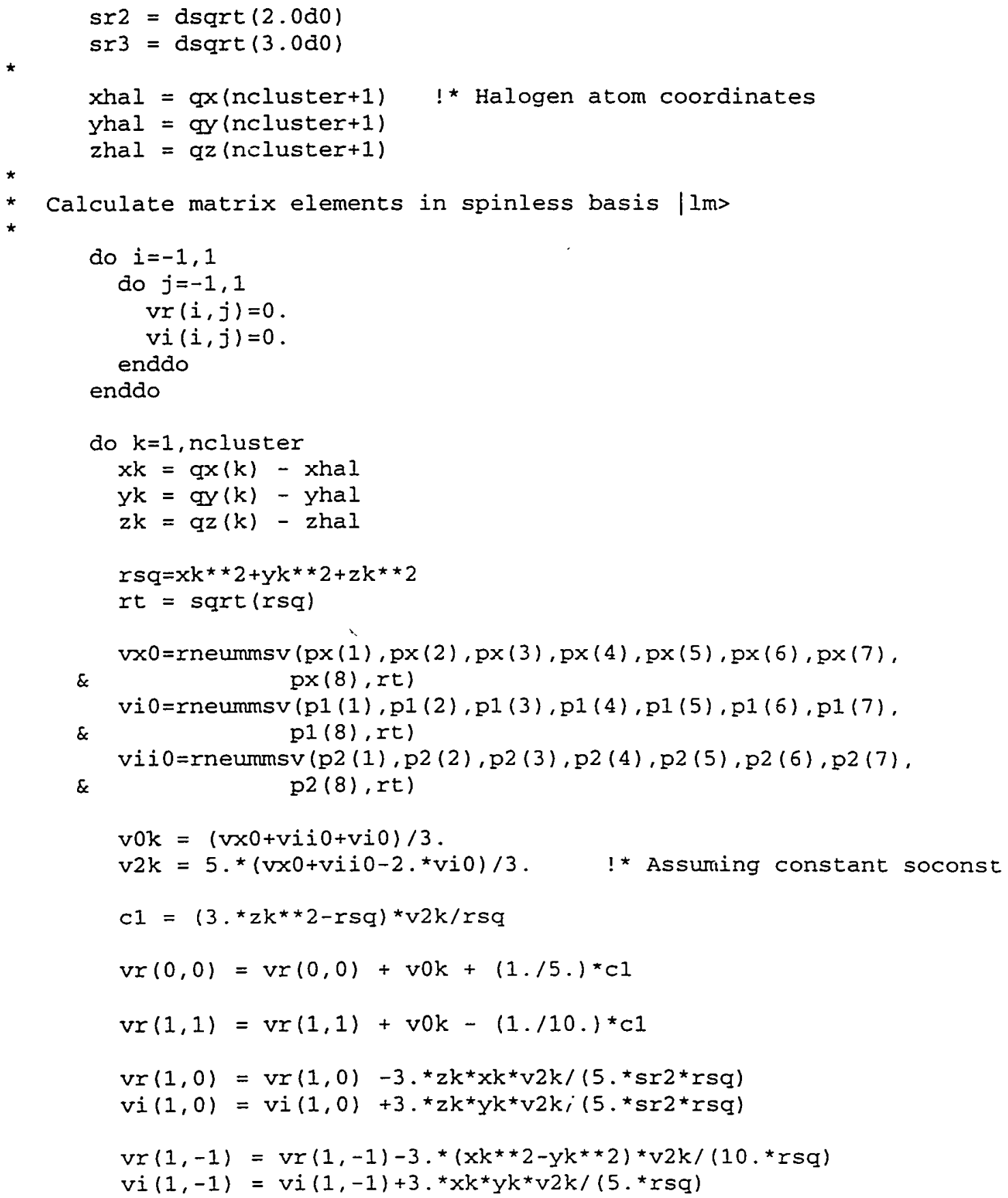




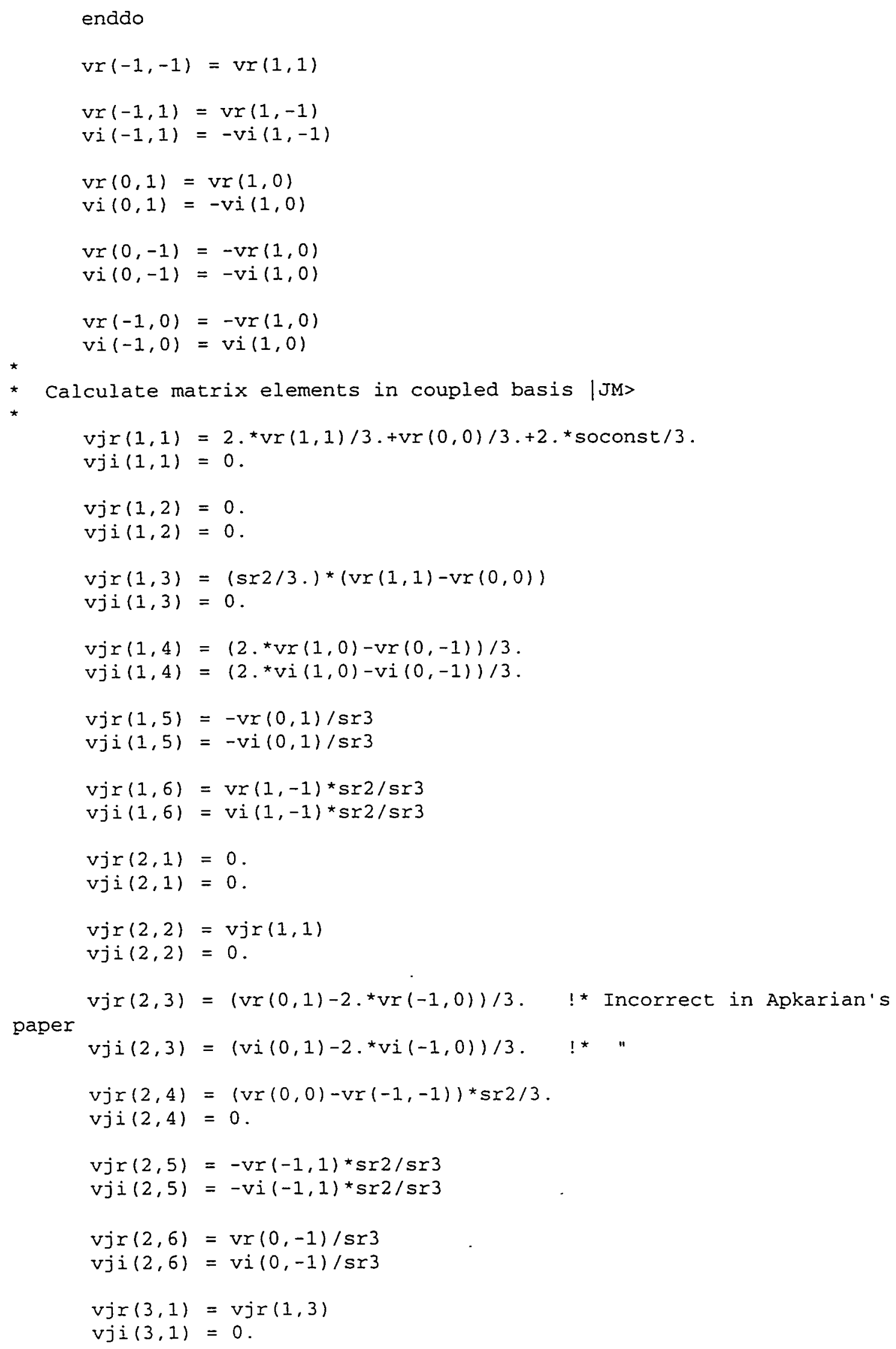




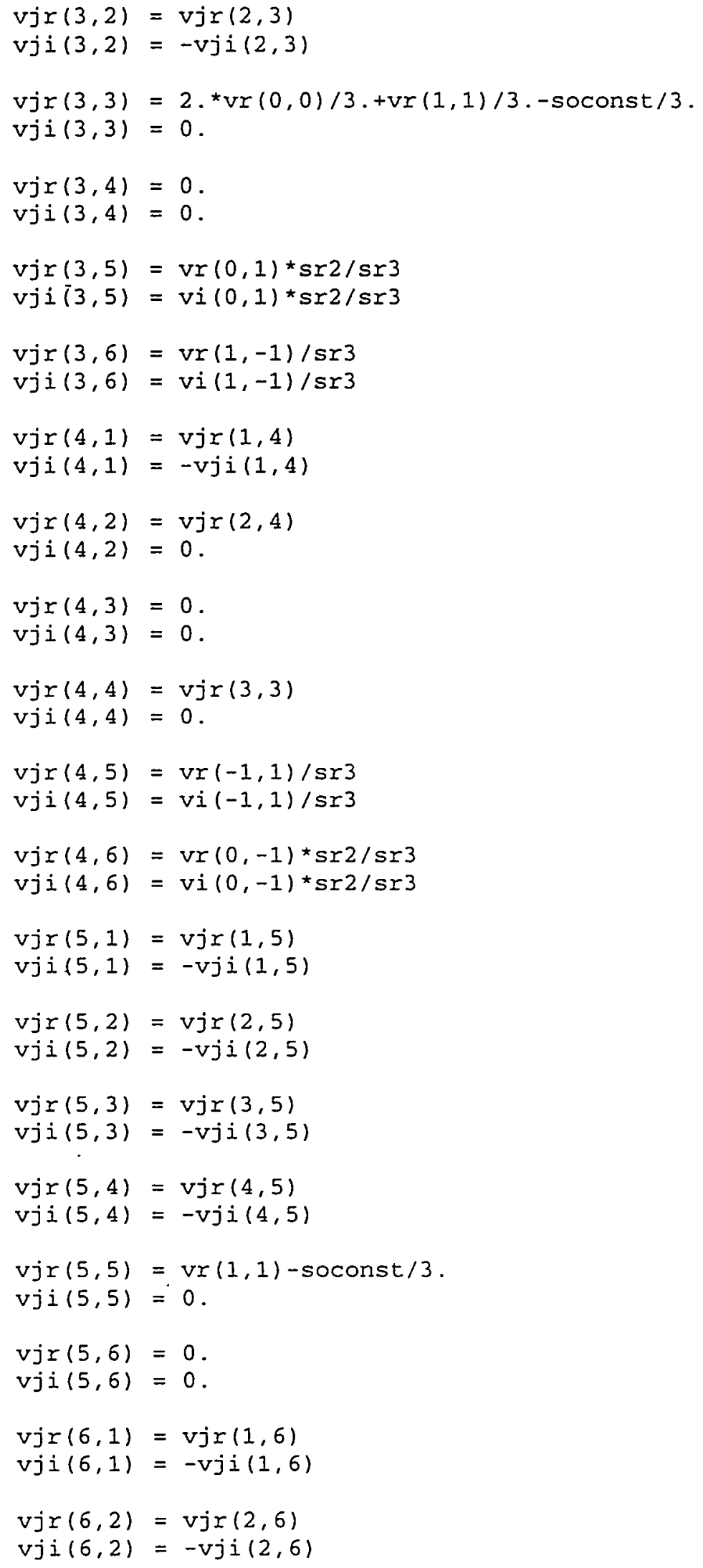




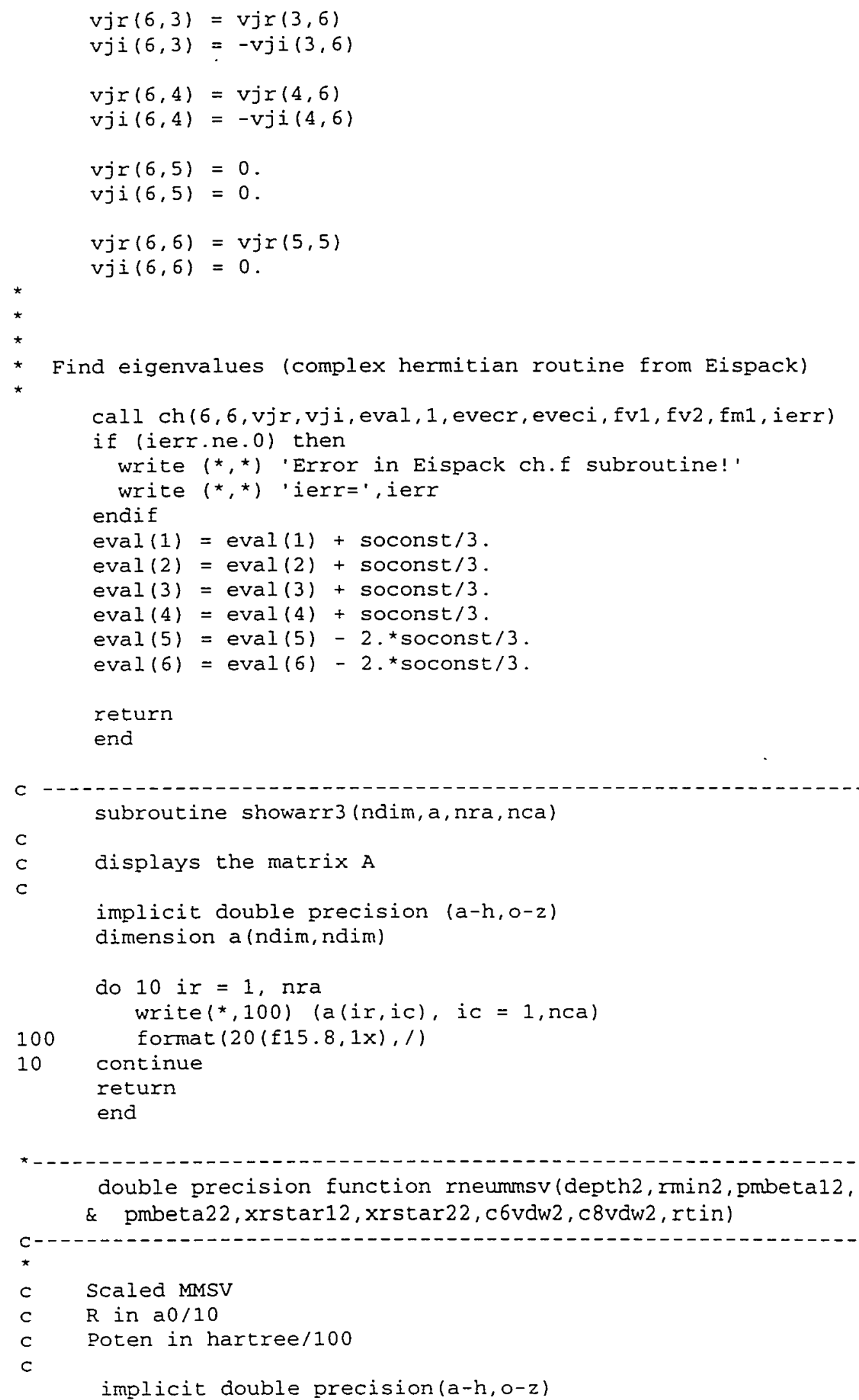




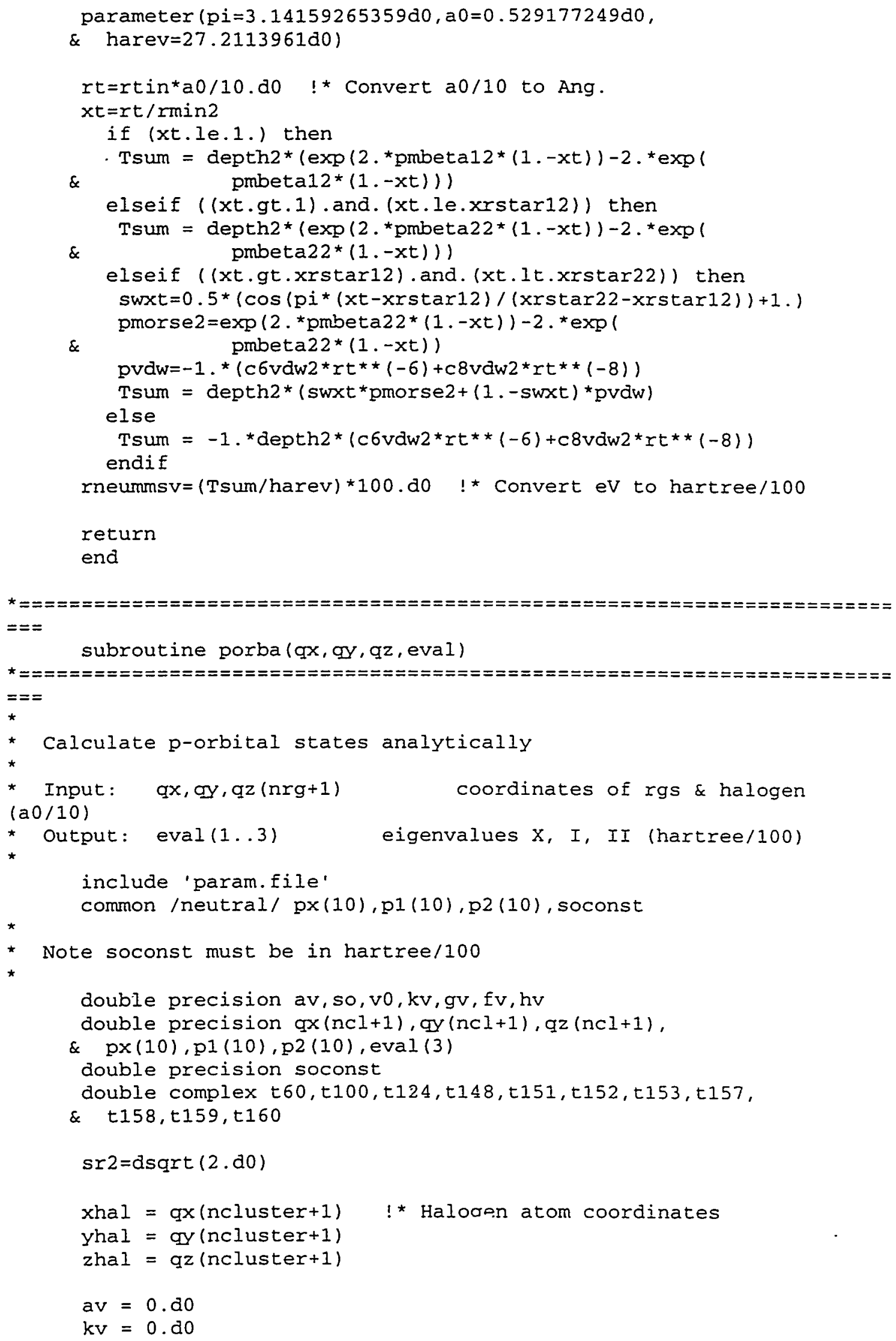




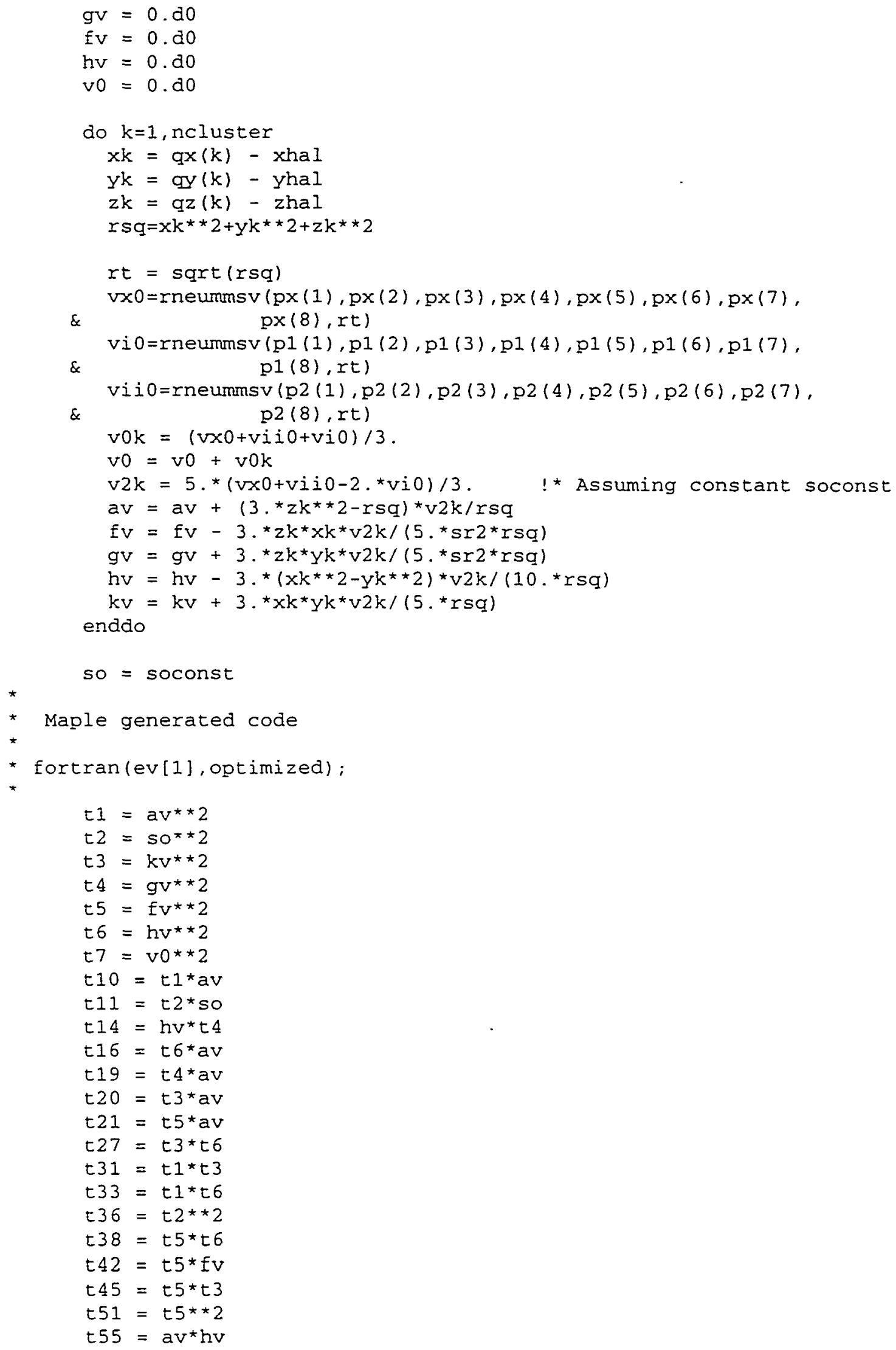




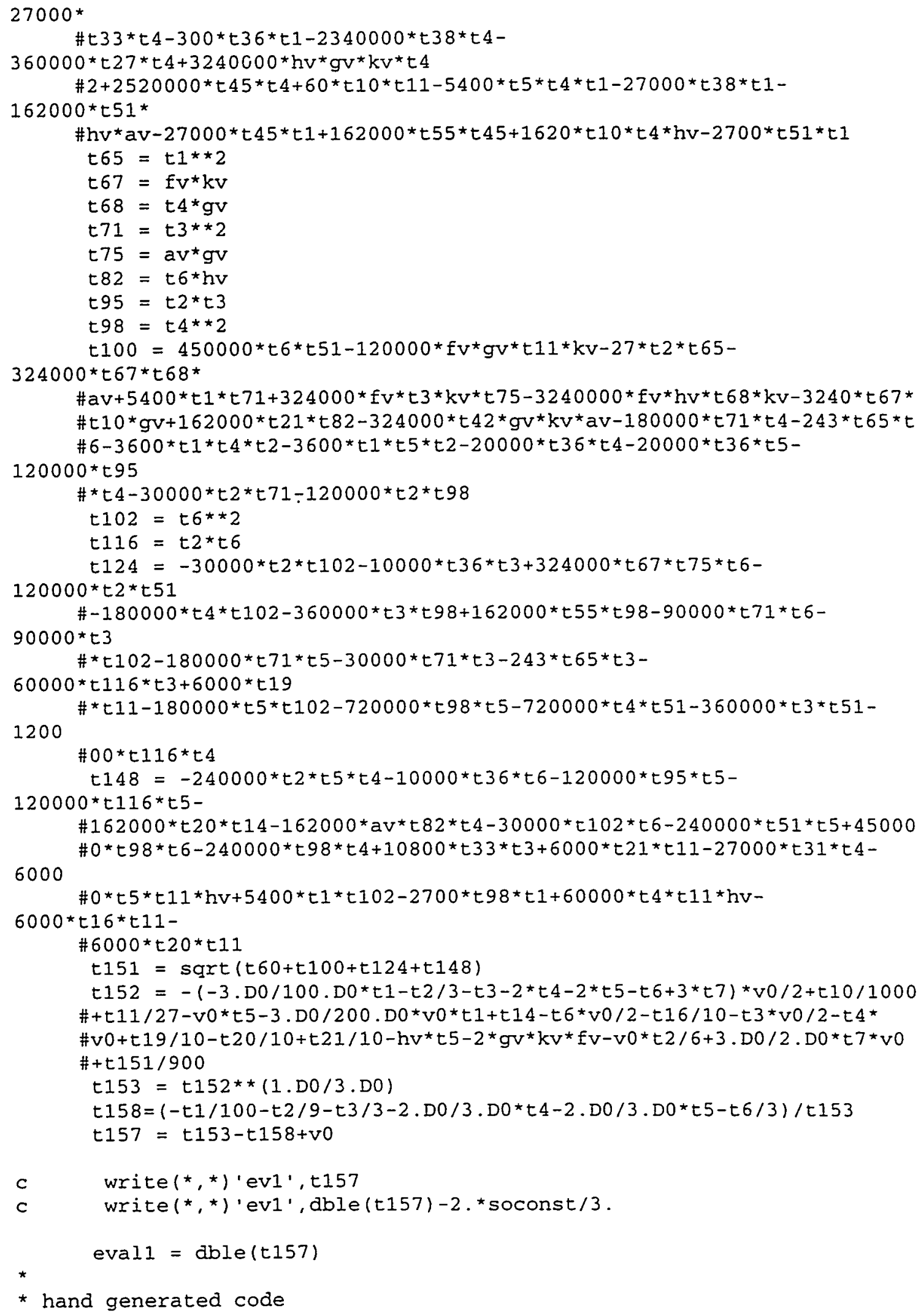




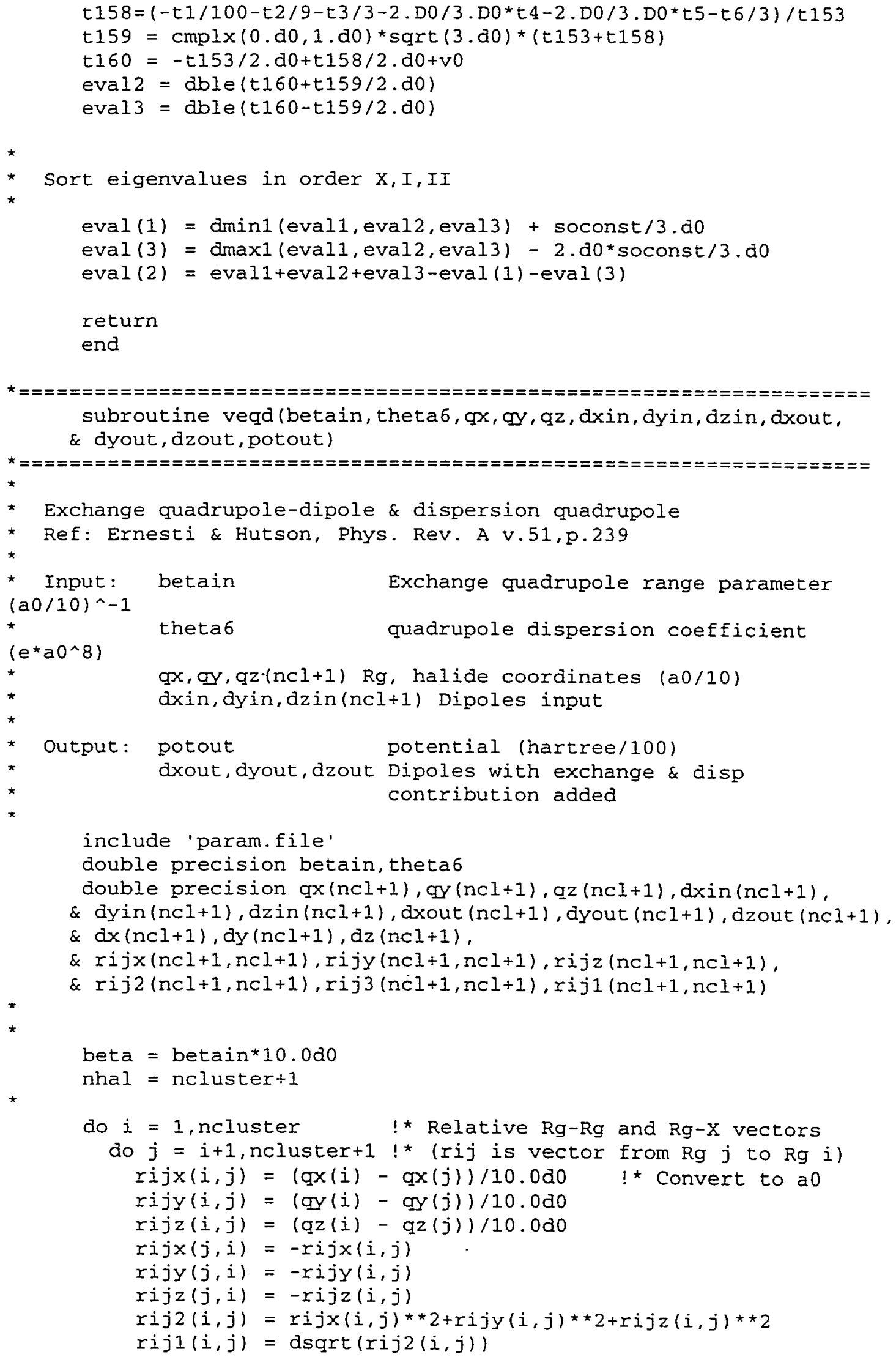




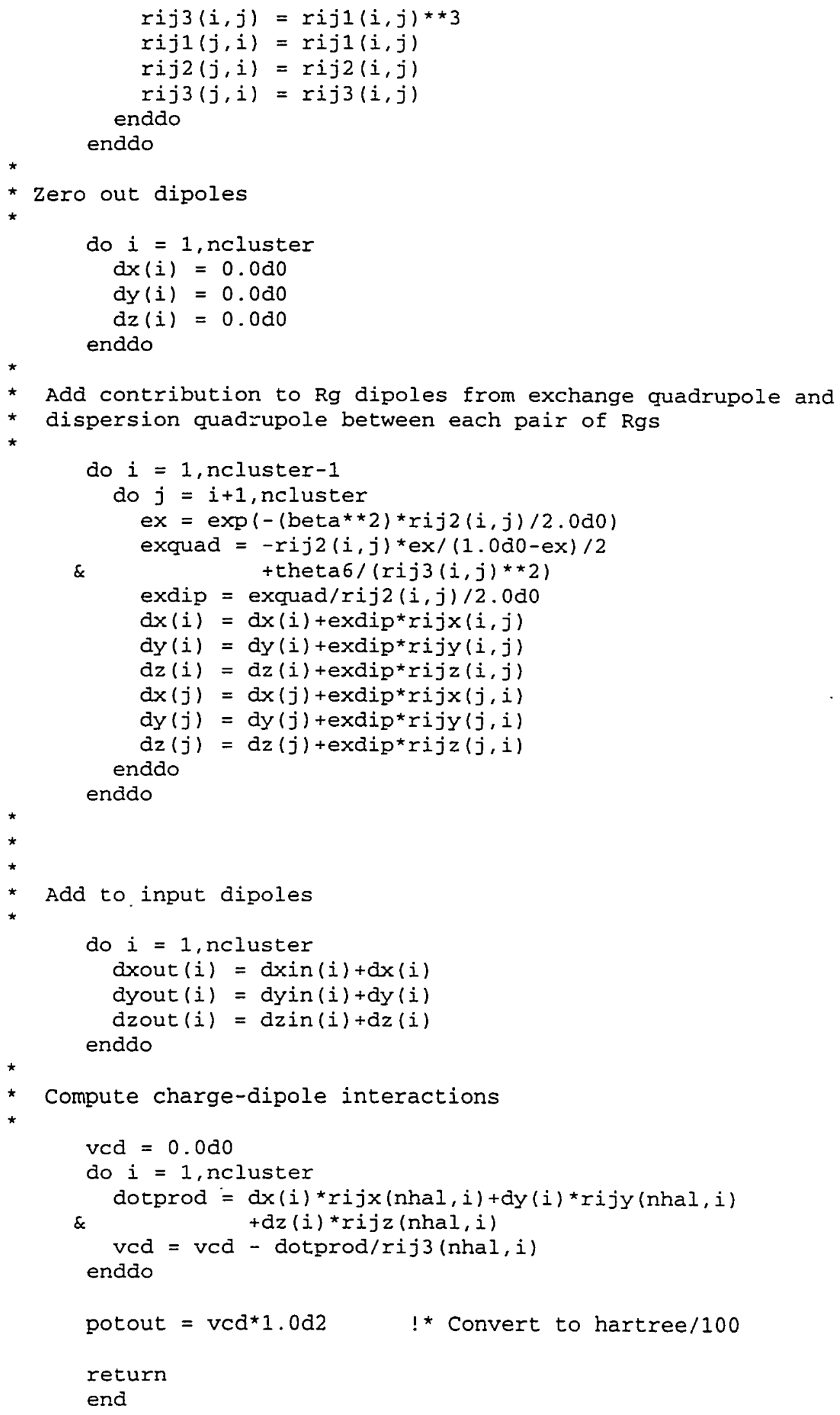




\section{B2.2. Running the program}

One runs the program in the usual Unix fashion, by piping in the input file. If one is calculating standard deviations as well as performing a fit it is usually desirable to run the program in the background, as in the following example using the input file "xebr_x_rfin" discussed above:

$>$ nice +19 rfit < xebr_x_Ifin > xebr_x_rfout \&

The output file "xebr_x_rfout" then contains the optimized parameters and standard deviations, and the convoluted spectrum is saved to the file "xebr_x_fit" named in the input file.

\section{B2.3. Outline of the program}

The program "rfit" is contained in only one file, "rfit.f." There is no makefile; the program is recompiled with a command such as:

$>\mp 77-0-0$ Ifit Ifit. $\pm-C$

The subroutines and functions used by the program are listed in Table B3. 
Table B3. Subroutines and functions used by the rotational fitting program "rfit".

\begin{tabular}{|c|l|}
\hline $\begin{array}{c}\text { Subroutine or } \\
\text { Function }\end{array}$ & main program \\
\hline rfit & general-purpose subroutine implementing the gradient \\
\hline optchi & calculates $\chi^{2}$ for a given set of fitting parameters \\
\hline chisquar & calculates a rotational stick spectrum given one or two \\
\hline rsticks & vibrational stick spectra, and the fitting parameters. \\
\hline be & function to calculate the rotational constant \\
\hline boltz & calculates the Boltzmann factor for a given rotational \\
& temperature and anion rotational state \\
\hline cp2 & convolutes the rotational stick spectrum with the $Z E K E$ line \\
& shape \\
\hline conzeke & compares the experimental and convoluted spectra and $\chi^{2}$ \\
\hline &
\end{tabular}




\section{B3. Source code for the rotational fitting program "rfit"}

program rfit

implicit undefined $(a-z)$

real frac, evtocm

parameter (frac $=0.1$, evtocm $=8065.541$ )

real vstk $(2,2,100)$

real temp, org, baseln

real beneu, bean, reneu (2), rean

real mass 1 , mass 2 , rmass

integer ninfil, $j, j, k$, state (2),j,nvstk (2), rct

integer mpts

character*25 infile(2), outfile, especfil

real relfcf $(2), \operatorname{scoef}(2)$

real fwhmcm, fwhmev, delta, deltawn

real smooth $(2,100000), \operatorname{espec}(2,100000), \operatorname{espec} 2(2,100000)$,

\& rstk $(2,500000)$

integer nspec

real dfwhmcm, dtemp, dscoef (2), drelfcf (2), dorg, dbaseln

real mfwhmom, mtemp, mscoef (2), mrelfcf (2), morg, mbaseln

real xfwhmcm, xtemp, xscoef (2), xrelfcf (2), xorg, xbaseln

real sl fwhm, sitemp, siscoef (2), slrelfcf(2), slorg, slbasein

real s2 fwhm, s2temp, s2scoef (2), s2relfcf (2), s2org, s2baseln

logical ufwhm, utemp, uscoef (2), urelfcf (2), uorg, ubaseln

real chisq, xinc, chiopt

character* 1 ans

logical vscoef(2), vrelfcf(2), vtemp, vfwhm, vorg, vbaseln integer ncomp

real a (30), da (30), ma (30), xa (30), aopt (30), abest (30)

real s1a(30), s2a(30)

logical va(30), ua(30), v1

integer nparm, mode, tout

logical pr

real ncounts, chilast, slast, slope

common /conv1/ fwhmcm, fwhmev, delta, deltawn

common / conv2/ mpts

common / func/ bean, beneu

common /rvars/ mass1, mass2, rmass, rean, temp, org, baseln

common /ivars/ rct, ninfil

common/arrays/reneu (2), state (2), nvstk (2),

$\& \quad r e l f c f(2), \operatorname{scoef}(2)$

common /comp/ chisq, ncomp

common /comp2/ ncounts

common lopti/ nparm

common /optr/ a (30), abest (30)

common /sticks/vstk $(2,2,100)$, rstk $(2,500000)$

common /spec1/ smooth $(2,100000)$, espec $(2,100000)$

common /spec2/ nspec

common /prl/ pr,tout

* Read in constants and fitting parameters 
vpair $=\operatorname{vpair}+\operatorname{drg} 2 /\left(2{ }^{\star} \operatorname{rjj} 2(\right.$ nhal, $\left.i)\right)$

enddo

potout $=($ vpol-vpair $) * 1.0 \mathrm{~d} 2 \quad ! *$ Convert to hartree $/ 100$

return

end

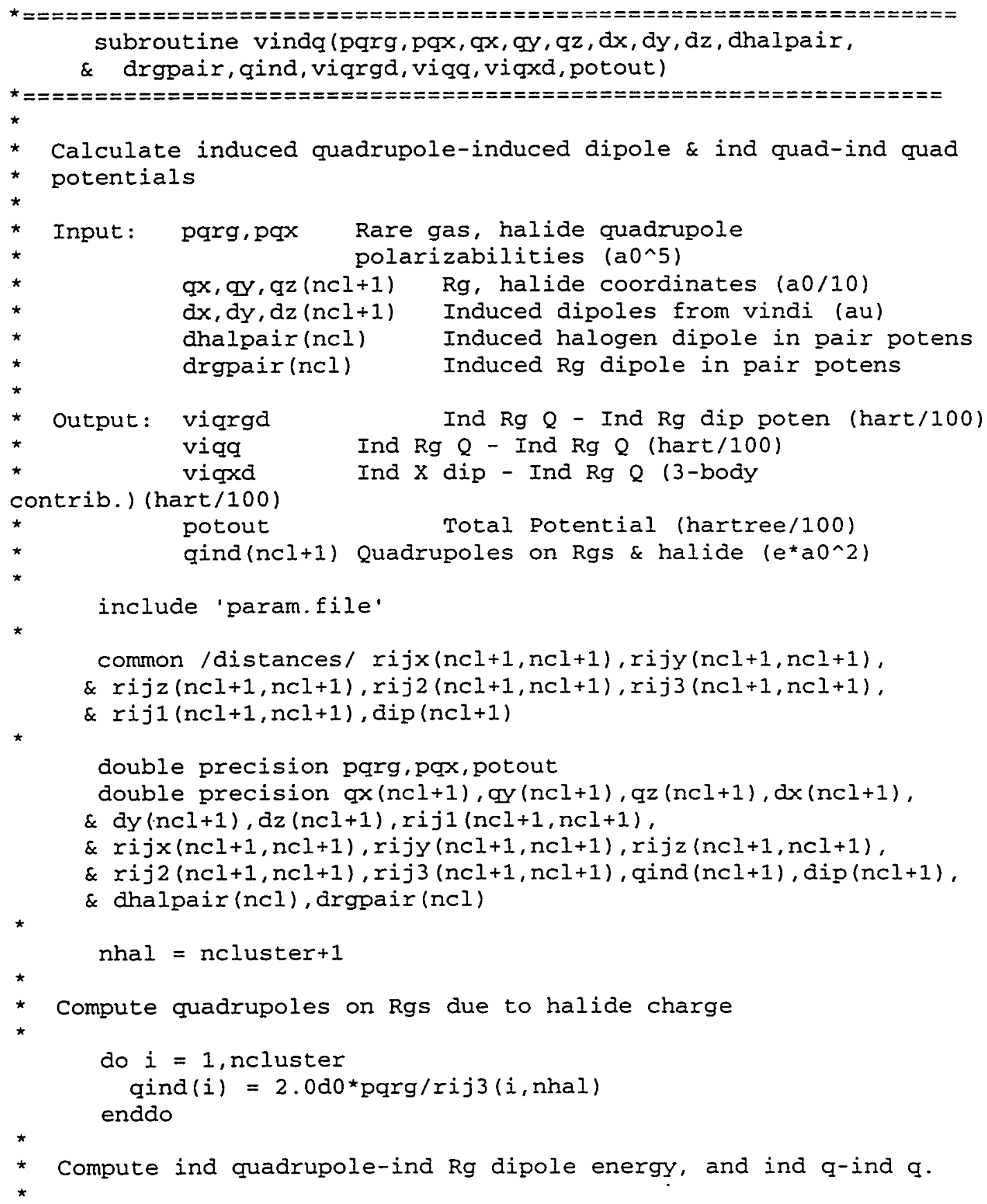




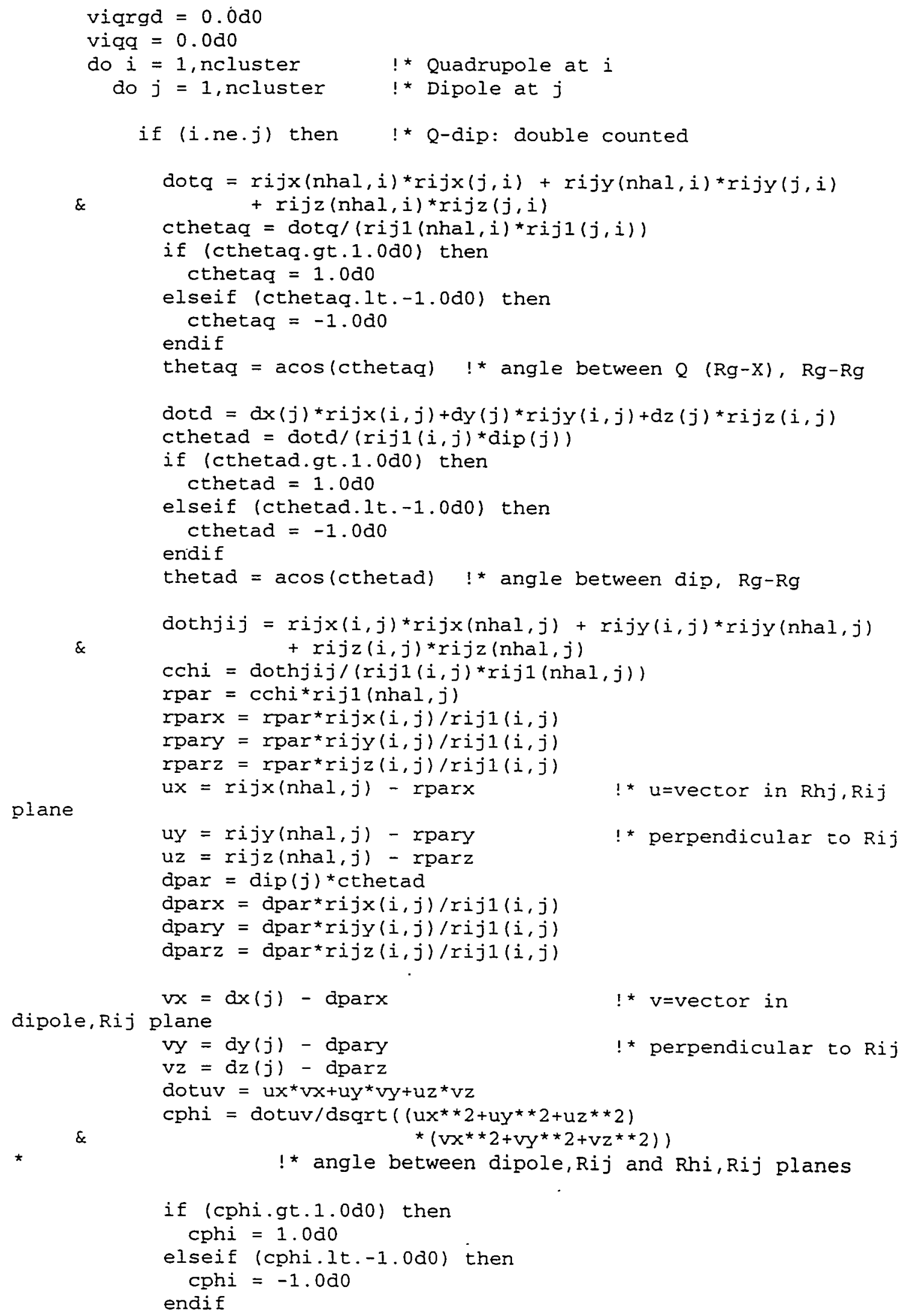




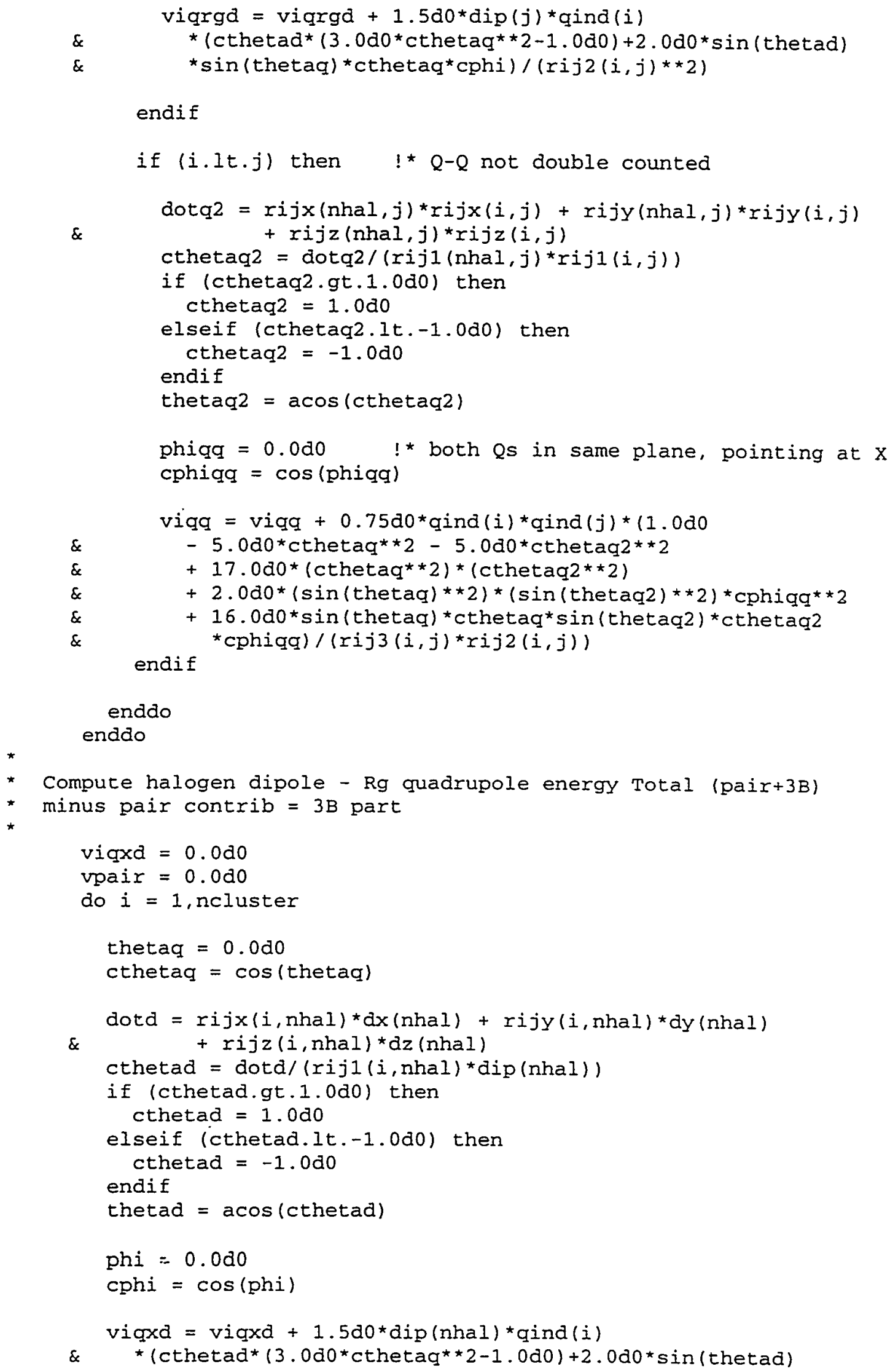




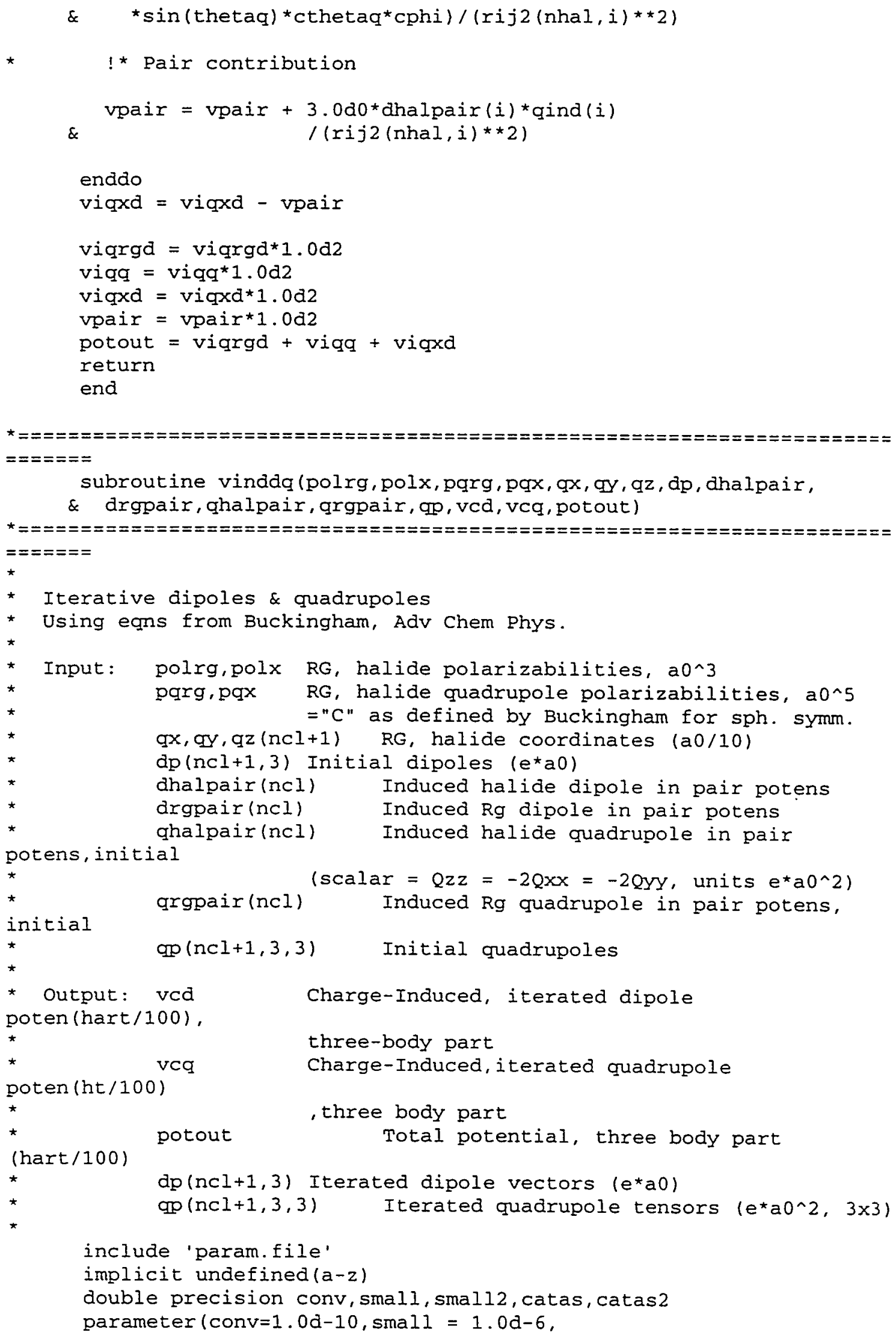


\& $\operatorname{sma} 112=\operatorname{sma} 11 * \operatorname{smal} 1$, catas $=10$, catas $2=$ catas $^{\star}$ catas $)$

double precision rmc, eec, eeint, sigc, sigint, delta, poten, \& rgrgpot, rgxpot, cddpot, exqpot, vindit, vexqd, rgqq, rgqxd,

\& viq, rgxxpot, rgxipot, rgxi ipot, potfin, tempfin, exqpot, rml ,

\& rgdrgq, cdpot, cqpot, cdqpot, gexpot, ddispot, qdispot, gextot,

$\&$ atpot

double precision dab, dag, dbg, dad, dbd, dgd, fgrg, ferg, vcdt,

\& vcqt, fex, fgx, vcdpair, vcqpair, drgold, dhalold, qrgold, qhalold

double precision polrg, polx,pqrg,pqx, vcd, vcq, potout double precision $q x(\mathrm{ncl}+1)$, qy $(\mathrm{ncl}+1), \mathrm{qz}(\mathrm{ncl}+1), \mathrm{dp}(\mathrm{ncl}+1,3)$,

\& dhalpair(ncl), drgpair(ncl), qhalpair(ncl), grgpair(ncl),

$\&$ qp $(n c l+1,3,3), \operatorname{rij}(\mathrm{ncl}+1, \mathrm{ncl}+1,3), \operatorname{rijl}(\mathrm{ncl}+1, \mathrm{ncl}+1)$.

\& $\operatorname{rij2}(\mathrm{ncl}+1, \mathrm{ncl}+1), \operatorname{rij3}(\mathrm{ncl}+1, \mathrm{ncl}+1), \operatorname{rij} 5(\mathrm{ncl}+1, \mathrm{ncl}+1)$,

$\& \operatorname{rij} 7(\mathrm{ncl}+1, \mathrm{ncl}+1), \operatorname{rij} 9(\mathrm{ncl}+1, \mathrm{ncl}+1), \mathrm{tl}(\mathrm{ncl}+1, \mathrm{ncl}+1,3)$,

\& $\mathrm{t} 2(\mathrm{ncl}+1, \mathrm{ncl}+1,3,3), \mathrm{t} 3(\mathrm{ncl}+1, \mathrm{ncl}+1,3,3,3), \mathrm{rij} 4(\mathrm{ncl}+1, \mathrm{ncl}+1)$,

$\& \mathrm{t} 4(\mathrm{ncl}+1, \mathrm{ncl}+1,3,3,3,3)$, fe $(\mathrm{ncl}+1,3), \mathrm{fg}(\mathrm{ncl}+1,3,3)$,

\& dmag $(n c l+1)$, gmag $(n c l+1), d \operatorname{dmagp}(n c l+1)$, qmagp $(\mathrm{ncl}+1)$

integer ncluster, natmax, ndim, neqm, neq, nrgpot, nhalpot, neigval, \&indflag, iexqflag, indi, i exqd, indq, indqi, iexg, iaxtel

integer nhal, $i, j, k$, iexit, niter, alp, bet, gam, del, iexsave

nhal $=$ ncluster +1

niter $=0$

* Calc initial dipole and quadrupole magnitudes

do $i=1$, ncluster +1

$\operatorname{dmagp}(i)=d p(i, 1) * \star 2+d p(i, 2) * \star 2+d p(i, 3) * \star 2$

gmagp $(i)=0.0 \mathrm{~d} 0$

do alp $=1,3$

do bet $=1,3$

qmagp $(i)=$ qmagp $(i)+q p(i$, alp, bet $) \star \star 2$

endoo

enddo

enddo

Set up position vectors and distances

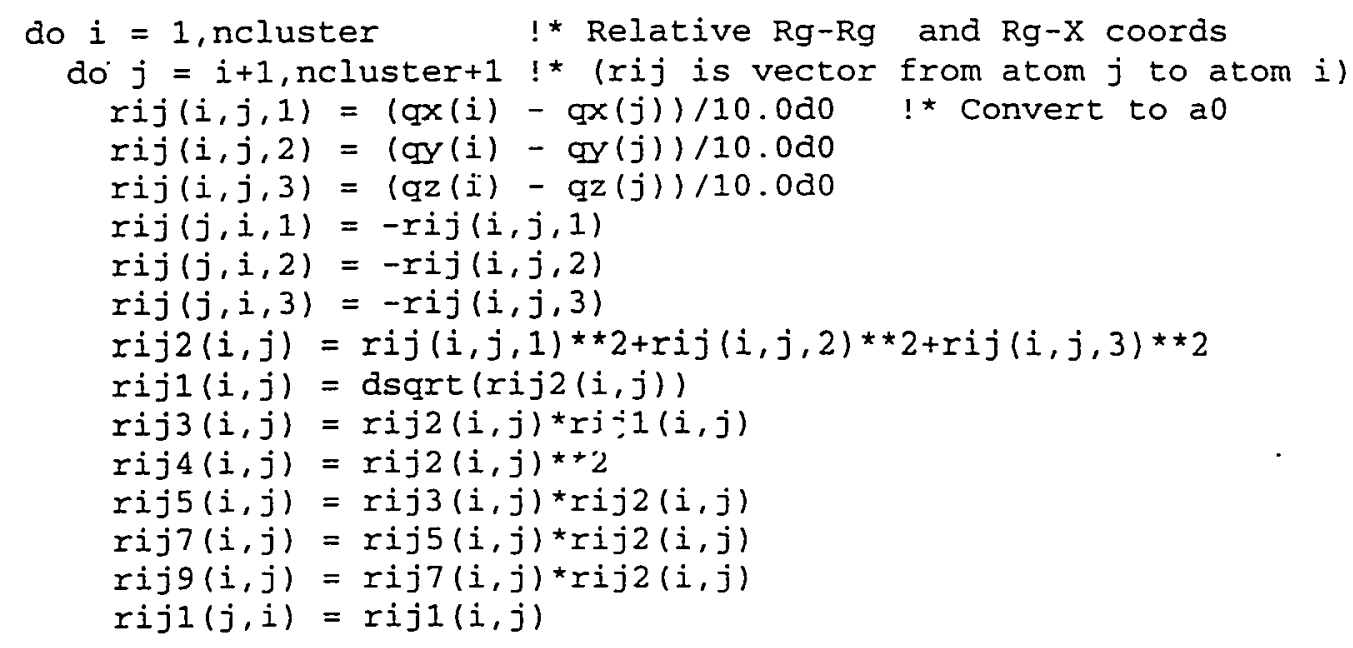




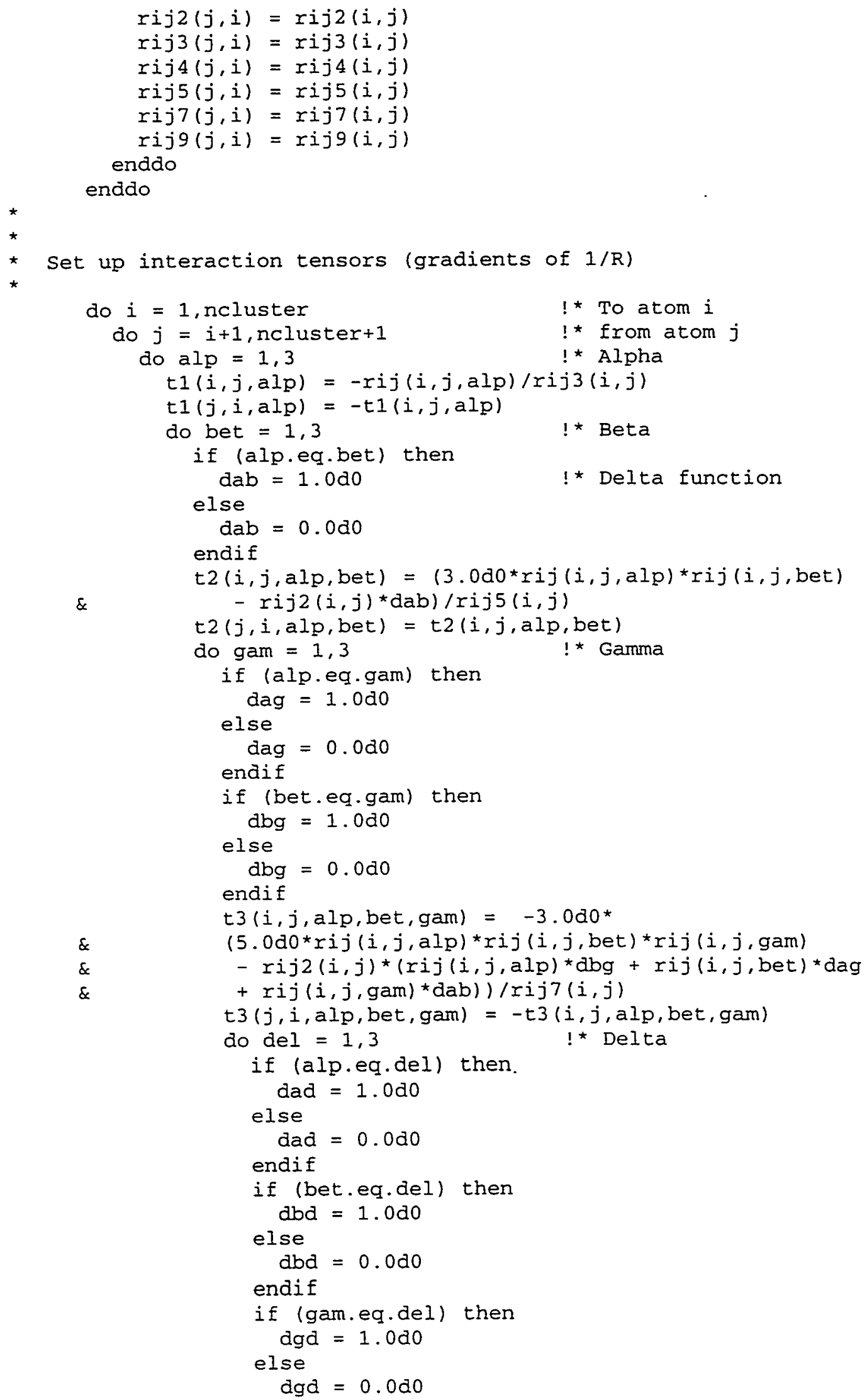




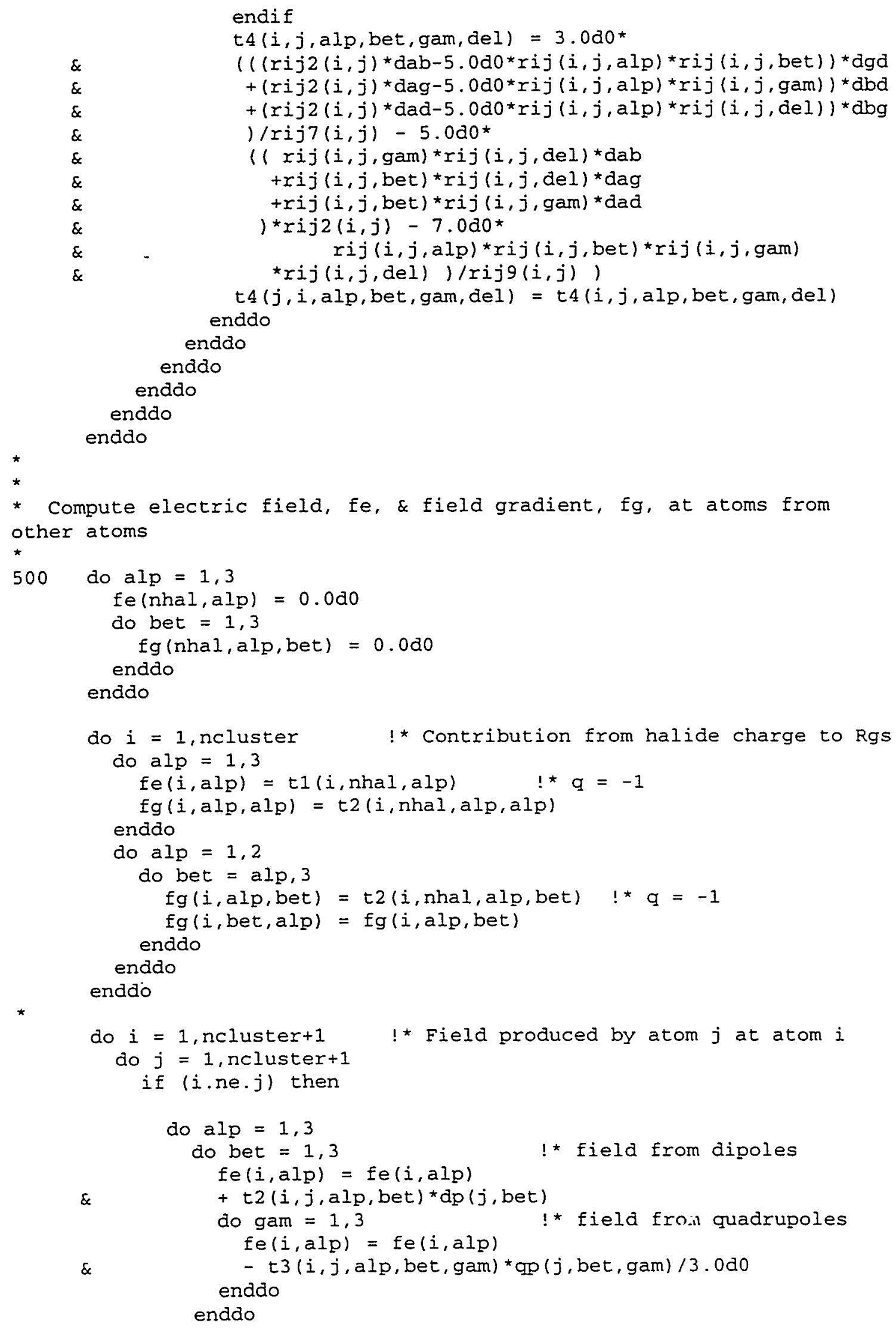




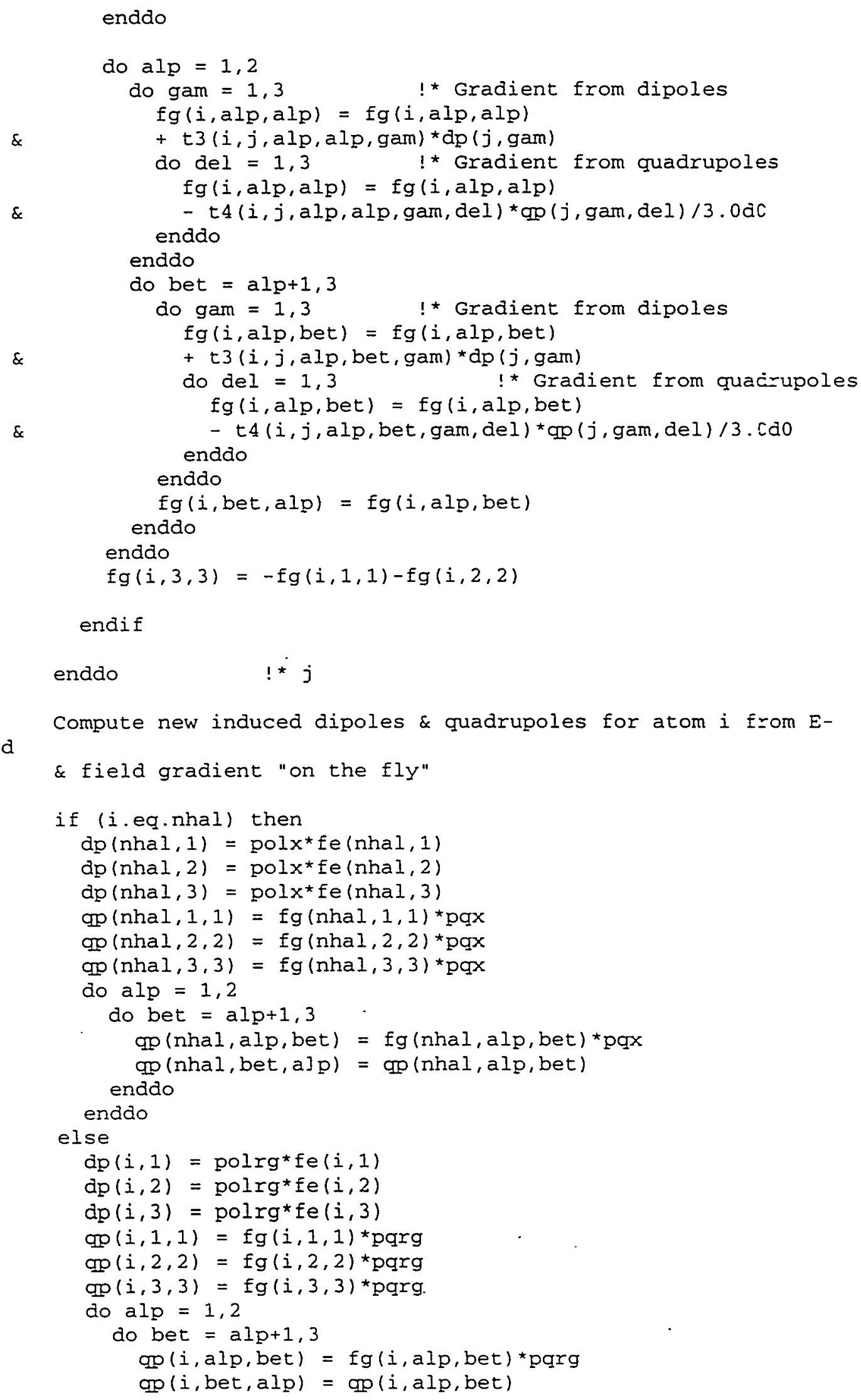




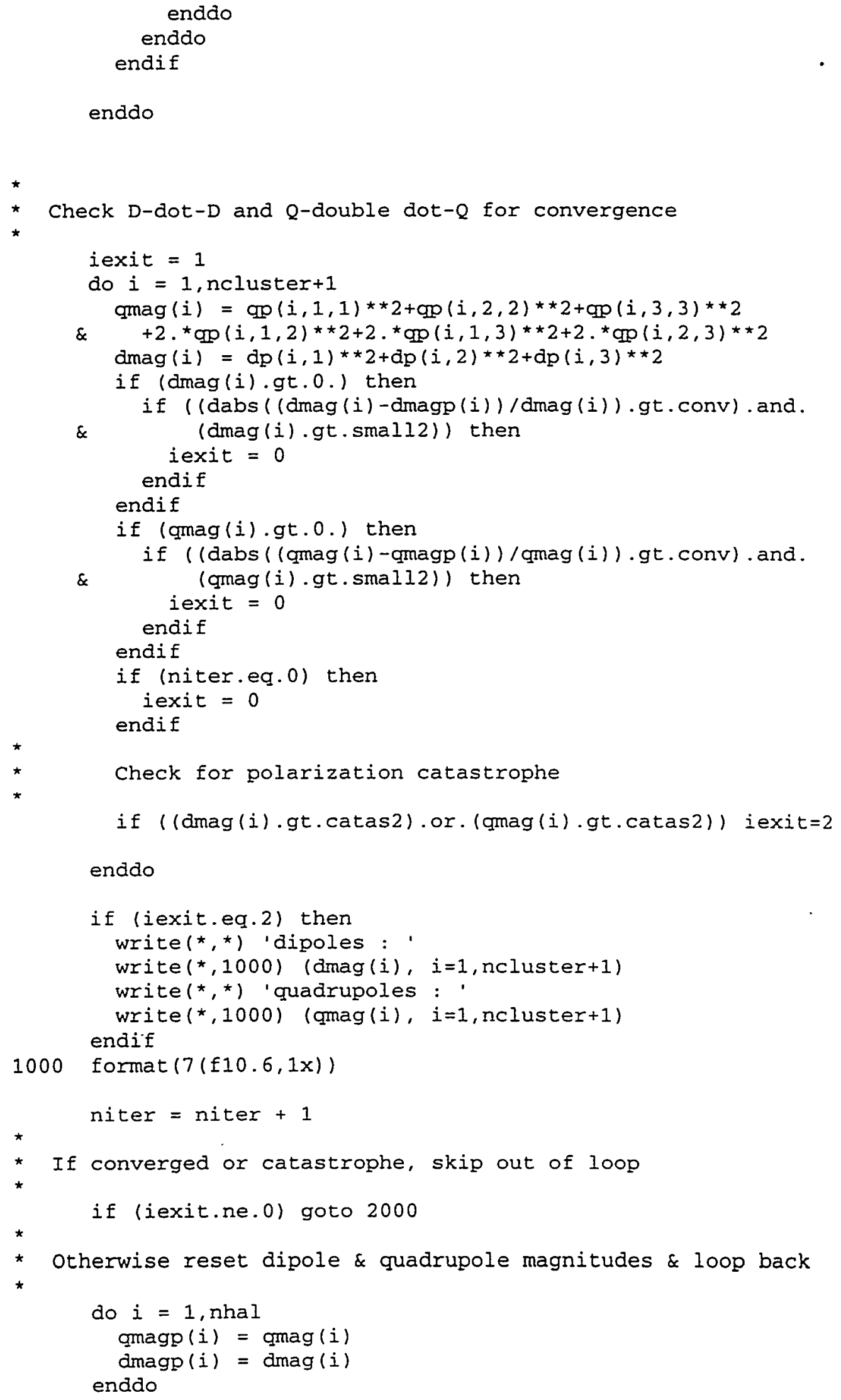




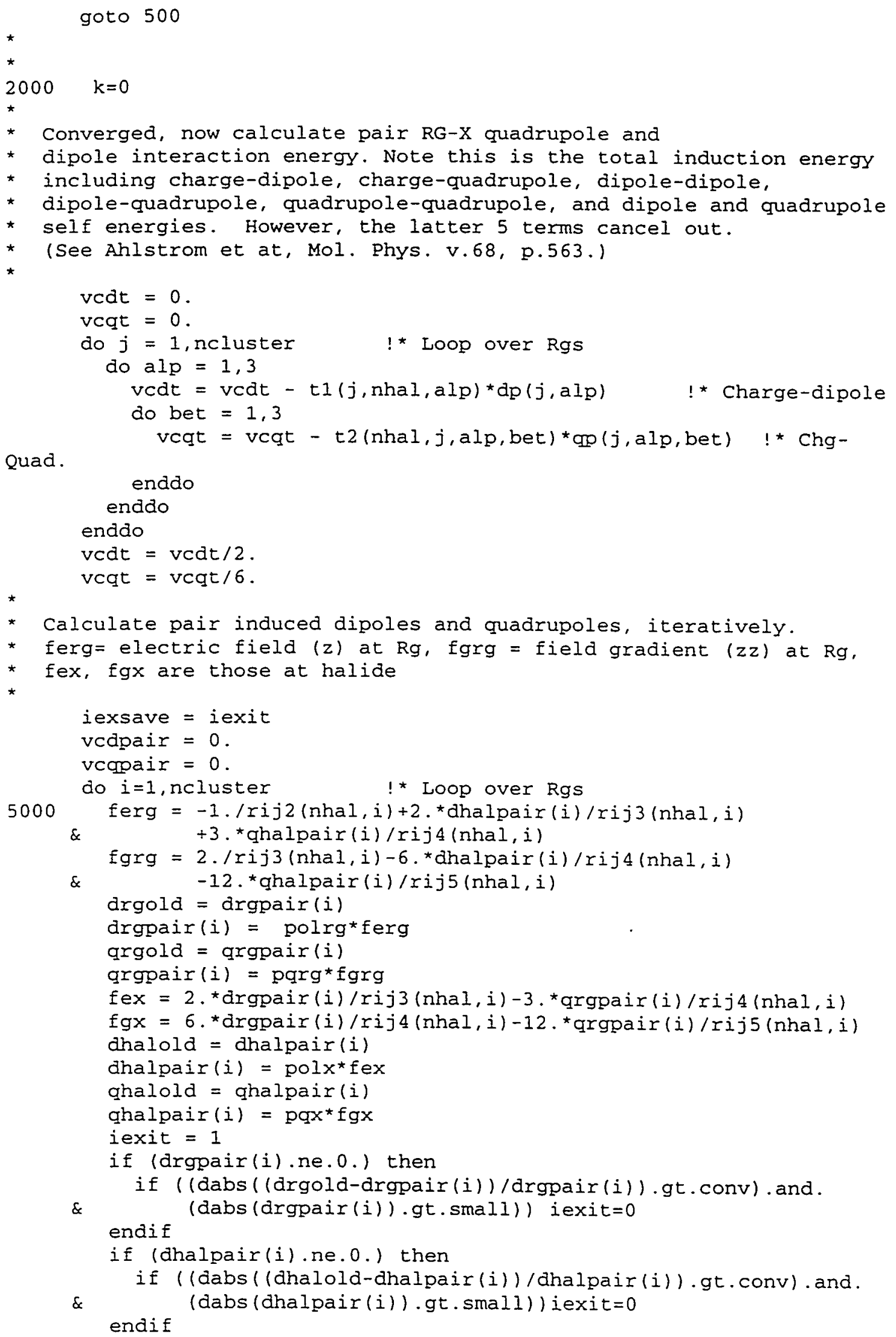




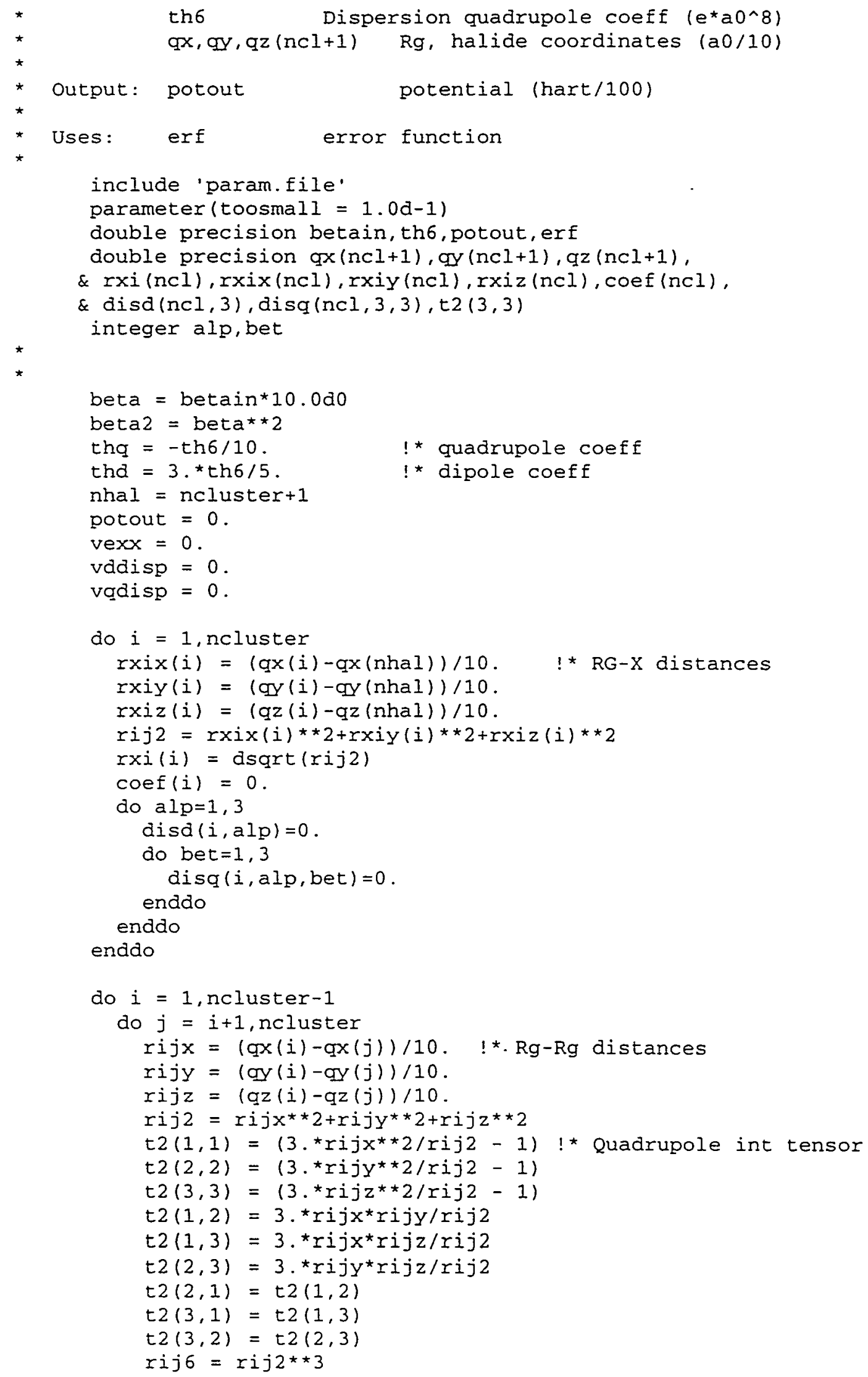




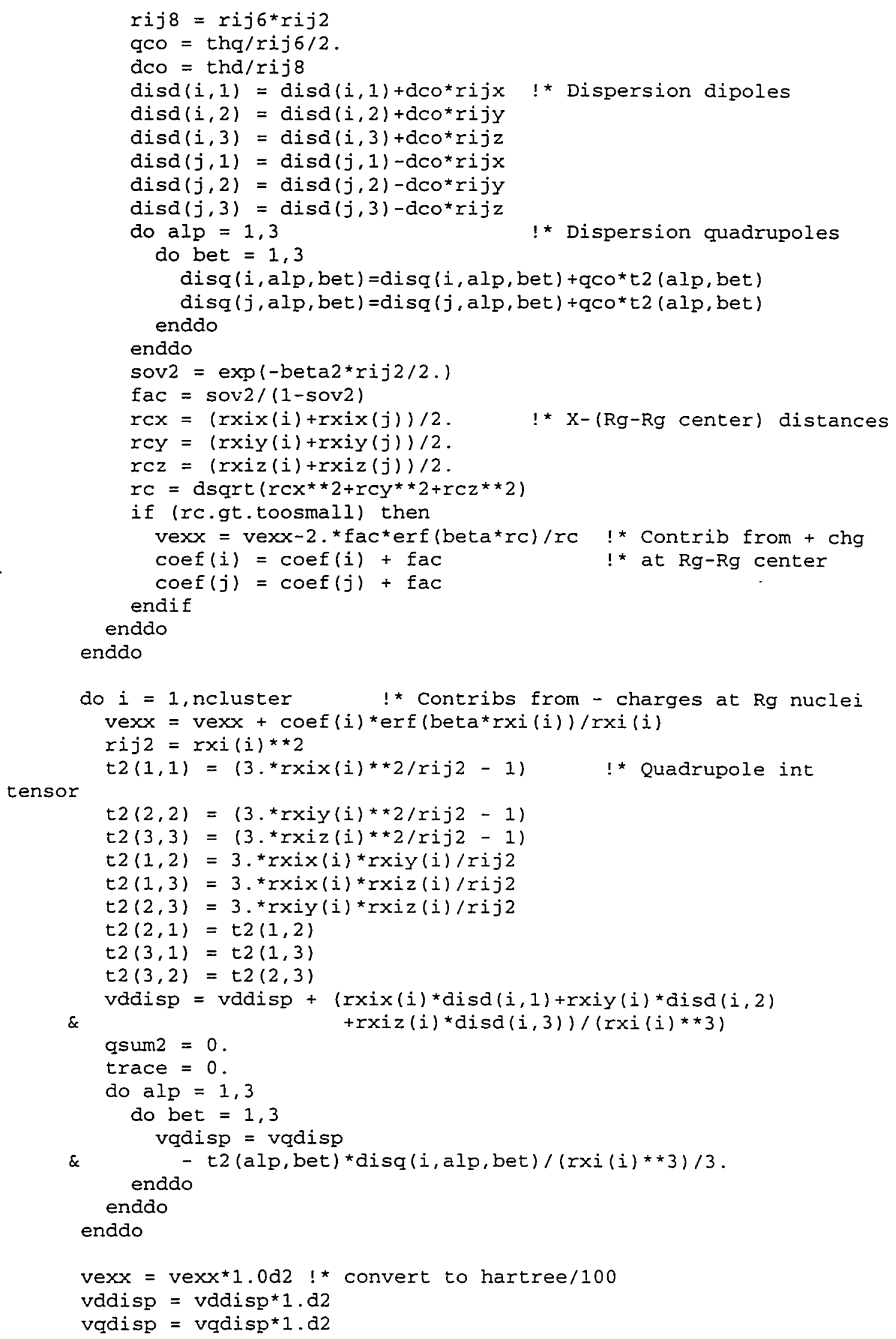




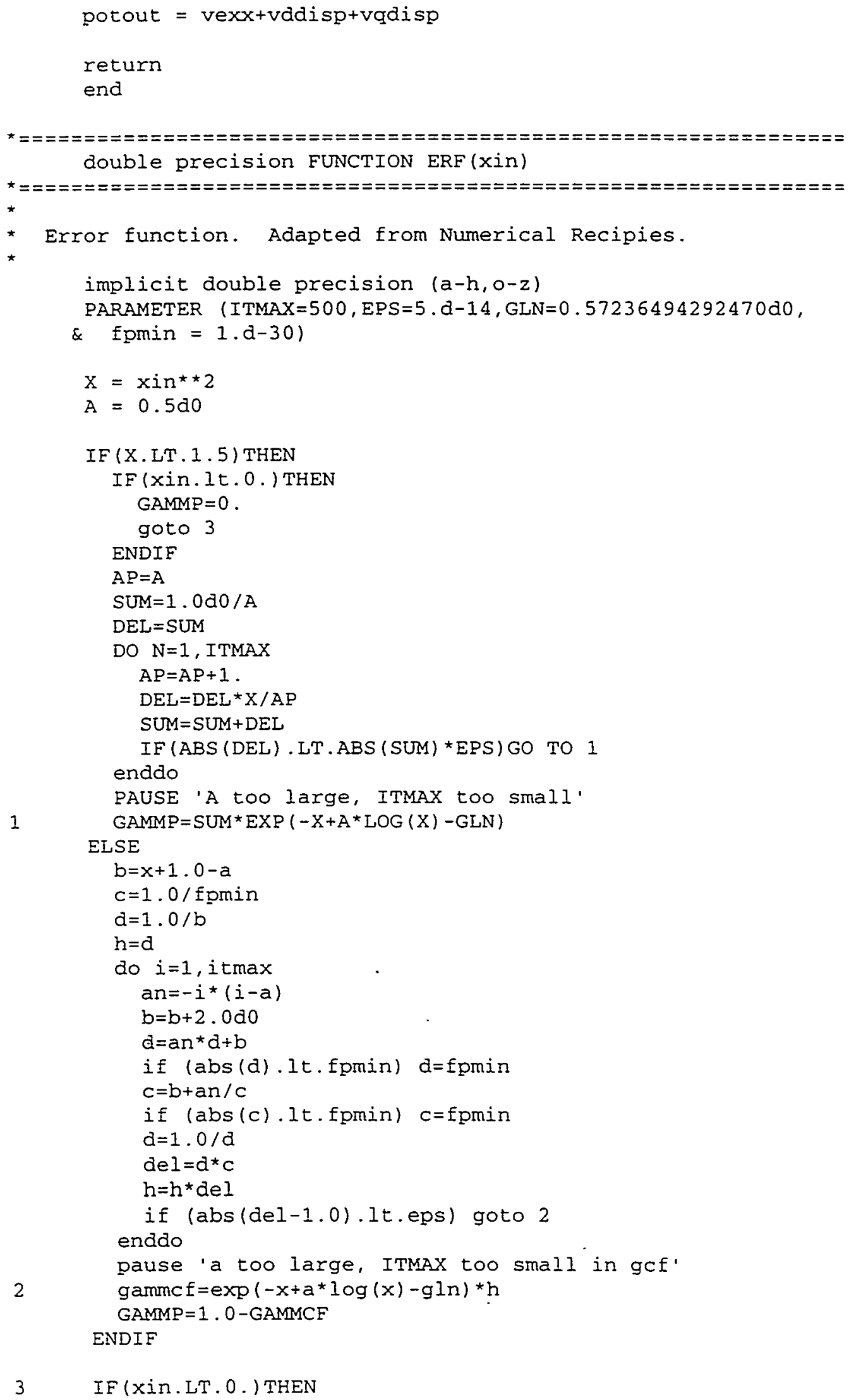




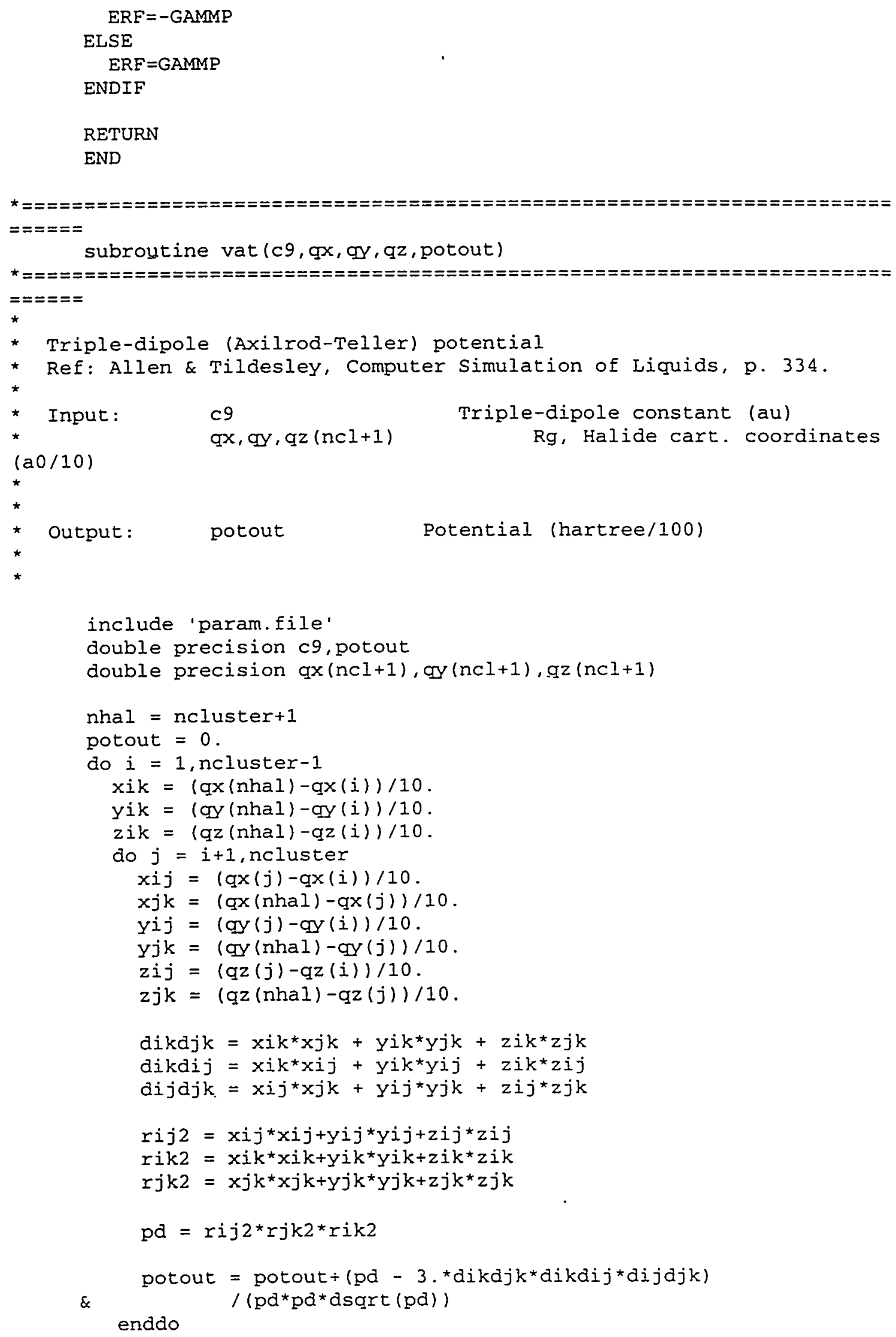


enddo

potout $=$ potout $* c 9 * 1 . d 2$

return

end

\section{C5.8. File "averages.f"}

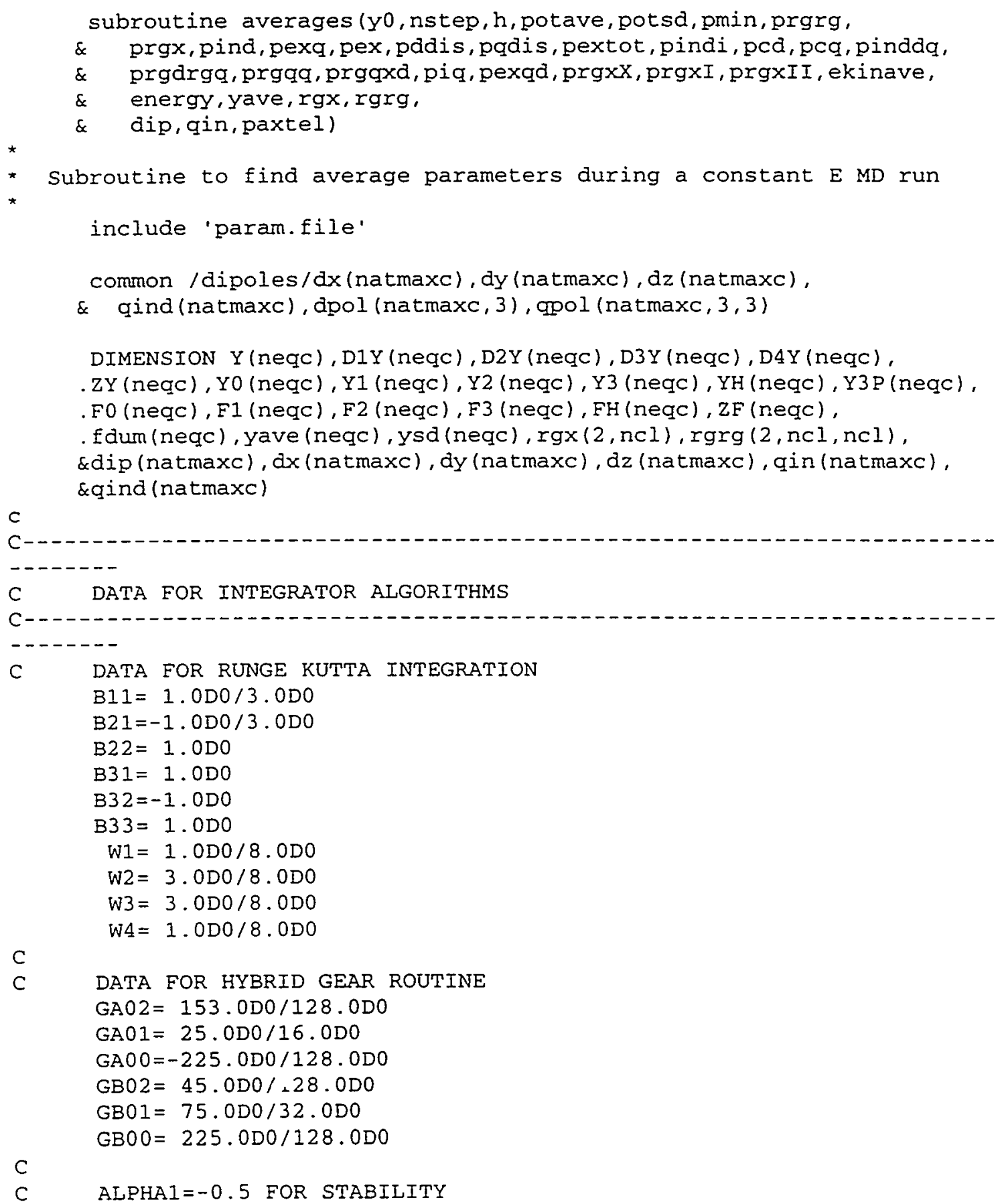


C

$$
\begin{aligned}
& \text { GA12 }=(15.0 D 0 / 16.0 D 0)-0.5 D 0 *(29.0 D 0 / 32.0 D 0) \\
& \text { GA11 }=(-1.0 D 0)-0.5 D 0 *(1.0 D 0) \\
& \text { GA10 }=(17.0 D 0 / 16.0 D 0)-0.5 D 0 *(-61.0 D 0 / 32.0 D 0) \\
& \text { GB12 }=(5.0 D 0 / 16.0 D 0)-0.5 D 0 *(43.0 D 0 / 160.0 D 0) \\
& \text { GB11 }=(11.0 D 0 / 12.0 D 0)-0.5 D 0 *(41.0 D 0 / 24.0 D 0) \\
& \text { GB10 }=(-11.0 D 0 / 16.0 D 0)-0.5 D 0 *(31.0 D 0 / 32.0 D 0) \\
& \text { GG10=(4.0D0/3.0D0)-0.5DO*(-2.0DO/15.0DO })
\end{aligned}
$$

$C$

C

C

ALPHA2 $=1.0, \quad B E T A=9 / 56$

$\mathrm{BETA}=9.0 \mathrm{DO} / 56.0 \mathrm{DO}$

$\mathrm{GA} 22=1.5 \mathrm{DO} *(29.0 \mathrm{D} 0 / 32.0 \mathrm{DO})+\mathrm{BETA} *(-45.0 \mathrm{D} 0 / 4.0 \mathrm{D} 0)$

$\mathrm{GA} 21=1.5 \mathrm{D} 0 *(1.0 \mathrm{D} 0)+\mathrm{BETA} *(0.0 \mathrm{DO})$

$\mathrm{GA} 20=1.5 \mathrm{DO} *(-61.0 \mathrm{DO} / 32.0 \mathrm{DO})+\mathrm{BETA} *(45.0 \mathrm{D} 0 / 4.0 \mathrm{D} 0)$

$\mathrm{GB} 22=1.5 \mathrm{DO} *(43.0 \mathrm{D} 0 / 160.0 \mathrm{D} 0)+\mathrm{BETA}^{*} *(-71.0 \mathrm{D} 0 / 20.0 \mathrm{D} 0)$

$\mathrm{GB} 21=1.5 \mathrm{DO} *(41.0 \mathrm{D} 0 / 24.0 \mathrm{DO})+\mathrm{BETA}^{*}(-16.0 \mathrm{DO})$

$\mathrm{GB} 20=1.5 \mathrm{DO} *(31.0 \mathrm{D} 0 / 32.0 \mathrm{DO})+\mathrm{BETA}^{*}(-3.0 \mathrm{D} 0 / 4.0 \mathrm{DO})$

$G G 20=1.5 D 0 *(-2.0 D 0 / 15.0 D 0)+B E T A *(-16.0 D 0 / 5.0 D 0)$

$c$

GG21 $=B E T A * 1.0 D 0$

call deriv $\left(f 0, y_{0}\right)$

call kinetic $(y 0$, ekin)

energy $=$ ekin + poten

*

* Zero averages

do $j j=1$, neq yave $(j j)=0$. $\operatorname{ysd}(j j)=0$.

enddo

do $i i=1$, ncluster

$\operatorname{rgx}(1, i i)=0$.

$\operatorname{rgx}(2, i i)=0$.

do $j j=1$,ncluster $\operatorname{rgrg}(1, i i, j j)=0$.

enddo $\operatorname{rgrg}(2, i i, j j)=0$.

enddo

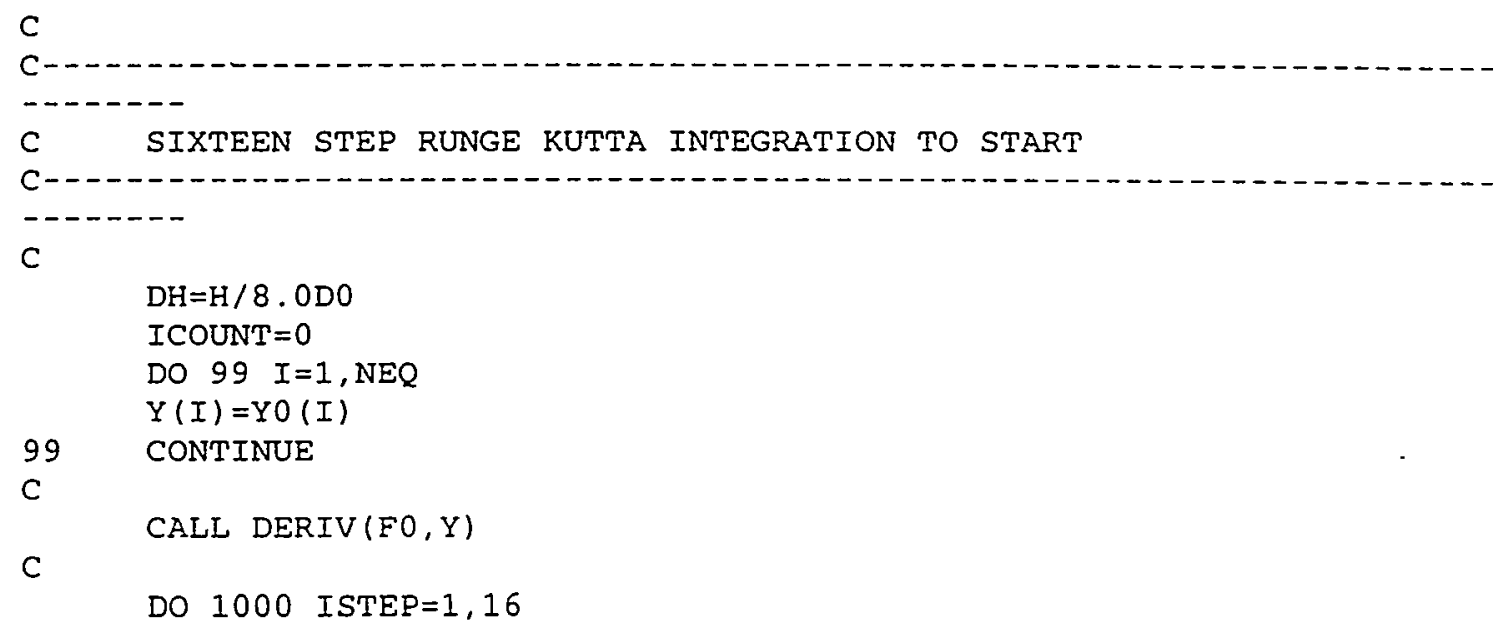




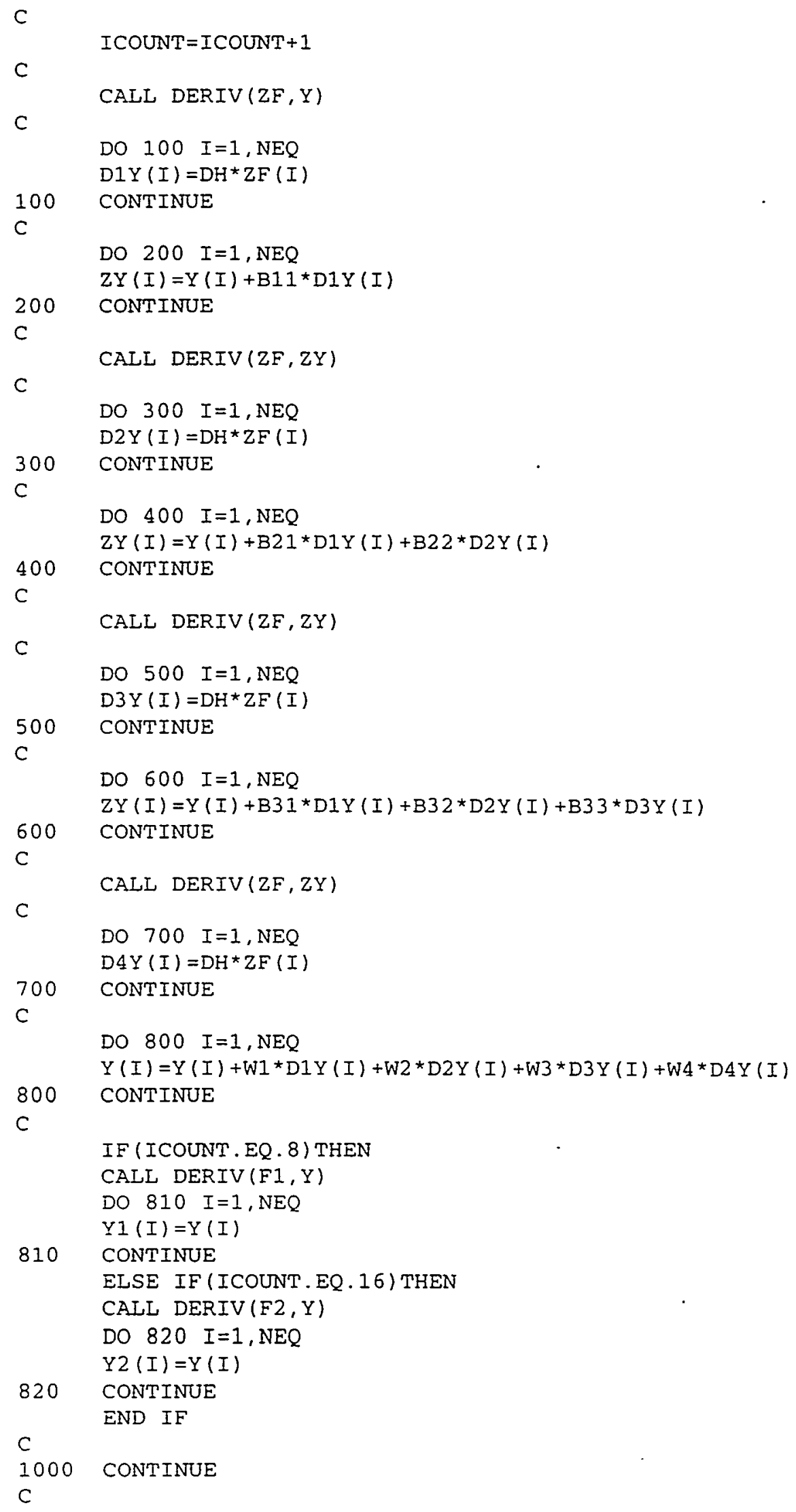




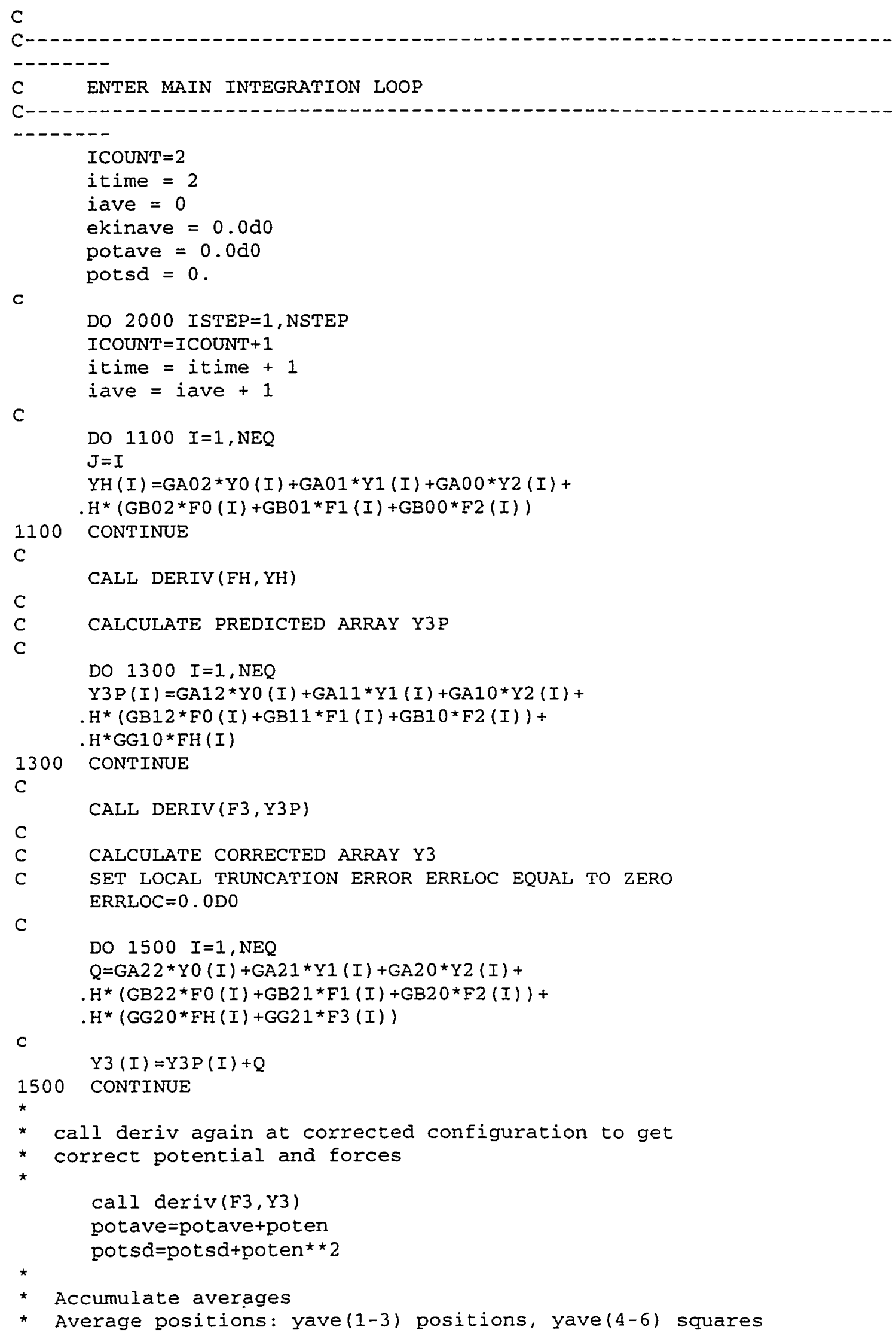




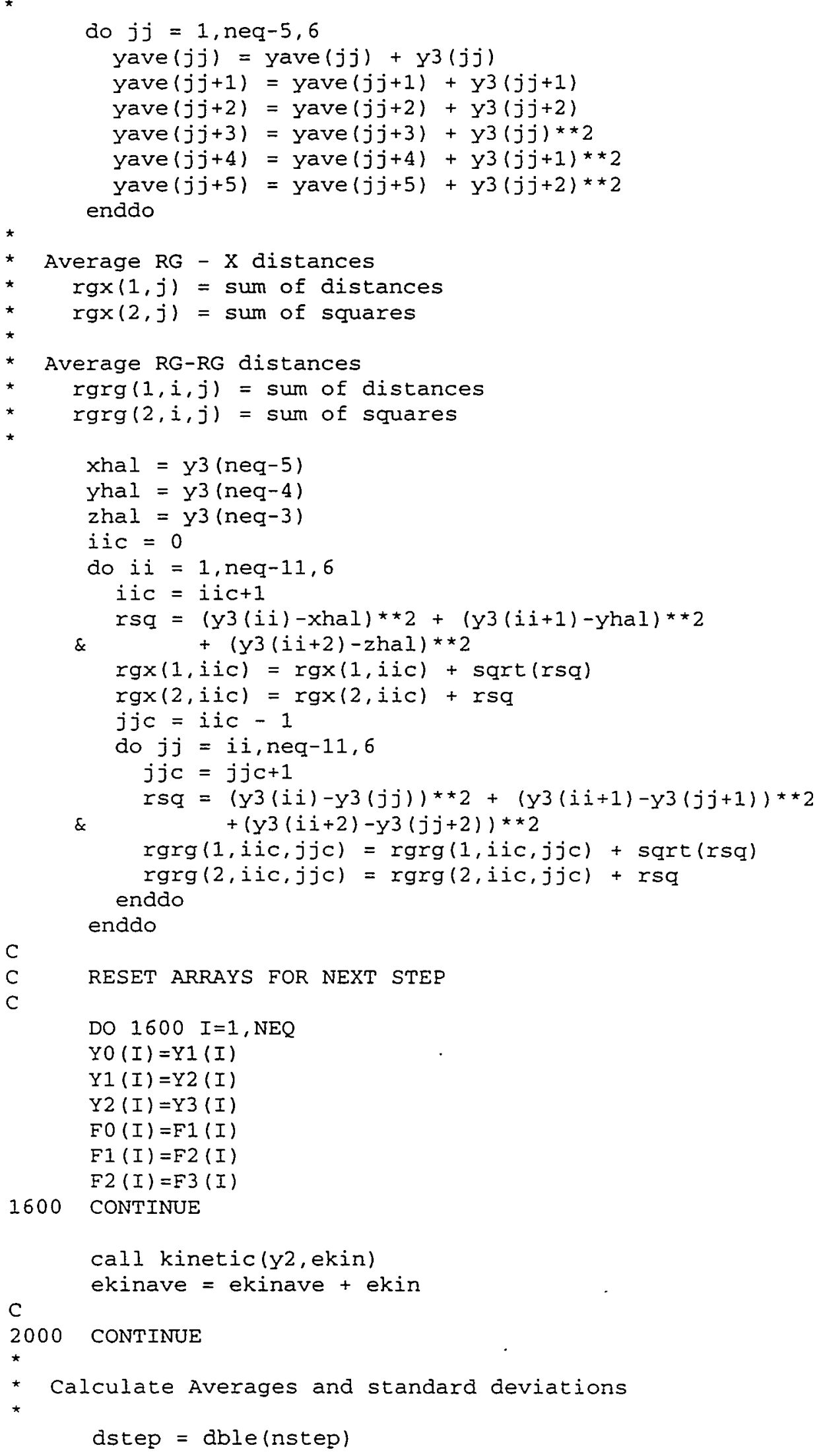




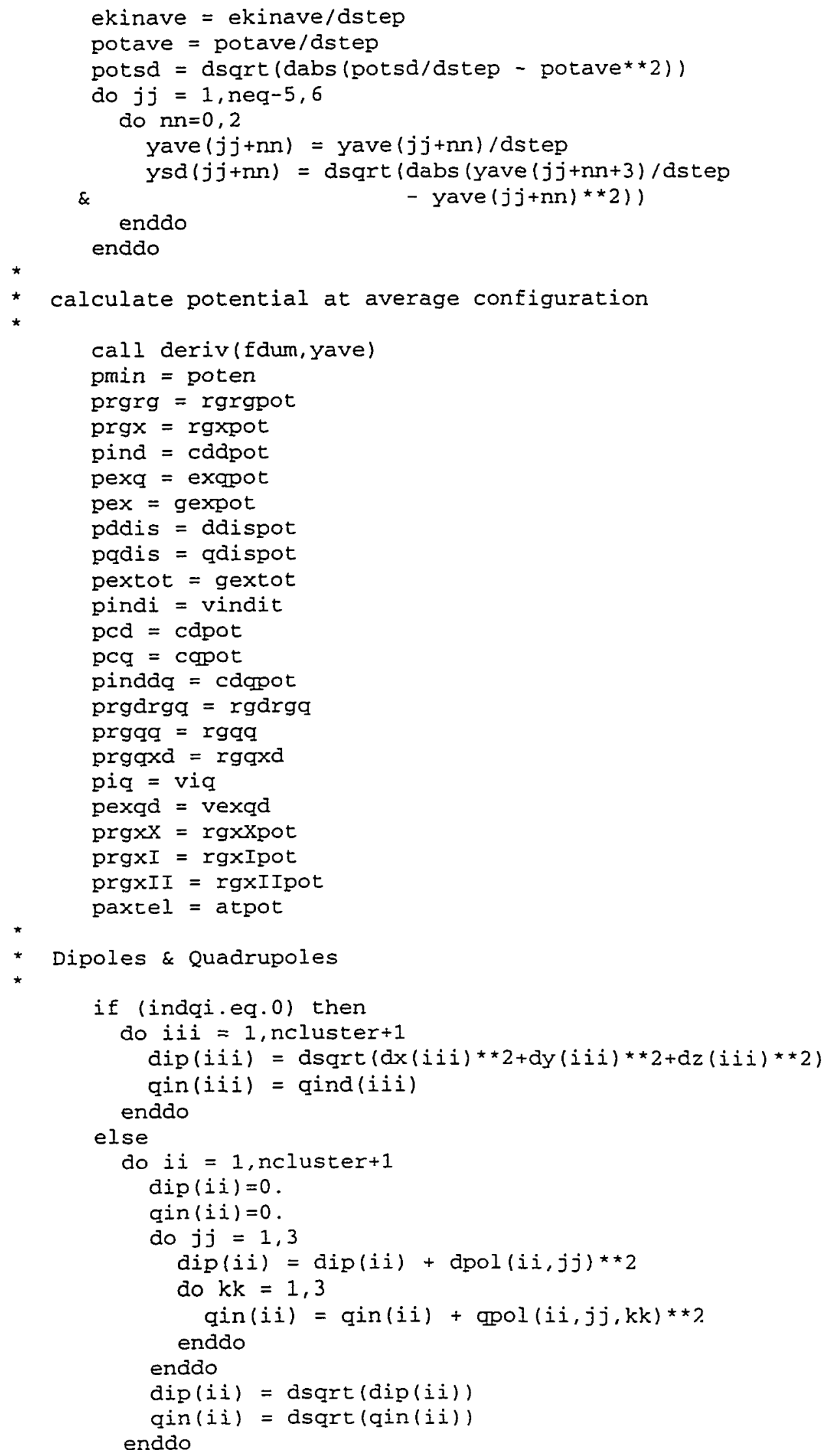


endif

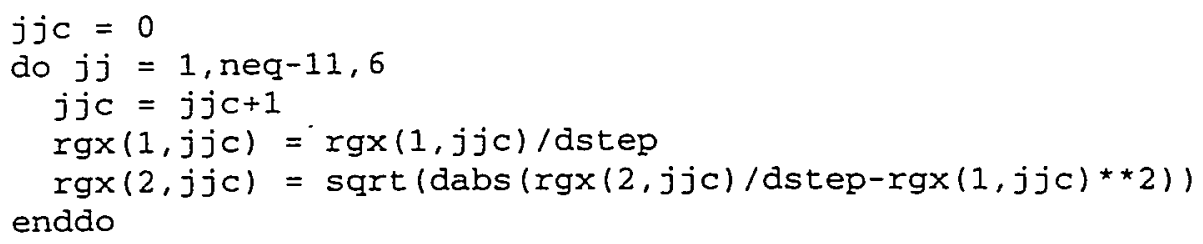

C

C

do $i=1$, neq

$\mathrm{y} 0(I)=\mathrm{y} 2$ (i)

end do

c

return

END

\section{C5.9. File "ch.f"'17}

subroutine $\operatorname{ch}(n m, n, a r, a i, w, m a t z, z r, z i, f v 1, f v 2, f m 1, i e r r)$

$c$

integer $i, j, n, n m$, ierr, matz

double precision $\operatorname{ar}(n m, n), a i(n m, n), w(n), \operatorname{zr}(n m, n), z i(n m, n)$, $x$ fv1 $(n), f v 2(n), f m 1(2, n)$

$c$

c

c

$c$

C

C

c

this subroutine calls the recommended sequence of subroutines from the eigensystem subroutine package (eispack) to find the eigenvalues and eigenvectors (if desired) of a complex hermitian matrix.

on input

nm must be set to the row dimension of the two-dimensional array parameters as declared in the calling program dimension statement.

$\mathrm{n}$ is the order of the matrix $\mathrm{a}=(a r, a i)$.

ar and ai contain the real and imaginary parts, respectively, of the complex hermitian matrix. 


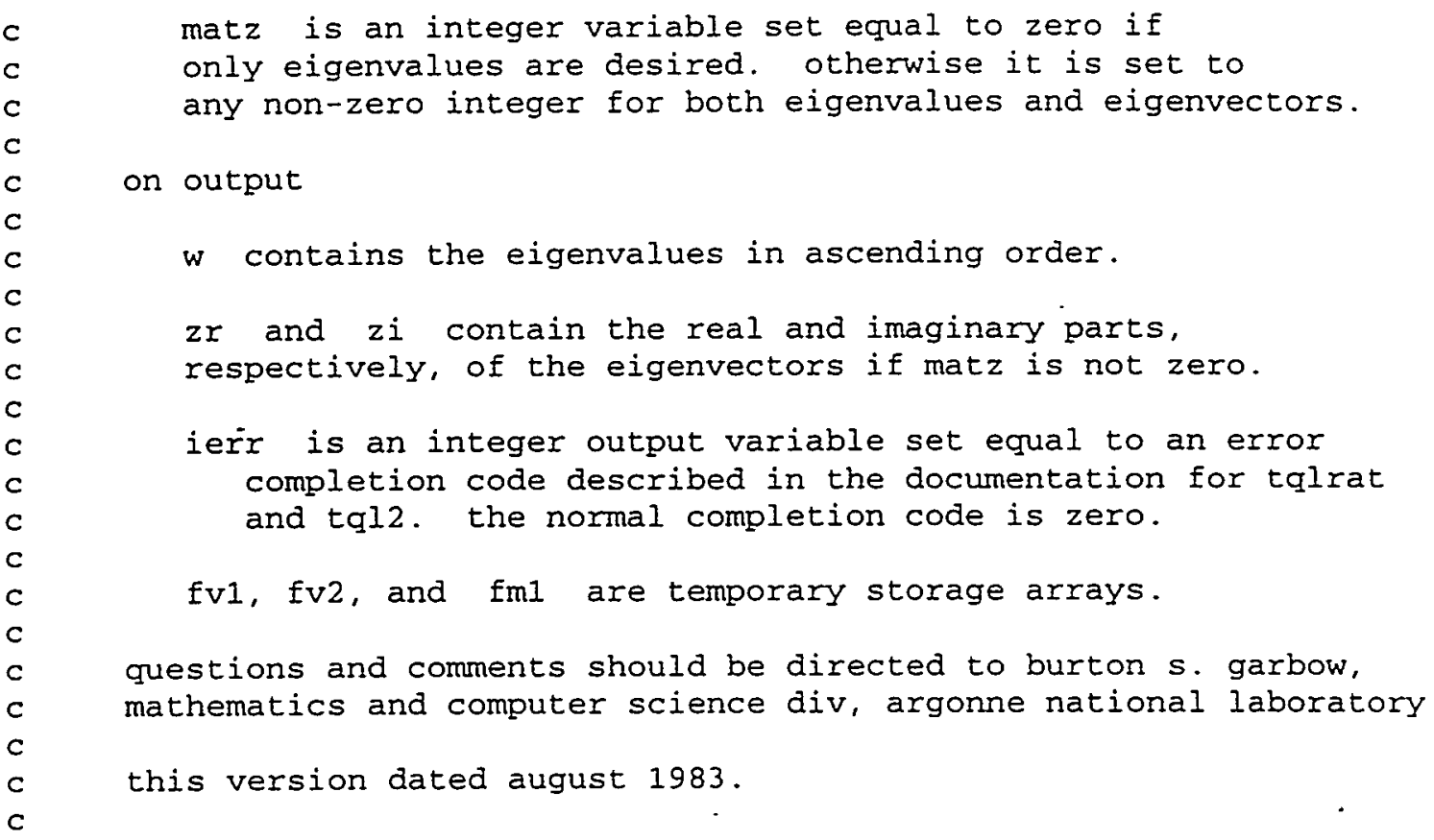

\section{C6. Documentation of the "normal" program for finding zero-point energies}

The "normal" program is used in conjunction with the simulated annealing program for finding the zero point energies of clusters. It accepts as input the cluster minimum energy configuration files produced by the simulated annealing program and calculates vibrational eigenvalues for each mode of the cluster as described in Section 4.4.3 (Chapter 4). The program also can be used for calculating higher vibrational eigenvalues and Franck-Condon factors.

\section{C6.1. Example: Zero-point energy calculation for $\mathrm{Ar}_{6} \mathrm{I}^{-}$}

\section{C6.1.1. The input file}


The input file for the "normal" program for the global minimum configuration of

$\mathrm{Ar}_{6} \mathrm{I}^{-}$found in section $\mathrm{C} 4.3$ is shown below. The line numbers in boldface are for

reference only and are not included in the actual input file. The name of the file is

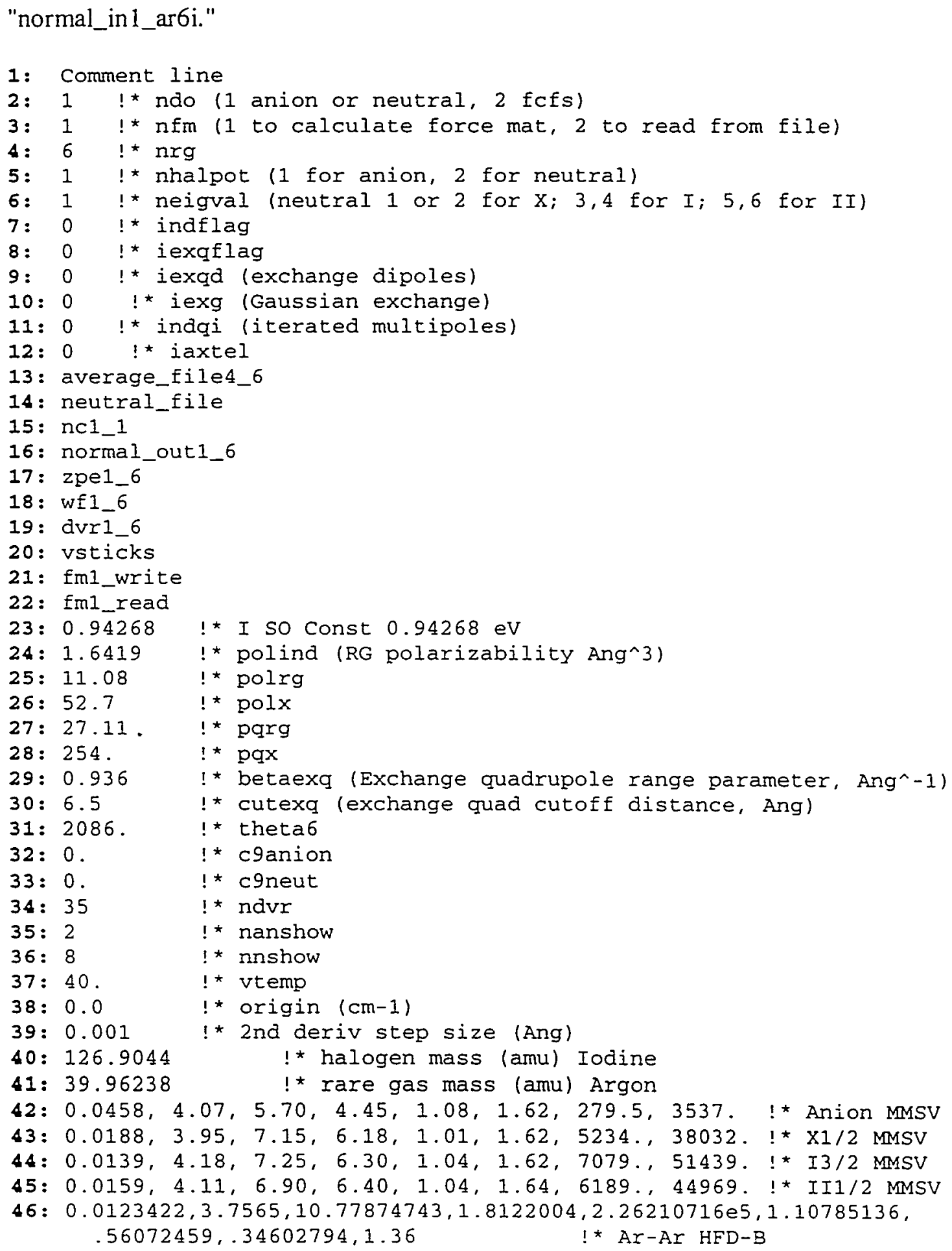


Line 1 is a comment line which is copied directly to the output file. Line 2 determines the mode of running the program: when this line is set to 1 , only the calculation for either the anion or neutral eigenvalues is performed; when it is 2 , the eigenvalues for both anion and neutral are calculated as well as Franck-Condon factors and a vibrational stick spectrum. The setting of line 3 determines whether the force matrix is calculated from the atomic configuration and potential (line $3=1$ ) or if a previously calculated force matrix is read in from in from a file (line $3=2$ ).

Lines 5-12, 23-33 and 42-46 describe the nature of the cluster and its potential and are identical to the corresponding lines of the input file "inl" to the simulated annealing program (see Section C4.1, above).

Lines 13 and 14 are the anion and neutral atomic configuration files generated by the simulated annealing program used as input by "normal." If the FCFs calculation is not performed, only one of the files is read in, but the input file must nonetheless contain both lines. In this example we are only interested in the zero point energies of the $\mathrm{Ar}_{6} \mathrm{I}^{*}$, and so have placed the $\mathrm{Ar}_{6} \mathrm{I}^{-}$global minimum configuration file "average_file4_6" in line 13 and a "dummy" file name in line $\mathbf{1 4}$. Line $\mathbf{1 5}$ is the name of a file to save the normal coordinates, which may be examined to ascertain the symmetry of each vibrational mode. Line $\mathbf{1 6}$ is the name of the program output file, which lists the individual mode vibrational frequencies and zero-point energies. If FCF mode is enabled, the FCFs are also listed in this file. Line $\mathbf{1 7}$ is the name of a file to save the total zero point energy. Line 18 is the name of a file to save the DVR eigenvalues and eigenvectors for each mode. As when running the Morse DVR program (Section A5.2), the eigenvectors 
should be examined for convergence. The file named in line 19 lists the DVR points for each vibrational mode. The file named in line $\mathbf{2 0}$ is the vibrational stick spectrum (if FCF mode is enabled). Line 21 is the name of the file to save the calculated force matrix. Line 22 is the name of the file from which to read in the force matrix if it is not to be calculated.

The program uses a harmonic oscillator-based DVR method ${ }^{18}$ for solving Hamiltonian along each normal coordinate. Line 34 is the number of harmonic oscillator basis functions to be used in the calculation. Lines 35 and 36 are the number of anion and neutral eigenvalues, respectively, one wishes to calculate. Line 37 is the vibrational temperature used to generate the vibrational stick spectrum. Line 38 is the origin, in wavenumbers, of the vibrational stick spectrum. Line 39 is the step size in $\AA$ used in calculating the second derivatives of the potential for the force matrix. Lines 40 and 41 are the halogen and rare gas masses in amu, respectively.

The frequency of the harmonic oscillator basis set for the DVR calculation is chosen to be the same for each mode as the harmonic frequency calculated numerically from the potential. In some cases, the DVR calculation does not converge well with this choice of basis frequency. In such cases one can specify the basis frequency for one or mode by setting line 47 of the input file to the number of basis frequencies to be set by hand. The user is then prompted for the numbers of the normal modes (as listed in the output file) and the basis frequencies. 


\section{C6.1.2. Running the program}

The program is run as in the following example, with user input shown in boldface.

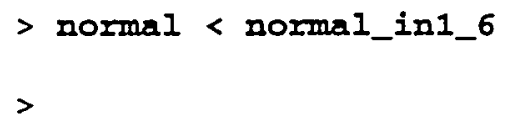

The output file "normal_out1_6" is then generated, and is excerpted here:

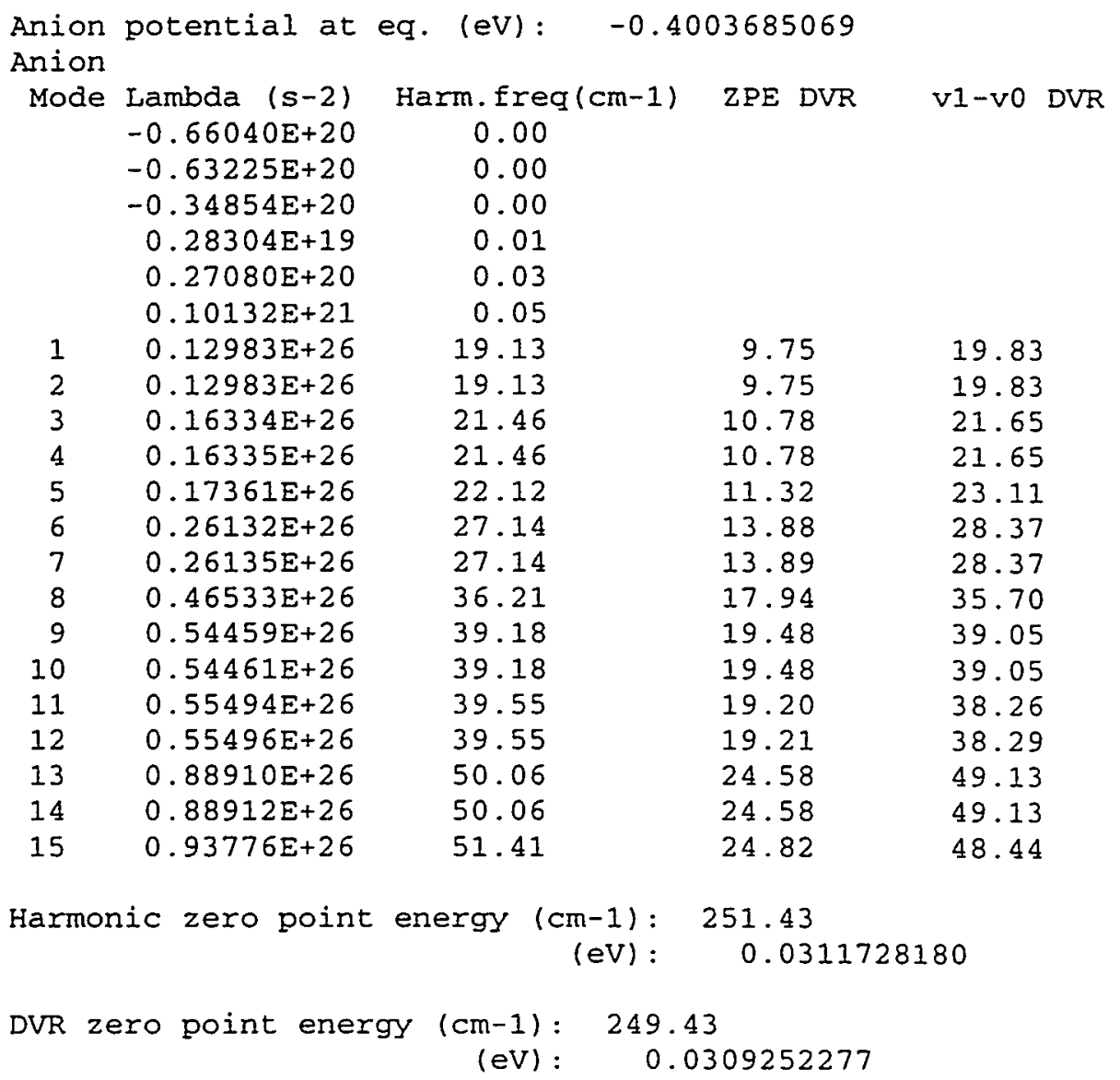

The first column of this file is the mode number, the second column is the harmonic mode frequency in $\mathrm{Hz}$, the third column is the harmonic mode frequency, the fourth column contains the zero point energies for each mode from the DVR calculation, and the fifth column contains the mode frequencies from the DVR calculation. 


\section{C6.2. Outline of the program}

The subroutines and functions used by the program "normal" are listed in Table C2, sorted according the file in which they reside. Some of the functions and subroutines are the same as those previously described for the simulated annealing program and Morse DVR program, and these are noted in the "Description" column of the table. 
Table C2. Subroutines and functions used by the program "normal"

\begin{tabular}{|c|c|c|}
\hline File & $\begin{array}{l}\text { Subroutine } \\
\text { or Function }\end{array}$ & Description \\
\hline param.file & & See Table C1 \\
\hline \multirow[t]{15}{*}{ normal.f } & normal & Main program \\
\hline & peval & Evaluates neutral potential of cluster \\
\hline & apeval & Evaluate anion potential of cluster \\
\hline & pind & $\begin{array}{l}\text { Calculates three-body induction potential from Eq. } \\
\text { (C10) }\end{array}$ \\
\hline & vexq & $\begin{array}{l}\text { Calculates three-body "exchange quadrupole" } \\
\text { potential from Eq. }(\mathrm{Cl} 2)\end{array}$ \\
\hline & hopts & $\begin{array}{l}\text { Calculates DVR points using harmonic oscillator } \\
\text { basis for a given vibrational mode }\end{array}$ \\
\hline & hokin & Transforms kinetic energy matrix to DVR basis \\
\hline & hoham & Calculated DVR Hamiltonian \\
\hline & prinaxes & $\begin{array}{l}\text { Transforms cluster coordinates to principal axis } \\
\text { system (used in FCF mode) }\end{array}$ \\
\hline & calcfcfs & $\begin{array}{l}\text { Calculates multi-mode Frank-Condon factors and } \\
\text { vibrational stick spectrum }\end{array}$ \\
\hline & veqd & $\begin{array}{l}\text { Calculated exchange-quadrupole potential using the } \\
\text { distributed dipole model. }\end{array}$ \\
\hline & vinddq & See Table C1 \\
\hline & exg & See Table C! \\
\hline & erf & See Table Cl \\
\hline & vat & See Table Cl \\
\hline porb.f & porb & See Table Cl \\
\hline poten2.f & & See Table A1 \\
\hline$\overline{\text { ch.f }}$ & $\mathrm{ch}$ & See Table $\mathrm{Cl}$ \\
\hline rs.f & rs & See Table A1 \\
\hline rsb.f & $\mathrm{rsb}$ & See Table A1 \\
\hline
\end{tabular}




\section{C6.3. Source code for the program "normal"}

The source code for the "normal" program is listed below. The files "rsb.f", "rs.f", and "poten2.f" are identical to those listed or described in Sections A6.3, A6.4 and A6.5 and hence are omitted here, as is the file "ch.f" which is identical to the EISPACK subroutine described in Section C5.9. Some of the subroutines and functions in the files "normal.f" and the subroutine in the file "porb.f" are identical with those in the simulated annealing program listing, and only the headers of these are reproduced below.

\subsubsection{File "makenormal"}

The program is recompiled with the command "make -f makenormal". The executable file "normal" is saved in the subdirectory "norm." The makefile is listed below.

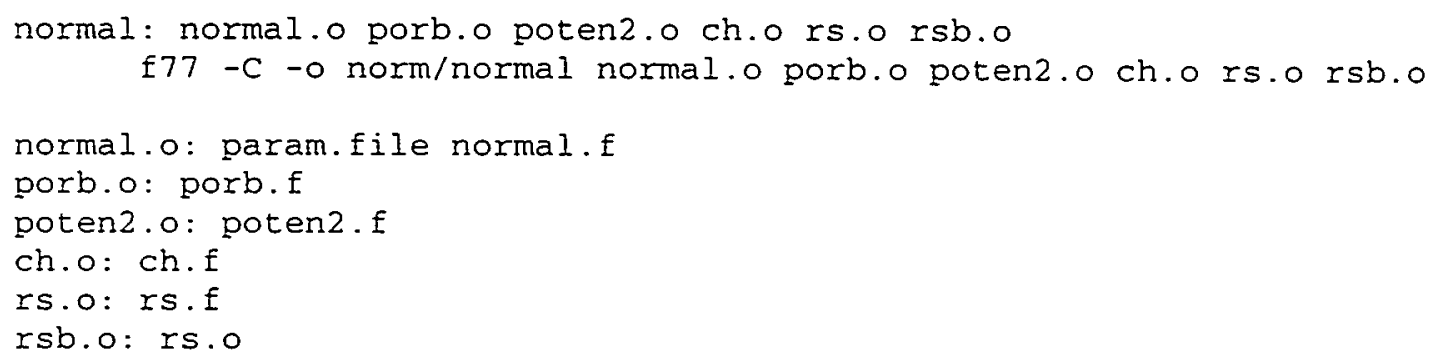

\subsubsection{File "'normal.f"}

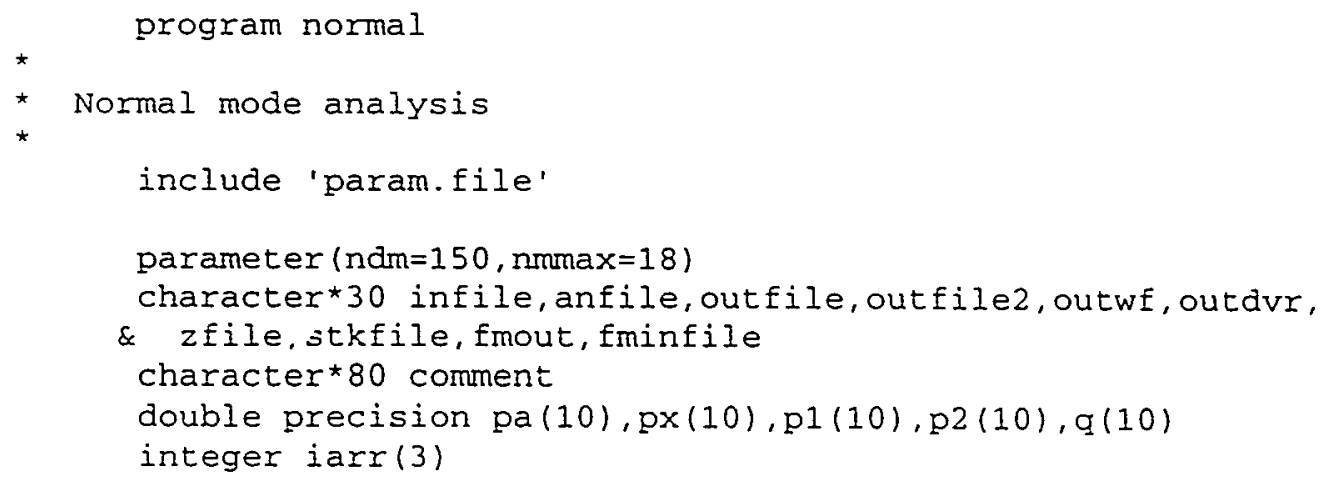


integer nmode (natmaxc*3-6)

double precision amass (natmaxc*3), fmat (natmaxc*3, natmaxc*3),

\& $\operatorname{co}($ natmaxc*3), co2 (natmaxc*3), co3 (natmaxc*3),

\& lambda (natmaxc*3), fv1 (natmaxc*3),

\& $\quad$ fv2 (natmaxc*3), evec (natmaxc*3, natmaxc*3), co1 (natmaxc*3),

\& $\operatorname{cdis}($ natmaxc*3), qdis (natmaxc*3), zpedvr (natmaxc*3-6),

\& frqdvr (natmaxc*3-6), alamhan (natmaxc*3-6)

double precision $\operatorname{rdvr}(n d m+1)$, tmat $(n d m+1, n d m+1)$,

$\& \operatorname{akin}(n d m+1, n d m+1)$, hamdvr $(n d m+1, n d m+1)$, evalm $(n d m+1)$,

\& $\operatorname{evalm} 2(n d m+1), \operatorname{evecm}(n d m+1, n d m+1), \operatorname{evecm} 2(n d m+1, n d m+1)$,

\& $\operatorname{fvlm}(n d m+1), f v 2 m(n d m+1), \operatorname{vdvr}(n d m+1), \operatorname{vdvr} 2(n d m+1)$

double precision wfs (nmmax, $n d m+1, n d m+1)$, wfs2 (nmmax, ndm+1, ndm+1),

\& evs ( $\operatorname{mmax}, n d m+1), \operatorname{evs} 2(n \operatorname{mmax}, n d m+1)$

double precision fcfs(nmmax, ndm+1, ndm+1), sticks (2, 1000),

\& totfcf (nmmax, ndm+1)

integer nma (2, nmmax, 1000)

common/anparams/polind, betaexq, cutexq, soconst,

\& pa,px,p1,p2,q,polrg,polx,pqrg,pqx, theta6,c9anion,

\& c9neut, nrg

write $\left({ }^{\star},{ }^{*}\right)$ 'Enter comment line'

read $(*, 80)$ comment

80 format $(a 80)$

write $(*, *)$ 'Enter mode: 1 anion or neutral frequencies.'

write $\left({ }^{\star},{ }^{\star}\right)$ ' 2 both, and fcfs (shifted normal coords).

coords).

write $(*, *)$ " 3 " (vertical normal

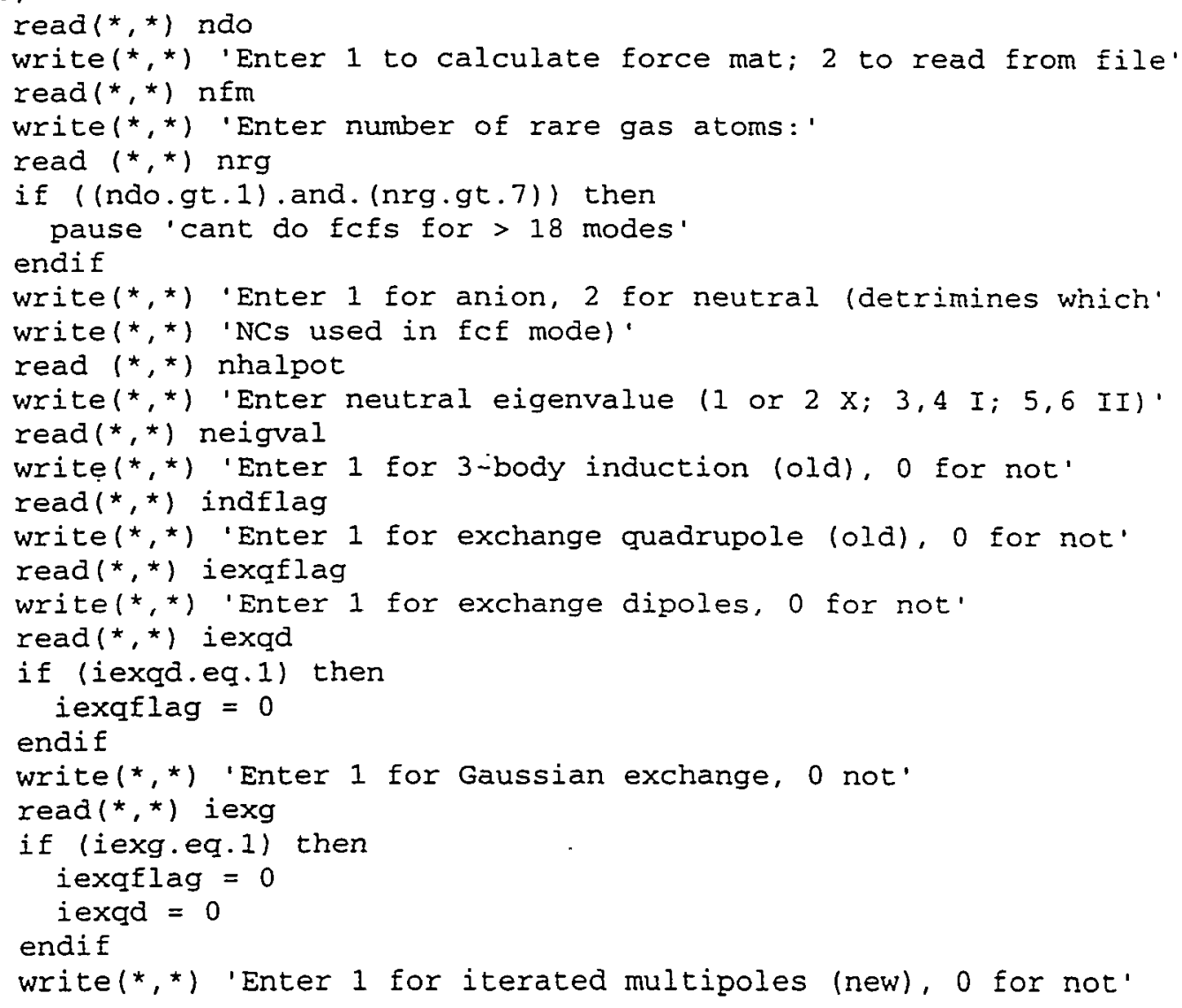




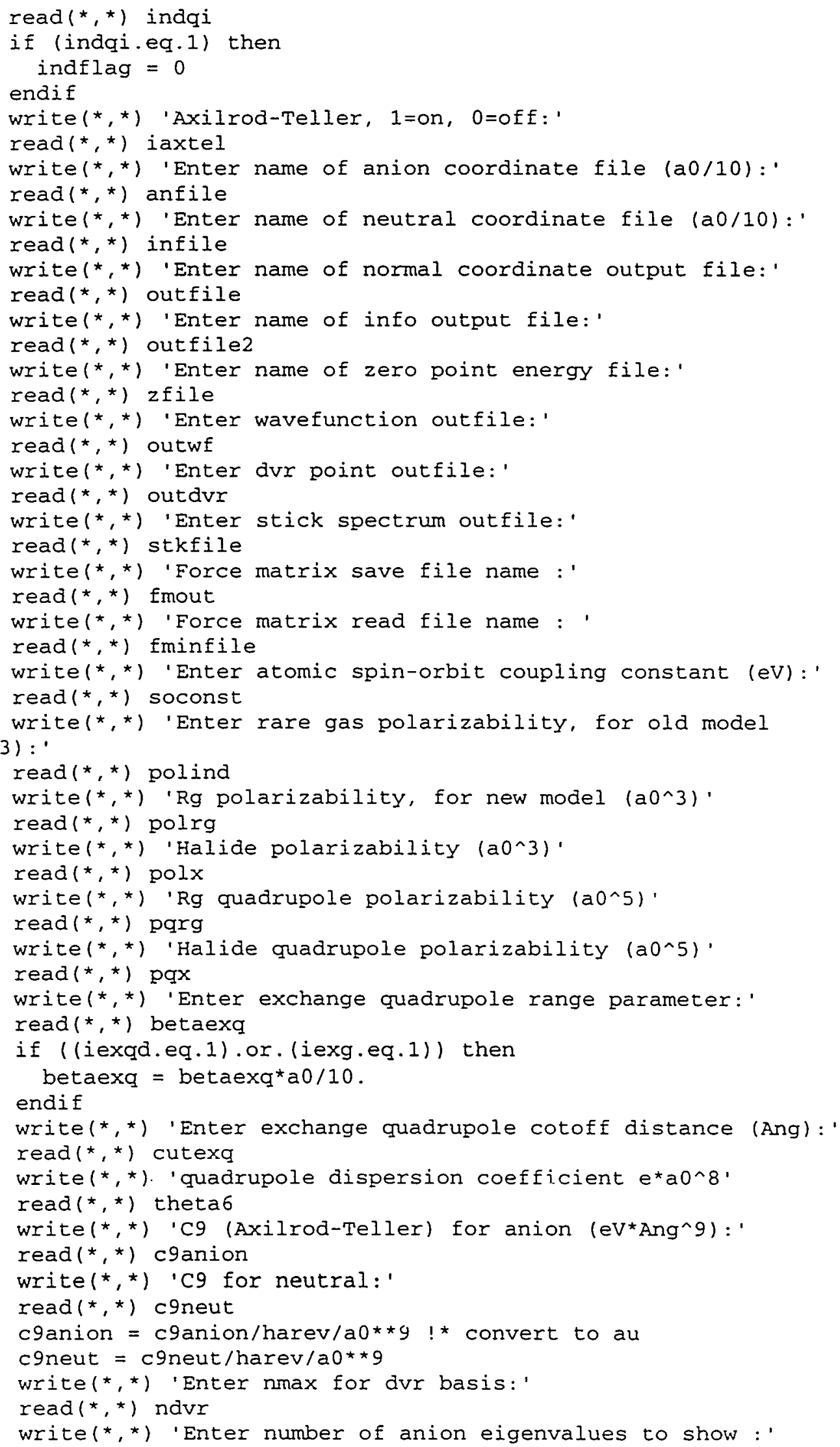




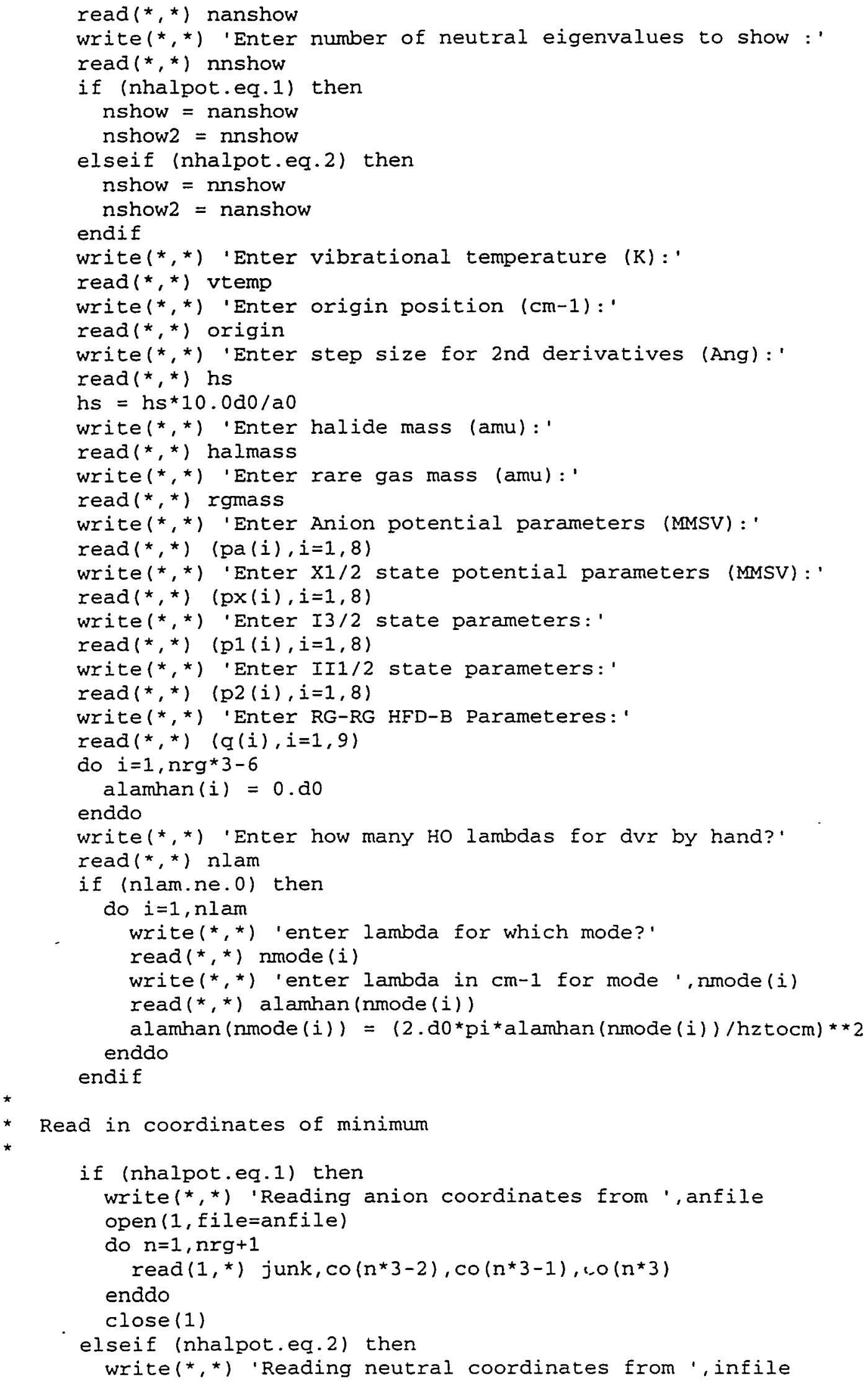




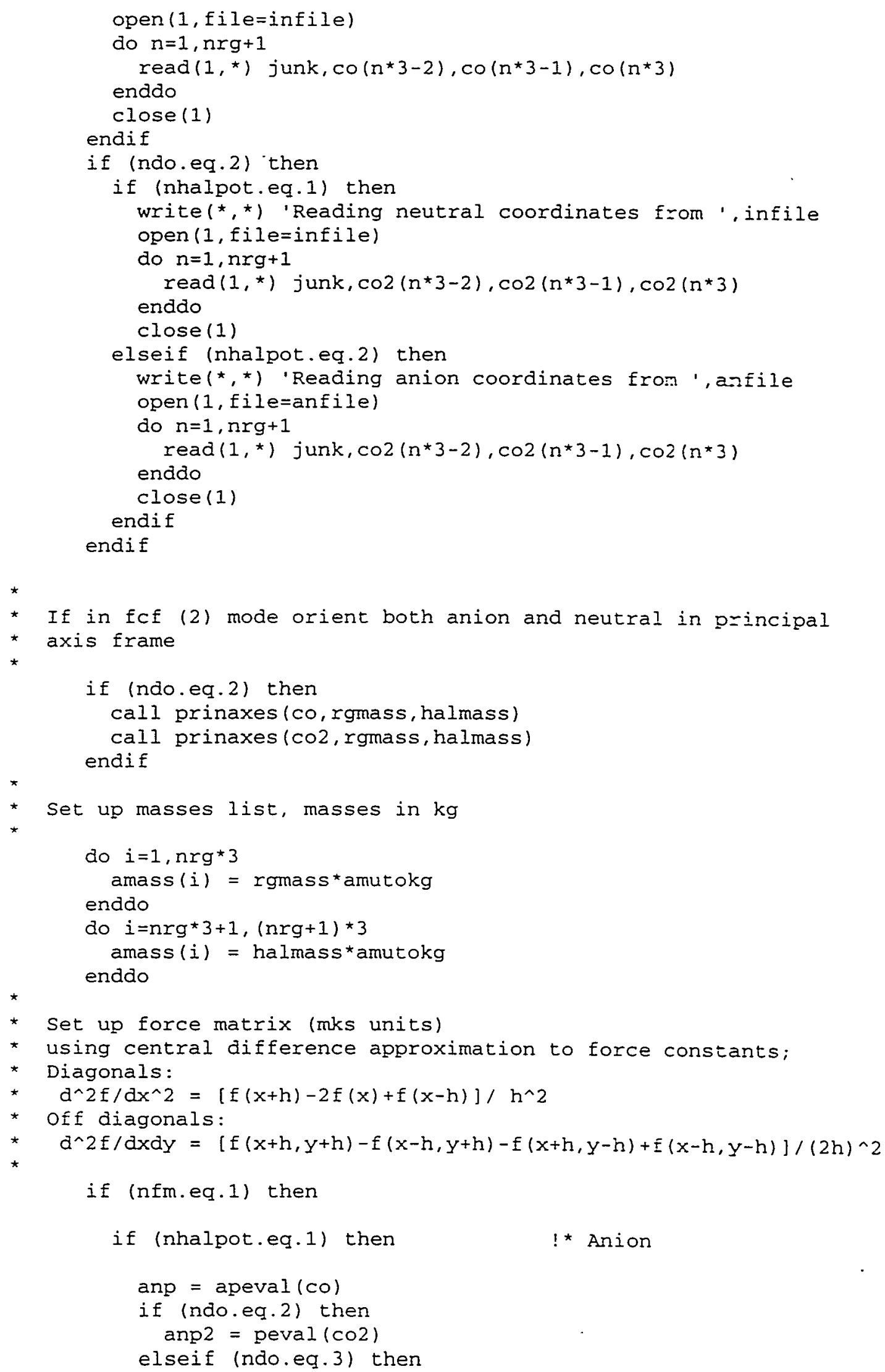




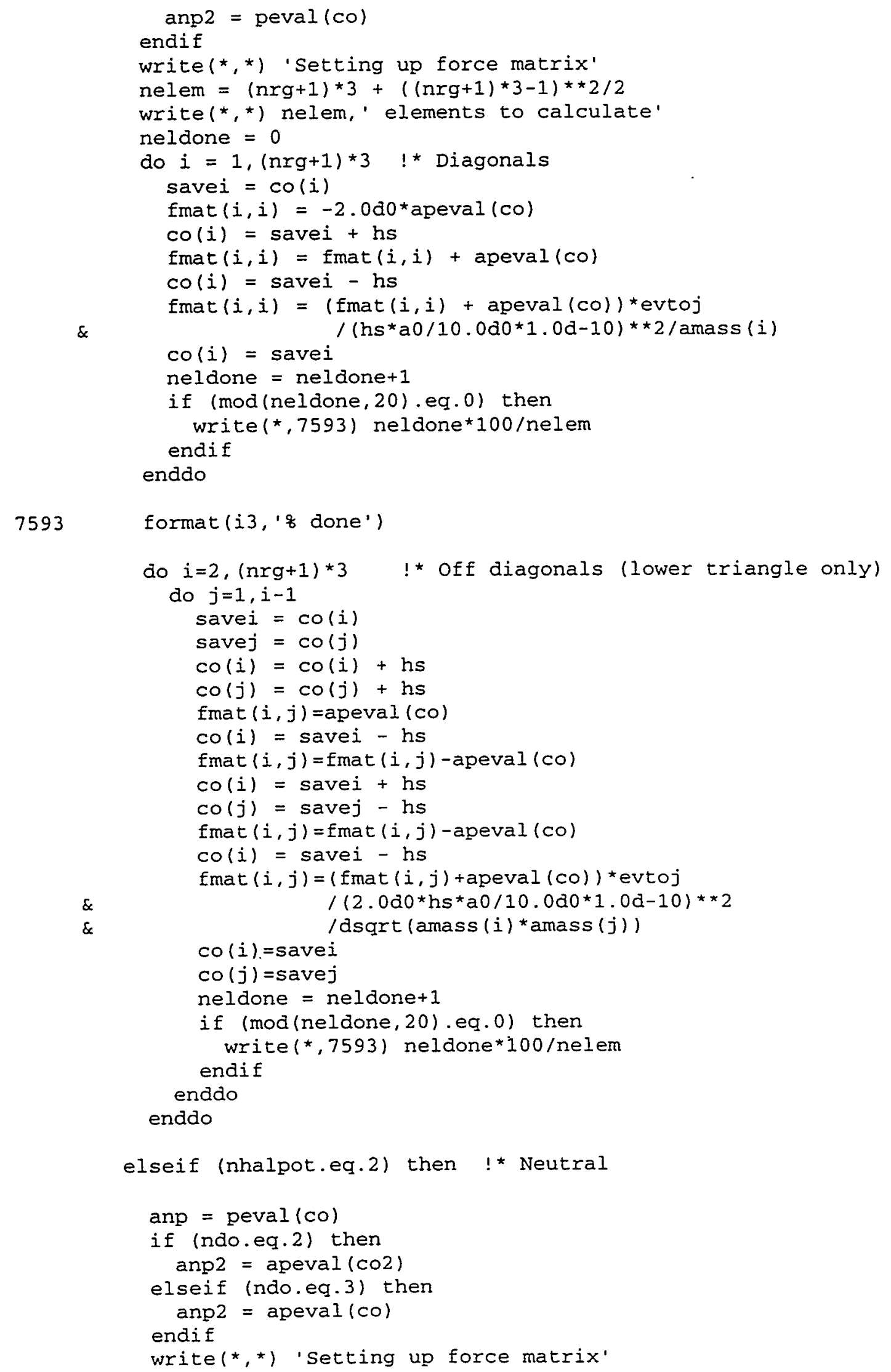




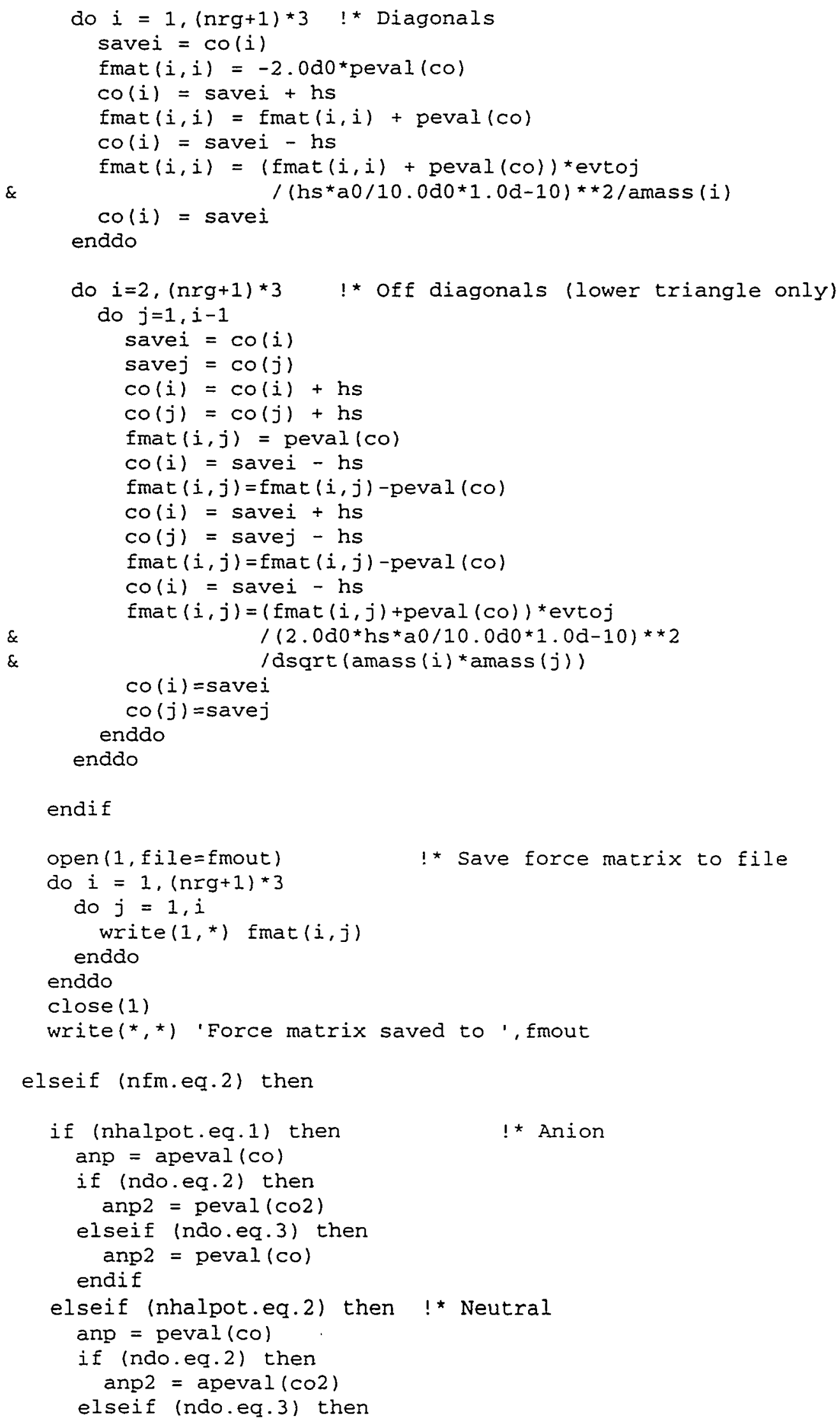




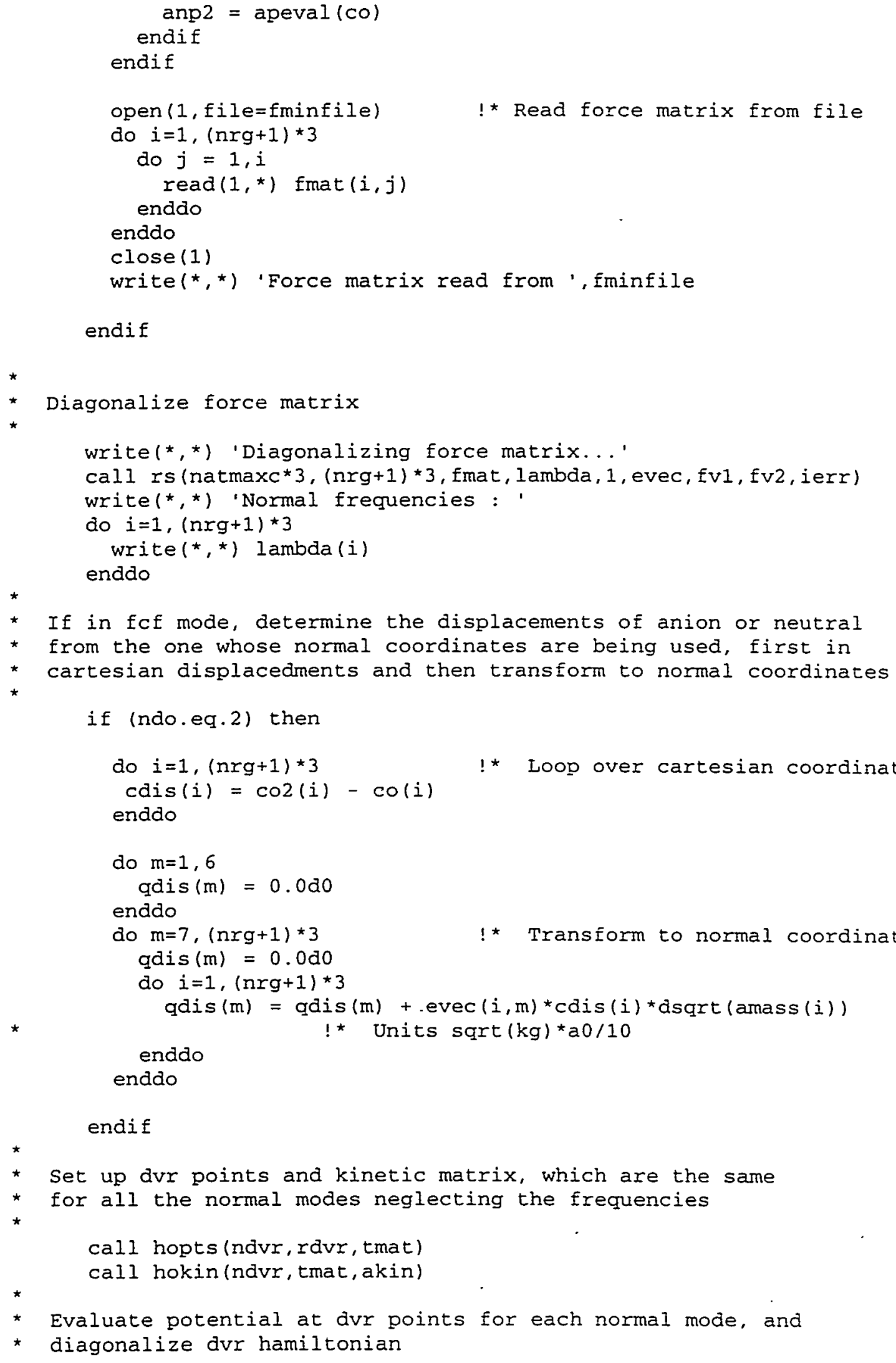




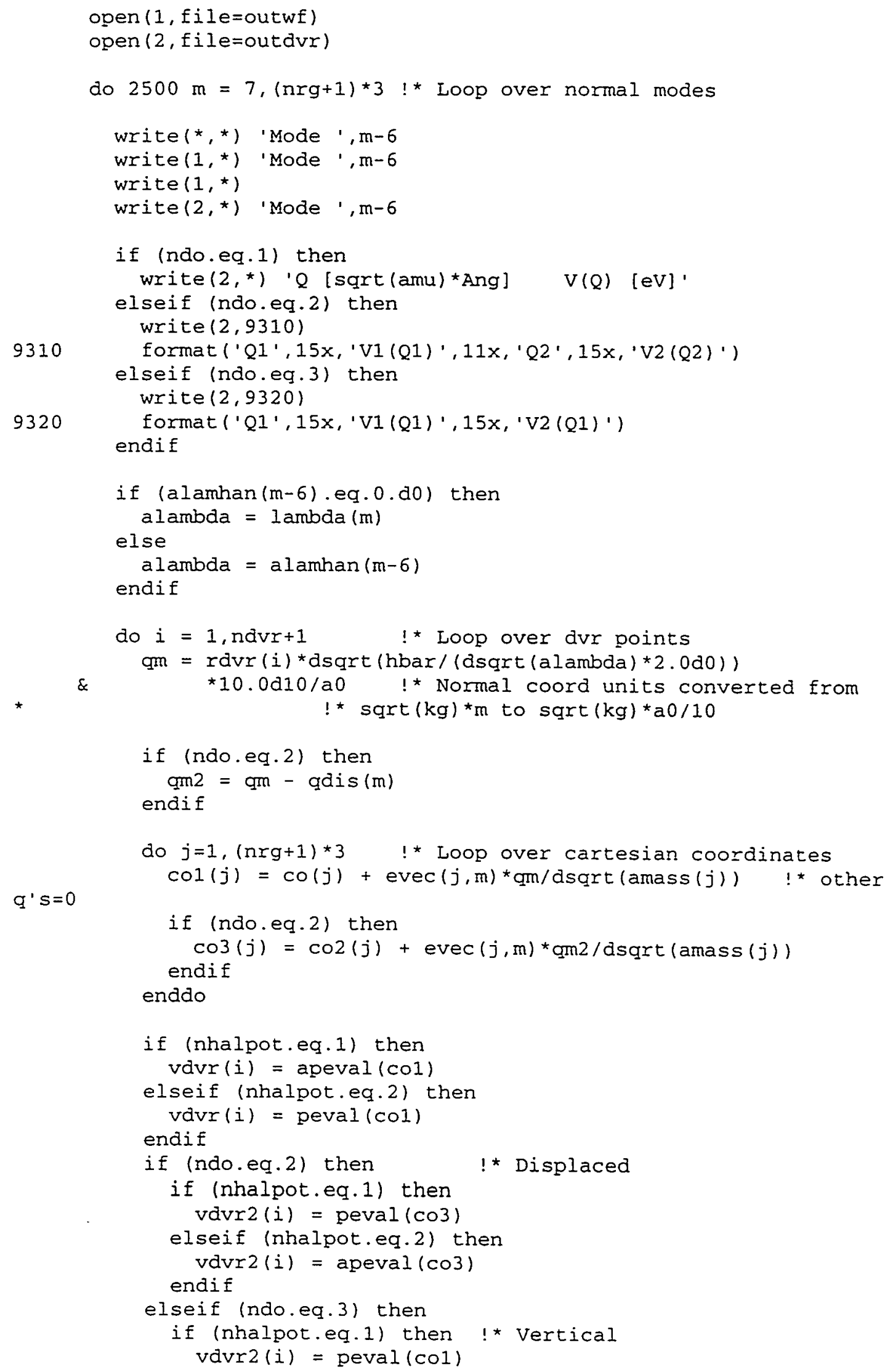




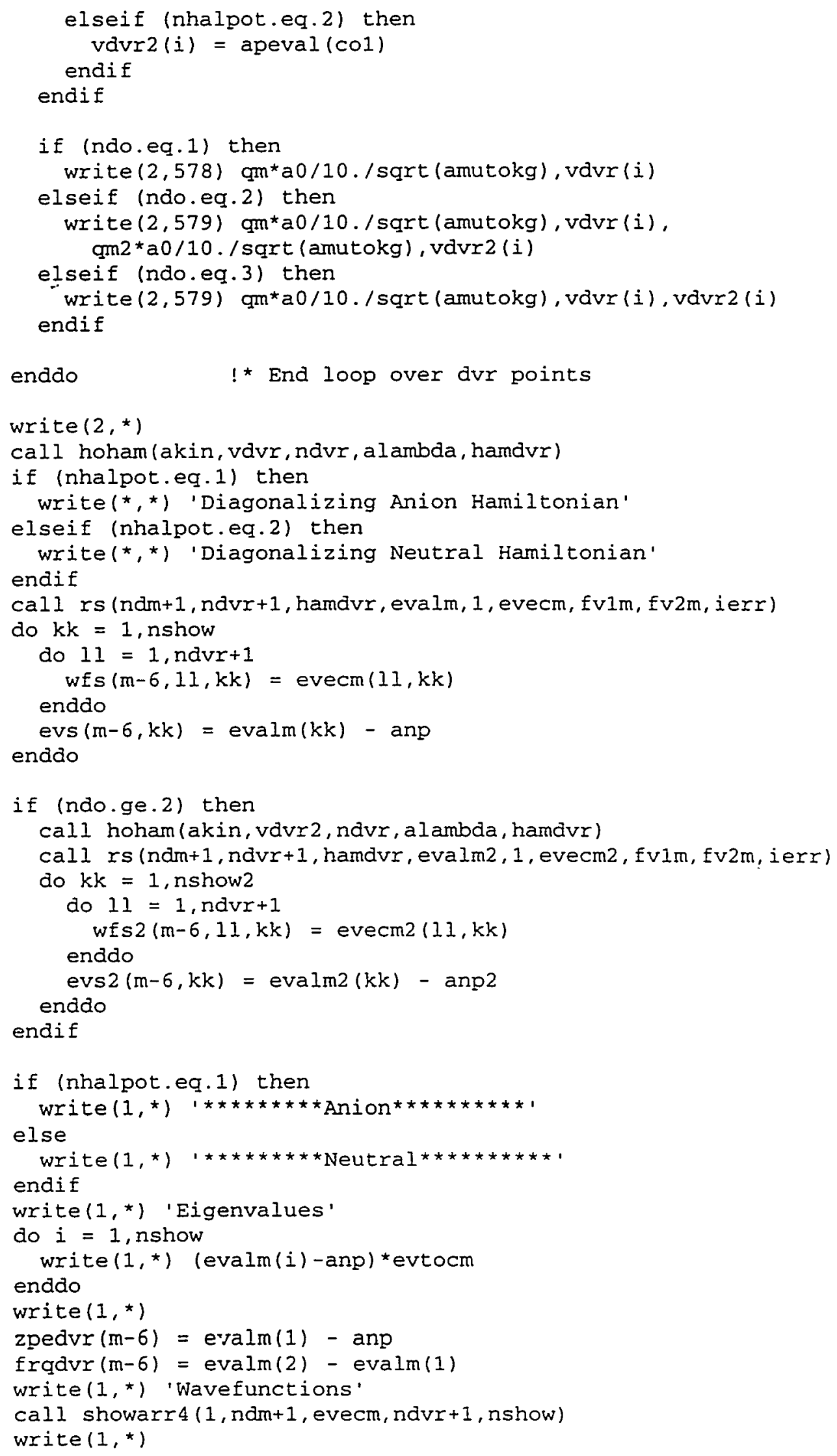




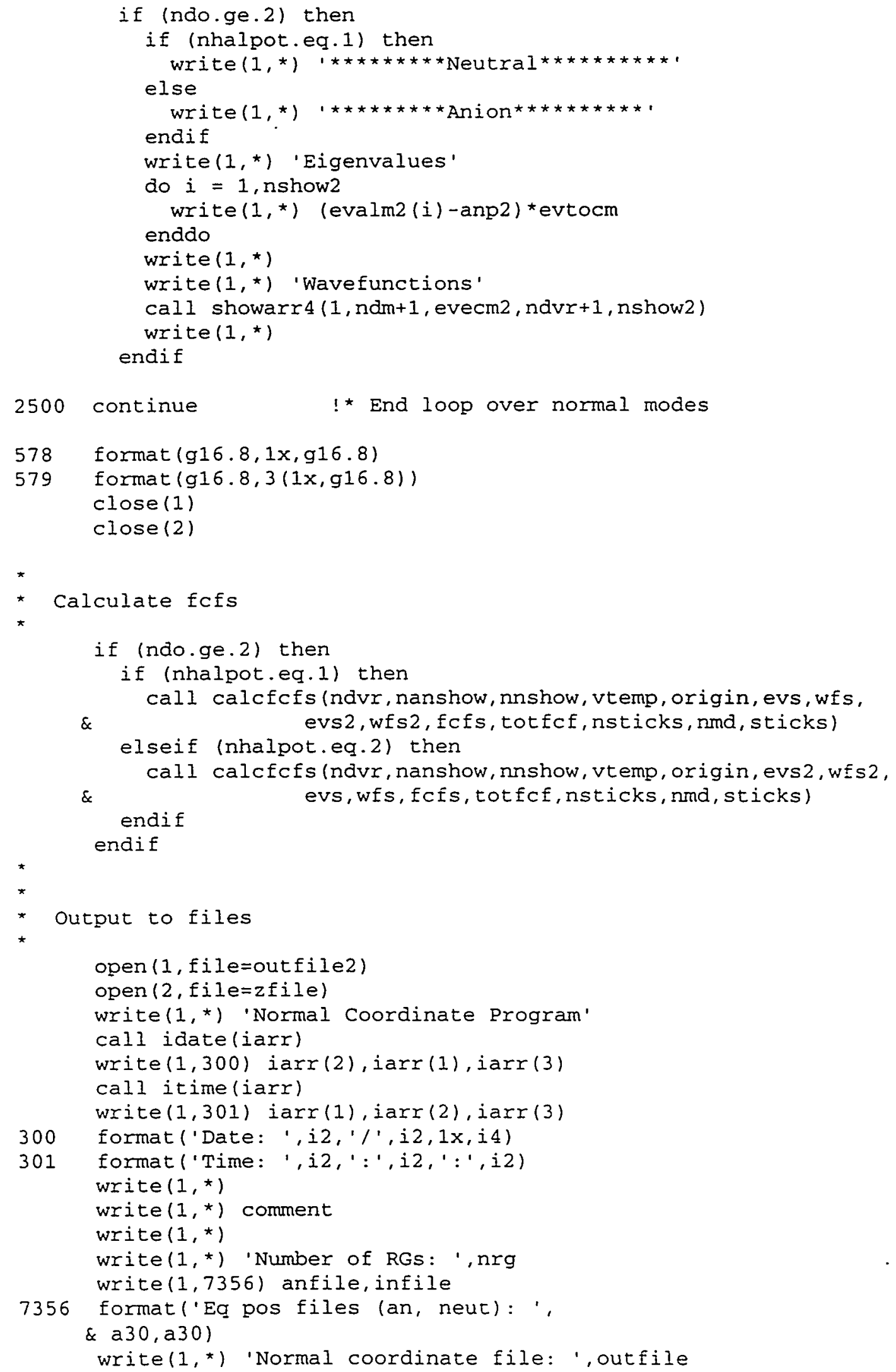




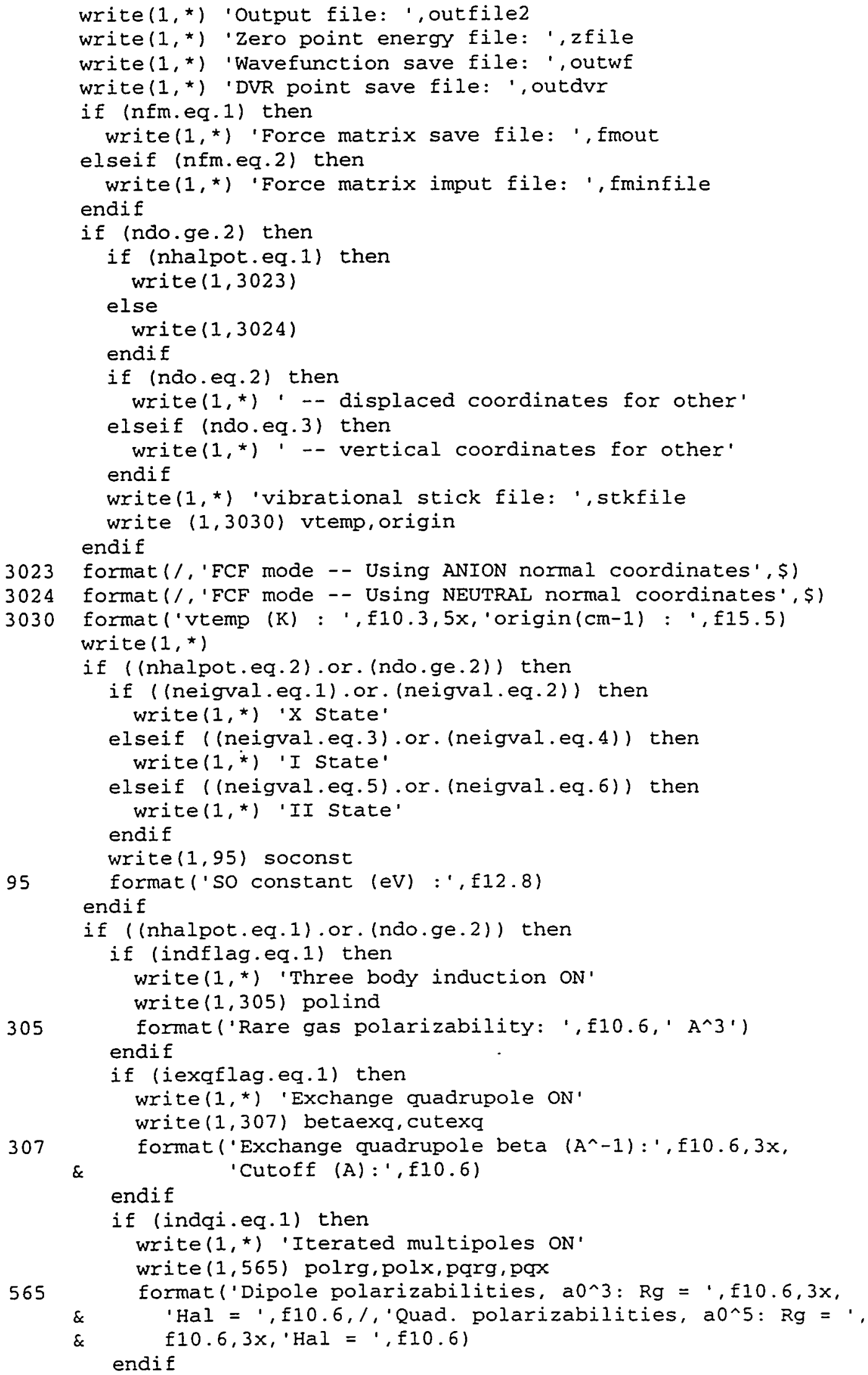




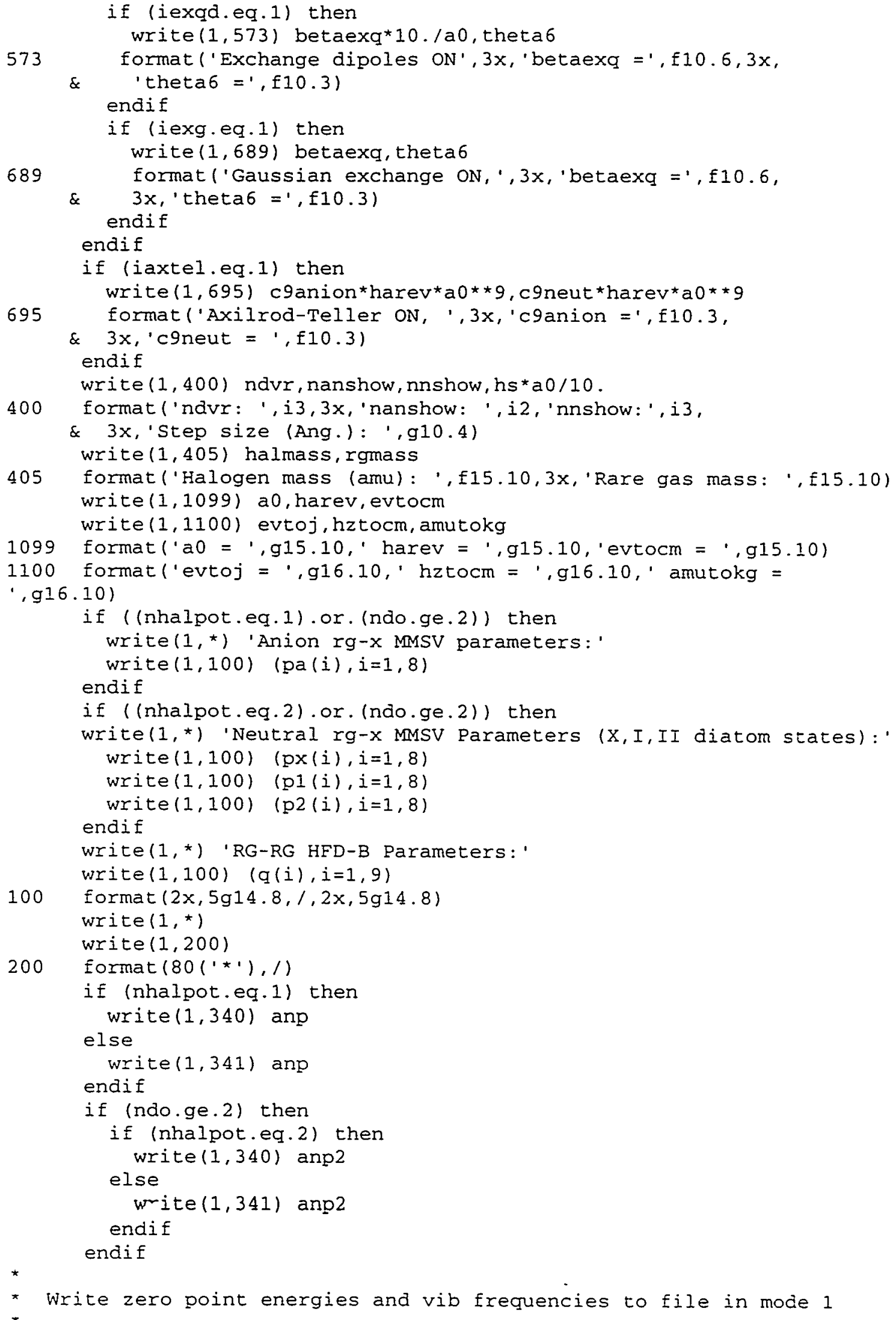




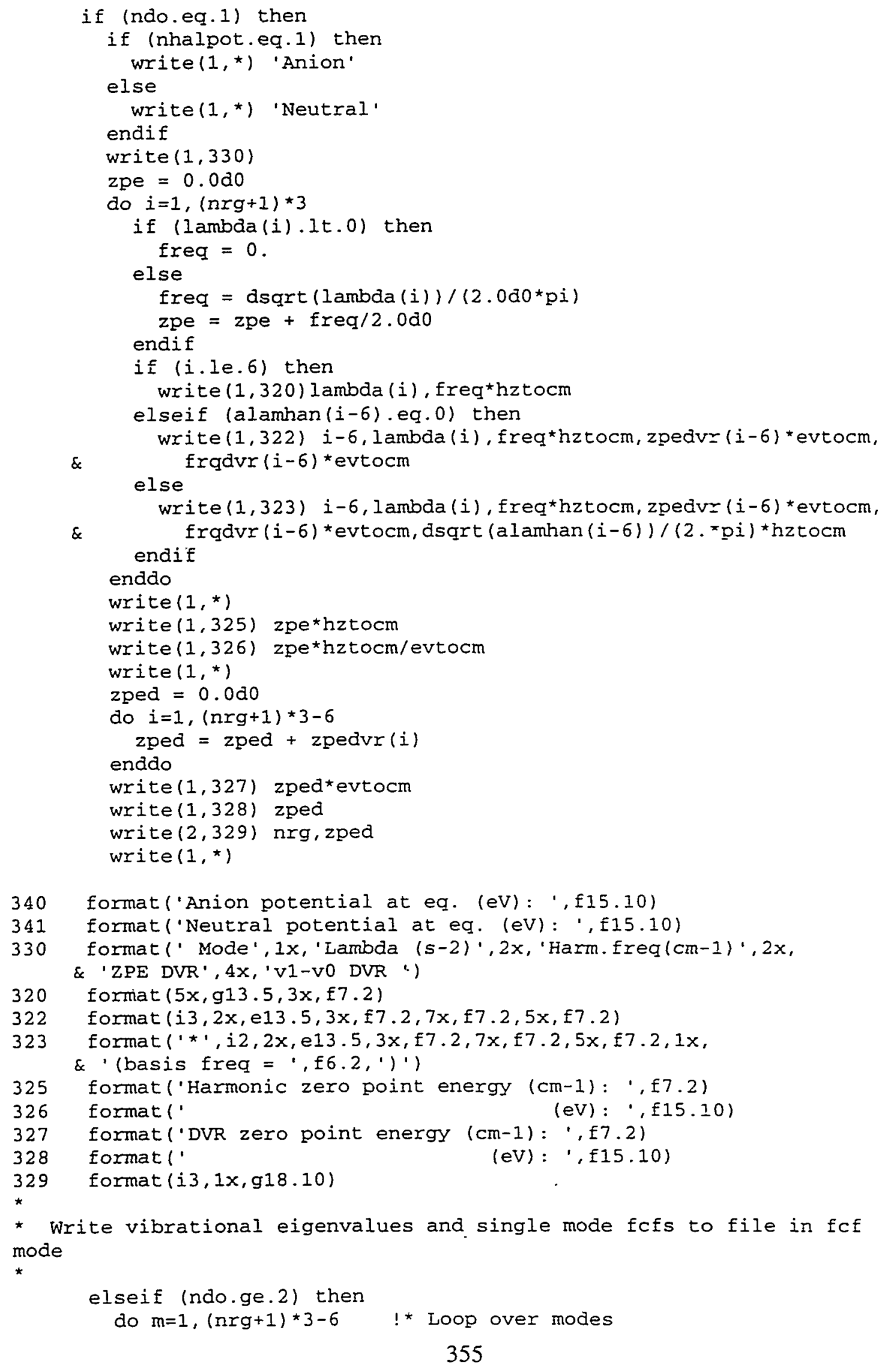




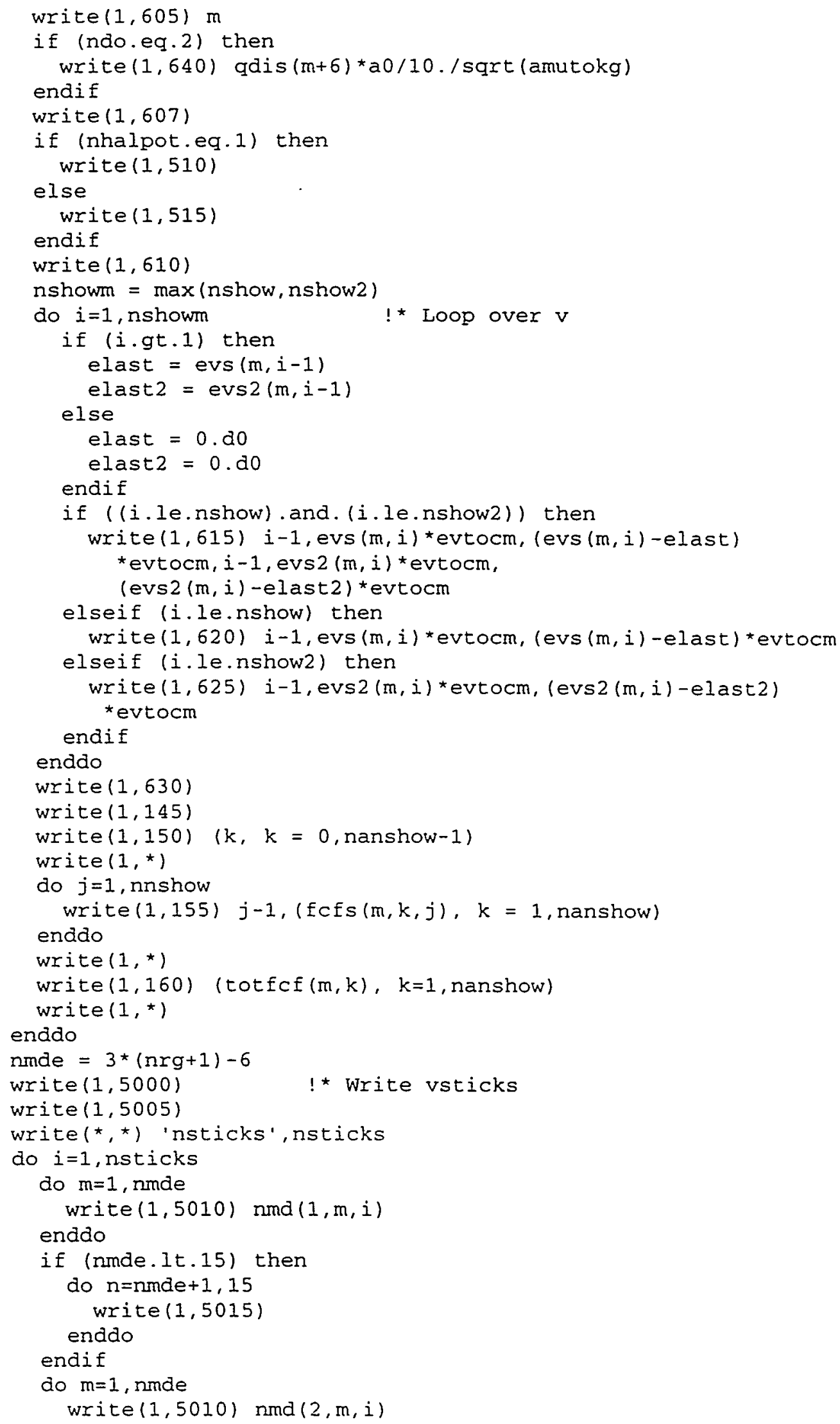




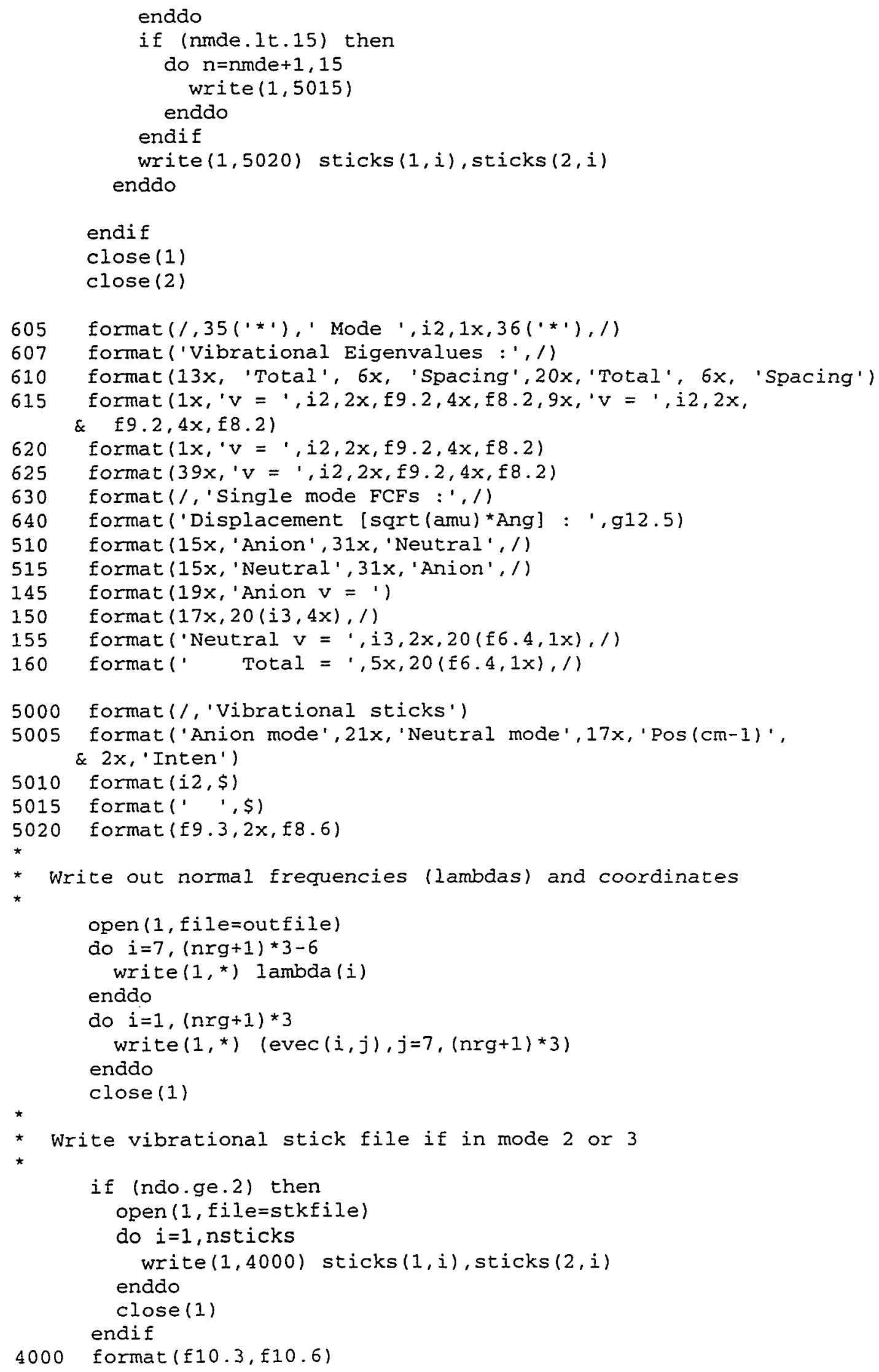


end

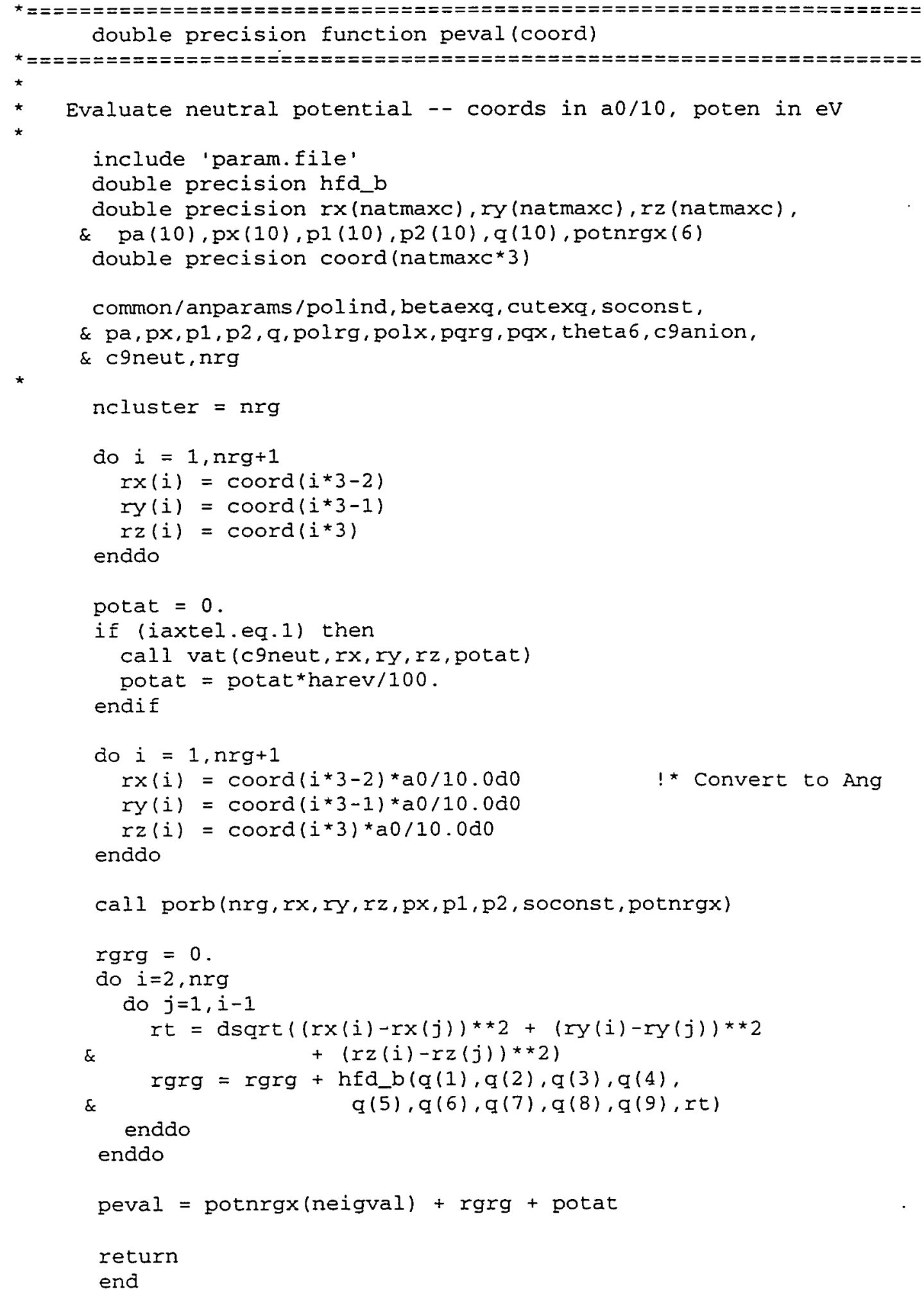




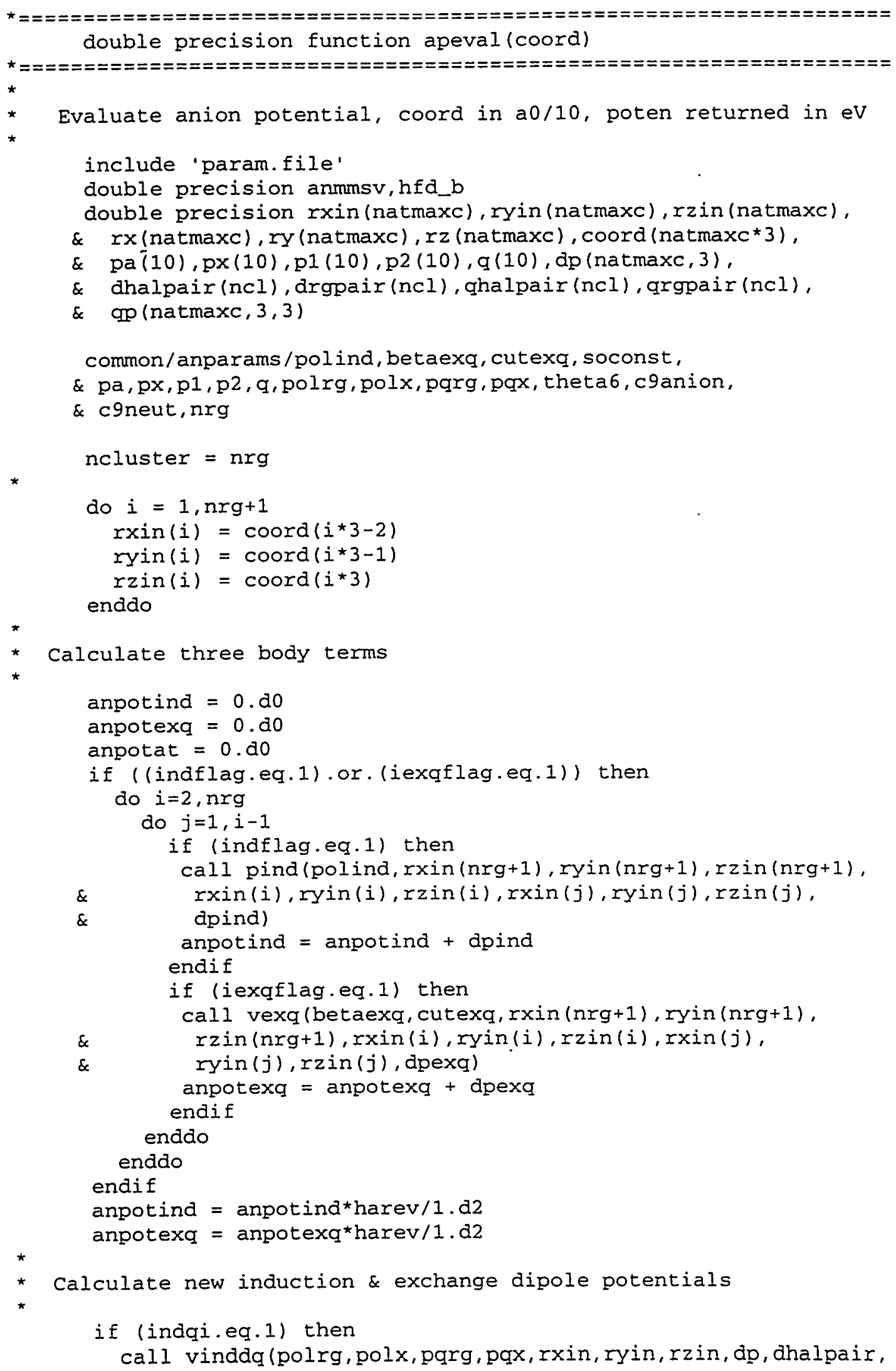


\& drgpair, ghalpair, qrgpair, qp, vcd, vcq, anpotind)

anpotind = anpotind*harev/1.d2

endif

if (iexqd.eq.1) then

call veqd (betaexq, theta6, rxin, ryin, rzin, anpotexq)

anpotexq $=$ anpotexq*harev/1.d2

elseif (iexg.eq.1) then

call exg (betaexq, theta6, rxin, ryin, rzin, anexx, anddisp,

\& angdisp, anpotexq)

anpotexg $=$ anpotexq ${ }^{\star}$ harev $/ 1 . d 2$

endif

if (iaxtel.eq.1) then

call vat (c9anion, rxin, ryin, rzin, anpotat)

anpotat $=$ anpotat*harev/1.d2

endif

* Convert from a0/10 to angstroms

do $\mathrm{n}=1$, $\mathrm{nrg}+1$

$r x(n)=r x i n(n) * 20 / 10$.

$r y(n)=r y i n(n) * a 0 / 10$.

$r z(n)=\operatorname{rzin}(n) * 20 / 10$.

enddo

* Calculate anion potential

xhal $=\operatorname{rx}(\mathrm{nrg}+1)$

yhal $=r y(n r g+1)$

zhal $=r z(n r g+1)$

anpotrgx $=0$.

do $i=1$, nrg

$r t=\operatorname{dsgrt}((\operatorname{xx}(i)-x h a l) * * 2+(\operatorname{ry}(i)-y h a l) * * 2$

$\&$

$+(r z(i)-z h a I) * * 2)$

anpotrgx $=$ anpotrgx $+\operatorname{anmmsv}(\mathrm{pa}(1), \mathrm{pa}(2), \mathrm{pa}(3), \mathrm{pa}(4)$, $\&$ pa (5), pa (6), pa(7), pa (8), rt)

enddo

anpotrgrg $=0$.

do $i=2, n r g$

do $j=1, i-1$

$r t=\operatorname{dsgrt}((\operatorname{rx}(i)-r x(j)) * \star 2+(r y(i)-r y(j)) * * 2$

$\& \quad+(r z(i)-r z(j)) * * 2)$

$\&$ anpotrgrg $=$ anpotrgrg $+h f d \_b(q(1), q(2), q(3), q(4)$, endao $q(5), q(6), q(7), q(8), q(9), r t)$

enddo

apeval $=$ anpotrgx+anpotrgrg+anpotind+anpotexq+anpotat

return

end

subroutine pind (pol, $x$

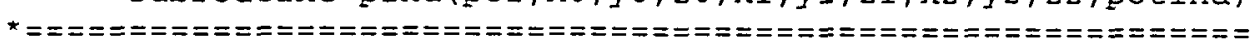


* Charge-Ind Dipole-Ind Dipole Three body potential

* Input: pol Rare gas polarizibility (A^3)

* $\quad x 0, y 0, z 0$ Halide cartesian coordinates $(a 0 / 10)$

*

Rare gas coordinates $(20 / 10)$

Output: potind Potential (hartree/100)

implicit double precision $(a-h, 0-z)$

parameter ( $\mathrm{p} i=3.14159265359 \mathrm{do}, \mathrm{a} 0=0.529177249 \mathrm{do}$,

\& harev $=27.2113961 \mathrm{do}$, evtocm $=8065.5410 \mathrm{~d} 0$ )

const $=(p \circ 1 /(a 0 * \star 3) \star 1 . d 3) * \star 2$

r10sqr $=(x 1-x 0) * \star 2+(y 1-y 0) * \star 2+(z 1-z 0) \star \star 2$

r20sqr $=(x 2-x 0) * \star 2+(y 2-y 0) * * 2+(z 2-z 0) * \star 2$

$r 12$ sqr $=(x 1-x 2) * \star 2+(y 1-y 2) * \star 2+(z 1-z 2) \star \star 2$

$\operatorname{dot} 1020=(x 1-x 0) *(x 2-x 0)+(y 1-y 0) *(y 2-y 0)+(z 1-z 0) *(z 2-z 0)$

$\operatorname{dot} 1012=(x 1-x 0) *(x 1-x 2)+(y 1-y 0) *(y 1-y 2)+(z 1-z 0) *(z 1-z 2)$

$\operatorname{dot} 1220=(x 1-x 2) *(x 2-x 0)+(y 1-y 2) *(y 2-y 0)+(z 1-z 2) *(z 2-z 0)$

$t I=\operatorname{dsgrt}(r 10 \mathrm{sgr})$

$t 2=r 10$ sqr $r \star 2$

$t 6=\operatorname{dsqrt}(r 12 \mathrm{sqr})$

$t 7=r 12 \mathrm{sqr} *{ }^{*} 2$

$t 10=\operatorname{dsqrt}(r 20 \mathrm{sqr})$

$t 11=r 20 s q r * 2$

potind $=$ const*t $1 / t 2 * t 6 / t 7 * t 10 / t 11 *(\operatorname{dot} 1020-$

\& $3 * \operatorname{dot} 1012 * \operatorname{dot} 1220 /$ r12sqr)

potind $=$ potind $* 1 \cdot d 3$

return

end

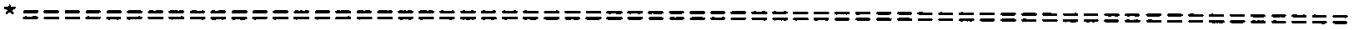
subroutine vexq (betain, cutoff, $x 0 i n, y 0 i n, z 0 i n$,

\& $\quad x 1$ in, $y 1$ in, zlin, $x 2$ in, $y 2$ in, z2in, pexq)

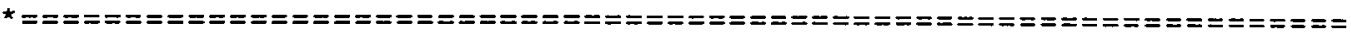

* Charge-exchange quadrupole interaction.

* Gaussian 1-electron model

* Input: betain Gaussian range parameter $\left(\right.$ Ang $\left.^{\wedge}-1\right)$

* cutoff RG-RG distance cutoff (Ang)

* $\quad$ xoin,yoin,z0 Halide cartesian coordinates $(a 0 / 10)$

* $x 1, y 1, z 1$ Rare gas 1 cart. coords. $(a 0 / 10)$

* $\mathrm{x} 2, \mathrm{y} 2, \mathrm{z} 2$ Rare gas 2 cart. coords. $(\mathrm{a} 0 / 10)$

* Returns: pexq potential in hartree/100

implicit double precision $(a-h, 0-z)$

parameter ( $\mathrm{p} i=3.14159265359 \mathrm{do}, \mathrm{a} 0=0.529177249 \mathrm{do}$,

\& harev $=27.2113961$ d0, evtocm $=8065.5410$ do)
} 


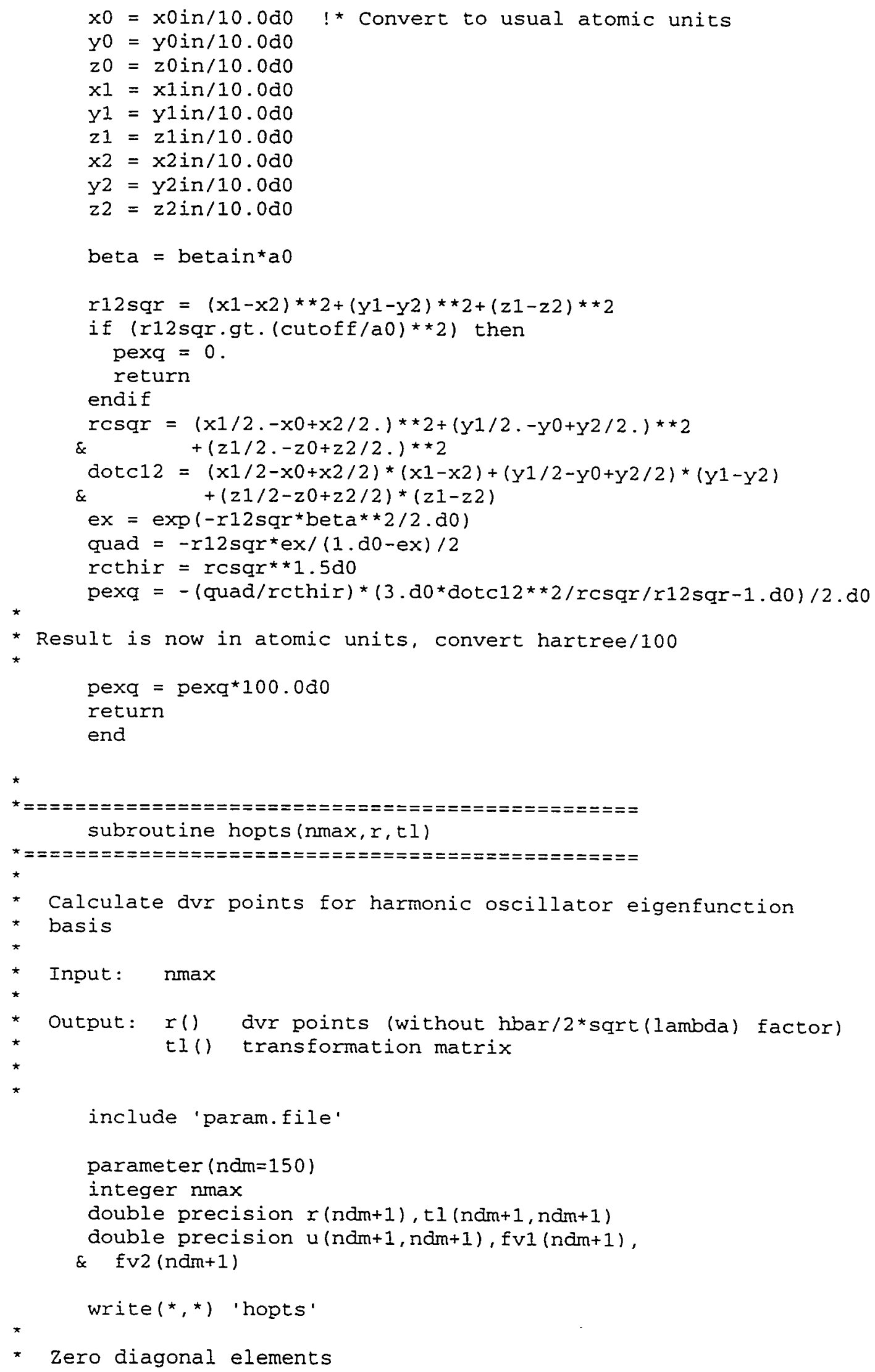




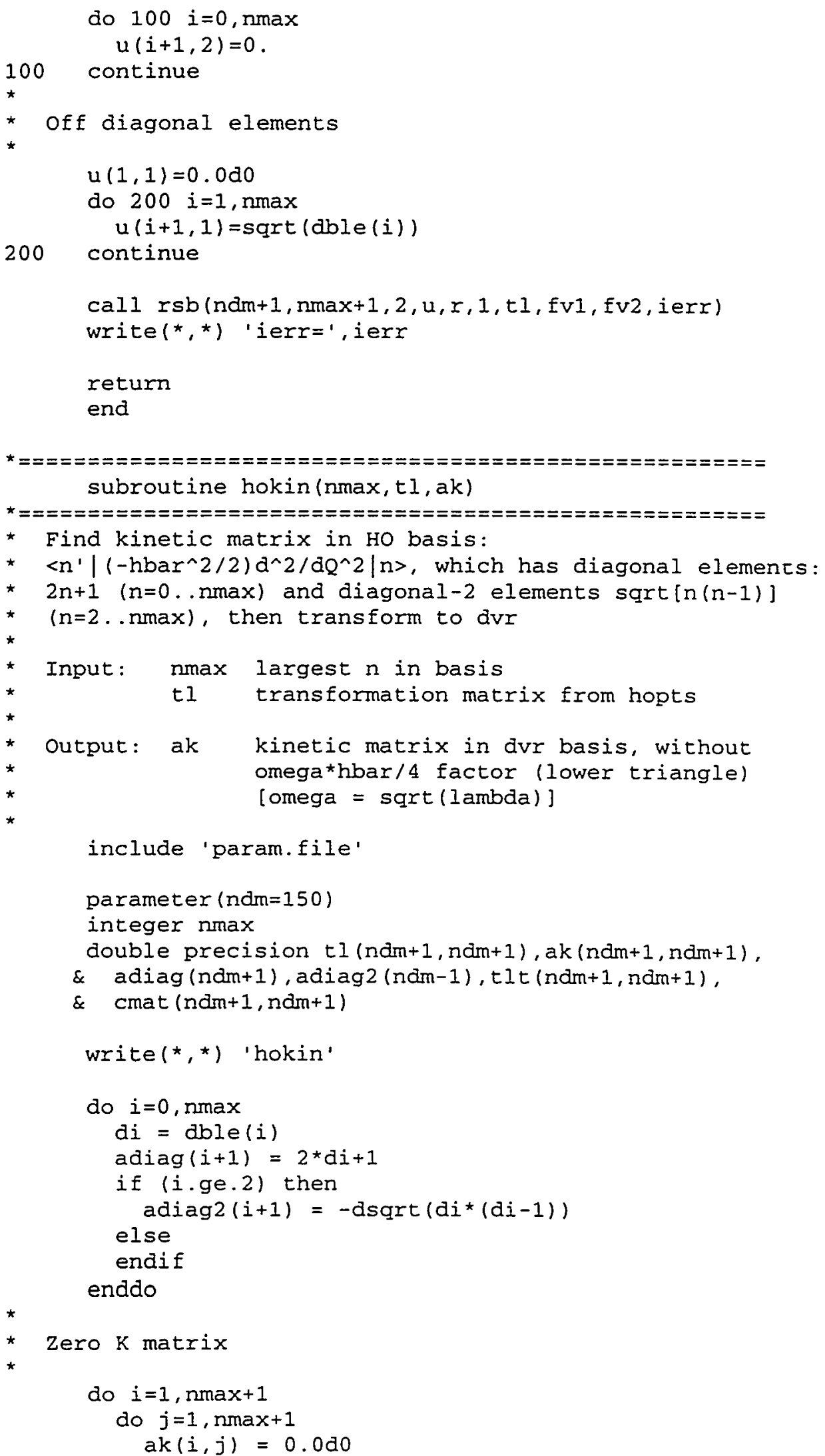




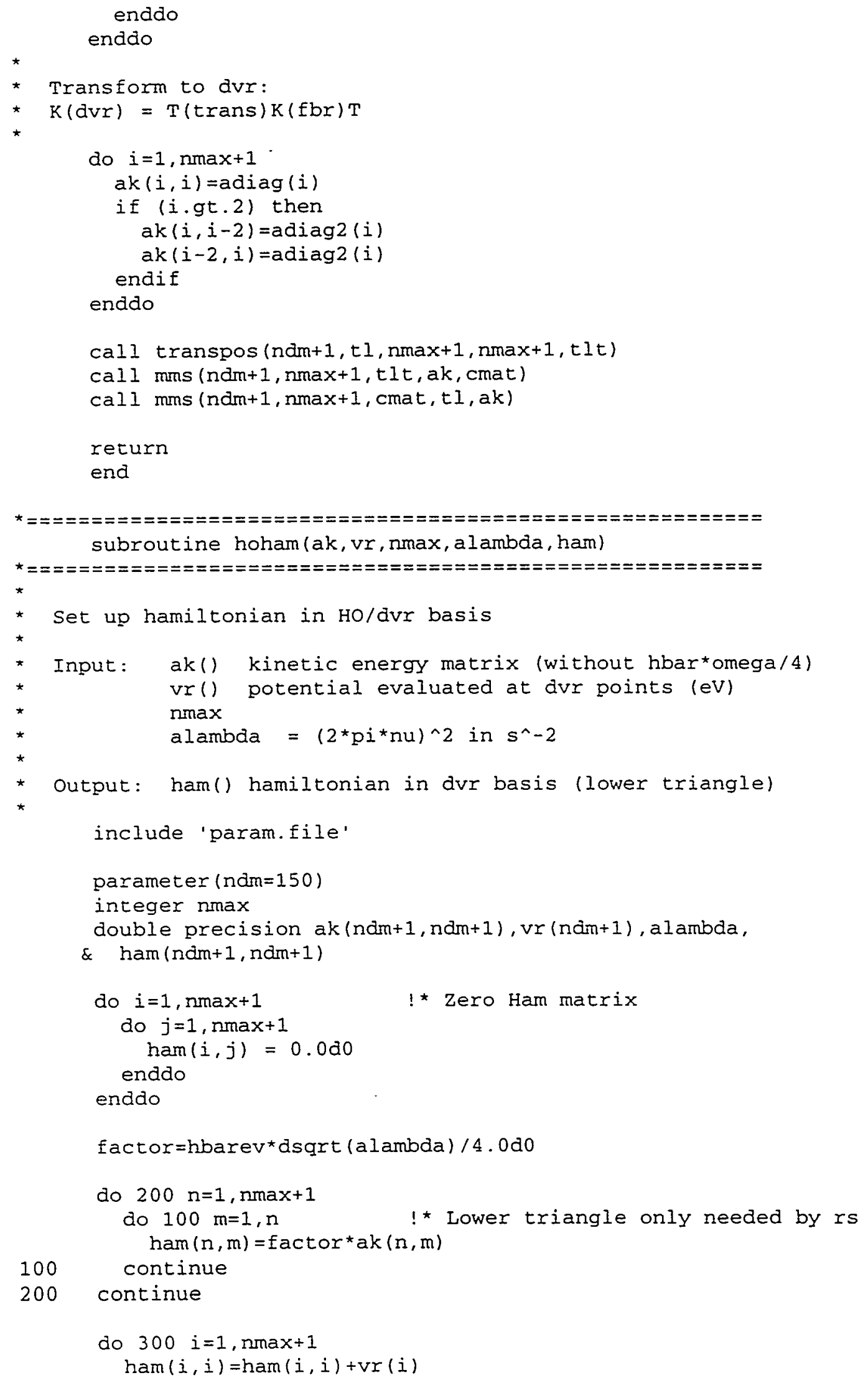




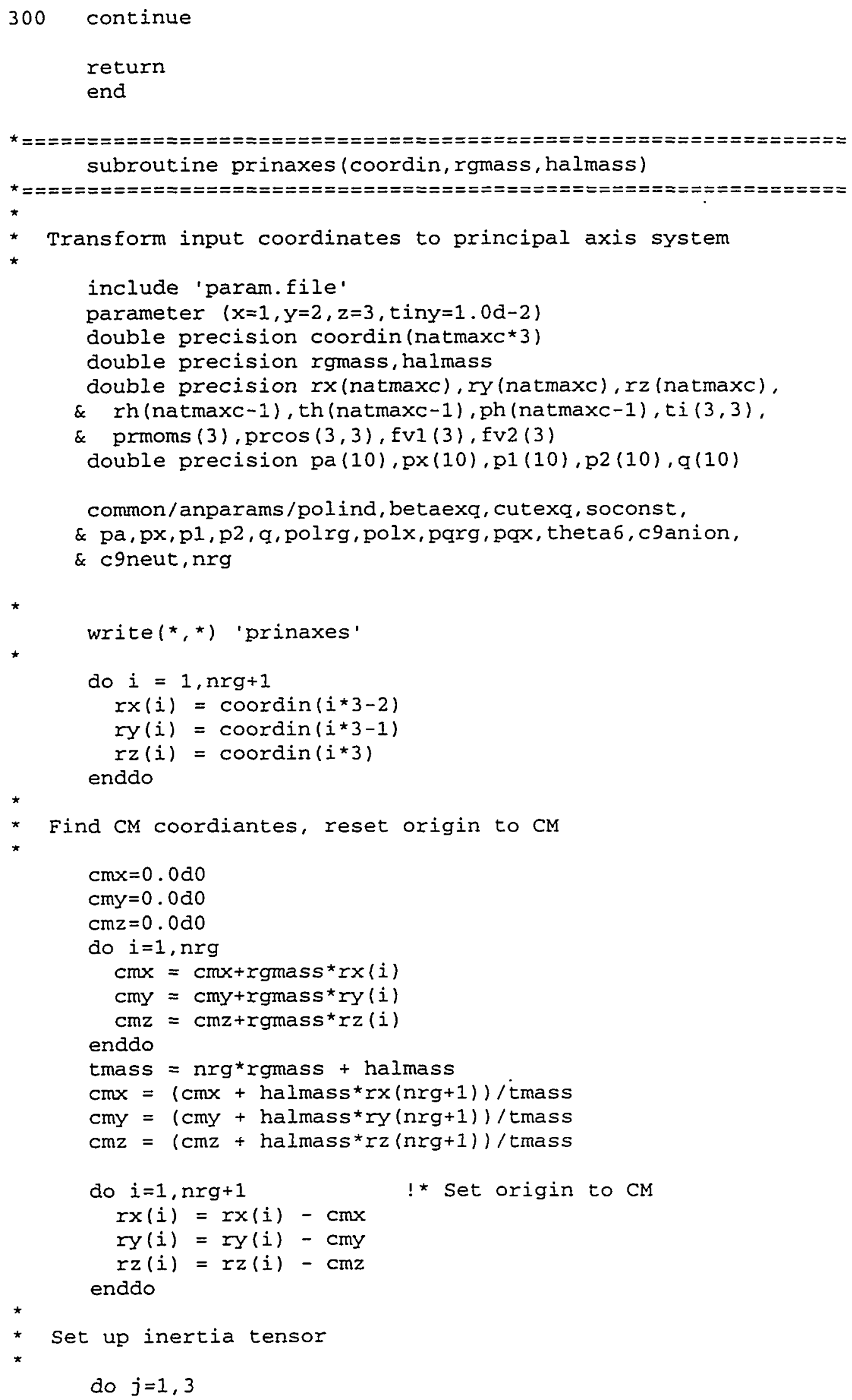




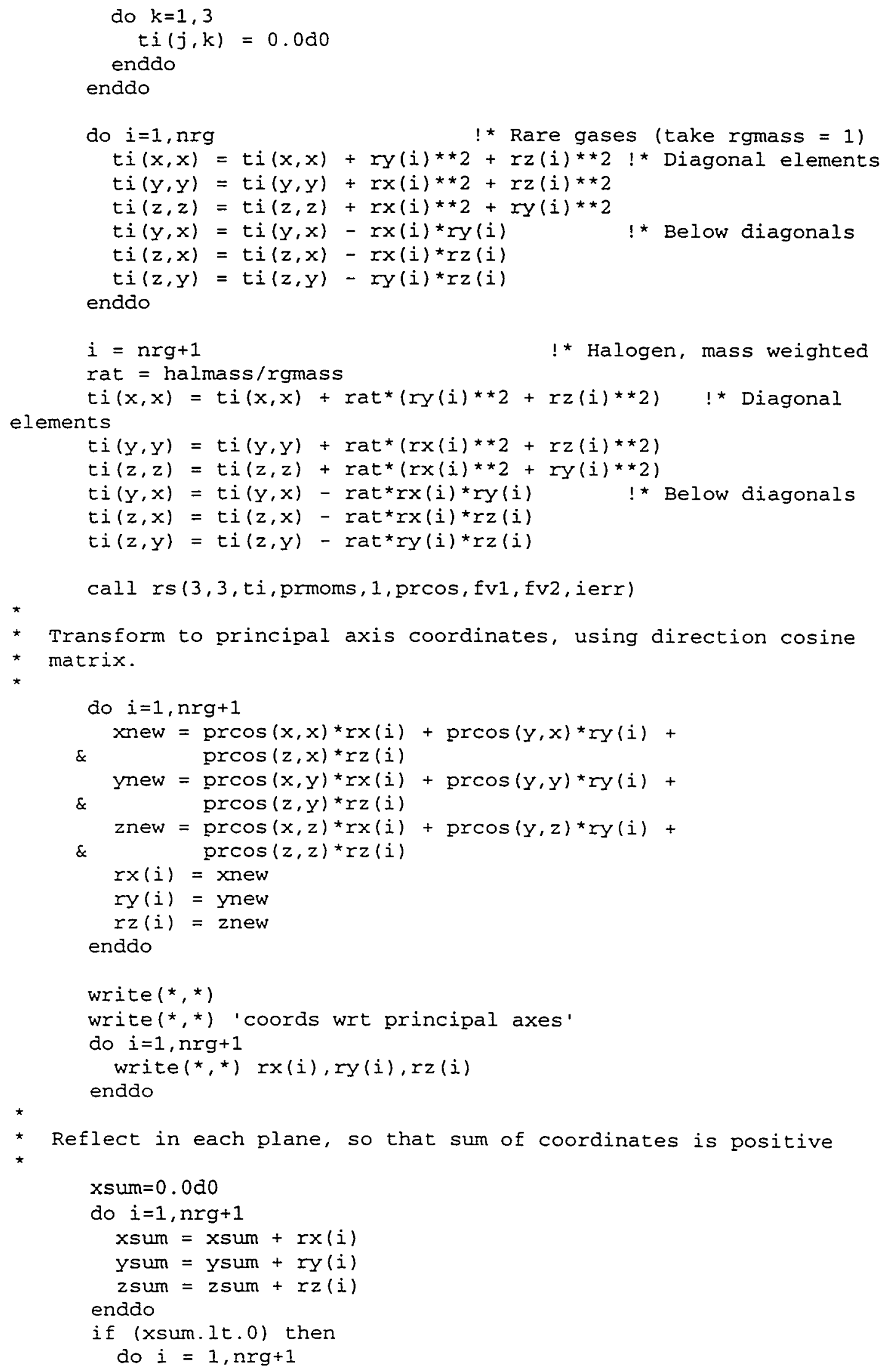




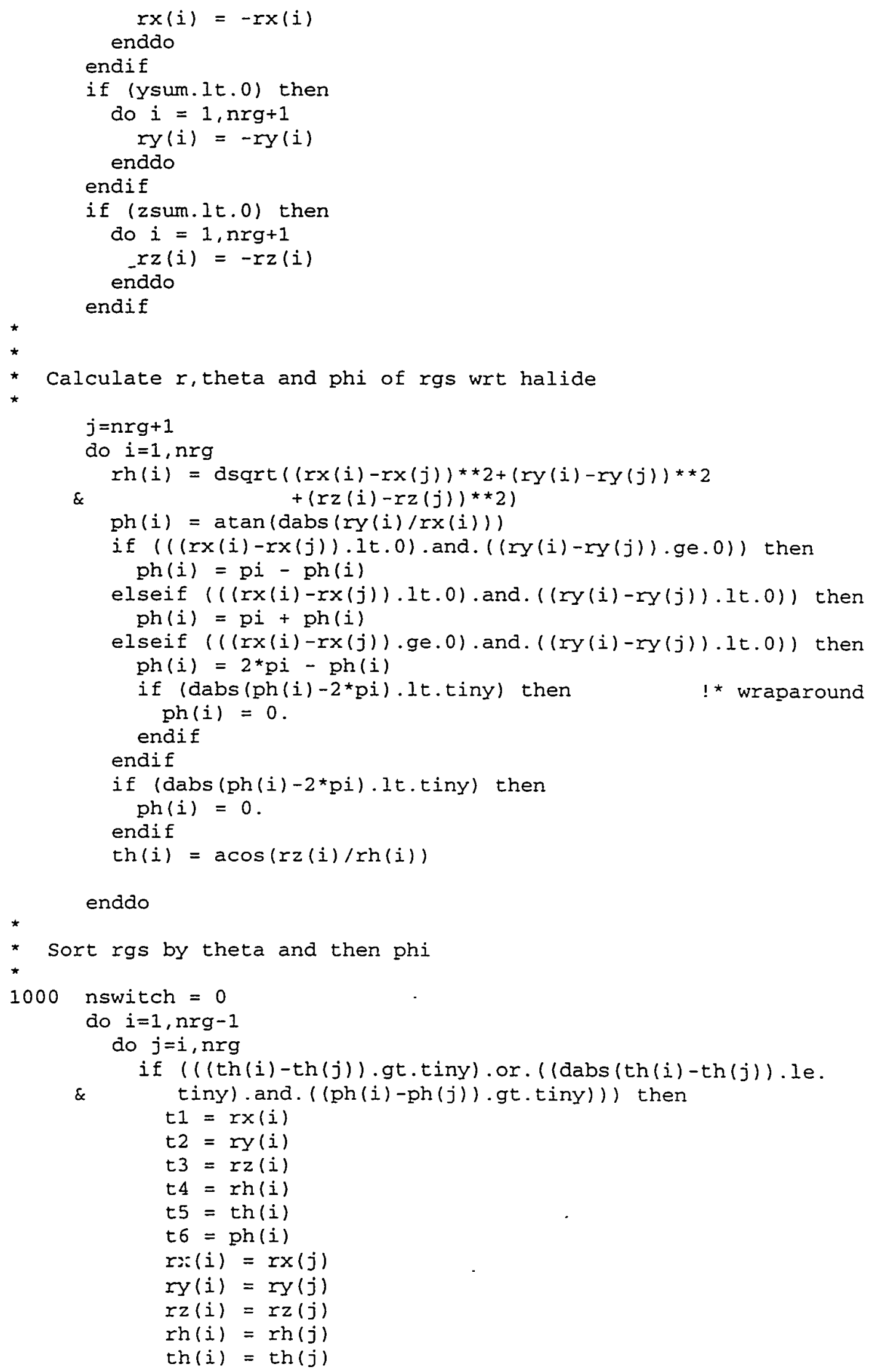




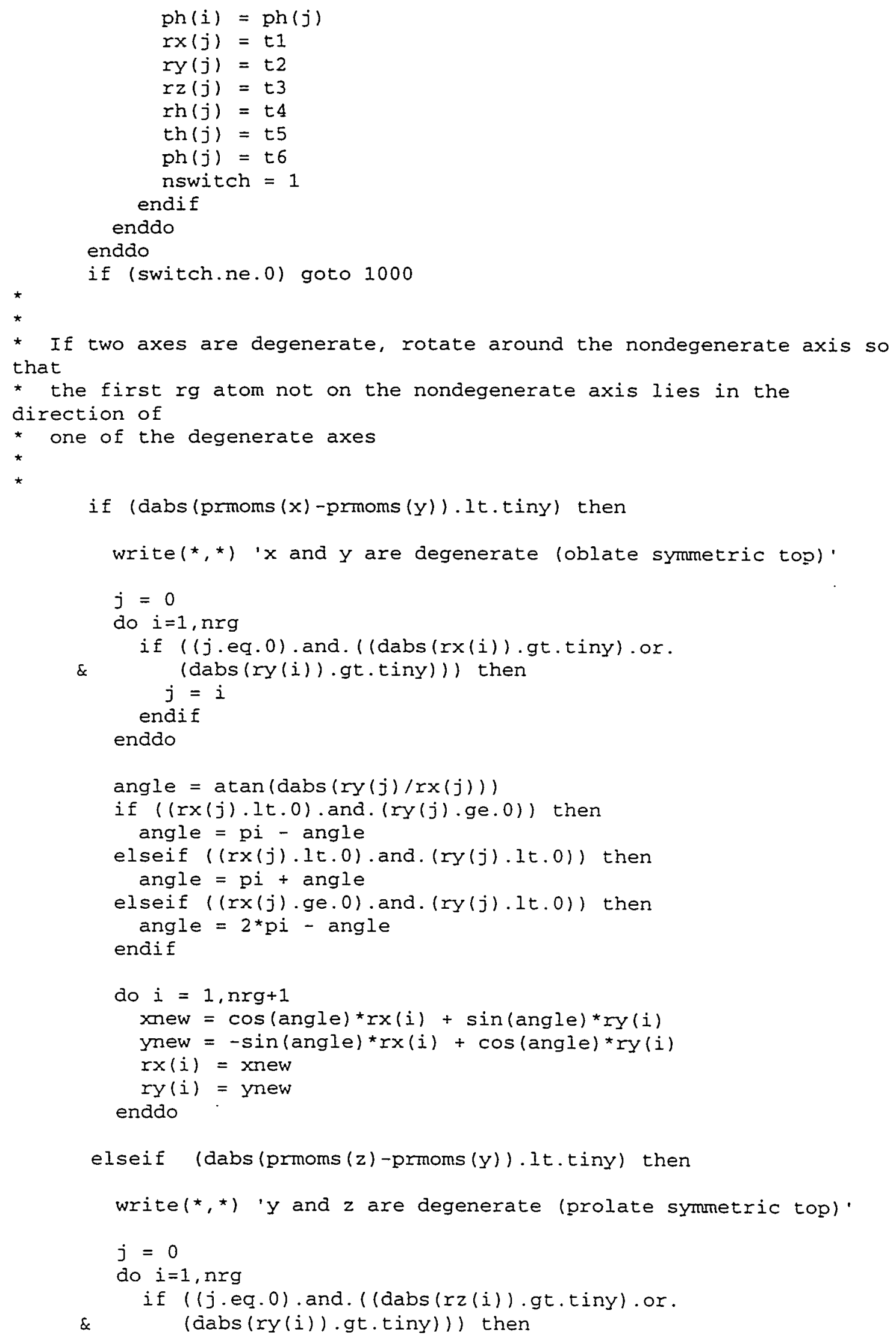




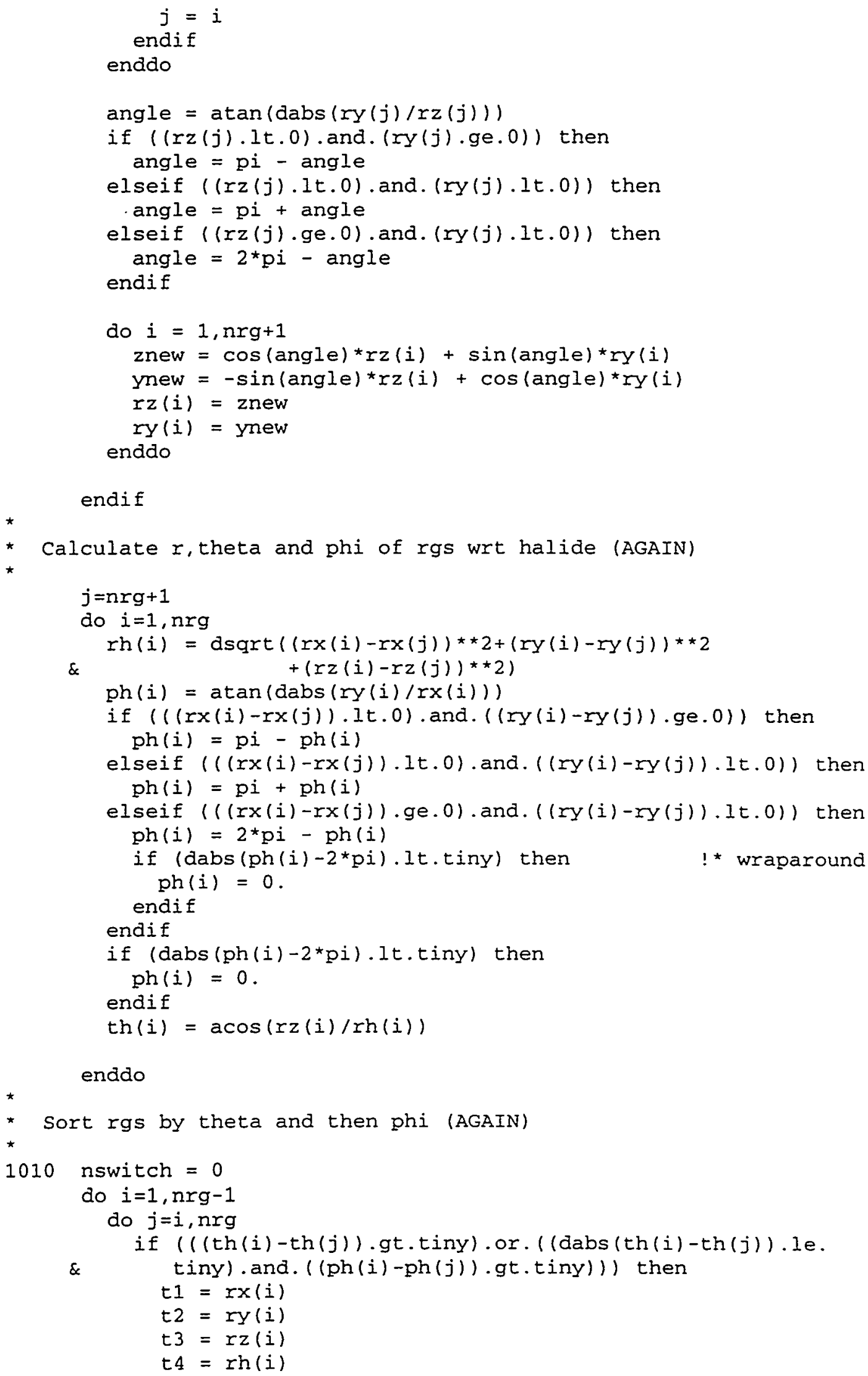




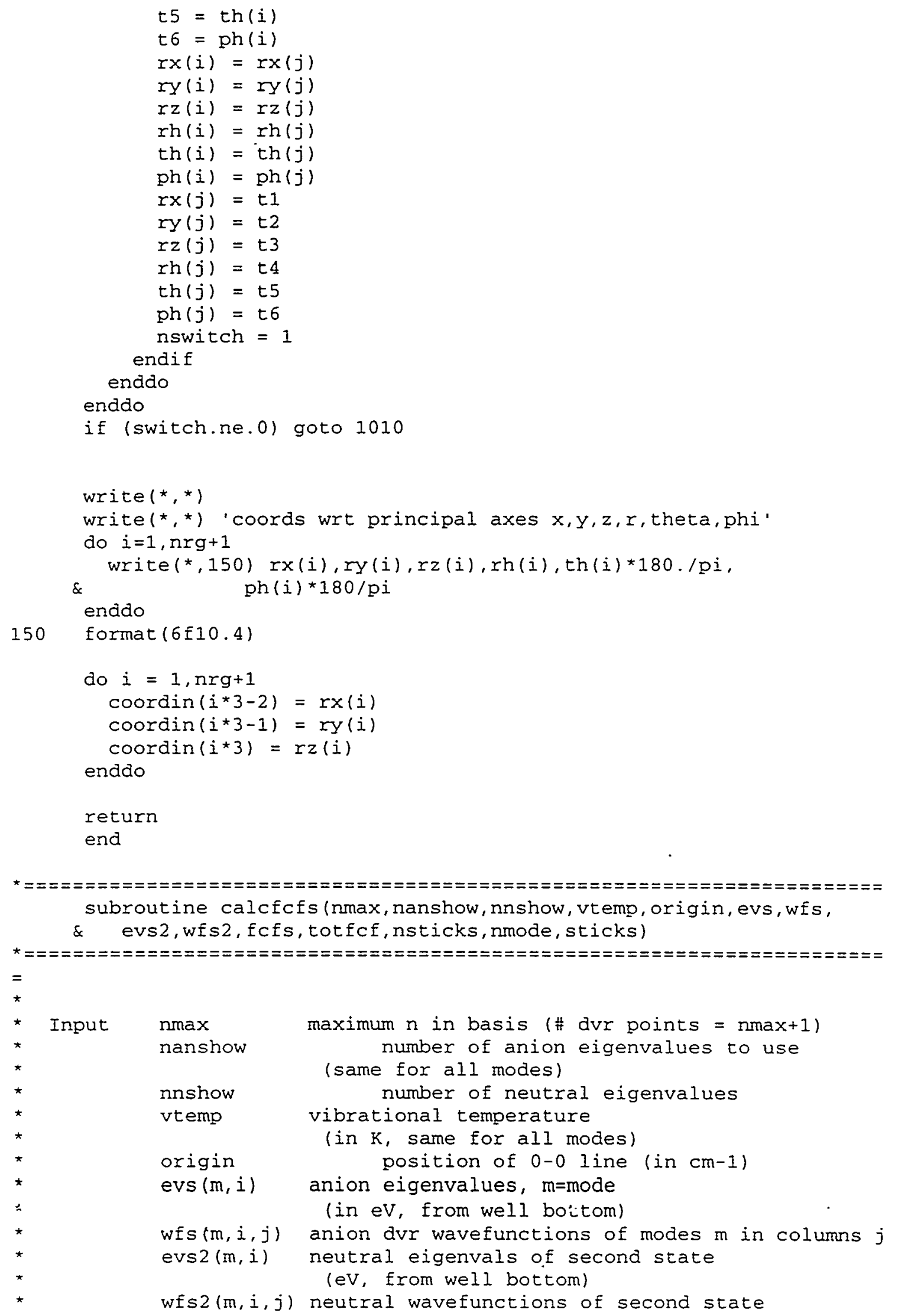




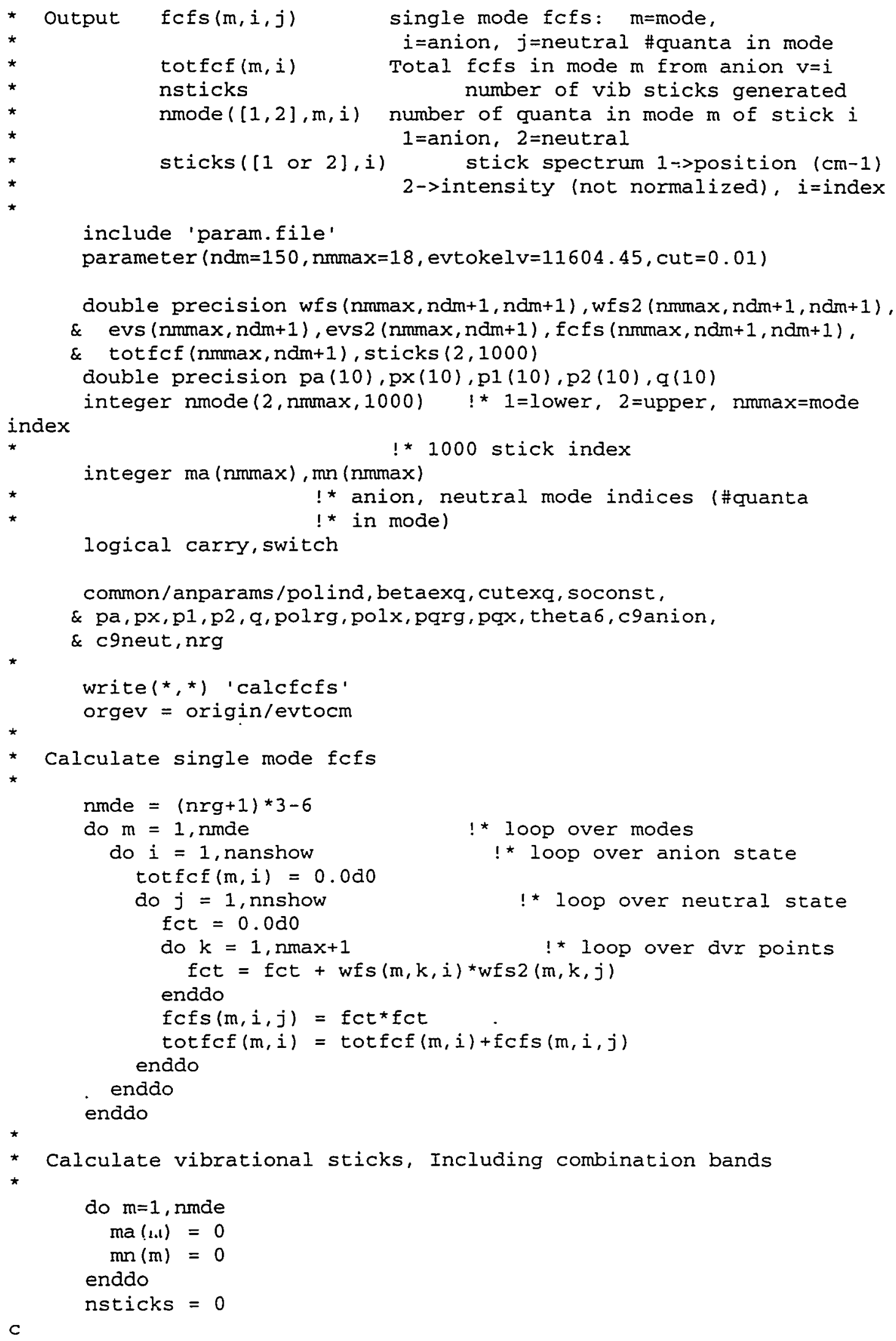

C 


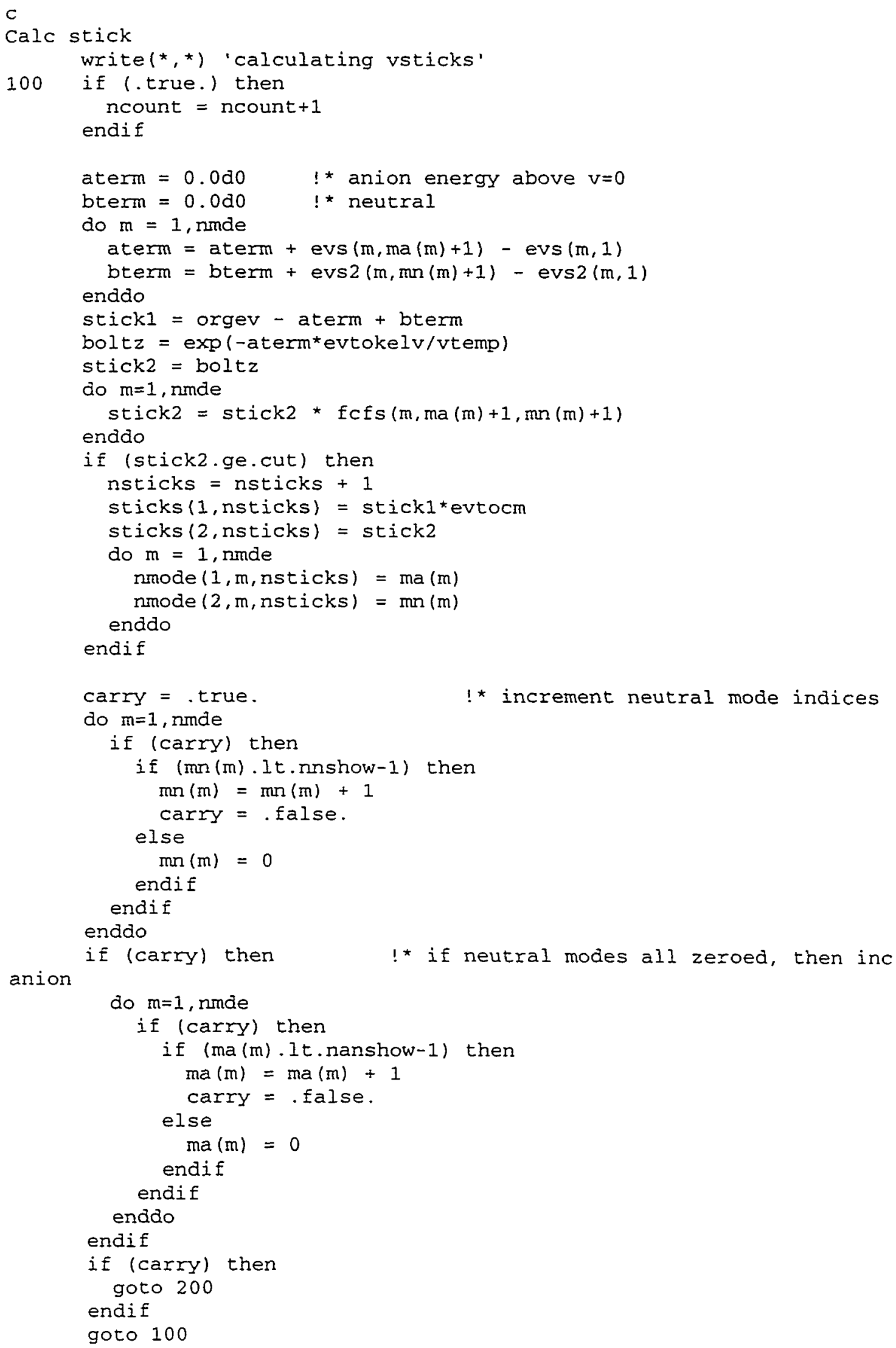




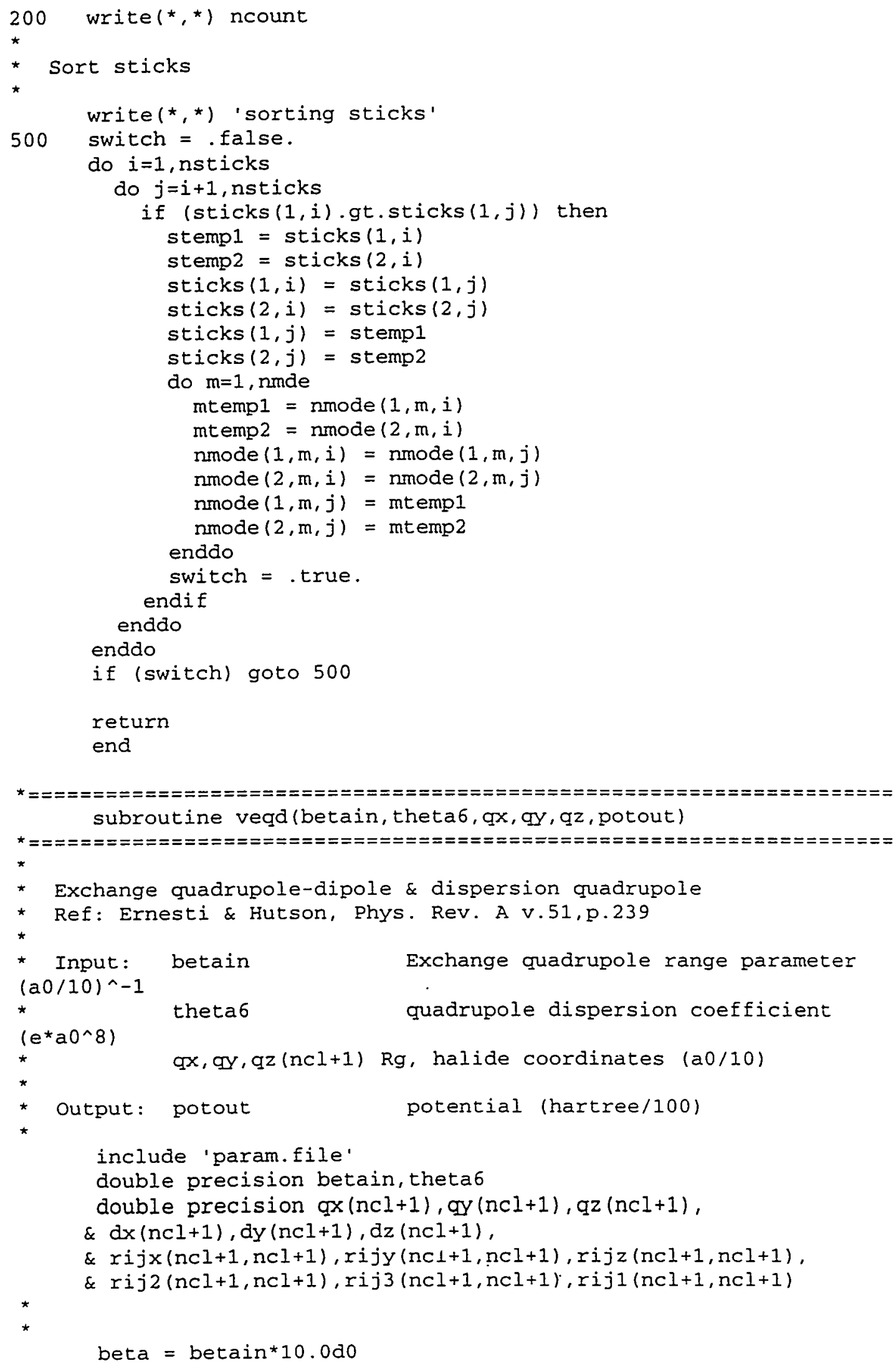




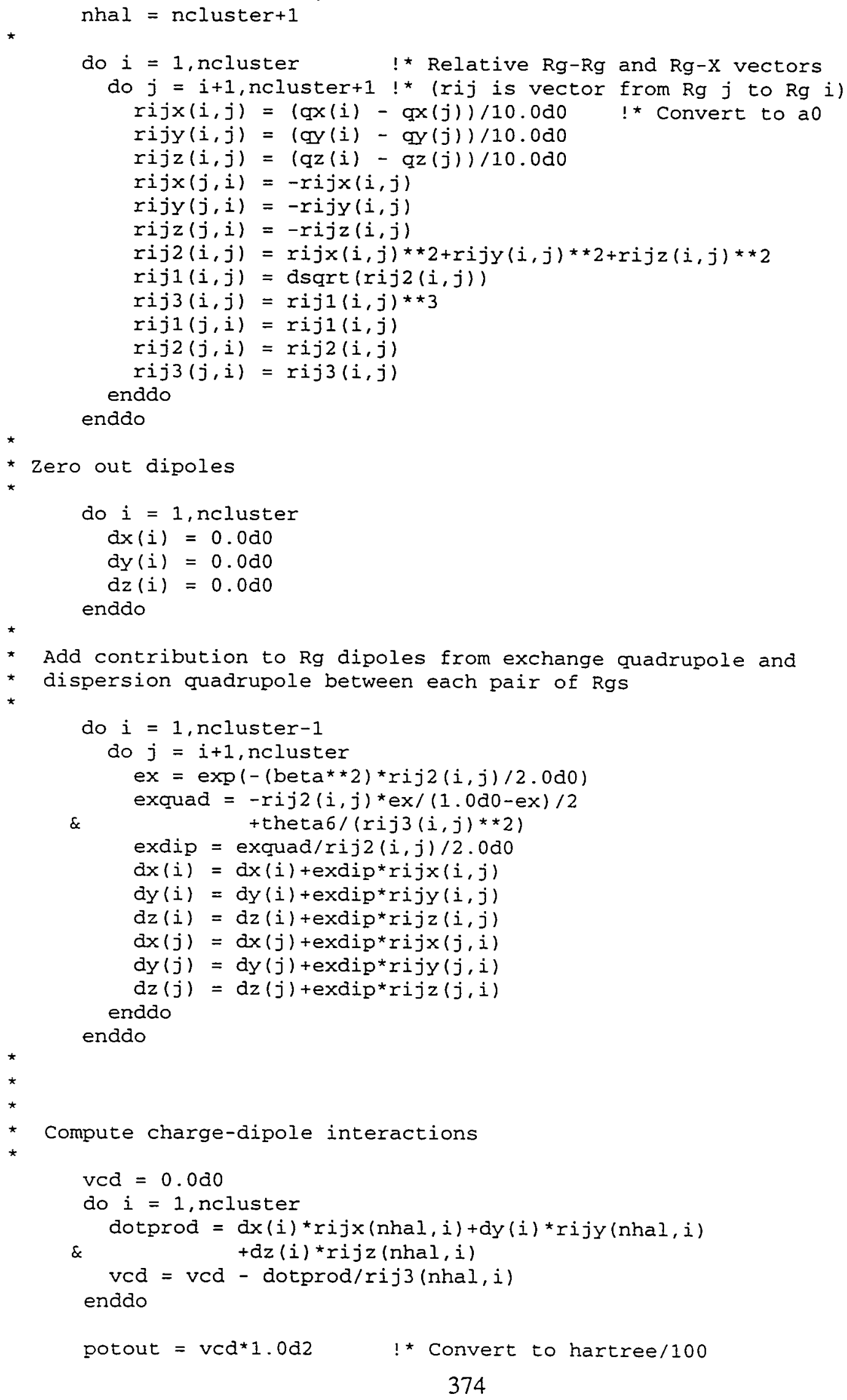


return

end

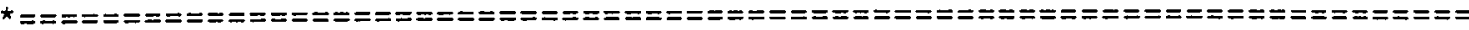
subroutine vinddq (polrg,polx,pqrg,pqx, qx, qy, qz, dp, dhalpair,

\& drgpair, qhalpair, qrgpair, qp, vcd, vcq, potout)

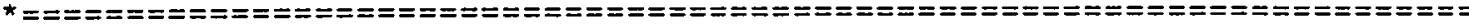

See Section C5.7 (file "pforce.f") for a complete listing of this subroutine

* subroutine exg (betain, th6, qx, $q y, q z$, vexx, vddisp, vqdisp, potout)

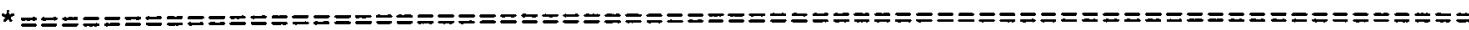

See Section C5.7 (file "pforce.f") for a complete listing of this subroutine

* double precision FUNCTION ERF(xin)

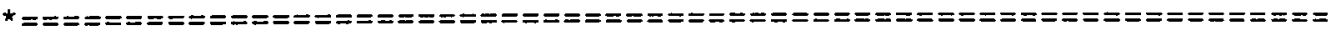

See Section C5.7 (file "pforce.f") for a complete listing of this subroutine

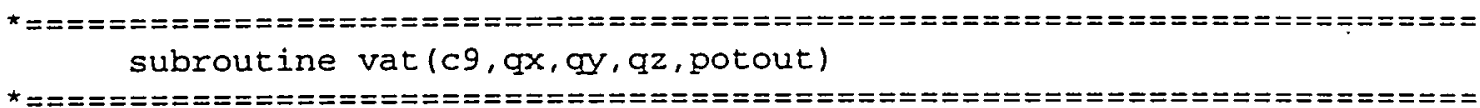

See Section C5.7 (file "pforce.f") for a complete listing of this subroutine 


\section{C7. References for Appendix C}

1 F. H. Stillinger and D. K. Stillinger, J. Chem. Phys. 93, 6106 (1990).

2 F. H. Stillinger and T. A. Weber, Phys. Rev. A 28, 2408 (1983).

3 W. H. Press, S. A. Teukolsky, W. T. Vetterling, and B. P. Flannery, Numerical Recipes, 2nd ed. (Cambridge University Press, Cambridge, 1992).

4 P. R. Bevington, Data Reduction and Error Analysis for the Physical Sciences (McGraw-Hill, New York, 1969).

5 S. Kirkpatrick, C. D. Gelatt, and M. P. Vecchi, Science 220,671 (1983).

6 N. Metropolis, A. W. Rosenbluth, M. N. Rosenbluth, A. H. Teller, and E. Teller, J. Chem. Phys. 21, 1087 (1953).

7 D. Vanderbilt and S. G. Louie, J. Comput. Phys. 56, 259 (1984).

8 I. M. Navon, F. B. Brown, and D. H. Robertson, Computers Chem. 14, 305 (1990).

9 M. P. Allen and D. J. Tildesley, Computer Simulation of Liquids (Clarendon Press, Oxford, 1987).

10 Z. Li, A. Borrmann, and C. C. Martens, J. Chem. Phys. 97 (1992).

11 R. A. Aziz and M. J. Slaman, Mol. Phys. 58, 679 (1986).

12 E. L. Pollock and B. J. Alder, Phys. Rev. Lett. 41, 903 (1978).

13 P. Jedlovszky and G. Palinkas, Mol. Phys. 84, 217 (1995).

14 R. L. Asher, D. A. Micha, and P. J. Brucat, J. Chem. Phys. 96, 9683 (1992).

15 A. D. Buckingham, Adv. Chem. Phys. 12, 107 (1967).

16 A. Ernesti and J. M. Hutson, Phys. Rev. A 51, 239 (1995). 
17 The EISPACK source code may be obtained by email from netlib@research.att.com or by FTP from the server "research.att.com" using the login name "netlib."

18 R. B. Metz, Ph.D., University of California, 1991. 